\title{
NONABELIAN HODGE THEORY IN CHARACTERISTIC $p$
}

\author{
by A. OGUS and $\mathrm{V}$. VOLOGODSKY
}

\begin{abstract}
Given a scheme in characteristic $p$ together with a lifting modulo $p^{2}$, we construct a functor from a category of suitably nilpotent modules with connection to the category of Higgs modules. We use this functor to generalize the decomposition theorem of Deligne-Illusie to the case of de Rham cohomology with coefficients.
\end{abstract}

\section{CONTENTS}

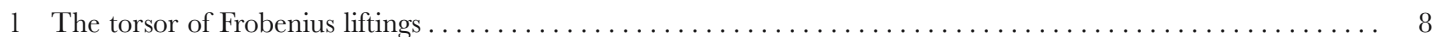

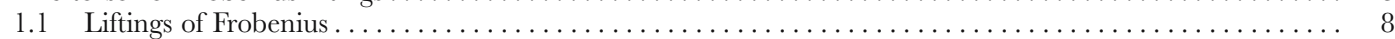

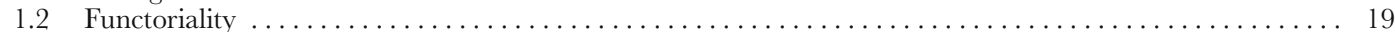

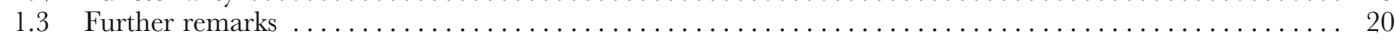

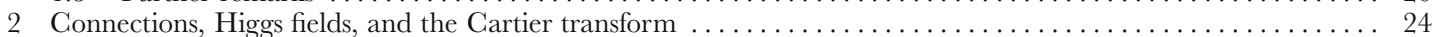

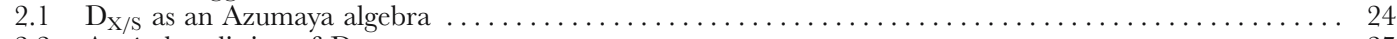

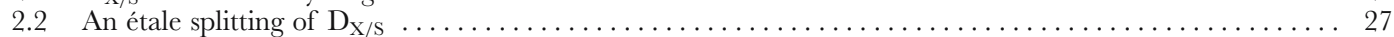

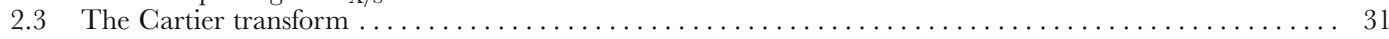

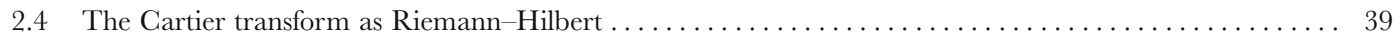

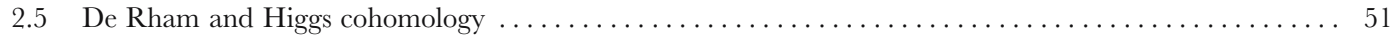

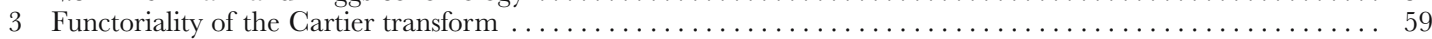

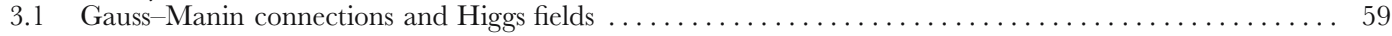

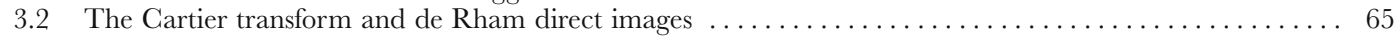

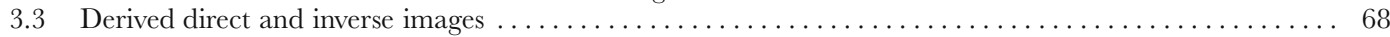

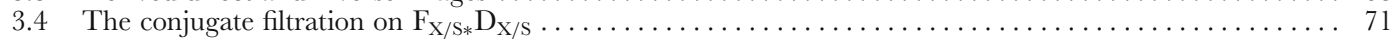

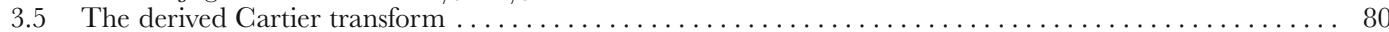

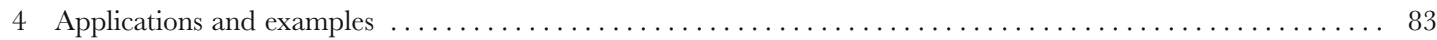

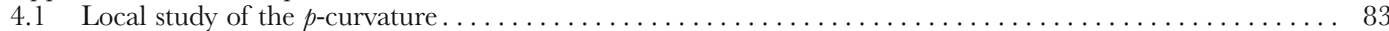

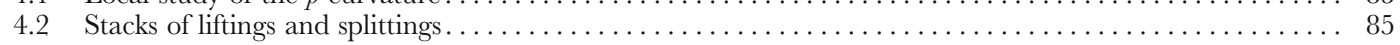

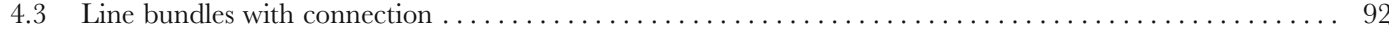

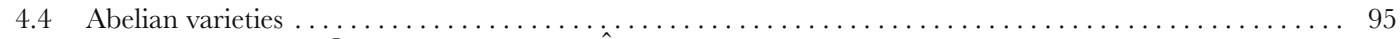

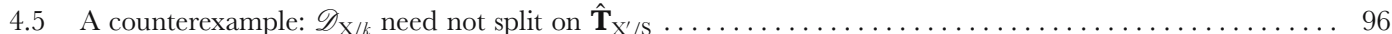

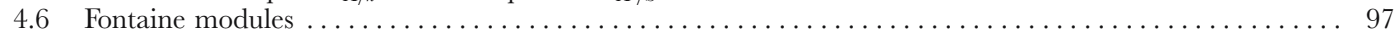

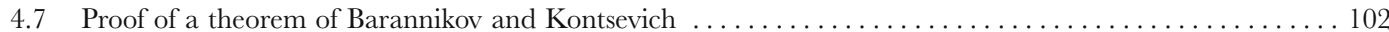

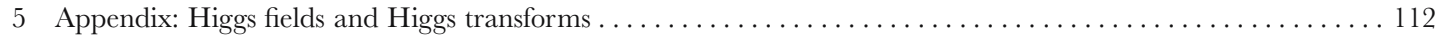

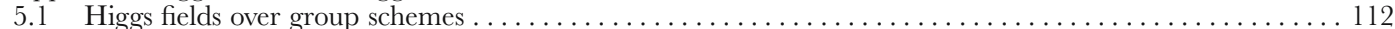

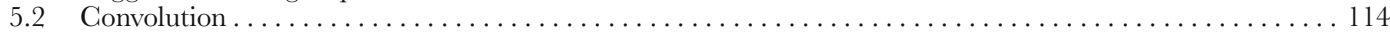

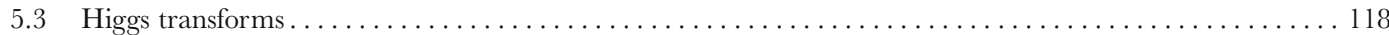

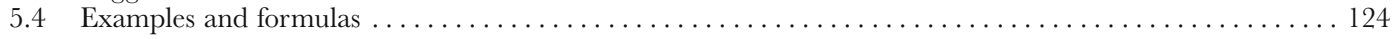

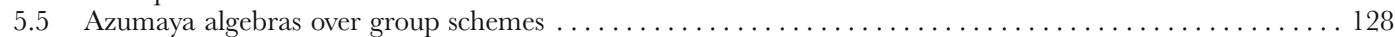

\section{Introduction}

Let $\mathrm{X} / \mathbf{G}$ be a smooth projective scheme over the complex numbers and let $\mathrm{X}^{a n}$ be the associated analytic space. Classical Hodge theory provides a canonical isomorphism:

$(\mathbf{0 . 0 . 1})$

$$
\mathrm{H}^{n}\left(\mathrm{X}, \Omega_{\mathrm{X} / \mathbf{G}}\right) \cong \mathrm{H}^{n}\left(\mathrm{X}^{a n}, \mathbf{C}\right) \cong \bigoplus_{i+j=n} \mathrm{H}^{i}\left(\mathrm{X}, \Omega_{\mathrm{X} / \mathbf{G}}^{j}\right)
$$


Carlos Simpson's "nonabelian Hodge theory" [36] provides a generalization of this decomposition to the case of cohomology with coefficients in a representation of the fundamental group of $\mathrm{X}^{a n}$. By the classical Riemann-Hilbert correspondence, such a representation can be viewed as a locally free sheaf $\mathrm{E}$ with integrable connection $(\mathrm{E}, \nabla)$ on $\mathrm{X}$. If $(\mathrm{E}, \nabla)$ satisfies suitable conditions, Simpson associates to it a Higgs bundle $\left(\mathrm{E}^{\prime}, \theta\right)$, i.e., a locally free sheaf $\mathrm{E}^{\prime}$ together with an $\mathscr{O}_{\mathrm{X}}$-linear map $\theta: \mathrm{E}^{\prime} \rightarrow \mathrm{E}^{\prime} \otimes \Omega_{\mathrm{X} / \mathbf{C}}^{1}$ such that $\theta \wedge \theta: \mathrm{E}^{\prime} \rightarrow \mathrm{E}^{\prime} \otimes \Omega_{\mathrm{X} / \mathbf{C}}^{2}$ vanishes. This integrability implies that the iterates of $\theta$ are zero, so that $\theta$ fits into a complex (the Higgs complex)

$$
\mathrm{E}^{\prime} \otimes \Omega_{\mathrm{X} / \mathbf{G}}^{\cdot}:=\mathrm{E}^{\prime} \rightarrow \mathrm{E}^{\prime} \otimes \Omega_{\mathrm{X} / \mathbf{G}}^{1} \rightarrow \mathrm{E}^{\prime} \otimes \Omega_{\mathrm{X} / \mathbf{G}}^{2} \cdots
$$

As a substitute for the Hodge decomposition (0.0.1), Simpson constructs a natural isomorphism:

$$
\mathrm{H}^{n}\left(\mathrm{X}, \mathrm{E} \otimes \Omega_{\mathrm{X} / \mathbf{C}}^{\cdot}, d\right) \cong \mathrm{H}^{n}\left(\mathrm{X}^{a n}, \mathrm{~V}\right) \cong \mathrm{H}^{n}\left(\mathrm{X}, \mathrm{E}^{\prime} \otimes \Omega_{\mathrm{X} / \mathbf{G}}^{\cdot}, \theta\right) .
$$

In general, there is no simple relation between $\mathrm{E}$ and $\mathrm{E}^{\prime}$, and in fact the correspondence $\mathrm{E} \mapsto \mathrm{E}^{\prime}$ is not holomorphic.

Our goal in this work is to suggest and investigate an analog of Simpson's theory for integrable connections in positive characteristics, as well as an extension of the paper [8] of Deligne and Illusie to the case of de Rham cohomology for modules with an integrable connection. Let $\mathrm{X}$ be a smooth scheme over the spectrum $\mathrm{S}$ of a perfect field $k$, and let $\mathrm{F}: \mathrm{X} \rightarrow \mathrm{X}^{\prime}$ be the relative Frobenius map. Assume as in [8] that there is a lifting $\tilde{\mathrm{X}}$ of $\mathrm{X}^{\prime}$ to $\mathrm{W}_{2}(k)$. Our main result is the construction of a functor $\mathrm{C}_{\tilde{\mathrm{X}}}$ (the Cartier transform) from the category $\mathrm{MIC}(\mathrm{X} / \mathrm{S})$ of modules with integrable connection on $\mathrm{X}$ to the category $\mathrm{HIG}\left(\mathrm{X}^{\prime} / \mathrm{S}\right)$ of Higgs modules on $\mathrm{X}^{\prime} / \mathrm{S}$, each subject to suitable nilpotence conditions.

The relative Frobenius morphism $\mathrm{F}$ and the $p$-curvature

$$
\psi: \mathrm{E} \rightarrow \mathrm{E} \otimes \mathrm{F}^{*} \Omega_{\mathrm{X}^{\prime}}^{1}
$$

of a module with integrable connection $(\mathrm{E}, \nabla)$ play a crucial role in the study of connections in characteristic $p$. A connection $\nabla$ on a sheaf of $\mathscr{O}_{\mathrm{X}}$-modules $\mathrm{E}$ can be viewed as an action of the sheaf $\mathrm{D}_{\mathrm{X}}$ of $\mathrm{PD}$-differential operators $[3,(4.4)]^{1}$ on $\mathrm{X}$. This sheaf of rings has a large center $\mathscr{Z}_{\mathrm{X}}$ : in fact, $\mathrm{F}_{*} \mathscr{Z}_{\mathrm{X}}$ is canonically isomorphic to the sheaf of functions on the cotangent bundle $\mathbf{T}_{\mathrm{X}^{\prime}}^{*}$ :

$$
c: \mathrm{S}^{\cdot} \mathrm{T}_{\mathrm{X}^{\prime}} \cong \mathrm{F}_{*} \mathscr{Z}_{\mathrm{X}}
$$

and $\mathrm{F}_{*} \mathrm{D}_{\mathrm{X}}$ is an Azumaya algebra over $\mathrm{S}^{\circ} \mathrm{T}_{\mathrm{X}^{\prime}}$ [4]. The map $c$ takes a vector field $\xi$ (i.e., a derivation of $\mathscr{O}_{\mathrm{X}}$ ) to $\xi^{p}-\xi^{(p)} \in \mathrm{D}_{\mathrm{X}}$, where $\xi^{(p)} \in \operatorname{Der}\left(\mathscr{O}_{\mathrm{X}}\right)$ is the $p$ th iterate of $\xi$

\footnotetext{
1 The name "differential operators" is perhaps misleading: although $\mathrm{D}_{\mathrm{X}}$ acts on $\mathscr{O}_{\mathrm{X}}$, the map $\mathrm{D}_{\mathrm{X}} \rightarrow \operatorname{End}\left(\mathscr{O}_{\mathrm{X}}\right)$ is not injective.
} 
and $\xi^{p}$ is the $p$ th power of $\xi$ in $\mathrm{D}_{\mathrm{X}}$. If $\nabla$ is an integrable connection on $\mathrm{E}$, then by definition $\psi_{\xi}$ is the $\mathscr{O}_{\mathrm{X}}$-linear endomorphism of $\mathrm{E}$ given by the action of $\nabla_{c(\xi)}$.

Let $\tilde{\mathrm{X}}$ be a lifting of $\mathrm{X}$. Our construction of the Cartier transform $\mathrm{C}_{\tilde{\mathrm{X}}}$ is based on a study of the sheaf of liftings of the relative Frobenius morphism $\mathrm{F}: \mathrm{X} \rightarrow \mathrm{X}^{\prime}$. For each open subset $\mathrm{U} \subseteq \mathrm{X}$, the set $\mathscr{L}_{\tilde{\mathrm{X}}}(\mathrm{U})$ of all Frobenius liftings $\tilde{\mathrm{F}}: \tilde{\mathrm{U}} \rightarrow \tilde{\mathrm{U}}^{\prime}$ is naturally a torsor under the group $\mathrm{F}^{*} \mathrm{~T}_{\mathrm{X}^{\prime}}$. Key to our construction is the fact that the $\mathrm{F}^{*} \mathrm{~T}_{\mathrm{X}^{\prime}}$-torsor $q: \mathscr{L}_{\tilde{\mathrm{X}}} \rightarrow \mathrm{X}$ has a canonical connection

$$
\nabla: \mathscr{L}_{\tilde{\mathrm{X}}} \rightarrow \mathrm{F}^{*} \mathrm{~T}_{\mathrm{X}^{\prime}} \otimes \Omega_{\mathrm{X}}^{1}
$$

compatible with the Frobenius descent connection on the vector bundle $\mathrm{F}^{*} \mathrm{~T}_{\mathrm{X}^{\prime}}$. If $\tilde{\mathrm{F}}$ is a local section of $\mathscr{L}_{\tilde{\mathrm{X}}}, \nabla(\tilde{\mathrm{F}}) \in \mathscr{H} 0 m\left(\mathrm{~F}^{*} \Omega_{\mathrm{X}^{\prime}}^{1}, \Omega_{\mathrm{X}}^{1}\right)$ is given by

$$
\zeta_{\tilde{\mathrm{F}}}: \mathrm{F}^{*} \Omega_{\mathrm{X}^{\prime}}^{1} \rightarrow \Omega_{\mathrm{X}}^{1}
$$

where $\zeta_{\tilde{\mathrm{F}}}:=p^{-1} d \tilde{\mathrm{F}}$ is the lifting of the inverse Cartier operator defined by $\tilde{\mathrm{F}}$. Thus the sheaf of functions $\mathscr{A}_{\tilde{\mathrm{X}}}:=q_{*} \mathscr{O}_{\mathscr{L}_{\tilde{\mathrm{X}}}}$ acquires a connection, as does its $\mathscr{O}_{\mathrm{X}}$-linear dual $\mathscr{B}_{\tilde{\mathrm{X}}}$. The torsor structure on $\mathscr{L}_{\tilde{\mathrm{X}}}$ induces an action of the completed PD symmetric algebra $\hat{\Gamma} \cdot \mathrm{F}^{*} \mathrm{~T}_{\mathrm{X}^{\prime}}$ on $\mathscr{A}_{\tilde{\mathrm{X}}}$ and $\mathscr{B}_{\tilde{\mathrm{X}}}$. We show that the induced action of $\mathrm{S}^{-} \mathrm{T}_{\mathrm{X}^{\prime}}$ coincides with the action of the center $\mathrm{S}^{*} \mathrm{~T}_{\mathrm{X}^{\prime}} \subset \mathrm{D}_{\mathrm{X}}$ defined by the $p$-curvature of the connection $\nabla$. Thus $\mathscr{B}_{\tilde{\mathrm{X}}}$ becomes a module over the algebra $\mathrm{D}_{\mathrm{X}}^{\gamma}:=\mathrm{D}_{\mathrm{X}} \otimes_{\mathrm{S}^{\bullet} \mathrm{T}_{\mathrm{X}}} \hat{\Gamma} \cdot \mathrm{T}_{\mathrm{X}^{\prime}}$.

We define the Cartier transform $\mathrm{C}_{\tilde{\mathrm{X}}}$ from the category of $\mathrm{D}_{\mathrm{X}}^{\gamma}$-modules to the category of $\hat{\Gamma} \cdot \mathrm{T}_{\mathrm{X}^{\prime}}$-modules by the formula:

$$
\mathrm{C}_{\tilde{\mathrm{X}}}(\mathrm{E})=\iota_{*} \mathscr{H} 0 m_{\mathrm{D}_{\mathrm{X}}^{\gamma}}\left(\mathscr{B}_{\tilde{\mathrm{X}}}, \mathrm{E}\right)
$$

where $\iota$ is the involution of $\mathrm{T}_{\mathrm{X}^{\prime}}$ sending $\xi^{\prime}$ to $-\xi^{\prime} .^{2}$ In fact, $\mathscr{B}_{\tilde{\mathrm{X}}}$ is a splitting module for the Azumaya algebra $\mathrm{D}_{\mathrm{X}}^{\gamma}$, and from this point of view, the Cartier transform is, up to the twist by $\iota$, just the equivalence of categories between the category of modules over a split Azumaya algebra and the category of $\mathscr{O}$-modules on the underlying space defined by the choice of a splitting module. In particular, the Cartier transform gives an equivalence between the category $\mathrm{MIC}_{p-1}(\mathrm{X})$ of nilpotent $\mathrm{D}$-modules of level less then or equal to $p-1$ and the category $\mathrm{HIG}_{p-1}\left(\mathrm{X}^{\prime}\right)$ of Higgs modules supported on the $(p-1)^{s t}$ infinitesimal neighborhood of the zero section $\mathrm{X}^{\prime} \hookrightarrow \mathbf{T}_{\mathrm{X}^{\prime}}^{*}$. The larger categories of locally nilpotent $\mathrm{D}_{\mathrm{X}}^{\gamma}$-modules and $\hat{\Gamma} \cdot \mathrm{T}_{\mathrm{X}^{\prime}}$-modules have the advantage of being tensor categories, and the Cartier transform is in fact compatible with the tensor structures.

We also obtain an analog of Simpson's isomorphism (0.0.2): if $\left(\mathbf{E}^{\prime}, \theta^{\prime}\right)$ is the Cartier transform of a module with connection $(\mathrm{E}, \nabla)$ whose level is less than the

2 The role of the involution $\iota$ is to insure that our constructions are compatible with the standard Cartier operator and with the decomposition of the de Rham complex constructed by Deligne and Illusie [8]. 
$p$ minus the dimension of $\mathrm{X}$, then we construct an isomorphism in the derived category between the de Rham complex of $(E, \nabla)$ and the Higgs complex of $\left(E^{\prime}, \theta^{\prime}\right)$. This result generalizes the decomposition theorem of Deligne-Illusie [8].

Let us describe the structure and content of the paper in more detail. We work with a smooth morphism $\mathrm{X} / \mathrm{S}$ of schemes in characteristic $p$. We shall see that the Cartier transform depends on a lifting $\tilde{\mathrm{X}}^{\prime} / \tilde{\mathrm{S}}$ of $\mathrm{X}^{\prime} / \mathrm{S}$ modulo $p^{2}$ rather than a lifting of $\mathrm{X} / \mathrm{S}$, and we write $\mathscr{X} / \mathscr{S}$ for the pair $\left(\mathrm{X} / \mathrm{S}, \tilde{\mathrm{X}}^{\prime} / \tilde{\mathrm{S}}\right)$. In Theorem 1.1 of Section 1.1 we construct the torsor $\mathscr{L}_{\mathscr{X} / \mathscr{S}}$ of liftings of Frobenius and compute its connection in Proposition 1.10 and its p-curvature in Proposition 1.5, using the geometric language of the crystalline site and in particular Mochizuki's geometric description of the $p$-curvature, which we recall in Proposition 1.7. We also discuss in Section 1.3 the relationship between $\mathscr{A}_{\mathscr{X} / \mathscr{S}}$ and some more familiar constructions in the literature.

Section 2 is devoted to the construction of the Cartier transform. We begin by reviewing in Theorem 2.1 the Azumaya property of the algebra of differential operators and the canonical fppf splitting module described in [4]. Then we discuss the global Cartier transform $\mathrm{C}_{\mathscr{X} / \mathscr{S}}$ as well as a local version which depends on a lifting $\tilde{\mathrm{F}}$ of the relative Frobenius morphism $\mathrm{F}_{\mathrm{X} / \mathrm{S}}$. Theorem 2.7 constructs from such a lifting $\tilde{\mathrm{F}}$, or just the corresponding splitting $\zeta$ of the inverse Cartier operator, a surjective étale endomorphism $\alpha_{\zeta}$ of $\mathbf{T}_{\mathrm{X}^{\prime}}^{*}$ and a splitting module $\mathscr{B}_{\zeta}$ of $\alpha_{\zeta}^{*} \mathrm{D}_{\mathrm{X} / \mathrm{S}}$. The restriction $\hat{\mathscr{B}}_{\zeta}$ of $\mathscr{B}_{\zeta}$ to the formal completion of $\mathbf{T}_{\mathrm{X}^{\prime}}^{*}$ along its zero section splits the ring $\hat{\mathrm{D}}_{\mathrm{X} / \mathrm{S}}:=\mathrm{D}_{\mathrm{X} / \mathrm{S}} \otimes_{\mathrm{S}^{\cdot}} \cdot \mathrm{T}_{\mathrm{X}^{\prime} / \mathrm{S}} \hat{\mathrm{S}}^{\cdot} \mathrm{T}_{\mathrm{X}^{\prime} / \mathrm{S}}$ of HPD differential operators, and this splitting module defines an equivalence, which we call in Theorem 2.11 the local Cartier transform, between the category of modules over $\hat{\mathrm{D}}_{\mathrm{X} / \mathrm{S}}$ and the category of modules over the ring $\hat{\mathrm{S}}^{\cdot} \mathrm{T}_{\mathrm{X}^{\prime} / \mathrm{S}}$. In fact, $\hat{\mathscr{B}}_{\zeta}$ is naturally isomorphic to the dual of the divided power envelope of $\mathscr{A}_{\tilde{\mathrm{X}}}$ along the ideal of the section of $\mathscr{L}_{\mathscr{X} / \mathscr{S}}$ defined by $\tilde{\mathrm{F}}$. This gives the compatibility between the local and global Cartier transforms.

In Theorem 2.23 we explain how the Cartier transform can be viewed as an analog of the Riemann-Hilbert correspondence, with the sheaf of $\mathscr{O}_{\mathrm{X}}$-algebras $\mathscr{A}_{\mathscr{X} / \mathscr{S}}$ playing the role of $\mathscr{O}_{\mathrm{X}^{a n}}$. We also discuss a filtered version of the construction, in which we study filtered $\mathrm{D}_{\mathrm{X} / \mathrm{S}}^{\gamma}$-modules $(\mathrm{E}, \mathrm{N}$.), where $\mathrm{N}$. is a filtration on $\mathrm{E}$ such that

$$
\left(\Gamma_{j} \mathrm{~T}_{\mathrm{X}^{\prime} / \mathrm{S}}\right) \mathrm{N}_{k} \mathrm{E} \subseteq \mathrm{N}_{k-j} \mathrm{E}
$$

for all $k$ and $j$. The algebra $\mathscr{A}_{\mathscr{X} / \mathscr{S}}$ has a canonical filtration with this property, and we show that the filtered object $\mathrm{C}_{\mathscr{X} / \mathscr{S}}(\mathrm{E}, \mathrm{N}$.), can be computed from the tensor product filtration on $\mathscr{A}_{\mathscr{X} / \mathscr{S}} \otimes \mathrm{E}$, which again satisfies (0.0.4). This construction will become important in our analog, Theorem 2.26, of the cohomological theorems of Simpson and Deligne-Illusie and in particular to our study of the "conjugate filtration" in cohomology.

Section 3 investigates the compatibility of the Cartier transform with direct and inverse images with respect to a morphism of smooth $\mathrm{S}$-schemes $h: \mathrm{X} \rightarrow \mathrm{Y}$. We begin 
with a review of the construction of the Gauss Manin connection on the relative de Rham cohomology $\mathrm{R}^{q} h_{*}\left(\mathrm{E} \otimes \Omega_{\mathrm{X} / \mathrm{Y}}^{\cdot}\right)$ when $h$ is smooth and discuss its analog for Higgs fields. Our review culminates with Theorem 3.4, which shows that $\mathrm{R}^{q} h_{*}$ increases the level of nilpotence of a connection by at most the relative dimension $d$ of $h$, strengthening the result $[18,5.10]$ of Katz. In particular, we show that if $\mathrm{N}$. is a filtration of $\mathrm{E}$ such that $\mathrm{Gr}^{\mathrm{N}} \mathrm{E}$ has zero $p$-curvature, then the filtration of $\mathrm{R}^{q} h_{*}\left(\mathrm{E} \otimes \Omega_{\mathrm{X} / \mathrm{Y}}^{*}\right)$ induced by Deligne's "filtration décalée" $\mathrm{N}^{\text {dec }}$ of $\mathrm{E} \otimes \Omega_{\mathrm{X} / \mathrm{Y}}^{\cdot}$ has the same property. Theorem 3.8 shows that the Cartier transform is compatible with direct image by constructing, given a lifting $\tilde{h}^{\prime}$ of of $h^{\prime}: \mathrm{X}^{\prime} \rightarrow \mathrm{Y}^{\prime}$, an isomorphism in $\mathrm{HIG}\left(\mathrm{Y}^{\prime} / \mathrm{S}\right)$

$$
\mathrm{R}^{q} h_{*}^{\prime \mathrm{HIG}} \mathrm{C}_{\mathscr{X} / \mathscr{S}} \mathrm{E} \cong \mathrm{C}_{\mathscr{Y} / \mathscr{S}} \mathrm{R}^{q} h_{*}^{\mathrm{DR}} \mathrm{E}
$$

if the level of $\mathrm{E}$ is less than $p-d$; we also show that this construction is compatible with the filtrations $\mathrm{N}^{\text {dec }}$. This result can be regarded as a relative version of the cohomology comparison Theorem 2.26.

The remainder of Section 3 is devoted to derived versions of these results in a certain filtered derived category of $\mathrm{D}_{\mathrm{X} / \mathrm{S}}$-modules. The first important ingredient of this approach is a new construction, described in Proposition 3.12, of the functors $\mathrm{L} h_{\mathrm{DR}}^{*}$ and $\mathrm{R} h_{*}^{\mathrm{DR}}$ in characteristic $p$, due to Bezrukavnikov and Braverman [5], based on the Azumaya property of the algebra $\mathrm{F}_{\mathrm{X} / \mathrm{S} *} \mathrm{D}_{\mathrm{X} / \mathrm{S}}$. This construction allows us to work locally over the cotangent bundle. Another ingredient is the conjugate filtration

$$
\begin{aligned}
& \cdots \subset \mathscr{I}_{\mathrm{X}}^{i} \subset \cdots \subset \mathscr{I}_{\mathrm{X}}^{1} \subset \mathrm{F}_{\mathrm{X} / \mathrm{S} *} \mathrm{D}_{\mathrm{X} / \mathrm{S}}, \\
& \text { where } \mathscr{I}_{\mathrm{X}}^{i}=\mathrm{S}^{i} \mathrm{~T}_{\mathrm{X}^{\prime} / \mathrm{S}}\left(\mathrm{F}_{\mathrm{X} / \mathrm{S} *} \mathrm{D}_{\mathrm{X} / \mathrm{S}}\right)
\end{aligned}
$$

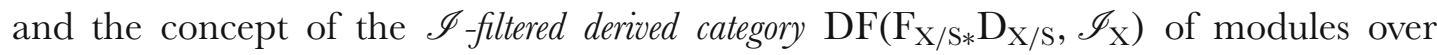
the filtered algebra $\mathrm{F}_{\mathrm{X} / \mathrm{S} *} \mathrm{D}_{\mathrm{X} / \mathrm{S}}$. Objects of this category are filtered complexes $\left(\mathrm{E}^{*}, \mathrm{~N}^{*}\right)$ of $\mathrm{F}_{\mathrm{X} / \mathrm{S} *} \mathrm{D}_{\mathrm{X} / \mathrm{S}}$-modules such that for every integer $i$

$$
\mathscr{I}_{\mathrm{X}} \mathrm{N}^{i} \mathrm{E}^{\cdot} \subset \mathrm{N}^{i+1} \mathrm{E}^{\cdot}
$$

or equivalently, such that the associated graded module has vanishing $p$-curvature. We lift the functors $\mathrm{R} h_{*}^{\mathrm{DR}}$ and $\mathrm{L} h_{\mathrm{DR}}^{*}$ to functors between the $\mathscr{I}$-filtered derived categories and prove in Proposition 3.16 that, for a smooth morphism $h: \mathrm{X} \rightarrow \mathrm{Y}$ of relative dimension $d$, the functor $\mathrm{R} h_{*}^{\mathrm{DR}}$ increases the range of the $\mathscr{I}$-filtration at most by $d$ :

$$
\mathrm{R} h_{*}^{\mathrm{DR}}\left(\mathrm{DF}_{[k, l]}\left(\mathrm{F}_{\mathrm{X} / \mathrm{S} *} \mathrm{D}_{\mathrm{X} / \mathrm{S}}, \mathscr{I}_{\mathrm{X}}\right)\right) \subset \mathrm{DF}_{[k-d, l]}\left(\mathrm{F}_{\mathrm{Y} / \mathrm{S} *} \mathrm{D}_{\mathrm{Y} / \mathrm{S}}, \mathscr{I}_{\mathrm{Y}}\right) .
$$

A different filtered derived category of $\mathrm{D}_{\mathrm{X} / \mathrm{S}}$-modules was defined by Laumon in [20]. Instead of the conjugate filtration (0.0.6) he considers the order filtration

$$
\mathscr{O}_{\mathrm{X}}=\mathrm{D}_{\mathrm{X} / \mathrm{S}, 0} \subset \mathrm{D}_{\mathrm{X} / \mathrm{S}, 1} \subset \cdots \subset \mathrm{D}_{\mathrm{X} / \mathrm{S}, i} \subset \cdots \subset \mathrm{D}_{\mathrm{X} / \mathrm{S}}
$$


An object of Laumon's category $\mathrm{D}\left(\mathrm{MF}\left(\mathrm{D}_{\mathrm{X} / \mathrm{S}}\right)\right)$ is a complex $\mathrm{E}^{\cdot}$ of $\mathrm{D}_{\mathrm{X} / \mathrm{S}}$-modules together with a filtration

$$
\cdots \subset \mathrm{F}^{i} \mathrm{E}^{\cdot} \subset \mathrm{F}^{i-1} \mathrm{E}^{\cdot} \subset \cdots \subset \mathrm{E}^{\cdot}
$$

by $\mathscr{O}_{\mathrm{X}}$-submodules satisfying Griffiths transversality:

$$
\mathrm{D}_{\mathrm{X} / \mathrm{S}, i}\left(\mathrm{~F}^{j} \mathrm{E}^{k}\right) \subset \mathrm{F}^{j-i} \mathrm{E}^{k}
$$

It is shown in [20] how the functors $\mathrm{R} h_{*}^{\mathrm{DR}}$ and $\mathrm{L} h_{\mathrm{DR}}^{*}$ lift to functors between Laumon's filtered derived categories. Laumon's construction makes sense over any base S, not necessarily of characteristic $p$.

We observe in Section 3.4 that the graded Azumaya algebra $\mathrm{Gr}_{\mathscr{I}} \mathrm{F}_{\mathrm{X} / \mathrm{S} *} \mathrm{D}_{\mathrm{X} / \mathrm{S}}$ has a canonical splitting which then defines an equivalence of categories

$$
\mathrm{C}_{\mathrm{X} / \mathrm{S}}^{\cdot}: \mathrm{D}\left(\operatorname{Mod}^{\bullet}\left(\mathrm{Gr}_{\mathscr{I}_{\mathrm{X}}} \mathrm{F}_{\mathrm{X} / \mathrm{S} *} \mathrm{D}_{\mathrm{X} / \mathrm{S}}\right)\right) \cong \mathrm{D}\left(\mathrm{HIG}^{\bullet}\left(\mathrm{X}^{\prime} / \mathrm{S}\right)\right) \text {. }
$$

We explain in Remark 3.19 how this observation combined with the formalism of filtered derived categories leads to a generalization of Katz's formula [19, Theorem 3.2] relating the $p$-curvature to the Kodaira-Spencer mapping. Namely, for any smooth morphism $h$, we have canonical quasi-isomorphisms

$$
\begin{aligned}
& \mathrm{C}_{\mathrm{Y} / \mathrm{S}}^{\cdot} \operatorname{Gr}_{\mathrm{N}} \mathrm{R} h_{*}^{\mathrm{DR}}\left(\mathscr{O}_{\mathrm{X}}\right) \simeq \mathrm{R} h_{*}^{\prime \mathrm{HIG}}\left(\mathscr{O}_{\mathrm{X}^{\prime}}\right) \simeq \operatorname{Gr}_{\mathrm{F}} \mathrm{R}_{h_{*}^{\prime}}{ }^{\mathrm{DR}}\left(\mathscr{O}_{\mathrm{X}^{\prime}}\right), \text { where } \\
& \mathrm{DF}\left(\mathrm{F}_{\mathrm{Y} / \mathrm{S} *} \mathrm{D}_{\mathrm{Y} / \mathrm{S}}, \mathscr{I}_{\mathrm{Y}}\right) \stackrel{\mathrm{C}_{\mathrm{Y} / \mathrm{S}} \mathrm{GrN}_{\mathrm{N}}}{\longrightarrow} \mathrm{D}\left(\mathrm{HIG}^{\bullet}\left(\mathrm{Y}^{\prime} / \mathrm{S}\right)\right) \stackrel{\mathrm{Gr}_{\mathrm{F}}}{\longleftarrow} \mathrm{D}\left(\mathrm{MF}\left(\mathrm{D}_{\mathrm{Y}^{\prime} / \mathrm{S}}\right)\right) .
\end{aligned}
$$

(The second quasi-isomorphism is constructed in [20].)

In Section 3.5 we explain how, when $l-k<p$, the Cartier transform lifts to an equivalence of triangulated categories

$$
\mathrm{G}_{\mathscr{X} / \mathrm{S}}: \mathrm{DF}_{[k, l]}\left(\mathrm{F}_{\mathrm{X} / \mathrm{S} *} \mathrm{D}_{\mathrm{X} / \mathrm{S}}, \mathscr{I}_{\mathrm{X}}\right) \cong \mathrm{DF}_{[k, l]}\left(\mathrm{S}^{*} \mathrm{~T}_{\mathrm{X}^{\prime} / \mathrm{S}}, \mathscr{J}_{\mathrm{X}^{\prime}}\right)
$$

between the category $\operatorname{DF}_{[k, l]}\left(\mathrm{F}_{\mathrm{X} / \mathrm{S} *} \mathrm{D}_{\mathrm{X} / \mathrm{S}}, \mathscr{I}_{\mathrm{X}}\right)$ and the $\mathscr{J}$-filtered derived category $\mathrm{DF}_{[k, l]}\left(\mathrm{S}^{\cdot} \mathrm{T}_{\mathrm{X}^{\prime} / \mathrm{S}}, \mathscr{J}_{\mathrm{X}^{\prime}}\right)$ of Higgs modules, where $\mathscr{J}_{\mathrm{X}^{\prime}} \subset \mathrm{S}^{\cdot} \mathrm{T}_{\mathrm{X}^{\prime} / \mathrm{S}}$ is the ideal generated by $\mathrm{T}_{\mathrm{X}^{\prime} / \mathrm{S}}$. We then show in Theorem 3.22 that, for a smooth morphism $h: \mathrm{X} \rightarrow \mathrm{Y}$, a lifting $\tilde{h}^{\prime}: \tilde{\mathrm{X}}^{\prime} \rightarrow \tilde{\mathrm{Y}}^{\prime}$ induces a filtered quasi-isomorphism

$$
\mathrm{C}_{\mathscr{X} / \mathrm{S}} \circ \mathrm{R} h_{*}^{\mathrm{DR}} \cong \mathrm{R} h_{*}^{\prime \mathrm{HIG}} \circ \mathrm{C}_{\mathscr{X} / \mathrm{S}}, \quad \text { for } \quad l-k+d<p .
$$

The exposition of Sections 3.3-3.5 does not depend on Sections 3.1-3.2, which obtain many of the same results on the level of cohomology by more explicit methods.

Section 4 is devoted to applications and examples. First we give a characterization of the local étale essential image of the $p$-curvature functor from the category $\mathrm{MIC}(\mathrm{X} / \mathrm{S})$ to the category of F-Higgs sheaves. We show in Theorem 4.1 that if $\mathrm{E}$ is 
coherent and $\psi: \mathrm{E} \rightarrow \mathrm{E} \otimes \mathrm{F}_{\mathrm{X} / \mathrm{S}}^{*} \Omega_{\mathrm{X}^{\prime} / \mathrm{S}}^{1}$ is an F-Higgs field, then, étale locally on $\mathrm{X}$, $(\mathrm{E}, \psi)$ comes from a connection if and only if, étale locally, $(\mathrm{E}, \psi)$ descends to $\mathrm{X}^{\prime}$. This can be regarded as a nonabelian analog of the exact sequence [23, 4.14]

$$
0 \longrightarrow \mathscr{O}_{\mathrm{X}^{\prime}}^{*} \stackrel{\mathrm{F}_{\mathrm{X} / \mathrm{S}}^{*}}{\longrightarrow} \mathrm{F}_{\mathrm{X} / \mathrm{S} *} \mathscr{O}_{\mathrm{X}}^{*} \stackrel{d l o g}{\longrightarrow} \mathrm{F}_{\mathrm{X} / \mathrm{S} *} \mathrm{Z}_{\mathrm{X} / \mathrm{S}}^{1} \stackrel{\pi_{\mathrm{X} / \mathrm{s}}^{*}-\mathrm{C}_{\mathrm{X} / \mathrm{S}}}{\longrightarrow} \Omega_{\mathrm{X}^{\prime} / \mathrm{S}}^{1} \longrightarrow 0
$$

where $\mathrm{C}_{\mathrm{X} / \mathrm{S}}$ is the Cartier operator and $\pi_{\mathrm{X} / \mathrm{S}}: \mathrm{X}^{\prime} \rightarrow \mathrm{X}$ is $\mathrm{id}_{\mathrm{X}} \times \mathrm{F}_{\mathrm{S}}$. Next in Theorem 4.5 and Proposition 4.4 come a comparison of the gerbes of liftings of $\mathrm{X}^{\prime}$ and of splittings of $\mathrm{F}_{\mathrm{X} / \mathrm{S} *} \mathrm{D}_{\mathrm{X} / \mathrm{S}}^{\gamma}$ and a cohomological formula for the class of $\mathrm{F}_{\mathrm{X} / \mathrm{S} *} \mathrm{D}_{\mathrm{X} / \mathrm{S}}$ in the Brauer group. We prove in Theorem 4.14 that if $\mathrm{X}$ is an abelian variety, then $\mathrm{F}_{\mathrm{X} / \mathrm{S} *} \mathrm{D}_{\mathrm{X} / \mathrm{S}}$ always splits over the formal completion of the zero section of its cotangent bundle, and in Section 4.5 we construct an example of a liftable surface for which $\mathrm{F}_{\mathrm{X} / \mathrm{S} *} \mathrm{D}_{\mathrm{X} / \mathrm{S}}$ does not have this property. Section 4.6 contains a discussion of $p$-torsion Fontaine modules, especially as developed in [11] and [28], from the point of view of the Cartier transform. As an application, we give a reduction modulo $p$ proof of the semistability of the Higgs bundles arising from Kodaira-Spencer mappings. Finally, in Section 4.7, we show how our nonabelian Hodge theory can be used to give a reduction modulo $p$ proof of a celebrated recent theorem of Barannikov and Kontsevich, answering a question of Sabbah [34].

We conclude with an appendix devoted to generalities about Higgs fields, and in particular to the study of the tensor product structure on the category of Higgs modules. This structure can be viewed as convolution with respect to the additive group law on the cotangent space and makes sense when restricted to the formal and divided power completions of the zero section. The tensor category of Higgs modules has an internal Hom, and an object $\mathscr{B}$ of $\mathrm{HIG}(\mathrm{X})$ defines what we call a "Higgs transform" $\mathrm{E} \mapsto \mathscr{H}_{\mathrm{HIG}}(\mathscr{B}, \mathrm{E})$ from the category of Higgs modules to itself. Our key technical result is Proposition 5.16, which shows that the Higgs transform with respect to a character sheaf on the cotangent space defines (after a change of sign) an involutive autoequivalence of tensor categories. In the last part of the appendix we introduce, using $\mathrm{D}_{\mathrm{X} / \mathrm{S}}$ as a model, the notion of a tensor structure on an Azumaya algebra $\mathscr{A}$ over a group scheme. Such a structure makes the category of $\mathscr{A}$-modules a tensor category.

Both authors would like to express their gratitude to Roman Bezrukavnikov. The second author would like to say that he learned the main idea of this work from him: in particular, he explained that the ring of differential operators in characteristic $p$ is an Azumaya algebra over the cotangent bundle and suggested that it might split over a suitable infinitesimal neighborhood of the zero section. The first author was blocked from realizing his vision (based on [29]) of a nonabelian Hodge theory in positive characteristics until he learned of this insight. Numerous conversations with Roman also helped us to overcome many of the technical and conceptual difficulties we encountered in the course of the work. The authors also benefited greatly from Pierre 
Berthelot, who in particular explained to the first author years ago how a lifting of Frobenius makes $\hat{\mathrm{D}}_{\mathrm{X} / \mathrm{S}}$ into a matrix algebra. Special thanks go to the referee who pointed out a mistake in an early draft as well as a simplification in our argument which offered a way around it. This led us to the realization that we could greatly strengthen one of our main results and allowed us to develop the filtered Cartier transform in the context of cohomology and derived categories. We are also extremely grateful to the referee for pointing out an enormous number of misprints and ambiguities in an early draft. We would also like to thank Alexander Beilinson, Alexander Braverman, Luc Illusie, and Ofer Gabber for the interest they showed and the advice they provided. Finally, we would like to alert the reader to a forthcoming work by Daniel Schepler which extends this theory to log geometry. ${ }^{3}$

\section{The torsor of Frobenius liftings}

\subsection{Liftings of Frobenius}

If $\mathrm{X}$ is a scheme in characteristic $p$, let $\mathrm{F}_{\mathrm{X}}$ denote its absolute Frobenius endomorphism, i.e., the map which is the identity on the underlying topological space and which takes each section of $\mathscr{O}_{\mathrm{X}}$ to its $p$ th power. For any morphism $f: \mathrm{X} \rightarrow \mathrm{S}$ of schemes in characteristic $p, \mathrm{~F}_{\mathrm{S}} \circ f=f \circ \mathrm{F}_{\mathrm{X}}$, and one has the relative Frobenius diagram:

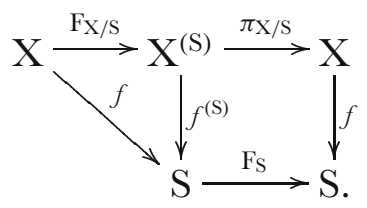

The square in this diagram is Cartesian, and the map $\mathrm{F}_{\mathrm{X} / \mathrm{S}}$ is the unique morphism over $\mathrm{S}$ such that $\pi_{\mathrm{X} / \mathrm{S}} \circ \mathrm{F}_{\mathrm{X} / \mathrm{S}}=\mathrm{F}_{\mathrm{X}}$. If no confusion seems likely to result, we may simplify the notation, writing $\mathrm{X}^{\prime}$ for $\mathrm{X}^{(\mathrm{S})}, \mathrm{F}$ for $\mathrm{F}_{\mathrm{X} / \mathrm{S}}$, etc. We also often write $\mathrm{X} / \mathrm{S}$ for the morphism $f: \mathrm{X} \rightarrow \mathrm{S}$, viewed as an S-scheme.

If $f: \mathrm{X} \rightarrow \mathrm{S}$ is any morphism of schemes in characteristic $p>0$ and $n$ is a positive integer, by a lifting of $f$ modulo $p^{n}$ we shall mean a morphism $\tilde{f}: \tilde{\mathrm{X}} \rightarrow \tilde{\mathrm{S}}$ of flat $\mathbf{Z} / p^{n} \mathbf{Z}$-schemes, together with a Cartesian diagram

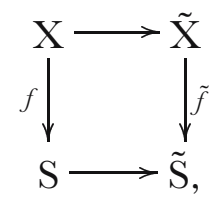

${ }^{3}$ Both authors would like to acknowledge the support this collaboration received from the Committee on Research at the University of California at Berkeley. The second author was partially supported by NSF grant DMS-0401164, but support for the team effort was denied by the National Science Foundation and the Miller Institute for Basic Research. 
where $\mathrm{S} \rightarrow \tilde{\mathrm{S}}$ is the closed subscheme defined by $p$. Note that if $\tilde{\mathrm{X}} / \tilde{\mathrm{S}}$ is such a lifting and $\mathrm{X} / \mathrm{S}$ is flat (resp. smooth), then so is $\tilde{\mathrm{X}} / \tilde{\mathrm{S}}$. We shall be primarily interested in the case $n=2$, and if $n$ is not specified, this is what we shall mean. If the absolute Frobenius endomorphism $\mathrm{F}_{\mathrm{S}}$ lifts to $\tilde{\mathrm{S}}$, then $\tilde{f}^{\prime}: \tilde{\mathrm{X}} \times_{\mathrm{F}_{\tilde{\mathrm{S}}}} \tilde{\mathrm{S}} \rightarrow \tilde{\mathrm{S}}$ lifts $\mathrm{X}^{\prime} / \mathrm{S}$. For example, if $\mathrm{S}$ is the spectrum of a perfect field $k$ and $\tilde{\mathrm{S}}$ the spectrum of its truncated Witt ring, then there is a unique such $\mathrm{F}_{\tilde{\mathrm{S}}}$, but in general there is no reason for a lifting of $\mathrm{F}_{\tilde{\mathrm{S}}}$ or of $\mathrm{X}^{\prime}$ to exist even locally on $\mathrm{S}$, unless $\mathrm{S}$ is smooth over a perfect field.

Throughout the rest of this section, let us fix a smooth $\mathrm{X} / \mathrm{S}$ as above. We assume that a lifting $\tilde{\mathrm{X}}^{\prime} / \tilde{\mathrm{S}}$ of $\mathrm{X}^{\prime} / \mathrm{S}$ modulo $p^{2}$ exists, and we denote the pair $\left(\mathrm{X} / \mathrm{S}, \tilde{\mathrm{X}}^{\prime} / \tilde{\mathrm{S}}\right)$ by $\mathscr{X} / \mathscr{S}$. Note that, given a lifting $\tilde{\mathrm{X}}$ of $\mathrm{X}$, it is very rare for there to exist a global lifting of $\mathrm{F}_{\mathrm{X} / \mathrm{S}}: \tilde{\mathrm{X}} \rightarrow \tilde{\mathrm{X}}^{\prime}$. (For example, no such lift can exist if $\mathrm{X}$ is a smooth proper curve of genus at least two over a perfect field, as is well known.) However it follows from the smoothness of $\mathrm{X}^{\prime} / \mathrm{S}$ that such lifts do exist locally, and we shall see that the sheaf of such liftings is crystalline in nature.

Let us fix a divided power structure on the ideal $p \mathscr{O}_{\tilde{\mathrm{S}}}$ and consider the crystalline site $\operatorname{Cris}(\mathrm{X} / \tilde{\mathrm{S}})$. If $(\mathrm{U}, \tilde{\mathrm{T}})$ is an object of $\operatorname{Cris}(\mathrm{X} / \tilde{\mathrm{S}})$, let $\mathrm{T}$ be the reduction of $\tilde{\mathrm{T}}$ modulo $p$. The ideal $\mathrm{J}_{\mathrm{T}}$ of the inclusion $i: \mathrm{U} \rightarrow \mathrm{T}$ is a divided power ideal, and so $a^{p}=0$ for every local section $a$ of $\mathrm{J}_{\mathrm{T}}$. Then the relative Frobenius map $\mathrm{F}_{\mathrm{T} / \mathrm{s}}$ factors through $\mathrm{U}^{\prime}$, and there is a unique and canonical morphism $f_{\mathrm{T} / \mathrm{S}}: \mathrm{T} \rightarrow \mathrm{X}^{\prime}$ such the following diagram commutes

$(\mathbf{1 . 0 . 1})$

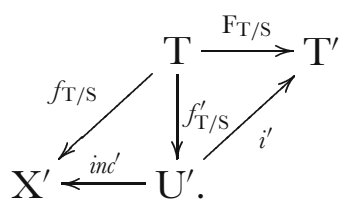

Let us note for future reference that the differential of $f_{\mathrm{T} / \mathrm{S}}$ vanishes:

$$
0=d f_{\mathrm{T} / \mathrm{S}}: \Omega_{\mathrm{X}^{\prime} / \mathrm{S}}^{1} \rightarrow f_{\mathrm{T} / \mathrm{S} *} \Omega_{\mathrm{T} / \mathrm{S}}^{1} .
$$

Indeed, $d f_{\mathrm{T} / \mathrm{S}}^{\prime} \circ d i^{\prime}=d \mathrm{~F}_{\mathrm{T} / \mathrm{S}}=0$, and since $d i^{\prime}$ is an epimorphism, $d f_{\mathrm{T} / \mathrm{S}}^{\prime}=0$.

If $g: \mathrm{T}_{1} \rightarrow \mathrm{T}_{2}$ is a morphism in Cris $(\mathrm{X} / \mathrm{S})$, then $f_{\mathrm{T}_{2} / \mathrm{S}} \circ g=f_{\mathrm{T}_{1} / \mathrm{S}}$. Hence if $\mathrm{E}^{\prime}$ is a sheaf of $\mathscr{O}_{\mathrm{X}^{\prime}}$-modules, there is a natural isomorphism

$$
\theta_{g}: g^{*} f_{\mathrm{T}_{2} / \mathrm{S}}^{*} \mathrm{E}^{\prime} \cong f_{\mathrm{T}_{1} / \mathrm{S}}^{*} \mathrm{E}^{\prime},
$$

and the collection $\left\{f_{\mathrm{T} / \mathrm{S}}^{*} \mathrm{E}^{\prime}, \theta_{g}\right\}$ defines a crystal of $\mathscr{O}_{\mathrm{X} / \mathrm{S}}$-modules. The corresponding object of MIC(X/S) is $\mathrm{F}_{\mathrm{X} / \mathrm{S}}^{*} \mathrm{E}^{\prime}$ with its Frobenius descent connection. (This is the unique connection $\nabla$ on $\mathrm{F}_{\mathrm{X} / \mathrm{S}}^{*} \mathrm{E}^{\prime}$ which annihilates the sections of $\mathrm{F}_{\mathrm{X} / \mathrm{S}}^{-1} \mathrm{E}^{\prime} \subseteq \mathrm{F}_{\mathrm{X} / \mathrm{S}}^{*} \mathrm{E}^{\prime}$.)

An extension of crystals

$$
0 \rightarrow \mathrm{E} \rightarrow \mathrm{H} \stackrel{h}{\rightarrow} \mathscr{O}_{\mathrm{X}} \rightarrow 0
$$


gives rise to a sheaf $h^{-1}(1) \subset \mathrm{H}$ of E-torsors on Cris $(\mathrm{X} / \mathrm{S})$; this construction defines an equivalence between the category of E-torsors and the category of extensions (1.0.3). Recall that giving a crystal $\mathrm{E}$ amounts to giving a quasi-coherent sheaf of $\mathscr{O}_{\mathrm{X}}$-modules with an integrable connection $\nabla_{\mathrm{E}}: \mathrm{E} \rightarrow \mathrm{E} \otimes \Omega_{\mathrm{X} / \mathrm{S}}^{1}$. Similarly, giving an E-torsor $\mathscr{L}$ on $\operatorname{Cris}(\mathrm{X} / \mathrm{S})$ is equivalent to giving an E-torsor $\mathscr{L}$ on $\operatorname{Zar}(\mathrm{X})$ together with a map

$$
\nabla_{\mathscr{L}}: \mathscr{L} \rightarrow \mathrm{E} \otimes \Omega_{\mathrm{X} / \mathrm{S}}^{1}
$$

such that $\nabla_{\mathscr{L}}(l+e)=\nabla_{\mathscr{L}}(l)+\nabla_{\mathrm{E}}(e)$ and such that the composition

$$
\mathscr{L} \stackrel{\nabla_{\mathscr{L}}}{\longrightarrow} \mathrm{E} \otimes \Omega_{\mathrm{X} / \mathrm{S}}^{1} \stackrel{\nabla_{\mathrm{E}}}{\longrightarrow} \mathrm{E} \otimes \Omega_{\mathrm{X} / \mathrm{S}}^{2}
$$

is equal to zero.

If $\mathrm{E}$ is a locally free crystal of $\mathscr{O}_{\mathrm{X} / \mathrm{S}}$-modules, we shall denote by $\mathbf{E}$ the corresponding crystal of affine group schemes over $\operatorname{Cris}(\mathrm{X} / \mathrm{S})$. That is, for each $\mathrm{T} \in \operatorname{Cris}(\mathrm{X} / \mathrm{S})$,

$$
\mathbf{E}_{\mathrm{T}}:=\operatorname{Spec}_{\mathrm{T}} \mathrm{S}^{*} \Omega_{\mathrm{T}}
$$

where $\Omega$ is the crystal of $\mathscr{O}_{\mathrm{X} / \mathrm{s}}$-modules dual to $\mathrm{E}$. In particular, a vector bundle $\mathrm{E}^{\prime}$ over $\mathrm{X}^{\prime}$ defines a crystal of affine schemes $\mathrm{F}_{\mathrm{X} / \mathrm{S}}^{*} \mathbf{E}^{\prime}$. More generally, for an E-torsor $\mathscr{L}$ on $\operatorname{Cris}(\mathrm{X} / \mathrm{S})$, we denote by $\mathbf{L}$ the corresponding crystal of affine schemes, which has a natural action $\mathbf{E} \times \mathbf{L} \rightarrow \mathbf{L}$.

Now let us fix a pair $\mathscr{X} / \mathscr{S}:=\left(\mathrm{X} / \mathrm{S}, \tilde{\mathrm{X}}^{\prime} / \tilde{\mathrm{S}}\right)$ as above. By a lifting of $f_{\mathrm{T} / \mathrm{S}}$ to $\tilde{\mathrm{T}}$ we shall mean a morphism $\tilde{\mathrm{F}}: \tilde{\mathrm{T}} \rightarrow \tilde{\mathrm{X}}^{\prime}$ lifting $f_{\mathrm{T} / \mathrm{S}}$. The sets of such liftings on open subsets of $\tilde{\mathrm{T}}$ form a sheaf $\mathscr{L}_{\mathscr{X} / \mathscr{S}, \tilde{\mathrm{T}}}$ on the Zariski topology of $\tilde{\mathrm{T}}$ (which coincides with the Zariski topology of $\mathrm{T}$ ). Since $\tilde{\mathrm{X}}^{\prime} / \tilde{\mathrm{S}}$ is smooth, such liftings exist locally, and by standard deformation theory, the sheaf $\mathscr{L}_{\mathscr{X} / \mathscr{S}, \tilde{T}}$ of such liftings forms a torsor under the abelian sheaf $\mathscr{H} o m\left(f_{\mathrm{T} / \mathrm{S}}^{*} \Omega_{\mathrm{X}^{\prime} / \mathrm{S}}^{1}, p \mathscr{O}_{\tilde{\mathrm{T}}}\right) \cong f_{\mathrm{T} / \mathrm{S}}^{*}\left(\mathrm{~T}_{\mathrm{X}^{\prime} / \mathrm{S}}\right)$.

Theorem 1.1. Let $\mathscr{X} / \mathscr{S}:=\left(\mathrm{X} / \mathrm{S}, \tilde{\mathrm{X}}^{\prime} / \tilde{\mathrm{S}}\right)$ be as above. Then there is a unique crystal of $\mathrm{F}_{\mathrm{X} / \mathrm{S}}^{*} \mathrm{~T}_{\mathrm{X}^{\prime} / \mathrm{S}}$-torsors $\mathscr{L}_{\mathscr{X} / \mathscr{S}}$ on $\mathrm{X} / \mathrm{S}$ with the following properties.

1. For each object $\mathrm{T}$ of $\mathrm{X} / \mathrm{S}$ admitting a flat lifting $\tilde{\mathrm{T}} \in \operatorname{Cris}(\mathrm{X} / \tilde{\mathrm{S}}), \mathscr{L}_{\mathscr{X} / \mathscr{S}, \mathrm{T}}$ is the sheaf of liftings of $f_{\mathrm{T} / \mathrm{S}}$ to $\tilde{\mathrm{T}}$.

2. For each morphism $\tilde{g}: \tilde{\mathrm{T}}_{1} \rightarrow \tilde{\mathrm{T}}_{2}$ of flat objects in $\mathrm{Cris}(\mathrm{X} / \tilde{\mathrm{S}})$ and each lifting $\tilde{\mathrm{F}}: \tilde{\mathrm{T}}_{2} \rightarrow \tilde{\mathrm{X}}^{\prime}$ of $f_{\mathrm{T}_{2} / \mathrm{S}}$, the transition map $\theta_{g}: g^{*} \mathscr{L}_{\mathscr{X} / \mathscr{S}, \mathrm{T}_{2}} \rightarrow \mathscr{L}_{\mathscr{X} / \mathscr{S}, \mathrm{T}_{1}}$ satisfies

$$
\theta_{g}(\tilde{\mathrm{F}})=\tilde{\mathrm{F}} \circ g: \tilde{\mathrm{T}}_{1} \rightarrow \tilde{\mathrm{X}}^{\prime} .
$$

We denote by $\mathbf{L}_{\mathscr{X} / \mathscr{S}}$ the crystal of relatively affine schemes Spec $\mathscr{A}_{\mathscr{X} / \mathscr{S}}$ corresponding to the

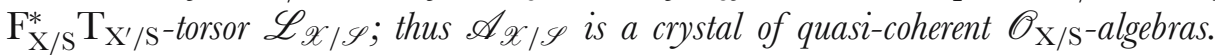

Remark 1.2. - We should point out that if $\tilde{\mathrm{T}}_{1}$ and $\tilde{\mathrm{T}}_{2}$ are two flat liftings of an object $\mathrm{T}$ of $\operatorname{Cris}(\mathrm{X} / \tilde{\mathrm{S}})$, then the set of liftings of $f_{\mathrm{T} / \mathrm{S}}$ to $\tilde{\mathrm{T}}_{1}$ and to $\tilde{\mathrm{T}}_{2}$ can be 
canonically identified. More precisely, let $\tilde{\mathrm{T}}_{1}$ and $\tilde{\mathrm{T}}_{2}$ be flat objects of Cris $(\mathrm{X} / \tilde{\mathrm{S}})$, and let $\tilde{g}$ and $\tilde{g}^{\prime}$ be two morphisms $\tilde{\mathrm{T}}_{1} \rightarrow \tilde{\mathrm{T}}_{2}$ with the same reduction modulo $p$. Then $\mathscr{L}_{\mathscr{X} / \mathscr{S}}(\tilde{g})=\mathscr{L}_{\mathscr{X} / \mathscr{S}}\left(\tilde{g}^{\prime}\right)$ as maps $\mathscr{L}_{\mathscr{X} / \mathscr{S}}\left(\tilde{\mathrm{T}}_{2}\right) \rightarrow \mathscr{L}_{\mathscr{X} / \mathscr{S}}\left(\tilde{\mathrm{T}}_{1}\right)$. This will follow from the proof of the theorem, but it can also be deduced from the following elementary argument. Let $g: \mathrm{T}_{1} \rightarrow \mathrm{T}_{2}$ be the common reduction modulo $p$ of $g_{1}$ and $g_{2}$. Then there is a map $h: \Omega_{\mathrm{T}_{2} / \mathrm{S}}^{1} \rightarrow g_{*} \mathscr{O}_{\mathrm{T}_{1}}$ such that $\tilde{g}^{\prime *}(\tilde{a})=\tilde{g}^{*}(\tilde{a})+[p] h(d a)$ for every section $\tilde{a}$ of $\mathscr{O}_{\tilde{\mathrm{T}}_{2}}$ lifting a section $a$ of $\mathscr{O}_{\mathrm{T}_{2}}$. Then if $\tilde{\mathrm{F}} \in \mathscr{L}_{\mathscr{X} / \mathscr{S}}\left(\tilde{\mathrm{T}}_{2}\right)$ is any lift of $f_{\mathrm{T}_{2} / \mathrm{S}}$ and $\tilde{b}$ is a section of $\mathscr{O}_{\tilde{\mathrm{X}}^{\prime}}$ with image $b$ in $\mathscr{O}_{\mathrm{X}^{\prime}}$,

$$
\left(\tilde{\mathrm{F}} \circ \tilde{g}^{\prime}\right)^{*}(\tilde{b})=(\tilde{\mathrm{F}} \circ \tilde{g})^{*}(\tilde{b})+[p] h\left(d f_{\mathrm{T}_{2} / \mathrm{S}}(d b)\right) .
$$

But we saw in (1.0.2) that $d f_{\mathrm{T}_{2} / \mathrm{S}}=0$, hence $\tilde{\mathrm{F}} \circ \tilde{g}=\tilde{\mathrm{F}} \circ \tilde{g}^{\prime}$.

Proof of Theorem 1.1. - We will need the following easy technical result.

Lemma 1.3. - Let $\operatorname{Cris}_{f}(\mathrm{X} / \tilde{\mathrm{S}})$ denote the full subsite of $\operatorname{Cris}(\mathrm{X} / \tilde{\mathrm{S}})$ consisting of those objects which are flat over $\tilde{\mathrm{S}}$. Then the morphism of sites $a: \operatorname{Cris}_{f}(\mathrm{X} / \tilde{\mathrm{S}}) \rightarrow \operatorname{Cris}(\mathrm{X} / \tilde{\mathrm{S}})$ induces an equivalence between the respective categories of crystals of $\mathscr{O}_{\mathrm{X} / \tilde{S}^{-m o d u l e s .}}$

Proof. - Indeed, the question is local on $\mathrm{X}$, so we may assume the existence of a lifting $\tilde{\mathrm{X}} / \tilde{\mathrm{S}}$. Then both categories can be identified with the category of pairs $(\mathrm{E}, \epsilon)$, where $\mathrm{E}$ is a quasi-coherent $\mathscr{O}_{\tilde{\mathrm{X}}}$-module and $\epsilon$ is an isomorphism between the two pullbacks of $\mathrm{E}$ to the divided power completion of $\tilde{\mathrm{X}} \times \tilde{\mathrm{X}}$ along the diagonal, satisfying the cocycle condition $[3, \S 6]$.

Thus we can identify the category of crystals of $\mathscr{O}_{\mathrm{X} / \mathrm{S}}-$ modules on Cris $(\mathrm{X} / \mathrm{S})$ and the category of $p$-torsion crystals of $\mathscr{O}_{\mathrm{X} / \tilde{\mathrm{S}}}$-modules on $\operatorname{Cris}_{f}(\mathrm{X} / \tilde{\mathrm{S}})$. The same is true for torsors over crystals of $\mathscr{O}_{\mathrm{X} / \mathrm{S}}$-modules.

It is clear that the family $\left\{\mathscr{L}_{\mathscr{X} / \mathscr{S}, \tilde{\mathrm{T}}}: \tilde{\mathrm{T}} \in \mathrm{Cris}_{f}(\mathrm{X} / \tilde{\mathrm{S}})\right\}$, together with the family of transition maps $\theta_{g}$ described in the theorem, forms a sheaf of sets on $\operatorname{Cris}_{f}(\mathrm{X} / \tilde{\mathrm{S}})$. Furthermore, as we saw above, this family naturally forms a sheaf of $\mathrm{F}_{\mathrm{X} / \mathrm{S}}^{*} \mathrm{~T}_{\mathrm{X}^{\prime} / \mathrm{S}}$-torsors. This proves the theorem.

Let us record some basic facts about vector groups which we will need later. Let $\pi_{\mathrm{T}}: \mathbf{T} \rightarrow \mathrm{X}$ be a vector group over $\mathrm{X}$ and let $\mathrm{T}$ be its sheaf of sections. Thus $\mathrm{T}$ is a locally free sheaf of $\mathscr{O}_{\mathrm{X}}$-modules of finite rank and $\mathbf{T}=\operatorname{Spec}_{\mathrm{X}} \mathrm{S} \cdot \Omega$, where $\Omega$ is the dual of $\mathrm{T}$. The pairing $\mathrm{T} \times \Omega \rightarrow \mathscr{O}_{\mathrm{X}}$ extends to a pairing $\mathrm{T} \times \mathrm{S}^{\circ} \Omega \rightarrow \mathrm{S}^{\circ} \Omega$, where sections of $\mathrm{T}$ act as derivations of $\mathrm{S}^{*} \Omega$. This action defines a map:

$$
\xi \mapsto \mathrm{D}_{\xi}: \mathrm{T} \rightarrow \pi_{\mathrm{T} *} \mathrm{~T}_{\mathbf{T} / \mathrm{X}},
$$

which identifies $\mathrm{T}$ with the sheaf of translation invariant vector fields of $\mathbf{T}$ relative to $\mathrm{X}$. It also induces an isomorphism $\pi_{\mathrm{T}}^{*} \mathrm{~T} \rightarrow \mathrm{T}_{\mathbf{T} / \mathrm{X}}$. Moreover, there is a canonical 
pairing of $\mathscr{O}_{\mathrm{X}}$-modules:

$$
\Gamma_{n} \mathrm{~T} \otimes \mathrm{S}^{n+m} \Omega \rightarrow \mathrm{S}^{m} \Omega
$$

which is perfect when $m=0$; see Section 5.4 and $[3, \mathrm{~A} 10]$. If we endow $\Gamma$. T with the topology defined by the PD-filtration of $\Gamma . \mathrm{T}$ and $\mathrm{S} \Omega$ with the discrete topology, this action is continuous. Thus it extends to a continuous action of the completion $\hat{\Gamma}$. T and identifies $\mathscr{H}_{0 m_{\mathscr{O}_{\mathrm{X}}}}\left(\mathrm{S} \cdot \Omega, \mathscr{O}_{\mathrm{X}}\right)$ with the completed divided power algebra $\hat{\Gamma}$.T of T. ${ }^{4}$ This action identifies the sheaf of divided power algebras $Г . T$ [33] with the subring of translation invariant elements in the full ring of differential operators [3, 2.1] of $\mathbf{T}$ relative to $\mathrm{X}$.

A section $\xi$ of $\mathrm{T}$ can be thought of as a section of the map $\pi_{\mathrm{T}}: \mathbf{T} \rightarrow \mathrm{X}$; let $t_{\xi}: \mathbf{T} \rightarrow \mathbf{T}$ be translation by $\xi$. Then the derivation $\mathrm{D}_{\xi}$ belongs to the divided power ideal of $\Gamma \cdot T, \exp \left(\mathrm{D}_{\xi}\right)$ makes sense as a differential operator of infinite order, and one has the formula (Taylor's theorem):

$$
t_{\xi}^{*}(f)=\left(\exp \mathrm{D}_{\xi}\right)(f)
$$

for the action of $t_{\xi}^{*}$ on $\mathrm{S}^{\cdot} \Omega$. The increasing filtration

$$
\mathrm{N}_{n} \mathrm{~S} \cdot \Omega:=\sum_{i \leq n} \mathrm{~S}^{i} \Omega \subseteq \mathrm{S} \cdot \Omega
$$

is invariant under $t_{\xi}^{*}$; furthermore $t_{\xi}^{*}$ acts trivially on the successive quotients.

Now let $\mathscr{L}$ be a T-torsor over $\mathrm{X}$ and let $\pi_{\mathscr{L}}: \mathbf{L} \rightarrow \mathrm{X}$ be the corresponding relatively affine scheme. It follows from the translation invariance of $\mathrm{D}_{\xi}$ that the action of $\hat{\Gamma}$.T on $\mathrm{S}^{\cdot} \Omega$ carries over to an action on $\pi_{\mathscr{L} *} \mathscr{O}_{\mathbf{L}}$. Similarly, there is a canonical filtration $\mathrm{N}$. on $\pi_{\mathscr{L} *} \mathscr{O}_{\mathbf{L}}$ and a canonical isomorphism

$$
\operatorname{Gr}^{\mathrm{N}}\left(\pi_{\mathscr{L} *} \mathscr{O}_{\mathbf{L}}\right) \cong \mathrm{S} \cdot \Omega .
$$

Note that $\mathrm{N}_{i} \pi_{\mathscr{L}_{*}} \mathscr{O}_{\mathbf{L}}$ can also be characterized as the annihilator of $\prod_{j>i} \Gamma_{j} \mathrm{~T}$. The bottom level $\mathrm{N}_{0} \mathrm{~S}^{\cdot} \Omega$ of $\mathrm{S}^{\cdot} \Omega$ corresponds to the translation invariant sections, so there is a canonical exact sequence

$$
0 \rightarrow \mathscr{O}_{\mathrm{X}} \rightarrow \mathscr{E} \rightarrow \Omega \rightarrow 0
$$

where $\mathscr{E}:=\mathrm{N}_{1} \pi_{\mathscr{L}_{*}} \mathscr{O}_{\mathbf{L}}$ is the set of affine functions on $\mathbf{L}$.

A section $\ell$ of $\mathscr{L}$ determines an isomorphism $s_{\ell}: \mathbf{L} \rightarrow \mathbf{T}: s_{\ell}\left(\ell^{\prime}\right):=\ell^{\prime}-\ell \in \mathbf{T}$ for all sections $\ell^{\prime}$ over all $\mathrm{X}$-schemes. This isomorphism determines an isomorphism

$$
\sigma_{\ell}:=s_{\ell}^{*}: \mathrm{S} \cdot \Omega \rightarrow \pi_{\mathscr{L} *} \mathscr{O}_{\mathbf{L}}
$$

${ }^{4}$ Thus the Cartier dual of $\mathbf{T}$ is the formal scheme $\hat{\mathbf{T}}_{\gamma}^{*}$ associated to the PD-algebra $\hat{\Gamma}$. T with the topology defined by the divided power filtration $\left\{\prod_{j \geq n} \Gamma_{j} \mathrm{~T}: n \in \mathbf{N}\right\}$. 
This is the unique isomorphism of filtered $\mathscr{O}_{\mathrm{X}}$-algebras with the property that $\sigma_{\ell}(\omega)\left(\ell^{\prime}\right)=\left\langle\omega, \ell^{\prime}-\ell\right\rangle$ for all local sections $\ell^{\prime}$ of $\mathscr{L}$ over $\mathrm{X}$ and $\omega$ of $\Omega$. (The uniqueness comes from the fact that any polynomial $\alpha \in \mathrm{A}\left[t_{1}, \ldots, t_{d}\right]$ of degree less than or equal to 1 is determined by its values on all A-valued points.) Note in particular that, as a $\hat{\Gamma}$.T-module, $\pi_{\mathscr{L}_{*}} \mathscr{O}_{\mathbf{L}}$ is locally coinvertible, i.e., its $\mathscr{O}_{\mathrm{X}}$-linear dual is, locally on $\mathrm{X}$, free of rank one over $\hat{\Gamma}$.T.

Finally, let us remark that if $\mathrm{T} \rightarrow \mathrm{T}^{\prime}$ is an $\mathscr{O}_{\mathrm{X}}$-linear map of locally free sheaves, and $\mathscr{L}^{\prime}$ is the $\mathbf{T}^{\prime}$-torsor deduced from $\mathscr{L}$ by pushout, then the morphism $\mathscr{L} \rightarrow \mathscr{L}^{\prime}$ induces an isomorphism

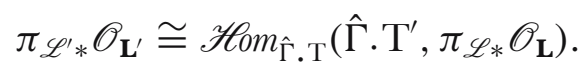

Let us summarize these remarks for our crystal of torsors $\mathscr{L}_{\mathscr{X} / \mathscr{S}}$.

Proposition 1.4. - Let $\mathscr{X} / \mathscr{S}:=\left(\mathrm{X}, \tilde{\mathrm{X}}^{\prime}\right)$ and $\mathscr{L}_{\mathscr{X} / \mathscr{S}}$ be as above, and let $\mathscr{A}_{\mathscr{X} / \mathscr{S}}$ denote the corresponding crystal of $\mathscr{O}_{\mathrm{X}}$-algebras.

1. There is a natural horizontal action of $\hat{\Gamma} \cdot \mathrm{F}_{\mathrm{X} / \mathrm{S}}^{*} \mathrm{~T}_{\mathrm{X}^{\prime} / \mathrm{S}}$ on $\mathscr{A}_{\mathscr{X} / \mathscr{S}}$, compatible with the action of $\mathrm{F}_{\mathrm{X} / \mathrm{S}}^{*} \mathrm{~T}_{\mathrm{X}^{\prime} / \mathrm{S}}$ by translation, as described in formula (1.3.1) above. As a sheaf of $\hat{\Gamma} \cdot \mathrm{F}_{\mathrm{X} / \mathrm{S}}^{*}\left(\mathrm{~T}_{\mathrm{X}^{\prime} / \mathrm{S}}\right)$-modules on $\mathrm{X}, \mathscr{A}_{\mathscr{X} / \mathscr{S}}$ is locally coinvertible.

2. There is a natural horizontal filtration $\mathrm{N}$. on $\mathscr{A}_{\mathscr{X} / \mathscr{S}}$, invariant under the action of $\mathrm{F}_{\mathrm{X} / \mathrm{S}}^{*} \mathrm{~T}_{\mathrm{X}^{\prime} / \mathrm{S}}$. In fact $\mathrm{N}_{i} \mathscr{A}_{\mathscr{X} / \mathscr{S}}$ is the annihilator of $\prod_{j>i} \Gamma_{j} \mathrm{~F}_{\mathrm{X} / \mathrm{S}}^{*} \mathrm{~T}_{\mathrm{X}^{\prime} / \mathrm{S}}$, and there is a canonical isomorphism:

$$
\mathrm{Gr}^{\mathrm{N}} \mathscr{A}_{\mathscr{X} / \mathscr{S}} \cong \mathrm{F}_{\mathrm{X} / \mathrm{S}}^{*} \mathrm{~S}^{\cdot} \Omega_{\mathrm{X}^{\prime} / \mathrm{S}}^{1} \cdot
$$

3. Let $\tilde{\mathrm{T}}$ be a flat object of $\operatorname{Cris}(\mathrm{X} / \tilde{\mathrm{S}})$ and let $\tilde{\mathrm{F}}: \tilde{\mathrm{T}} \rightarrow \tilde{\mathrm{X}}^{\prime}$ be a lift of $f_{\mathrm{T} / \mathrm{s}}$. Then there is a unique isomorphism of (filtered) $\mathscr{O}_{\mathrm{T}}$-algebras

$$
\sigma_{\tilde{\mathrm{F}}}: f_{\mathrm{T} / \mathrm{S}}^{*} \mathrm{~S} \cdot \Omega_{\mathrm{X}^{\prime} / \mathrm{S}}^{1} \stackrel{\cong}{\longrightarrow} \mathscr{A}_{\mathscr{X} / \mathscr{S}, \mathrm{T}}
$$

with the following property. For every section $\tilde{a}^{\prime}$ of $\mathscr{O}_{\tilde{\mathrm{X}}^{\prime}}$ lifting a section $a^{\prime}$ of $\mathscr{O}_{\mathrm{X}^{\prime}}$, $\sigma_{\tilde{\mathrm{F}}}\left(f_{\mathrm{T} / \mathrm{S}}^{*} d a^{\prime}\right) \in \mathrm{N}_{1} \mathscr{A}_{\mathscr{X} / \mathscr{S}, \mathrm{T}}$ is the $\mathscr{O}_{\mathrm{T}}$-valued function on $\mathscr{L}_{\mathscr{X} / \mathscr{S}}(\tilde{\mathrm{T}})$ such that for each $\tilde{\mathrm{F}}^{\prime}$,

$$
[p] \sigma_{\tilde{\mathrm{F}}}\left(f_{\mathrm{X} / \mathrm{S}}^{*} d a^{\prime}\right)\left(\tilde{\mathrm{F}}^{\prime}\right)=\tilde{\mathrm{F}}^{*}\left(\tilde{a}^{\prime}\right)-\tilde{\mathrm{F}}^{*}\left(\tilde{a}^{\prime}\right) .
$$

Furthermore $\mathrm{Gr}_{\mathrm{N}} \sigma_{\tilde{\mathrm{F}}}$ is the isomorphism of (2).

In particular we have a fundamental exact sequence: ${ }^{5}$

$$
0 \rightarrow \mathscr{O}_{\mathrm{X}} \rightarrow \mathscr{E}_{\mathscr{X} / \mathscr{S}} \rightarrow \mathrm{F}_{\mathrm{X} / \mathrm{S}}^{*} \Omega_{\mathrm{X}^{\prime} / \mathrm{S}}^{1} \rightarrow 0
$$

where

$$
\mathscr{E}_{\mathscr{X} / \mathscr{S}}:=\mathrm{N}_{1} \mathscr{A}_{\mathscr{X} / \mathscr{S}}
$$

\footnotetext{
${ }^{5}$ The first explicit construction of this sequence was given in [37].
} 
A section $\tilde{\mathrm{F}}$ of $\mathscr{L}_{\mathscr{X} / \mathscr{S}}$ determines as above a homomorphism $\sigma_{\tilde{\mathrm{F}}}$ which induces a splitting (not compatible with the connections) of this sequence, and in fact the set of splittings is bijective with the set of sections.

Since $\pi_{\mathscr{L}}: \mathscr{L}_{\mathscr{X} / \mathscr{S}} \rightarrow \mathrm{X}$ is an $\mathrm{F}_{\mathrm{X} / \mathrm{S}}^{*} \mathrm{~T}_{\mathrm{X}^{\prime} / \mathrm{S}}$-torsor over $\mathrm{X}$, there is a natural identification $\Omega_{\mathscr{L} / \mathrm{X}}^{1} \cong \pi_{\mathscr{L}}^{*} \mathrm{~F}_{\mathrm{X} / \mathrm{S}}^{*} \Omega_{\mathrm{X}^{\prime} / \mathrm{S}}^{1}$. (Here we are omitting the distinction between $\mathscr{L}$ and $\mathbf{L}$ in the notation.) The following result is the key to our theory; it shows that the $p$-curvature of the connection on $\mathscr{A}_{\mathscr{X} / \mathscr{S}}$ is very rich.

Proposition 1.5. - The action described in part (1) of Proposition 1.4 of $\mathrm{F}_{\mathrm{X} / \mathrm{S}}^{*} \mathrm{~T}_{\mathrm{X}^{\prime} / \mathrm{S}} \subseteq$ $\hat{\Gamma} \mathrm{F}_{\mathrm{X} / \mathrm{S}}^{*} \mathrm{~T}_{\mathrm{X}^{\prime} / \mathrm{S}}$ on $\mathscr{A}_{\mathscr{X} / \mathscr{S}}$ is the same as the action given by the $p$-curvature $\psi$ of the connection $\nabla$ on $\mathscr{A}_{\mathscr{X} / \mathscr{S}}$. That is, the diagram

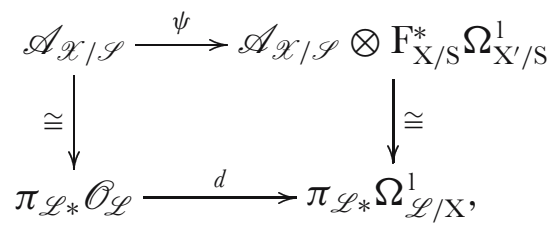

where $d$ is the usual exterior derivative and $\psi$ is the p-curvature of the connection on $\mathscr{A}_{\mathscr{X} / \mathscr{S}}$, is commutative.

This formula can be proved by explicit calculation (see Remark 1.11 below). We prefer to give here a conceptual proof based on a geometric construction of the $p$-curvature due to Mochizuki and communicated to us by Brian Osserman; see [30]. This construction begins with the following crystalline interpretation of $\mathrm{F}_{\mathrm{X} / \mathrm{S}}^{*} \Omega_{\mathrm{X}^{\prime} / \mathrm{S}}^{1}$.

Proposition 1.6. - Let $\mathrm{X} / \mathrm{S}$ be a smooth morphism of schemes in characteristic $p$, let $\mathrm{X}(1):=\mathrm{X} \times{ }_{\mathrm{S}} \mathrm{X}$, and let $(\mathrm{D}(1), \overline{\mathrm{I}}, \gamma)$ denote the divided power envelope of the ideal $\mathrm{I}$ of the diagonal immersion $\mathrm{X} \rightarrow \mathrm{X}(1)$. Then there is a unique and functorial isomorphism

$$
\xi_{p}: \mathrm{F}_{\mathrm{X} / \mathrm{S}}^{*} \Omega_{\mathrm{X}^{\prime} / \mathrm{S}}^{1} \rightarrow \overline{\mathrm{I}} /\left(\overline{\mathrm{I}}^{[p+1]}+\mathrm{I} \mathscr{O}_{\mathrm{D}(1)}\right)
$$

such that, for every local section a of $\mathscr{O}_{\mathrm{X}}$,

$$
\xi_{p}\left(d \pi^{*}(a)\right)=d_{p}(a):=((1 \otimes a)-(a \otimes 1))^{[p]} \quad\left(\bmod \overline{\mathrm{I}}^{[p+1]}+\mathrm{I} \mathscr{O}_{\mathrm{D}(1)}\right) .
$$

Proof. - For each section $a$ of $\mathscr{O}_{\mathrm{X}}$, let $\xi(a):=1 \otimes a-a \otimes 1 \in \mathrm{I} \mathscr{O}_{\mathrm{D}(1)} \subseteq \overline{\mathrm{I}}$. Note that $\xi(a)$ annihilates $\overline{\mathrm{I}} /\left(\overline{\mathrm{I}}^{[p+1]}+\mathrm{I} \mathscr{O}_{\mathrm{D}(1)}\right)$, and hence that the actions of $(a \otimes 1)$ and of $(1 \otimes a)$ on $\overline{\mathrm{I}} /\left(\overline{\mathrm{I}}^{[p+1]}+\mathrm{I} \mathscr{O}_{\mathrm{D}(1)}\right)$ are the same. Thus this quotient can be viewed as a sheaf of $\mathscr{O}_{\mathrm{X}}$-modules. If $b$ is another section of $\mathscr{O}_{\mathrm{X}}$, then

$$
\begin{aligned}
\xi(a+b)^{[p]} & =(\xi(a)+\xi(b))^{[p]}=\xi(a)^{[p]}+\sum_{i=1}^{p-1} \xi(a)^{[i]} \xi(b)^{[p-i]}+\xi(b)^{[p]} \\
& =\xi(a)^{[p]}+\xi(b)^{[p]} \quad\left(\bmod \mathrm{I} \mathscr{O}_{\mathrm{D}(1)}\right) .
\end{aligned}
$$


Furthermore, $\xi(a b)=(1 \otimes a) \xi(b)+(b \otimes 1) \xi(a)$, so a similar calculation shows that

$$
\xi(a b)^{[p]}=a^{p} \xi(b)^{[p]}+b^{p} \xi(a)^{[p]} \quad\left(\bmod \mathrm{I} \mathscr{O}_{\mathrm{D}(1)}\right) .
$$

Finally, if $a$ is a local section of $f^{-1}\left(\mathscr{O}_{\mathrm{S}}\right), \xi(a)=0$. These properties imply that $d_{p}$ is a derivation $\mathscr{O}_{\mathrm{X}} \rightarrow \mathrm{F}_{\mathrm{X} *}\left(\overline{\mathrm{I}} /\left(\overline{\mathrm{I}}^{[p+1]}+\mathrm{I} \mathscr{O}_{\mathrm{D}(1)}\right)\right)$, and hence that $d_{p}$ factors through an $\mathscr{O}_{\mathrm{X}}$-linear $\xi_{p}$ as claimed. To see that $\xi_{p}$ is an isomorphism, we may work with the aid of a system of local coordinates $t_{1}, \ldots, t_{m}$ for $\mathrm{X} / \mathrm{S}$. Let $\xi_{i}:=\xi\left(t_{i}\right)$, so that, in multiindex notation, $\left\{\xi^{[I]}: \mathrm{I} \in \mathbf{N}^{m}\right\}$ forms a basis for $h_{1 *} \mathscr{O}_{\mathrm{D}(1)}$, where $h_{1}: \mathrm{D}(1) \rightarrow \mathrm{X}$ is the first projection. Note that $\overline{\mathrm{I}} \subseteq \overline{\mathrm{I}}^{[p]}+\mathrm{I}_{\mathscr{O}_{\mathrm{D}(1)}}$ and that $\xi^{[\mathrm{I}]} \in \mathrm{I} \mathscr{O}_{\mathrm{D}(1)}$ if any $\mathrm{I}_{j}<p$. It follows that $\overline{\mathrm{I}} /\left(\overline{\mathrm{I}}^{[p+1]}+\mathrm{I} \mathscr{O}_{\mathrm{D}(1)}\right)$ is freely generated by $\xi_{1}^{[p]}, \ldots, \xi_{m}^{[p]}$, and hence that $\xi_{p}$ is an isomorphism.

Proposition 1.7 (Mochizuki). - Let $\mathrm{E}$ be a crystal of $\mathscr{O}_{\mathrm{X}}$-modules on $\mathrm{X} / \mathrm{S}$, Let $h_{1}$ and $h_{2}$ be the canonical maps $\mathrm{D}(1) \rightarrow \mathrm{X}$, and let $\epsilon: h_{2}^{*} \mathrm{E} \rightarrow h_{1}^{*} \mathrm{E}$ be the canonical isomorphism. Then the p-curvature $\psi$ of $\mathrm{E}$ identifies, via the isomorphism $\xi_{p}$ of Proposition 1.6, with the map sending each local section e of $\mathrm{E}_{\mathrm{X}}$ to the class of $\epsilon\left(h_{2}^{*}(e)\right)-h_{1}^{*}(e)$ in $\overline{\mathrm{I}} /\left(\overline{\mathrm{I}}^{[p+1]}+\mathrm{I} \mathscr{O}_{\mathrm{D}(1)}\right) \otimes \mathrm{E}$.

Proof. - We verify this formula with the aid of a system of local coordinates $\left(t_{1}, \ldots, t_{m}\right)$, using the notation above. Then if $\mathrm{D}_{i}:=\partial / \partial t_{i}$,

$$
\epsilon\left(h_{2}^{*}(e)\right)=\sum_{\mathrm{I}} \xi^{[\Gamma]} \nabla_{\mathrm{D}}^{\mathrm{I}} h_{1}^{*}(e)
$$

note that $\mathrm{D}_{i}^{(p)}=0$. Thus, modulo $\left.\overline{\mathrm{I}}^{[p+1]}+\mathrm{I} \mathscr{O}_{\mathrm{D}(1)}, \epsilon\left(h_{2}^{*}(e)\right)-h_{1}^{*}(e)\right)$ reduces to

$$
\sum_{i} \xi_{i}^{[p]} \nabla_{\mathrm{D}_{i}}^{p} h_{1}^{*}(e)=\sum_{i} \xi_{p}\left(d \pi^{*}\left(t_{i}\right)\right) \nabla_{\mathrm{D}_{i}}^{p} h_{1}^{*}(e)=\left(\mathrm{id} \otimes \xi_{p}\right)(\psi(e)) .
$$

Remark 1.8. - Let $h: \mathrm{X} \rightarrow \mathrm{Y}$ be a morphism of smooth S-schemes, let $\mathrm{E} \in \mathrm{MIC}(\mathrm{Y} / \mathrm{S})$ be a module with an integrable connection, and let $\psi_{\mathrm{Y}}: \mathrm{E} \rightarrow$ $\mathrm{E} \otimes_{\mathscr{O}_{\mathrm{Y}}} \mathrm{F}_{\mathrm{Y} / \mathrm{S}}^{*} \Omega_{\mathrm{Y}^{\prime} / \mathrm{S}}^{1}$ be its $p$-curvature. Then the $p$-curvature of $h^{*} \mathrm{E}$ is the composition:

$$
h^{*} \mathrm{E} \stackrel{h^{*} \psi_{\mathrm{Y}}}{\longrightarrow} h^{*} \mathrm{E} \otimes_{\mathscr{O}_{\mathrm{X}}} \mathrm{F}_{\mathrm{X} / \mathrm{S}}^{*} h^{\prime *} \Omega_{\mathrm{Y}^{\prime} / \mathrm{S}}^{1} \stackrel{i d \otimes h^{*}}{\longrightarrow} h^{*} \mathrm{E} \otimes_{\mathscr{O}_{\mathrm{X}}} \mathrm{F}_{\mathrm{X} / \mathrm{S}}^{*} \Omega_{\mathrm{X}^{\prime} / \mathrm{S}}^{1}
$$

This follows immediately from Proposition 1.7; it was first proved years ago by O. Gabber, using an indirect method.

Proof of Proposition 1.5. - Let $\tilde{\mathrm{F}}: \tilde{\mathrm{X}} \rightarrow \tilde{\mathrm{X}}^{\prime}$ be a local lift of $\mathrm{F}_{\mathrm{X} / \mathrm{S}}$. Let $(\tilde{\mathrm{D}}(1), \overline{\mathrm{J}}, \gamma)$ denote the PD-envelope of the diagonal ideal $\mathrm{J}$ of $\tilde{\mathrm{X}}(1)$, let $(\mathrm{D}(1), \overline{\mathrm{I}}, \gamma)$ denote its reduction modulo $p$, and denote again by $\tilde{\mathrm{F}}$ the induced maps $\tilde{\mathrm{X}}(1) \rightarrow \tilde{\mathrm{X}}^{\prime}(1)$ and 
$\tilde{\mathrm{D}}(1) \rightarrow \tilde{\mathrm{D}}^{\prime}(1)$. Since $\overline{\mathrm{J}}$ is flat over $\tilde{\mathrm{S}}$, multiplication by $p$ induces an injective map

$$
[p]: \overline{\mathrm{I}} / \mathrm{I} \mathscr{O}_{\mathrm{D}(1)} \rightarrow \overline{\mathrm{J}} / p \mathrm{~J} \mathscr{O}_{\tilde{\mathrm{D}}(1)}
$$

Since $\overline{\mathrm{J}} / \overline{\mathrm{J}}^{[p+1]}$ is flat over $\tilde{\mathrm{S}},\left(p \mathrm{~J} \mathscr{O}_{\tilde{\mathrm{D}}(1)}+\overline{\mathrm{J}}^{[p+1]}\right) \cap p \mathscr{O}_{\tilde{\mathrm{D}}(1)}=p\left(\mathrm{~J} \mathscr{O}_{\tilde{\mathrm{D}}(1)}+\overline{\mathrm{J}}^{[p+1]}\right)$, so multiplication by $p$ induces an injective map

$$
[p]: \overline{\mathrm{I}} /\left(\mathrm{I} \mathscr{O}_{\mathrm{D}(1)}+\overline{\mathrm{I}}^{[p+1]}\right) \rightarrow \overline{\mathrm{J}} /\left(p \mathrm{~J} \mathscr{O}_{\tilde{\mathrm{D}}(1)}+\overline{\mathrm{J}}^{[p+1]}\right) .
$$

If $\tilde{a}$ is a local section of $\mathscr{O}_{\tilde{\mathrm{X}}}$, we let $\xi(\tilde{a}):=1 \otimes \tilde{a}-\tilde{a} \otimes 1$.

Claim 1.9. - Let $\tilde{\mathrm{F}}: \tilde{\mathrm{X}} \rightarrow \tilde{\mathrm{X}}^{\prime}$ be a local lift of Frobenius, let $a$ be a local section of $\mathscr{O}_{\mathrm{X}}$, let $a^{\prime}:=\pi^{*}(a)$, and let $\tilde{a}^{\prime}$ be a local lift of $a^{\prime}$ to $\mathscr{O}_{\tilde{\mathrm{X}}^{\prime}}$. Then

$$
\begin{aligned}
\xi\left(\tilde{\mathrm{F}}^{*}\left(\tilde{a}^{\prime}\right)\right) & =-[p] \xi(a)^{[p]} \quad\left(\bmod p \mathrm{~J} \mathscr{O}_{\tilde{\mathrm{D}}(1)}\right) \\
& =-[p] d_{p}(a) \quad\left(\bmod p \mathrm{~J} \mathscr{O}_{\tilde{\mathrm{D}}(1)}+\overline{\mathrm{J}}^{[p+1]}\right) .
\end{aligned}
$$

Proof. - We may prove this claim with the aid of a local lifting $\tilde{a}$ of $a$. Then $\tilde{\mathrm{F}}^{*}\left(\tilde{a}^{\prime}\right)=\tilde{a}^{p}+p \tilde{b}$ for some section $\tilde{b}$ of $\mathscr{O}_{\tilde{\mathrm{X}}}$. Since $p \xi(\tilde{b}) \in p \mathrm{~J} \mathscr{O}_{\tilde{\mathrm{D}}(1)}$,

$$
\xi\left(\tilde{\mathrm{F}}^{*}\left(\tilde{a}^{\prime}\right)\right)=1 \otimes \tilde{a}^{p}-\tilde{a}^{p} \otimes 1 \quad\left(\bmod p \mathrm{~J} \mathscr{O}_{\tilde{\mathrm{D}}(1)}\right) .
$$

Now $1 \otimes \tilde{a}=\tilde{a} \otimes 1+\xi(\tilde{a})$, so

$$
1 \otimes \tilde{a}^{p}=\tilde{a}^{p} \otimes 1+p \zeta+(\xi(\tilde{a}))^{p}
$$

where $p \zeta=\sum_{i=1}^{p-1}\left(\begin{array}{c}p \\ i\end{array}\right) \tilde{a}^{i} \xi(\tilde{a})^{p-i} \in p \mathrm{~J} \mathscr{O}_{\tilde{\mathrm{D}}(1)}$. Since $(\xi(\tilde{a}))^{p}=p !(\xi(\tilde{a}))^{[p]}$ and $(p-1) ! \equiv$ $-1(\bmod p)$, this proves the claim.

Let $\sigma_{\tilde{\mathrm{F}}}$ be the splitting associated with $\tilde{\mathrm{F}}$ described in Proposition 1.4, and let $\alpha:=\sigma_{\tilde{\mathrm{F}}}\left(d \pi^{*}(a)\right) \in \mathscr{A}_{\mathscr{X} / \mathscr{S}}$. Then $\psi(\alpha) \in \mathscr{A}_{\mathscr{X} / \mathscr{S}} \otimes \mathrm{F}_{\mathrm{X} / \mathrm{S}}^{*} \Omega_{\mathrm{X}^{\prime} / \mathrm{S}}^{1}$, and by the $p$-curvature formula of Proposition 1.7, $\left(\mathrm{id} \otimes \xi_{p}\right) \psi(\alpha)$ is the class of $h_{2}^{*}(\alpha)-h_{1}^{*}(\alpha)$ in $\mathscr{A}_{\mathscr{X} / \mathscr{S}} \otimes$ $\left(\overline{\mathrm{I}} / \mathrm{I} \mathscr{O}_{\mathrm{D}(1)}+\overline{\mathrm{I}}^{[p+1]}\right)$. If $\tilde{\mathrm{F}}^{\prime}: \tilde{\mathrm{D}}(1) \rightarrow \tilde{\mathrm{X}}^{\prime}$ is any section of $\mathscr{L}_{\mathscr{X} / \mathscr{S}}(\tilde{\mathrm{D}}(1))$ and $\tilde{\mathrm{F}}_{i}:=\tilde{\mathrm{F}} \circ \tilde{h}_{i}$,

$$
\begin{aligned}
{[p]\left(\mathrm{id} \otimes \xi_{p}\right) \psi(\alpha)\left(\tilde{\mathrm{F}}^{\prime}\right) } & =[p]\left(h_{2}^{*}(\alpha)\left(\tilde{\mathrm{F}}^{\prime}\right)-h_{1}^{*}(\alpha)\left(\tilde{\mathrm{F}}^{\prime}\right)\right) \\
& =[p] \sigma_{\tilde{\mathrm{F}}_{2}}\left(\mathrm{~F}_{\mathrm{X} / \mathrm{S}}^{*} d a^{\prime}\right)\left(\tilde{\mathrm{F}}^{\prime}\right)-[p] \sigma_{\tilde{\mathrm{F}}_{1}}\left(\mathrm{~F}_{\mathrm{X} / \mathrm{S}}^{*} d a^{\prime}\right)\left(\tilde{\mathrm{F}}^{\prime}\right) \\
& =\left(\tilde{\mathrm{F}}^{*}\left(\tilde{a}^{\prime}\right)-\tilde{\mathrm{F}}_{2}^{*}\left(\tilde{a}^{\prime}\right)\right)-\left(\tilde{\mathrm{F}}^{\prime *}\left(\tilde{a}^{\prime}\right)-\tilde{\mathrm{F}}_{1}^{*}\left(\tilde{a}^{\prime}\right)\right) \\
& =\tilde{h}_{1}^{*} \tilde{\mathrm{F}}^{*}\left(\tilde{a}^{\prime}\right)-\tilde{h}_{2}^{*} \tilde{\mathrm{F}}^{*}\left(\tilde{a}^{\prime}\right) \\
& =-\xi\left(\tilde{\mathrm{F}}^{*}\left(\tilde{a}^{\prime}\right)\right) \\
& =[p]\left(d_{p} a\right) \\
& =[p] \xi_{p}\left(d \pi^{*} a\right) .
\end{aligned}
$$


Since [p] is injective, it follows that $\psi(\alpha)=d \pi(a)$. This proves the formula for elements of the form $\alpha=\sigma_{\widetilde{\mathrm{F}}}\left(d a^{\prime}\right)$. The general case follows from the fact that both $\psi$ and the action described in Proposition 1.4.1 annihilate $\mathscr{O}_{\mathrm{X}} \subseteq \mathscr{A}_{\mathscr{X} / \mathscr{S}}$ and both are compatible with the algebra structure.

It is helpful to have at our disposal an explicit formula for the connection on $\mathscr{A}_{\mathscr{X} / \mathscr{S}}$. Recall from [18] that the inverse Cartier isomorphism $\mathrm{G}_{\mathrm{X} / \mathrm{S}}^{-1}$ is a canonical $\mathscr{O}_{\mathrm{X}^{\prime}}$ linear map:

$$
\mathrm{C}_{\mathrm{X} / \mathrm{S}}^{-1}: \Omega_{\mathrm{X}^{\prime} / \mathrm{S}}^{i} \rightarrow \mathscr{H}^{i}\left(\mathrm{~F}_{\mathrm{X} / \mathrm{S} *} \Omega_{\mathrm{X} / \mathrm{S}}^{\cdot}\right)
$$

if $i=1$ and $a$ is a local section of $\mathscr{O}_{\mathrm{X}}$, then $\mathrm{C}_{\mathrm{X} / \mathrm{S}}^{-1}\left(d \pi_{\mathrm{X} / \mathrm{S}}^{*}(a)\right)$ is the cohomology class of $a^{p-1} d a$. Let $\mathrm{Z}_{\mathrm{X} / \mathrm{S}}^{i}$ denote the sheaf of closed $i$-forms on $\mathrm{X} / \mathrm{S}$. Then the Cartier operator is the composite

$$
\mathrm{C}_{\mathrm{X} / \mathrm{S}}: \mathrm{F}_{\mathrm{X} / \mathrm{S} *} \mathrm{Z}_{\mathrm{X} / \mathrm{S}}^{i} \rightarrow \mathscr{H}^{i}\left(\mathrm{~F}_{\mathrm{X} / \mathrm{S} *} \Omega_{\mathrm{X} / \mathrm{S}}^{i}\right) \rightarrow \Omega_{\mathrm{X}^{\prime} / \mathrm{S}}^{i}
$$

where the first map is the natural projection and the second is the inverse of $\mathrm{C}_{\mathrm{X} / \mathrm{S}}^{-1}$. Since $\Omega_{\mathrm{X}^{\prime} / \mathrm{S}}^{1}$ is locally free, locally on $\mathrm{X}$ there exists a section of $\mathrm{C}_{\mathrm{X} / \mathrm{S}}$ (in degree one), giving rise to a commutative diagram:

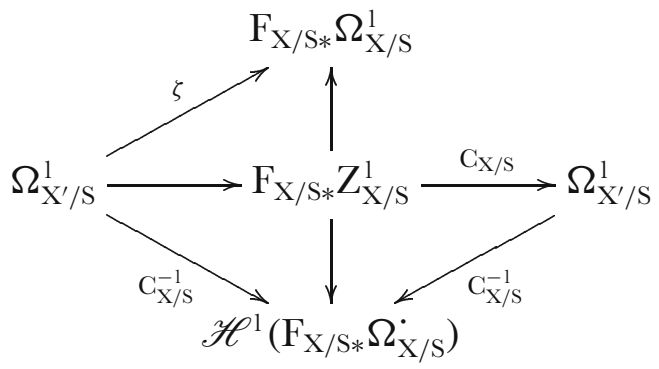

Mazur's formula [21] shows that a lifting $\tilde{\mathrm{F}}$ of the relative Frobenius morphism $\mathrm{F}_{\mathrm{X} / \mathrm{S}}: \mathrm{X} \rightarrow \mathrm{X}^{\prime}$ determines such a splitting $\zeta$. Suppose that $\tilde{\mathrm{F}}: \tilde{\mathrm{X}} \rightarrow \tilde{\mathrm{X}}^{\prime}$ is a lifting of $\mathrm{F}_{\mathrm{X} / \mathrm{S}}$ modulo $p^{2}$. Since

$$
d \tilde{\mathrm{F}}: \Omega_{\tilde{\mathrm{X}}^{\prime} / \tilde{\mathrm{S}}}^{1} \rightarrow \mathrm{F}_{\mathrm{X} / \mathrm{S} *} \Omega_{\tilde{\mathrm{X}} / \tilde{\mathrm{S}}}^{1}
$$

is divisible by $p$, there is a unique map $\zeta_{\tilde{\mathrm{F}}}$ making the following diagram commute:

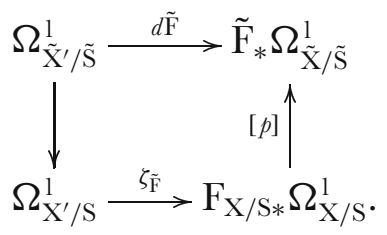

Then $\zeta_{\tilde{F}}$ is a splitting of the inverse Cartier operator in the sense of diagram (1.9.2). Let us recall the proof. Let $\tilde{a}$ be lift of a section $a$ of $\mathscr{O}_{\mathrm{X}}$ and let $\tilde{a}^{\prime}$ be a lift of $\pi^{*} a$. 
Then $\tilde{\mathrm{F}}^{*}\left(\tilde{a}^{\prime}\right)=\tilde{a}^{p}+p \tilde{b}$ for some $\tilde{b} \in \mathscr{O}_{\tilde{\mathrm{X}}}$. Hence

$$
[p] \zeta_{\tilde{\mathrm{F}}}\left(d \pi^{*} a\right)=d \tilde{\mathrm{F}}^{*}\left(\tilde{a}^{\prime}\right)=[p] a^{p-1} d a+[p] d b,
$$

where $b$ is the image of $\tilde{b}$ in $\mathscr{O}_{\mathrm{X}}$. Then $\zeta_{\tilde{\mathrm{F}}}\left(d \pi^{*} a\right)=a^{p-1} d a+d b$ is closed, and its image in $\mathscr{H}^{1}\left(\mathrm{~F}_{\mathrm{X} / \mathrm{S} *} \Omega_{\mathrm{X} / \mathrm{S}}\right)$ is the class of $a^{p-1} d a$, as required.

Proposition 1.10. - Let $\mathscr{X} / \mathscr{S}$ be as above and let $\tilde{\mathrm{U}}$ be a lift of some open subset of $\mathrm{X}$, let $\tilde{\mathrm{F}}$ be an element of $\mathscr{L}_{\mathscr{X} / \mathscr{S}}(\tilde{\mathrm{U}})$, and let $\sigma_{\tilde{\mathrm{F}}}$ be the corresponding splitting of $\mathscr{E}_{\mathscr{X} / \mathscr{S}, \mathrm{U}}$ described in Proposition 1.4. Then for any local section $\omega^{\prime}$ of $\Omega_{\mathrm{X}^{\prime} / \mathrm{S}}^{1}$,

$$
\nabla\left(\sigma_{\tilde{\mathrm{F}}}\left(1 \otimes \omega^{\prime}\right)\right)=-\zeta_{\tilde{\mathrm{F}}}\left(\omega^{\prime}\right)
$$

where $\nabla$ is the connection $(1.4 .1)$ on $\mathscr{E}_{\mathscr{X} / \mathscr{S}}$.

Proof. - Since both sides are linear over $\mathscr{O}_{\mathrm{X}^{\prime}}$, it suffices to prove the formula if $\omega^{\prime}=d a^{\prime}$, where $a^{\prime}$ is a section of $\mathscr{O}_{\mathrm{X}^{\prime}}$. Let $\tilde{\mathrm{T}}$ be the first infinitesimal neighborhood of $\tilde{\mathrm{U}}$ in $\tilde{\mathrm{U}} \times \tilde{\mathrm{S}} \tilde{\mathrm{U}}$ with its two natural projection $\tilde{h}_{i}: \tilde{\mathrm{T}} \rightarrow \tilde{\mathrm{U}}$, and let $\mathrm{T}$ be the reduction of $\tilde{\mathrm{T}}$ modulo $p$, so that $\mathscr{O}_{\mathrm{T}} \cong \mathscr{O}_{\mathrm{U}} \oplus \Omega_{\mathrm{U} / \mathrm{S}}^{1}$. The crystal structure on $\mathscr{E}_{\mathscr{X} / \mathscr{S} \text { gives us }}$ isomorphisms

$$
h_{2}^{*} \mathscr{E} \mathscr{X} / \mathscr{S}, \mathrm{U} \stackrel{\cong}{\cong} \mathscr{E}_{\mathscr{X} / \mathscr{S}, \mathrm{T}} \stackrel{\cong}{\cong} h_{1}^{*} \mathscr{E} \mathscr{X} / \mathscr{S}, \mathrm{U}
$$

reducing to the identity modulo the ideal $\Omega_{\mathrm{U} / \mathrm{S}}^{1}$ of $\mathscr{O}_{\mathrm{T}}$. Using the resulting identifications,

$$
\begin{aligned}
& \nabla\left(\sigma_{\tilde{\mathrm{F}}}\left(d a^{\prime}\right)\right):= \\
& \quad h_{2}^{*}\left(\sigma_{\tilde{\mathrm{F}}}\left(\mathrm{F}_{\mathrm{X} / \mathrm{S}}^{*} d a^{\prime}\right)\right)-h_{1}^{*}\left(\sigma_{\tilde{\mathrm{F}}}\left(\mathrm{F}_{\mathrm{X} / \mathrm{S}}^{*} d a^{\prime}\right)\right) \in \mathscr{A}_{\mathscr{X} / \mathscr{S}, \mathrm{U}} \otimes \Omega_{\mathrm{X} / \mathrm{S}}^{1} \subseteq \mathscr{A}_{\mathscr{X} / \mathscr{S}, \mathrm{T}} .
\end{aligned}
$$

Let us evaluate this section on an arbitrary section $\tilde{\mathrm{F}}^{\prime}: \tilde{\mathrm{T}} \rightarrow \tilde{\mathrm{X}}^{\prime}$ of $\mathscr{L}_{\mathscr{X} / \mathscr{S}}(\tilde{\mathrm{T}})$. Let $\tilde{\mathrm{F}}_{i}:=\tilde{\mathrm{F}} \circ \tilde{h}_{i} \in \mathscr{L}_{\mathscr{X} / \mathscr{S}}(\tilde{\mathrm{T}})$. If $\tilde{a}^{\prime}$ is a lift of $a^{\prime}$, then by Proposition 1.4,

$$
\begin{aligned}
{[p] \nabla\left(\sigma_{\tilde{\mathrm{F}}}\left(\mathrm{F}_{\mathrm{X} / \mathrm{S}}^{*} d a^{\prime}\right)\right)\left(\tilde{\mathrm{F}}^{\prime}\right) } & =[p] h_{2}^{*}\left(\sigma_{\tilde{\mathrm{F}}}\left(\mathrm{F}_{\mathrm{X} / \mathrm{S}}^{*} d a^{\prime}\right)\right)\left(\tilde{\mathrm{F}}^{\prime}\right)-[p] h_{1}^{*}\left(\sigma_{\tilde{\mathrm{F}}}\left(\mathrm{F}_{\mathrm{X} / \mathrm{S}}^{*} d a^{\prime}\right)\right)\left(\tilde{\mathrm{F}}^{\prime}\right) \\
& =[p] \sigma_{\tilde{\mathrm{F}}_{2}}\left(\mathrm{~F}_{\mathrm{X} / \mathrm{S}}^{*} d a^{\prime}\right)\left(\tilde{\mathrm{F}}^{\prime}\right)-[p] \sigma_{\tilde{\mathrm{F}}_{1}}\left(\mathrm{~F}_{\mathrm{X} / \mathrm{S}}^{*} d a^{\prime}\right)\left(\tilde{\mathrm{F}}^{\prime}\right) \\
& =\left(\tilde{\mathrm{F}}^{\prime *}\left(\tilde{a}^{\prime}\right)-\tilde{\mathrm{F}}_{2}^{*}\left(\tilde{a}^{\prime}\right)\right)-\left(\tilde{\mathrm{F}}^{\prime *}\left(\tilde{a}^{\prime}\right)-\tilde{\mathrm{F}}_{1}^{*}\left(\tilde{a}^{\prime}\right)\right) \\
& =\tilde{h}_{1}^{*} \tilde{\mathrm{F}}^{*}\left(\tilde{a}^{\prime}\right)-\tilde{h}_{2}^{*} \tilde{\mathrm{F}}^{*}\left(\tilde{a}^{\prime}\right) \\
& =-d \tilde{\mathrm{F}}^{*}\left(\tilde{a}^{\prime}\right) \\
& =-[p] \tilde{\mathrm{F}}_{\tilde{\mathrm{F}}}\left(d a^{\prime}\right) .
\end{aligned}
$$

Remark 1.11. - Somewhat more generally, let $\zeta$ be a section of $\mathrm{C}_{\mathrm{X} / \mathrm{S}}^{-1}$ as in (1.9.2), and let

$$
\left(\mathscr{E}_{\zeta}, \nabla\right):=\mathscr{O}_{\mathrm{X}} \oplus \mathrm{F}_{\mathrm{X} / \mathrm{S}}^{*} \Omega_{\mathrm{X}^{\prime} / \mathrm{S}}^{1},
$$


where $\nabla: \mathscr{E}_{\zeta} \rightarrow \mathscr{E}_{\zeta} \otimes \Omega_{\mathrm{X} / \mathrm{S}}^{1}$ is the map

$$
\left(f, g \otimes \omega^{\prime}\right) \mapsto\left(d f-g \otimes \zeta\left(\omega^{\prime}\right), \omega^{\prime} \otimes d g\right)
$$

Then $\nabla$ is an integrable connection on $\mathscr{E}_{\zeta}$, and one can simply compute that its $p$-curvature is the map

$$
\psi: \mathscr{E}_{\zeta} \rightarrow \mathscr{E}_{\zeta} \otimes \mathrm{F}_{\mathrm{X} / \mathrm{S}}^{*} \Omega_{\mathrm{X}^{\prime} / \mathrm{S}}^{1} \quad\left(f, g \otimes \omega^{\prime}\right) \mapsto(g, 0) \otimes \omega^{\prime}
$$

(See for example $[26,2.10]$.) If $\tilde{\mathrm{F}}$ is a lift of Frobenius, then $\sigma_{\tilde{\mathrm{F}}}$ provides a splitting of the fundamental exact sequence (1.4.1) and hence an isomorphism $\mathscr{E}_{\mathscr{X} / \mathscr{S}} \cong \mathscr{E}_{\zeta}$ inducing the identity maps on $\mathscr{O}_{\mathrm{X}}$ and $\mathrm{F}_{\mathrm{X} / \mathrm{S}}^{*} \Omega_{\mathrm{X}^{\prime} / \mathrm{S}}^{1}$. The formula of Proposition 1.10 shows that this morphism is horizontal, and hence provides another proof of Proposition 1.5.

\subsection{Functoriality}

The geometric construction of $\mathscr{L}_{\mathscr{X} / \mathscr{S}}$ we have given makes it quite straightforward to check its functoriality. Note first that a morphism $h: \mathrm{X} \rightarrow \mathrm{Y}$ of smooth S-schemes induces a morphism of schemes $h^{\prime}: \mathrm{X}^{\prime} \rightarrow \mathrm{Y}^{\prime}$, a morphism of $\mathscr{O}_{\mathrm{X}^{\prime}}$-modules $\mathrm{T}_{\mathrm{X}^{\prime} / \mathrm{S}} \rightarrow h^{*} \mathrm{~T}_{\mathrm{Y}^{\prime} / \mathrm{S}}$, and hence a morphism of crystals of vector bundles:

$$
\mathbf{T}_{h^{\prime}}: \mathrm{F}_{\mathrm{X} / \mathrm{S}}^{*} \mathbf{T}_{\mathrm{X}^{\prime} / \mathrm{S}} \rightarrow h^{*} \mathrm{~F}_{\mathrm{Y} / \mathrm{S}}^{*} \mathbf{T}_{\mathrm{Y}^{\prime} / \mathrm{S}} .
$$

Proposition 1.12. — Let $h: \mathrm{X} \rightarrow \mathrm{Y}$ be a morphism of smooth $\mathrm{S}$-schemes and let $\tilde{h}^{\prime}$ be a lift of $h^{\prime}$. Then the pair $\tilde{h}:=\left(h, \tilde{h}^{\prime}\right)$ induces a morphism of crystals of torsors:

$$
\mathscr{L}_{\mathscr{X} \mid \mathscr{S}} \stackrel{\mathscr{L}_{\tilde{h}}}{\rightarrow} h^{*} \mathscr{L}_{\mathscr{Y} \mid \mathscr{S}}
$$

compatible with the actions of $\mathrm{F}_{\mathrm{X} / \mathrm{S}}^{*} \mathbf{T}_{\mathrm{X}^{\prime} / \mathrm{S}}$ and $h^{*} \mathrm{~F}_{\mathrm{Y} / \mathrm{S}}^{*} \mathbf{T}_{\mathrm{Y}^{\prime} / \mathrm{S}}$ ira the morphism $\mathbf{T}_{h^{\prime}}$. This induces an isomorphism of crystals of $h^{*} \mathrm{~F}_{\mathrm{Y} / \mathrm{S}}^{*} \mathbf{T}_{\mathrm{Y}^{\prime} / \mathrm{S}}$-torsors,

$$
h^{*} \mathrm{~F}_{\mathrm{Y} / \mathrm{S}}^{*} \mathbf{T}_{\mathrm{Y}^{\prime} / \mathrm{S}} \times_{\mathrm{F}_{\mathrm{X} / \mathrm{S}}^{*} \mathbf{T}_{\mathrm{X}^{\prime} / \mathrm{S}}} \mathscr{L}_{\mathscr{X} / \mathscr{S}} \stackrel{\mathscr{L}_{\hat{h}}}{\longrightarrow} h^{*} \mathscr{L}_{\mathscr{Y} / \mathscr{S}},
$$

a horizontal morphism of filtered $\mathscr{O}_{\mathrm{X}}$-algebras:

$$
\theta_{\tilde{h}}:\left(h^{*} \mathscr{A}_{\mathscr{Y} / \mathscr{S}}, \mathrm{N} .\right) \rightarrow\left(\mathscr{A}_{\mathscr{X} / \mathscr{S}}, \mathrm{N} .\right)
$$

and a horizontal isomorphism of $h^{*} \mathrm{~F}_{\mathrm{Y} / \mathrm{S}}^{*} \Gamma . \mathrm{T}_{\mathrm{Y}^{\prime} / \mathrm{S}^{-}}$algebras

$$
h^{*} \mathscr{A}_{\mathscr{Y} / \mathscr{S}} \stackrel{\cong}{\longrightarrow} \mathscr{H}_{0} m_{\mathrm{F}_{\mathrm{X} / \mathrm{S}}^{*} \hat{\Gamma} \cdot \mathrm{T}_{\mathrm{X}^{\prime} / \mathrm{S}}}\left(h^{*} \mathrm{~F}_{\mathrm{Y} / \mathrm{S}}^{*} \hat{\Gamma} \cdot \mathrm{T}_{\mathrm{Y}^{\prime} / \mathrm{S}}, \mathscr{A}_{\mathscr{X} / \mathscr{S}}\right) .
$$

Proof. - Recall from [3, 6.5] and [3, 5.11] that if $\mathrm{E}$ is a crystal on Y/S, then $h^{*} \mathrm{E}$ is the unique sheaf such that for each morphism $g: \mathrm{T}_{1} \rightarrow \mathrm{T}_{2}$ from an object 
in $\operatorname{Cris}(\mathrm{X} / \mathrm{S})$ to an object in $\operatorname{Cris}(\mathrm{Y} / \mathrm{S}),\left(h^{*} \mathrm{E}\right)_{\mathrm{T}_{1}}=g^{*}\left(\mathrm{E}_{\mathrm{T}_{2}}\right)$. Now if $\tilde{\mathrm{T}}$ is an object of $\operatorname{Cris}_{f}(\mathrm{X} / \tilde{\mathrm{S}}), h^{\prime} \circ f_{\mathrm{T} / \mathrm{S}}$ is a map $\mathrm{T} \rightarrow \mathrm{Y}^{\prime}$, and the set $\mathscr{L}_{h, \mathrm{~T}}$ of its liftings $\tilde{\mathrm{T}} \rightarrow \tilde{\mathrm{Y}}^{\prime}$ is a torsor under $f_{\mathrm{T} / \mathrm{S}}^{*} h^{\prime *} \mathrm{~T}_{\mathrm{Y}^{\prime} / \mathrm{S}}$. We claim first of all that $\mathrm{T} \mapsto f_{\mathrm{T} / \mathrm{S}}^{*} h^{\prime *} \mathrm{~T}_{\mathrm{Y}^{\prime} / \mathrm{S}}$ can be identified with

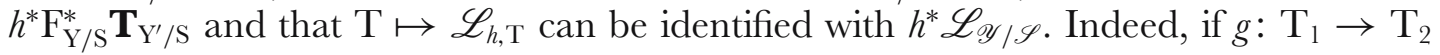
is as above, then

$$
\left(h^{*} \mathrm{~F}_{\mathrm{Y} / \mathrm{S}}^{*} \mathrm{~T}_{\mathrm{Y}^{\prime} / \mathrm{S}}\right)_{\mathrm{T}_{1}}=g^{*}\left(\left(\mathrm{~F}_{\mathrm{Y} / \mathrm{S}}^{*} \mathrm{~T}_{\mathrm{Y}^{\prime} / \mathrm{S}}\right)_{\mathrm{T}_{2}}\right)=g^{*} f_{\mathrm{T}_{2} / \mathrm{S}}^{*} \mathrm{~T}_{\mathrm{Y} / \mathrm{S}}=f_{\mathrm{T}_{1}}^{*} h^{\prime *} \mathrm{~T}_{\mathrm{Y} / \mathrm{S}},
$$

proving the first part of the claim. Suppose further that $\tilde{\mathrm{T}}_{1} \in \operatorname{Cris}_{f}(\mathrm{X} / \tilde{\mathrm{S}}), \tilde{\mathrm{T}}_{2} \in$ $\operatorname{Cris}_{f}(\mathrm{Y} / \tilde{\mathrm{S}})$, and $\tilde{g}: \tilde{\mathrm{T}}_{1} \rightarrow \tilde{\mathrm{T}}_{2}$ is a PD-morphism, compatible with $h$, and let $g: \mathrm{T}_{1} \rightarrow \mathrm{T}_{2}$

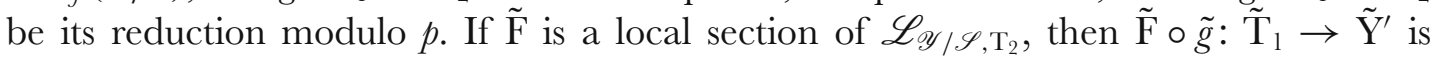
a lift of $f_{\mathrm{T}_{2}} \circ g=h^{\prime} \circ f_{\mathrm{T}_{1} / \mathrm{S}}$, and the sheaf of such lifts forms a $g^{*} f_{\mathrm{T}_{2} / \mathrm{S}}^{*} \mathbf{T}_{\mathrm{Y}^{\prime} / \mathrm{S}}$-torsor. Thus $\tilde{\mathrm{F}} \mapsto \tilde{\mathrm{F}} \circ \tilde{g}$ defines an isomorphism of torsors from $\mathscr{L}_{\mathscr{Y} / \mathscr{S}, \mathrm{T}_{2}} \times_{\mathrm{T}_{2}} \mathrm{~T}_{1}$ to the torsor of such liftings, proving the second part of the claim. Now if $\tilde{\mathrm{F}}_{1}: \tilde{\mathrm{T}}_{1} \rightarrow \tilde{\mathrm{X}}^{\prime}$ is a local section of $\mathscr{L}_{\mathscr{X} / \mathscr{S}, \mathrm{T}_{1}}$, then $\tilde{h}^{\prime} \circ \tilde{\mathrm{F}}_{1}$ is such a lifting. Thus composition with $\tilde{h}^{\prime}$ defines a morphism $\mathscr{L}_{\mathscr{X} / \mathscr{S}, \mathrm{T}_{1}} \rightarrow g^{*} \mathscr{L}_{\mathscr{Y} / \mathscr{S}, \mathrm{T}_{2}}$, which is evidently compatible with the torsor actions.

Corollary 1.13. - Let $h: \mathrm{X} \rightarrow \mathrm{Y}$ is a morphism of smooth $\mathrm{S}$-schemes. Then a lift $\tilde{h}^{\prime}: \tilde{\mathrm{X}}^{\prime} \rightarrow \tilde{\mathrm{Y}}^{\prime}$ of $h^{\prime}$ induces an exact sequence

$$
h^{*} \mathscr{E}_{\mathscr{Y} / \mathscr{S}} \rightarrow \mathscr{E}_{\mathscr{X} / \mathscr{S}} \rightarrow \mathrm{F}_{\mathrm{X} / \mathrm{S}}^{*} \Omega_{\mathrm{X}^{\prime} / \mathrm{Y}^{\prime}}^{1} \rightarrow 0 .
$$

If $h$ is smooth, this sequence is short exact (and locally split).

\subsection{Further remarks}

If $\mathrm{F}_{\mathrm{S}}$ lifts to $\tilde{\mathrm{S}}$ and $\tilde{\mathrm{X}} / \tilde{\mathrm{S}}$ lifts $\mathrm{X} / \mathrm{S}$, then $\tilde{\mathrm{X}}^{\prime}:=\tilde{\mathrm{X}} \times_{\mathrm{F}_{\tilde{\mathrm{S}}}} \tilde{\mathrm{S}}$ lifts $\mathrm{X}^{\prime}$. In this case there is a lifting $\tilde{\pi}^{\prime}: \tilde{\mathrm{X}}^{\prime} \rightarrow \tilde{\mathrm{X}}$ of $\pi: \mathrm{X}^{\prime} \rightarrow \mathrm{X}$, and the following proposition applies.

Proposition 1.14. - Suppose that $\tilde{\pi}: \tilde{\mathrm{X}}^{\prime} \rightarrow \tilde{\mathrm{X}}$ lifts $\pi: \mathrm{X}^{\prime} \rightarrow \mathrm{X}$. Then for each section $\tilde{a}$ of $\mathscr{O}_{\tilde{\mathrm{X}}}$, there is a unique section $\delta_{\tilde{\pi}}(\tilde{a})$ of $\mathscr{E}_{\mathscr{X} / \mathscr{S}} \subseteq \mathscr{A}_{\mathscr{X} / \mathscr{S}}$ such that for every lifting $\tilde{\mathrm{F}}: \tilde{\mathrm{U}} \rightarrow \tilde{\mathrm{X}}^{\prime}$ of $\mathrm{F}_{\mathrm{X} / \mathrm{S}}$ over some open subset $\mathrm{U}$ of $\mathrm{X}$,

$$
[p] \delta_{\tilde{\pi}}(\tilde{a})(\tilde{\mathrm{F}})=\tilde{\mathrm{F}}^{*} \tilde{\pi}^{*}(\tilde{a})-\tilde{a}^{p}
$$

on $\tilde{U}$. Furthermore, $\delta_{\tilde{\pi}}$ has the following properties.

1. The following diagram commutes:

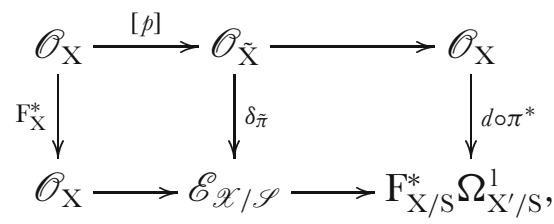

where the bottom row is the fundamental extension (1.4.1). 
2. The set of all $\delta_{\tilde{\pi}}(\tilde{a})$ for $\tilde{a} \in \mathscr{O}_{\tilde{\mathrm{X}}}$ generates $\mathscr{E}_{\mathscr{X} / \mathscr{S}}$ as an $\mathscr{O}_{\mathrm{X}}$-module.

3. If $\tilde{\mathrm{F}}: \tilde{\mathrm{X}} \rightarrow \tilde{\mathrm{X}}^{\prime}$ is a lift of $\mathrm{F}$,

$$
\delta_{\tilde{\pi}}(\tilde{a})=\delta_{\tilde{\pi}}(\tilde{a})(\tilde{\mathrm{F}})+\sigma_{\tilde{\mathrm{F}}}\left(\mathrm{F}_{\mathrm{X}}^{*} d a\right)
$$

where $\sigma_{\tilde{\mathrm{F}}}$ is the splitting defined in Proposition 1.4.

4. For every local section $\tilde{a}$ of $\mathscr{O}_{\tilde{\mathrm{X}}}$ lifting some $a \in \mathscr{O}_{\mathrm{X}}$,

$$
\begin{aligned}
& \nabla \delta_{\tilde{\pi}}(a)=-1 \otimes a^{p-1} d a \in \mathscr{A}_{\mathscr{X} / \mathscr{S}} \otimes \Omega_{\mathrm{X} / \mathrm{S}}^{1} \text { and } \\
& \psi \delta_{\tilde{\pi}}(a)=1 \otimes \mathrm{F}_{\mathrm{X}}^{*}(d a) \in \mathscr{A}_{\mathscr{X} / \mathscr{S}} \otimes \mathrm{F}_{\mathrm{X} / \mathrm{S}}^{*} \Omega_{\mathrm{X}^{\prime} / \mathrm{S}}^{1} .
\end{aligned}
$$

5. If $\tilde{a}$ and $\tilde{b}$ are sections of $\mathscr{O}_{\tilde{\mathrm{X}}}$ reducing to sections $a$ and $b$ of $\mathscr{O}_{\mathrm{X}}$,

$$
\begin{aligned}
\delta_{\tilde{\pi}}(\tilde{a} \tilde{b}) & =a^{p} \delta_{\tilde{\pi}}(\tilde{b})+b^{p} \delta_{\tilde{\pi}}(\tilde{a}), \\
\delta_{\tilde{\pi}}(\tilde{a}+\tilde{b}) & =\delta_{\tilde{\pi}}(\tilde{a})+\delta_{\tilde{\pi}}(b)+\sum_{0<i<p} \frac{a^{i}}{i !} \frac{b^{p-i}}{(p-i) !} .
\end{aligned}
$$

Proof. - First of all, note that $\pi \circ \mathrm{F}_{\mathrm{X} / \mathrm{S}}=\mathrm{F}_{\mathrm{X}}$, which takes any section $a$ of $\mathscr{O}_{\mathrm{X}}$ to $a^{p}$. Hence if $\tilde{\mathrm{F}}$ is a lift of $\mathrm{F}_{\mathrm{X} / \mathrm{S}}$ and $\tilde{a}$ is a lift of $a, \tilde{\mathrm{F}}^{*} \tilde{\pi}^{*}(\tilde{a})-\tilde{a}^{p}$ is divisible by $p$. Thus the formula defining $\delta_{\tilde{\pi}}$ as a function $\mathscr{L}_{\mathscr{X} / \mathscr{S}}(\tilde{\mathrm{U}}) \rightarrow \mathscr{O}_{\mathrm{X}}$ makes sense. Now if $\tilde{\mathrm{F}}^{\prime}$ is another lift of $\mathrm{F}_{\mathrm{X} / \mathrm{S}}$,

$$
\begin{aligned}
{[p] \delta_{\tilde{\pi}}(\tilde{a})\left(\tilde{\mathrm{F}}^{\prime}\right) } & =\tilde{\mathrm{F}}^{*} \tilde{\pi}^{*}(\tilde{a})-\tilde{a}^{p} \\
& =\tilde{\mathrm{F}}^{*} \tilde{\pi}^{*}(\tilde{a})-\tilde{a}^{p}+\tilde{\mathrm{F}}^{*} \tilde{\pi}^{*}(\tilde{a})-\tilde{\mathrm{F}}^{*} \tilde{\pi}^{*}(\tilde{a}) \\
& =[p] \delta_{\tilde{\pi}}(\tilde{a})(\tilde{\mathrm{F}})+[p] \sigma_{\tilde{\mathrm{F}}}\left(\mathrm{F}_{\mathrm{X}}^{*}(d a)\left(\tilde{\mathrm{F}}^{\prime}\right),\right.
\end{aligned}
$$

by Proposition 1.4. This proves that, as functions on $\mathscr{L}_{\mathscr{X} / \mathscr{S}}(\tilde{\mathrm{U}})$,

$$
\delta_{\tilde{\pi}}(\tilde{a})=\delta_{\tilde{\pi}}(\tilde{a})(\tilde{\mathrm{F}})+\sigma_{\tilde{\mathrm{F}}}\left(\mathrm{F}_{\mathrm{X}}^{*} d a\right) .
$$

This proves that $\delta_{\tilde{\pi}}$ is well defined and satisfies (3). If $\tilde{a}=[p] b$ for some $b \in \mathscr{O}_{\mathrm{X}}$, then $\tilde{a}^{p}=0$, and $[p] \delta_{\tilde{\pi}}(\tilde{a})(\tilde{\mathrm{F}})=\mathrm{F}_{\mathrm{X} / \mathrm{S}}^{*} \tilde{\pi}^{*}(p b)=[p] b^{p}$. This proves the commutativity of the first square in the diagram, and shows that the sub- $\mathscr{O}_{\mathrm{X}}$-module of $\mathscr{E}_{\mathscr{X} / \mathscr{S}}$ generated by the image of $\delta_{\tilde{\pi}}$ contains $\mathscr{O}_{\mathrm{X}}$. We have already proved (3), which implies the commutativity of the second square and the fact the set of images of all the $\delta_{\tilde{\pi}}(\tilde{a})$ 's generates $\mathscr{E}_{\mathscr{X} / \mathscr{S}}$. To prove (4), we may assume that a lifting $\tilde{\mathrm{F}}$ of $\mathrm{F}_{\mathrm{X} / \mathrm{S}}$ exists. Then by (3) and Proposition 1.10,

$$
\begin{aligned}
\nabla \delta_{\tilde{\pi}}(\tilde{a}) & =d \delta_{\tilde{\pi}}(\tilde{a})(\tilde{\mathrm{F}})+\nabla \sigma_{\tilde{\mathrm{F}}} \mathrm{F}_{\mathrm{X}}^{*}(d a) \\
& =d \delta_{\tilde{\pi}}(\tilde{a})(\tilde{\mathrm{F}})-\zeta_{\tilde{\mathrm{F}}}\left(d \pi^{*}(a)\right)
\end{aligned}
$$


hence

$$
\begin{aligned}
{[p] \nabla \delta_{\tilde{\pi}}(\tilde{a}) } & =d\left([p] \delta_{\tilde{\pi}}(\tilde{a})(\tilde{\mathrm{F}})\right)-[p] \zeta_{\tilde{\mathrm{F}}}\left(d \pi^{*}(a)\right) \\
& =d\left(\tilde{\mathrm{F}}^{*} \tilde{\pi}^{*}(\tilde{a})-\tilde{a}^{p}-(\tilde{\mathrm{F}} \circ \tilde{\pi})^{*}(\tilde{a})\right) \\
& =-d \tilde{a}^{p} \\
& =-[p] a^{p-1} d a .
\end{aligned}
$$

This proves the first equation in (1.14.4). The second follows from the formula for the $p$-curvature $\psi$ in Proposition 1.5; see also Remark 1.11.

The proofs of the formulas of (1.14.5) are straightforward calculations which we leave to the reader.

Remark 1.15. - We have seen that if $\mathrm{F}_{\tilde{\mathrm{S}}}: \tilde{\mathrm{S}} \rightarrow \tilde{\mathrm{S}}$ lifts $\mathrm{F}_{\mathrm{S}}$ and $\tilde{\mathrm{X}}^{\prime}=\tilde{\mathrm{X}} \times_{\mathrm{F}_{\tilde{\mathrm{S}}}} \tilde{\mathrm{S}}$, then the projection $\tilde{\mathrm{X}}^{\prime} \rightarrow \tilde{\mathrm{X}}$ is a natural global choice of a lifting $\tilde{\pi}$ as above. If $\tilde{\mathrm{X}}^{\prime}$ is some other lifting of $\mathrm{X}^{\prime}$, then such a lift $\tilde{\pi}$ will exist locally on $\mathrm{X}$. However in general there may be no lift of $\mathrm{F}_{\mathrm{S}}$ even locally on $\mathrm{S}$, and consequently there may be no lift $\tilde{\pi}$ even locally on $\mathrm{X}$. However, if $\tilde{a} \in \mathscr{O}_{\tilde{\mathrm{X}}}$ is a local lift of $a \in \mathscr{O}_{\mathrm{X}}$, then we can choose a local lift $\tilde{a}^{\prime} \in \mathscr{O}_{\tilde{\mathrm{X}}^{\prime}}$ of $\pi^{*}(a)$. Then the analogs of the formulas in Proposition 1.14 hold with $\tilde{a}^{\prime}$ in place of $\delta_{\tilde{\pi}}(\tilde{a})$.

Let us describe another construction of the fundamental exact sequence (1.4.1). For each $\mathrm{T} \in \operatorname{Cris}(\mathrm{X} / \tilde{\mathrm{S}})$, let $\Gamma$ be the graph of $f_{\mathrm{T} / \mathrm{S}}: \mathrm{T} \rightarrow \mathrm{X}^{\prime}$, and for each lifting $\tilde{\mathrm{F}}: \tilde{\mathrm{T}} \rightarrow \tilde{\mathrm{X}}^{\prime}$ of $f_{\mathrm{T} / \mathrm{S}}$ let $\tilde{\Gamma}$ be the graph of $\tilde{\mathrm{F}}$. Let $\mathrm{J}_{\tilde{\mathrm{T}}}$ be the ideal of the of the immersion

$$
j: \mathrm{T} \stackrel{\Gamma}{\rightarrow} \mathrm{T} \times_{\mathrm{S}} \mathrm{X}^{\prime} \stackrel{i n c}{\rightarrow} \tilde{\mathrm{T}} \times_{\tilde{\mathrm{S}}} \tilde{\mathrm{X}}^{\prime} .
$$

A morphism $\tilde{g}: \tilde{\mathrm{T}}_{1} \rightarrow \tilde{\mathrm{T}}_{2}$ in $\operatorname{Cris}_{f}(\mathrm{X} / \tilde{\mathrm{T}})$ induces a corresponding morphism of conormal sheaves: $g^{*} \mathrm{~J}_{\tilde{\mathrm{T}}_{2}} / \mathrm{J}_{\tilde{\mathrm{T}}_{2}}^{2} \rightarrow \mathrm{J}_{\tilde{\mathrm{T}}_{1}} / \mathrm{J}_{\tilde{\mathrm{T}}_{1}}^{2}$, and so the family $\left\{\mathrm{J}_{\tilde{\mathrm{T}}} / \mathrm{J}_{\tilde{\mathrm{T}}}^{2}: \tilde{\mathrm{T}} \in \operatorname{Cris}_{f}(\mathrm{X} / \tilde{\mathrm{T}})\right\}$ forms a sheaf on $\operatorname{Cris}_{f}(\mathrm{X} / \tilde{\mathrm{S}})$. If $\tilde{\mathrm{F}}: \tilde{\mathrm{T}} \rightarrow \tilde{\mathrm{X}}^{\prime}$ is a lifting of $f_{\mathrm{T} / \mathrm{S}}$ and $\tilde{c}$ is a section of $\mathrm{J}_{\tilde{\mathrm{T}}}$, $\tilde{\Gamma}^{*}(\tilde{c}) \in \mathscr{O}_{\tilde{\mathrm{T}}}$ vanishes on $\mathrm{T}$, and hence is divisible by $p$.

Proposition 1.16. - For each $\tilde{\mathrm{T}} \in \mathrm{Cris}_{f}(\mathrm{X} / \tilde{\mathrm{S}})$, there is a unique morphism

$$
\tilde{\beta}: \mathrm{J}_{\tilde{\mathrm{T}}} \rightarrow \mathrm{J}_{\tilde{\mathrm{T}}} / \mathrm{J}_{\tilde{\mathrm{T}}}^{2} \stackrel{\beta}{\rightarrow} \mathscr{E}_{\mathscr{X} / \mathscr{S}, \tilde{\mathrm{T}}} \quad \tilde{c} \mapsto \tilde{\beta}_{\tilde{c}}
$$

such that for every local lift $\tilde{\mathrm{F}}$ of $f_{\mathrm{T} / \mathrm{S}}$ and every section $\tilde{c}$ of $\mathrm{J}_{\tilde{\mathrm{T}}}$,

$$
[p] \tilde{\beta}_{\tilde{c}}(\tilde{\mathrm{F}})=\Gamma_{\tilde{\mathrm{F}}}^{*}(\tilde{c}) \in \mathscr{O}_{\tilde{\mathrm{T}}} \text {. }
$$

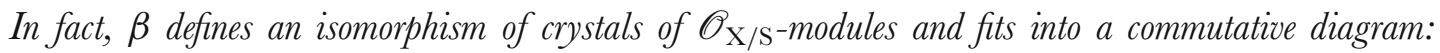

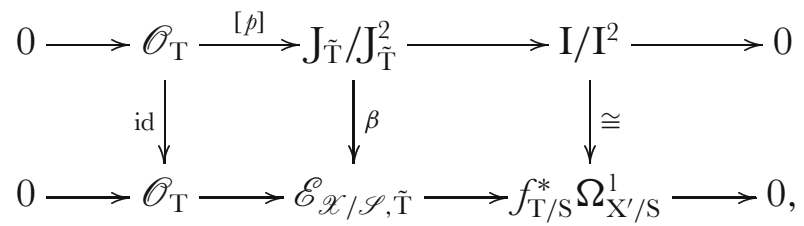

where $\mathrm{I}$ is the ideal of $\Gamma: \mathrm{T} \subseteq \mathrm{T} \times \mathrm{X}^{\prime}$ and the bottom row is the exact sequence (1.4.1). 
Proof. - Suppose for example that $\tilde{a}^{\prime}$ is a local section of $\mathscr{O}_{\tilde{\mathrm{X}}^{\prime}}$ and that $\tilde{b}$ is a local section of $\mathscr{O}_{\tilde{\mathrm{T}}}$ such that $f_{\mathrm{T} / \mathrm{S}}^{*}\left(a^{\prime}\right)=i n c^{*}(\tilde{b})$. Then $\tilde{c}:=1 \otimes \tilde{a}^{\prime}-\tilde{b} \otimes 1$ is a section of $\mathrm{J}_{\tilde{T}}$, and $\mathrm{J}_{\tilde{T}}$ is locally generated by such elements. If $\tilde{\mathrm{F}}$ is any local lift of $f_{\mathrm{T} / \mathrm{S}},[p] \beta_{\tilde{c}}(\tilde{\mathrm{F}})=$ $\tilde{\mathrm{F}}^{*}\left(\tilde{a}^{\prime}\right)-\tilde{b}$. If $\xi^{\prime}$ is a local section of $f_{\mathrm{T} / \mathrm{S}}^{*} \mathrm{~T}_{\mathrm{X}^{\prime} / \mathrm{S}}$ and $\tilde{\mathrm{F}}^{\prime}=\xi^{\prime}+\tilde{\mathrm{F}}^{\prime}$, then $\tilde{\beta}_{\tilde{c}}\left(\tilde{\mathrm{F}}^{\prime}\right)=\tilde{\beta}_{\tilde{c}}(\tilde{\mathrm{F}})+$ $\left\langle\xi^{\prime}, d a^{\prime}\right\rangle$. This shows that $\tilde{\beta}_{\tilde{c}}$ defines a section of $\mathscr{E}_{\mathscr{X} / \mathscr{S}, \mathrm{T}}$. It is clear that $\tilde{\beta}_{\tilde{c}}$ depends only on the class of $\tilde{c} \bmod \mathrm{J}_{\tilde{T}}^{2}$, and so $\tilde{c} \mapsto \beta_{\tilde{c}}$ defines a map $\beta: \mathrm{J}_{\tilde{\mathrm{T}}} / \mathrm{J}_{\tilde{\mathrm{T}}}^{2} \rightarrow \mathscr{E}_{\mathscr{X} / \mathscr{S}, \mathrm{T}}$.

Let us check that the diagram commutes. We may assume that a lifting $\tilde{\mathrm{F}}$ of $f_{\mathrm{T} / \mathrm{S}}$ exists. By definition $\mathrm{I}:=\mathrm{J}_{\tilde{\mathrm{T}}} /(p)$ is the ideal of $\Gamma$. Then $\mathrm{I} / \mathrm{I}^{2} \cong f_{\mathrm{T} / \mathrm{S}}^{*} \Omega_{\mathrm{X}^{\prime} / \mathrm{S}}^{1}$, and the image of $\sigma_{\widetilde{\mathrm{F}}}\left(d a^{\prime}\right)$ in $\mathrm{I} / \mathrm{I}^{2}$ is the class of

$$
1 \otimes a^{\prime}-f_{\mathrm{T} / \mathrm{S}}^{*}\left(a^{\prime}\right) \otimes 1=\left(f_{\mathrm{T} / \mathrm{S}} \times \mathrm{id}\right)^{*}\left(1 \otimes a^{\prime}-a^{\prime} \otimes 1\right),
$$

which corresponds to $f_{\mathrm{T} / \mathrm{S}}^{*}\left(d a^{\prime}\right)$ in $f_{\mathrm{T} / \mathrm{S}}^{*} \Omega_{\mathrm{X}^{\prime} / \mathrm{S}}^{1}$, so that the right square of the diagram commutes. Furthermore, if $\tilde{a}$ is a local section of $\mathscr{O}_{\tilde{T}}$, then $p \tilde{a} \in \mathrm{J}_{\tilde{T}}$ and $\tilde{\beta}_{p \tilde{a}}(\tilde{\mathrm{F}})=$ $\Gamma_{\tilde{\mathrm{F}}}^{*}(p \tilde{a})=[p] a$, where $a$ is the image of $\tilde{a}$ in $\mathscr{O}_{\mathrm{T}}$. This shows that the left square of the diagram also commutes. This implies that the arrow $[p]$ in the diagram is injective. The exactness of the rest of the top row is formal, and it follows that $\beta$ is an isomorphism.

Remark 1.17. - The isomorphism class of the extension of connections in (1.4.1) is an element of $\operatorname{Ext}_{\mathrm{MIC}}^{1}\left(\mathrm{~F}_{\mathrm{X} / \mathrm{S}}^{*} \Omega_{\mathrm{X}^{\prime} / \mathrm{S}}^{1}, \mathscr{O}_{\mathrm{X}}\right)$, and there is a spectral sequence with

$$
\mathrm{E}_{2}^{i, j} \cong \mathrm{H}^{i}\left(\mathrm{X}, \mathscr{E} x t_{\mathrm{MIC}}^{j}\left(\Omega_{\mathrm{X}^{\prime} / \mathrm{S}}^{1}, \mathscr{O}_{\mathrm{X}}\right)\right) \cong \mathrm{H}^{i}\left(\mathrm{X}^{\prime}, \mathrm{T}_{\mathrm{X}^{\prime} / \mathrm{S}} \otimes \Omega_{\mathrm{X}^{\prime} / \mathrm{S}}^{j}\right)
$$

In particular, there is an exact sequence

$$
0 \rightarrow \mathrm{H}^{1}\left(\mathrm{X}^{\prime}, \mathrm{T}_{\mathrm{X}^{\prime} / \mathrm{S}}\right) \rightarrow \mathrm{Ext}_{\mathrm{MIC}}^{1}\left(\mathrm{~F}^{*} \Omega_{\mathrm{X}^{\prime} / \mathrm{S}}^{1}, \mathscr{O}_{\mathrm{X}}\right) \rightarrow \mathrm{H}^{0}\left(\mathrm{X}^{\prime}, \mathrm{T}_{\mathrm{X}^{\prime} / \mathrm{S}} \otimes \Omega_{\mathrm{X}^{\prime} / \mathrm{S}}^{1}\right) .
$$

The extension (1.4.1) has the property that its image in $\mathrm{H}^{0}\left(\mathrm{X}^{\prime}, \mathrm{T}_{\mathrm{X}^{\prime} / \mathrm{S}} \otimes \Omega_{\mathrm{X}^{\prime} / \mathrm{S}}^{1}\right)$ is the identity, and the above exact sequence shows that the set of extension classes with this property is a (pseudo)-torsor under $\mathrm{H}^{1}\left(\mathrm{X}^{\prime}, \mathrm{T}_{\mathrm{X}^{\prime} / \mathrm{S}}\right)$. Note that the same is true of the set of isomorphism classes of liftings of $\mathrm{X}^{\prime} / \mathrm{S}$. We shall investigate this further in Section 4.2.

It is perhaps worthwhile to elucidate the relationship between the fundamental extension (1.4.1) and some more familiar exact sequences. Since the relative Frobenius morphism $\mathrm{F}_{\mathrm{X} / \mathrm{S}}: \mathrm{X} \rightarrow \mathrm{X}^{\prime}$ is a homeomorphism, (1.4.1) remains exact when pushed forward by $\mathrm{F}_{\mathrm{X} / \mathrm{S}}$. Pulling the resulting sequence back by means of the canonical map $\Omega_{\mathrm{X}^{\prime} / \mathrm{S}}^{1} \rightarrow \mathrm{F}_{\mathrm{X} / \mathrm{S} *} \mathrm{~F}_{\mathrm{X} / \mathrm{S}}^{*}\left(\Omega_{\mathrm{X}^{\prime} / \mathrm{S}}^{1}\right)$, one gets an exact sequence

$$
0 \rightarrow \mathrm{F}_{\mathrm{X} / \mathrm{S} *}\left(\mathscr{O}_{\mathrm{X}}\right) \rightarrow \mathscr{E}_{\mathscr{X} / \mathscr{S}}^{\prime} \rightarrow \Omega_{\mathrm{X}^{\prime} / \mathrm{S}}^{1} \rightarrow 0
$$


of locally free sheaves on $\mathrm{X}^{\prime}$. Each local section $e^{\prime}$ of $\mathscr{E}_{\mathscr{X} / \mathscr{S}}$ maps to a horizontal section of $\mathrm{F}_{\mathrm{X} / \mathrm{S}}^{*} \Omega_{\mathrm{X}^{\prime} / \mathrm{S}}^{1}$, and hence $\nabla\left(e^{\prime}\right)$ lies in $\mathscr{O}_{\mathrm{X}} \otimes \Omega_{\mathrm{X}^{\prime} / \mathrm{S}}^{1} \subseteq \mathscr{E}_{\mathscr{X} / \mathscr{S}} \otimes \Omega_{\mathrm{X}^{\prime} / \mathrm{S}}^{1}$. Since $\nabla$ is integrable, in fact $\nabla\left(e^{\prime}\right) \in Z_{\mathrm{X}^{\prime} / \mathrm{S}}^{1}$. Thus, the connection $\nabla$ on $\mathscr{E}_{\mathscr{X} / \mathscr{S}}$ induces an $\mathscr{O}_{\mathrm{X}^{\prime-}}$ linear map $\mathscr{E}_{\mathscr{X} / \mathscr{S}} \rightarrow \mathrm{F}_{\mathrm{X} / \mathrm{S} *} \mathrm{Z}_{\mathrm{X} / \mathrm{S}}^{1}$, which fits into the commutative diagram below:

$(\mathbf{1 . 1 7 . 2})$

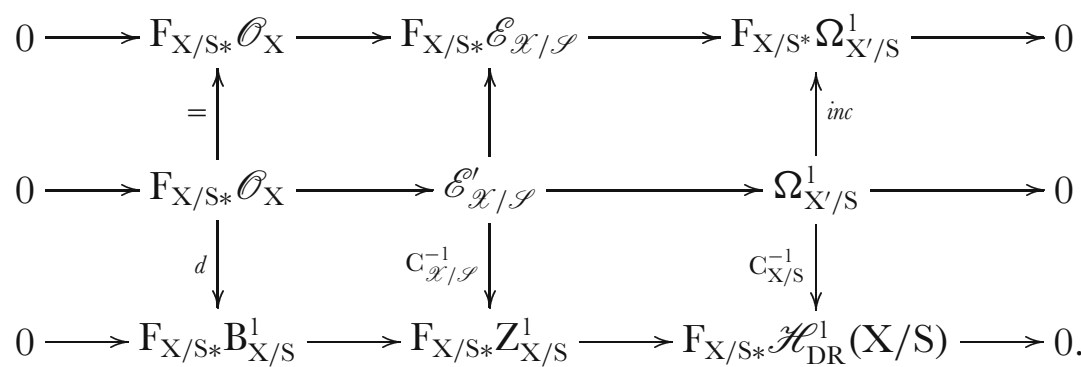

Here the middle row is the pullback of the top row along inc and the familiar bottom row is the pushout of the middle row along $d: \mathrm{F}_{\mathrm{X} / \mathrm{S} *}\left(\mathscr{O}_{\mathrm{X}}\right) \rightarrow \mathrm{F}_{\mathrm{X} / \mathrm{S} *} \mathrm{~B}_{\mathrm{X} / \mathrm{S}}$. Recall that the bottom row is rarely split. Indeed, a splitting would induce an injective map $\Omega_{\mathrm{X}^{\prime} / \mathrm{S}}^{1} \rightarrow \mathrm{F}_{\mathrm{X} / \mathrm{S} *} \mathrm{Z}_{\mathrm{X} / \mathrm{S}}^{1} \rightarrow \mathrm{F}_{\mathrm{X} / \mathrm{S} *} \Omega_{\mathrm{X} / \mathrm{S}}^{1}$ and in particular a nonzero map $\mathrm{F}_{\mathrm{X} / \mathrm{S}}^{*} \Omega_{\mathrm{X}^{\prime} / \mathrm{S}}^{1} \rightarrow \Omega_{\mathrm{X} / \mathrm{S}}^{1}$. For example, no such map can exist on a complete curve of genus at least two over a field.

Note that there is also an exact sequence

$$
0 \rightarrow \mathscr{O}_{\mathrm{X}^{\prime}} \rightarrow \mathrm{F}_{\mathrm{X} / \mathrm{S} *} \mathscr{O}_{\mathrm{X}} \rightarrow \mathrm{F}_{\mathrm{X} / \mathrm{S} *} \mathrm{~B}_{\mathrm{X} / \mathrm{S}} \rightarrow 0
$$

When pulled back to $\mathrm{X}$ this sequence is split by the natural map

$$
s: \mathrm{F}_{\mathrm{X} / \mathrm{S}}^{*} \mathrm{~F}_{\mathrm{X} / \mathrm{S} *} \mathscr{O}_{\mathrm{X}} \rightarrow \mathscr{O}_{\mathrm{X}}
$$

Thus $\mathrm{F}_{\mathrm{X} / \mathrm{S}}^{*} \mathrm{~F}_{\mathrm{X} / \mathrm{S} *}\left(\mathscr{O}_{\mathrm{X}}\right) \cong \mathscr{O}_{\mathrm{X}} \oplus \mathrm{F}_{\mathrm{X} / \mathrm{S}}^{*} \mathrm{~F}_{*}\left(\mathrm{~B}_{\mathrm{X} / \mathrm{S}}\right)$. Furthermore, (1.4.1) is the pushout by $s$ of the pullback by $\mathrm{F}_{\mathrm{X} / \mathrm{S}}^{*}$ of (1.17.1) along s. Warning: the map $s$ is not compatible with the natural connections on the source and target. An S-scheme X/S for which sequence (1.17.3) splits is called F-split [16].

\section{Connections, Higgs fields, and the Cartier transform}

\section{1 $\mathrm{D}_{\mathrm{X} / \mathrm{S}}$ as an Azumaya algebra}

Let $\mathrm{X} / \mathrm{S}$ be a smooth morphism of schemes in characteristic $p>0$. Let $\Omega_{\mathrm{X} / \mathrm{S}}^{1}$ be its sheaf of Kahler differentials, let $\mathrm{T}_{\mathrm{X} / \mathrm{S}}$ be its dual, and let $\mathrm{D}_{\mathrm{X} / \mathrm{S}}$ denote the ring of $\mathrm{PD}$-differential operators of $\mathrm{X} / \mathrm{S}[3, \S 2]$. A section $\mathrm{D}$ of $\mathrm{T}_{\mathrm{X} / \mathrm{S}}$ can be viewed as a derivation of $\mathscr{O}_{\mathrm{X}}$ relative to $\mathrm{S}$ and hence as a $\mathrm{PD}$-differential operator of order less than or equal to 1 , and $\mathrm{D}_{\mathrm{X} / \mathrm{S}}$ is generated as a sheaf of rings over $\mathscr{O}_{\mathrm{X}}$ by $\mathrm{T}_{\mathrm{X} / \mathrm{S}}$. If $\mathrm{E}$ 
is a sheaf of $\mathscr{O}_{\mathrm{X}}$-modules, then to give an integrable connection $\nabla: \mathrm{E} \rightarrow \mathrm{E} \otimes \Omega_{\mathrm{X} / \mathrm{S}}^{1}$ is the same as to give an extension of the action of $\mathscr{O}_{\mathrm{X}}$ on $\mathrm{E}$ to an action of $\mathrm{D}_{\mathrm{X} / \mathrm{S}}$ $[3,4.8]$, which we continue to denote by $\nabla$. The $p$ th iterate $\mathrm{D}^{(p)}$ of a derivation is again a derivation, hence a section of $\mathrm{T}_{\mathrm{X} / \mathrm{S}}$ and an operator of order less than or equal to 1 . This is in general not the same as the $p$ th power $\mathrm{D}^{p}$ of $\mathrm{D}$, which is an operator of order less than or equal to $p$, even though $\mathrm{D}^{(p)}$ and $\mathrm{D}^{p}$ have the same effect on sections of $\mathscr{O}_{\mathrm{X}}$. For each derivation $\mathrm{D}$, let

$$
c(\mathrm{D}):=\mathrm{D}^{p}-\mathrm{D}^{(p)} \text {. }
$$

One can show either by calculating in local coordinates [4] or by means of techniques from noncommutative algebra [18], that $c$ is an $\mathrm{F}_{\mathrm{X}}^{*}$-linear map from $\mathrm{T}_{\mathrm{X} / \mathrm{S}}$ to the center $\mathscr{Z}_{\mathrm{X} / \mathrm{S}}$ of $\mathrm{D}_{\mathrm{X} / \mathrm{S}}$. By adjunction, one deduces from $c$ an $\mathscr{O}_{\mathrm{X}^{\prime} / \mathrm{S}}$-linear map

$$
c^{\prime}: \mathrm{T}_{\mathrm{X}^{\prime} / \mathrm{S}} \rightarrow \mathrm{F}_{\mathrm{X} / \mathrm{S} *} \mathscr{Z}_{\mathrm{X} / \mathrm{S}} \quad: \quad \mathrm{D}^{\prime} \mapsto c^{\prime}\left(\mathrm{D}^{\prime}\right):=\left(1 \otimes \mathrm{D}^{\prime}\right)^{p}-\left(1 \otimes \mathrm{D}^{\prime}\right)^{(p)} .
$$

Let $\nabla$ be an integrable connection on $\mathrm{E}$ and $\psi: \mathrm{E} \rightarrow \mathrm{E} \otimes \mathrm{F}_{\mathrm{X} / \mathrm{S}}^{*} \Omega_{\mathrm{X}^{\prime} / \mathrm{S}}^{1}$ be its $p$-curvature. It follows from the definitions that for every local section $\mathrm{D}^{\prime}$ of $\mathrm{T}_{\mathrm{X}^{\prime} / \mathrm{S}}, \psi_{\mathrm{D}^{\prime}}$ is the endomorphism of $\mathrm{E}$ induced by the differential operator $c^{\prime}\left(\mathrm{D}^{\prime}\right)$. This mapping satisfies the linearity and integrability conditions of a Higgs field with $\mathrm{F}_{\mathrm{X} / \mathrm{S}}^{*} \Omega_{\mathrm{X}^{\prime} / \mathrm{S}}^{1}$ in place of $\Omega_{\mathrm{X} / \mathrm{S}}^{1}$. We refer to such a map as an F-Higgs field on $\mathrm{E}$, and we denote by

$$
\Psi: \mathrm{MIC}(\mathrm{X} / \mathrm{S}) \rightarrow \mathrm{F}-\mathrm{HIG}(\mathrm{X} / \mathrm{S})
$$

the functor taking $(\mathrm{E}, \nabla)$ to $(\mathrm{E}, \psi)$.

Since $c^{\prime}$ maps to the center of $\mathrm{F}_{\mathrm{X} / \mathrm{S} *} \mathrm{D}_{\mathrm{X} / \mathrm{S}}$, it extends to a map from the symmetric algebra $\mathrm{S}^{\cdot} \mathrm{T}_{\mathrm{X}^{\prime} / \mathrm{S}}$ to $\mathscr{Z}_{\mathrm{X} / \mathrm{S}}$, and in particular makes $\mathrm{F}_{\mathrm{X} / \mathrm{S} *} \mathrm{D}_{\mathrm{X} / \mathrm{S}}$ into a sheaf of $\mathrm{S}^{\bullet} \mathrm{T}_{\mathrm{X}^{\prime} / \mathrm{S}}$-modules. Let $\mathbf{T}_{\mathrm{X}^{\prime} / \mathrm{S}}^{*}:=\mathrm{Spec}_{\mathrm{X}^{\prime}} \mathrm{S}^{\cdot} \mathrm{T}_{\mathrm{X}^{\prime} / \mathrm{S}}$ be the cotangent bundle of $\mathrm{X}^{\prime} / \mathrm{S}$. Since $\mathrm{F}_{\mathrm{X} / \mathrm{S} *} \mathrm{D}_{\mathrm{X} / \mathrm{S}}$ is quasi-coherent as a sheaf of $\mathscr{O}_{\mathrm{X}^{\prime}}$-modules, it defines a quasi-coherent sheaf $\mathscr{D} \mathrm{X} / \mathrm{S}$ on $\mathbf{T}_{\mathrm{X}^{\prime} / \mathrm{S}}^{*}$.

Recall that an Azumaya algebra over a scheme $\mathrm{Y}$ is a sheaf of associative algebras A such that locally for the fppf topology, A is isomorphic to $\operatorname{End}_{\mathscr{O}_{\mathrm{Y}}}\left(\mathscr{O}_{\mathrm{Y}}^{n}\right)$. More generally, if $\mathrm{Y}$ is a topological space, $\mathrm{R}$ is a sheaf of commutative rings on $\mathrm{Y}$, and $\mathrm{A}$ is a sheaf of associative R-algebras which is locally free and finite rank as an R-module, we say that $\mathrm{A}$ is an Azumaya algebra over $\mathrm{R}$ if the canonical map $\mathrm{A} \otimes \mathrm{A}^{o p} \rightarrow \operatorname{End}_{\mathrm{R}}(\mathrm{A})$ is an isomorphism. One can show that if $\mathrm{Y}$ is a scheme and $\mathrm{R}=\mathscr{O}_{\mathrm{Y}}$, then these definitions agree. (See Chapter 4 of [23] for a quick review.)

Our starting point in this section is the following theorem of [4], which asserts that $\mathscr{D} \mathrm{X} / \mathrm{S}$ is an Azumaya algebra on $\mathbf{T}_{\mathrm{X}^{\prime} / \mathrm{S}}^{*}$.

Theorem 2.1. - Let X/S be a smooth $\mathrm{S}$-scheme of relative dimension d. Then the map (2.0.2) induces an isomorphism:

$$
\mathrm{S}^{\cdot} \mathrm{T}_{\mathrm{X}^{\prime} / \mathrm{S}} \stackrel{\cong}{\longrightarrow} \mathrm{F}_{\mathrm{X} / \mathrm{S} *} \mathscr{Z}_{\mathrm{X} / \mathrm{S}} .
$$


This morphism makes $\mathrm{F}_{\mathrm{X} / \mathrm{S} *} \mathrm{D}_{\mathrm{X} / \mathrm{S}}$ an Azumaya algebra over $\mathrm{S}^{\cdot} \mathrm{T}_{\mathrm{X}^{\prime} / \mathrm{S}}$ of rank $p^{2 d}$. The corresponding sheaf $\mathscr{D}_{\mathrm{X} / \mathrm{S}}$ of $\mathscr{O}_{\mathbf{T}_{\mathrm{X}^{\prime} / \mathrm{s}}^{*}}$-algebras on $\mathbf{T}_{\mathrm{X}^{\prime} / \mathrm{S}}^{*}$ is canonically split (isomorphic to a matrix algebra) when pulled back via the map $\pi_{\mathrm{T}}$ in the diagram below:

$(\mathbf{2 . 1 . 1})$

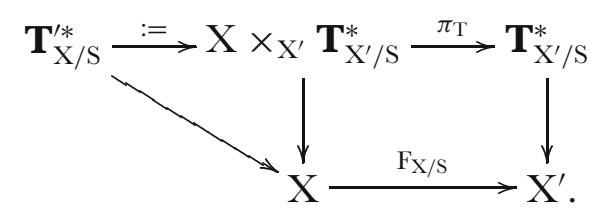

Proof. - We recall here only the main idea of the proof, referring to [4] for the details. Let $\mathrm{M}_{\mathrm{X} / \mathrm{S}}:=\mathrm{F}_{\mathrm{X} / \mathrm{S} *} \mathrm{D}_{\mathrm{X} / \mathrm{S}}$ which we can view as a module over $\mathscr{O}_{\mathbf{T}_{\mathrm{X} / \mathrm{S}}^{\prime *}}=$ $\mathrm{F}_{\mathrm{X} / \mathrm{S} *} \mathscr{Z}_{\mathrm{X} / \mathrm{S}} \otimes_{\mathscr{O}_{\mathrm{X}}}, \mathrm{F}_{\mathrm{X} / \mathrm{S} *} \mathscr{O}_{\mathrm{X}}$ via right multiplication and the inclusion $\mathscr{O}_{\mathrm{X}} \rightarrow \mathrm{D}_{\mathrm{X} / \mathrm{S}}$ as well as a left module over itself. These left and right actions agree on the center $\mathscr{Z}_{\mathrm{X} / \mathrm{s}}$, and hence they define a homomorphism of sheaves of rings

$$
\mathrm{F}_{\mathrm{X} / \mathrm{S} *} \mathrm{D}_{\mathrm{X} / \mathrm{S}} \otimes_{\mathrm{S}^{\cdot} \mathrm{T}_{\mathrm{X}^{\prime} / \mathrm{S}}} \mathscr{O}_{\mathrm{T}_{\mathrm{X} / \mathrm{S}}^{\prime *}} \rightarrow \mathscr{E}^{n} d_{\mathscr{O}_{\mathrm{T}_{\mathrm{X} / \mathrm{S}}^{\prime *}}}\left(\mathrm{M}_{\mathrm{X} / \mathrm{S}}\right)
$$

which one can check is an isomorphism in local coordinates.

Observe that if $\operatorname{dim} \mathrm{X} / \mathrm{S}>0$, then $\mathscr{D}_{\mathrm{X} / \mathrm{S}}$ is not split locally in the Zariski topology of $\mathbf{T}_{\mathrm{X}^{\prime} / \mathrm{S}}^{*}$. It suffices to check this when $\mathrm{S}$ is the spectrum of a field and $\mathrm{X}$ is affine. Then $\Gamma\left(\mathrm{X}, \mathrm{D}_{\mathrm{X} / \mathrm{S}}\right)$ has no zero divisors, because its associated graded sheaf with respect to the filtration by order is canonically isomorphic to the symmetric algebra $\mathrm{S}^{\cdot} \mathrm{T}_{\mathrm{X} / \mathrm{S}}$. Since $\mathbf{T}_{\mathrm{X}^{\prime} / \mathrm{S}}^{*}$ is integral and $\mathscr{D} \mathrm{X} / \mathrm{S}$ is locally free as an $\mathscr{O}_{\mathbf{T}_{\mathrm{X}^{\prime} / \mathrm{S}}^{*}}$-module, it also has no zero divisors and hence is not split.

Remark 2.2. - The power of Theorem 2.1 can be seen from its application to Cartier descent [18]. Consider the action of $\mathrm{D}_{\mathrm{X} / \mathrm{S}}$ on $\mathscr{O}_{\mathrm{X}}$. Since $\mathrm{D}^{p}$ and $\mathrm{D}^{(p)}$ agree on $\mathscr{O}_{\mathrm{X}}$, this action kills the ideal $\mathrm{S}^{+} \mathrm{T}_{\mathrm{X}^{\prime} / \mathrm{S}}$ of $\mathrm{S}^{\cdot} \mathrm{T}_{\mathrm{X}^{\prime} / \mathrm{S}}$. Thus $\mathrm{F}_{\mathrm{X} / \mathrm{S} *}\left(\mathscr{O}_{\mathrm{X}}\right)$ can be viewed as a sheaf of $i^{*} \mathscr{D}_{\mathrm{X} / \mathrm{S}}$ modules, where $i: \mathrm{X}^{\prime} \rightarrow \mathbf{T}_{\mathrm{X}^{\prime} / \mathrm{S}}^{*}$ is the zero section. Since $i^{*} \mathscr{D} \mathrm{X} / \mathrm{S}$ is an Azumaya algebra over $\mathrm{X}^{\prime}$ of rank $p^{2 d}$ and $\mathrm{F}_{\mathrm{X} / \mathrm{S} *}\left(\mathscr{O}_{\mathrm{X}}\right)$ has $\operatorname{rank} p^{d}$, this shows that $i^{*} \mathscr{D}_{\mathrm{X} / \mathrm{S}}$ is split, and that tensoring with the splitting module $\mathrm{F}_{\mathrm{X} / \mathrm{S} *}\left(\mathscr{O}_{\mathrm{X}}\right)$ induces an equivalence between the category of $\mathscr{O}_{\mathrm{X}^{\prime}}$-modules and the category of $\mathrm{D}_{\mathrm{X} / \mathrm{S}}$-modules for which the action of $\mathrm{S}^{+} \mathrm{T}_{\mathrm{X}^{\prime} / \mathrm{S}}$ is zero. This is just the category of $\mathscr{O}_{\mathrm{X}}$-modules endowed with an integrable connection whose $p$-curvature is zero.

Let $\mathrm{D}_{\mathrm{X} / \mathrm{S}}^{\ell}$ be the commutative subalgebra of $\mathrm{D}_{\mathrm{X} / \mathrm{S}}$ generated by the left inclusion $\mathscr{O}_{\mathrm{X}} \rightarrow \mathrm{D}_{\mathrm{X} / \mathrm{S}}$ and its center. Then $\mathrm{F}_{\mathrm{X} / \mathrm{S} *} \mathrm{D}_{\mathrm{X} / \mathrm{S}}^{\ell}$ defines a quasi-coherent sheaf of algebras $\mathscr{D}_{\mathrm{X} / \mathrm{S}}^{\ell}$ on $\mathbf{T}_{\mathrm{X}^{\prime} / \mathrm{S}}^{*}$. In fact, it is easy to check that the natural map $\mathrm{F}_{\mathrm{X} / \mathrm{S}}^{*} \mathrm{~S}^{\cdot} \mathrm{T}_{\mathrm{X}^{\prime} / \mathrm{S}} \rightarrow \mathscr{D}_{\mathrm{X} / \mathrm{S}}^{\ell}$ is an isomorphism, so that $\operatorname{Spec}_{\mathbf{T}_{\mathrm{X}^{\prime} / \mathrm{S}}^{*}} \mathscr{D}_{\mathrm{X} / \mathrm{S}}^{\ell} \cong \mathbf{T}_{\mathrm{X} / \mathrm{S}}^{*}$ (see Diagram (2.1.1)). In particular, a sheaf $\mathrm{M}$ of $\mathrm{D}_{\mathrm{X} / \mathrm{S}}$-modules which is quasi-coherent over $\mathrm{X}$ can be viewed as a quasicoherent sheaf of $\mathscr{O}_{\mathbf{T}_{\mathrm{X} / \mathrm{S}}^{*}}$-modules. 
Proposition 2.3. - Let $f: \mathrm{Z} \rightarrow \mathbf{T}_{\mathrm{X}^{\prime} / \mathrm{S}}^{*}$ be a morphism and suppose $\mathrm{L}$ is a splitting module for $f^{*} \mathscr{D}_{\mathrm{X} / \mathrm{s}}$. Then $\mathrm{L}$, viewed as a sheaf of $f^{*} \mathscr{D}_{\mathrm{X} / \mathrm{S}}^{\ell}-$ modules, is locally free of rank one.

Proof. - First let us prove this when $f=\pi_{\mathrm{T}}$ and $\mathrm{L}=\mathrm{M}_{\mathrm{X} / \mathrm{S}}$. Our claim is that $\mathrm{M}_{\mathrm{X} / \mathrm{S}}:=\mathscr{D}_{\mathrm{X} / \mathrm{S}}$ is locally free of rank one over $\pi_{\mathrm{T}}^{*} \mathscr{D}_{\mathrm{X} / \mathrm{S}}^{\ell}=\mathscr{O}_{\mathrm{X}} \otimes \mathrm{S}^{*} \mathrm{~T}_{\mathrm{X}^{\prime} / \mathrm{S}} \otimes \mathscr{O}_{\mathrm{X}}$, where the first $\mathscr{O}_{\mathrm{X}}$ acts by multiplication on the left and the second on the right and the tensor products are taken over $\mathscr{O}_{\mathrm{X}^{\prime}}$. We may assume that we have a system of local coordinates $\left(t_{1}, \ldots, t_{d}\right)$ for $\mathrm{X} / \mathrm{S}$, with a corresponding set of generators $\mathrm{D}_{i}$ for $\mathrm{D}_{\mathrm{X} / \mathrm{s}}$. Then the product $\mathrm{D}_{1}^{p-1} \cdots \mathrm{D}_{d}^{p-1}$ generates $\mathrm{M}_{\mathrm{X} / \mathrm{S}}$ as a module over $\pi_{\mathrm{T}}^{*} \mathscr{D}_{\mathrm{X} / \mathrm{S}}^{\ell}$, as one sees from the fact that $\left[\mathrm{D}_{i}, t_{j}\right]=\delta_{i j}$. This generator defines a surjective map $\pi_{\mathrm{T}}^{*} \mathscr{D}_{\mathrm{X} / \mathrm{S}}^{\ell} \rightarrow \mathrm{M}_{\mathrm{X} / \mathrm{S}}$, and since the source and target of this map are locally free $\mathscr{O}_{\mathrm{X}^{\prime}}$-modules of the same rank, it is an isomorphism.

To deduce the general statement, note that it is enough to prove the claim about $\mathrm{L}$ after a faithfully flat cover, and in particular after a base extension induced by $\pi_{\mathrm{T}}$. Thus we can replace $\mathrm{Z}$ by $\mathrm{Z} \times_{\mathbf{T}_{\mathrm{X}^{\prime} / \mathrm{S}}^{*}} \mathbf{T}_{\mathrm{X} / \mathrm{S}}^{*} \cong \mathrm{Z} \times_{\mathrm{X}^{\prime}} \mathrm{X}$. The pullback of $\mathrm{M}_{\mathrm{X} / \mathrm{S}}$ to this space has the desired property, and $\mathrm{L}$ is necessarily locally isomorphic to $\mathrm{M}_{\mathrm{X} / \mathrm{S}}$. This concludes the proof.

Let us recall that the category of left $\mathrm{D}_{\mathrm{X} / \mathrm{S}}$-modules is equipped with a tensor structure. In Section 5.5 we will discuss this structure from the point of view of Azumaya algebras.

\subsection{An étale splitting of $\mathrm{D}_{\mathrm{X} / \mathrm{S}}$}

The proof of Theorem 2.1 gives an explicit flat covering of $\mathbf{T}_{\mathrm{X}^{\prime} / \mathrm{S}}^{*}$ which splits $\mathscr{D}_{\mathrm{X} / \mathrm{S}}$. It follows from the general theory of Azumaya algebras that there exist étale coverings over which it is split. In this section we will give an explicit construction of such a covering, which in fact is a surjective étale endomorphism of the group scheme $\mathbf{T}_{\mathrm{X}^{\prime} / \mathrm{S}}^{*}$.

The construction of the splitting depends on a choice $\zeta$ of a splitting of the Cartier operator $\mathrm{C}_{\mathrm{X} / \mathrm{S}}$, as exhibited in Diagram (1.9.2). In order to express the formulas we shall encounter geometrically, we introduce the following notation. The map $\zeta$ induces by adjunction a map $\mathrm{F}_{\mathrm{X} / \mathrm{S}}^{*} \Omega_{\mathrm{X}^{\prime} / \mathrm{S}}^{1} \rightarrow \Omega_{\mathrm{X} / \mathrm{S}}^{1}$ whose dual is a map $\phi: \mathrm{T}_{\mathrm{X} / \mathrm{S}} \rightarrow$ $\mathrm{F}_{\mathrm{X} / \mathrm{S}}^{*} \mathrm{~T}_{\mathrm{X}^{\prime} / \mathrm{S}}$. Pulling back by $\pi_{\mathrm{X} / \mathrm{S}}$, we find an $\mathscr{O}_{\mathrm{X}^{\prime}}$-linear map $\phi^{\prime}: \mathrm{T}_{\mathrm{X}^{\prime} / \mathrm{S}} \rightarrow \mathrm{F}_{\mathrm{X}^{\prime}}^{*} \mathrm{~T}_{\mathrm{X}^{\prime} / \mathrm{S}}$. We let $h_{\zeta}$ be the composite of the map of vector bundles induced by $\phi^{\prime}$ with the relative Frobenius map for the $\mathrm{X}^{\prime}$-scheme $\mathbf{T}_{\mathrm{X}^{\prime} / \mathrm{S}}^{*}$, as displayed in the diagram below.

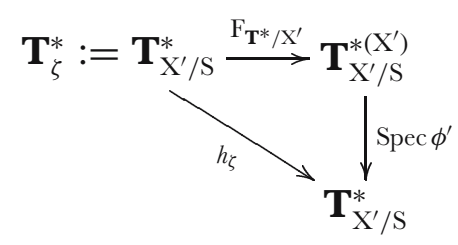


This morphism is a homomorphism of affine group schemes over $\mathrm{X}^{\prime}$, but it is not compatible with the vector bundle structures. We shall see that $\alpha_{\zeta}:=h_{\zeta}-$ id is surjective and étale and that the Azumaya algebra $\mathscr{D}_{\mathrm{X} / \mathrm{S}}$ splits when pulled back via $\alpha_{\zeta}$.

Recall from Remark 1.11 that associated to a splitting $\zeta$ there is an object $\left(\mathscr{E}_{\zeta}, \nabla\right)$ of $\mathrm{MIC}(\mathrm{X} / \mathrm{S})$, where $\mathscr{E}_{\zeta}=\mathscr{O}_{\mathrm{X}} \oplus \mathrm{F}_{\mathrm{X} / \mathrm{S}}^{*} \Omega_{\mathrm{X}^{\prime} / \mathrm{S}}^{1}$. The connection $\nabla$ on $\mathscr{E}_{\zeta}$ induces a connection on each $\mathrm{S}^{n} \mathscr{E}_{\zeta}$, compatibly with the inclusion maps $\mathrm{S}^{n} \mathscr{E}_{\zeta} \rightarrow \mathrm{S}^{n+1} \mathscr{E}_{\zeta}$ induced by the map $\mathscr{O}_{\mathrm{X}} \rightarrow \mathscr{E}_{\zeta}$, and hence also on the direct limit $\mathscr{A}_{\zeta}:=\lim _{\rightarrow} \mathrm{S}^{n} \mathscr{E}_{\zeta}$. The splitting $\sigma: \mathscr{E}_{\zeta} \rightarrow \mathscr{O}_{\mathrm{X}}$ defines an isomorphism of $\mathscr{O}_{\mathrm{X}}$-algebras $\mathscr{A}_{\zeta} \cong \mathrm{F}_{\mathrm{X} / \mathrm{S}}^{*} \mathrm{~S} \Omega_{\mathrm{X}^{\prime} / \mathrm{S}}^{1}$ and the submodule $\mathrm{F}_{\mathrm{X} / \mathrm{S}}^{*} \Omega_{\mathrm{X}^{\prime} / \mathrm{S}}^{1}$ generates an ideal $\mathrm{I}_{\zeta}$ of $\mathscr{A}_{\zeta}$, which we can identify with $\mathrm{S}^{+} \mathrm{F}_{\mathrm{X} / \mathrm{S}}^{*} \Omega_{\mathrm{X}^{\prime} / \mathrm{S}}^{1}$. By $[3,6.2]$, the completed PD-envelope $\hat{\mathscr{A}}_{\zeta}^{\gamma}$ of this ideal has a natural structure of a crystal of $\mathscr{O}_{\mathrm{X} / \mathrm{S}}$-modules, so the connection $\nabla$ on $\mathscr{A}_{\zeta}$ extends canonically to a connection $\nabla_{\zeta}$ on $\hat{\mathscr{A}}_{\zeta}^{\gamma}$. Furthermore, if $a$ is a local section of $\overline{\mathrm{I}}_{\zeta}$, then $\nabla_{\zeta} a^{[n]}=a^{[n-1]} \nabla_{\zeta}(a)$, and $\nabla_{\zeta}$ maps $\overline{\mathrm{I}}_{\zeta}^{[n]}$ to $\overline{\mathrm{I}}_{\zeta}^{[n-1]} \otimes \Omega_{\mathrm{X} / \mathrm{S}}^{1}$. The algebra $\mathscr{A}_{\zeta} \cong \mathrm{F}_{\mathrm{X} / \mathrm{S}}^{*} \mathrm{~S}^{\cdot} \Omega_{\mathrm{X}^{\prime} / \mathrm{S}}^{1}$ also has a canonical F-Higgs field $\theta$ : if $\xi$ is a local section of $\mathrm{T}_{\mathrm{X}^{\prime} / \mathrm{s}}$ and $\omega^{\prime}$ a local section of $\Omega_{\mathrm{X}^{\prime} / \mathrm{S}}^{1}, \theta_{\xi}\left(\omega^{\prime}\right)=\left\langle\xi, \omega^{\prime}\right\rangle$, and the action of $\theta_{\xi}$ on the higher symmetric powers is determined by the Leibnitz rule. In fact, as we saw in Remark 1.11, this F-Higgs field is also the $p$-curvature of the connection $\mathscr{A}_{\zeta} \cong \mathrm{S}^{\cdot} \mathrm{F}_{\mathrm{X} / \mathrm{S}}^{*} \Omega_{\mathrm{X}^{\prime} / \mathrm{S}}^{1}$. This field extends to the divided power envelope $\mathscr{A}_{\zeta}^{\gamma}$ and its completion $\hat{\mathscr{A}}_{\zeta}^{\gamma}$ : the pairing

$$
\mathrm{S}^{n} \mathrm{~F}_{\mathrm{X} / \mathrm{S}}^{*} \mathrm{~T}_{\mathrm{X}^{\prime} / \mathrm{S}} \otimes \Gamma_{n+m} \mathrm{~F}_{\mathrm{X} / \mathrm{S}}^{*} \Omega_{\mathrm{X}^{\prime} / \mathrm{S}}^{1} \rightarrow \Gamma_{m} \mathrm{~F}_{\mathrm{X} / \mathrm{S}}^{*} \Omega_{\mathrm{X}^{\prime} / \mathrm{S}}^{1}
$$

comes from the multiplication on the symmetric algebra and the duality between the symmetric and divided power algebras explained for example in [3, A10]. In particular, if $\xi \in \mathrm{T}_{\mathrm{X}^{\prime} / \mathrm{S}}$ and $\omega \in \Omega_{\mathrm{X}^{\prime} / \mathrm{S}}^{1}$, one has

$$
\xi \omega^{[i]}=\langle\xi, \omega\rangle \omega^{[i-1]} \quad \text { and hence } \quad \xi^{p} \omega^{[i]}=\langle\xi, \omega\rangle^{p} \omega^{[i-p]} \text {. }
$$

Let

$$
\mathscr{B}_{\zeta}:=\lim _{\longrightarrow} \mathscr{H}_{0} m_{\mathscr{O}_{\mathrm{X}}}\left(\mathscr{A}_{\zeta}^{\gamma} / \overline{\mathrm{I}}^{[n]}, \mathscr{O}_{\mathrm{X}}\right)
$$

be the topological dual of $\hat{\mathscr{A}}_{\zeta}^{\gamma}$, equipped with the dual connection and F-Higgs field (5.5.1). Thus $\mathscr{B}_{\zeta} \cong \bigoplus \mathrm{S}^{n} \mathrm{~F}_{\mathrm{X} / \mathrm{S}}^{*} \mathrm{~T}_{\mathrm{X}^{\prime} / \mathrm{S}}$ as an $\mathscr{O}_{\mathrm{X}}$-module. Because of the sign in the definition of the dual Higgs field, a section of $\xi$ of $\mathrm{T}_{\mathrm{X}^{\prime} / \mathrm{S}}$ acts on $\mathrm{B}_{\zeta}$ as multiplication by $-\xi$. The $\mathrm{F}_{\mathrm{X} / \mathrm{S}}^{*} \mathrm{~S}^{\cdot} \mathrm{T}_{\mathrm{X}^{\prime} / \mathrm{S}}$-structure of $\mathrm{B}_{\zeta}$ corresponding to this field identifies it with $\iota_{*} \mathrm{~F}_{\mathrm{X} / \mathrm{S}}^{*} \mathrm{~S}^{\cdot} \mathrm{T}_{\mathrm{X}^{\prime} / \mathrm{S}}$, where $\iota: \mathbf{T}_{\mathrm{X}^{\prime} / \mathrm{S}}^{*} \rightarrow \mathbf{T}_{\mathrm{X}^{\prime} / \mathrm{S}}^{*}$ is the involution $t \rightarrow-t$ of the vector group $\mathbf{T}_{\mathrm{X}^{\prime} / \mathrm{S}}$. Note that $\nabla$ is compatible with the algebra structure of $\mathscr{A}_{\zeta}$ and with the divided power algebra structure of $\hat{\mathscr{A}}_{\zeta}^{\gamma}$. It is not, however, compatible with the algebra structure of $\mathscr{B}_{\zeta}$, but rather with its coalgebra structure. 
Remark 2.4. - If $\zeta$ comes from a lifting $\tilde{\mathrm{F}}$ of $\mathrm{F}_{\mathrm{X} / \mathrm{S}}$ as in (1.9.1), we can give a geometric interpretation of the construction of $\mathscr{B}_{\zeta}$ as follows. Let $\left(\mathscr{A}_{\tilde{\mathrm{F}}}^{\gamma}, \overline{\mathrm{I}}\right)$ be the divided power envelope of the the ideal I of the section of $\mathscr{L}_{\mathscr{X} / \mathscr{S}}$ corresponding to $\tilde{\mathrm{F}}$. Recall from Proposition 1.4 that $\mathscr{A}_{\mathscr{X} / \mathscr{S}}$ has a connection $\nabla$ as well as an action of $\mathrm{F}_{\mathrm{X} / \mathrm{S}}^{*} \mathrm{~S}^{\cdot} \mathrm{T}_{\mathrm{X}^{\prime} / \mathrm{S}}$, the latter via its identification with the ring of translation invariant PDdifferential operators. Both the connection $\nabla$ and the action of $\mathrm{F}_{\mathrm{X} / \mathrm{S}}^{*} \mathrm{~T}_{\mathrm{X}^{\prime} / \mathrm{S}}$ extend naturally to $\mathscr{A}_{\tilde{\mathrm{F}}}^{\gamma}$ and to its PD-completion $\hat{\mathscr{A}}_{\tilde{\mathrm{F}}}^{\gamma}$. Then $\hat{\mathscr{A}}_{\zeta}^{\gamma}$ can be identified with the $\hat{\mathscr{A}}_{\tilde{\mathrm{F}}}^{\gamma}$ and $\mathscr{B}_{\zeta}$ with its topological dual. It is clear from the definitions that these identifications are compatible with the $\mathrm{F}_{\mathrm{X} / \mathrm{S}}^{*} \mathrm{~S}^{\cdot} \mathrm{T}_{\mathrm{X}^{\prime} / \mathrm{S}}$-module structure, and Proposition 1.10 shows that they are also compatible with the connections.

Proposition 2.5. - Let $\mathrm{X} / \mathrm{S}$ be a smooth morphism of schemes in characteristic $p>0$ with a splitting $\zeta$ of $\mathrm{C}_{\mathrm{X} / \mathrm{S}}^{-1}$, and let $h_{\zeta}$ and $\mathscr{B}_{\zeta}:=\mathrm{F}_{\mathrm{X} / \mathrm{S}}^{*} \mathrm{~S}^{\cdot} \mathrm{T}_{\mathrm{X}^{\prime} / \mathrm{S}}$ with the connection $\nabla_{\zeta}$ described above.

1. The map:

$$
\alpha_{\zeta}:=\mathrm{id}-h_{\zeta}: \mathbf{T}_{\zeta}^{*}:=\mathbf{T}_{\mathrm{X}^{\prime} / \mathrm{S}}^{*} \rightarrow \mathbf{T}_{\mathrm{X}^{\prime} / \mathrm{S}}^{*}
$$

is a surjective étale morphism of affine group schemes over $\mathrm{X}^{\prime}$.

2. The action of an element $\xi^{\prime}$ of $\mathrm{S}^{\prime} \mathrm{T}_{\mathrm{X}^{\prime} / \mathrm{S}}$ on $\mathscr{B}_{\zeta}$ defined by its $p$-curvature is multiplication by $\alpha_{\zeta}^{*}\left(\xi^{\prime}\right)$.

Proof. - We have already observed that $h_{\zeta}$ is a morphism of group schemes, and consequently so is $\alpha_{\zeta}$. Since $h_{\zeta}$ factors through the relative Frobenius map, its differential vanishes, and it follows that $\alpha_{\zeta}$ is étale. Then the images under $\alpha_{\zeta}$ of the geometric fibers of $\mathbf{T}_{\zeta}^{*} / \mathrm{X}^{\prime}$ are open subgroups of the fibers of $\mathbf{T}_{\mathrm{X}^{\prime} / \mathrm{S}}^{*} / \mathrm{X}^{\prime}$. Hence the image of each fiber of $\mathbf{T}_{\zeta}^{*} / \mathrm{X}^{\prime}$ must contain the entire corresponding fiber of $\mathbf{T}_{\mathrm{X}^{\prime} / \mathrm{S}}^{*} / \mathrm{X}^{\prime}$, and so $\alpha_{\zeta}$ is surjective. Thus $\alpha_{\zeta}$ is an étale covering (but not necessarily an étale cover, since it need not be a finite morphism).

We must next compute the $p$-curvature of the divided power envelope $\mathscr{A}_{\zeta}^{\gamma} \cong$ $\Gamma . \mathrm{F}_{\mathrm{X} / \mathrm{S}}^{*} \Omega_{\mathrm{X}^{\prime} / \mathrm{S}}^{1}$ of $\mathscr{A}_{\zeta}$. Let $\omega^{\prime}$ be a local section of $\Omega_{\mathrm{X}^{\prime} / \mathrm{S}}^{1}$, so that $x:=\left(0,1 \otimes \omega^{\prime}\right)$ belongs to the divided power ideal of $\mathscr{A}_{\zeta}^{\gamma}$. Let $\mathrm{D}$ be a local section of $\mathrm{T}_{\mathrm{X} / \mathrm{S}}$ and let $\xi^{\prime}:=$ $\pi_{\mathrm{X} / \mathrm{S}}^{*} \mathrm{D} \in \mathrm{T}_{\mathrm{X}^{\prime} / \mathrm{S}}$. Then $\phi(\mathrm{D}) \in \mathrm{F}_{\mathrm{X} / \mathrm{S}}^{*} \mathrm{~T}_{\mathrm{X}^{\prime} / \mathrm{S}}$, and we shall need the following formula.

Claim 2.6. - $\phi(\mathrm{D})^{p}=\mathrm{F}_{\mathrm{X} / \mathrm{S}}^{*} h_{\zeta}^{*}\left(\xi^{\prime}\right) \in \mathrm{S}^{\cdot} \mathrm{F}_{\mathrm{X} / \mathrm{S}}^{*} \mathrm{~T}_{\mathrm{X}^{\prime} / \mathrm{S}}$.

To check this, let $\mathbf{T}^{*}:=\mathrm{Spec}_{\mathrm{X}^{\prime}} \mathrm{S}^{\cdot} \mathrm{T}_{\mathrm{X}^{\prime} / \mathrm{S}}$ and let $\tilde{\mathbf{T}}^{*}$ denote its pullback to $\mathrm{X}$ via the map $\mathrm{F}_{\mathrm{X} / \mathrm{S}}$, i.e., $\tilde{\mathbf{T}}^{*}=\mathrm{Spec}_{\mathrm{X}} \mathrm{F}_{\mathrm{X} / \mathrm{S}}^{*} \mathrm{~S}^{\cdot} \mathrm{T}_{\mathrm{X}^{\prime} / \mathrm{S}}$. Then there is a commutative diagram:

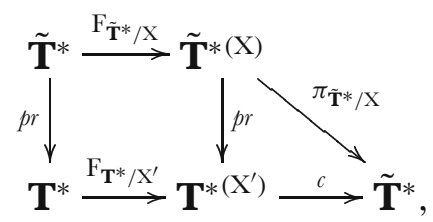


where the morphism $c$ is the projection

$$
\mathbf{T}^{*\left(\mathrm{X}^{\prime}\right)}:=\mathbf{T}^{*} \times_{\mathrm{F}_{\mathrm{X}^{\prime}}} \mathrm{X}^{\prime} \cong \tilde{\mathbf{T}}^{*} \times_{\pi_{\mathrm{X} / \mathrm{S}}} \mathrm{X}^{\prime} \rightarrow \tilde{\mathbf{T}}^{*} .
$$

Let us view $\phi(\mathrm{D})$ as a section of $\mathscr{O}_{\tilde{\mathbf{T}}^{*}}$. Then $c^{*} \phi(\mathrm{D})=\phi^{\prime}\left(\xi^{\prime}\right)$, so

$$
\begin{aligned}
(\phi(\mathrm{D}))^{p} & =\mathrm{F}_{\mathbf{T}^{*}}^{*}(\phi(\mathrm{D})) \\
& =\mathrm{F}_{\tilde{\mathbf{T}}^{*} / \mathrm{X}^{\prime}}^{*} \pi_{\tilde{\mathbf{T}}^{*} / \mathrm{X}}^{*}(\phi(\mathrm{D})) \\
& =\mathrm{F}_{\widetilde{\mathbf{T}}^{*} / \mathrm{X}^{\prime}}^{*} p r^{*}\left(\phi^{\prime}\left(\xi^{\prime}\right)\right) \\
& =p r^{*} \mathrm{~F}_{\mathbf{T}^{*} / \mathrm{X}^{\prime}}^{*}\left(\phi^{\prime}\left(\xi^{\prime}\right)\right) \\
& =p r^{*} h_{\zeta}^{*}\left(\xi^{\prime}\right) .
\end{aligned}
$$

Since the map $p r$ in the diagram corresponds to pullback by $\mathrm{F}_{\mathrm{X} / \mathrm{S}}$, the claim is proved.

By the definition of the connection on $\mathscr{E}_{\zeta} \subseteq \mathscr{A}_{\zeta}^{\gamma}$ given in Remark 1.11 and of the morphism $\phi$,

$$
\begin{aligned}
\nabla_{\mathrm{D}}(x) & =\nabla_{\mathrm{D}}\left(0,1 \otimes \omega^{\prime}\right)=\left(\left\langle\mathrm{D},-\zeta\left(1 \otimes \omega^{\prime}\right)\right\rangle, 0\right) \\
& =-\left(\left\langle\phi(\mathrm{D}), 1 \otimes \omega^{\prime}\right\rangle, 0\right) \\
& =-\phi(\mathrm{D}) x \in \mathscr{A}_{\zeta}^{\gamma} .
\end{aligned}
$$

The formula $[29,6.1 .1]$ for the $p$-curvature of divided powers and the computation of the $p$-curvature of $\mathscr{E}_{\zeta}$ (Proposition 1.5), then say:

$$
\begin{aligned}
\psi_{\xi^{\prime}}\left(x^{[i]}\right) & =x^{[i-1]} \otimes \psi_{\xi^{\prime}}(x)+x^{[i-p]}\left(\nabla_{\mathrm{D}}(x)\right)^{p} \\
& =\left\langle\xi^{\prime}, x\right\rangle x^{[i-1]}-x^{[i-p]}(\phi(\mathrm{D}) x)^{p} \\
& =\xi^{\prime} x^{[i]}-(\phi(\mathrm{D}))^{p}\left(x^{[i]}\right) \\
& =\left(\xi^{\prime}-h_{\zeta}^{*}\left(\xi^{\prime}\right)\right) x^{[i]} \\
& =-\alpha_{\zeta}^{*}\left(\xi^{\prime}\right) x^{[i]} .
\end{aligned}
$$

Since $\mathscr{B}_{\zeta} \subseteq \mathscr{H} 0 m\left(\mathscr{A}_{\zeta}^{\gamma}, \mathscr{O}_{\mathrm{X}}\right)$ as a module with connection, the second part of Proposition 2.5 follows from the formula for the $p$-curvature of the dual of a connection; see for example Lemma 5.27.

We can now show that $\mathscr{D} \mathrm{X} / \mathrm{S}$ splits when pulled back by $\alpha_{\zeta}$. Since $\mathbf{T}_{\zeta}^{*}=\mathbf{T}_{\mathrm{X}^{\prime} / \mathrm{S}}^{*}$, $\mathrm{F}_{\mathrm{X} / \mathrm{S} *} \mathscr{B}_{\zeta}$ can also be viewed as a quasi-coherent sheaf on $\mathbf{T}_{\zeta}^{*}$.

Theorem 2.7. - There is a unique action of $\alpha_{\zeta}^{*}\left(\mathscr{D}_{\mathrm{X} / \mathrm{S}}\right)$ on $\mathrm{F}_{\mathrm{X} / \mathrm{S} *} \mathscr{B}_{\zeta}$ extending the actions of $\alpha_{\zeta}^{-1}\left(\mathscr{D}_{\mathrm{X} / \mathrm{S}}\right)$ and of $\mathscr{O}_{\mathbf{T}_{\zeta}^{*}}$. The resulting module splits the Azumaya algebra $\alpha_{\zeta}^{*}\left(\mathscr{D}_{\mathrm{X} / \mathrm{S}}\right)$.

Proof. - Proposition 2.5 shows that the actions of $\mathrm{S}^{\cdot} \mathrm{T}_{\mathrm{X}^{\prime} / \mathrm{S}}$ on $\mathrm{F}_{\mathrm{X} / \mathrm{S} *} \mathscr{B}_{\zeta}$ defined on the one hand through the $p$-curvature homomorphism $\mathrm{S}^{\cdot} \mathrm{T}_{\mathrm{X}^{\prime} / \mathrm{S}} \rightarrow \mathrm{D}_{\mathrm{X} / \mathrm{S}}$ and through $\alpha_{\zeta}$ agree, and hence that the action of $\mathscr{D} \mathrm{X} / \mathrm{s}$ extends canonically to an action 
of $\alpha_{\zeta}^{*} \mathscr{D} \mathrm{X} / \mathrm{S}$. Since $\mathscr{B}_{\zeta}=\mathrm{F}_{\mathrm{X} / \mathrm{S}}^{*} \mathscr{B}_{\zeta}^{\prime}$ and $\mathscr{B}_{\zeta}^{\prime} \cong \mathrm{S}^{\cdot} \mathrm{T}_{\mathrm{X}^{\prime} / \mathrm{S}}, \mathscr{B}_{\zeta}$ is locally free of rank $p^{d}$ over $\mathbf{T}_{\zeta}^{*}$. Hence it is a splitting module for the Azumaya algebra $\alpha_{\zeta}^{*} \mathscr{D} \mathrm{x} / \mathrm{s}$.

\subsection{The Cartier transform}

In this section we explain how a lifting of $\mathrm{F}_{\mathrm{X} / \mathrm{S}}: \mathrm{X} \rightarrow \mathrm{X}^{\prime}$ or just of $\mathrm{X}^{\prime} / \mathrm{S}$ modulo $p^{2}$ determines splittings of $\mathscr{D} \mathrm{x} / \mathrm{S}$ on suitable neighborhoods of the zero section of $\mathbf{T}_{\mathrm{X}^{\prime} / \mathrm{S}}^{*}$. We then use these splittings to define characteristic $p$ analogs of the Simpson correspondence.

Let us begin with the global construction. Suppose we are given a lifting $\tilde{\mathrm{X}}^{\prime} / \tilde{\mathrm{S}}$ of $\mathrm{X}^{\prime} / \mathrm{S}$; and as before, let $\mathscr{X} / \mathscr{S}$ denote the pair $\left(\mathrm{X} / \mathrm{S}, \tilde{\mathrm{X}}^{\prime} / \tilde{\mathrm{S}}\right)$. The sheaf $\Gamma \cdot \mathrm{T}_{\mathrm{X}^{\prime} / \mathrm{S}}$ has a canonical divided power structure and can be identified with the divided power envelope $\mathbf{T}_{\mathrm{X}^{\prime} / \mathrm{S}}^{* \gamma}$ of the zero section of the cotangent bundle $\mathbf{T}_{\mathrm{X}^{\prime} / \mathrm{S}}^{*}$ of $\mathrm{X}^{\prime} / \mathrm{S}$. Its completion $\hat{\Gamma} . \mathrm{T}_{\mathrm{X}^{\prime} / \mathrm{S}}$ with respect to the PD-filtration $\left\{\overline{\mathrm{I}}^{[n]}: n \in \mathbf{N}\right\}$ can be viewed as the sheaf of functions on the formal scheme $\hat{\mathbf{T}}_{\mathrm{X}^{\prime} / \mathrm{S}}^{* \gamma}$. The topology on the structure sheaf is defined by the PD-filtration and is admissible $[14,7.1 .2]$ but not adic, and its underlying topological space is $\mathrm{X}^{\prime}$. It inherits the structure of a formal group scheme from the group structure of $\mathbf{T}_{\mathrm{X}^{\prime} / \mathrm{S}}^{*}$, and the group law is a PD-morphism. If $\mathbf{T}_{n}^{* \gamma}$ is the closed subscheme defined by $\overline{\mathrm{I}}^{[n+1]}$, the group law factors through maps $\mathbf{T}_{n}^{* \gamma} \times \mathbf{T}_{m}^{* \gamma} \rightarrow \mathbf{T}_{n+m}^{* \gamma}$ for all $n, m$. We shall denote by $\mathrm{HIG}_{\gamma}\left(\mathrm{X}^{\prime} / \mathrm{S}\right)$ the category of sheaves of $\hat{\Gamma} \cdot \mathrm{T}_{\mathrm{X}^{\prime} / \mathrm{S}}$-modules and by $\mathrm{HIG}_{\gamma}^{\cdot}\left(\mathrm{X}^{\prime} / \mathrm{S}\right)$ the full subcategory of locally PD-nilpotent modules, i.e., those with the property that each local section is annihilated by some $\overline{\mathrm{I}}^{[n]}$. As explained in Definition 5.3 and (more abstractly) in Section 5.5 of the appendix, the group law on $\hat{\mathbf{T}}_{\mathrm{X}^{\prime} / \mathrm{S}}^{* \gamma}$ defines a tensor structure (convolution) on the category $\mathrm{HIG}_{\gamma}\left(\mathrm{X}^{\prime} / \mathrm{S}\right)$. If $\mathrm{HIG}_{\gamma}^{n}$ denotes the category of $\mathscr{O}_{\mathbf{T}_{n}^{* \gamma}}$-modules, the convolution factors through functors

$$
\mathrm{HIG}_{\gamma}^{m}\left(\mathrm{X}^{\prime} / \mathrm{S}\right) \times \mathrm{HIG}_{\gamma}^{n}\left(\mathrm{X}^{\prime} / \mathrm{S}\right) \rightarrow \mathrm{HIG}_{\gamma}^{m+n}\left(\mathrm{X}^{\prime} / \mathrm{S}\right) .
$$

If $\mathrm{E}_{1}$ and $\mathrm{E}_{2}$ are objects of $\mathrm{HIG}_{\gamma}(\mathrm{X} / \mathrm{S})$ and $\xi$ is a local section of $\mathrm{T}_{\mathrm{X}^{\prime} / \mathrm{S}}$, then the total PD-Higgs field on the tensor product satisfies

$$
\psi_{\xi^{[n]}}=\sum_{i+j=n} \psi_{\xi^{[i]}} \otimes \psi_{\xi^{[j]}} .
$$

Note that $\psi_{\left.\xi^{[}[]\right]}$can be nonzero even if $\mathrm{E}_{1}$ and $\mathrm{E}_{2}$ have level less than $p$. Note also that this total PD-Higgs field commutes with the Higgs fields id $\otimes \psi$ and $\psi \otimes$ id. If $\mathrm{E}_{1} \in \mathrm{HIG}_{\gamma}^{m}\left(\mathrm{X}^{\prime} / \mathrm{S}\right)$ and $\mathrm{E}_{2} \in \mathrm{HIG}_{\gamma}^{n}\left(\mathrm{X}^{\prime} / \mathrm{S}\right)$, then $\mathscr{H}_{0 m_{\mathbb{O}_{\mathrm{X}}}}\left(\mathrm{E}_{1}, \mathrm{E}_{2}\right) \in \mathrm{HIG}_{\gamma}^{m+n}(\mathrm{X} / \mathrm{S})$, with the unique PD-Higgs field satisfying:

$$
\psi_{\xi^{[n]}}(h)=\sum_{i+j=n}(-1)^{j} \psi_{\xi^{[i]}} \circ h \circ \psi_{\xi^{[j]}} .
$$


See Section 5.5 for a geometric explanation of this formula. More generally, if $\mathrm{E}_{1}$ is locally PD-nilpotent, then $\mathrm{E}_{1}=\underset{\lim }{\longrightarrow} \mathrm{N}_{k} \mathrm{E}_{1}$, where $\mathrm{N}_{k} \mathrm{E}_{1}$ is the subsheaf of sections annihilated by $\overline{\mathrm{I}}^{[k+1]}$, and if $\mathrm{E}_{2} \in \mathrm{HIG}_{\gamma}^{n}(\mathrm{X} / \mathrm{S})$ for some $n$, then

$$
\mathscr{H}_{0} m_{\mathscr{O}_{\mathrm{X}}}\left(\mathrm{E}_{1}, \mathrm{E}_{2}\right) \cong \lim \mathscr{H}_{0} m_{\mathscr{O}_{\mathrm{X}}}\left(\mathrm{N}_{k} \mathrm{E}_{1}, \mathrm{E}_{2}\right)
$$

has a natural structure of a $\hat{\Gamma} \cdot T_{\mathrm{X}^{\prime} / \mathrm{S}}$ module, but it may not be locally PD-nilpotent.

Let $\mathrm{D}_{\mathrm{X} / \mathrm{S}}^{\gamma}$ denote the tensor product

$$
\mathrm{D}_{\mathrm{X} / \mathrm{S}}^{\gamma}:=\mathrm{D}_{\mathrm{X} / \mathrm{S}} \otimes_{\mathrm{S}} \cdot \mathrm{T}_{\mathrm{X}^{\prime} / \mathrm{S}} \hat{\Gamma} \cdot\left(\mathrm{T}_{\mathrm{X}^{\prime} / \mathrm{S}}\right)
$$

via the map $\mathrm{S}^{\cdot} \mathrm{T}_{\mathrm{X}^{\prime} / \mathrm{S}} \rightarrow \mathrm{D}_{\mathrm{X} / \mathrm{S}}$ induced by the $p$-curvature mapping $c^{\prime}$ (2.0.2). The category $\mathrm{MIC}_{\gamma}(\mathrm{X} / \mathrm{S})$ of $\mathrm{D}_{\mathrm{X} / \mathrm{S}}^{\gamma}$-modules on $\mathrm{X}$ is equivalent to the category of sheaves of $\mathscr{O}_{\mathrm{X}}$-modules $\mathrm{E}$ equipped with a connection $\nabla$ and a horizontal homomorphism

$$
\psi: \hat{\Gamma} \cdot\left(\mathrm{T}_{\mathrm{X}^{\prime} / \mathrm{S}}\right) \rightarrow \mathrm{F}_{\mathrm{X} / \mathrm{S} *} \mathscr{E} n d_{\mathscr{O}_{\mathrm{X}}}(\mathrm{E}, \nabla)
$$

which extends the Higgs field

$$
\mathrm{S}^{\bullet} \mathrm{T}_{\mathrm{X}^{\prime} / \mathrm{S}} \rightarrow \mathrm{F}_{\mathrm{X} / \mathrm{S} *} \mathscr{E}_{n} d_{\mathscr{O}_{\mathrm{X}}}(\mathrm{E}, \nabla)
$$

given by the $p$-curvature of $\nabla$. We write $\mathrm{MIC}_{\gamma}(\mathrm{X} / \mathrm{S})$ for the full subcategory of locally nilpotent objects, those for which each local section is locally annihilated by $\Gamma^{i} \mathrm{~T}_{\mathrm{X}^{\prime} / \mathrm{S}}$ for $i \gg 0$. For example, $\mathscr{O}_{\mathrm{X}}$ has an obvious structure of a $\mathrm{D}_{\mathrm{X} / \mathrm{S}}^{\gamma}$-module. More generally, if $(\mathrm{E}, \nabla)$ is a module with integrable connection whose $p$-curvature is nilpotent of level less than $p,(\mathrm{E}, \nabla)$ can be viewed as an object of $\mathrm{MIC}_{\gamma}(\mathrm{X} / \mathrm{S})$ by letting the $p$ th divided power of the ideal $\Gamma^{+} \mathrm{T}_{\mathrm{X}^{\prime} / \mathrm{S}}$ act as zero.

The convolution product on $\mathrm{HIG}_{\gamma}^{\cdot}\left(\mathrm{X}^{\prime} / \mathrm{S}\right)$ allows us to make the category $\mathrm{MIC}_{\gamma}(\mathrm{X} / \mathrm{S})$ into a tensor category. If $\mathrm{E}_{1}$ and $\mathrm{E}_{2}$ are objects of $\mathrm{MIC}_{\gamma}(\mathrm{X} / \mathrm{S})$ and $\xi$ is a local section of $\mathrm{T}_{\mathrm{X}^{\prime} / \mathrm{S}}$, then the total PD-Higgs field on the tensor product satisfies Equation (2.7.1). Since these endomorphisms are horizontal and since this formula agrees with the $p$-curvature of a tensor product when $n=1$, it does indeed define an object of $\mathrm{MIC}_{\gamma}(\mathrm{X} / \mathrm{S})$. If $\mathrm{E}_{1} \in \mathrm{MIC}_{\gamma}(\mathrm{X} / \mathrm{S})$ and $\mathrm{E}_{2} \in \mathrm{MIC}_{\gamma}^{n}(\mathrm{X} / \mathrm{S})$ for some $n$, then $\mathscr{H}_{0} m_{\mathscr{O}_{\mathrm{X}}}\left(\mathrm{E}_{1}, \mathrm{E}_{2}\right) \in \mathrm{MIC}_{\gamma}(\mathrm{X} / \mathrm{S})$, with the usual connection rule and the action of $\hat{\Gamma} \cdot\left(\mathrm{T}_{\mathrm{X}^{\prime} / \mathrm{S}}\right)$ defined above.

In order to keep our sign conventions consistent with other constructions ${ }^{6}$, we have found it convenient to introduce a twist. Let $\iota: \mathbf{T}_{\mathrm{X}^{\prime} / \mathrm{S}}^{*} \rightarrow \mathbf{T}_{\mathrm{X}^{\prime} / \mathrm{S}}^{*}$ be the inverse operation in the group law. Then $\iota^{*}=\iota_{*}$ is an involutive autoequivalence of the tensor category $\operatorname{HIG}\left(\mathrm{X}^{\prime} / \mathrm{S}\right)$. If $\left(\mathrm{E}^{\prime}, \psi^{\prime}\right) \in \operatorname{HIG}\left(\mathrm{X}^{\prime} / \mathrm{S}\right)$,

$$
\left(\mathrm{E}^{\prime}, \psi^{\prime}\right)^{\iota}:=\iota_{*}\left(\mathrm{E}^{\prime}, \psi^{\prime}\right)=\iota^{*}\left(\mathrm{E}^{\prime}, \psi^{\prime}\right)=\left(\mathrm{E}^{\prime},-\psi^{\prime}\right)
$$

\footnotetext{
${ }^{6}$ See for example Remark 2.15 below.
} 
Recall that in Theorem 1.1 we constructed an algebra $\mathscr{A}_{\mathscr{X} / \mathscr{S}}$ from the torsor of Frobenius liftings $\mathscr{L}_{\mathscr{X} / \mathscr{S}}$. We have seen in Proposition 1.5 that the $p$-curvature of $\left(\mathscr{A}_{\mathscr{X} / \mathscr{S}}, \nabla_{\mathscr{A}}\right)$ coincides with the action of $\mathrm{S}^{\cdot} \mathrm{T}_{\mathrm{X}^{\prime} / \mathrm{S}}$ coming from the torsor structure and hence that it extends naturally to a continuous divided power Higgs field $\psi_{\mathscr{A}}$. Thus $\mathscr{A}_{\mathscr{X} / \mathscr{S}}$ can be regarded as an element of $\mathrm{MIC}_{\gamma}(\mathrm{X} / \mathrm{S})$. Let $\mathscr{B}_{\mathscr{X} / \mathscr{S}}$ be its $\mathscr{O}_{\mathrm{X}}-$ linear dual, which makes sense as an object of $\mathrm{MIC}_{\gamma}(\mathrm{X} / \mathrm{S})$ (although it does not lie in $\left.\mathrm{MIC}_{\gamma}(\mathrm{X} / \mathrm{S})\right)$.

Theorem 2.8. - Let $\mathscr{X} / \mathscr{S}:=\left(\mathrm{X} / \mathrm{S}, \tilde{\mathrm{X}}^{\prime} / \tilde{\mathrm{S}}\right)$ be a smooth morphism together with a lift of $\mathrm{X}^{\prime} / \mathrm{S}$ modulo $p^{2}$.

1. The $\mathrm{D}_{\mathrm{X} / \mathrm{S}}^{\gamma}$-module $\mathscr{B}_{\mathscr{X} / \mathscr{S}}$ described above is a splitting module for the Azumaya algebra $\mathrm{F}_{\mathrm{X} / \mathrm{S} *}\left(\mathrm{D}_{\mathrm{X} / \mathrm{S}}^{\gamma}\right)$ over $\hat{\Gamma} \cdot\left(\mathrm{T}_{\mathrm{X}^{\prime} / \mathrm{S}}\right)$.

2. The functor

$$
\begin{aligned}
& \mathrm{C}_{\mathscr{X} / \mathscr{S}}: \mathrm{MIC}_{\gamma}(\mathrm{X} / \mathrm{S}) \rightarrow \mathrm{HIG}_{\gamma}\left(\mathrm{X}^{\prime} / \mathrm{S}\right) \\
& \mathrm{E} \mapsto \iota^{*} \mathscr{H}_{0} m_{\mathrm{D}_{\mathrm{X} / \mathrm{S}}^{\gamma}}\left(\mathscr{B}_{\mathscr{X} / \mathscr{S}}, \mathrm{E}\right)
\end{aligned}
$$

defines an equivalence of categories, with quasi-inverse

$$
\begin{aligned}
& \mathrm{C}_{\mathscr{X} / \mathscr{S}}^{-1}: \mathrm{HIG}_{\gamma}\left(\mathrm{X}^{\prime} / \mathrm{S}\right) \rightarrow \mathrm{MIC}_{\gamma}(\mathrm{X} / \mathrm{S}) \\
& \mathrm{E}^{\prime} \mapsto \mathscr{B}_{\mathscr{X} / \mathscr{S}} \otimes_{\hat{\Gamma} \cdot \mathrm{T}_{\mathrm{X}^{\prime} / \mathrm{S}}} \iota^{*} \mathrm{E}^{\prime} .
\end{aligned}
$$

Furthermore, $\mathrm{C}_{\mathscr{X} / \mathscr{S}}$ induces an equivalence of tensor categories:

$$
\mathrm{MIC}_{\gamma}^{\cdot}(\mathrm{X} / \mathrm{S}) \rightarrow \mathrm{HIG}_{\gamma}^{\cdot}\left(\mathrm{X}^{\prime} / \mathrm{S}\right)
$$

3. Let $(\mathrm{E}, \nabla)$ be an object of $\mathrm{MIC}_{\gamma}(\mathrm{X} / \mathrm{S})$, let $\psi$ be its $p$-curvature, and let $\left(\mathrm{E}^{\prime}, \psi^{\prime}\right):=$ $\mathrm{C}_{\mathscr{X} / \mathscr{S}}(\mathrm{E}, \nabla) . A$ lifting $\tilde{\mathrm{F}}$ of $\mathrm{F}_{\mathrm{X} / \mathrm{S}}$, if it exists, induces a natural isomorphism

$$
\eta_{\tilde{\mathrm{F}}}:(\mathrm{E}, \psi) \cong \mathrm{F}_{\mathrm{X} / \mathrm{S}}^{*}\left(\mathrm{E}^{\prime},-\psi^{\prime}\right) .
$$

Proof. - To prove that $\mathscr{B}_{\mathscr{X} / \mathscr{S}}$ is a splitting module for $\mathrm{D}_{\mathrm{X} / \mathrm{S}}^{\gamma}$, it suffices to show that it is locally free of rank $p^{d}$ over the center $\hat{\Gamma} \cdot \mathrm{T}_{\mathrm{X}^{\prime} / \mathrm{S}}$ of $\mathrm{D}_{\mathrm{X} / \mathrm{S}}^{\gamma}$ As we have already observed, the action of this center coincides with the action coming from the torsor structure as described in Proposition 1.4. Since $\mathscr{A}_{\mathscr{X} / \mathscr{S}}$ is coinvertible by op. cit., $\mathscr{B}_{\mathscr{X} / \mathscr{S}}$ is locally free of rank one over $\mathrm{F}_{\mathrm{X} / \mathrm{S}}^{*} \hat{\Gamma} \cdot \mathrm{T}_{\mathrm{X}^{\prime} / \mathrm{S}}$, and hence is locally free of rank $p^{d}$ over $\hat{\Gamma} \cdot \mathrm{T}_{\mathrm{X}^{\prime} / \mathrm{S}}$. It then follows from the general theory of matrix algebras that $\mathscr{H}_{0} m_{\mathrm{D}_{\mathrm{X} / \mathrm{S}}^{\gamma}}\left(\mathscr{B}_{\mathscr{X} / \mathscr{S}},\right)$ and $\mathscr{B}_{\mathscr{X} / \mathscr{S}} \otimes_{\hat{\Gamma} . \mathrm{T}_{\mathrm{X}^{\prime} / \mathrm{s}}}$ are quasi-inverse equivalences of categories. Since $\iota_{*}$ is an involutive equivalence, the functors $\mathrm{C}_{\mathscr{X} / \mathscr{S}}$ and $\mathrm{C}_{\mathscr{X} / \mathscr{S}}^{-1}$ are also quasiinverse equivalences.

The algebra structure of $\mathscr{A}_{\mathscr{X} / \mathscr{S}}$ endows $\mathscr{B}_{\mathscr{X} / \mathscr{S}}$ with the structure of a coalgebra with counit. As explained in Proposition 5.29, this gives $\mathscr{B}_{\mathscr{X} / \mathscr{S}}$ the structure of 
a tensor splitting and makes $\mathscr{H} 0 m\left(\mathscr{B}_{\mathscr{X} / \mathscr{S}}, \quad\right)$ a tensor functor; the compatibility isomorphism

$$
\mathrm{C}_{\mathscr{X} / \mathscr{S}}\left(\mathrm{E}_{1}\right) \otimes \mathrm{G}_{\mathscr{X} / \mathscr{S}}\left(\mathrm{E}_{2}\right) \rightarrow \mathrm{G}_{\mathscr{X} / \mathscr{S}}\left(\mathrm{E}_{1} \otimes \mathrm{E}_{2}\right)
$$

comes from the diagram:

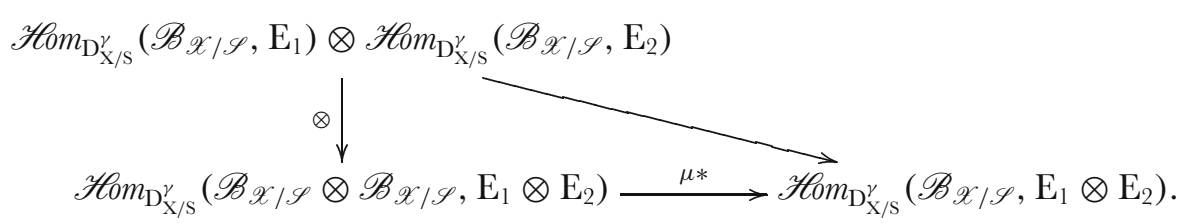

Since $\iota$ is a group morphism, $\iota_{*}$ is also compatible with the tensor structure.

A lifting $\tilde{\mathrm{F}}$ of $\mathrm{F}_{\mathrm{X}^{\prime} / \mathrm{S}}$ defines a trivialization of the torsor $\mathscr{L}_{\mathscr{X} / \mathscr{S}}$ and hence isomorphisms of $\hat{\Gamma} \cdot \mathrm{T}_{\mathrm{X}^{\prime} / \mathrm{S}}$-modules

$$
\mathscr{A}_{\mathscr{X} / \mathscr{S}} \cong \mathrm{F}_{\mathrm{X} / \mathrm{S}}^{*} \mathrm{~S}^{\cdot} \Omega_{\mathrm{X}^{\prime} / \mathrm{S}}, \quad \mathscr{B}_{\mathscr{X} / \mathscr{S}} \cong \mathrm{F}_{\mathrm{X} / \mathrm{S}}^{*} \hat{\Gamma} \cdot \mathrm{T}_{\mathrm{X}^{\prime} / \mathrm{S}}
$$

Then

$$
\mathrm{E} \cong \iota_{*} \mathrm{E}^{\prime} \otimes_{\hat{\Gamma} \cdot \mathrm{T}_{\mathrm{X}^{\prime} / \mathrm{S}}} \mathscr{B}_{\mathscr{X} / \mathscr{S}} \cong \iota_{*} \mathrm{E}^{\prime} \otimes_{\hat{\Gamma} \cdot \mathrm{T}_{\mathrm{X}^{\prime} / \mathrm{S}}} \mathrm{F}_{\mathrm{X} / \mathrm{S}}^{*} \hat{\Gamma} \cdot \mathrm{T}_{\mathrm{X}^{\prime} / \mathrm{S}} \cong \mathrm{F}_{\mathrm{X} / \mathrm{S}}^{*} \iota_{*} \mathrm{E}^{\prime}
$$

as $\mathrm{F}_{\mathrm{X} / \mathrm{S}}^{*} \hat{\Gamma}^{\cdot} \mathrm{T}_{\mathrm{X}^{\prime} / \mathrm{S}}$-modules. Statement (3) follows.

Corollary 2.9. - With the notation of Theorem 2.8, the Azumaya algebra $\mathrm{F}_{\mathrm{X} / \mathrm{S} *} \mathrm{D}_{\mathrm{X} / \mathrm{S}}$ splits on the $(p-1)$ st infinitesimal neighborhood of the zero section of $\mathbf{T}_{\mathrm{X}^{\prime} / \mathrm{S}}^{*}$.

Remark 2.10. - Although the source and target of the isomorphism $\eta_{\tilde{\mathrm{F}}}$ in part (3) of Theorem 2.8, are independent of $\tilde{\mathrm{F}}, \eta_{\tilde{\mathrm{F}}}$ itself is not. Indeed, let $\tilde{\mathrm{F}}_{2}$ and $\tilde{\mathrm{F}}_{1}$ be two liftings of $\mathrm{F}_{\mathrm{X} / \mathrm{S}}$, differing by a section $\xi$ of $\mathrm{F}_{\mathrm{X} / \mathrm{S}}^{*} \mathrm{~T}_{\mathrm{X}^{\prime} / \mathrm{S}}$. Then one can form $e^{\xi}$ in the completed divided power envelope $\mathrm{F}_{\mathrm{X} / \mathrm{S}}^{*} \hat{\Gamma} \mathrm{T}_{\mathrm{X}^{\prime} / \mathrm{S}}$. Since $\mathrm{E}^{\prime} \in \mathrm{HIG}_{\gamma}\left(\mathrm{X}^{\prime} / \mathrm{S}\right), e^{\xi}$ acts naturally on $\mathrm{F}_{\mathrm{X} / \mathrm{S}}^{*} \mathrm{E}^{\prime}$, and we have the formula

$$
\eta_{\tilde{\mathrm{F}}_{2}}=e^{\xi} \circ \eta_{\tilde{\mathrm{F}}_{1}}
$$

This follows from the fact that the isomorphism of Theorem 2.8 is induced by the section of $\mathscr{L}_{\mathscr{X} / \mathscr{S}}$ defined by $\tilde{\mathrm{F}}$ and the formula (1.3.1) for the action by translation of $\mathrm{F}_{\mathrm{X} / \mathrm{S}}^{*} \mathrm{~T}_{\mathrm{X}^{\prime} / \mathrm{S}}$ on $\mathscr{A}_{\mathscr{X} / \mathscr{S}}$.

A lifting $\tilde{\mathrm{F}}$ of $\mathrm{F}_{\mathrm{X} / \mathrm{S}}$, if it exists, allows us to extend the equivalence of Theorem 2.8 to the category $\mathrm{MIC}^{*}(\mathrm{X} / \mathrm{S})$ of all locally nilpotent connections. As explained in $[3,4.4,4.12]$, objects of this category give rise to modules over the ring $\hat{\mathrm{D}}_{\mathrm{X} / \mathrm{S}}$ of 
hyper-PD-differential operators. This ring can be identified with the tensor product of $\mathrm{D}_{\mathrm{X} / \mathrm{S}}$ with the completion $\hat{\mathrm{S}}^{\cdot} \mathrm{T}_{\mathrm{X}^{\prime} / \mathrm{S}}$ of $\mathrm{S}^{\cdot} \mathrm{T}_{\mathrm{X}^{\prime} / \mathrm{S}}$ along the ideal of the zero section, and $\mathrm{F}_{\mathrm{X} / \mathrm{S} *} \hat{\mathrm{D}}_{\mathrm{X} / \mathrm{S}}$ can be viewed as an Azumaya algebra over the sheaf of rings $\hat{\mathrm{S}}^{\cdot} \mathrm{T}_{\mathrm{X}^{\prime} / \mathrm{S}}$, or equivalently, over the formal completion $\hat{\mathbf{T}}_{\mathrm{X}^{\prime} / \mathrm{S}}$ of the cotangent space of $\mathrm{X}^{\prime} / \mathrm{S}$ along its zero section. Let $\mathrm{MIC}_{\infty}(\mathrm{X} / \mathrm{S})$ denote the category of sheaves of $\hat{\mathrm{D}}_{\mathrm{X} / \mathrm{S}}$-modules on $\mathscr{O}_{\mathrm{X}}$, and let $\mathrm{HIG}_{\infty}\left(\mathrm{X}^{\prime} / \mathrm{S}\right)$ denote the category of sheaves of $\hat{\mathrm{S}}^{\cdot} \mathrm{T}_{\mathrm{X}^{\prime} / \mathrm{S}}$-modules on $\mathscr{O}_{\mathrm{X}^{\prime}}$. The subcategories $\mathrm{MIC}^{\cdot}(\mathrm{X} / \mathrm{S})$ and $\mathrm{HIG}^{\cdot}\left(\mathrm{X}^{\prime} / \mathrm{S}\right)$ are tensor categories. The natural map $\hat{\mathrm{S}} \cdot \mathrm{T}_{\mathrm{X}^{\prime} / \mathrm{S}} \rightarrow \hat{\Gamma} \cdot \mathrm{T}_{\mathrm{X}^{\prime} / \mathrm{S}}$ induces a pair of adjoint functors

$$
\begin{aligned}
\gamma_{*}: \mathrm{HIG}_{\gamma}\left(\mathrm{X}^{\prime} / \mathrm{S}\right) & \rightarrow \mathrm{HIG}_{\infty}\left(\mathrm{X}^{\prime} / \mathrm{S}\right) \\
\gamma^{*}: \mathrm{HIG}_{\infty}\left(\mathrm{X}^{\prime} / \mathrm{S}\right) & \rightarrow \mathrm{HIG}_{\gamma}\left(\mathrm{X}^{\prime} / \mathrm{S}\right),
\end{aligned}
$$

and similarly for MIC(X/S).

Let $\mathscr{A}_{\tilde{\mathrm{F}}}$ be the divided power envelope of the augmentation ideal of $\mathscr{A}_{\mathscr{X} / \mathscr{S}}$ defined by the section of $\mathscr{L}_{\mathscr{X} / \mathscr{S}}$ given by $\tilde{\mathrm{F}}$, and let $\hat{\mathscr{B}}_{\tilde{\mathrm{F}}}$ be its $\mathscr{O}_{\mathrm{X}}$-linear dual. Recall from Remark 2.4 that it has a natural $\hat{\mathrm{D}}_{\mathrm{X} / \mathrm{S}}$-module structure. There are natural maps

$$
\mathscr{A}_{\mathscr{X} / \mathscr{S}} \rightarrow \mathscr{A}_{\overline{\mathrm{F}}} ; \quad \mathrm{D}_{\mathrm{X} / \mathrm{S}}^{\gamma} \otimes_{\hat{D}_{\mathrm{X} / \mathrm{S}}} \hat{\mathscr{B}}_{\tilde{\mathrm{F}}} \cong \mathscr{B}_{\mathscr{X} / \mathscr{S}}
$$

Theorem 2.11. - Let $\mathrm{X} / \mathrm{S}$ be a smooth morphism of schemes endowed with a lift $\tilde{\mathrm{F}}: \tilde{\mathrm{X}} \rightarrow \tilde{\mathrm{X}}^{\prime}$ of the relative Frobenius morphism $\mathrm{F}_{\mathrm{X} / \mathrm{S}}$.

1. The $\hat{\mathscr{D}}_{\mathrm{X} / \mathrm{S}}$-module $\hat{\mathscr{B}}_{\tilde{\mathrm{F}}}$ described above is a splitting module for the Azumaya algebra $\mathrm{F}_{\mathrm{X} / \mathrm{S} *} \hat{\mathrm{D}}_{\mathrm{X} / \mathrm{S}}$ over its center $\mathrm{F}_{\mathrm{X} / \mathrm{S} *} \hat{\mathcal{Z}}_{\mathrm{X} / \mathrm{S}} \cong \hat{\mathrm{S}}^{\cdot} \mathrm{T}_{\mathrm{X}^{\prime} / \mathrm{S}}$.

2. The functor

$$
\begin{aligned}
& \mathrm{C}_{\tilde{\mathrm{F}}}: \mathrm{MIC}_{\infty}(\mathrm{X} / \mathrm{S}) \rightarrow \mathrm{HIG}_{\infty}\left(\mathrm{X}^{\prime} / \mathrm{S}\right) \\
& \mathrm{E} \mapsto \iota_{*} \mathscr{H}_{0 m_{\hat{\mathrm{D}}_{\mathrm{X} / \mathrm{S}}}}\left(\hat{\mathscr{B}}_{\tilde{\mathrm{F}}}, \mathrm{E}\right)
\end{aligned}
$$

defines an equivalence of categories, with quasi-inverse

$$
\begin{aligned}
& \mathrm{C}_{\tilde{\mathrm{F}}}^{-1}: \mathrm{HIG}_{\infty}\left(\mathrm{X}^{\prime} / \mathrm{S}\right) \rightarrow \mathrm{MIC}_{\infty}(\mathrm{X} / \mathrm{S}) \\
& \mathrm{E}^{\prime} \mapsto \hat{\mathscr{B}}_{\tilde{\mathrm{F}}} \otimes_{\hat{\mathcal{Z}}_{\mathrm{X}^{\prime} / \mathrm{S}}} \iota_{*} \mathrm{E}^{\prime} .
\end{aligned}
$$

Furthermore, $\mathrm{C}_{\tilde{\mathrm{F}}}$ induces an equivalence of tensor categories

$$
\mathrm{MIC}^{\cdot}(\mathrm{X} / \mathrm{S}) \rightarrow \mathrm{HIG}^{\cdot}\left(\mathrm{X}^{\prime} / \mathrm{S}\right) \text {. }
$$

3. The map $\hat{\mathscr{B}}_{\tilde{\mathrm{F}}} \rightarrow \mathscr{B}_{\mathscr{X} / \mathscr{S}}(2.10 .1)$ induces isomorphisms of functors

$$
\mathrm{C}_{\tilde{\mathrm{F}}} \circ \gamma_{*} \cong \gamma_{*} \circ \mathrm{C}_{\mathscr{X} / \mathscr{S}} \text { and } \mathrm{C}_{\mathscr{X} / \mathscr{S}} \circ \gamma^{*} \cong \gamma^{*} \circ \mathrm{C}_{\tilde{\mathrm{F}}} \text {. }
$$


Proof. - Let $\zeta: \Omega_{\mathrm{X}^{\prime} / \mathrm{S}}^{1} \rightarrow \mathrm{F}_{\mathrm{X} / \mathrm{S} *} \Omega_{\mathrm{X} / \mathrm{S}}^{1}$ be the splitting of Cartier associated to $\tilde{\mathrm{F}}$ (1.9.3). Recall that we constructed in Proposition 2.5 a module with connection $\mathscr{B}_{\zeta}$ together with a horizontal action of $\mathrm{F}_{\mathrm{X} / \mathrm{S}}^{*} \mathrm{~S}^{\cdot} \mathrm{T}_{\mathrm{X}^{\prime} / \mathrm{S}}$; as a module over this sheaf of rings, $\mathscr{B}_{\zeta}$ is free of rank one. As we have already noted in Remark 2.4, we can identify $\hat{\mathscr{B}}_{\tilde{\mathrm{F}}}$ with the formal completion $\hat{\mathscr{B}}_{\zeta}$ of $\mathscr{B}_{\zeta}$; this identification is compatible with the connections and the actions of $\mathrm{F}_{\mathrm{X} / \mathrm{S}}^{*} \hat{\mathrm{S}}^{\cdot} \mathrm{T}_{\mathrm{X}^{\prime} / \mathrm{S}}$. In particular, $\hat{\mathscr{B}}_{\zeta}$ is an invertible (even free) sheaf of $\mathrm{F}_{\mathrm{X} / \mathrm{S}}^{*} \hat{\mathrm{S}}^{\cdot} \mathrm{T}_{\mathrm{X}^{\prime} / \mathrm{S}}$-modules, and hence is locally free of rank $p^{d}$ over $\hat{\mathrm{S}}^{\cdot} \mathrm{T}_{\mathrm{X}^{\prime} / \mathrm{S}}$. Recall from Proposition 2.5 that there is a surjective étale group morphism $\alpha_{\zeta}=\mathrm{id}-h_{\zeta}: \mathbf{T}_{\mathrm{X}^{\prime} / \mathrm{S}}^{*} \rightarrow \mathbf{T}_{\mathrm{X}^{\prime} / \mathrm{S}}^{*}$, and note that its restriction $\hat{\alpha}_{\zeta}$ to $\hat{\mathbf{T}}_{\mathrm{X}^{\prime} / \mathrm{S}}^{*}$ is an isomorphism, with inverse

$$
\hat{\alpha}_{\zeta}^{-1}=\mathrm{id}+h_{\zeta}+h_{\zeta}^{2}+\cdots
$$

According to Proposition 2.5, the $p$-curvature action of $\mathrm{S}^{*} \mathrm{~T}_{\mathrm{X}^{\prime} / \mathrm{S}}$ on $\hat{\mathscr{B}}_{\zeta}$ is given by $\hat{\alpha}_{\zeta}^{*}$ followed by the standard action. Since $\hat{\alpha}_{\zeta}$ is an isomorphism, $\hat{\alpha}_{\zeta *} \hat{\mathscr{B}}_{\zeta}$ is locally free of rank $p^{d}$. Thus $\hat{\mathscr{B}}_{\zeta}$ is an $\mathrm{F}_{\mathrm{X} / \mathrm{S} *} \hat{\mathrm{D}}_{\mathrm{X} / \mathrm{S}}$-module which is locally free of rank $p^{d}$ over the center $\hat{\mathrm{S}}^{\bullet} \mathrm{T}_{\mathrm{X}^{\prime} / \mathrm{S}}$, and hence is a splitting module. This proves (1), and (2) follows as before. The compatibilities stated in (3) follow immediately from the constructions and the morphisms (2.10.1).

Let us give a more explicit description of the local Cartier transform $\mathrm{C}_{\tilde{\mathrm{F}}}$. Given a splitting $\zeta$ and a Higgs module $\left(\mathrm{E}^{\prime}, \psi^{\prime}\right)$ we define a module with integrable connection

$(2.11 .1)$

$(2.11 .2)$

$$
\begin{aligned}
\Psi_{\zeta}^{-1}\left(\mathrm{E}^{\prime}, \psi^{\prime}\right) & :=\left(\mathrm{F}_{\mathrm{X} / \mathrm{S}}^{*} \mathrm{E}^{\prime}, \nabla\right) \\
\nabla & :=\nabla_{0}+\left(\mathrm{id}_{\mathrm{E}^{\prime}} \otimes \zeta\right) \circ \mathrm{F}_{\mathrm{X} / \mathrm{S}}^{*}\left(\psi^{\prime}\right),
\end{aligned}
$$

where $\nabla_{0}$ is the Frobenius descent connection and $\left(\operatorname{id}_{\mathrm{E}^{\prime}} \otimes \zeta\right) \circ \mathrm{F}_{\mathrm{X} / \mathrm{S}}^{*}\left(\psi^{\prime}\right)$ is the $\mathscr{O}_{\mathrm{X}}$-linear map

$$
\mathrm{F}_{\mathrm{X} / \mathrm{S}}^{*} \mathrm{E}^{\prime} \stackrel{\mathrm{F}_{\mathrm{X} / \mathrm{S}}^{*}\left(\psi^{\prime}\right)}{\longrightarrow} \mathrm{F}_{\mathrm{X} / \mathrm{S}}^{*} \mathrm{E}^{\prime} \otimes \mathrm{F}_{\mathrm{X} / \mathrm{S}}^{*} \Omega_{\mathrm{X}^{\prime} / \mathrm{S}}^{1} \stackrel{\mathrm{id}_{\mathrm{E}^{\prime}} \otimes \zeta}{\longrightarrow} \mathrm{F}_{\mathrm{X} / \mathrm{S}}^{*} \mathrm{E}^{\prime} \otimes \Omega_{\mathrm{X}^{\prime} / \mathrm{S}}^{1} \cdot
$$

Let $\mathscr{B}_{\mathrm{X} / \mathrm{S}}^{\prime}:=\iota_{*} \mathrm{~S}^{\cdot} \mathrm{T}_{\mathrm{X}^{\prime} / \mathrm{S}}$, viewed as an object of $\mathrm{HIG}\left(\mathrm{X}^{\prime} / \mathrm{S}\right)$.

Lemma 2.12. - The isomorphism $\mathscr{B}_{\zeta} \cong \iota^{*} \mathrm{~S}^{\cdot} \mathrm{F}_{\mathrm{X} / \mathrm{S}}^{*} \mathrm{~T}_{\mathrm{X}^{\prime} / \mathrm{S}}$ induces an isomorphism

$$
\Psi_{\zeta}^{-1}\left(\mathscr{B}_{\mathrm{X} / \mathrm{S}}^{\prime}\right) \cong \mathscr{B}_{\zeta}
$$

compatible with the connections.

Proof. — For each $n$, the ideal $\mathscr{B}_{\mathrm{X} / \mathrm{S}}^{\prime>n}:=\bigoplus_{j>n} \mathrm{~S}^{j} \mathrm{~T}_{\mathrm{X}^{\prime} / \mathrm{S}}$ also defines an object of $\mathrm{HIG}\left(\mathrm{X}^{\prime} / \mathrm{S}\right)$, as does the quotient $\mathscr{B}_{n}^{\prime}$ of $\mathscr{B}_{\mathrm{X} / \mathrm{S}}^{\prime}$ by $\mathscr{B}_{\mathrm{X} / \mathrm{S}}^{\prime>n}$. Let $\mathscr{A}_{n}^{\prime \gamma}$ denote the dual of 
$\mathscr{B}_{n}^{\prime}$ in $\operatorname{HIG}\left(\mathrm{X}^{\prime} / \mathrm{S}\right)$ and let $\mathscr{A}_{\mathrm{X} / \mathrm{S}}^{\prime}:=\lim _{\longrightarrow} \mathscr{A}_{n}^{\prime \gamma}$. For example,

$$
\mathscr{A}_{1}^{\prime \gamma}=\mathscr{E}_{\mathrm{X} / \mathrm{S}}^{\prime}:=\mathscr{O}_{\mathrm{X}^{\prime}} \oplus \Omega_{\mathrm{X}^{\prime} / \mathrm{S}}^{1},
$$

and if $\xi \in \mathrm{T}_{\mathrm{X}^{\prime} / \mathrm{S}}, a^{\prime} \in \mathscr{O}_{\mathrm{X}^{\prime}}$, and $\omega^{\prime} \in \Omega_{\mathrm{X}^{\prime} / \mathrm{S}}^{1}$,

$$
\xi\left(a^{\prime}, \omega^{\prime}\right)=\left(\left\langle\xi, \omega^{\prime}\right\rangle, 0\right) \text {. }
$$

Furthermore, $\mathscr{A}_{\mathrm{X} / \mathrm{S}}^{\prime \gamma} \cong \Gamma \cdot \Omega_{\mathrm{X}^{\prime} / \mathrm{S}}^{1}$, and if $\omega_{j}^{\prime} \in \Omega_{\mathrm{X}^{\prime} / \mathrm{S}}^{1}$ for $j=1, \ldots, r$, then each $x_{j}:=\left(0, \omega_{j}^{\prime}\right)$ belongs to the divided power ideal of $\mathscr{A}_{\mathrm{X} / \mathrm{S}}^{\prime \gamma}$, and

$$
\xi\left(x_{1}^{\left[i_{1}\right]} x_{2}^{\left[i_{2}\right]} \cdots x_{r}^{\left[i_{r}\right]}\right)=\sum_{j}\left\langle\xi, \omega_{j}^{\prime}\right\rangle x_{1}^{\left[i_{1}\right]} x_{2}^{\left[i_{2}\right]} \cdots x_{j}^{\left[j_{j}-1\right]} x_{r}^{\left[i_{r}\right]} .
$$

It follows from the definitions that $\left(\mathscr{E}_{\zeta}, \nabla\right)=\Psi_{\zeta}^{-1}\left(\mathscr{E}_{\mathrm{X} / \mathrm{S}}^{\prime \prime}\right)$. Then by the formula above for the action of $\mathrm{T}_{\mathrm{X}^{\prime} / \mathrm{S}}$ on divided powers and the similar formula for the action of a connection on divided powers, it follows that $\left(\mathscr{A}_{\zeta}^{\gamma}, \nabla\right) \cong \Psi_{\zeta}^{-1}\left(\mathscr{A}_{\mathrm{X} / \mathrm{S}}^{\prime \gamma}\right)$. Hence by the compatibility of $\Psi_{\zeta}^{-1}$ with duality, $\left(\mathscr{B}_{\zeta}, \nabla\right) \cong \Psi_{\zeta}^{-1}\left(\mathscr{B}_{\mathrm{X} / \mathrm{S}}^{\prime}\right)$.

Let $\left(\mathrm{E}^{\prime}, \psi^{\prime}\right)$ be an object of $\mathrm{HIG}\left(\mathrm{X}^{\prime} / \mathrm{S}\right)$. Then the isomorphism in the previous lemma induces isomorphisms:

$$
\begin{aligned}
& \Psi_{\zeta}^{-1}\left(\iota_{*} \mathrm{E}^{\prime}\right) \cong \Psi_{\zeta}^{-1}\left(\mathrm{E}^{\prime} \otimes_{\mathrm{S}} \cdot \mathrm{T}_{\mathrm{X}^{\prime} / \mathrm{S}} \mathscr{B}_{\mathrm{X} / \mathrm{S}}^{\prime}\right) \\
& \cong \mathrm{E}^{\prime} \otimes_{\mathrm{S}^{\cdot} \mathrm{T}_{\mathrm{X}^{\prime} / \mathrm{S}}} \Psi_{\zeta}^{-1}\left(\mathscr{B}_{\mathrm{X} / \mathrm{S}}^{\prime}\right) \cong \mathrm{E}^{\prime} \otimes_{\mathrm{S}^{\cdot} \cdot \mathrm{T}_{\mathrm{X}^{\prime} / \mathrm{S}}} \mathscr{B}_{\zeta} .
\end{aligned}
$$

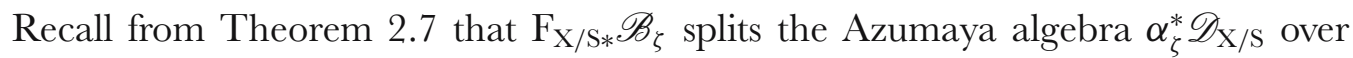

$$
\mathbf{T}_{\zeta}^{*}:=\mathbf{T}_{\mathrm{X}^{\prime} / \mathrm{S}}^{*} \stackrel{\alpha_{\zeta}}{\rightarrow} \mathbf{T}_{\mathrm{X}^{\prime} / \mathrm{S}}^{*} \cdot
$$

This, together with (2.12.1), implies the following result.

Theorem 2.13. - Let $\zeta$ be a lift of $\mathrm{C}_{\mathrm{X} / \mathrm{S}}^{-1}$, let

$$
\alpha_{\zeta}^{*}: \mathrm{F}_{\mathrm{X} / \mathrm{S} *} \mathscr{Z}_{\mathrm{X} / \mathrm{S}} \cong \mathrm{S}^{\cdot} \mathrm{T}_{\mathrm{X}^{\prime} / \mathrm{S}} \rightarrow \mathrm{S}^{\cdot} \mathrm{T}_{\mathrm{X}^{\prime} / \mathrm{S}}:=\mathscr{Z}_{\zeta}
$$

be the map described in Proposition 2.5, and let

$$
\mathrm{D}_{\zeta}:=\mathrm{S}^{\cdot} \mathrm{T}_{\mathrm{X}^{\prime} / \mathrm{S}} \otimes_{\alpha_{\zeta}^{*}} \mathrm{~F}_{\mathrm{X} / \mathrm{S} *} \mathrm{D}_{\mathrm{X} / \mathrm{S}} .
$$

Let $\mathrm{MIC}_{\zeta}(\mathrm{X} / \mathrm{S})$ denote the category of sheaves of $\mathrm{D}_{\zeta}$-modules on $\mathrm{X}$. For each $\xi \in \mathrm{T}_{\mathrm{X}^{\prime} / \mathrm{S}}$, the p-curvature $\psi_{\xi}$ on $\Psi_{\zeta}^{-1}\left(\iota_{*} \mathrm{E}^{\prime}\right)$ is induced by the action of $\alpha_{\zeta}^{*}(\xi)$ on $\mathrm{E}^{\prime}$, i.e., $\psi_{\xi}=\mathrm{F}_{\mathrm{X} / \mathrm{S}}^{*}\left(-\alpha_{\zeta}^{*}(\xi)\right)$. This makes $\Psi_{\zeta}^{-1}\left(\iota_{*} \mathrm{E}^{\prime}\right)$ a $\mathrm{D}_{\zeta}$-module. Furthermore, the functors

$$
\begin{aligned}
\operatorname{HIG}\left(\mathrm{X}^{\prime} / \mathrm{S}\right) & \rightarrow \mathrm{MIC}_{\zeta}(\mathrm{X} / \mathrm{S}) \\
\left(\mathrm{E}^{\prime}, \psi^{\prime}\right) & \mapsto \mathrm{E}^{\prime} \otimes_{\mathscr{Z}_{\zeta}} \mathscr{B}_{\zeta} \\
\left(\mathrm{E}^{\prime}, \psi^{\prime}\right) & \mapsto \Psi_{\zeta}^{-1}\left(\iota_{*} \mathrm{E}^{\prime}\right)
\end{aligned}
$$


are isomorphic equivalences of categories, with quasi-inverse given by

$$
\mathrm{E} \mapsto \mathscr{H}_{0 m_{\mathscr{D}_{\zeta}}}\left(\mathscr{B}_{\zeta}, \mathrm{E}\right)
$$

Corollary 2.14. - Let $(\mathrm{E}, \nabla)$ be an object of $\mathrm{MIC}_{\infty}(\mathrm{X} / \mathrm{S})$, let $\psi$ be its p-curvature, and let $\left(\mathrm{E}^{\prime}, \psi^{\prime}\right):=\mathrm{C}_{\tilde{\mathrm{F}}}(\mathrm{E}, \nabla)$, and let $\zeta$ be the splitting of Cartier determined by $\tilde{\mathrm{F}}$. Then there is canonical isomorphism:

$$
(\mathrm{E}, \nabla) \cong \Psi_{\zeta}^{-1} \alpha_{\zeta *}^{-1}\left(\mathrm{E}^{\prime}, \psi^{\prime}\right)
$$

Remark 2.15. - The appearance of the involution $\iota$ in Definition 2.8 insures the compatibility of the Cartier transform with the usual Cartier operator. Let us explain this in the context of extensions. The group $\operatorname{Ext}_{\mathrm{MIC}}^{1}\left(\mathscr{O}_{\mathrm{X}}, \mathscr{O}_{\mathrm{X}}\right)$ of isomorphism classes of the category $\operatorname{EXT}_{\mathrm{MIC}}^{1}\left(\mathscr{O}_{\mathrm{X}}, \mathscr{O}_{\mathrm{X}}\right)$ of extensions of $\mathscr{O}_{\mathrm{X}}$ by $\mathscr{O}_{\mathrm{X}}$ in the category $\mathrm{MIC}(\mathrm{X} / \mathrm{S})$ is canonically isomorphic to the de Rham cohomology group $\mathrm{H}_{d \mathrm{R}}^{1}(\mathrm{X} / \mathrm{S})$. Similarly, the group $\operatorname{Ext}_{\mathrm{HIG}}^{1}\left(\mathscr{O}_{\mathrm{X}^{\prime}}, \mathscr{O}_{\mathrm{X}^{\prime}}\right)$ of isomorphism classes of the category $\operatorname{EXT}_{\mathrm{HIG}}^{1}\left(\mathscr{O}_{\mathrm{X}^{\prime}}, \mathscr{O}_{\mathrm{X}^{\prime}}\right)$ of extensions of $\mathscr{O}_{\mathrm{X}^{\prime}}$ by $\mathscr{O}_{\mathrm{X}^{\prime}}$ in $\mathrm{HIG}\left(\mathrm{X}^{\prime} / \mathrm{S}\right)$ is canonically isomorphic to

$$
\mathrm{H}_{\mathrm{H} d g}^{1}\left(\mathrm{X}^{\prime} / \mathrm{S}\right) \cong \mathrm{H}^{1}\left(\mathrm{X}^{\prime}, \mathscr{O}_{\mathrm{X}^{\prime}} \oplus \Omega_{\mathrm{X}^{\prime} / \mathrm{S}}^{1}[-1]\right)
$$

The inverse Cartier transform defines an equivalence of categories

$$
\mathrm{C}_{\mathscr{X} / \mathscr{S}}^{-1}: \operatorname{EXT}_{\mathrm{HIG}}^{1}\left(\mathscr{O}_{\mathrm{X}^{\prime}}, \mathscr{O}_{\mathrm{X}^{\prime}}\right) \rightarrow \operatorname{EXT}_{\mathrm{MIC}}^{1}\left(\mathscr{O}_{\mathrm{X}}, \mathscr{O}_{\mathrm{X}}\right)
$$

and hence an isomorphism of groups

$$
c_{\mathscr{X} / \mathscr{S}}^{-1}: \mathrm{H}_{\mathrm{H} d g}^{1}\left(\mathrm{X}^{\prime} / \mathrm{S}\right) \rightarrow \mathrm{H}_{d \mathrm{R}}^{1}(\mathrm{X} / \mathrm{S})
$$

Let us consider the following diagram.

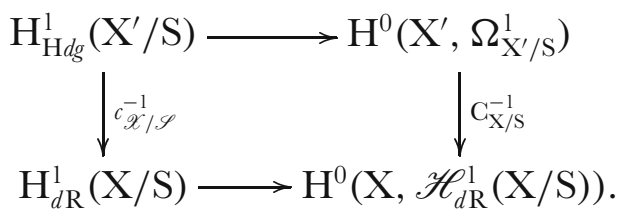

Thanks to our definition, the diagram is commutative. It suffices to verify this when $\mathrm{F}_{\mathrm{X} / \mathrm{S}}$ lifts and for extensions $\mathscr{O}_{\mathrm{X}^{\prime}} \rightarrow \mathrm{E} \rightarrow \mathscr{O}_{\mathrm{X}^{\prime}}$ which split in the category of $\mathscr{O}_{\mathrm{X}^{\prime}}$ modules. Then $\mathrm{E}^{\prime}$ has a basis $\left(e_{0}^{\prime}, e_{1}^{\prime}\right)$ such that $\psi\left(e_{0}^{\prime}\right)=0$ and $\psi\left(e_{1}^{\prime}\right)=e_{0} \otimes \omega^{\prime}$, where $\omega^{\prime} \in \Omega_{\mathrm{X}^{\prime} / \mathrm{S}}^{1}$. Then one can check that $\mathrm{E}:=\mathrm{C}_{\mathscr{X} / \mathscr{S}}^{-1}\left(\mathrm{E}^{\prime}\right)$ has a basis $\left(e_{0}, e_{1}\right)$ such that $\nabla\left(e_{0}\right)=0$ and $\nabla\left(e_{1}\right)=e_{0} \otimes \zeta\left(\omega^{\prime}\right)$, where $\zeta$ is the splitting of $\mathrm{C}_{\mathscr{X} / \mathscr{S}}^{-1}$ defined by the lifting of $\mathrm{F}_{\mathrm{X} / \mathrm{S}}$. This implies that the diagram commutes. 


\subsection{The Cartier transform as Riemann-Hilbert} gories:

In the previous section we defined a pair of inverse quasi-equivalences of cate-

$$
\begin{array}{ll}
\mathrm{C}_{\mathscr{X} / \mathscr{S}}: \mathrm{MIC}_{\gamma}^{*}(\mathrm{X} / \mathrm{S}) \rightarrow \mathrm{HIG}_{\gamma}^{*}\left(\mathrm{X}^{\prime} / \mathrm{S}\right) & : \quad \mathrm{E} \mapsto \iota^{*} \operatorname{Hom}_{\mathscr{D}_{\mathrm{X} / \mathrm{S}}^{\prime}}\left(\mathscr{B}_{\mathscr{X} / \mathscr{S}}, \mathrm{E}\right) \\
\mathrm{C}_{\mathscr{X} / \mathscr{S}}^{-1}: \mathrm{HIG}_{\gamma}^{\cdot}\left(\mathrm{X}^{\prime} / \mathrm{S}\right) \rightarrow \mathrm{MIC}_{\gamma}^{\cdot}(\mathrm{X} / \mathrm{S}) \quad: \quad \mathrm{E}^{\prime} \mapsto \mathscr{B}_{\mathscr{C} / \mathscr{S}} \otimes_{\hat{\Gamma} \cdot \mathrm{T}_{\mathrm{X}^{\prime} / \mathrm{S}}} \iota^{*} \mathrm{E}^{\prime} .
\end{array}
$$

Our goal here is to show how the ring structure on the dual $\mathscr{A}_{\mathscr{X} / \mathscr{S}}$ of $\mathscr{B}_{\mathscr{X} / \mathscr{S}}$ can be used to give an alternative and more symmetric description of these functors. This viewpoint sharpens the analogy between the Cartier transform and the Riemann Hilbert and Higgs correspondences, with the sheaf of $\mathscr{O}_{\mathrm{X}}$-algebras $\mathscr{A}_{\mathscr{X} / \mathscr{S}}$ playing the role of the sheaf of analytic or $\mathrm{C}^{\infty}$ functions. This construction of the Cartier transform relies on the "Higgs transforms" described in (5.9) and ordinary Frobenius descent instead of the theory of Azumaya algebras.

Roughly speaking, the idea is the following. The algebra $\mathscr{A}_{\mathscr{X} / \mathscr{S}}$ is endowed with a connection $\nabla_{\mathscr{A}}$ and a PD-Higgs field $\theta_{\mathscr{A}}$. If $(\mathrm{E}, \nabla)$ is an object of $\mathrm{MIC}_{\gamma}(\mathrm{X} / \mathrm{S})$, the tensor product connection on $\mathrm{E} \otimes \mathscr{A}_{\mathscr{X} / \mathscr{S}}$ commutes with the PD-Higgs field id $\otimes \theta_{\mathscr{A}}$. Hence id $\otimes \theta_{\mathscr{A}}$ induces a PD-Higgs field on the sheaf of horizontal sections of $\mathrm{E} \otimes \mathscr{A}_{\mathscr{X} / \mathscr{S}}$, and it turns out that the corresponding object of $\mathrm{HIG}_{\gamma}^{\cdot}\left(\mathrm{X}^{\prime} / \mathrm{S}\right)$ is $\mathrm{C}_{\mathscr{X} / \mathscr{S}}(\mathrm{E})$. Similarly, if $\left(\mathrm{E}^{\prime}, \theta^{\prime}\right)$ is an object of $\mathrm{HIG}_{\gamma}^{\cdot}\left(\mathrm{X}^{\prime} / \mathrm{S}\right)$, then the total PD-Higgs field $\theta_{t o t}^{\prime}$ of $\mathrm{E}^{\prime} \otimes \mathscr{A}_{\mathscr{X} / \mathscr{S}}$ commutes with the connection induced by $\nabla_{\mathscr{A}}$. Hence the subsheaf of sections annihilated by $\theta_{t o t}^{\prime}$ inherits a connection, and the corresponding object of $\mathrm{MIC}_{\gamma}(\mathrm{X} / \mathrm{S})$ is $\mathrm{C}_{\mathscr{X} / \mathscr{S}}^{-1}\left(\mathrm{E}^{\prime}\right)$.

To make this precise, we begin with some notation and a slightly more general setting. Let $\Omega$ be a locally free sheaf of $\mathscr{O}_{\mathrm{X}}$-modules, let $\mathrm{T}$ be its dual, and let $\mathbf{T}$ be the vector group $\operatorname{Spec}_{\mathrm{X}} \mathrm{S} \Omega$. Let $\mathscr{G}$ be the group scheme $\operatorname{Spec}_{\mathrm{X}} \Gamma$.T, and let us write $\mathscr{O}_{\mathscr{G}}$ for the sheaf $\Gamma$.T, $\mathscr{I}$ for the divided power ideal $\Gamma_{+} \mathrm{T}$ of $\Gamma$.T, and $\mathscr{O}_{\mathscr{G}_{n}}:=\mathscr{O}_{\mathscr{G}} / \mathscr{I}^{[n+1]}$ if $n \in \mathbf{N}$. Recall from the discussion preceding Proposition 1.4 that if $\pi_{\mathscr{L}}: \mathscr{L} \rightarrow \mathrm{X}$ is any $\mathbf{T}$-torsor, then there is natural action of $\mathscr{O}_{\mathscr{G}}$ on the filtered algebra $\left(\mathscr{A}_{\mathscr{L}}, \mathrm{N}\right.$. $):=$ $\left(\pi_{\mathscr{L} *} \mathscr{O}_{\mathscr{L}}, \mathrm{N}\right.$.), and that $\mathrm{N}_{n} \mathscr{A}_{\mathscr{L}}$ is the annihilator of the ideal $\mathscr{I}^{[n+1]}$. Thus there is a natural map

$$
\mathscr{O}_{\mathscr{S}_{n}} \times \mathrm{N}_{n} \mathscr{A}_{\mathscr{L}} \rightarrow \mathrm{N}_{n} \mathscr{A}_{\mathscr{L}}
$$

We shall find it both useful and convenient to study filtered $\mathscr{O}_{\mathscr{g}}$-modules. We denote by $\mathscr{I}^{\cdot}$ the divided power filtration on $\mathscr{O}_{\mathscr{G}}$ (although we should perhaps really write $\left.\mathscr{I}^{[\cdot]}\right)$.

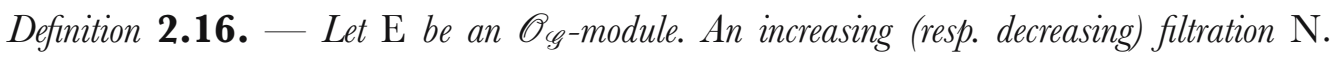
(resp $\mathrm{N}^{*}$ ) of $\mathrm{E}$ by sub $\mathscr{O}_{\mathscr{g}}$-modules is said to be $\mathscr{I}^{-}$-saturated, or just an $\mathscr{I}$-filtration, if for all $j$ and $k$,

$$
\mathrm{I}^{[j]} \mathrm{N}_{k} \mathrm{E} \subseteq \mathrm{N}_{k-j} \mathrm{E}, \quad\left(r e s p . \mathrm{I}^{[j]} \mathrm{N}^{k} \mathrm{E} \subseteq \mathrm{N}^{k+j} \mathrm{E}\right) .
$$


For example, the filtrations N. of $\mathscr{A}_{\mathscr{L}}$ and $\mathscr{I} \cdot$ of $\mathscr{O}_{\mathscr{G}}$ are $\mathscr{I}^{\circ}$-filtrations. If $\mathrm{E}$ is any $\mathscr{O}_{\mathscr{G}}$-module, then the canonical filtration

$$
\mathrm{N}_{k} \mathrm{E}:=\left\{e \in \mathrm{E}: \mathscr{I}^{[k]} e=0\right\}
$$

is $\mathscr{I}^{\circ}$-saturated, and $\mathrm{E}$ is locally nilpotent if and only if this filtration is exhaustive. If $\left(\mathrm{E}_{1}, \mathrm{~N}\right.$. $)$ and $\left(\mathrm{E}_{2}, \mathrm{~N}\right)$ are $\mathscr{O}_{\mathscr{G}}$-modules with $\mathscr{I}^{\circ}$-saturated filtrations, then the tensor product filtration

$$
\mathrm{N}_{c}\left(\mathrm{E}_{1} \otimes \mathrm{E}_{2}\right):=\sum_{a+b=c} \operatorname{Im}\left(\mathrm{N}_{a} \mathrm{E}_{1} \otimes \mathrm{N}_{b} \mathrm{E}_{2} \rightarrow \mathrm{E}_{1} \otimes \mathrm{E}_{2}\right)
$$

is again $\mathscr{I}^{\cdot}$-saturated, because the group law induces maps

$$
\mu^{*}: \mathscr{I}^{[j]} \rightarrow \sum_{a+b=j} \operatorname{Im}\left(\mathscr{I}^{[a]} \otimes \mathscr{I}^{[b]} \rightarrow \mathscr{O}_{\mathscr{G}} \otimes \mathscr{O}_{\mathscr{G}}\right)
$$

If $\mathrm{E}$ is any $\mathscr{O}_{\mathrm{X}}$-module, let $\theta_{0}$ denote the $\mathscr{O}_{\mathscr{G}}$-module structure on $\mathrm{E}$ for which the ideal $\mathscr{I}$ acts as zero. That is, $\left(\mathrm{E}, \theta_{0}\right)=i_{*} \mathrm{E}$, where $i^{*}: \mathscr{O}_{\mathscr{G}} \rightarrow \mathscr{O}_{\mathrm{X}}$ is restriction along the zero section. If $\mathrm{E}$ any $\mathscr{O}_{\mathrm{X}}$-module and $\theta$ is an $\mathscr{O}_{\mathscr{G}}$-module structure on $\mathrm{E}$, let

$$
\mathrm{E}^{\theta}:=\mathscr{H}_{0 m_{\mathscr{G}}}\left(i_{*} \mathscr{O}_{\mathrm{X}}, \mathrm{E}\right)
$$

i.e., $\mathrm{E}^{\theta}$ is the sub $\mathscr{O}_{\mathrm{X}}$-module of $\mathrm{E}$ consisting of all the elements annihilated by the ideal $\mathscr{I}$.

Now let $\mathrm{E}$ be an $\mathscr{O}_{\mathrm{X}}$-module equipped with an $\mathscr{O}_{\mathscr{G}}$-module structure $\theta$ and an $\mathscr{I}^{*}$-saturated filtration $\mathrm{N}$. The $\mathscr{O}_{\mathrm{X}}$-module

$$
\mathscr{A}_{\mathscr{L}}(\mathrm{E}):=\mathrm{E} \otimes_{\mathscr{O}_{\mathrm{x}}} \mathscr{A}_{\mathscr{L}}
$$

has three natural $\mathscr{O}_{\mathscr{G}}$-module structures: the action by transport of structure via E, the action by transport of structure via $\mathscr{A}_{\mathscr{L}}$, and the convolution structure defined in (2.7.1). We shall denote these by

$$
\begin{aligned}
& \theta_{\mathrm{E}}:=\theta_{\mathrm{E}} \otimes \mathrm{id}_{\mathscr{A}}=\theta_{\mathrm{E}} \circledast \theta_{0} \\
& \theta_{\mathscr{A}}:=\mathrm{id}_{\mathrm{E}} \otimes \theta_{\mathscr{A}}=\theta_{0} \circledast \theta_{\mathscr{A}} \\
& \theta_{t o t}:=\theta_{\mathrm{E}} \circledast \theta_{\mathscr{A}} .
\end{aligned}
$$

We endow it with the total (tensor product) filtration N. (2.16.2). It follows from Formula (2.7.1) that $\theta_{\mathscr{A}}$ and $\theta_{\text {tot }}$ commute. Define

$$
\mathscr{T}_{\mathscr{L}}(\mathrm{E}):=\left(\mathscr{A}_{\mathscr{L}}(\mathrm{E})\right)^{\theta_{t o t}},
$$


with the $\mathscr{O}_{\mathscr{G}}$-structure $\theta_{\mathscr{T}}$ induced by $\theta_{\mathscr{A}}$ and the filtration induced by $\mathrm{N}$. We have natural maps, compatible with the $\mathscr{O}_{\mathscr{G}}$-structures shown:

\section{(2.16.3 $\quad\left(\mathrm{E}, \theta_{0}, \theta_{\mathrm{E}}\right) \stackrel{i_{\mathrm{E}}}{\longrightarrow}\left(\mathscr{A}_{\mathscr{L}}(\mathrm{E}), \theta_{\mathscr{A}}, \theta_{t o t}\right) \stackrel{j \mathrm{E}}{\leftarrow}\left(\mathscr{T}_{\mathscr{L}}(\mathrm{E}), \theta_{\mathscr{T}}, \theta_{0}\right)$,}

where $i_{\mathrm{E}}(e):=1 \otimes e$ and $j_{\mathrm{E}}$ is the inclusion. Note that $i_{\mathrm{E}}$ factors through $\left(\mathscr{A}_{\mathscr{L}}(\mathrm{E})\right)^{\theta_{\mathscr{A}}}$ and $j_{\mathrm{E}}$ factors through $\left(\mathscr{A}_{\mathscr{L}}(\mathrm{E})\right)^{\theta_{\text {tot }}}$. Endow $\mathscr{A}_{\mathscr{L}}\left(\mathscr{T}_{\mathscr{L}}(\mathrm{E})\right)$ with the tensor product filtration, and let

$$
h: \mathscr{A}_{\mathscr{L}}\left(\mathscr{T}_{\mathscr{L}}(\mathrm{E})\right) \rightarrow \mathscr{A}_{\mathscr{L}}(\mathrm{E})
$$

be the map defined by the commutative diagram:

\section{$(2.16 .4)$}

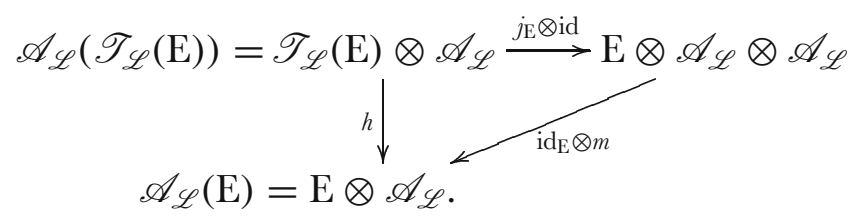

Proposition 2.17. - Let $\mathrm{E}$ be an $\mathscr{O}_{\mathrm{X}}$-module with a locally nilpotent $\mathscr{O}_{G \text {-module structure }}$ $\theta$ and an $\mathscr{I}^{\cdot}$-filtration N. which is bounded below.

1. The map $i_{\mathrm{E}}$ of (2.16.3) is injective and strictly compatible with the filtrations, and its image is $\left(\mathscr{A}_{\mathscr{L}}(\mathrm{E})\right)^{\theta_{\mathscr{A}}}$.

2. The map $j_{\mathrm{E}}$ of (2.16.3) is injective and strictly compatible with the filtrations, and its image is $\left(\mathscr{A}_{\mathscr{L}}(\mathrm{E})\right)^{\theta_{\text {tot }}}$.

3. The map $h$ of (2.16.4) fits in a commutative diagram

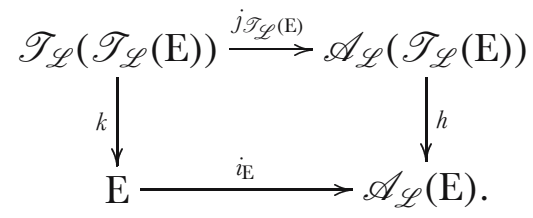

Furthermore, $h$ and $k$ are strict filtered isomorphisms, compatible with $\mathscr{O}_{\mathscr{G} \text {-module structures }}$ as shown:

$$
\begin{aligned}
h:\left(\mathscr{A}_{\mathscr{L}}\left(\mathscr{T}_{\mathscr{L}}(\mathrm{E})\right), \theta_{\mathscr{A}}, \theta_{t o t}\right) & \rightarrow\left(\mathscr{A}_{\mathscr{L}}(\mathrm{E}), \theta_{t o t}, \theta_{\mathscr{A}}\right) \\
k:\left(\mathscr{T}_{\mathscr{L}}\left(\mathscr{T}_{\mathscr{L}}(\mathrm{E})\right), \theta_{\mathscr{T}}\right) & \rightarrow\left(\mathrm{E}, \theta_{\mathrm{E}}\right) .
\end{aligned}
$$

4. If $s$ is a section of $\mathscr{L}$, then $s^{*} \circ j_{\mathrm{E}}$ induces a strict isomorphism

$$
\eta_{s}:\left(\mathscr{T}_{\mathscr{L}}(\mathrm{E}), \theta_{\mathscr{T}}\right) \rightarrow\left(\mathrm{E}, \iota_{*} \theta_{\mathrm{E}}\right)
$$

where $\iota$ is the inversion mapping of the group scheme $\mathscr{G}$. 
Proof. - This result can be interpreted and proved in many ways. For example, it is a special case of the theory of Higgs transforms on affine group schemes as explained in Section 5. Here we give a simpler version. Indeed, all of the statements of the proposition can be verified locally on $\mathrm{X}$, and so we may and shall assume without loss of generality that $\mathscr{L}$ has a section $s$ defining an isomorphism $\mathscr{L} \cong \mathbf{T}$ and hence $\mathscr{A}_{\mathscr{L}} \cong \mathrm{S} \cdot \Omega$.

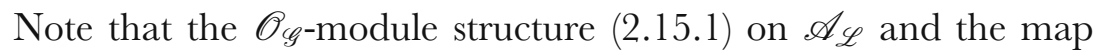

$$
s^{*}: \mathscr{A}_{\mathscr{L}} \rightarrow \mathscr{O}_{\mathrm{X}}
$$

defined by the section $s$ of $\mathscr{L}$ together define a perfect pairing

$$
\mathscr{O}_{\mathscr{G}_{n}} \times \mathrm{N}_{n} \mathscr{A}_{\mathscr{L}} \rightarrow \mathscr{O}_{\mathrm{X}}
$$

If $\mathrm{E}$ is any $\mathscr{O}_{\mathrm{X}}$-module, let

$$
\mathscr{H} .\left(\mathscr{O}_{\mathscr{G}}, \mathrm{E}\right):=\lim _{\longrightarrow} \mathscr{H}_{0 m_{\mathscr{O}_{\mathrm{X}}}}\left(\mathscr{O}_{\mathscr{G}_{n}}, \mathrm{E}\right) \subseteq \mathscr{H}_{0 m_{\mathscr{O}_{\mathrm{X}}}}\left(\mathscr{O}_{\mathscr{G}}, \mathrm{E}\right)
$$

Then the pairing (2.17.1) defines an isomorphism:

$$
\mathscr{A}_{\mathscr{L}}(\mathrm{E}):=\mathscr{A}_{\mathscr{L}} \otimes \mathrm{E} \cong \mathscr{H}_{.}\left(\mathscr{O}_{\mathscr{G}}, \mathrm{E}\right) .
$$

Let us denote by $\theta_{\mathscr{A}}$ and $\theta_{\text {tot }}$ the $\mathscr{O}_{\mathscr{G}}$-module structures on $\mathscr{H} .\left(\mathscr{O}_{\mathscr{G}}, \mathrm{E}\right)$ deduced from the corresponding structures on $\mathscr{A}_{\mathscr{L}}(\mathrm{E})$. These can be described explicitly as follows. If $\mathrm{E}_{1}$ and $\mathrm{E}_{2}$ are two $\mathscr{O}_{\mathscr{G}}$-modules, then $\mathscr{H}_{0 m_{\mathscr{O}_{\mathrm{X}}}}\left(\mathrm{E}_{1}, \mathrm{E}_{2}\right)$ can be give an $\mathscr{O}_{\mathscr{G}} \otimes \mathscr{O}_{\mathscr{G}}$-module structure by the rule

$$
\left(b_{1}, b_{2}\right)(\phi)\left(e_{1}\right):=b_{2} \phi\left(b_{1} e_{1}\right) .
$$

Then $\theta_{\text {tot }}$ corresponds to the $\mathscr{O}_{\mathscr{G}}$-structure induced by $\mu_{*}$ and $\theta_{\mathscr{A}}$ to the structure induced by $p r_{1 *}{ }^{7}$ The total filtration $\mathrm{N}$. of $\mathscr{A}_{\mathscr{L}}(\mathrm{E})$ corresponds to the filtration $\mathrm{N}$. of $\mathscr{H} .\left(\mathscr{O}_{\mathscr{G}}, \mathrm{E}\right)$ defined by

$$
\mathrm{N}_{k} \mathscr{H} \cdot\left(\mathscr{O}_{\mathscr{G}}, \mathrm{E}\right):=\left\{\phi: \phi\left(\mathscr{I}^{[j]}\right) \subseteq \mathrm{N}_{k-j} \mathrm{E}\right\}
$$

Now if $\mathrm{E}$ is a locally nilpotent $\mathscr{O}_{\mathscr{G}}$-module, let us consider the following maps:

$$
\begin{array}{ll}
i_{\mathrm{E}}: \mathrm{E} \rightarrow \mathscr{H}_{.}\left(\mathscr{O}_{\mathscr{G}}, \mathrm{E}\right) & i_{\mathrm{E}}(e)(b):=i_{\mathscr{G}}^{*}(b) e \\
\sigma: \mathscr{H} .\left(\mathscr{O}_{\mathscr{G}}, \mathrm{E}\right) \rightarrow \mathrm{E} & \phi \mapsto \phi(1) .
\end{array}
$$

The map $i_{\mathscr{G}}$ is the identity section of $\mathscr{G}$ and the map $i_{\mathrm{E}}$ corresponds to the map $i_{\mathrm{E}}$ defined in (2.16.3). Similarly the map $\sigma$ corresponds to the map $\eta_{s}$ of statement (4) of

\footnotetext{
${ }^{7}$ In the systematic treatment in the appendix, we use $\mu_{*}^{\prime}$ instead of $\mu_{*}$.
} 
Proposition 2.17. Note that $\sigma$ is compatible with the filtrations and that $\sigma \circ i_{\mathrm{E}}=\mathrm{id}_{\mathrm{E}}$. This shows that $i_{\mathrm{E}}$ is injective and strictly compatible with the filtrations. The image of $i_{\mathrm{E}}$ is just the set of homomorphisms which factor through $\mathscr{O}_{\mathscr{G}_{0}}=i_{\mathscr{G}_{*}}\left(\mathscr{O}_{\mathrm{X}}\right)$, which corresponds to $(\mathscr{A}(\mathrm{E}))^{\theta_{\mathscr{A}}}$. This proves (1) of Proposition 2.17, and (2) is a tautological consequence of the definitions.

Define

$$
\tau: \mathrm{E} \rightarrow \mathscr{H} \cdot\left(\mathscr{O}_{\mathscr{G}}, \mathrm{E}\right) \text { by } \tau(e)(b):=\iota^{*}(b) e,
$$

where $\iota: \mathscr{G} \rightarrow \mathscr{G}$ is the inverse mapping in the group $\mathscr{G}$. If $e \in \mathrm{E}$, then a priori $\tau(e)$ is just an element of $\mathscr{H}_{0 m_{\mathscr{O}_{\mathrm{X}}}}\left(\mathscr{O}_{\mathscr{G}}, \mathrm{E}\right)$, but if $e$ is annihilated by $\mathrm{I}^{[n+1]}$ then $\tau(e) \in$ $\mathscr{H} \operatorname{lom}\left(\mathscr{O}_{\mathscr{G}_{n}}, \mathrm{E}\right)$. Thus $\tau$ is well-defined if $\mathrm{E}$ is locally nilpotent. Note that $\sigma \circ \tau=\mathrm{id}_{\mathrm{E}}$, so $\tau$ is also injective. If $e \in \mathrm{N}_{k} \mathrm{E}$ and $b \in \mathscr{I}^{[j]}$, then $\iota^{*}(b) e \in \mathrm{N}_{k-j} \mathrm{E}$, so $\tau(e) \in \mathrm{N}_{k} \mathscr{H} \cdot\left(\mathscr{O}_{\mathscr{G}}, \mathrm{E}\right)$. Thus $\tau$ is compatible with the filtrations, and in fact is strictly compatible because $\sigma$ is also compatible.

It is clear that the image of $\tau$ consists precisely of the elements of $\mathscr{H}_{\text {. }}\left(\mathscr{O}_{\mathscr{G}}, \mathrm{E}\right)$ which are $\iota$-linear. We claim that these are the elements which correspond to elements of $\mathscr{T}_{\mathscr{L}}(\mathrm{E}) \subseteq \mathscr{A}_{\mathscr{L}}(\mathrm{E})$. Indeed, if $\phi: \mathscr{O}_{\mathscr{G}} \rightarrow \mathrm{E}$ is $\iota$-linear then it follows from the commutativity of the diagram

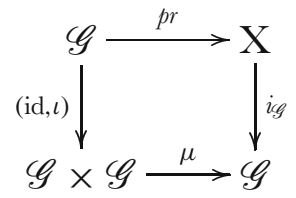

that $\theta_{t o t}(\phi)=0$, and the converse follows from the fact that the diagram is Cartesian.

Thus we can write $\tau=j_{\mathrm{E}} \circ \bar{\tau}$, where $\bar{\tau}: \mathrm{E} \rightarrow \mathscr{T}_{\mathscr{L}}(\mathrm{E})$ is an isomorphism of $\mathscr{O}_{\mathrm{X}^{-}}$ modules, inverse to the mapping $\sigma \circ j$. It is clear from the definitions of $\tau$ and $\theta_{\mathscr{A}}$ that $\tau$ takes $\theta_{\mathrm{E}}$ to $\iota_{*} \theta_{\mathscr{A}}$, and this proves (4) of Proposition 2.17 .

It remains for us the prove statement (3). First let us check that $h$ is compatible with the Higgs fields as described there. As we have observed in equation (2.16.3), $j_{\mathrm{E}}$ takes $\theta_{0}$ to $\theta_{t o t}$. More precisely, but perhaps somewhat cryptically: $\theta_{t o t} \circ j_{\mathrm{E}}=$ $j_{\mathrm{E}} \circ \theta_{0}$, where for example we are writing $\theta_{t o t}$ for the endomorphism of $\mathrm{E} \otimes \mathscr{A}_{\mathscr{L}}$ induced by some element of $\mathscr{O}_{\mathscr{G}}$ corresponding to the $\mathscr{O}_{\mathscr{G}}$-module structure given by $\theta_{\text {tot }}$. Hence:

$$
\begin{aligned}
\left(\theta_{t o t} \circledast \theta_{\mathscr{A}}\right) \circ\left(j_{\mathrm{E}} \otimes \mathrm{id}_{\mathscr{A}}\right) & =\left(j_{\mathrm{E}} \otimes \mathrm{id}_{\mathscr{A}}\right) \circ\left(\theta_{0} \circledast \theta_{\mathscr{A}}\right) \\
\left(\mathrm{id}_{\mathrm{E}} \otimes m\right) \circ\left(\theta_{t o t} \circledast \theta_{\mathscr{A}}\right) \circ\left(j_{\mathrm{E}} \otimes \mathrm{id}_{\mathscr{A}}\right) & =\left(\mathrm{id}_{\mathrm{E}} \otimes m\right) \circ\left(j_{\mathrm{E}} \otimes \mathrm{id}_{\mathscr{A}}\right) \circ\left(\theta_{0} \circledast \theta_{\mathscr{A}}\right) \\
\theta_{t o t} \circ\left(\mathrm{id}_{\mathrm{E}} \otimes m\right) \circ\left(j_{\mathrm{E}} \otimes \mathrm{id}_{\mathscr{A}}\right) & =\left(\mathrm{id}_{\mathrm{E}} \otimes m\right) \circ\left(j_{\mathrm{E}} \otimes \mathrm{id}_{\mathscr{A}}\right) \circ \theta_{\mathscr{A}} \\
\theta_{\text {tot }} \circ h & =h \circ \theta_{\mathscr{A}} .
\end{aligned}
$$


Similarly, $\left(\theta_{0} \circledast \theta_{\mathscr{A}}\right) \circ j_{\mathrm{E}}=j_{\mathrm{E}} \circ \theta_{\mathscr{T}}$, so

$$
\begin{aligned}
\left(\theta_{0} \circledast \theta_{\mathscr{A}} \circledast \theta_{\mathscr{A}}\right) \circ\left(j_{\mathrm{E}} \otimes \mathrm{id}_{\mathscr{A}}\right) & =\left(j_{\mathrm{E}} \otimes \mathrm{id}_{\mathscr{A}}\right) \circ\left(\theta_{\mathscr{T}} \circledast \theta_{\mathscr{A}}\right) \\
\left(\mathrm{id}_{\mathrm{E}} \otimes m\right) \circ\left(\theta_{0} \circledast \theta_{\mathscr{A}} \circledast \theta_{\mathscr{A}}\right) \circ\left(j_{\mathrm{E}} \otimes\right. & \left.\operatorname{id}_{\mathscr{A}}\right) \\
& =\left(\mathrm{id}_{\mathrm{E}} \otimes m\right) \circ\left(j_{\mathrm{E}} \otimes \mathrm{id}_{\mathscr{A}}\right) \circ\left(\theta_{\mathscr{T}} \circledast \theta_{\mathscr{A}}\right) \\
\left(\theta_{0} \circledast \theta_{\mathscr{A}}\right) \circ\left(\mathrm{id}_{\mathrm{E}} \otimes m\right) \circ\left(j_{\mathrm{E}} \otimes \mathrm{id}_{\mathscr{A}}\right) & =h \circ\left(\theta_{\mathscr{T}} \circledast \theta_{\mathscr{A}}\right) \\
\theta_{\mathscr{A}} \circ h & =h \circ \theta_{t o t} .
\end{aligned}
$$

Thus $h$ takes $\theta_{\mathscr{A}}$ to $\theta_{\text {tot }}$ and $\theta_{\text {tot }}$ to $\theta_{\mathscr{A}}$ as claimed. Since $h$ takes $\theta_{\text {tot }}$ to $\theta_{\mathscr{A}}$, it induces the map $k$ :

$$
\mathscr{T}_{\mathscr{L}}\left(\mathscr{T}_{\mathscr{L}}(\mathrm{E})\right):=\left(\mathscr{A}_{\mathscr{L}}\left(\mathscr{T}_{\mathscr{L}}\right)(\mathrm{E})\right)^{\theta_{t o t}} \rightarrow \mathscr{A}_{\mathscr{L}}(\mathrm{E})^{\theta_{\mathscr{A}}}=\mathrm{E}
$$

Let us check that $h$ is compatible with the filtrations. By definition,

$$
\mathrm{N}_{c}^{t o t} \mathscr{A}_{\mathscr{L}}\left(\mathscr{T}_{\mathscr{L}}(\mathrm{E})\right)=\sum_{a} \operatorname{Im}\left(\mathrm{N}_{a} \mathscr{T}_{\mathscr{L}}(\mathrm{E}) \otimes \mathrm{N}_{c-a} \mathscr{A}_{\mathscr{L}} \rightarrow \mathscr{T}_{\mathscr{L}}(\mathrm{E}) \otimes \mathscr{A}_{\mathscr{L}}(\mathrm{E})\right)
$$

The definition of $\mathrm{N}_{a} \mathscr{T}_{\mathscr{L}}(\mathrm{E})$ shows that its image under $j_{\mathrm{E}}$ is contained in the sum of the images of $\mathrm{N}_{b} \mathrm{E} \otimes \mathrm{N}_{a-b} \mathscr{A}_{\mathscr{L}}$. Hence $h$ maps $\mathrm{N}_{c}^{\text {tot }} \mathscr{A}_{\mathscr{L}}\left(\mathscr{T}_{\mathscr{L}}(\mathrm{E})\right)$ into the sum of the images of

$$
\mathrm{N}_{b} \mathrm{E} \otimes \mathrm{N}_{a-b} \mathscr{A}_{\mathscr{L}} \otimes \mathrm{N}_{c-a} \mathscr{A}_{\mathscr{L}} \stackrel{m}{\rightarrow} \mathrm{E} \otimes \mathscr{A}_{\mathscr{L}}(\mathrm{E})
$$

which is contained in $\mathrm{N}_{c}^{\text {tot }} \mathscr{A}_{\mathscr{L}}(\mathrm{E})$.

Note that if $h$ is a strict isomorphism, then it induces a strict isomorphism from the annihilator of $\theta_{\text {tot }}$ to the annihilator of $\theta_{\mathscr{A}}$, i.e., from $\mathscr{T}_{\mathscr{L}}\left(\mathscr{T}_{\mathscr{L}}(\mathrm{E})\right)$ to E. Thus $k$ is also a strict isomorphism.

Thus it remains only to show that $h$ is a strict isomorphism. Suppose first that

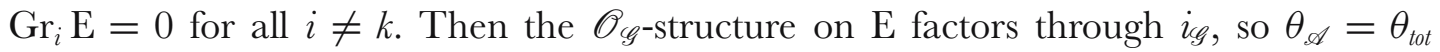
and $\mathrm{E}=\mathscr{T}_{\mathscr{L}}(\mathrm{E}) \subseteq \mathscr{A}_{\mathscr{L}}(\mathrm{E})$. Then the map $h$ is:

$$
e \otimes a \mapsto e \otimes 1 \otimes a \mapsto e \otimes a
$$

i.e., the identity map. Now we can proceed by dévissage. Statement (4) shows that the functor $\mathrm{E} \mapsto \mathscr{T}_{\mathscr{L}}(\mathrm{E})$ preserves strict exact sequences, and since $\mathrm{Gr} \mathscr{A}_{\mathscr{L}}$ is locally free, the same is true of the functors $\mathrm{E} \mapsto \mathscr{A}_{\mathscr{L}}(\mathrm{E})$ and $\mathrm{E} \mapsto \mathscr{A}_{\mathscr{L}}\left(\mathscr{T}_{\mathscr{L}}(\mathrm{E})\right)$. Now suppose that $\mathrm{N}_{a} \mathrm{E}=0$ and that $h$ induces an isomorphism for $\mathrm{E}^{\prime}:=\mathrm{N}_{b-1} \mathrm{E}$. Then we have a strict exact sequence

$$
0 \rightarrow \mathrm{E}^{\prime} \rightarrow \mathrm{N}_{b} \mathrm{E} \rightarrow \mathrm{E}^{\prime \prime} \rightarrow 0
$$


where $\mathrm{E}^{\prime \prime}:=\mathrm{N}_{b} \mathrm{E} / \mathrm{N}_{b-1} \mathrm{E}$. We have seen above that the theorem is true for $\mathrm{E}^{\prime \prime}$, and it holds for $\mathrm{E}^{\prime}$ by the induction assumption. Then it also holds for $\mathrm{N}_{b} \mathrm{E}$ by the strict exactness of the functors $\mathscr{A}_{\mathscr{L}}\left(\right.$ ) and $\mathscr{A}_{\mathscr{L}}\left(\mathscr{T}_{\mathscr{L}}()\right)$. It follows by induction that $h$ is a strict isomorphism whenever the filtration on $\mathrm{E}$ is bounded, and, by taking direct limits, whenever the filtration is bounded below and exhaustive. This completes the proof.

Remark 2.18. - It is easy to see that the filtration of $\mathscr{T}_{\mathscr{L}}(\mathrm{E})$ induced by the total filtration $\mathrm{N}_{\text {tot }}$ on $\mathscr{A}_{\mathscr{L}}(\mathrm{E})$ is the same as the filtration induced by the filtration $\mathscr{A}_{\mathscr{L}} \otimes$ N.E. The total filtration has the advantage of being again $\mathscr{I}^{\circ}$-saturated, a fact we will exploit in our cohomology computations in the next section.

Remark 2.19. - A similar result holds for standard Higgs fields if one works with the divided power completion of $\mathscr{A}_{\mathscr{L}}$ along the ideal of a section. More abstractly, suppose that $\mathrm{T}$ and $\Omega$ be as above, let $\theta: \mathrm{E} \rightarrow \mathrm{E} \otimes \Omega$ be a locally nilpotent T-Higgs field on $\mathrm{E}$. Let I be the ideal of the symmetric algebra $\mathrm{S}^{*} \mathrm{~T}$ generated by $\mathrm{T}$. Then an I-saturated filtration on $\mathrm{E}$ is just a filtration $\mathrm{N}$ such that $\mathrm{IN}_{k} \mathrm{E} \subseteq \mathrm{N}_{k-1} \mathrm{E}$. Let $\mathscr{A}_{\Omega}$ be the divided power algebra $\Gamma \cdot \Omega$, and define $\mathscr{A}_{\Omega}(\mathrm{E}):=\mathrm{E} \otimes \mathscr{A}_{\Omega}$ and $\mathscr{T}_{\Omega}(\mathrm{E}):=\left(\mathscr{A}_{\Omega}(\mathrm{E})\right)^{\theta_{\text {tot }}}$. Then the evident analog of Proposition 2.17 holds.

We will sometimes want to consider graded Higgs fields and PD-Higgs modules, i.e., graded modules over the graded ring $\mathscr{O}_{\mathscr{G}}$, where $\mathscr{O}_{\mathscr{G}}=\Gamma . \mathrm{T}$ or $\mathrm{S}{ }^{*} \mathrm{~T}$. There is an evident functor $\mathrm{Gr}$ from the category of $\mathscr{I}^{\circ}$-filtered (resp. $\mathscr{I}$-filtered) modules to the category of graded $\mathscr{O}_{\mathscr{G}}$-modules, compatible with the convolution tensor product. In particular, if $\mathscr{L}$ is a $\mathbf{T}$-torsor, then $\operatorname{Gr} \mathscr{A}_{\mathscr{L}} \cong \mathrm{S} \Omega=\mathrm{S}_{\mathbf{T}}^{*}$, as a graded $\Gamma$.T-modules (note that the multiplication sends $\Gamma_{a} \mathrm{~T} \otimes \mathrm{S}^{b} \Omega$ to $\mathrm{S}_{b-a} \Omega$ ); furthermore its divided power envelope $\Gamma . \Omega$ is in a natural way a graded $\mathrm{S}^{*} \Omega$-module. If $\mathrm{E}$ is an $\mathscr{I}^{\circ}$-filtered $\mathscr{O}_{\mathrm{G}}$-module, the natural map

$$
\mathrm{GrE} \otimes \mathscr{A}_{\mathbf{T}} \cong \mathrm{GrE} \otimes \mathrm{Gr} \mathscr{A}_{\mathscr{L}} \rightarrow \mathrm{Gr} \mathscr{A}_{\mathscr{L}}(\mathrm{E})
$$

is an isomorphism, since $\operatorname{Gr} \mathscr{A}_{\mathscr{L}}$ is locally free over $\mathscr{O}_{\mathrm{X}}$, and it is compatible both with $\theta_{\mathscr{A}}$ and $\theta_{\text {tot }}$. In particular, if $\mathrm{E}$ satisfies the hypothesis of Proposition 2.17, the map $\mathscr{T}_{\mathscr{L}}(\mathrm{E}) \rightarrow \mathscr{A}_{\mathscr{L}}(\mathrm{E})$ induces a map

$$
\operatorname{Gr} \mathscr{T}_{\mathscr{L}}(\mathrm{E}) \rightarrow \operatorname{Gr}\left(\mathscr{A}_{\mathscr{L}}(\mathrm{E})\right) \cong \operatorname{Gr}(\mathrm{E}) \otimes \mathscr{A}_{\mathbf{T}}(\mathrm{E})
$$

whose image is annihilated by $\theta_{\text {tot }}$ and hence induces a map

$$
\operatorname{Gr} \mathscr{T}_{\mathscr{L}}(\mathrm{E}) \rightarrow \mathscr{T}_{\mathbf{T}}(\operatorname{Gr}(\mathrm{E}))
$$

Corollary 2.20. - Let $\left(\mathrm{E}, \theta, \mathrm{N}\right.$.) be an $\mathscr{O}_{\mathrm{X}}$-module $\mathrm{E}$ equipped with an $\mathscr{O}_{\mathscr{G} \text {-module struc- }}$ ture $\theta$ and an $\mathscr{I}^{\circ}$-filtration N., as in Proposition 2.17. 
1. The map $\operatorname{Gr} \mathscr{T}_{\mathscr{L}}(\mathrm{E}) \rightarrow \mathscr{T}_{\mathbf{T}}(\mathrm{Gr}(\mathrm{E}))$ above is an isomorphism. In fact there is a commutative diagram of isomorphisms:

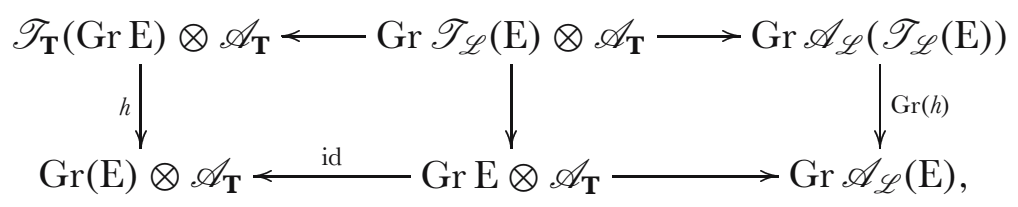

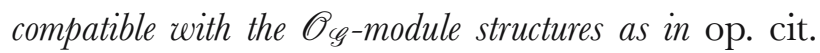

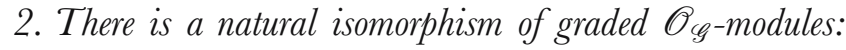

$$
\operatorname{Gr} \mathscr{T}_{\mathscr{L}}(\mathrm{E}) \cong \iota_{*} \mathrm{GrE} .
$$

Proof. - The existence and the commutativity of the diagram is clear, as is the fact that the arrows are compatible with the $\mathscr{O}_{\mathscr{G}}$-module structures. Furthermore, it follows from Proposition 2.17 that $h$ and $\operatorname{Gr}(h)$ are isomorphisms. It follows that the middle vertical arrow is an isomorphism, and that the image of $\operatorname{Gr} \mathscr{T}_{\mathscr{L}}(\mathrm{E})$ in $\mathrm{GrE} \otimes \mathscr{A}_{\mathbf{T}}$ is exactly the annihilator of $\theta_{\text {tot }}$, i.e., $\mathscr{T}_{\mathbf{T}}(\mathrm{Gr}(\mathrm{E}))$. This proves (1). Then (2) follows by applying (4) of Proposition 2.17 with $\mathrm{E}$ replaces by $\mathrm{GrE}$ and $\mathscr{L}$ replaced by $\mathbf{T}$.

There is a useful cohomological complement to the construction of Remark 2.19. Recall that associated to a T-Higgs module $(\mathrm{E}, \theta)$ is its Higgs (Koszul) complex

$$
\mathrm{E} \rightarrow \mathrm{E} \otimes \Omega \rightarrow \mathrm{E} \otimes \Omega^{2} \rightarrow \cdots,
$$

where $\Omega^{i}:=\Lambda^{i} \Omega$.

Proposition 2.21. - Let $\mathrm{E}$ be a graded $\mathscr{O}_{\mathrm{X}}$-module with a graded $\mathrm{T}$-Higgs field $\theta$ :

$$
\theta: \mathrm{E} \rightarrow \mathrm{E} \otimes \Omega
$$

where $\Omega$ is in degree 1 . Using the notation of Remark 2.19, let

$$
\mathscr{A}_{\Omega}^{i, j}(\mathrm{E}):=\mathscr{A}_{\Omega}(\mathrm{E}) \otimes \Omega^{i} \otimes \Omega^{j}
$$

and let

$$
\begin{aligned}
& d_{\mathscr{A}}: \mathscr{A}_{\Omega}^{i, j}(\mathrm{E}) \rightarrow \mathscr{A}_{\Omega}^{i+1, j}(\mathrm{E}) \\
& d_{t o t}: \mathscr{A}_{\Omega}^{i, j}(\mathrm{E}) \rightarrow \mathscr{A}_{\Omega}^{i, j+1}(\mathrm{E})
\end{aligned}
$$

be the boundary maps associated to the fields $\theta_{\mathscr{A}}$ and $\theta_{\text {tot }}$ respectively, tensored with the identity. Then these maps fit into a graded double complex $\mathscr{A}_{\Omega}^{\cdot}(\mathrm{E})$, and the maps $i$ and $j$ of Remark 2.19 define augmentations of the double complex

$$
\begin{aligned}
\mathrm{E} & \otimes \Omega^{\cdot} \rightarrow \mathscr{A}_{\Omega}^{\cdot}(\mathrm{E}) \\
\mathscr{T}_{\Omega}(\mathrm{E}) \otimes \Omega^{\cdot} & \rightarrow \mathscr{A}_{\Omega}^{\cdot}(\mathrm{E}) .
\end{aligned}
$$

For each $i, \mathscr{A}_{\Omega}^{i, \cdot}(\mathrm{E})$ is a resolution of $\mathrm{E} \otimes \Omega^{i}$ and for each $j \mathscr{A}_{\Omega}^{\cdot, j}(\mathrm{E})$ is a resolution of $\mathscr{T}_{\Omega}(\mathrm{E}) \otimes \Omega^{j}$. 
Proof. - It is immediate to verify that the boundary maps commute and hence define a double complex. The fact that $\mathscr{A}_{\Omega}^{i, \cdot}(\mathrm{E})$ is a graded resolution of $\mathrm{E} \otimes \Omega^{i}$ follows from the filtered Poincaré lemma $[3,6.13]$ for the divided power algebra $\Gamma$. $(\Omega)$. Since $h$ is an isomorphism transforming $\theta_{\text {tot }}$ into $\theta_{\mathscr{A}}$, the second statement follows.

Let us now return to our discussion of the Cartier transform. Recall that the center of $\mathrm{D}_{\mathrm{X} / \mathrm{S}}^{\gamma}$ can be identified with the divided power algebra $\Gamma \cdot \mathrm{T}_{\mathrm{X}^{\prime} / \mathrm{S}}$. Let $\mathscr{I}_{\mathrm{X}}$ denote the divided power filtration of the divided power ideal $\mathscr{I}_{\mathrm{X}}$ of $\Gamma \cdot \mathrm{T}_{\mathrm{X}^{\prime} / \mathrm{S}}$. Let $\mathrm{MICN}_{\gamma}(\mathrm{X} / \mathrm{S})$ denote the category of $\mathrm{D}_{\mathrm{X} / \mathrm{S}}^{\gamma}$-modules $\mathrm{E}$ equipped with an exhaustive, horizontal, and bounded below filtration $\mathscr{I}^{\circ}$-filtration N. (see Definition 2.16). Similarly, let $\mathrm{HIGN}_{\gamma}\left(\mathrm{X}^{\prime} / \mathrm{S}\right)$ denote the category of $\Gamma^{\cdot} \mathrm{T}_{\mathrm{X}^{\prime} / \mathrm{S}}$-modules $\mathrm{E}^{\prime}$ equipped with an exhaustive and bounded below $\mathscr{I}^{\circ}$-saturated filtration $\mathrm{N}^{\prime}$.

If $\left(\mathrm{E}, \nabla, \mathrm{N}\right.$.) is an object of $\mathrm{MICN}_{\gamma}(\mathrm{X} / \mathrm{S})$, let

$$
\begin{aligned}
\mathrm{E}^{\nabla, \gamma} & :=\mathscr{H}_{0} m_{\mathrm{D}_{\mathrm{X} / \mathrm{s}}^{\gamma}}\left(\mathscr{O}_{\mathrm{X}}, \mathrm{E}\right), \text { and } \\
\mathrm{E}^{\nabla} & :=\operatorname{Ker}\left(\mathrm{E} \stackrel{\nabla}{\rightarrow} \mathrm{E} \otimes \Omega_{\mathrm{X} / \mathrm{S}}^{1}\right) .
\end{aligned}
$$

The action of the center $\Gamma \cdot \mathrm{T}_{\mathrm{X}^{\prime} / \mathrm{S}}$ of $\mathrm{D}_{\mathrm{X} / \mathrm{S}}^{\gamma}$ defines a PD-Higgs field on $\mathrm{F}_{\mathrm{X} / \mathrm{S} *} \mathrm{E}$ and hence an F-PD Higgs field $\psi$ on $\mathrm{E}$; note that $\mathrm{E}^{\psi}$ is invariant under the connection $\nabla: \mathrm{E} \rightarrow \mathrm{E} \otimes \Omega_{\mathrm{X} / \mathrm{S}}^{1}$. Furthermore, $\mathrm{E}^{\nabla, \gamma}=\mathrm{E}^{\psi^{\nabla}}$, since $\mathrm{D}_{\mathrm{X} / \mathrm{S}}^{\gamma}$ is generated as a topological ring by $\mathrm{T}_{\mathrm{X} / \mathrm{S}}$ and $\Gamma \cdot \mathrm{T}_{\mathrm{X}^{\prime} / \mathrm{S}}$.

We endow $\mathscr{A}_{\mathscr{L}}(\mathrm{E}):=\mathrm{E} \otimes \mathscr{A}_{\mathscr{L}}$ with the tensor product $\mathrm{D}_{\mathrm{X} / \mathrm{S}}^{\gamma}$-module structure $\nabla$ coming from the given structures on $\mathrm{E}$ and on $\mathscr{A}_{\mathscr{X} / \mathscr{S}}$ and with the tensor product filtration coming from the filtrations $\mathrm{N}$. of $\mathrm{E}$ and $\mathscr{A}_{\mathscr{X} / \mathscr{S}}$. We also endow it with the F-PD-Higgs field $\theta_{\mathscr{A}}:=\mathrm{id} \otimes \theta_{\mathscr{A}}$, where $\theta_{\mathscr{A}}$ is the F-PD-Higgs field of $\mathscr{A}_{\mathscr{X} / \mathscr{S}}$.

Lemma 2.22. - The action $\theta_{\mathscr{A}}$ of $\Gamma$. $\left(\mathrm{T}_{\mathrm{X}^{\prime} / \mathrm{S}}\right)$ on $\mathscr{A}_{\mathscr{X} / \mathscr{S}}(\mathrm{E})$ commutes with the action of $\mathrm{D}_{\mathrm{X} / \mathrm{S}}^{\gamma}$ corresponding to the tensor product $\mathrm{D}_{\mathrm{X} / \mathrm{S}}^{\gamma}$-module structure.

Proof. - As we have already observed, it follows from the formula (2.7.1) that the $p$-curvature PD-Higgs field of $\mathscr{A}_{\mathscr{X} / \mathscr{S}}(\mathrm{E})$ commutes with id $\otimes \theta_{\mathscr{A}}$. That is, the action of $\Gamma \cdot \mathrm{T}_{\mathrm{X}^{\prime} / \mathrm{S}} \subseteq \mathrm{D}_{\mathrm{X} / \mathrm{S}}^{\gamma}$ commutes with id $\otimes \theta_{\mathscr{A}}$. Furthermore, if $\mathrm{D} \in \mathrm{T}_{\mathrm{X} / \mathrm{S}}, \xi^{\prime} \in \Gamma \cdot \mathrm{T}_{\mathrm{X}^{\prime} / \mathrm{S}}$,

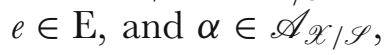

$$
\begin{aligned}
\left(\mathrm{id} \otimes \theta_{\xi^{\prime}}\right) \nabla_{\mathrm{D}}(e \otimes a) & =\nabla_{\mathrm{D}}(e) \otimes \theta_{\xi^{\prime}}(a)+e \otimes \theta_{\xi^{\prime}} \nabla_{\mathrm{D}}(a) \\
& =\nabla_{\mathrm{D}}(e) \otimes \theta_{\xi^{\prime}}(a)+e \otimes \nabla_{\mathrm{D}} \theta_{\xi^{\prime}}(a) \\
& =\nabla_{\mathrm{D}}\left(\operatorname{id} \otimes_{\theta_{\xi^{\prime}}}\right)(e \otimes a) .
\end{aligned}
$$

Since $\mathrm{D}_{\mathrm{X} / \mathrm{S}}^{\gamma}$ is generated by $\mathrm{T}_{\mathrm{X} / \mathrm{S}}$ and $\Gamma \cdot \mathrm{T}_{\mathrm{X}^{\prime} / \mathrm{S}}$, it follows that $\nabla_{\mathrm{D}}$ commutes with $\theta_{\xi^{\prime}}$ for every $\mathrm{D} \in \mathrm{D}_{\mathrm{X} / \mathrm{S}}^{\gamma}$. 
Now recall that, by definition, $\iota_{*} \mathrm{C}_{\mathscr{X} / \mathscr{S}}(\mathrm{E})=\mathscr{H}_{0} m_{\mathrm{D}_{\mathrm{X} / \mathrm{s}}^{\gamma}}\left(\mathscr{B}_{\mathscr{X} / \mathscr{S}}, \mathrm{E}\right)$, with the $\Gamma \cdot \mathrm{T}_{\mathrm{X}^{\prime} / \mathrm{S}}$-module structure coming from $\mathrm{E}$, where $\mathscr{B}_{\mathscr{X} / \mathscr{S}}:=\mathscr{H}_{0} m_{\mathscr{O}_{\mathrm{X}}}\left(\mathscr{A}_{\mathscr{X} / \mathscr{S}}, \mathscr{O}_{\mathrm{X}}\right)$ in the category of $\mathrm{D}_{\mathrm{X} / \mathrm{S}}^{\gamma}$-modules. Thus when $\mathrm{E}$ is locally nilpotent,

$$
\begin{aligned}
\iota_{*} \mathrm{C}_{\mathscr{X} / \mathscr{S}}(\mathrm{E}):=\mathscr{H}_{0} m_{\mathrm{D}_{\mathrm{X} / \mathrm{S}}^{\gamma}}\left(\mathscr{B}_{\mathscr{X} / \mathscr{S}}, \mathrm{E}\right) & \cong\left(\mathrm{E} \otimes \mathscr{A}_{\mathscr{X} / \mathscr{S}}\right)^{\nabla, \gamma} \\
& =\left(\left(\mathscr{A}_{\mathscr{X} / \mathscr{S}}(\mathrm{E})\right)^{\psi}\right)^{\nabla} .
\end{aligned}
$$

Of course, the total PD-Higgs field on $\mathrm{C}_{\mathscr{X} / \mathscr{S}}(\mathrm{E})$ is zero, but because of the commutation of $\mathrm{D}_{\mathrm{X} / \mathrm{S}}^{\gamma}$ and id $\otimes \theta_{\mathscr{A}}, \iota_{*} \mathrm{C}_{\mathscr{X} / \mathscr{S}}(\mathrm{E})$ is stable under the PD-Higgs field id $\otimes \theta_{\mathscr{A}}$ of $\mathscr{A}_{\mathscr{X} / \mathscr{S}}$. In fact, the induced PD-Higgs field induced by $\theta_{\mathscr{A}}$ on is $\iota_{*}$ of the PD-Higgs field induced by $\theta_{\mathrm{E}}$. A geometric explanation of this fact is given in the appendix after Definition 5.9; it can also be checked by direct computation. Thus it follows that

$$
\mathrm{C}_{\mathscr{X} / \mathscr{S}}(\mathrm{E}):=\iota_{*} \mathscr{H}_{0} m_{\mathrm{D}_{\mathrm{X} / \mathrm{S}}^{\gamma}}\left(\mathscr{B}_{\mathscr{X} / \mathscr{S}}, \mathrm{E}\right) \cong\left(\mathscr{T}_{\mathscr{X} / \mathscr{S}}(\mathrm{E})\right)^{\nabla}
$$

where $\mathscr{T}_{\mathscr{X} / \mathscr{S}}(\mathrm{E}):=\left(\mathscr{A}_{\mathscr{X} / \mathscr{S}}(\mathrm{E})\right)^{\theta_{\text {tot }}}$ as in Proposition 2.17.

It is clear from the construction that there are natural maps, compatible with the connections and F-PD-Higgs fields shown:

$$
\left(\mathrm{E}, \theta_{0}, \nabla\right) \stackrel{i}{\rightarrow}\left(\mathscr{A}_{\mathscr{X} / \mathscr{S}}(\mathrm{E}), \theta_{\mathscr{A}}, \nabla\right) \stackrel{j}{\leftarrow}\left(\mathrm{F}_{\mathrm{X} / \mathrm{S}}^{*} \mathrm{C}_{\mathscr{X} / \mathscr{S}}(\mathrm{E}), \theta, \nabla_{0}\right) .
$$

Here $\nabla_{0}$ is the Frobenius descent connection on $\mathrm{F}_{\mathrm{X} / \mathrm{S}}^{*} \mathrm{G}_{\mathscr{X} / \mathscr{S}}(\mathrm{E})$. Since N. is an $\mathscr{I}^{*}$-filtration on $\mathrm{E}$, the filtration on $\mathrm{F}_{\mathrm{X} / \mathrm{S}}^{*} \mathrm{C}_{\mathscr{X} / \mathscr{S}}(\mathrm{E})$ induced by the total filtration of $\mathscr{A}_{\mathscr{X} / \mathscr{S}}(\mathrm{E})$ is horizontal and is also an $\mathscr{I}^{\circ}$-filtration with respect to the action of $\theta_{\mathscr{A}}$. It follows that it descends to an $\mathscr{I}^{\circ}$-filtration on $\mathrm{C}_{\mathscr{X} / \mathscr{S}}(\mathrm{E})$. Thus we obtain a filtered version of the Cartier transform:

$$
\mathrm{C}_{\mathscr{X} / \mathscr{S}}: \mathrm{MICN}_{\gamma}(\mathrm{X} / \mathrm{S}) \rightarrow \mathrm{HIGN}_{\gamma}\left(\mathrm{X}^{\prime} / \mathrm{S}\right)
$$

On the other hand, if $\left(\mathrm{E}^{\prime}, \theta^{\prime}, \mathrm{N}^{\prime}\right.$.) is an object of $\mathrm{HIGN}_{\gamma}\left(\mathrm{X}^{\prime} / \mathrm{S}\right)$, we can endow $(2.22 .4)$

$$
\mathscr{A}_{\mathscr{X} / \mathscr{S}}^{\prime}\left(\mathrm{E}^{\prime}\right):=\mathrm{F}_{\mathrm{X} / \mathrm{S}}^{*} \mathrm{E}^{\prime} \otimes_{\mathscr{O}_{\mathrm{X}}} \mathscr{A}_{\mathscr{X} / \mathscr{S}}
$$

with the tensor product F-PD-Higgs field $\theta_{t o t}^{\prime}$. It follows as in Lemma 2.22 that $\theta_{t o t}^{\prime}$ commutes with the tensor product connection on $\mathrm{F}_{\mathrm{X} / \mathrm{S}}^{*} \mathrm{E}^{\prime} \otimes \mathscr{A}_{\mathscr{X} / \mathscr{S}}$, where $\mathrm{F}_{\mathrm{X} / \mathrm{S}}^{*} \mathrm{E}^{\prime}$ is given the Frobenius descent connection $\nabla_{0}$. Thus

$$
\mathrm{C}_{\mathscr{X} / \mathscr{S}}^{\prime}\left(\mathrm{E}^{\prime}\right):=\left(\mathscr{A}_{\mathscr{X} / \mathscr{S}}^{\prime}\left(\mathrm{E}^{\prime}\right)\right)^{\theta_{t o t}^{\prime}}
$$

inherits a nilpotent $\mathrm{D}_{\mathrm{X} / \mathrm{S}}^{\gamma}$-module structure from $\mathscr{A}_{\mathscr{X} / \mathscr{S}}$, which we denote by $\nabla_{\mathscr{A}}$. We have natural maps

$$
\mathrm{F}_{\mathrm{X} / \mathrm{S}}^{*}\left(\mathrm{E}^{\prime}, \theta^{\prime}, \nabla_{0}\right) \stackrel{i^{\prime}}{\rightarrow}\left(\mathscr{A}_{\mathscr{X} / \mathscr{S}}^{\prime}\left(\mathrm{E}^{\prime}\right), \theta_{t o t}^{\prime}, \nabla_{\mathscr{A}^{\prime}}\right) \stackrel{j^{\prime}}{\longleftarrow}\left(\mathrm{C}_{\mathscr{X} / \mathscr{S}}^{\prime}\left(\mathrm{E}^{\prime}\right), \theta_{t o t}^{\prime}, \nabla_{\mathscr{A}}\right)
$$


where $i^{\prime}$ takes the PD-Higgs field of $\mathrm{E}^{\prime}$ As before, the $\mathscr{I}^{\cdot}$-filtration $\mathrm{N}^{\prime}$. on $\mathrm{E}^{\prime}$ induces an $\mathscr{I}^{\circ}$-filtration on $\mathrm{C}_{\mathscr{X} / \mathscr{S}}^{\prime}\left(\mathrm{E}^{\prime}\right)$, and we get a functor:

$$
\mathrm{C}_{\mathscr{X} / \mathscr{S}}^{\prime}: \mathrm{HIGN}_{\gamma}^{*}\left(\mathrm{X}^{\prime} / \mathrm{S}\right) \rightarrow \mathrm{MICN}_{\gamma}(\mathrm{X} / \mathrm{S})
$$

The commutative diagram

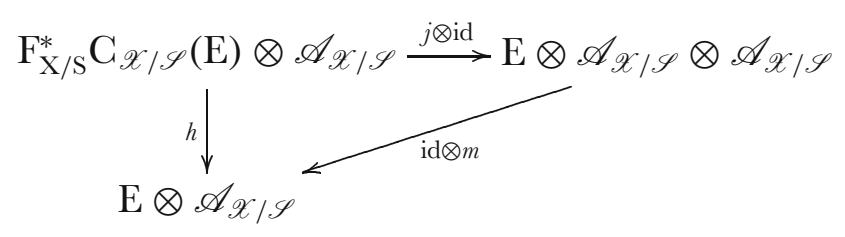

defines a horizontal map

$(2.22 .8)$

$$
h:\left(\mathscr{A}_{\mathscr{X} / \mathscr{S}}^{\prime}\left(\mathrm{C}_{\mathscr{X} / \mathscr{S}}(\mathrm{E})\right), \theta_{\mathscr{A}}, \theta_{t o t}, \mathrm{~N} .\right) \rightarrow\left(\mathscr{A}_{\mathscr{X} / \mathscr{S}}(\mathrm{E}), \theta_{t o t}, \theta_{\mathscr{A}}, \mathrm{N} .\right)
$$

A similar construction defines a horizontal map

$$
h^{\prime}:\left(\mathscr{A}_{\mathscr{X} / \mathscr{S}}\left(\mathrm{C}_{\mathscr{X} / \mathscr{S}}^{\prime}\left(\mathrm{E}^{\prime}\right)\right), \theta_{\mathscr{A}}^{\prime}, \theta_{t o t}^{\prime}, \mathrm{N}^{\prime}\right) \rightarrow\left(\mathscr{A}_{\mathscr{X} / \mathscr{S}}^{\prime}\left(\mathrm{E}^{\prime}\right), \theta_{t o t}^{\prime}, \theta_{\mathscr{A}}^{\prime}, \mathrm{N}^{\prime} .\right)
$$

Theorem 2.23. - Let $\mathscr{X} / \mathscr{S}:=\left(\mathrm{X} / \mathrm{S}, \tilde{\mathrm{X}}^{\prime} / \tilde{\mathrm{S}}\right)$ be a smooth morphism with a lifting of $\mathrm{X}^{\prime} \bmod p^{2}$ as described above.

1. Let $(\mathrm{E}, \nabla, \mathrm{N}$.$) be an object of \mathrm{MICN}_{\gamma}^{*}(\mathrm{X} / \mathrm{S})$ and let $\left(\mathrm{E}^{\prime}, \theta^{\prime}, \mathrm{N}^{\prime}.\right)$ denote $\mathrm{C}_{\mathscr{X} / \mathscr{S}}(\mathrm{E}, \nabla, \mathrm{N}$.$) . Then the map h(2.22 .8)$ is a filtered isomorphism, and fits into a commutative diagram:

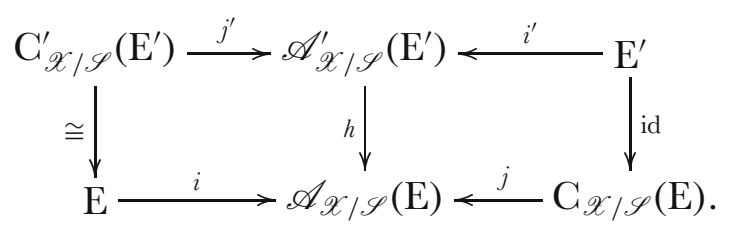

2. Let $\left(\mathrm{E}^{\prime}, \theta^{\prime}, \mathrm{N}^{\prime}\right)$ be an object of $\operatorname{HIGN}_{\gamma}^{\cdot}\left(\mathrm{X}^{\prime} / \mathrm{S}\right)$, and let $(\mathrm{E}, \nabla, \mathrm{N}$. $)$ denote $\mathrm{C}_{\mathscr{X} / \mathscr{S}}^{\prime}\left(\mathrm{E}^{\prime}, \theta^{\prime}, \mathrm{N}^{\prime}\right.$.). Then the map $h^{\prime}(2.22 .9)$ is a filtered isomorphism and fits into a commutative diagram:

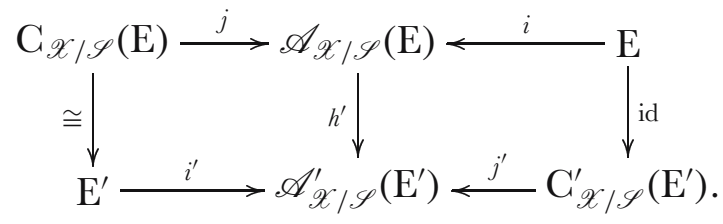


Consequently, $\mathrm{C}_{\mathscr{X} / \mathscr{S}}^{\prime}$ is quasi-inverse to the Cartier transform $\mathrm{C}_{\mathscr{X} / \mathscr{S}}$ and is therefore isomorphic to the functor $\mathrm{C}_{\mathscr{X} / \mathscr{S}}^{-1}$ of Theorem 2.8 (ignoring the filtrations).

Proof. - This theorem is an immediate consequence of Proposition 2.17 and Cartier descent. The $p$-curvature of the connection on $\mathscr{A}_{\mathscr{X} / \mathscr{S}}(\mathrm{E})$ is the total Higgs field $\theta_{t o t}$. Hence

$$
\mathrm{E}^{\prime}:=\mathrm{C}_{\mathscr{X} / \mathscr{S}}(\mathrm{E})=\left(\left(\mathscr{A}_{\mathscr{X} / \mathscr{S}}(\mathrm{E})\right)^{\theta_{t o t}}\right)^{\nabla}=\mathscr{T}_{\mathscr{X} / \mathscr{S}}(\mathrm{E})^{\nabla},
$$

in the notation of op. cit. Since the $p$-curvature of the connection $\mathscr{T}_{\mathscr{X} / \mathscr{S}}(\mathrm{E})$ vanishes, standard Cartier descent implies that the natural map

$$
\mathrm{F}_{\mathrm{X} / \mathrm{S}}^{*} \mathrm{E}^{\prime} \rightarrow \mathscr{T}_{\mathscr{X} / \mathscr{S}}(\mathrm{E})
$$

is a filtered isomorphism. Thus we have a commutative diagram

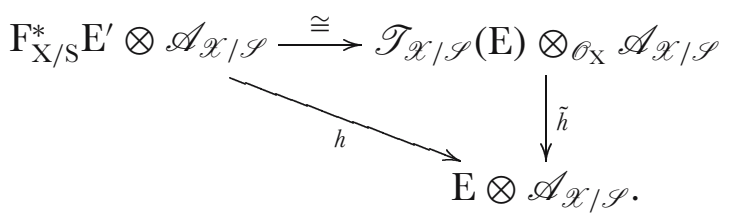

Proposition 2.17 implies that $\tilde{h}$ is a filtered isomorphism and hence so is $h$, and it is also horizontal. The vertical left arrow in the diagram of (1) corresponds to the map $k$ of Proposition 2.17 and is also a horizontal filtered isomorphism, compatible with the PD-Higgs fields, i.e., an isomorphism in the category $\mathrm{MICN}_{\gamma}^{*}(\mathrm{X} / \mathrm{S})$. A similar argument works if we start with an object $\left(\mathrm{E}^{\prime}, \theta^{\prime}, \mathrm{N}^{\prime}\right.$.) of $\mathrm{HIGN}_{\gamma}\left(\mathrm{X}^{\prime} / \mathrm{S}\right)$. This shows that $\mathrm{C}_{\mathscr{X} / \mathscr{S}}$ and $\mathrm{C}_{\mathscr{X} / \mathscr{S}}^{\prime}$ are quasi-inverse equivalences.

Corollary 2.24. - Let $(\mathrm{E}, \nabla, \mathrm{N}$.$) be an object of \mathrm{MICN}_{\gamma}^{*}(\mathrm{X} / \mathrm{S})$ and let

$$
\left(\mathrm{E}^{\prime}, \theta^{\prime}, \mathrm{N}^{\prime}\right):=\mathrm{G}_{\mathscr{X} / \mathscr{S}}(\mathrm{E}, \nabla, \mathrm{N} .)
$$

Then there is a natural isomorphism in the category of graded $\Gamma \cdot \mathrm{T}_{\mathrm{X}^{\prime} / \mathrm{S}}$-modules:

$$
\left(\operatorname{Gr}\left(\mathrm{E}^{\prime}, \theta^{\prime}, \mathrm{N}^{\prime} .\right)\right) \cong \iota_{*}(\mathrm{Gr}(\mathrm{E}, \psi, \mathrm{N} .))^{\nabla},
$$

where $\psi$ is the action of $\Gamma . \mathrm{T}_{\mathrm{X}^{\prime} / \mathrm{S}} \subseteq \mathrm{D}_{\mathrm{X} / \mathrm{S}}^{\gamma}$ and $\iota$ is the inversion involution of $\Gamma . \mathrm{T}_{\mathrm{X}^{\prime} / \mathrm{S}}$.

Proof. — Using Corollary 2.20, we have

$$
\begin{aligned}
\operatorname{Gr} \mathrm{E}^{\prime} & \cong \operatorname{Gr}\left(\left(\mathscr{T}_{\mathscr{X} / \mathscr{S}}(\mathrm{E})\right)^{\nabla}\right) \\
& \cong\left(\operatorname{Gr} \mathscr{T}_{\mathscr{X} / \mathscr{S}}(\mathrm{E})\right)^{\nabla} \\
& \cong\left(\iota_{*} \operatorname{Gr}(\mathrm{E})\right)^{\nabla} .
\end{aligned}
$$


Remark 2.25. - A similar formalism works when there is a lifting $\tilde{\mathrm{F}}$ of $\mathrm{F}_{\mathrm{X} / \mathrm{S}}$. Let $\mathrm{MICN}(\mathrm{X} / \mathrm{S})$ denote the category of modules with connection $(\mathrm{E}, \nabla)$ endowed with a horizontal filtration $\mathrm{N}$. such that $\mathrm{Gr}^{\mathrm{N}}(\mathrm{E})$ is constant. We assume also that $\mathrm{N}$. is exhaustive and bounded below. As before, let $\mathscr{A}_{\tilde{\mathrm{F}}}$ be the nilpotent divided power completion of $\mathscr{A}_{\mathscr{X} / \mathscr{S}}$ along the ideal of the corresponding augmentation $\mathscr{A}_{\mathscr{X} / \mathscr{S}} \rightarrow \mathscr{O}_{\mathrm{X}}$. Then if $(\mathrm{E}, \nabla, \mathrm{N}$.$) is an object of \operatorname{MICN}(\mathrm{X} / \mathrm{S})$, its $p$-curvature $\psi$ gives $\left(\mathrm{F}_{\mathrm{X} / \mathrm{S} *} \mathrm{E}, \mathrm{N}\right.$. $)$ an I-saturated Higgs field as discussed in Remark 2.19. Then we define:

$$
\mathscr{A}_{\tilde{\mathrm{F}}}(\mathrm{E}):=\mathrm{E} \otimes_{\mathscr{O}_{\mathrm{X}}} \mathscr{A}_{\tilde{\mathrm{F}}} \quad \text { and } \mathscr{T}_{\widetilde{\mathrm{F}}}(\mathrm{E}):=\left(\mathscr{A}_{\tilde{\mathrm{F}}}(\mathrm{E})\right)^{\theta_{t o t}}
$$

where $\mathscr{T}_{\widetilde{\mathrm{F}}}(\mathrm{E})$ has the Higgs field $\theta_{\mathscr{T}}$ induced by $\theta_{\mathscr{A}}$. Then

$$
\mathrm{C}_{\tilde{\mathrm{F}}}(\mathrm{E}):=\left(\mathscr{A}_{\tilde{\mathrm{F}}}(\mathrm{E})\right)^{\nabla}=\left(\mathscr{T}_{\widetilde{\mathrm{F}}}(\mathrm{E})\right)^{\nabla}
$$

with it inherits a Higgs field and filtration. Thus we obtain a functor

$$
\mathrm{C}_{\tilde{\mathrm{F}}}: \operatorname{MICN}(\mathrm{X} / \mathrm{S}) \rightarrow \mathrm{HIGN}\left(\mathrm{X}^{\prime} / \mathrm{S}\right) .
$$

On the other hand, if $\left(\mathrm{E}^{\prime}, \theta^{\prime}, \mathrm{N}^{\prime}\right.$. $)$ is an object of $\operatorname{HIGN}\left(\mathrm{X}^{\prime} / \mathrm{S}\right)$, let

$$
\mathscr{A}_{\tilde{\mathrm{F}}}\left(\mathrm{E}^{\prime}\right):=\mathrm{E}^{\prime} \otimes_{\mathscr{O}_{\mathrm{X}}^{\prime}} \mathscr{A}_{\overline{\mathrm{F}}}
$$

Then the total Higgs field $\theta^{\prime}$ on $\mathscr{A}_{\hat{\mathrm{F}}}\left(\mathrm{E}^{\prime}\right)$ commutes with the connection id $\otimes \nabla_{\mathscr{A}}$. Let

$$
\mathrm{C}_{\tilde{\mathrm{F}}}^{-1}:=\left(\mathscr{A}_{\tilde{\mathrm{F}}}\left(\mathrm{E}^{\prime}\right)\right)^{\theta^{\prime}},
$$

which inherits a connection from the action of id $\otimes \nabla_{\mathscr{A}}$ and a filtration $\mathrm{N}$. from the total filtration $\mathrm{N}_{t o t}^{\prime}$. Thus $\mathrm{C}_{\tilde{\mathrm{F}}}^{-1}$ is a functor

$$
\mathrm{C}_{\tilde{\mathrm{F}}}^{-1}: \operatorname{HIGN}\left(\mathrm{X}^{\prime} / \mathrm{S}\right) \rightarrow \mathrm{MICN}(\mathrm{X} / \mathrm{S}) \text {. }
$$

These functors are quasi-inverse equivalences, compatible with the tensor structures and with the global functors $\mathrm{C}_{\mathscr{X} / \mathscr{S}}$ considered above.

\subsection{De Rham and Higgs cohomology}

Let us continue to denote by $\mathscr{X} / \mathscr{S}$ a smooth morphism $\mathrm{X} / \mathrm{S}$ of schemes in characteristic $p$, together with a lifting $\tilde{\mathrm{X}}^{\prime} / \tilde{\mathrm{S}}$ of $\mathrm{X}^{\prime} / \mathrm{S}$. Let $(\mathrm{E}, \nabla)$ be a module with integrable connection on $\mathrm{X} / \mathrm{S}$, nilpotent of level $\ell$. Our goal in this section is to compare the de Rham cohomology of $(\mathrm{E}, \nabla)$ with the Higgs cohomology of its Cartier transform $\left(\mathrm{E}^{\prime}, \theta^{\prime}\right)$. We shall do this by constructing a canonical filtered double complex $\left(\mathscr{A}_{\mathscr{X} / \mathscr{S}}(\mathrm{E}), \mathrm{N}.\right)$ of $\mathscr{O}_{\mathrm{X}^{\prime}}$-modules and quasi-isomorphisms

$$
\mathrm{F}_{\mathrm{X} / \mathrm{S} *}\left(\mathrm{E} \otimes \Omega_{\mathrm{X} / \mathrm{S}}^{\cdot}, d\right) \rightarrow \mathrm{N}_{n} \mathscr{A}_{\mathscr{X} / \mathscr{S}}^{\cdot}(\mathrm{E}) \leftarrow\left(\mathrm{E}^{\prime} \otimes \Omega_{\mathrm{X}^{\prime} / \mathrm{S}}^{\cdot}, \theta^{\prime}\right),
$$


whenever $\ell+d \leq n<p$, where $\mathscr{A}_{\mathscr{X} / \mathscr{S}}$ is the total complex associated to the double complex $\mathscr{A}_{\mathscr{X} / \mathscr{S}}^{\cdot}$.

In fact,

$$
\begin{aligned}
\mathscr{A}_{\mathscr{X} / \mathscr{S}}^{\ddot{j}}(\mathrm{E}): & =\mathrm{F}_{\mathrm{X} / \mathrm{S} *}\left(\mathrm{E} \otimes \mathscr{A}_{\mathscr{X} / \mathscr{S}} \otimes \mathrm{F}_{\mathrm{X} / \mathrm{S}}^{*} \Omega_{\mathrm{X}^{\prime} / \mathrm{S}}^{i} \otimes \Omega_{\mathrm{X} / \mathrm{S}}^{j}\right) \\
& \cong \mathrm{F}_{\mathrm{X} / \mathrm{S} *}\left(\mathrm{E} \otimes \mathscr{A}_{\mathscr{X} / \mathscr{S}} \otimes \Omega_{\mathrm{X} / \mathrm{S}}^{j}\right) \otimes \Omega_{\mathrm{X}^{\prime} / \mathrm{S}}^{i}
\end{aligned}
$$

with boundary maps constructed from the de Rham differentials of $(\mathrm{E}, \nabla)$ and the $p$-curvature of $\mathscr{A}_{\mathscr{X} / \mathscr{S}}$. In the case $(\mathrm{E}, \nabla)=\left(\mathscr{O}_{\mathrm{X}}, d\right)$ we obtain an isomorphism in the derived category

$$
\mathrm{F}_{\mathrm{X} / \mathrm{S} *}\left(\mathrm{E} \otimes \Omega_{\mathrm{X} / \mathrm{S}}^{\cdot}, d\right) \sim\left(\Omega_{\mathrm{X}^{\prime} / \mathrm{S}}^{\cdot}, 0\right)
$$

between the de Rham complex of $\mathrm{X} / \mathrm{S}$ and the Hodge complex of $\mathrm{X}^{\prime} / \mathrm{S}$, when $d<p$. This is the result of Deligne and Illusie [8] (with a loss of one dimension). For general E it can be regarded as an analog of Simpson's "formality" theorem [36].

We shall find it convenient to work with filtered connections and their de Rham complexes. Let $(\mathrm{E}, \nabla)$ be a module with integrable connection endowed with a horizontal filtration $\mathrm{N}$. such that $\left(\mathrm{Gr}_{\mathrm{N}}(\mathrm{E}), \nabla\right)$ is constant, i.e., has zero $p$-curvature. We assume that $\mathrm{N}_{-1} \mathrm{E}=0$ and $\mathrm{N}_{p-1} \mathrm{E}=\mathrm{E}$, so that $(\mathrm{E}, \nabla, \mathrm{N}$.) defines an object of $\mathrm{MICN}_{\gamma}(\mathrm{X} / \mathrm{S})$. Let $\mathrm{N}^{\text {tot }}$ be the tensor product filtration on $\mathrm{E} \otimes \mathscr{A}_{\mathscr{X} / \mathscr{S}}$ induced by $\mathrm{N}$. and the filtration $\mathrm{N}$. of $\mathscr{A}_{\mathscr{X} / \mathscr{S}}$. Let $\left(\mathrm{E}^{\prime}, \mathrm{N}^{\prime}\right.$.) be the Cartier transform of $(\mathrm{E}, \mathrm{N}$.) with the filtration induced by $\mathbf{N}^{\text {tot }}$, as explained in Theorem 2.23. For fixed $i$, the de Rham complex of the module with connection $\mathscr{A}_{\mathscr{X} / \mathscr{S}}(\mathrm{E}) \otimes \mathrm{F}_{\mathrm{X} / \mathrm{S}}^{*} \Omega_{\mathrm{X}^{\prime} / \mathrm{S}}^{i}$ is the complex:

$$
\mathscr{A}_{\mathscr{X} / \mathscr{S}}^{i, 0}(\mathrm{E}) \stackrel{d^{i, 0}}{\rightarrow} \mathscr{A}_{\mathscr{X} / \mathscr{S}}^{i, 1}(\mathrm{E}) \stackrel{d^{i, 1}}{\rightarrow} \cdots .
$$

Similarly, for fixed $j$, the Higgs complex of $\left(\mathscr{A}_{\mathscr{X} / \mathscr{S}}, \theta_{\mathscr{A}}\right)$ tensored with $\mathrm{E} \otimes \Omega_{\mathrm{X} / \mathrm{S}}^{j}$, is the complex

$$
\mathscr{A}_{\mathscr{X} / \mathscr{S}}^{\cdot, j}(\mathrm{E}):=\mathscr{A}_{\mathscr{X} / \mathscr{S}}^{0, j}(\mathrm{E}) \stackrel{d^{10, j}}{\longrightarrow} \mathscr{A}_{\mathscr{X} / \mathscr{S}}^{1, j}(\mathrm{E}) \stackrel{d^{1, j}}{\longrightarrow} \cdots .
$$

It follows from Lemma 2.22 that the differentials $d$ and $d^{\prime}$ commute. Thus we can form the double complex $\mathscr{A}_{\mathscr{X} / \mathscr{S}}(\mathrm{E})$ and the associated simple complex $\mathscr{A}_{\mathscr{X} / \mathscr{S}}(\mathrm{E})$.

For each $i$ there is a natural map from $\mathrm{E}^{\prime} \otimes \Omega_{\mathrm{X}^{\prime} / \mathrm{S}}^{i}$ to $\operatorname{Ker}\left(d^{i, 0}\right)$, which can be regarded as a morphism of filtered complexes,

$(2.25 .3)$

$$
\left(\mathrm{E}^{\prime} \otimes \Omega_{\mathrm{X}^{\prime} / \mathrm{S}}^{i}, \mathrm{~N}^{\prime}\right) \rightarrow\left(\mathscr{A}_{\mathscr{X} / \mathscr{S}}^{i, \cdot}(\mathrm{E}), \mathrm{N}^{t o t}\right)
$$

compatible with the Higgs boundary maps:

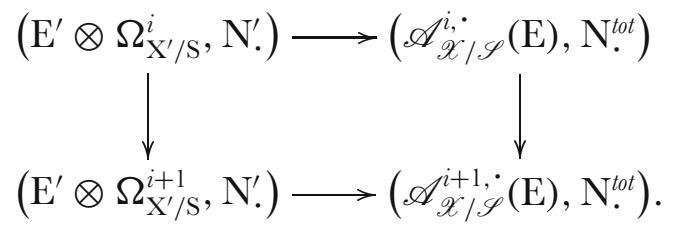


In the same way we find for each $j$ a morphism

$$
\mathrm{F}_{\mathrm{X} / \mathrm{S} *}\left(\mathrm{E} \otimes \Omega_{\mathrm{X} / \mathrm{S}}^{j}, \mathrm{~N} .\right) \rightarrow\left(\mathscr{A}_{\mathscr{X} / \mathscr{S}}^{\cdot, j}(\mathrm{E}), \mathrm{N}^{t o t}\right)
$$

compatible with the de Rham boundary maps

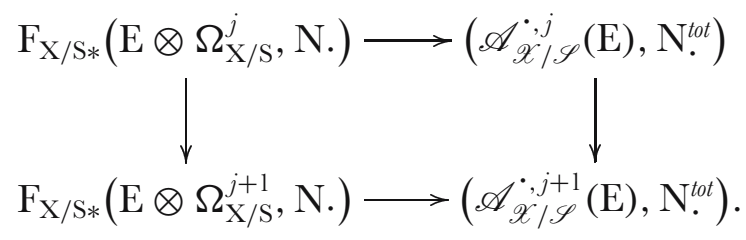

These assemble into morphisms of filtered complexes:

$$
\left(\mathrm{E}^{\prime} \otimes \Omega_{\mathrm{X}^{\prime} / \mathrm{S}}^{\cdot}, \mathrm{N}_{.}^{\prime}\right) \stackrel{a_{\mathscr{X} / \mathscr{S}}}{\longrightarrow}\left(\mathscr{A}_{\mathscr{X} / \mathscr{S}}^{\cdot}, \mathrm{N}^{t o t}\right) \stackrel{b_{\mathscr{X} / \mathscr{S}}}{\longleftarrow}\left(\mathrm{E} \otimes \Omega_{\mathrm{X} / \mathrm{S}}^{\cdot}, \mathrm{N} .\right) .
$$

If there is a lifting $\tilde{\mathrm{F}}$ of $\mathrm{F}_{\mathrm{X} / \mathrm{S}}$, we can make the analogous construction with $\mathscr{A}_{\tilde{\mathrm{F}}}$ in place of $\mathscr{A}_{\mathscr{X} / \mathscr{S}}$, and we use the analogous notation. Then there is a natural morphism of double complexes $\mathscr{A}_{\mathscr{X} / \mathscr{S}}(\mathrm{E}) \rightarrow \mathscr{A}_{\tilde{\mathrm{F}}}(\mathrm{E})$. Taking associated simple complexes, we find a commutative diagram:

$(2.25 .6)$

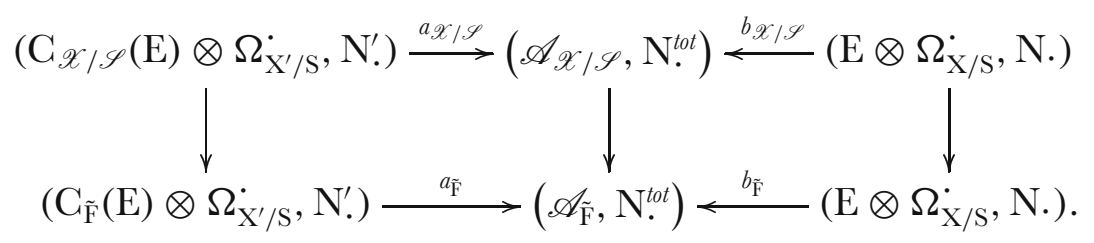

Before stating the main theorem, let us recall that if $\mathrm{C}^{*}$ is a complex with an increasing filtration $\mathrm{N}$., then as explained in [7], the filtration décalée $\mathrm{N}^{\text {dec }}$ on $\mathrm{C}^{*}$ is defined by

$$
\mathrm{N}_{k}^{d e c} \mathrm{C}^{q}:=\mathrm{N}_{k-q} \mathrm{C}^{q}+d\left(\mathrm{~N}_{k-q+1} \mathrm{C}^{q-1}\right) .
$$

Theorem 2.26. - Let $\mathrm{X} / \mathrm{S}$ be a smooth morphism in characteristic p. Let $\mathrm{E}:=(\mathrm{E}, \nabla, \mathrm{N})$ be an object of $\mathrm{MICN}(\mathrm{X} / \mathrm{S})$ with $\mathrm{N}_{-1} \mathrm{E}=0$ and $\mathrm{N}_{p-1} \mathrm{E}=\mathrm{E}$.

1. If $\mathscr{X} / \mathscr{S}$ is a lifting of $\mathrm{X} / \mathrm{S}$, then the maps $a_{\mathscr{X} / \mathscr{S}}$ and $b_{\mathscr{X} / \mathscr{S}}(2.25 .5)$ induce filtered quasi-isomorphisms:

$$
\begin{aligned}
\left(\mathrm{N}_{p-1}^{\prime d e c}\left(\mathrm{C}_{\mathscr{X} / \mathscr{S}}(\mathrm{E}) \otimes \Omega_{\mathrm{X}^{\prime} / \mathrm{S}}^{\cdot}\right), \mathrm{N}^{\prime d e c}\right) & \rightarrow \mathrm{F}_{\mathrm{X} / \mathrm{S} *}\left(\mathrm{~N}_{p-1}^{d e c} \mathscr{A}_{\mathscr{X} / \mathscr{S}}(\mathrm{E}), \mathrm{N}^{d e c}\right) \\
\mathrm{F}_{\mathrm{X} / \mathrm{S} *}\left(\mathrm{~N}_{p-1}^{d e c}\left(\mathrm{E} \otimes \Omega_{\mathrm{X} / \mathrm{S}}\right), \mathrm{N}^{d e c}\right) & \rightarrow\left(\mathrm{N}_{p-1}^{d e c} \mathscr{A}_{\mathscr{X} / \mathscr{S}}(\mathrm{E}), \mathrm{N}^{d e c}\right) .
\end{aligned}
$$

Consequently they assemble into an isomorphism in the filtered derived category of $O_{\mathrm{X}^{\prime}}$-modules:

$$
\mathrm{F}_{\mathrm{X} / \mathrm{S} *}\left(\mathrm{~N}_{p-1}^{d e c}\left(\mathrm{E} \otimes \Omega_{\mathrm{X} / \mathrm{S}}^{\cdot}\right), \mathrm{N}^{d e c}, d\right) \cong\left(\mathrm{N}_{p-1}^{\prime d e c}\left(\mathrm{C}_{\mathscr{X} / \mathscr{S}}(\mathrm{E}) \otimes \Omega_{\mathrm{X}^{\prime} / \mathrm{S}}^{\cdot}\right), \mathrm{N}^{\prime d e c}, \theta^{\prime}\right) .
$$


2. If $\tilde{\mathrm{F}}$ is a lifting of $\mathrm{F}_{\mathrm{X} / \mathrm{S}}$, then the maps $a_{\tilde{\mathrm{F}}}$ and $b_{\tilde{\mathrm{F}}}$ (2.25.6) induce filtered quasi-isomorphisms:

$$
\begin{aligned}
& \left(\mathrm{C}_{\tilde{\mathrm{F}}}(\mathrm{E}) \otimes \Omega_{\mathrm{X}^{\prime} / \mathrm{S}}, \mathrm{N}^{\prime d e c}\right) \rightarrow \mathrm{F}_{\mathrm{X} / \mathrm{S} *}\left(\mathscr{A}_{\tilde{\mathrm{F}}}(\mathrm{E}), \mathrm{N}^{d e c}\right) \\
& \mathrm{F}_{\mathrm{X} / \mathrm{S} *}\left(\mathrm{E} \otimes \Omega_{\mathrm{X} / \mathrm{S}}^{\cdot}, \mathrm{N}^{d e c}\right) \rightarrow\left(\mathscr{A}_{\tilde{\mathrm{F}}}(\mathrm{E}), \mathrm{N}^{d e c}\right) .
\end{aligned}
$$

These assemble into an isomorphism in the filtered derived category of $\mathscr{O}_{\mathrm{X}^{\prime}}$-modules

$$
\left(\mathrm{F}_{\mathrm{X} / \mathrm{S} *}\left(\mathrm{E} \otimes \Omega_{\mathrm{X} / \mathrm{S}}^{\cdot}\right), \mathrm{N}^{d e c}\right) \cong\left(\mathrm{C}_{\tilde{\mathrm{F}}}(\mathrm{E}) \otimes \Omega_{\mathrm{X}^{\prime} / \mathrm{S}}^{\cdot}, \mathrm{N}^{\prime d e c}\right) .
$$

Corollary 2.27. - Let $(\mathrm{E}, \nabla)$ be an object of $\mathrm{MIC}(\mathrm{X} / \mathrm{S})$ which is nilpotent of level $\ell<p$. Then a lifting $\mathscr{X} / \mathscr{S}$ induces isomorphisms in the derived category:

$$
\mathrm{F}_{\mathrm{X} / \mathrm{S}^{*}}\left(\tau_{<p-\ell}\left(\mathrm{E} \otimes \Omega_{\mathrm{X} / \mathrm{S}}^{\cdot}\right)\right) \cong \tau_{<p-\ell}\left(\mathrm{C}_{\mathscr{X} / \mathscr{S}}(\mathrm{E}) \otimes \Omega_{\mathrm{X}^{\prime} / \mathrm{S}}^{\cdot}\right)
$$

and if $\ell+\operatorname{dim}(\mathrm{X} / \mathrm{S})<p$,

$$
\mathrm{F}_{\mathrm{X} / \mathrm{S}^{*}}\left(\mathrm{E} \otimes \Omega_{\mathrm{X} / \mathrm{S}}^{\cdot}\right) \cong\left(\mathrm{C}_{\mathscr{X} / \mathscr{S}}(\mathrm{E}) \otimes \Omega_{\mathrm{X}^{\prime} / \mathrm{S}}^{\cdot}\right) .
$$

Applying (2) of Theorem 2.26 to the canonical filtration (2.16.1) of a locally nilpotent connection, we obtain the following result.

Corollary 2.28. - Let $(\mathrm{E}, \nabla)$ be an object of $\mathrm{MIC}(\mathrm{X} / \mathrm{S})$. Assume that the connection $\nabla$ is locally nilpotent (quasi-nilpotent in the terminology of [3]). Then a lifting $\tilde{\mathrm{F}}$ of $\mathrm{F}_{\mathrm{X} / \mathrm{S}}$ induces isomorphisms in the derived category

$$
\mathrm{F}_{\mathrm{X} / \mathrm{S} *}\left(\mathrm{E} \otimes \Omega_{\mathrm{X} / \mathrm{S}}^{\cdot}\right) \cong \mathrm{C}_{\tilde{\mathrm{F}}}(\mathrm{E}) \otimes \Omega_{\mathrm{X}^{\prime} / \mathrm{S}}^{\cdot}
$$

Before beginning the proof of Theorem 2.26, let us remark that it is not true that the maps

$$
\begin{aligned}
a_{\tilde{\mathrm{F}}}:\left(\mathrm{E}^{\prime} \otimes \Omega_{\mathrm{X}^{\prime} / \mathrm{S}}^{\cdot}, \mathrm{N} .\right) & \rightarrow \mathrm{F}_{\mathrm{X} / \mathrm{S} *}\left(\mathscr{A}_{\tilde{\mathrm{F}}}(\mathrm{E}), \mathrm{N} .\right) \\
b_{\tilde{\mathrm{F}}}: \mathrm{F}_{\mathrm{X} / \mathrm{S} *}\left(\mathrm{E} \otimes \Omega_{\mathrm{X} / \mathrm{S}}^{\cdot}, \mathrm{N} .\right) & \rightarrow\left(\mathscr{A}_{\tilde{\mathrm{F}}}^{*}(\mathrm{E}), \mathrm{N} .\right)
\end{aligned}
$$

are filtered quasi-isomorphisms. However, these maps induce maps of spectral sequences, which on the $\mathrm{E}_{1}$ level are maps of complexes of sheaves:

$$
\mathscr{H}\left(\mathrm{Gr} a_{\tilde{\mathrm{F}}}\right):\left(\mathscr{H}\left(\mathrm{Gr} \mathrm{E}^{\prime} \otimes \Omega_{\mathrm{X}^{\prime} / \mathrm{S}}^{\cdot}\right), d_{1}\right) \rightarrow\left(\mathrm{F}_{\mathrm{X} / \mathrm{S} *}\left(\mathscr{H}\left(\mathrm{Gr} \mathscr{A}_{\tilde{\mathrm{F}}}^{\cdot}(\mathrm{E})\right), d_{1}\right)\right.
$$

$$
\mathscr{H}\left(\mathrm{Gr} b_{\tilde{\mathrm{F}}}\right):\left(\mathrm{F}_{\mathrm{X} / \mathrm{S} *} \mathscr{H}\left(\mathrm{Gr} \mathrm{E} \otimes \Omega_{\mathrm{X} / \mathrm{S}}^{\cdot}\right), d_{1}\right) \rightarrow\left(\mathscr{H}\left(\mathrm{Gr} \mathscr{A}_{\tilde{\mathrm{F}}}(\mathrm{E})\right), d_{1}\right)
$$

where $d_{1}$ is the differential of the spectral sequences. We shall prove that these maps are quasi-isomorphisms (not isomorphisms), and hence induce isomorphisms on the $\mathrm{E}_{2}$-terms of the spectral sequence. 
Lemma 2.29. - In the situation of (2) in Theorem 2.26, the maps (2.28.3) and (2.28.4) above are quasi-isomorphisms.

Proof. - Since the p-curvature of Gr E vanishes, the classical Cartier isomorphism induces a canonical isomorphism:

$$
\mathrm{E}_{1}\left(\mathrm{E} \otimes \Omega_{\mathrm{X} / \mathrm{S}}^{\cdot}, \mathrm{N}\right)=\mathscr{H}^{q}\left(\mathrm{GrE} \otimes \Omega_{\mathrm{X} / \mathrm{S}}^{\cdot}\right) \cong(\mathrm{GrE})^{\nabla} \otimes \Omega_{\mathrm{X}^{\prime} / \mathrm{S}}^{q} \cdot
$$

Corollary (5.1.1) of [29] allows us to compute the differential $d_{1}$ of this spectral sequence. It asserts that the diagram below is anticommutative, thus identifying the (negative of) the differential $d_{1}^{q}$ with the graded map $\operatorname{Gr}(\psi)$ induced by the $p$-curvature of $\mathrm{E}$ :

$$
\mathscr{H}^{q}\left(\mathrm{Gr}_{i} \mathrm{E} \otimes \Omega_{\mathrm{X} / \mathrm{S}}^{\cdot}\right) \stackrel{d_{1}^{q}}{\longrightarrow} \mathscr{H}^{q+1}\left(\mathrm{Gr}_{i-1} \mathrm{E} \otimes \Omega_{\mathrm{X} / \mathrm{S}}^{\cdot}\right)
$$

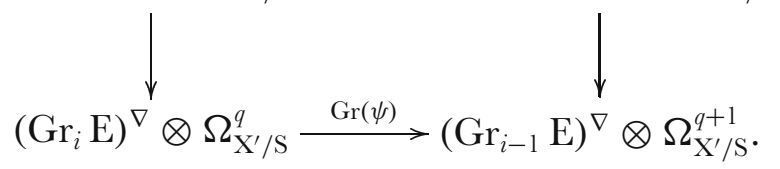

Thus there is an isomorphism of complexes

$$
\left(\mathrm{F}_{\mathrm{X} / \mathrm{S} *} \mathrm{E}_{1}^{\cdot}\left(\mathrm{E} \otimes \Omega_{\mathrm{X} / \mathrm{S}}^{\cdot}, \mathrm{N} .\right), d_{1}\right) \cong\left(\mathrm{Gr}^{\prime} \otimes \Omega_{\mathrm{X}^{\prime} / \mathrm{S}}^{\cdot}, \mathrm{Gr}(\psi)\right) .
$$

We apply the same method to analyze the $\mathrm{E}_{1}$ term of the spectral sequence of the filtered complex $\left(\mathscr{A}_{\tilde{\mathrm{F}}}(\mathrm{E}), \mathrm{N}\right.$.). The total differential of the double complex $\mathscr{A}_{\tilde{\mathrm{F}}} \cdot(\mathrm{E})$ induces a map

$$
\mathrm{N}_{k} \mathscr{A}_{\tilde{\mathrm{F}}}^{i, j} \rightarrow \mathrm{N}_{k-1} \mathscr{A}_{\tilde{\mathrm{F}}}^{i+1, j} \oplus \mathrm{N}_{k} \mathscr{A}_{\tilde{\mathrm{F}}}^{i, j+1}
$$

so the differential on $\operatorname{Gr} \mathscr{A}_{\tilde{\mathrm{F}}}(\mathrm{E})$ is just the de Rham differential of the module with connection

$$
\operatorname{Gr} \mathscr{A}_{\tilde{\mathrm{F}}}^{\cdot, 0}(\mathrm{E})=\bigoplus_{i} \operatorname{Gr} \mathscr{A}_{\tilde{\mathrm{F}}}(\mathrm{E}) \otimes \mathrm{F}_{\mathrm{X} / \mathrm{S}}^{*} \Omega_{\mathrm{X}^{\prime} / \mathrm{S}}^{i}
$$

Since this connection has vanishing $p$-curvature, the classical Cartier isomorphism provides an isomorphism:

$$
\mathrm{H}^{\cdot}\left(\operatorname{Gr} \mathscr{A}_{\tilde{\mathrm{F}}}^{\cdot, 0}(\mathrm{E}) \otimes \mathrm{F}_{\mathrm{X} / \mathrm{S}}^{*} \Omega_{\mathrm{X}^{\prime} / \mathrm{S}}^{i}, d\right) \cong\left(\operatorname{Gr}\left(\mathscr{A}_{\tilde{\mathrm{F}}}(\mathrm{E})\right)^{\nabla} \otimes \Omega_{\mathrm{X}^{\prime} / \mathrm{S}}^{\cdot} \otimes \Omega_{\mathrm{X}^{\prime} / \mathrm{S}}^{i} \cdot\right.
$$

The differential $d_{1}$ of the spectral sequence is then a sum of maps

$$
\begin{aligned}
& \left(\mathrm { Gr } ( \mathscr { A } _ { \tilde { \mathrm { F } } } ( \mathrm { E } ) ) ^ { \nabla } \otimes \Omega _ { \mathrm { X } ^ { \prime } / \mathrm { S } } ^ { j } \otimes \Omega _ { \mathrm { X } ^ { \prime } / \mathrm { S } } ^ { i } \rightarrow \left(\operatorname{Gr}\left(\mathscr{A}_{\tilde{\mathrm{F}}}(\mathrm{E})\right)^{\nabla} \otimes \Omega_{\mathrm{X}^{\prime} / \mathrm{S}}^{j} \otimes \Omega_{\mathrm{X}^{\prime} / \mathrm{S}}^{i+1}\right.\right. \\
& \left(\operatorname { G r } ( \mathscr { A } _ { \tilde { \mathrm { F } } } ( \mathrm { E } ) ) ^ { \nabla } \otimes \Omega _ { \mathrm { X } ^ { \prime } / \mathrm { S } } ^ { j } \otimes \Omega _ { \mathrm { X } ^ { \prime } / \mathrm { S } } ^ { i } \rightarrow \left(\operatorname{Gr}\left(\mathscr{A}_{\tilde{\mathrm{F}}}(\mathrm{E})\right)^{\nabla} \otimes \Omega_{\mathrm{X}^{\prime} / \mathrm{S}}^{j+1} \otimes \Omega_{\mathrm{X}^{\prime} / \mathrm{S}}^{i} .\right.\right.
\end{aligned}
$$


The first of these is the map induced by differential $d^{\prime}$ of $\mathscr{A}_{\tilde{\mathrm{F}}} \cdot(\mathrm{E})$, which comes from the $p$-curvature of $\mathscr{A}$, and [29] identifies the second as the map coming from the $p$-curvature of the connection $\nabla$ on $\mathscr{A}_{\mathrm{F}}(\mathrm{E})$. Thus we have an isomorphism of complexes:

$$
\left(\mathrm{E}_{1}^{*}\left(\mathscr{A}_{\tilde{\mathrm{F}}}(\mathrm{E}), \mathrm{N} ., d_{1}\right) \cong\left(\operatorname{Gr} \mathscr{A}_{\tilde{\mathrm{F}}}(\mathrm{E})^{\nabla} \otimes \Omega_{\mathrm{X}^{\prime} / \mathrm{S}}^{\cdot} \otimes \Omega_{\mathrm{X}^{\prime} / \mathrm{S}}^{\cdot}, d\right),\right.
$$

where the differential on the right is the differential of the simple complex associated to the double complex whose term in degree $i, j$ is

$$
\left(\mathrm{Gr} \mathscr{A}_{\tilde{\mathrm{F}}}(\mathrm{E})\right)^{\nabla} \otimes \Omega_{\mathrm{X}^{\prime} / \mathrm{S}}^{i} \otimes \Omega_{\mathrm{X}^{\prime} / \mathrm{S}}^{j}
$$

and whose differential is the graded map induced by the Higgs fields $\theta_{\mathscr{A}}$ and $\theta_{t o t}$. In fact, by Corollary $2.20, \operatorname{Gr} \mathscr{A}_{\widetilde{\mathrm{F}}}(\mathrm{E}) \cong \mathrm{Gr} \mathrm{E} \otimes \mathrm{Gr} \mathscr{A}_{\tilde{\mathrm{F}}}(\mathrm{E})$, compatibly with the connections and Higgs fields. Furthermore,

$$
(\mathrm{GrE} \otimes \mathrm{Gr} \mathscr{A} \tilde{\mathrm{F}})^{\nabla} \cong(\mathrm{GrE})^{\nabla} \otimes\left(\mathrm{Gr} \mathscr{A}_{\tilde{\mathrm{F}}}\right)^{\nabla} \cong(\mathrm{GrE})^{\nabla} \otimes \Gamma \cdot \Omega_{\mathrm{X}^{\prime} / \mathrm{S}}^{1} .
$$

Let us write $\Omega$ for $\Omega_{\mathrm{X}^{\prime} / \mathrm{S}}^{1}$ and $\mathrm{T}$ for its dual. According to Corollary 2.20, Gr $\mathrm{E}^{\prime}$ is the Higgs transform of $\mathrm{GrE}$ with respect to the T-Higgs module $\Gamma$. $\Omega$. Thus the maps $\operatorname{Gr} a_{\tilde{\mathrm{F}}}$ and $\mathrm{Gr} b_{\tilde{\mathrm{F}}}$ become identified with maps of complexes which term by term are the mappings

$$
\begin{gathered}
(\mathrm{GrE})^{\nabla} \otimes \Omega^{j} \rightarrow(\mathrm{GrE})^{\nabla} \otimes \Gamma . \Omega \otimes \Omega^{\cdot} \otimes \Omega^{j} \\
\operatorname{Gr}\left(\mathrm{E}^{\prime}\right) \otimes \Omega^{i} \rightarrow \operatorname{Gr}\left(\mathrm{E}^{\prime}\right) \otimes \Gamma . \Omega \otimes \Omega^{i} \otimes \Omega^{\cdot}
\end{gathered}
$$

constructed in the same way as $a_{\tilde{\mathrm{F}}}$ and $b_{\tilde{\mathrm{F}}}$. This is exactly the situation discussed in Proposition 2.21, so the lemma follows.

Proof of Theorem 2.26. — To prove that the arrows in (1) of the theorem are isomorphisms is a local question, so we may without loss of generality assume that there is a lifting $\tilde{\mathrm{F}}$ of Frobenius. For $i<p$, the map $\mathrm{N}_{i} \mathscr{A}_{\mathscr{X} / \mathscr{S}} \rightarrow \mathrm{N}_{i} \mathscr{A}_{\tilde{\mathrm{F}}}$ is an isomorphism. Furthermore, since $\mathrm{N}_{-1} \mathrm{E}=0$,

$$
\mathrm{N}_{i}^{t o t} \mathscr{A}_{\mathscr{X} / \mathscr{S}}(\mathrm{E})=\sum_{j=0}^{i} \mathrm{~N}_{j} \mathrm{E} \otimes \mathrm{N}_{i-j} \mathscr{A}_{\mathscr{X} / \mathscr{S}}=\sum_{j=0}^{i} \mathrm{~N}_{j} \mathrm{E} \otimes \mathrm{N}_{i-j} \mathscr{A}_{\tilde{\mathrm{F}}}=\mathrm{N}_{i}^{t o t} \mathscr{A}_{\tilde{\mathrm{F}}}(\mathrm{E})
$$

when $i<p$. Thus the map

$$
\left(\mathscr{A}_{\mathscr{X} / \mathscr{S}}^{\cdot}(\mathrm{E}), \mathrm{N}^{t o t}\right) \rightarrow\left(\mathscr{A}_{\tilde{\mathrm{F}}}^{\cdot}(\mathrm{E}), \mathrm{N}^{t o t}\right)
$$

is a filtered isomorphism when restricted to $\mathrm{N}_{p-1}$. Thus statement (1) will follow from statement (2). 
Since the filtration $\mathrm{N}$. on $\mathrm{E}$ is exhaustive and formation of direct limits in the category of sheaves on $\mathrm{X}$ is exact, we may and shall assume that $\mathrm{N}$. is finite. It will suffice for us to prove that the maps of complexes

$$
\begin{aligned}
& \mathrm{F}_{\mathrm{X} / \mathrm{S} *} \operatorname{Gr}^{\mathrm{N}^{\text {dec }}}\left(\mathrm{E} \otimes \Omega_{\mathrm{X} / \mathrm{S}}^{\cdot}\right) \rightarrow \operatorname{Gr}^{\mathrm{N}^{\text {dec }}}\left(\mathscr{A}_{\tilde{\mathrm{F}}}(\mathrm{E})\right) \\
& \mathrm{Gr}^{\mathrm{N}^{d e c}}\left(\mathrm{G}_{\tilde{\mathrm{F}}}(\mathrm{E}) \otimes \Omega_{\mathrm{X}^{\prime} / \mathrm{S}}^{\cdot}\right) \rightarrow \operatorname{Gr}^{\mathrm{N}^{d e c}}\left(\mathscr{A}_{\tilde{\mathrm{F}}}(\mathrm{E})\right)
\end{aligned}
$$

are quasi-isomorphisms. Recall from [7] that there are natural injections $\mathrm{H}^{q}\left(\mathrm{Gr}^{\mathrm{N}} \mathrm{C}^{\cdot}\right)$ $\rightarrow \mathrm{Gr}^{\mathrm{N}^{\text {dec }}} \mathrm{C}^{q}$ which assemble to form a quasi-isomorphism

$$
\left(\mathrm{E}_{1}\left(\mathrm{G}^{\cdot}, \mathrm{N} \cdot\right), d\right) \cong\left(\mathrm{H}^{\cdot}\left(\mathrm{Gr}^{\mathrm{N}} \mathrm{C}^{\cdot}\right), d\right) \rightarrow \mathrm{Gr}^{\mathrm{N}^{d e c}} \mathrm{G}^{\cdot} \cong\left(\mathrm{E}_{0}\left(\mathrm{C}^{\cdot}, \mathrm{N}^{\text {dec }}\right), d\right)
$$

Thus the theorem follows from Lemma 2.29.

Remark 2.30. - Let $(\mathrm{E}, \nabla)$ be an object of MIC. (X/S), suppose that there exists a global lifting of $\mathrm{F}_{\mathrm{X} / \mathrm{S}}$, and let $\left(\mathrm{E}^{\prime}, \psi^{\prime}\right)$ denote the Cartier transform of $(\mathrm{E}, \nabla)$. By Remark 2.10, there is a canonical isomorphism $\mathrm{F}_{\mathrm{X} / \mathrm{S}}^{*}\left(\mathrm{E}^{\prime}, \psi^{\prime}\right) \cong(\mathrm{E},-\psi)$, where $\psi$ is the $p$-curvature of $\nabla$. This induces isomorphisms

$$
\mathrm{F}_{\mathrm{X} / \mathrm{S}}^{*} \mathscr{H}^{i}\left(\mathrm{E}^{\prime}, \psi^{\prime}\right) \cong \mathscr{H}^{i}(\mathrm{E},-\psi)
$$

for all $i$. Recall from [29] that the sheaves of $\mathscr{O}_{\mathrm{X}}$-modules $\mathscr{H}^{i}(\mathrm{E},-\psi)$ carry a canonical integrable connection $\nabla$ whose $p$-curvature is zero, induced by the given connection on $\mathrm{E}$ and the Frobenius descent connection on $\mathrm{F}_{\mathrm{X} / \mathrm{S}}^{*} \Omega_{\mathrm{X}^{\prime} / \mathrm{S}}^{q}$. It follows easily that the above isomorphisms are horizontal and hence descend to isomorphisms of $\mathscr{O}_{\mathrm{X}^{\prime}}$-modules

$$
\mathscr{H}^{i}\left(\mathrm{E}^{\prime}, \psi^{\prime}\right) \cong \mathscr{H}^{i}(\mathrm{E},-\psi)^{\nabla}
$$

On the other hand, (2.26) gives us isomorphisms $\mathscr{H}^{i}\left(\mathrm{E}^{\prime}, \psi^{\prime}\right) \cong \mathscr{H}^{i}(\mathrm{E}, \nabla)$. Combining these, we find the "generalized Cartier isomorphism"

$$
\mathscr{H}^{i}(\mathrm{E}, \nabla) \cong \mathscr{H}^{i}(\mathrm{E},-\psi)^{\nabla} .
$$

Another construction of such an isomorphisms was given in [29], independent of any lifting of $\mathrm{X}$ or $\mathrm{F}_{\mathrm{X} / \mathrm{S}}$ or nilpotence condition on $\nabla$. One can easily see that these two isomorphisms are the same, because they agree when $i=0$ and because both sides are effaceable cohomological delta functors in the category MIC. (X/S).

Suppose that $\mathrm{X}$ is noetherian and $\mathrm{E}$ is coherent. A consequence of the isomorphisms discussed in Remark 2.30 is the fact that the de Rham complex of $(\mathrm{E}, \nabla)$ with an integrable connection $\nabla$ is determined, as an object in the derived category, by its 
formal completion along a closed subset determined by its $p$-curvature $\psi$. Recall that $(\mathrm{E}, \psi)$ gives rise to a coherent sheaf $\tilde{\mathrm{E}}$ on $\mathbf{T}_{\mathrm{X}^{\prime} / \mathrm{S}}^{*}$. Define the essential support of $(\mathrm{E}, \nabla)$ to be the set-theoretic intersection of the support of $\tilde{\mathrm{E}}$ with the zero section of $\mathbf{T}_{\mathrm{X}^{\prime} / \mathrm{S}}^{*}$. We should perhaps recall that $\mathrm{F}_{\mathrm{X} / \mathrm{S}}: \mathrm{X} \rightarrow \mathrm{X}^{\prime}$ is a homeomorphism and from [29, 2.3.1] that the essential support of $(\mathrm{E}, \nabla)$ corresponds via $\mathrm{F}_{\mathrm{X} / \mathrm{S}}$ to the support in $\mathrm{X}$ of the Higgs cohomology sheaves of the $p$-curvature of $(\mathrm{E}, \nabla)$. (In fact, the $d$ th cohomology sheaf suffices.)

Proposition 2.31. - Let X/S be a smooth morphism of noetherian schemes in characteristic $p>0$ of relative dimension $d$. Let $(\mathrm{E}, \nabla)$ be a coherent sheaf with integrable connection on $\mathrm{X} / \mathrm{S}$, and let $\mathrm{Z} \subseteq \mathrm{X}$ be a closed subscheme containing the essential support of $(\mathrm{E}, \nabla)$. Let $i_{\mathrm{Z}}: \mathrm{X}_{\mathrm{Z}} \rightarrow \mathrm{X}$ denote the natural map from the formal completion of $\mathrm{X}$ along $\mathrm{Z}$ to $\mathrm{X}$. Then the natural map of de Rham complexes:

$$
a: \mathrm{E} \otimes \Omega_{\mathrm{X} / \mathrm{S}}^{\cdot} \rightarrow i_{\mathrm{Z} *} \mathrm{E}_{/ \mathrm{Z}} \otimes \Omega_{\mathrm{X} / \mathrm{S}}
$$

is a quasi-isomorphism.

Proof. - It suffices to prove that the map above induces an isomorphism on cohomology sheaves. The generalized Cartier isomorphism [29] is an isomorphism of sheaves of $\mathscr{O}_{\mathrm{X}^{\prime}}$-modules

$$
\mathscr{H}^{q}\left(\mathrm{~F}_{\mathrm{X} / \mathrm{S} *} \mathrm{E} \otimes \Omega_{\mathrm{X} / \mathrm{S}}^{\cdot}\right) \cong \mathrm{F}_{\mathrm{X} / \mathrm{S} *} \mathscr{H}^{q}\left(\mathrm{E} \otimes \mathrm{F}_{\mathrm{X} / \mathrm{S}}^{*} \Omega_{\mathrm{X}^{\prime} / \mathrm{S}}^{\cdot}\right)^{\nabla}
$$

where the complex on the right is the Higgs complex of the F-Higgs field given by the $p$-curvature of $\nabla$. Now one has a commutative diagram

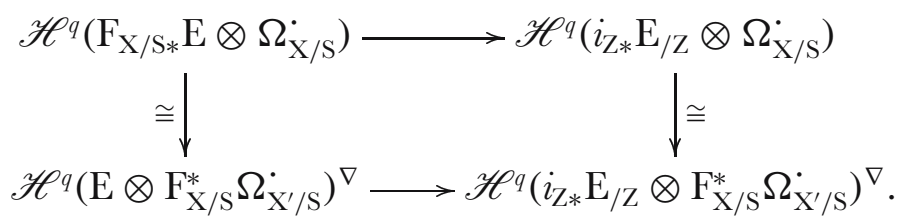

Thus it suffices to prove that the natural map

$$
\mathscr{H}^{q}\left(\mathrm{E} \otimes \mathrm{F}_{\mathrm{X} / \mathrm{S}}^{*} \Omega_{\mathrm{X}^{\prime} / \mathrm{S}}^{\cdot}\right) \rightarrow \mathscr{H}^{q}\left(i_{\mathrm{Z} *} \mathrm{E}_{/ \mathrm{Z}} \otimes \mathrm{F}_{\mathrm{X} / \mathrm{S}}^{*} \Omega_{\mathrm{X}^{\prime} / \mathrm{S}}^{\cdot}\right)
$$

is an isomorphism of $\mathscr{O}_{\mathrm{X}}$-modules. Since the completion functor is exact, and since the cohomology sheaves $\mathscr{H}^{q}\left(\mathrm{E} \otimes \mathrm{F}_{\mathrm{X} / \mathrm{S}}^{*} \Omega_{\mathrm{X}^{\prime} / \mathrm{S}}^{*}\right)$ have support in $\mathrm{Z}$, this is clear.

Let us also remark that in the situation of Proposition 2.31, we can define a formal Cartier transform as follows. Let $\mathrm{I} \subseteq \mathscr{O}_{\mathrm{X}^{\prime}}$ be an ideal of definition of the essential support Z of $\tilde{\mathrm{E}}$. For each $n$, let $\mathrm{E}_{n}:=\mathrm{E} / \mathrm{F}_{\mathrm{X} / \mathrm{S}}^{*} \mathrm{I}^{n} \mathrm{E}$, which inherits an integrable connection from the connection on $\mathrm{E}$. Then the $p$-curvature of $\left(\mathrm{E}_{n}, \nabla\right)$ is nilpotent and 
hence, given a lifting $\tilde{\mathrm{F}}$ of $\mathrm{F}_{\mathrm{X} / \mathrm{S}}$, it has has Cartier transform $\mathrm{C}_{\tilde{\mathrm{F}}}\left(\mathrm{E}_{n}\right)$. These Cartier transforms are compatible with change in $n$, and they fit together to define a coherent sheaf on the formal scheme $\mathrm{X}_{/ \mathrm{Z}}^{\prime}$, which we (slightly abusively) still denote by $\mathrm{C}_{\tilde{\mathrm{F}}}(\mathrm{E})$. The double complex constructions used in the proof of Theorem 2.26 also fit together into a formal double complex. The following statement is a consequence of this and the previous proposition.

Proposition 2.32. - Suppose that $\mathrm{X}$ is noetherian and that $(\mathrm{E}, \nabla)$ is a coherent sheaf on $\mathrm{X}$ with integrable connection. Let $\tilde{\mathrm{F}}$ be a lifting of $\mathrm{F}_{\mathrm{X} / \mathrm{S}}$ and let $\mathrm{C}_{\tilde{\mathrm{F}}}(\mathrm{E})$ denote the formal Cartier transform of $\mathrm{E}$ described above. Then the maps of Proposition 2.31 and statement (2) of Theorem 2.26 fit together to define an isomorphism in the derived category of $\mathscr{O}_{\mathrm{X}^{\prime}}$-modules

$$
\mathrm{F}_{\mathrm{X} / \mathrm{S} *}\left(\mathrm{E} \otimes \Omega_{\mathrm{X} / \mathrm{S}}^{\cdot}, d\right) \cong\left(\mathrm{C}_{\tilde{\mathrm{F}}}(\mathrm{E}) \otimes \Omega_{\mathrm{X}^{\prime} / \mathrm{S}}^{\cdot}, \theta^{\prime}\right) .
$$

\section{Functoriality of the Cartier transform}

\subsection{Gauss-Manin connections and Higgs fields}

In this section we review the definitions of higher direct images of modules with connections and Higgs fields. We show that their formation with respect to a smooth morphism of relative dimension $d$ increases the level of nilpotence of a connection (resp. of a Higgs field) by at most $d$. This result strengthens the nilpotence theorem of Katz [18, 5.10] and will be used in our discussion of the compatibility of the Cartier transform with higher direct images.

Recall that if $h: \mathrm{X} \rightarrow \mathrm{Y}$ is a smooth morphism of smooth $\mathrm{S}$-schemes and if $(\mathrm{E}, \nabla)$ is a module with integrable connection on $\mathrm{X} / \mathrm{S}$, then the sheaves

$$
\mathrm{R}^{n} h_{*}^{\mathrm{DR}}(\mathrm{E}, \nabla):=\mathrm{R}^{n} h_{*}\left(\mathrm{E} \otimes \Omega_{\mathrm{X} / \mathrm{Y}}^{\cdot}, d\right)
$$

are endowed with a canonical connection, called the Gauss-Manin connection. By the same token, if $(\mathrm{E}, \theta)$ is a module with a Higgs field $\theta$, then the sheaves

$$
\mathrm{R}^{n} h_{\mathrm{HIG} *}(\mathrm{E}, \theta):=\mathrm{R}^{n} h_{*}\left(\mathrm{E} \otimes \Omega_{\mathrm{X} / \mathrm{Y}}^{\cdot}, \theta\right)
$$

are endowed with a canonical Higgs field, which we shall call the Gauss-Manin field. Each of these can be constructed in many ways. For the reader's convenience we explain one of these here; a variant of the "explicit" construction explained in [18, 3.4]. We write out the details in the de Rham case only; the Higgs case is analogous but easier.

Let $(\mathrm{E}, \nabla)$ be a module with integrable connection on $\mathrm{X} / \mathrm{S}$ and let $\xi$ be a local section of $\mathrm{T}_{\mathrm{X} / \mathrm{S}}$. Then interior multiplication by $\xi$ defines a map of graded sheaves

$$
i_{\xi}: \mathrm{E} \otimes \Omega_{\mathrm{X} / \mathrm{S}} \rightarrow \mathrm{E} \otimes \Omega_{\mathrm{X} / \mathrm{S}}^{\circ}
$$


of degree -1 . The Lie derivative with respect to $\xi$ is by definition the map

$$
\mathrm{L}_{\xi}:=d i_{\xi}+i_{\xi} d
$$

which has degree zero. By construction $\mathrm{L}_{\xi}$ is a morphism of complexes, homotopic to zero. Now recall that a smooth morphism $h$ induces exact sequences

$$
\begin{aligned}
& 0 \rightarrow h^{*} \Omega_{\mathrm{Y} / \mathrm{S}}^{1} \rightarrow \Omega_{\mathrm{X} / \mathrm{S}}^{1} \rightarrow \Omega_{\mathrm{X} / \mathrm{Y}}^{1} \rightarrow 0 \\
& 0 \rightarrow \mathrm{T}_{\mathrm{X} / \mathrm{Y}} \rightarrow \mathrm{T}_{\mathrm{X} / \mathrm{S}} \rightarrow h^{*} \mathrm{~T}_{\mathrm{Y} / \mathrm{S}} \rightarrow 0 .
\end{aligned}
$$

Pull the second of these sequences back via the map $h^{-1} \mathrm{~T}_{\mathrm{Y} / \mathrm{S}} \rightarrow h^{*} \mathrm{~T}_{\mathrm{Y} / \mathrm{S}}$ to obtain an exact sequence of sheaves of $h^{-1}\left(\mathscr{O}_{\mathrm{Y}}\right)$-modules:

$$
0 \rightarrow \mathrm{T}_{\mathrm{X} / \mathrm{Y}} \rightarrow \mathrm{T}_{\mathrm{X} / \mathrm{S}}^{\mathrm{Y}} \rightarrow h^{-1} \mathrm{~T}_{\mathrm{Y} / \mathrm{S}} \rightarrow 0 .
$$

Let us note that $\mathrm{T}_{\mathrm{X} / \mathrm{S}}^{\mathrm{Y}} \subseteq \mathrm{T}_{\mathrm{X} / \mathrm{S}}$ is closed under the bracket operation and that the inclusion $\mathrm{T}_{\mathrm{X} / \mathrm{Y}} \rightarrow \mathrm{T}_{\mathrm{X} / \mathrm{S}}^{\mathrm{Y}}$ is compatible with the bracket operations. Moreover, if $g$ is a local section of $h^{-1}\left(\mathscr{O}_{\mathrm{Y}}\right)$ and $\xi$ is a local section of $\mathrm{T}_{\mathrm{X} / \mathrm{S}}^{\mathrm{Y}}$, then $\xi(g)$ also belongs to $h^{-1}\left(\mathscr{O}_{\mathrm{Y}}\right)$, and if $\eta$ is a local section of $\mathrm{T}_{\mathrm{X} / \mathrm{Y}}$, then

$$
[\eta, \xi](g)=\eta(\xi(g))-\xi(\eta(g))=0 .
$$

It follows that $[\eta, \xi] \in \mathrm{T}_{\mathrm{X} / \mathrm{Y}}$, so that $\mathrm{T}_{\mathrm{X} / \mathrm{Y}}$ is an ideal in the Lie algebra $\mathrm{T}_{\mathrm{X} / \mathrm{S}}^{\mathrm{Y}}$ and the map $\mathrm{T}_{\mathrm{X} / \mathrm{S}}^{\mathrm{Y}} \rightarrow h^{-1} \mathrm{~T}_{\mathrm{Y} / \mathrm{S}}$ is a Lie algebra homomorphism.

Lemma 3.1. - If $\xi$ is a local section of $\mathrm{T}_{\mathrm{X} / \mathrm{S}}^{\mathrm{Y}}$, then $\mathrm{L}_{\xi}$ preserves the Koszul filtration $\mathrm{K}^{\cdot}$ of $\mathrm{E} \otimes \Omega_{\mathrm{X} / \mathrm{S}}$ induced by the exact sequence (3.0.1). In particular, $\mathrm{L}_{\xi}$ induces a morphism of complexes

$$
\mathrm{L}_{\xi}: \mathrm{E} \otimes \Omega_{\mathrm{X} / \mathrm{Y}}^{\cdot} \rightarrow \mathrm{E} \otimes \Omega_{\mathrm{X} / \mathrm{Y}}^{\cdot}
$$

Furthermore, if $\xi$ and $\xi^{\prime}$ are local sections of $\mathrm{T}_{\mathrm{X} / \mathrm{S}}^{\mathrm{Y}}$, then $\left[\mathrm{L}_{\xi}, \mathrm{L}_{\xi^{\prime}}\right]=\mathrm{L}_{\left[\xi, \xi^{\prime}\right]}$ in $\operatorname{End}\left(\mathrm{E} \otimes \Omega_{\mathrm{X} / \mathrm{Y}}\right)$.

Proof. — By definition,

$$
\mathrm{K}^{i}\left(\mathrm{E} \otimes \Omega_{\mathrm{X} / \mathrm{S}}^{q}\right)=\operatorname{Im}\left(h^{*} \Omega_{\mathrm{Y} / \mathrm{S}}^{i} \otimes \mathrm{E} \otimes \Omega_{\mathrm{X} / \mathrm{S}}^{q-i}\right) \rightarrow \mathrm{E} \otimes \Omega_{\mathrm{X} / \mathrm{S}}^{q}
$$

Let $\xi$ be a local section of $\mathrm{T}_{\mathrm{X} / \mathrm{S}}^{\mathrm{Y}}$. Since $\mathrm{L}_{\xi}$ acts as a derivation with respect to multiplication by $\Omega_{\mathrm{X} / \mathrm{S}}$, it suffices to check that if $\omega$ is a local section of $h^{*} \Omega_{\mathrm{Y} / \mathrm{S}}^{1}$, then $\mathrm{L}_{\xi}(\omega)$ also belongs to $h^{*} \Omega_{\mathrm{Y} / \mathrm{S}}^{1}$. Again using the fact that $\mathrm{L}_{\xi}$ is a derivation, we see that it suffices to check this when $\omega$ lies in $h^{-1} \Omega_{\mathrm{Y} / \mathrm{S}}^{1}$. But if $\omega \in h^{-1} \Omega_{\mathrm{Y} / \mathrm{S}}^{1}$ and if the image of $\xi$ in $h^{*} \mathrm{~T}_{\mathrm{Y} / \mathrm{S}}$ lies in $h^{-1} \mathrm{~T}_{\mathrm{Y} / \mathrm{S}}, \mathrm{L}_{\xi}(\omega)=d i_{\xi}(\omega)+i_{\xi} d \omega \in h^{-1} \Omega_{\mathrm{Y} / \mathrm{S}}^{1}$.

The fact that the action of $\mathrm{T}_{\mathrm{X} / \mathrm{S}}$ on $\mathrm{E}$ by Lie derivative is compatible with the bracket follows from the integrability of $\nabla$, and it is well-known that the same is true for its action on $\Omega_{\mathrm{X} / \mathrm{S}}$. Since $\mathrm{L}_{\xi}, \mathrm{L}_{\xi^{\prime}}$ and $\mathrm{L}_{\left[\xi, \xi^{\prime}\right]}$ act as derivations with respect to multiplication by forms, it follows that $\left[\mathrm{L}_{\xi}, \mathrm{L}_{\xi^{\prime}}\right]=\mathrm{L}_{\left[\xi, \xi^{\prime}\right]}$ on $\mathrm{E} \otimes \Omega_{\mathrm{X} / \mathrm{S}}^{\circ}$ and hence also on $\mathrm{E} \otimes \Omega_{\mathrm{X} / \mathrm{Y}}$ 
Now let

$$
\mathrm{T}_{\mathrm{X} \rightarrow \mathrm{Y}}^{\cdot}:=\mathrm{T}_{\mathrm{X} / \mathrm{Y}} \stackrel{d}{\rightarrow} \mathrm{T}_{\mathrm{X} / \mathrm{S}}^{\mathrm{Y}}
$$

regarded as a complex in degrees -1 and 0 , where the boundary map is the inclusion. We can give $\mathrm{T}_{\mathrm{X} \rightarrow \mathrm{Y}}^{\cdot}$ the structure of a differential graded Lie algebra by defining $\left[\eta, \eta^{\prime}\right]:=0$ if $\eta, \eta^{\prime} \in \mathrm{T}_{\mathrm{X} / \mathrm{Y}},[\eta, \xi]:=[d \eta, \xi] \in \mathrm{T}_{\mathrm{X} / \mathrm{Y}}$ if $\eta \in \mathrm{T}_{\mathrm{X} / \mathrm{Y}}$ and $\xi \in \mathrm{T}_{\mathrm{X} / \mathrm{S}}^{\mathrm{Y}}$, and $\left[\xi, \xi^{\prime}\right]$ the usual bracket if $\xi, \xi^{\prime} \in \mathrm{T}_{\mathrm{X} / \mathrm{Y}}^{\mathrm{Y}}$. The exact sequence (3.0.3) defines an isomorphism in the derived category of $f^{-1} \mathscr{O}_{\mathrm{Y}}$-modules:

\section{(3.1.1)}

$$
\mathrm{T}_{\mathrm{X} \rightarrow \mathrm{Y}}^{\cdot} \rightarrow h^{-1} \mathrm{~T}_{\mathrm{Y} / \mathrm{S}}
$$

which is compatible with the bracket structure on $h^{-1} \mathrm{~T}_{\mathrm{X} / \mathrm{Y}}$.

If $\eta$ is a local section of $\mathrm{T}_{\mathrm{X} / \mathrm{Y}}$, then $i_{\eta}$ defines a section of degree -1 of the complex $\mathscr{E n d}\left(\mathrm{E} \otimes \Omega_{\mathrm{X} / \mathrm{Y}}^{*}\right)$, which we denote by $\nabla^{-1}(\eta)$. If $\xi$ is a local section of $\mathrm{T}_{\mathrm{X} / \mathrm{S}}^{\mathrm{Y}}$, then Lemma 3.1 tells us that $\mathrm{L}_{\xi}$ defines a section $\nabla^{0}(\xi)$ of degree 0 of $\mathscr{E}$ d $\left(\mathrm{E} \otimes \Omega_{\mathrm{X} / \mathrm{Y}}^{*}\right)$. Let us observe that $\nabla^{-1}$ and $\nabla^{0}$ assemble into a morphism of complexes:

$$
\nabla \cdot \mathrm{T}_{\mathrm{X} \rightarrow \mathrm{Y}}^{\cdot} \rightarrow \operatorname{End}\left(\mathrm{E} \otimes \Omega_{\mathrm{X} / \mathrm{Y}}^{\cdot}\right)
$$

Indeed, if $\xi \in \mathrm{T}_{\mathrm{X} / \mathrm{S}}^{\mathrm{Y}}$, then $\nabla^{0}(\xi)$ is a morphism of complexes, so it is annihilated by the total differential of $\mathscr{E} d\left(\mathrm{E} \otimes \Omega_{\mathrm{X} / \mathrm{Y}}\right)$. If $\eta \in \mathrm{T}_{\mathrm{X} / \mathrm{Y}}$, then $\nabla^{-1}(\eta)$ has degree -1 , so

$$
d \nabla^{-1}(\eta)=d \circ \nabla^{-1}(\eta)+\nabla^{-1}(\eta) \circ d=d \circ i_{\eta}+i_{\eta} \circ d=\mathrm{L}_{\eta}=\nabla^{0}(d \eta) .
$$

Let us also check that $\nabla^{\cdot}$ is a morphism of differential graded Lie algebras. If $\xi, \xi^{\prime} \in \mathrm{T}_{\mathrm{X} / \mathrm{S}}^{\mathrm{Y}}$, then we saw in Lemma 3.1 that

$$
\left[\nabla^{0}(\xi), \nabla^{0}\left(\xi^{\prime}\right)\right]:=\left[\mathrm{L}_{\xi}, \mathrm{L}_{\xi^{\prime}}\right]=\mathrm{L}_{\left[\xi, \xi^{\prime}\right]}=\nabla^{0}\left(\left[\xi, \xi^{\prime}\right]\right) .
$$

We must also check that if $\xi \in \mathrm{T}_{\mathrm{X} / \mathrm{S}}^{\mathrm{Y}}$ and $\eta \in \mathrm{T}_{\mathrm{X} / \mathrm{Y}}$, then

$$
\left[\nabla^{-1}(\eta), \nabla^{0}(\xi)\right]=\nabla^{-1}([\eta, \xi]), \text { i.e. },
$$

that $\left[i_{\eta}, \mathrm{L}_{\xi}\right]=i_{[\eta, \xi]}$. Observe first that both sides are derivations of $\mathrm{E} \otimes \Omega_{\mathrm{X} / \mathrm{Y}}$ of degree -1 with respect to multiplication by forms, and in particular are $\mathscr{O}_{\mathrm{X}}$-linear. Thus it suffices to check the formula for closed 1-forms. In fact, if $\omega \in \Omega_{\mathrm{X} / \mathrm{S}}^{1}$ is closed, then

$$
\begin{aligned}
{\left[\nabla^{-1}(\eta), \nabla^{0}(\xi)\right](\omega) } & =i_{\eta} \mathrm{L}_{\xi}(\omega)-\mathrm{L}_{\xi} i_{\eta}(\omega) \\
& =i_{\eta}(d\langle\xi, \omega\rangle)-\mathrm{L}_{\xi}\langle\eta, \omega\rangle \\
& =\eta\langle\xi, \omega\rangle-\xi\langle\eta, \omega\rangle \\
& =\langle[\eta, \xi], \omega\rangle \\
& =\nabla^{-1}([\eta, \xi]),
\end{aligned}
$$

as required. Finally, let us observe that $\nabla^{\cdot}$ is a derivation with respect to multiplication by sections of $h^{-1}\left(\mathscr{O}_{Y}\right)$. 
Definition 3.2. - Let $h: \mathrm{X} \rightarrow \mathrm{Y}$ be a smooth morphism of smooth $\mathrm{S}$-schemes and let $(\mathrm{E}, \nabla)$ (resp. $(\mathrm{E}, \theta))$ be a module with integrable connection (resp. Higgs field) on X/S. Then the Gauss-Manin connection (resp. Higgs field) on $\mathrm{R}^{n} h_{*}\left(\mathrm{E} \otimes \Omega_{\mathrm{X} / \mathrm{Y}}\right)$ is the map

$$
\mathrm{T}_{\mathrm{Y} / \mathrm{S}} \rightarrow \operatorname{End~}^{n} h_{*}\left(\mathrm{E} \otimes \Omega_{\mathrm{X} / \mathrm{Y}}^{*}\right)
$$

obtained by composing the adjunction map

$$
\mathrm{T}_{\mathrm{Y} / \mathrm{S}} \rightarrow h_{*} h^{-1} \mathrm{~T}_{\mathrm{Y} / \mathrm{S}}=\mathrm{R}^{0} h_{*} h^{-1} \mathrm{~T}_{\mathrm{Y} / \mathrm{S}}
$$

with the inverse of the isomorphism $\mathrm{R}^{0} h_{*} \mathrm{~T}_{\mathrm{X} \rightarrow \mathrm{Y}} \rightarrow \mathrm{R}^{0} h_{*} h^{-1} \mathrm{~T}_{\mathrm{Y} / \mathrm{S}}$ defined by (3.1.1) and the maps

$$
\mathrm{R}^{0} h_{*}\left(\nabla^{*}\right): \mathrm{R}^{0} h_{*}\left(\mathrm{~T}_{\mathrm{X} \rightarrow \mathrm{Y}}^{\cdot}\right) \rightarrow \mathrm{R}^{0} h_{*} \mathscr{E} n d\left(\mathrm{E} \otimes \Omega_{\mathrm{X} / \mathrm{Y}}^{\cdot}\right) \rightarrow \operatorname{End} \mathrm{R}^{n} h_{*}\left(\mathrm{E} \otimes \Omega_{\mathrm{X} / \mathrm{Y}}^{\cdot}\right) .
$$

Remark 3.3. - The integrability of the Gauss-Manin connection defined here follows from the compatibility of the maps (3.1.1) and $\nabla^{\cdot}$ with the bracket operations. A similar construction defines the Gauss-Manin Higgs field, and thus we obtain sequence of functors

$$
\begin{aligned}
& \mathrm{R}^{n} h_{*}^{\mathrm{DR}}: \operatorname{MIG}(\mathrm{X} / \mathrm{S}) \rightarrow \operatorname{MIG}(\mathrm{Y} / \mathrm{S}) \\
& \mathrm{R}^{n} h_{*}^{\mathrm{HIG}}: \operatorname{HIG}(\mathrm{X} / \mathrm{S}) \rightarrow \mathrm{HIG}(\mathrm{Y} / \mathrm{S}) .
\end{aligned}
$$

It is straightforward to check that these fit into sequences of exact effaceable $\delta$-functors and hence are derived functors. This makes it easy to compare this construction with the many others which appear in the literature and in particular with the derived category constructions appearing in Section 3.3.

Now suppose that $\mathrm{N}$. is an increasing filtration on $\mathrm{E}$ which is stable under the connection (resp. Higgs field). Then the filtrations $\mathrm{N}$. and $\mathrm{N}^{\text {dec }}$ of $\mathrm{E} \otimes \Omega_{\mathrm{X} / \mathrm{Y}}$ are stable under the action of $\mathrm{T}_{\mathrm{X} \rightarrow \mathrm{Y}}$, and hence the higher direct images of the corresponding filtered pieces and the graded objects inherit Gauss-Manin connections.

Theorem 3.4. - Let $h: \mathrm{X} \rightarrow \mathrm{Y}$ be a smooth morphism of smooth $\mathrm{S}$-schemes. Let $\mathrm{E}$ be a sheaf of $\mathscr{O}_{\mathrm{X}}$-modules endowed with an integrable connection $\nabla$ (resp. a Higgs field $\theta$ ). Suppose that $\mathrm{N}$. is a filtration on $\mathrm{E}$ such that $\mathrm{Gr}^{\mathrm{N}} \nabla$ is constant (resp., such that $\mathrm{Gr}^{\mathrm{N}} \theta=0$ ). Then for each $n$ and $i$, the action of the Gauss-Manin connection (resp. Higgs field) on $\mathrm{R}^{n} h_{*}\left(\mathrm{Gr}_{i}^{\mathrm{N}^{\text {dec }}}\left(\mathrm{E} \otimes \Omega_{\mathrm{X} / \mathrm{Y}}^{*}\right)\right.$ ) is constant (resp. trivial). ${ }^{8}$

Proof. - If $\theta$ is a Higgs field such that $\operatorname{Gr}^{\mathrm{N}}(\theta)=0$, then $\theta$ maps $\mathrm{N}_{i} \mathrm{E}$ to $\mathrm{N}_{i-1} \mathrm{E} \otimes \Omega_{\mathrm{X} / \mathrm{S}}^{1}$. It follows that the actions of $\mathrm{T}_{\mathrm{X} / \mathrm{Y}}$ and $\mathrm{T}_{\mathrm{X} / \mathrm{S}}^{\mathrm{Y}}$ on $\mathrm{E} \otimes \Omega_{\mathrm{X} / \mathrm{Y}}^{\cdot}$ by interior multiplication and Lie derivative map $\mathrm{N}_{i}^{d e c}$ to $\mathrm{N}_{i-1}^{d e c}$. Hence $\mathrm{T}_{\mathrm{X} \rightarrow \mathrm{Y}}^{\cdot}$ acts trivially on $\mathrm{Gr}_{i}^{\mathrm{N}^{\text {dec }}}\left(\mathrm{E} \otimes \Omega_{\mathrm{X} / \mathrm{Y}}^{\cdot}\right)$.

${ }^{8}$ We should point out that the statement for connections, but not for Higgs fields, requires that $\mathrm{S}$ have characteristic $p>0$. 
Now suppose that $\nabla$ is a connection on $\mathrm{E}$ and $\mathrm{N}$ is a horizontal filtration on $\mathrm{E}$. Recall that we have a natural quasi-isomorphism (2.29.2) of complexes

$$
a:\left(\mathrm{E}_{1}^{\cdot, j}\left(\mathrm{E} \otimes \Omega_{\mathrm{X} / \mathrm{Y}}^{\cdot}, \mathrm{N}\right), d_{1}\right) \rightarrow \mathrm{Gr}_{j}^{\mathrm{N} d e c}\left(\mathrm{E} \otimes \Omega_{\mathrm{X} / \mathrm{Y}}^{\cdot}, d\right) .
$$

Here $\mathrm{E}_{1}^{i, j}\left(\mathrm{E} \otimes \Omega_{\mathrm{X} / \mathrm{Y}}, \mathrm{N}\right)=\mathscr{H}^{j-i}\left(\mathrm{Gr}_{i}^{\mathrm{N}} \mathrm{E} \otimes \Omega_{\mathrm{X} / \mathrm{Y}}^{*}\right)$. Note that if $\xi \in \mathrm{T}_{\mathrm{X} / \mathrm{Y}} \subseteq \mathrm{T}_{\mathrm{X} / \mathrm{S}}^{\mathrm{Y}}$, then $i_{\xi}$ is well-defined on $\mathrm{E} \otimes \Omega_{\mathrm{X} / \mathrm{Y}}^{\circ}$, and hence $\mathrm{L}_{\xi}=d i_{\xi}+i_{\xi} d$ acts as zero on $\mathscr{H}^{q}(\mathrm{E} \otimes$ $\left.\Omega_{\mathrm{X} / \mathrm{Y}}\right)$. Thus the action of $\mathrm{T}_{\mathrm{X} \rightarrow \mathrm{Y}}^{*}$ factors through $h^{-1} \mathrm{~T}_{\mathrm{Y} / \mathrm{S}}$; the boundary maps $d_{1}$ are compatible with this action. Thus $\mathrm{R}^{n} h_{*}\left(\mathrm{E}_{1}^{\cdot}{ }^{j}, d_{1}\right)$ has a connection also, and we claim that $\mathrm{R}^{n} h_{*}(a)$ is compatible with the connections. To see this, it is convenient to recall the "dual" version of the filtration décalée:

$$
\mathrm{N}_{i}^{*}\left(\mathrm{E} \otimes \Omega_{\mathrm{X} / \mathrm{Y}}^{q}\right):=\mathrm{N}_{i-q} \mathrm{E} \otimes \Omega_{\mathrm{X} / \mathrm{Y}}^{q} \cap d^{-1}\left(\mathrm{~N}_{i-q-1} \mathrm{E} \otimes \Omega_{\mathrm{X} / \mathrm{Y}}^{q}\right) .
$$

Then there is also a natural quasi-isomorphism

$$
a^{*}:\left(\mathrm{Gr}_{j}^{\mathrm{N}^{*}}\left(\mathrm{E} \otimes \Omega_{\mathrm{X} / \mathrm{Y}}^{\cdot}\right), d\right) \rightarrow\left(\mathrm{E}_{1}^{\cdot j}\left(\mathrm{E} \otimes \Omega_{\mathrm{X} / \mathrm{Y}}^{\cdot}, \mathrm{N}\right), d_{1}\right) .
$$

Then $a^{*}$ and $a a^{*}$ are compatible with the actions of $\mathrm{T}_{\mathrm{X} \rightarrow \mathrm{Y}}^{\cdot}$. Although $a$ is not compatible with the action of $\mathrm{T}_{\mathrm{X} \rightarrow \mathrm{Y}}$ on the level of complexes, it follows that it is compatible with the induced action of $\mathrm{T}_{\mathrm{Y} / \mathrm{S}}$ on hyper direct images.

Now suppose that $\mathrm{Gr} E:=\mathrm{Gr}^{\mathrm{N}} \mathrm{E}$ is constant. The theorem will follow if we prove that the Gauss-Manin connection on $\mathrm{R}^{n} h_{*}\left(\mathrm{E}_{1}^{\cdot}\left(\mathrm{E} \otimes \Omega_{\mathrm{X} / \mathrm{Y}}^{*}, \mathrm{~N}\right), d_{1}\right)$ is constant. Let us consider the relative Frobenius diagram:

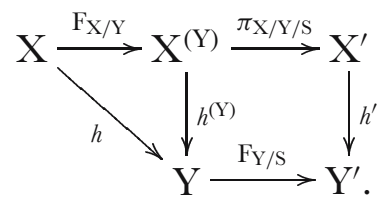

Here $\mathrm{F}_{\mathrm{X} / \mathrm{S}}=\pi_{\mathrm{X} / \mathrm{Y} / \mathrm{S}} \circ \mathrm{F}_{\mathrm{X} / \mathrm{Y}}$ and he square is Cartesian, so $\Omega_{\mathrm{X}^{(\mathrm{Y})} / \mathrm{Y}}^{q} \cong \pi_{\mathrm{X} / \mathrm{Y} / \mathrm{S}}^{*} \Omega_{\mathrm{X}^{\prime} / \mathrm{Y}^{\prime}}^{q}$.

The morphism of filtered complexes

$$
\left(\mathrm{E} \otimes \Omega_{\mathrm{X} / \mathrm{S}}^{\cdot}, \mathrm{N} .\right) \rightarrow\left(\mathrm{E} \otimes \Omega_{\mathrm{X} / \mathrm{Y}}^{\cdot}, \mathrm{N} .\right)
$$

induces a morphism of spectral sequences, which on the $\mathrm{E}_{1}$-level corresponds to the top row of the following commutative diagram:

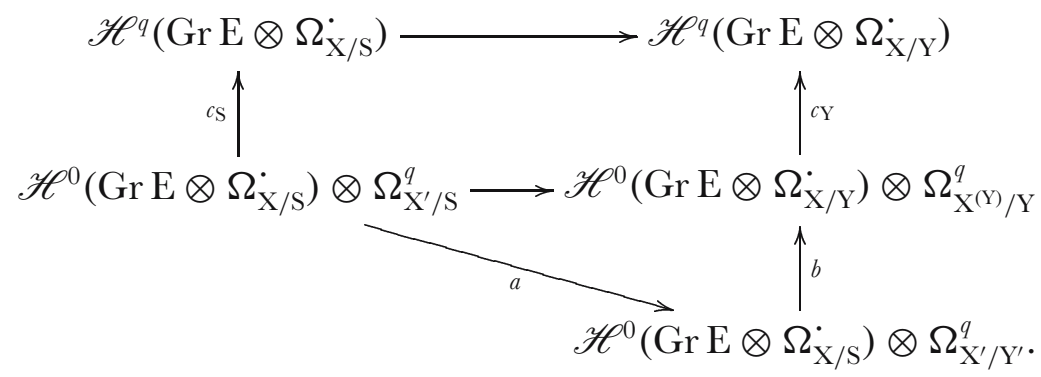


The vertical maps $c_{\mathrm{S}}$ and $c_{\mathrm{Y}}$ induced by the inverse Cartier isomorphism are isomorphisms because GrE is constant, the map $a$ is surjective, and the map $b$ is injective. Thus $\mathscr{H}^{0}\left(\mathrm{GrE} \otimes \Omega_{\mathrm{X} / \mathrm{S}}^{\circ}\right) \otimes \Omega_{\mathrm{X}^{\prime} / \mathrm{Y}^{\prime}}^{q}$ can be identified with the image of the arrow at the top of the diagram. Since the differentials of the spectral sequence leave this image invariant, they induce maps

$$
\mathscr{H}^{0}\left(\mathrm{GrE} \otimes \Omega_{\mathrm{X} / \mathrm{S}}^{\cdot}\right) \otimes \Omega_{\mathrm{X}^{\prime} / \mathrm{Y}^{\prime}}^{q} \rightarrow \mathscr{H}^{0}\left(\mathrm{Gr} \mathrm{E} \otimes \Omega_{\mathrm{X} / \mathrm{S}}^{\cdot}\right) \otimes \Omega_{\mathrm{X}^{\prime} / \mathrm{Y}^{\prime}}^{q+1}
$$

and define a complex $\mathscr{H}^{0}\left(\mathrm{GrE} \otimes \Omega_{\mathrm{X} / \mathrm{S}}^{\cdot}\right) \otimes \Omega_{\mathrm{X}^{\prime} / \mathrm{Y}^{\prime}}$ of sheaves of $\mathscr{O}_{\mathrm{X}^{\prime}}$-modules on $\mathrm{X}^{\prime}$. Since the natural map

$$
\pi_{\mathrm{X} / \mathrm{Y} / \mathrm{S}}^{*}\left(\mathscr{H}^{0}\left(\mathrm{Gr} \mathrm{E} \otimes \Omega_{\mathrm{X} / \mathrm{S}}^{\cdot}\right) \otimes \Omega_{\mathrm{X}^{\prime} / \mathrm{Y}^{\prime}}^{q}\right) \rightarrow \mathscr{H}^{0}\left(\mathrm{GrE} \otimes \Omega_{\mathrm{X} / \mathrm{Y}}^{\cdot}\right) \otimes \Omega_{\mathrm{X}^{(\mathrm{Y})} / \mathrm{Y}}^{q}
$$

is an isomorphism, we see that the complex $\mathrm{E}_{1}(\mathrm{E}, \mathrm{N}$.) descends to a complex of $\mathscr{O}_{\mathrm{X}^{\prime}}$-modules on $\mathrm{X}^{\prime}$.

Note that if $\xi \in \mathrm{T}_{\mathrm{X} / \mathrm{Y}} \subseteq \mathrm{T}_{\mathrm{X} / \mathrm{S}}^{\mathrm{Y}}$, then $i_{\xi}$ is well-defined on $\mathrm{E} \otimes \Omega_{\mathrm{X} / \mathrm{Y}}$, and hence $\mathrm{L}_{\xi}=d i_{\xi}+i_{\xi} d$ acts as zero on $\mathscr{H}^{q}\left(\mathrm{E} \otimes \Omega_{\mathrm{X} / \mathrm{Y}}^{*}\right)$. Thus the action of $\mathrm{T}_{\mathrm{X} \rightarrow \mathrm{Y}}$ on $\mathscr{H}^{q}\left(\mathrm{E} \otimes \Omega_{\mathrm{X} / \mathrm{Y}}\right)$ factors through $h^{-1} \mathrm{~T}_{\mathrm{Y} / \mathrm{S}}$. For the same reason, $h^{-1} \mathrm{~T}_{\mathrm{Y} / \mathrm{S}}$ acts as zero on the image of $\mathscr{H}^{q}\left(\mathrm{Gr} \mathrm{E} \otimes \Omega_{\mathrm{X} / \mathrm{S}}\right)$ in $\mathscr{H}^{q}\left(\mathrm{GrE} \otimes \Omega_{\mathrm{X} / \mathrm{Y}}\right)$ and it follows that the action of $h^{-1} \mathrm{~T}_{\mathrm{Y} / \mathrm{S}}$ on $\mathrm{E}_{1}(\mathrm{E}, \mathrm{N}$.) is nothing but the Frobenius descent connection. It follows that the Gauss-Manin connection on $\mathrm{R}^{n} h_{*}^{(\mathrm{Y})}\left(\mathscr{H}^{\cdot} \mathrm{Gr}^{\mathrm{N}}\left(\mathrm{E} \otimes \Omega_{\mathrm{X} / \mathrm{Y}}^{\cdot}\right), d_{1}\right)$ is the Frobenius descent connection.

The following result is an improvement of the result [18, 5.10] of Katz, which gives a multiplicative instead of an additive estimate for the level of nilpotence of higher direct images.

Corollary 3.5. - In the situation of the previous theorem, suppose that $h: \mathrm{X} \rightarrow \mathrm{Y}$ has relative dimension $d$, and denote by $\mathrm{MICN}_{\ell}(\mathrm{X} / \mathrm{S})$ the category of objects of $\mathrm{MICN}(\mathrm{X} / \mathrm{S})$ of level $\ell$, i.e., such that there exists an integer $k$ such that $\mathrm{N}_{k} \mathrm{E}=0$ and $\mathrm{N}_{k+\ell} \mathrm{E}=\mathrm{E}$. Then for each $q, \mathrm{R}^{q} h_{*}^{\mathrm{DR}}\left(\mathrm{E} \otimes \Omega_{\mathrm{X} / \mathrm{Y}}^{\cdot}, \mathrm{N}^{\text {dec }}\right)$ lies in $\mathrm{MICN}_{d+\ell}(\mathrm{Y} / \mathrm{S})$, and the analogous statement for Higgs modules also holds.

Remark 3.6. - In the case of connections, we can use the diagram (2.29.1), which computes the boundary maps of the complex $\mathrm{E}_{1}^{\cdot, j}\left(\mathrm{E} \otimes \Omega_{\mathrm{X} / \mathrm{Y}}^{\cdot}, \mathrm{N}\right.$.), to see that

$$
\mathrm{R}^{n} h_{*} \mathrm{Gr}_{i}^{\mathrm{N}^{\text {ddc }}}\left(\mathrm{E} \otimes \Omega_{\mathrm{X} / \mathrm{Y}}^{\cdot}, d\right) \cong \mathrm{F}_{\mathrm{Y} / \mathrm{S}}^{*} \mathrm{R}^{n} h_{*}^{\prime} \mathrm{Gr}_{.}^{\mathrm{N}}\left(\mathrm{E} \otimes \Omega_{\mathrm{X}^{\prime} / \mathrm{Y}^{\prime}}^{\cdot}, \bar{\psi}\right)
$$

where $\bar{\psi}$ is the map induced by the $p$-curvature.

Example 3.7. - Let $k$ be a field of characteristic $p, \mathrm{~S}:=\operatorname{Spec} k, \mathrm{Y}:=\operatorname{Spec} k[t]$. If $d$ is a positive integer, let $m:=d+2$, assume $(p, m)=1$, and consider the hypersurface 
$\mathrm{X}$ in $\mathbf{P}^{n+1}$ over $\mathrm{S}$ defined by $\mathrm{X}_{0}^{m}+\mathrm{X}_{1}^{m}+\cdots \mathrm{X}_{d+1}^{m}+t \mathrm{X}_{0} \mathrm{X}_{1} \cdots \mathrm{X}_{d+1}$. Once $\mathrm{Y}$ is replaced by a suitable affine neighborhood of the origin, $\mathrm{X} / \mathrm{Y}$ will be smooth, and the iterated Kodaira-Spencer mapping

$$
\left(\kappa_{\partial / \partial t}\right)^{d}: \mathrm{H}^{0}\left(\mathrm{X}, \Omega_{\mathrm{X} / \mathrm{Y}}^{d}\right) \rightarrow \mathrm{H}^{d}\left(\mathrm{X}, \mathscr{O}_{\mathrm{X}}\right)
$$

is an isomorphism [27, 3.4]. Then Katz's formula [19, Theorem 3.2] implies that the iterated $p$-curvature mapping

$$
\left(\psi_{\partial / \partial t^{\prime}}\right)^{d}: \mathrm{H}^{0}\left(\mathrm{X}, \mathscr{H}^{d}\left(\Omega_{\mathrm{X} / \mathrm{Y}}^{\cdot}\right)\right) \rightarrow \mathrm{H}^{d}\left(\mathrm{X}, \mathscr{H}^{0}\left(\Omega_{\mathrm{X} / \mathrm{Y}}^{\cdot}\right)\right)
$$

is also an isomorphism. This implies that the level of the Gauss-Manin connection on $\mathrm{R}^{d} h_{*}\left(\Omega_{\mathrm{X} / \mathrm{Y}}\right)$ is $d$. Moreover, if $d>p$, the action of the center of $\mathrm{D}_{\mathrm{Y} / \mathrm{S}}$ on $\mathrm{R}^{d} h_{*}\left(\Omega_{\mathrm{X} / \mathrm{Y}}\right)$ does not factor through the divided power neighborhood of the zero section.

\subsection{The Cartier transform and de Rham direct images}

Let $h: \mathrm{X} / \mathrm{S} \rightarrow \mathrm{Y} / \mathrm{S}$ be a smooth morphism of smooth $\mathrm{S}$-schemes, endowed with liftings $\tilde{\mathrm{X}}^{\prime} / \mathscr{S}$ and $\tilde{\mathrm{Y}}^{\prime} / \mathscr{S}$. We shall explain how a lifting $\tilde{h}^{\prime}: \tilde{\mathrm{X}}^{\prime} \rightarrow \tilde{\mathrm{Y}}^{\prime}$ of $h^{\prime}$ defines a compatibility isomorphism between the Cartier transform of the de Rham direct image of a module with connection and the Higgs direct image of its Cartier transform.

It is convenient to work with filtered categories as described in Corollary 3.5. If $\ell<p$, an object $\left(\mathrm{E}, \nabla, \mathrm{N}\right.$.) of $\mathrm{MICN}_{\ell}(\mathrm{X} / \mathrm{S})$ can be viewed as an object of $\mathrm{MICN}_{\gamma}(\mathrm{X} / \mathrm{S})$ and we apply the filtered Cartier transform of Theorem 2.23 to obtain an object $\left(\mathrm{E}^{\prime}, \theta^{\prime}, \mathrm{N}^{\prime}\right.$.) of $\mathrm{HIGN}_{\ell}\left(\mathrm{X}^{\prime} / \mathrm{S}\right)$.

Theorem 3.8. — Let $h: \mathrm{X} / \mathrm{S} \rightarrow \mathrm{Y} / \mathrm{S}$ be a smooth morphism of smooth $\mathrm{S}$-schemes, endowed with liftings $\tilde{\mathrm{X}}^{\prime} / \mathscr{S}$ and $\tilde{\mathrm{Y}}^{\prime} / \mathscr{S}$. Let $\ell$ be an integer less than $p-d$, where $d$ is the relative dimension of $h$. Then a lifting $\tilde{h}^{\prime}: \tilde{\mathrm{X}}^{\prime} / \mathrm{S} \rightarrow \tilde{\mathrm{Y}}^{\prime} / \mathrm{S}$ of $h^{\prime}: \mathrm{X}^{\prime} / \mathrm{S} \rightarrow \mathrm{Y}^{\prime} / \mathrm{S}$ induces an isomorphism of functors (made explicit below):

$$
\Theta_{\tilde{h}^{\prime}}^{q}: \mathrm{R}^{q} h_{*}^{\prime \mathrm{HIG}} \circ \mathrm{C}_{\mathscr{X} / \mathscr{S}} \Rightarrow \mathrm{C}_{\mathscr{Y} / \mathscr{S}} \circ \mathrm{R}^{q} h_{*}^{\mathrm{DR}}
$$

making the diagram below 2-commutative:

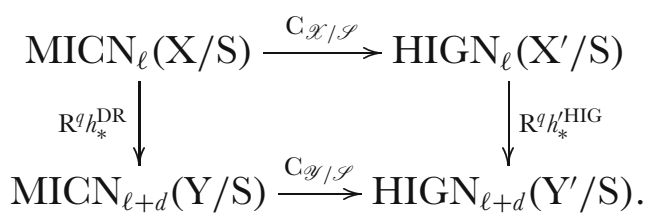


We shall construct the compatibility isomorphism of Theorem 3.8 from a canonical filtered double complex, a relative version of the double complex we used in the construction of the comparison isomorphism in Theorem 2.26. For any $(\mathrm{E}, \nabla, \mathrm{N}) \in$ $\operatorname{MICN}(\mathrm{X} / \mathrm{S})$, define

$$
\begin{aligned}
\mathscr{A}_{\mathscr{X} / \mathscr{Y} / \mathscr{S}}^{i j}(\mathrm{E}): & =\mathrm{F}_{\mathrm{X} / \mathrm{S} *}\left(\mathrm{E} \otimes \mathscr{A}_{\mathscr{X} / \mathscr{S}} \otimes \mathrm{F}_{\mathrm{X} / \mathrm{S}}^{*} \Omega_{\mathrm{X}^{\prime} / \mathrm{Y}^{\prime}}^{i} \otimes \Omega_{\mathrm{X} / \mathrm{Y}}^{j}\right) \\
& \cong \mathrm{F}_{\mathrm{X} / \mathrm{S} *}\left(\mathrm{E} \otimes \mathscr{A}_{\mathscr{X} / \mathscr{S}} \otimes \Omega_{\mathrm{X} / \mathrm{Y}}^{j}\right) \otimes \Omega_{\mathrm{X}^{\prime} / \mathrm{Y}^{\prime}}^{i} .
\end{aligned}
$$

The de Rham and Higgs boundary maps then form a double complex $\left(\mathscr{A}_{\mathscr{X} / \mathscr{Y} / \mathrm{S}}(\mathrm{E}), d^{\prime}, d\right)$, which we endow with the total filtration $\mathrm{N} .:=\mathrm{N}^{\text {tot }}$. There is a canonical morphism

$$
\left(\mathscr{A}_{\mathscr{X} / \mathscr{S}}(\mathrm{E}), \mathrm{N}^{t o t}\right) \rightarrow\left(\mathscr{A}_{\mathscr{X} / \mathscr{Y} / \mathscr{S}}(\mathrm{E}), \mathrm{N}^{t o t}\right)
$$

Let us recall from the diagram (3.4.1) that we have a morphism $h^{(\mathrm{Y})}: \mathrm{X}^{(\mathrm{Y})} \rightarrow \mathrm{Y}$ and a homeomorphism $\pi_{\mathrm{X} / \mathrm{Y} / \mathrm{S}}: \mathrm{X}^{(\mathrm{Y})} \rightarrow \mathrm{X}^{\prime}$, which we will sometimes allow ourselves to view as an identification to simplify the notation. The terms of the complex $\mathscr{A}_{\mathscr{X} / \mathscr{Y} / \mathscr{S}}(\mathrm{E})$ are $\mathrm{F}_{\mathrm{X} / \mathrm{S} *} \mathscr{O}_{\mathrm{X}}$-modules and the boundary maps are $\pi_{\mathrm{X} / \mathrm{Y} / \mathrm{S} *} \mathscr{O}_{\mathrm{X}}{ }^{(\mathrm{Y})}$-linear.

Recall from Proposition 1.12 that the lifting $\tilde{h}^{\prime}$ of $h$ defines a morphism of filtered algebras with connection

$$
\theta_{\tilde{h}^{\prime}}:\left(h^{*} \mathscr{A}_{\mathscr{Y} / \mathscr{S}}, \mathrm{N} .\right) \rightarrow\left(\mathscr{A}_{\mathscr{X} / \mathscr{S}}, \mathrm{N} .\right)
$$

Then we have a morphism of filtered relative de Rham complexes:

$$
\left(\mathrm{E} \otimes h_{\mathrm{DR}}^{*} \mathscr{A}_{\mathscr{Y} / \mathscr{S}} \otimes \Omega_{\mathrm{X} / \mathrm{Y}}^{\cdot}, \mathrm{N}^{t o t}\right) \rightarrow\left(\mathrm{E} \otimes \mathscr{A}_{\mathscr{X} / \mathscr{S}} \otimes \Omega_{\mathrm{X} / \mathrm{Y}}, \mathrm{N}^{t o t}\right)
$$

Since $h_{\mathrm{DR}}^{*} \mathscr{A}_{\mathscr{Y} / \mathscr{S}}$ comes from $\mathrm{Y}$, its $p$-curvature relative to $\mathrm{Y}$ vanishes, so for each $j$, the map

$$
\mathrm{E} \otimes h^{*} \mathscr{A}_{\mathscr{Y} / \mathscr{S}} \otimes \Omega_{\mathrm{X} / \mathrm{Y}}^{j} \rightarrow \mathrm{E} \otimes \mathscr{A}_{\mathscr{X} / \mathscr{S}} \otimes \Omega_{\mathrm{X} / \mathrm{Y}}^{j}
$$

is annihilated by the differential:

$$
d^{\prime}: \mathrm{E} \otimes \mathscr{A}_{\mathscr{X} / \mathscr{S}} \otimes \Omega_{\mathrm{X} / \mathrm{Y}}^{j} \rightarrow \mathrm{E} \otimes \mathscr{A}_{\mathscr{X} / \mathscr{S}} \otimes \Omega_{\mathrm{X} / \mathrm{Y}}^{j} \otimes \mathrm{F}_{\mathrm{X} / \mathrm{S}}^{*} \Omega_{\mathrm{X}^{\prime} / \mathrm{Y}^{\prime}}^{1}
$$

Let $\mathscr{A}_{\mathscr{Y} / \mathscr{S}}(\mathrm{E}):=\mathrm{E} \otimes h_{\mathrm{DR}}^{*} \mathscr{A}_{\mathscr{Y} / \mathscr{S}} \in \mathrm{MIC}(\mathrm{X} / \mathrm{S})$. It follows that the maps (3.8.1) define a morphism of filtered complexes:

$$
b: \mathrm{F}_{\mathrm{X} / \mathrm{S} *}\left(\mathscr{A}_{\mathscr{Y} / \mathscr{S}}(\mathrm{E}) \otimes \Omega_{\mathrm{X} / \mathrm{Y}}, \mathrm{N}^{t o t}\right) \rightarrow\left(\mathscr{A}_{\mathscr{X} / \mathscr{Y} / \mathscr{S}}(\mathrm{E}), \mathrm{N}^{\mathrm{t} t}\right) .
$$

Let $\mathrm{E}^{\prime}$ be the Cartier transform of $\mathrm{E}$. Since formation of $p$-curvature is compatible with de Rham pullback (see Remark 1.8), the map $\theta_{\tilde{h}^{\prime}}$ is also compatible with the 
F-Higgs fields. Thus we have a morphism of filtered relative F-Higgs complexes:

$$
\left(\mathrm{E}^{\prime} \otimes h^{*} \mathscr{A}_{\mathscr{Y} / \mathscr{S}} \otimes \mathrm{F}_{\mathrm{X} / \mathrm{S}}^{*} \Omega_{\mathrm{X}^{\prime} / \mathrm{Y}^{\prime}}^{\cdot}, \mathrm{N}^{t o t}\right) \rightarrow\left(\mathrm{E} \otimes \mathscr{A}_{\mathscr{X} / \mathscr{S}} \otimes \mathrm{F}_{\mathrm{X} / \mathrm{S}}^{*} \Omega_{\mathrm{X}^{\prime} / \mathrm{Y}^{\prime}}^{\cdot}, \mathrm{N}^{t o t}\right)
$$

Note that there is an isomorphism of $\mathscr{O}_{\mathrm{X}^{(\mathrm{Y})}}$-modules

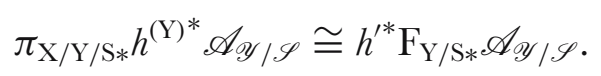

Since $h^{(\mathrm{Y})^{*}} \mathscr{A}_{\mathscr{Y} / \mathscr{S}}$ and the Cartier transform $\mathrm{E}^{\prime}$ of $\mathrm{E}$ are both annihilated by the relative de Rham differential $\mathscr{A}_{\mathscr{X} / \mathscr{S}}(\mathrm{E}) \rightarrow \mathscr{A}_{\mathscr{X} / \mathscr{S}}(\mathrm{E}) \otimes \Omega_{\mathrm{X} / \mathrm{Y}}^{1}$, the same is true of the tensor product

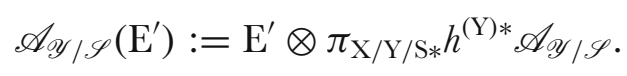

Thus we find a morphism of filtered complexes:

$$
a:\left(\mathscr{A}_{\mathscr{Y} / \mathscr{S}}\left(\mathrm{E}^{\prime}\right) \otimes \Omega_{\mathrm{X}^{\prime} / \mathrm{Y}^{\prime}}, \mathrm{N}^{t o t}\right) \rightarrow\left(\mathscr{A}_{\mathscr{X} / \mathscr{Y} / \mathscr{S}}(\mathrm{E}), \mathrm{N}^{t o t}\right)
$$

We shall deduce Theorem 3.8 from the following result on the level of complexes.

Theorem 3.9. - Suppose that $\mathrm{E}$ is an object of $\mathrm{MICN}(\mathrm{X} / \mathrm{S})$ such that $\mathrm{N}_{p-1} \mathrm{E}=\mathrm{E}$ and $\mathrm{N}_{-1} \mathrm{E}=0$. Then the morphisms $a$ and $b$ above induce filtered quasi-isomorphisms

$$
\begin{aligned}
a:\left(\mathrm{N}_{p-1}^{d e c}\left(\mathscr{A}_{\mathscr{Y} / \mathscr{S}}\left(\mathrm{E}^{\prime}\right) \otimes \Omega_{\mathrm{X}^{\prime} / \mathrm{Y}^{\prime}}^{\cdot}\right), \mathrm{N}^{d e c}\right) & \rightarrow\left(\mathrm{N}_{p-1}^{d e c} \mathscr{A}_{\mathscr{X} / \mathscr{Y} / \mathscr{S}}(\mathrm{E}), \mathrm{N}^{d e c}\right) \\
b: \mathrm{F}_{\mathrm{X} / \mathrm{S} *}\left(\mathrm{~N}_{p-1}^{d e c}\left(\mathscr{A}_{\mathscr{Y} / \mathscr{S}}(\mathrm{E}) \otimes \Omega_{\mathrm{X} / \mathrm{Y}}^{\cdot}\right), \mathrm{N}^{d e c}\right) & \rightarrow\left(\mathrm{N}_{p-1}^{d e c} \mathscr{A}_{\mathscr{X} / \mathscr{Y} / \mathscr{S}}(\mathrm{E}), \mathrm{N}^{d e c}\right) .
\end{aligned}
$$

The map $a$ is compatible with the Gauss-Manin connections and the map $b$ is compatible with the Gauss-Manin Higgs fields defined in (3.2). Moreover, the Gauss-Manin connection annihilates the map

$$
\mathrm{E}^{\prime} \otimes \Omega_{\mathrm{X}^{\prime} / \mathrm{Y}^{\prime}}^{\cdot} \rightarrow \mathscr{A}_{\mathscr{X} / \mathscr{Y} / \mathscr{S}}(\mathrm{E})
$$

and the Gauss-Manin Higgs field annihilates the map

$$
\mathrm{F}_{\mathrm{X} / \mathrm{S} *} \mathrm{E} \otimes \Omega_{\mathrm{X} / \mathrm{Y}}^{\cdot} \rightarrow \mathscr{A}_{\mathscr{X} / \mathscr{Y} / \mathscr{S}}(\mathrm{E})
$$

Proof. - The compatibilities with the Gauss-Manin connections and fields are straightforward. To prove that the maps in the theorem are filtered quasi-isomorphisms, we follow the outline of the proof of Theorem 2.26. In particular, we may work locally on $\mathrm{X}$ and $\mathrm{Y}$, and we may assume that there are compatible Frobenius lifts $\tilde{\mathrm{G}}: \tilde{\mathrm{Y}} \rightarrow \tilde{\mathrm{Y}}^{\prime}$ and $\tilde{\mathrm{F}}: \tilde{\mathrm{X}} \rightarrow \tilde{\mathrm{X}}^{\prime}$. Then we work with the local Cartier transforms, using complexes $\mathscr{A}_{\tilde{\mathrm{F}} / \tilde{\mathrm{G}} / \mathscr{S}}(\mathrm{E}), \mathscr{A}_{\tilde{\mathrm{G}} / \mathscr{S}}(\mathrm{E})$, and $\mathscr{A}_{\tilde{\mathrm{G}} / \mathscr{S}}\left(\mathrm{E}^{\prime}\right)$. It will suffice to show that the maps 
of complexes

$$
\begin{gathered}
a:\left(\mathrm{E}_{1}^{\cdot j}\left(\mathscr{A}_{\tilde{\mathrm{G}} / \mathscr{S}}\left(\mathrm{E}^{\prime}\right), \mathrm{N} .\right), d_{1}\right) \rightarrow\left(\mathrm{E}_{1}^{\cdot j}\left(\mathscr{A}_{\tilde{\mathrm{F}} / \tilde{\mathrm{G}} / \mathscr{S}}(\mathrm{E}), \mathrm{N} .\right), d_{1}\right) \\
b:\left(\mathrm{E}_{1}^{\cdot, j}\left(\mathscr{A}_{\tilde{\mathrm{G}} / \mathrm{S}}(\mathrm{E}), \mathrm{N} .\right), d_{1}\right) \rightarrow\left(\mathrm{E}_{1}^{\cdot j}\left(\mathscr{A}_{\tilde{\mathrm{F}} / \tilde{\mathrm{G}} / \mathscr{S}}(\mathrm{E}), \mathrm{N} .\right), d_{1}\right)
\end{gathered}
$$

are quasi-isomorphisms. As in the proof of Theorem 2.26, we find that these become maps

$$
\begin{aligned}
& \operatorname{Gr} a:\left(\mathrm{GrE}^{\prime} \otimes \Gamma \cdot \Omega_{\mathrm{Y}^{\prime} / \mathrm{S}} \otimes \Omega_{\mathrm{X}^{\prime} / \mathrm{Y}^{\prime}}^{\cdot}, d_{1}\right) \rightarrow \operatorname{Gr}\left(\mathrm{E} \otimes \Gamma \cdot \Omega_{\mathrm{X}^{\prime} / \mathrm{S}} \otimes \Omega_{\mathrm{X}^{\prime} / \mathrm{Y}^{\prime}}^{\cdot}, d_{1}\right) \\
& \operatorname{Gr} b:\left(\mathrm{GrE} \otimes \Gamma \cdot \Omega_{\mathrm{Y}^{\prime} / \mathrm{S}} \otimes \Omega_{\mathrm{X}^{\prime} / \mathrm{Y}^{\prime}}^{\cdot}, d_{1}\right) \rightarrow \operatorname{Gr}\left(\mathrm{E} \otimes \Gamma \cdot \Omega_{\mathrm{X}^{\prime} / \mathrm{S}} \otimes \Omega_{\mathrm{X}^{\prime} / \mathrm{Y}^{\prime}}^{\cdot}, d_{1}\right) .
\end{aligned}
$$

Working locally on $\mathrm{X}$, we may assume that the sequence $0 \rightarrow h^{*} \Omega_{\mathrm{Y} / \mathrm{S}}^{1} \rightarrow \Omega_{\mathrm{X}^{\prime} / \mathrm{S}}^{1} \rightarrow$ $\Omega_{\mathrm{X} / \mathrm{Y}}^{1} \rightarrow 0$ splits. Then we can identify $\Gamma \cdot \Omega_{\mathrm{X}^{\prime} / \mathrm{S}}^{1}$ with the tensor product $h^{*} \Gamma \cdot \Omega_{\mathrm{Y}^{\prime} / \mathrm{S}}^{1} \otimes$ $\Gamma \cdot \Omega_{\mathrm{X}^{\prime} / \mathrm{Y}^{\prime}}^{1}$, and the result follows from the filtered Poincaré lemma, as in Proposition 2.21 .

Proof of Theorem 3.8. - We may assume without loss of generality that $\mathrm{N}_{-1} \mathrm{E}=0$. For each $q$, let $\left(\mathrm{E}_{\mathrm{DR}}^{q}, \mathrm{~N}\right.$. $):=\mathrm{R}^{q} h_{*}^{\mathrm{DR}} \mathrm{E}$ with the filtration induced by the filtration $\mathrm{N}$. of $\mathrm{E} \otimes \Omega_{\mathrm{X} / \mathrm{Y}}^{\circ}$, and let $\left(\mathrm{E}_{\mathrm{HIG}}^{\prime q}, \mathrm{~N}.\right):=\mathrm{R}^{q} h^{\prime}{ }_{*}^{\mathrm{HIG}} \mathrm{E}^{\prime}$ with the filtration induced by $\mathrm{N}^{\prime}{ }^{d e c}$.

Since the pieces of $\mathrm{Gr} \mathscr{A}_{\mathscr{Y} / \mathscr{S}}$ consists of locally free sheaves of finite rank, the projection formula gives filtered isomorphisms

$$
\begin{aligned}
\left(\mathscr{A}_{\mathscr{Y} / \mathscr{S}} \otimes \mathrm{E}_{\mathrm{DR}}^{q}, \mathrm{~N}^{t o t}\right) & \cong\left(\mathrm{R}^{q} h_{*}^{\mathrm{DR}} \mathscr{A}_{\mathscr{Y} / \mathscr{S}}(\mathrm{E}), \mathrm{N} .\right) \\
\left(\mathrm{F}_{\mathrm{Y} / \mathrm{S} *} \mathscr{A}_{\mathscr{Y} / \mathscr{S}} \otimes \mathrm{E}_{\mathrm{HIG}}^{\prime q}, \mathrm{~N}^{t o t}\right) & \cong\left(\mathrm{R}^{q} h_{*}^{\mathrm{HIG}} \mathscr{A}_{\mathscr{Y} / \mathscr{S}}\left(\mathrm{E}^{\prime}\right), \mathrm{N} .\right)
\end{aligned}
$$

where the filtrations on the right are induced by the filtration $\mathrm{N}^{d e c}$. Furthermore, these maps are compatible with the Higgs fields and connections. Theorem 3.9 then gives us an isomorphism

$$
\left(\mathrm{N}_{p-1}^{t o t}\left(\mathscr{A}_{\mathscr{Y} / \mathscr{S}} \otimes \mathrm{E}_{\mathrm{DR}}^{q}\right), \mathrm{N}^{t o t}\right) \cong\left(\mathrm{N}_{p-1}^{t o t}\left(\mathscr{A}_{\mathscr{Y} / \mathscr{S}} \otimes \mathrm{E}_{\mathrm{HIG}}^{q q}\right), \mathrm{N}^{t o t}\right)
$$

compatible with the filtrations, connections, and Higgs fields. Since $\mathrm{E}_{\mathrm{DR}}^{q}$ has level at most $p-1$, its Cartier transform is obtained by taking the horizontal sections of $\mathrm{N}_{p-1} \mathscr{A}_{\mathscr{Y} / \mathscr{S}}\left(\mathrm{E}^{q}\right)$, which by the above isomorphism is $\left(\mathrm{E}_{\mathrm{HIG}}^{q}, \mathrm{~N}.\right)$.

Remark 3.10. - When $\mathrm{Y}=\mathrm{S}$, the categories $\mathrm{MIC}_{\ell}(\mathrm{Y} / \mathrm{S})$ and $\mathrm{HIG}_{\ell}\left(\mathrm{Y}^{\prime} / \mathrm{S}\right)$ reduce to the category of $\mathscr{O}_{\mathrm{S}}$-modules, and Theorem 3.8 above reduces to Theorem 2.26.

\subsection{Derived direct and inverse images}

Let $\mathrm{S}$ be a noetherian scheme of finite Krull dimension, $h: \mathrm{X} \rightarrow \mathrm{Y}$ a morphism of smooth schemes over S. Let $\mathbf{T}_{\mathrm{X}^{\prime} \rightarrow \mathrm{Y}^{\prime}}^{*}$ be the pullback of $\mathbf{T}_{\mathrm{Y}^{\prime} / \mathrm{S}}^{*}$ to $\mathrm{X}^{\prime}$, which fits into 
the following diagram:

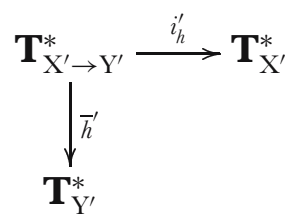

Note that $i_{h}^{\prime}$ is a closed embedding if and only if $h^{\prime}$ is smooth.

Let $\mathrm{HIG}\left(\mathrm{X}^{\prime} \rightarrow \mathrm{Y}^{\prime}\right)$ denote the category of sheaves of $h^{\prime *} \mathrm{~S}^{\cdot} \mathrm{T}_{\mathrm{Y}^{\prime} / \mathrm{S}}$-modules on $\mathrm{X}^{\prime}$.

We define the derived inverse image

$$
\mathrm{L}_{\mathrm{HIG}}^{\prime *}: \mathrm{D}\left(\mathrm{HIG}\left(\mathrm{Y}^{\prime} / \mathrm{S}\right)\right) \rightarrow \mathrm{D}\left(\mathrm{HIG}\left(\mathrm{X}^{\prime} / \mathrm{S}\right)\right)
$$

to be the composition

$$
\mathrm{D}\left(\mathrm{HIG}\left(\mathrm{Y}^{\prime} / \mathrm{S}\right)\right) \stackrel{\mathrm{L} \bar{h}^{*}}{\longrightarrow} \mathrm{D}\left(\mathrm{HIG}\left(\mathrm{X}^{\prime} \rightarrow \mathrm{Y}^{\prime}\right)\right) \stackrel{i_{h *}^{\prime}}{\longrightarrow} \mathrm{D}\left(\mathrm{HIG}\left(\mathrm{X}^{\prime} / \mathrm{S}\right)\right) .
$$

Since $h^{\prime}$ is a morphism between smooth S-schemes, ${\overline{h^{\prime}}}^{*}$ has bounded cohomological dimension, and so takes $\mathrm{D}^{b}\left(\mathrm{HIG}\left(\mathrm{Y}^{\prime} / \mathrm{S}\right)\right)$ to $\mathrm{D}^{b}\left(\mathrm{HIG}\left(\mathrm{X}^{\prime} / \mathrm{S}\right)\right)$.

Similarly, for a smooth morphism $h$, the derived direct image

$$
\mathrm{R} h_{*}^{\prime \mathrm{HIG}}: \mathrm{D}\left(\mathrm{HIG}\left(\mathrm{X}^{\prime} / \mathrm{S}\right)\right) \rightarrow \mathrm{D}\left(\mathrm{HIG}\left(\mathrm{Y}^{\prime} / \mathrm{S}\right)\right)
$$

is the composition

$$
\mathrm{D}\left(\mathrm{HIG}\left(\mathrm{X}^{\prime} / \mathrm{S}\right)\right) \stackrel{\mathrm{R} i{ }_{h}^{\prime}}{\longrightarrow} \mathrm{D}\left(\mathrm{HIG}\left(\mathrm{X}^{\prime} \rightarrow \mathrm{Y}^{\prime}\right)\right) \stackrel{\mathrm{R} \bar{h}_{*}^{\prime}}{\longrightarrow} \mathrm{D}\left(\mathrm{HIG}\left(\mathrm{Y}^{\prime} / \mathrm{S}\right)\right)
$$

where $\mathrm{R} i^{\prime}$ sends a complex $\mathrm{E}$ in $\mathrm{D}\left(\mathrm{HIG}\left(\mathrm{X}^{\prime} / \mathrm{S}\right)\right)$ to

$$
\mathrm{R} i^{\prime !}(\mathrm{E})=\mathrm{R} \mathscr{H} o m_{\mathrm{S}^{\bullet}} \mathrm{T}_{\mathrm{X}^{\prime} / \mathrm{S}}\left(\mathrm{S}^{\bullet} h^{\prime *} \mathrm{~T}_{\mathrm{Y}^{\prime} / \mathrm{S}}, \mathrm{E}\right)
$$

It is again true that this functor takes bounded complexes to bounded complexes. Note that $\mathrm{R} h^{\prime H}$ is is right adjoint to $\mathrm{L} h_{\mathrm{HIG}}^{\prime *}$.

Let us pass to the direct and inverse images of D-modules. Proposition 3.12 below is a reformulation, based on the Azumaya property of the algebra of differential operators in characteristic $p$, of the usual definition of the functors

$$
\begin{aligned}
& \mathrm{L} h_{\mathrm{DR}}^{*}: \mathrm{D}(\mathrm{MIC}(\mathrm{Y} / \mathrm{S})) \rightarrow \mathrm{D}(\mathrm{MIC}(\mathrm{X} / \mathrm{S})) \\
& \mathrm{R} h_{*}^{\mathrm{DR}}: \mathrm{D}(\mathrm{MIC}(\mathrm{X} / \mathrm{S})) \rightarrow \mathrm{D}(\mathrm{MIC}(\mathrm{Y} / \mathrm{S})) .
\end{aligned}
$$

Recall that $\mathscr{D}_{\mathrm{X} / \mathrm{S}}$ is the sheaf of algebras on the cotangent space of $\mathrm{X}^{\prime} / \mathrm{S}$ associated to $\mathrm{F}_{\mathrm{X} / \mathrm{S} *} \mathrm{D}_{\mathrm{X} / \mathrm{S}}$. We first need the following result. 
Theorem $3.11([5])$. - Let $h: \mathrm{X} \rightarrow \mathrm{Y}$ be a morphism of smooth $\mathrm{S}$-schemes. Then the $\mathrm{D}_{\mathrm{X} / \mathrm{S}} \otimes h^{-1} \mathrm{D}_{\mathrm{Y} / \mathrm{S}}^{\text {opmodule }} \mathrm{D}_{\mathrm{X} \rightarrow \mathrm{Y}}:=h^{*} \mathrm{D}_{\mathrm{Y} / \mathrm{S}}$ induces an equivalence of Azumaya algebras on $\mathbf{T}_{\mathrm{X}^{\prime} \rightarrow \mathrm{Y}^{\prime}}^{*}$ :

$$
i_{h}^{* *} \mathscr{D}_{\mathrm{X} / \mathrm{S}} \sim{\overline{h^{\prime}}}^{*} \mathscr{D}_{\mathrm{Y} / \mathrm{S}}
$$

Proof. - To prove the theorem consider $\mathrm{D}_{\mathrm{Y} / \mathrm{S}}$ as a left module over itself. Remark 1.8 shows that the left action of $\mathrm{D}_{\mathrm{X} / \mathrm{S}}$ on $h^{*} \mathrm{D}_{\mathrm{Y} / \mathrm{S}}$ and the right action of $h^{-1} \mathrm{D}_{\mathrm{Y} / \mathrm{S}}$ together define a left action of

$$
\mathrm{F}_{\mathrm{X} / \mathrm{S} *} \mathrm{D}_{\mathrm{X} / \mathrm{S}} \otimes_{\mathrm{S}^{*} \mathrm{~T}_{\mathrm{X}^{\prime} / \mathrm{S}}} h^{\prime *} \mathrm{~F}_{\mathrm{Y} / \mathrm{S} *} \mathrm{D}_{\mathrm{Y} / \mathrm{S}}^{o p},
$$

where $\mathrm{S}^{\cdot} \mathrm{T}_{\mathrm{X}^{\prime} / \mathrm{S}}$ acts on $h^{\prime *} \mathrm{~F}_{\mathrm{Y} / \mathrm{S} *} \mathrm{D}_{\mathrm{Y} / \mathrm{S}}^{o p}$ via $i_{h}^{\prime *}$ and the evident action of $h^{\prime *} \mathrm{~S}^{\cdot} \mathrm{T}_{\mathrm{Y}^{\prime} / \mathrm{S}}$. This

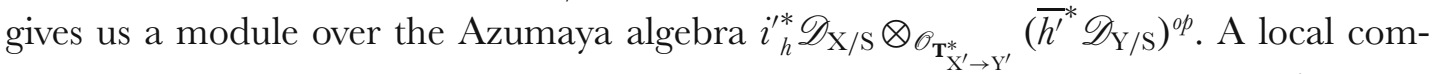
putation shows that this module is locally free over $\mathscr{O}_{\mathbf{T}_{\mathrm{X}^{\prime} \rightarrow \mathrm{Y}^{\prime}}^{*}}$ of rank $\operatorname{ind}\left(i_{h}^{\prime *} \mathscr{D}_{\mathrm{X} / \mathrm{S}}\right)$. ind $\left({\overline{h^{\prime}}}^{*} \mathscr{D}_{\mathrm{Y} / \mathrm{S}}\right)$.

As a corollary, we get an equivalence of categories:

$$
\mathrm{C}_{\mathrm{X}^{\prime} \rightarrow \mathrm{Y}^{\prime}}: \operatorname{Mod}\left(i_{h}^{* *} \mathrm{~F}_{\mathrm{X} / \mathrm{S} *} \mathrm{D}_{\mathrm{X} / \mathrm{S}}\right) \simeq \operatorname{Mod}\left(h^{\prime *} \mathrm{~F}_{\mathrm{Y} / \mathrm{S} *} \mathrm{D}_{\mathrm{Y} / \mathrm{S}}\right),
$$

where $\operatorname{Mod}(\mathscr{A})$ denotes the category of $\mathscr{A}$-modules. Note that, since $\mathrm{F}_{\mathrm{X} / \mathrm{S}}$ is a homeomorphism, the functor

$$
\operatorname{MIC}(\mathrm{X} / \mathrm{S})=\operatorname{Mod}\left(\mathrm{D}_{\mathrm{X} / \mathrm{S}}\right) \stackrel{\mathrm{Fx}_{\mathrm{X} / \mathrm{S} *}}{\longrightarrow} \operatorname{Mod}\left(\mathrm{F}_{\mathrm{X} / \mathrm{S} *} \mathrm{D}_{\mathrm{X} / \mathrm{S}}\right)
$$

is an equivalence of categories. Thus the following result determines $\mathrm{L} h_{\mathrm{DR}}^{*}$ and $\mathrm{R} h_{*}^{\mathrm{DR}}$.

Proposition 3.12 ([5]). - For any morphism $h: \mathrm{X} \rightarrow \mathrm{Y}$ there is a canonical isomorphism:

$$
\mathrm{F}_{\mathrm{X} / \mathrm{S} *} \circ \mathrm{L} h_{\mathrm{DR}}^{*} \cong i_{h *}^{\prime} \circ \mathrm{C}_{\mathrm{X}^{\prime} \rightarrow \mathrm{Y}^{\prime}}^{-1} \circ \mathrm{L}^{\prime *} \circ \mathrm{F}_{\mathrm{Y} / \mathrm{S} *} \cdot
$$

If $h$ is smooth we also have

$$
\mathrm{F}_{\mathrm{Y} / \mathrm{S} *} \circ \mathrm{R} h_{*}^{\mathrm{DR}} \cong \mathrm{R} h_{*}^{\prime} \circ \mathrm{C}_{\mathrm{X}^{\prime} \rightarrow \mathrm{Y}^{\prime}} \circ \mathrm{R} i_{h}^{\prime !} \circ \mathrm{F}_{\mathrm{X} / \mathrm{S} *} .
$$

Proof. — We shall just explain the second formula. By definition, for any $\mathrm{E} \in \mathrm{D}(\mathrm{MIC}(\mathrm{X} / \mathrm{S}))$, we have

$$
\begin{aligned}
& \mathrm{C}_{\mathrm{X}^{\prime} \rightarrow \mathrm{Y}^{\prime}} \circ \mathrm{R} i_{h}^{\prime !}\left(\mathrm{F}_{\mathrm{X} / \mathrm{S} *} \mathrm{E}\right)
\end{aligned}
$$

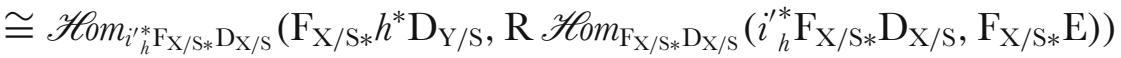

$$
\begin{aligned}
& \cong \mathrm{R} \mathscr{H}_{0} m_{\mathrm{F} / \mathrm{S} *} \mathrm{D}_{\mathrm{X} / \mathrm{S}}\left(\mathrm{F}_{\mathrm{X} / \mathrm{S} *} h^{*} \mathrm{D}_{\mathrm{Y} / \mathrm{S}}, \mathrm{F}_{\mathrm{X} / \mathrm{S} *} \mathrm{E}\right) \text {. }
\end{aligned}
$$

It follows then that

$$
\mathrm{R} h_{*}^{\prime} \circ \mathrm{C}_{\mathrm{X}^{\prime} \rightarrow \mathrm{Y}^{\prime}} \circ \mathrm{R} i_{h}^{\prime}\left(\mathrm{F}_{\mathrm{X} / \mathrm{S} *} \mathrm{E}\right)=\mathrm{F}_{\mathrm{Y} / \mathrm{S} *} \mathrm{R} h_{*}\left(\mathrm{R} \mathscr{H}_{0 m_{\mathrm{DX} / \mathrm{S}}}\left(h^{*} \mathrm{D}_{\mathrm{Y} / \mathrm{S}}, \mathrm{E}\right)\right) .
$$

When $h$ is smooth this is the standard definition of $\mathrm{F}_{\mathrm{Y} / \mathrm{S} *} \circ \mathrm{R} h_{*}^{\mathrm{DR}}$. 
As an application of the new construction of $\mathrm{R} h h_{*}^{\mathrm{DR}}$ let us observe that if $\mathrm{E} \in \mathrm{MIC}(\mathrm{X} / \mathrm{S})$ and the Zariski closure of $\operatorname{supp} \mathrm{F}_{\mathrm{X} / \mathrm{S} *} \mathrm{E} \subset \mathbf{T}_{\mathrm{X}^{\prime}}^{*}$ does not intersect $\mathbf{T}_{\mathrm{X}^{\prime} \rightarrow \mathrm{Y}^{\prime}}^{*} \subset \mathbf{T}_{\mathrm{X}^{\prime}}^{*}$, then $\mathrm{R} h_{*}^{\mathrm{DR}} \mathrm{E}=0$. (This follows also from Proposition 2.31).

3.4 The conjugate filtration on $\mathrm{F}_{\mathrm{X} / \mathrm{S} *} \mathrm{D}_{\mathrm{X} / \mathrm{S}}$

The algebra of differential operators in characteristic $p$, besides the order filtration, has another natural filtration by ideals:

$$
\begin{aligned}
& \cdots \subset \mathscr{I}_{\mathrm{X}}^{i} \subset \cdots \subset \mathscr{I}_{\mathrm{X}}^{1} \subset \mathrm{F}_{\mathrm{X} / \mathrm{S} *} \mathrm{D}_{\mathrm{X} / \mathrm{S}}, \\
& \mathscr{I}_{\mathrm{X}}^{i}=\mathrm{S}^{i} \mathrm{~T}_{\mathrm{X}^{\prime}}\left(\mathrm{F}_{\mathrm{X} / \mathrm{S} *} \mathrm{D}_{\mathrm{X} / \mathrm{S}}\right) .
\end{aligned}
$$

We shall call (3.12.1) the conjugate filtration since, as we will explain in (3.17) below, it induces the conjugate filtration on the de Rham cohomology groups. The associated graded algebra $\operatorname{Gr}\left(\mathrm{F}_{\mathrm{X} / \mathrm{S} *} \mathrm{D}_{\mathrm{X} / \mathrm{S}}\right)$ is a canonically split tensor Azumaya algebra. In this section we shall study a certain filtered derived category of modules over the filtered algebra $\mathrm{F}_{\mathrm{X} / \mathrm{S} *} \mathrm{D}_{\mathrm{X} / \mathrm{S}}$. We will see how the splitting property of $\operatorname{Gr}\left(\mathrm{F}_{\mathrm{X} / \mathrm{S} *} \mathrm{D}_{\mathrm{X} / \mathrm{S}}\right)$ together with some general results in homological algebra lead to generalizations and simple proofs of some of the fundamental results of Katz, including the $p$-curvature formula for the Gauss-Manin connection. Our main application is the functoriality of the Cartier transform with respect to the direct images.

The following construction plays a central role in this subsection.

Definition 3.13. - Let $\mathscr{A}$ be a sheaf of algebras over a scheme $\mathrm{Z}$ and $\mathscr{I} \subset \mathscr{A}$ be a twosided ideal. Denote by $\mathrm{CF}(\mathscr{A}, \mathscr{I})$ the category of (unbounded) filtered complexes of $\mathscr{A}$-modules

$$
\cdots \subset\left(\mathrm{N}^{i+1} \mathrm{E}^{*}, d\right) \subset\left(\mathrm{N}^{i} \mathrm{E}^{*}, d\right) \subset \cdots \subset\left(\mathrm{E}^{*}, d\right),
$$

satisfying the following conditions:

1. $\bigcup_{i \in \mathbf{Z}} \mathrm{N}^{i} \mathrm{E}^{j}=\mathrm{E}^{j}$ and $\bigcap_{i \in \mathbf{Z}} \mathrm{N}^{i} \mathrm{E}^{j}=0$,

2. The filtration $\mathrm{N}^{\cdot}$ on each $\mathrm{E}^{j}$ is an $\mathscr{I}$-filtration, that is:

$$
\mathscr{I} \mathrm{N}^{i} \mathrm{E}^{j} \subseteq \mathrm{N}^{i+1} \mathrm{E}^{j} .
$$

(see also Definition 2.16).

The $\mathscr{I}$-filtered derived category $\operatorname{DF}(\mathscr{A}, \mathscr{I})$ is the Verdier quotient of the homotopy category $\operatorname{Ho}(\mathrm{CF}(\mathscr{A}, \mathscr{I}))$ of $\mathrm{CF}(\mathscr{A}, \mathscr{I})$ by the subcategory $\operatorname{Ho}\left(\mathrm{CF}^{a c}(\mathscr{A}, \mathscr{I})\right)$ of acyclic complexes.

In the context of this definition, a filtered complex is said to be acyclic if for every $i$ the complex $\left(\mathrm{N}^{i} \mathrm{E}^{*}, d\right)$ is acyclic. Recall that by the definition of the Verdier quotient there is a triangulated functor

$$
\mathrm{L}: \operatorname{Ho}(\mathrm{CF}(\mathscr{A}, \mathscr{I})) \rightarrow \operatorname{DF}(\mathscr{A}, \mathscr{I}),
$$


such that $\mathrm{L}\left(\operatorname{Ho}\left(\mathrm{CF}^{a c}(\mathscr{A}, \mathscr{I})\right)\right)=0$. The pair $(\mathrm{DF}(\mathscr{A}, \mathscr{I}), \mathrm{L})$ has the following universal property: for every triangulated category $\mathrm{T}$, the composition with $\mathrm{L}$ induces an equivalence of categories between the full subcategory of triangulated functors $\Phi: \operatorname{Ho}(\mathrm{CF}(\mathscr{A}, \mathscr{I})) \rightarrow \mathrm{T}$, such that $\Phi\left(\operatorname{Ho}\left(\mathrm{CF}^{a c}(\mathscr{A}, \mathscr{I})\right)\right)=0$, and the category of triangulated functors $\Phi^{\prime}: \operatorname{DF}(\mathscr{A}, \mathscr{I}) \rightarrow$ T. Explicitly, $\operatorname{DF}(\mathscr{A}, \mathscr{I})$ can be constructed as the category whose objects are those of $\operatorname{Ho}(\mathrm{CF}(\mathscr{A}, \mathscr{I}))$ and morphisms $\operatorname{Hom}_{\mathrm{DF}(\mathscr{A}, \mathscr{A})}(\mathrm{X}, \mathrm{Y})$ are represented by diagrams

$$
\mathrm{X} \stackrel{\alpha}{\longrightarrow} \mathrm{Y}^{\prime} \stackrel{s}{\longleftarrow} \mathrm{Y}
$$

where $\alpha$ and $s$ are morphisms in $\operatorname{Ho}(\mathrm{CF}(\mathscr{A}, \mathscr{I}))$ and cones $\in \operatorname{Ho}\left(\mathrm{CF}^{a c}(\mathscr{A}, \mathscr{I})\right)$. We refer the reader to [25] for a detailed discussion. In the case when $\mathscr{I}=0$, the filtered derived category $\operatorname{DF}(\mathscr{A}):=\operatorname{DF}(\mathscr{A}, 0)$ was first considered by Illusie in his thesis [15].

Given a filtered complex $\mathrm{E}^{*}$, we denote by $\mathrm{E}^{*}(r)$ the same complex but with the shifted filtration: $\mathrm{N}^{i}\left(\mathrm{E}^{*}(r)\right)=\mathrm{N}^{i+r} \mathrm{E}^{*}$. Let $\mathrm{CF}_{\leq l}(\mathscr{A}, \mathscr{I})$ be the full subcategory of $\mathrm{CF}(\mathscr{A}, \mathscr{I})$ whose objects are filtered complexes with $\mathrm{N}^{l+1} \mathrm{E}^{\cdot}=0$, let $\mathrm{CF}_{\geq k}(\mathscr{A}, \mathscr{I})$ be the full subcategory whose objects satisfy $\mathrm{N}^{k} \mathrm{E}^{\cdot}=\mathrm{E}$, and let be $\mathrm{CF}_{[k, l]}(\mathscr{A}, \mathscr{I})$ the intersection of $\mathrm{CF}_{\leq l}(\mathscr{A}, \mathscr{I})$ and $\mathrm{CF}_{\geq k}(\mathscr{A}, \mathscr{I})$. Denote by $\mathrm{DF}_{\leq l}(\mathscr{A}, \mathscr{I}), \mathrm{DF}_{\geq k}(\mathscr{A}, \mathscr{I})$, and $\mathrm{DF}_{[k, l]}(\mathscr{A}, \mathscr{I})$ the quotients of the corresponding homotopy categories.

Lemma 3.14. - The functor $c_{\geq k}: \mathrm{DF}_{\geq k}(\mathscr{A}, \mathscr{I}) \rightarrow \operatorname{DF}(\mathscr{A}, \mathscr{I})$ has a right adjoint functor

$$
w_{\geq k}: \operatorname{DF}(\mathscr{A}, \mathscr{I}) \rightarrow \operatorname{DF}_{\geq k}(\mathscr{A}, \mathscr{I}) \quad: \quad w_{\geq k}\left(\mathrm{E}^{\cdot}\right)=\mathrm{N}^{k} \mathrm{E}^{\cdot}
$$

The functor $c_{\leq l}: \mathrm{DF}_{\leq l}(\mathscr{A}, \mathscr{I}) \rightarrow \operatorname{DF}(\mathscr{A}, \mathscr{I})$ has a left adjoint functor

$$
w_{\leq l}: \operatorname{DF}(\mathscr{A}, \mathscr{I}) \rightarrow \mathrm{DF}_{\leq l}(\mathscr{A}, \mathscr{I}) \quad: \quad w_{\leq l}\left(\mathrm{E}^{*}\right)=\mathrm{E}^{*} / \mathrm{N}^{l+1} \mathrm{E}^{*}
$$

Moreover, $w_{\geq k} c_{\geq k} \simeq \mathrm{Id}, w_{\leq l} c_{\leq l} \simeq \mathrm{Id}$.

The proof is straightforward.

Corollary 3.15. - The functors $c_{\geq k}, c_{\leq l}$ and $c_{[k, l]}: \operatorname{DF}_{[k, l]}(\mathscr{A}, \mathscr{I}) \rightarrow \operatorname{DF}(\mathscr{A}, \mathscr{I})$ are fully faithful. The essential image of $c_{\geq k}$ consists of those objects $\left(\mathrm{E}^{*}, \mathrm{~N}^{*} \mathrm{E}^{*}\right)$ such that each $\mathrm{N}^{j} \mathrm{E}^{\cdot} \rightarrow \mathrm{E}^{\cdot}$ is a quasi-isomorphism for all $j \leq k$, and the essential image of $c_{\leq l}$ consists of those objects such that $\mathrm{N}^{j} \mathrm{E}^{\cdot}$ is acyclic for all $j>l$.

Proof. — Indeed, for $\mathrm{E}^{*}, \mathrm{E}^{\prime} \in \mathrm{DF}_{\geq k}(\mathscr{A}, \mathscr{I})$ we have

$$
\begin{aligned}
& \operatorname{Hom}_{\mathrm{DC}_{\geq k}(\mathscr{A}, \mathscr{I})}\left(\mathrm{E}^{\cdot}, \mathrm{E}^{\prime \cdot}\right) \simeq \operatorname{Hom}_{\mathrm{DF}_{\geq k}(\mathscr{A}, \mathscr{I})}\left(\mathrm{E}^{\cdot}, w_{\geq k} c_{\geq k} \mathrm{E}^{\prime *}\right) \\
& \simeq \operatorname{Hom}_{\mathrm{DF}(\mathscr{A}, \mathscr{A})}\left(c_{\geq k} \mathrm{E}^{\cdot}, c_{\geq k} \mathrm{E}^{\prime \cdot}\right),
\end{aligned}
$$


where the first isomorphism is induced by $w_{\geq k} c_{\geq k} \simeq \mathrm{Id}$ and the second one comes from the adjointness property from the lemma. The proofs for $c_{\leq l}$ and $c_{[k, l]}$ are similar. If $\left(\mathrm{E}^{*}, \mathrm{~N}^{*} \mathrm{E}^{*}\right)$ is an object of $\operatorname{DF}(\mathrm{A}, \mathscr{I})$ and each $\mathrm{N}^{j} \mathrm{E}^{*} \rightarrow \mathrm{E}^{*}$ is a quasi-isomorphism for all $j \leq k$, then the natural map $c_{\geq k} w_{\geq k}\left(\mathrm{E}^{*}, \mathrm{~N}^{*} \mathrm{E}^{*}\right) \rightarrow\left(\mathrm{E}^{*}, \mathrm{~N}^{*} \mathrm{E}^{*}\right)$ is an isomorphism in $\operatorname{DF}(\mathrm{A}, \mathscr{I})$, so that $\left(\mathrm{E}^{*}, \mathrm{~N}^{*} \mathrm{E}^{*}\right)$ is in the essential image of $c_{\geq k}$. The proof for $c_{\leq l}$ is similar.

Let $p: \mathbf{V} \rightarrow \mathrm{Z}$ be a vector bundle over a scheme $\mathrm{Z}, \mathrm{V}$ the corresponding sheaf of sections (thus, $\mathrm{V}$ is a locally free sheaf of $\mathscr{O}_{\mathrm{Z}}$-modules), and let $\mathscr{A}$ be a flat sheaf of algebras over $p_{*} \mathscr{O}_{\mathbf{v}} \cong \mathrm{S}^{*} \mathrm{~V}^{*}$. Let $\mathscr{I}$ be the sheaf of ideals in $\mathscr{A}$ generated by $\mathrm{V}^{*}$. Denote by $\operatorname{Gr} \mathscr{A}=\bigoplus_{j \geq 0} \mathscr{I}^{j} / \mathscr{I}^{j+1}$ the sheaf of graded algebras over $\mathrm{S}^{*} \mathrm{~V}^{*}$. Since $\mathscr{A}$ is flat over $\mathrm{S}^{\cdot} \mathrm{V}^{*}$ the morphism:

$$
\mathrm{S}^{*} \mathrm{~V}^{*} \otimes_{\mathscr{O}_{\mathrm{Z}}} \mathscr{A} / \mathscr{I} \rightarrow \operatorname{Gr} \mathscr{A}, f \otimes a \longrightarrow f a
$$

is an isomorphism and $\operatorname{Gr} \mathscr{A}$ is a flat $\mathrm{S}^{*} \mathrm{~V}^{*}$-module. Denote by $\mathrm{D}(\operatorname{Mod}(\operatorname{Gr} \mathscr{A}))$ the derived category of graded $\operatorname{Gr} \mathscr{A}$-modules. We then have a functor:

$$
\begin{aligned}
\operatorname{Gr}: \operatorname{DF}(\mathscr{A}, \mathscr{I}) & \rightarrow \mathrm{D}\left(\operatorname{Mod}^{\bullet}(\operatorname{Gr} \mathscr{A})\right) \\
\left(\mathrm{E}^{*}\right) & \mapsto \bigoplus_{-\infty<j<+\infty} \mathrm{N}^{j} \mathrm{E}^{\cdot} / \mathrm{N}^{j+1} \mathrm{E}^{\cdot} .
\end{aligned}
$$

Let $q: \mathbf{W} \rightarrow \mathrm{Z}$ be another vector bundle over $\mathrm{Z}$ and $i: \mathbf{W} \hookrightarrow \mathbf{V}$ a linear embedding. Set $\mathscr{B}=\mathscr{A} \otimes_{\mathrm{S}^{*} \mathrm{~V}^{*}} \mathrm{~S}^{*} \mathrm{~W}^{*}$ and $\mathscr{I}^{\prime}=\mathrm{W}^{*} \mathscr{B} \subset \mathscr{B}$. Then $\mathscr{B}$ is a sheaf of algebras over $\mathrm{S}^{*} \mathrm{~W}^{*}$ and $\mathscr{I}^{\prime} \subset \mathscr{B}$ is a subsheaf of ideals.

Proposition 3.16. - Assume that $\mathrm{Z}$ is a noetherian scheme of finite Krull dimension.

1. The functor $i_{*}: \operatorname{DF}\left(\mathscr{B}, \mathscr{I}^{\prime}\right) \rightarrow \operatorname{DF}(\mathscr{A}, \mathscr{I})$ has a right adjoint

$$
\mathrm{R} i ! \mathrm{DF}(\mathscr{A}, \mathscr{I}) \rightarrow \operatorname{DF}\left(\mathscr{B}, \mathscr{I}^{\prime}\right)
$$

and the functor $i_{*}: \mathrm{D}\left(\operatorname{Mod}^{\bullet}(\operatorname{Gr} \mathscr{B})\right) \rightarrow \mathrm{D}\left(\operatorname{Mod}^{\bullet}(\operatorname{Gr} \mathscr{A})\right)$ has a right adjoint:

$$
\mathrm{R} i^{!}: \mathrm{D}\left(\operatorname{Mod}^{\bullet}(\operatorname{Gr}, \mathscr{A})\right) \rightarrow \mathrm{D}(\operatorname{Mod}(\operatorname{Gr} \mathscr{B})) .
$$

2. The functor $\mathrm{R} i$ takes the essential image of $\mathrm{DF}_{\leq l}(\mathscr{A}, \mathscr{I})$ into the essential image of $\mathrm{DF}_{\leq l}\left(\mathscr{B}, \mathscr{I}^{\prime}\right)$ and the essential image of $\mathrm{DF}_{\geq k}(\mathscr{A}, \mathscr{I})$ into the essential image of $\mathrm{DF}_{\geq k-d}\left(\mathscr{B}, \mathscr{I}^{\prime}\right)$, where $d:=r k \mathbf{V}-r k \mathbf{W}$.

3. For every $\mathscr{I}$-filtered $\mathscr{A}$ complex $\mathrm{E}^{*}$, the morphism $\mathrm{Gr} \mathrm{R} i \mathrm{E}^{*} \rightarrow \mathrm{R} i \mathrm{Gr}^{*}$ defined by adjunction:

$$
\begin{aligned}
\operatorname{Id} \in \operatorname{Hom}\left(\mathrm{E}^{*}, \mathrm{E}^{*}\right) & \rightarrow \operatorname{Hom}\left(\operatorname{Gr} i_{*} \operatorname{R} i^{!} \mathrm{E}^{*}, \operatorname{Gr} \mathrm{E}^{*}\right) \\
& \rightarrow \operatorname{Hom}\left(\operatorname{Gr} \operatorname{R} i^{\prime} \mathrm{E}^{*}, \operatorname{R} i^{!} \operatorname{Gr} \mathrm{E}^{*}\right)
\end{aligned}
$$

is an isomorphism. 
4. $\mathrm{R} i$ ' commutes with the forgetful functors

$$
\Psi: \mathrm{DF}(\mathscr{A}, \mathscr{I}) \rightarrow \mathrm{D}(\operatorname{Mod}(\mathscr{A})) \text { and } \Psi^{\prime}: \mathrm{DF}\left(\mathscr{B}, \mathscr{I}^{\prime}\right) \rightarrow \mathrm{D}(\operatorname{Mod}(\mathscr{B})),
$$

i.e. the canonical morphism $\Psi^{\prime} \mathrm{R} i^{\prime} \mathrm{E}^{*} \rightarrow \mathrm{R} i^{\prime} \Psi \mathrm{E}^{*}$, defined by adjunction, is an isomorphism.

Proof. - For (1) we use the technique from [24]. The Brown representability theorem (loc.cit., Theorem 4.1.) asserts that the existence of the adjoint functor $\mathrm{R} i ! \mathrm{DF}(\mathscr{A}, \mathscr{I}) \rightarrow \operatorname{DF}\left(\mathscr{B}, \mathscr{I}^{\prime}\right)$ would follow if we prove that

1. the categories $\operatorname{DF}(\mathscr{A}, \mathscr{I})$ and $\operatorname{DF}\left(\mathscr{B}, \mathscr{I}^{\prime}\right)$ have arbitrary direct sums

2. the functor $i_{*}$ commutes with arbitrary direct sums

3. the category $\operatorname{DF}\left(\mathscr{B}, \mathscr{I}^{\prime}\right)$ is compactly generated ${ }^{9}$.

The first two properties are immediate. Let us check the third. Given an open subset $j: \mathrm{U} \hookrightarrow \mathrm{Z}$, denote by $\mathscr{B}_{\mathrm{U}}$ the filtered $\mathscr{B}$-module such that $\mathrm{N}^{i} \mathscr{B}_{\mathrm{U}}=j ! \mathscr{B}$ for $i \leq 0$ and $\mathrm{N}^{i} \mathscr{B}_{\mathrm{U}}=j_{!}\left(\mathrm{S}^{i} \mathrm{~W}^{*} \mathscr{B}\right)$ for $i>0$. For any $\mathrm{E} \cdot \in \mathrm{DF}\left(\mathscr{B}, \mathscr{I}^{\prime}\right)$, one has

$$
\operatorname{Hom}_{\mathrm{DF}(\mathscr{B}, \mathscr{J})}\left(\mathscr{B}_{\mathrm{U}}(l), \mathrm{E}^{\cdot}[j]\right) \simeq \mathrm{R}^{j} \Gamma\left(\mathrm{U}, \mathrm{N}^{-l} \mathrm{E}^{*}\right) .
$$

It follows that $\operatorname{DF}(\mathscr{B}, \mathscr{J})$ is generated by objects of the form $\mathscr{B}_{\mathrm{U}}(l)$. It is known that for any noetherian space $\mathrm{U}$ of finite Krull dimension the functor $\mathrm{R} \Gamma(\mathrm{U}$,$) commutes$ with arbitrary direct sums (see, for example [38]). Thus the objects $\mathscr{B}_{\mathrm{U}}(l)$ are compact.

The second claim in (1) is proven by a similar argument.

For $(2)$, let $\mathrm{E}^{*} \in \mathrm{DF}_{\leq l}(\mathscr{A}, \mathscr{I})$. We want to show that $w_{\geq l+l} \mathrm{R} i^{!}\left(c_{\leq l} \mathrm{E}^{*}\right)=0$. Indeed, for every $\mathrm{E}^{\prime} \in \mathrm{DF}_{\geq l+1}\left(\mathscr{B}, \mathscr{I}^{\prime}\right)$ we have

$$
\begin{aligned}
\operatorname{Hom}\left(\mathrm{E}^{\prime}, w_{\geq l+1} \operatorname{R} i^{\prime}\left(c_{\leq l} \mathrm{E}^{*}\right)\right) & \simeq \operatorname{Hom}\left(c_{\geq l+1} \mathrm{E}^{\prime}, \operatorname{R} i^{\prime}\left(c_{\leq l} \mathrm{E}^{*}\right)\right) \\
& \simeq \operatorname{Hom}\left(w_{\leq l} i_{*}\left(c_{\geq l+l} \mathrm{E}^{\prime *}\right), \mathrm{E}^{*}\right) \\
& \simeq \operatorname{Hom}\left(w_{\leq l} c_{\geq l+l} i_{*}\left(\mathrm{E}^{\prime}\right), \mathrm{E}^{\cdot}\right) \\
& =0 .
\end{aligned}
$$

To prove the second statement consider the forgetful functor

$$
\Phi: \operatorname{DF}\left(\mathscr{B}, \mathscr{I}^{\prime}\right) \rightarrow \operatorname{DF}\left(\mathscr{O}_{\mathrm{Z}}\right):=\operatorname{DF}\left(\mathscr{O}_{\mathrm{Z}}, 0\right)
$$

to the filtered derived category of $\mathscr{O}_{\mathrm{Z}}$-modules. By Corollary 3.15, we will be done if we show that $\Phi \mathrm{R} i^{!}\left(\mathrm{E}^{*}\right) \in \mathrm{DF}_{\geq k-d}\left(\mathscr{O}_{\mathrm{Z}}\right)$ for every object $\mathrm{E}^{\cdot}$ of $\mathrm{DF}_{\geq k}(\mathscr{A}, \mathscr{I})$. Consider

\footnotetext{
${ }^{9}$ Recall that an object $\mathrm{X} \in \mathrm{DF}\left(\mathscr{B}, \mathscr{I}^{\prime}\right)$ is called compact if for every set of objects $\left\{\mathrm{Y}_{\alpha}\right\}$ one has $\bigoplus \operatorname{Hom}\left(\mathrm{X}, \mathrm{Y}_{\alpha}\right) \simeq \operatorname{Hom}\left(\mathrm{X}, \oplus \mathrm{Y}_{\alpha}\right)$. A category is said to be compactly generated if there exists a set $\mathrm{T}$ of compact objects such that for every nonzero $\mathrm{Y} \in \mathrm{DF}\left(\mathscr{B}, \mathscr{I}^{\prime}\right)$ there exists $\mathrm{X} \in \mathrm{T}$ such that $\operatorname{Hom}(\mathrm{X}, \mathrm{Y}) \neq 0$.
} 
the Koszul complex

$$
0 \rightarrow \Lambda^{d} \mathrm{~T} \otimes_{\mathscr{O}_{\mathrm{Z}}} \mathscr{A}(-d) \rightarrow \cdots \rightarrow \mathrm{T} \otimes_{\mathscr{O}_{\mathrm{Z}}} \mathscr{A}(-1) \rightarrow \mathscr{A} \rightarrow i_{*} i^{*} \mathscr{A} \rightarrow 0
$$

where $\mathrm{T}:=\operatorname{ker}\left(\mathrm{V}^{*} \stackrel{i^{*}}{\rightarrow} \mathrm{W}^{*}\right)$, and where the $\mathscr{I}$-filtration on $\Lambda^{m} \mathrm{~T} \otimes_{\mathscr{O}_{\mathrm{Z}}} \mathscr{A}(-m)$ is defined by

$$
\mathrm{N}^{i}\left(\Lambda^{m} \mathrm{~T} \otimes_{\mathscr{O}_{\mathrm{Z}}} \mathscr{A}(-m)\right)=\Lambda^{m} \mathrm{~T} \otimes_{\mathscr{O}_{\mathrm{Z}}} \mathscr{I}^{i-m}
$$

Then (3.16.1) is an acyclic complex in $\mathrm{CF}(\mathscr{A}, \mathscr{I})$. It yields a functorial isomorphism

$$
\Phi \operatorname{R} i^{!}\left(\mathrm{E}^{*}\right) \simeq \mathscr{H}_{0 m_{\mathscr{A}}}\left(\Lambda^{\cdot} \mathrm{T} \otimes_{\mathscr{O}_{\mathrm{Z}}} \mathscr{A}(-\cdot), \mathrm{E}^{*}\right)
$$

This is the filtered complex $\mathrm{C}^{\cdot}$ whose term in degree $i$ is

$$
\mathrm{C}^{i}:=\bigoplus_{p+q=i} \Lambda^{p} \mathrm{~T}^{*} \otimes_{\mathscr{O}_{\mathrm{Z}}} \mathrm{E}^{q}(p) .
$$

Since $\mathrm{E}^{q}(p) \in \operatorname{DF}_{\geq k-p}(\mathscr{A}, \mathscr{I})$ and $\mathrm{T}^{*}$ has rank $d$, this completes the proof.

For the last two statements, it will be enough to prove that $\operatorname{Gr} \mathrm{R}^{2} \mathrm{E}^{*} \rightarrow \operatorname{R} i ! \mathrm{GrE}$ : (resp. $\Psi \mathrm{R} i i^{\prime} \mathrm{E}^{*} \rightarrow \mathrm{R} i^{!} \Psi \mathrm{E}^{*}$ ) becomes an isomorphism after the projection to the derived category of graded $\mathscr{O}_{\mathrm{Z}}$-modules (resp. the derived category of $\mathscr{O}_{\mathrm{Z}}$-modules). In turn, this follows from the Koszul computation in (2).

Let $h: \mathrm{X} \rightarrow \mathrm{Y}$ be a smooth morphism of relative dimension $d$ of smooth schemes over a noetherian scheme $\mathrm{S}$ of finite Krull dimension. We shall apply the above construction to the linear morphism

$$
\mathbf{T}_{\mathrm{X}^{\prime} \rightarrow \mathrm{Y}^{\prime}}^{*} \stackrel{i_{h}^{\prime}}{\longrightarrow} \mathbf{T}_{\mathrm{X}^{\prime}}^{*}
$$

and to $\mathscr{A} \supset \mathscr{I}$ being either $\mathrm{S}^{\cdot} \mathrm{T}_{\mathrm{X}^{\prime}} \supset \mathscr{J}_{\mathrm{X}^{\prime}}:=\bigoplus_{i>0} \mathrm{~S}^{i} \mathrm{~T}_{\mathrm{X}^{\prime}}$ or the Azumaya algebra $\mathrm{F}_{\mathrm{X} / \mathrm{S} *} \mathrm{D}_{\mathrm{X} / \mathrm{S}} \supset \mathscr{I}_{\mathrm{X}}:=\mathrm{T}_{\mathrm{X}^{\prime}}\left(\mathrm{F}_{\mathrm{X} / \mathrm{S} *} \mathrm{D}_{\mathrm{X} / \mathrm{S}}\right)$. We then have the filtered derived image functors

$$
\begin{aligned}
& \mathrm{R} h_{*}^{\prime \mathrm{HIG}}=\mathrm{R} h_{*}^{\prime} \circ \mathrm{R} i_{h}^{\prime !}: \operatorname{DF}\left(\mathrm{S}^{\bullet} \mathrm{T}_{\mathrm{X}^{\prime}}, \mathscr{J}_{\mathrm{X}^{\prime}}\right) \rightarrow \operatorname{DF}\left(\mathrm{S}^{\circ} \mathrm{T}_{\mathrm{Y}^{\prime}}, \mathscr{J}_{\mathrm{Y}^{\prime}}\right) \\
& \mathrm{R} h_{*}^{\mathrm{DR}}=\mathrm{R} h_{*}^{\prime} \circ \mathrm{C}_{\mathrm{X}^{\prime} \rightarrow \mathrm{Y}^{\prime}} \circ \mathrm{R} i_{h}^{\prime !}: \operatorname{DF}\left(\mathrm{F}_{\mathrm{X} / \mathrm{S} *} \mathrm{D}_{\mathrm{X} / \mathrm{S}}, \mathscr{I}_{\mathrm{X}}\right) \rightarrow \operatorname{DF}\left(\mathrm{F}_{\mathrm{Y} / \mathrm{S} *} \mathrm{D}_{\mathrm{Y} / \mathrm{S}}, \mathscr{I}_{\mathrm{Y}}\right)
\end{aligned}
$$

and by the previous proposition

$$
\begin{aligned}
\mathrm{R} h_{*}^{\prime \mathrm{HIG}}: \mathrm{DF}_{[k, l]}\left(\mathrm{S}^{*} \mathrm{~T}_{\mathrm{X}^{\prime}}, \mathscr{J}_{\mathrm{X}^{\prime}}\right) & \rightarrow \mathrm{DF}_{[k-d, l]}\left(\mathrm{S}^{*} \mathrm{~T}_{\mathrm{Y}^{\prime}}, \mathscr{J}_{\mathrm{Y}^{\prime}}\right) \\
\mathrm{R} h_{*}^{\mathrm{DR}}: \mathrm{DF}_{[k, l]}\left(\mathrm{F}_{\mathrm{X} / \mathrm{S} *} \mathrm{D}_{\mathrm{X} / \mathrm{S}}, \mathscr{I}_{\mathrm{X}}\right) & \rightarrow \mathrm{DF}_{[k-d, l]}\left(\mathrm{F}_{\mathrm{Y} / \mathrm{S} *} \mathrm{D}_{\mathrm{Y} / \mathrm{S}}, \mathscr{I}_{\mathrm{Y}}\right) .
\end{aligned}
$$

In particular, this gives another proof of Corollary 3.5. 
Example 3.17. - Consider the $\mathrm{D}_{\mathrm{X} / \mathrm{S}}$-module $\mathscr{O}_{\mathrm{X}} \in \mathrm{DF}\left(\mathrm{F}_{\mathrm{X} / \mathrm{S} *} \mathrm{D}_{\mathrm{X} / \mathrm{S}}, \mathscr{I}_{\mathrm{X}}\right)$ endowed with the trivial filtration. Then the filtration on

$$
\mathrm{R} h_{*}^{\mathrm{DR}} \mathscr{O}_{\mathrm{X}} \in \mathrm{DF}_{[-d, 0]}\left(\mathrm{F}_{\mathrm{Y} / \mathrm{S} *} \mathrm{D}_{\mathrm{Y} / \mathrm{S}}, \mathscr{I}_{\mathrm{Y}}\right)
$$

coincides with the "conjugate" filtration. Indeed, we will construct a canonical quasiisomorphism in the filtered derived category $\mathrm{DF}\left(i_{h}^{\prime *}\left(\mathrm{~F}_{\mathrm{X} / \mathrm{S} *} \mathrm{D}_{\mathrm{X} / \mathrm{S}}\right)\right)$ :

$$
\left(\mathrm{R} i i_{h}^{\prime !}\left(\mathrm{F}_{\mathrm{X} / \mathrm{S} *} \mathscr{O}_{\mathrm{X}}\right), \mathrm{N}^{*}\right) \simeq\left(\mathrm{R} i_{h}^{\prime !}\left(\mathrm{F}_{\mathrm{X} / \mathrm{S} *} \mathscr{O}_{\mathrm{X}}\right), \mathrm{T}^{*}\right),
$$

where for any complex $\mathrm{C}$;

$$
\mathrm{T}^{i} \mathrm{C}^{q}:= \begin{cases}\mathrm{C}^{q} & \text { if } q \leq-i \\ \operatorname{Im}\left(d^{q}\right) & \text { if } q=-i+1 \\ 0 & \text { if } q>-i+1 .\end{cases}
$$

That is, $\mathrm{T}^{i} \mathrm{C}^{\cdot}=\tau_{\leq-i} \mathrm{C}^{\cdot}$, where $\tau_{\leq}$is the canonical filtration. Note that by (3.16.2),

$$
\mathrm{Gr}^{-m} \mathrm{R}_{h}^{\prime !}\left(\mathrm{F}_{\mathrm{X} / \mathrm{S} *} \mathscr{O}_{\mathrm{X}}\right) \simeq \Omega_{\mathrm{X}^{\prime} / \mathrm{Y}^{\prime}}^{m} \otimes \mathrm{F}_{\mathrm{X} / \mathrm{S} *} \mathscr{O}_{\mathrm{X}}[-m]
$$

Thus the result follows from the following lemma, whose proof is straightforward.

Lemma 3.18. - Let $\left(\mathrm{E}^{*}, \mathrm{~N}^{*}\right)$ be a filtered complex in an abelian category. Assume that the filtration is finite and that for every $m$

$$
\mathrm{H}^{i}\left(\mathrm{Gr}^{-m} \mathrm{E}^{*}\right)=0, \text { for every } i \neq m \text {. }
$$

For each $i$, let $\mathrm{T}_{\mathrm{N}}^{i} \mathrm{E}^{\cdot}:=\mathrm{T}^{i} \mathrm{~N}^{i} \mathrm{E}^{\cdot} \subseteq \mathrm{N}^{i} \mathrm{E}^{\cdot}$. Then the morphisms

$$
\left(\mathrm{E}^{*}, \mathrm{~N}^{*}\right) \leftarrow\left(\mathrm{E}^{*}, \mathrm{~T}_{\mathrm{N}}^{*}\right) \rightarrow\left(\mathrm{E}^{*}, \mathrm{~T}^{*}\right)
$$

are filtered quasi-isomorphisms.

Observe that the graded Azumaya algebra

$$
\mathrm{Gr} \mathrm{F}_{\mathrm{X} / \mathrm{S} *} \mathrm{D}_{\mathrm{X} / \mathrm{S}} \simeq\left(\mathrm{F}_{\mathrm{X} / \mathrm{S} *} \mathrm{D}_{\mathrm{X} / \mathrm{S}} / \mathscr{I}_{\mathrm{X}}\right) \otimes_{\mathscr{O}_{\mathrm{X}^{\prime}}} \mathrm{S}^{\cdot} \mathrm{T}_{\mathrm{X}^{\prime}}
$$

over $\mathrm{S}^{\cdot} \mathrm{T}_{\mathrm{X}^{\prime}}$ splits canonically: $\mathrm{F}_{\mathrm{X} / \mathrm{S} *} \mathscr{O}_{\mathrm{X}} \otimes_{\mathscr{O}_{\mathrm{X}^{\prime}}} \mathrm{S}^{\cdot} \mathrm{T}_{\mathrm{X}^{\prime}}$ is the graded splitting module. This defines an equivalence of categories:

$$
\begin{aligned}
& \mathrm{C}_{\mathrm{X} / \mathrm{S}}^{\bullet-1}: \mathrm{D}\left(\mathrm{HIG}^{\bullet}\left(\mathrm{X}^{\prime} / \mathrm{S}\right)\right) \rightarrow \mathrm{D}\left(\operatorname{Mod}^{\bullet}\left(\mathrm{Gr} \mathrm{F}_{\mathrm{X} / \mathrm{S} *} \mathrm{D}_{\mathrm{X} / \mathrm{S}}\right)\right) \\
& \mathrm{C}_{\mathrm{X} / \mathrm{S}}^{\bullet-1}\left(\mathrm{E}^{\bullet}\right)=\mathrm{E}^{\bullet} \otimes_{\mathrm{S}^{*} \mathrm{~T}_{\mathrm{X}^{\prime} / \mathrm{S}}}\left(\mathrm{F}_{\mathrm{X} / \mathrm{S} *} \mathscr{O}_{\mathrm{X}} \otimes \mathrm{S}^{\cdot} \mathrm{T}_{\mathrm{X}^{\prime} / \mathrm{S}}\right) \\
& \cong \mathrm{E}^{\cdot} \otimes_{\mathscr{O}_{\mathrm{X}^{\prime}}} \mathrm{F}_{\mathrm{X} / \mathrm{S} *} \mathscr{O}_{\mathrm{X}}
\end{aligned}
$$


Observe that $\mathrm{C}_{\mathrm{X} / \mathrm{S}}^{\cdot-1}$ and its quasi-inverse $\mathrm{C}_{\mathrm{X} / \mathrm{S}}^{\cdot}$ commute with $\mathrm{R} i_{h}^{\prime !}$. By Part (3) of Proposition 3.16 we have a functorial isomorphism

$$
\mathrm{C}_{\mathrm{X} / \mathrm{S}} \operatorname{Gr} \operatorname{R}_{h}^{\prime !}\left(\mathrm{E}^{*}\right) \simeq \mathrm{R}_{h}^{\prime !}\left(\mathrm{C}_{\mathrm{X} / \mathrm{S}}^{\cdot} \mathrm{GrE}^{*}\right)
$$

and its direct image to $\mathrm{Y}^{\prime}$

$$
\mathrm{C}_{\mathrm{Y} / \mathrm{S}} \operatorname{Gr} \mathrm{R} h_{*}^{\mathrm{DR}}\left(\mathrm{E}^{*}\right) \simeq \mathrm{R}_{*}^{\prime \mathrm{HIG}}\left(\mathrm{C}_{\mathrm{X} / \mathrm{S}}^{\cdot} \operatorname{GrE} \mathrm{E}^{*}\right)
$$

Let $\mathrm{E}^{\cdot}$ be an object of $\mathrm{DF}_{[k, l]}\left(\mathrm{F}_{\mathrm{X} / \mathrm{S} *} \mathrm{D}_{\mathrm{X} / \mathrm{S}}, \mathscr{I}_{\mathrm{X}}\right)$. Then the filtered complex $\mathrm{R} h_{*}^{\mathrm{DR}}\left(\mathrm{E}^{*}\right)$ yields a spectral sequence:

$$
\mathrm{E}_{1}^{p, q}=\mathrm{H}^{p+q}\left(\operatorname{Gr}^{p} \mathrm{R}_{*}^{\mathrm{DR}}\left(\mathrm{E}^{*}\right)\right) \Rightarrow \mathrm{H}^{*}\left(\mathrm{R} h_{*}^{\mathrm{DR}}\left(\mathrm{E}^{*}\right)\right)
$$

We shall call it the conjugate spectral sequence ${ }^{10}$ (c.f. Example 3.17).

Assume that the conjugate spectral sequence degenerates at $\mathrm{E}_{1}$. Then the quasiisomorphism (3.18.2) induces an isomorphism of graded Higgs modules:

$$
\mathrm{C}_{\mathrm{Y} / \mathrm{S}} \operatorname{GrR}^{j} h_{*}^{\mathrm{DR}}\left(\mathrm{E}^{\cdot}\right) \simeq \mathrm{R}^{j} h_{*}^{\prime \mathrm{HIG}}\left(\mathrm{C}_{\mathrm{X} / \mathrm{S}}^{\cdot} \mathrm{Gr} \mathrm{E}^{\cdot}\right) .
$$

Remark 3.19. - Let us explain how formulas (3.18.2) and (3.18.3) can be viewed as generalizations of Katz's formula [19, Theorem 3.2] relating $p$-curvature and the Kodaira-Spencer mapping. Recall from [20] that the complex $\mathrm{M}^{\cdot}=\mathrm{R} h^{\prime}{ }_{*}^{\mathrm{DR}}\left(\mathscr{O}_{\mathrm{X}^{\prime}}\right)$ of $\mathrm{D}_{\mathrm{Y}^{\prime} / \mathrm{S}}$-modules has another natural filtration

$$
\cdots \subset \mathrm{F}^{i} \mathrm{M}^{\cdot} \subset \mathrm{F}^{i-1} \mathrm{M}^{\cdot} \subset \cdots \subset \mathrm{M}^{\cdot}
$$

where $\mathrm{F}^{j} \mathrm{M}^{k} \subset \mathrm{M}^{k}$ are $\mathscr{O}_{\mathrm{Y}^{\prime}}$-submodules satisfying Griffiths transversality:

$$
\mathrm{T}_{\mathrm{Y}^{\prime} / \mathrm{S}}\left(\mathrm{F}^{j} \mathrm{M}^{k}\right) \subset \mathrm{F}^{j-1} \mathrm{M}^{k}
$$

The last property makes $\mathrm{Gr}_{\mathrm{F}} \mathrm{M}^{k}$ a Higgs module on $\mathrm{Y}^{\prime}$ and there is canonical quasiisomorphism

$$
\operatorname{Gr}_{\mathrm{F}} \mathrm{R} h_{*}^{\prime \mathrm{DR}}\left(\mathscr{O}_{\mathrm{X}^{\prime}}\right) \simeq \mathrm{R} h_{*}^{\prime \mathrm{HIG}}\left(\mathscr{O}_{\mathrm{X}^{\prime}}\right)
$$

in the derived category of graded Higgs modules [20, Construction 5.6.1]. If the morphism $h^{\prime}$ is smooth and proper, the filtration $\mathrm{F}^{*}$ induces the Hodge filtration on

${ }^{10}$ Let us note that, when $\mathrm{E}=\mathscr{O}_{\mathrm{X}}$ the $\mathrm{E}_{r}$-terms of our spectral sequence correspond to the $\mathrm{E}_{r+1}$ terms of the usual conjugate spectral sequence, after a suitable renumbering. 
$\mathrm{R} h^{\prime \mathrm{DR}}\left(\mathscr{O}_{\mathrm{X}^{\prime}}\right)$ and the spectral sequence associated to $\mathrm{F}^{*}$ is the Hodge spectral sequence. Combining Laumon's formula (3.19.1) with (3.18.2) ( for $\mathrm{E}^{*}=\mathscr{O}_{\mathrm{X}}$ ) we obtain a canonical quasi-isomorphism

$$
\mathrm{C}_{\mathrm{Y} / \mathrm{S}}^{\cdot} \operatorname{Gr}_{\mathrm{N}} \mathrm{R} h_{*}^{\mathrm{DR}}\left(\mathscr{O}_{\mathrm{X}}\right) \simeq \operatorname{Gr}_{\mathrm{F}} \operatorname{R}^{\prime \mathrm{DR}}\left(\mathscr{O}_{\mathrm{X}^{\prime}}\right)
$$

in $\mathrm{D}\left(\mathrm{HIG}^{\bullet}\left(\mathrm{Y}^{\prime} / \mathrm{S}\right)\right)$.

Assume that $h$ is smooth, proper, and that the Hodge spectral sequence and the conjugate spectral sequence for the de Rham direct image of $\mathscr{O}_{\mathrm{X}}$ degenerate at $\mathrm{E}_{1}$. Then (3.19.2) yields an isomorphism of Higgs modules

$$
\mathrm{C}_{\mathrm{Y} / \mathrm{S}} \operatorname{Gr}_{\mathrm{N}} \mathrm{R}^{j} h_{*}^{\mathrm{DR}}\left(\mathscr{O}_{\mathrm{X}}\right) \simeq\left(\operatorname{Gr}_{\mathrm{F}} \mathrm{R}^{j} h_{*}^{\prime \mathrm{DR}}\left(\mathscr{O}_{\mathrm{X}^{\prime}}\right), \kappa\right),
$$

where $\mathrm{Gr}_{\mathrm{F}}$ denotes the associated graded object with respect to the Hodge filtration on $\mathrm{R}^{j} h_{*}^{\prime \mathrm{DR}}\left(\mathscr{O}_{\mathrm{X}^{\prime}}\right)$ and $\kappa$ is the Kodaira-Spencer operator viewed as a Higgs field on $\operatorname{Gr}_{\mathrm{F}} \mathrm{R} h_{*}^{\mathrm{DR}}\left(\mathscr{O}_{\mathrm{X}^{\prime}}\right)$. This is Katz's $p$-curvature formula ${ }^{11}$. See Example 3.17 for an explication of the left side which relates it to Katz's original formulation. We refer the reader to Section 4.6 (Formula (4.16.2)) for a generalization of this remark.

Remark 3.20. - Example 3.17 can be generalized as follows. Let $\mathscr{A}$ be sheaf of algebras flat over $\mathrm{S}^{*} \mathrm{~V}^{*}$ and $i: \mathbf{W} \hookrightarrow \mathbf{V}$ a linear embedding. Consider the functors

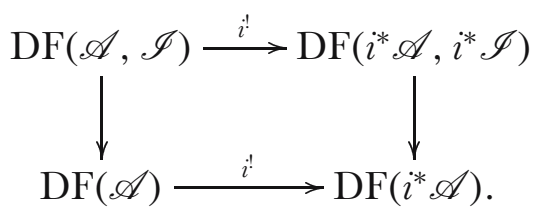

This diagram is not commutative. However, we will show that for every $\mathscr{A}$-module $\mathrm{E}$ with a finite $\mathscr{I}$-filtration $\left(\mathrm{E}=\mathrm{N}^{0} \mathrm{E} \supset \cdots \supset \mathrm{N}^{n} \mathrm{E} \supset \mathrm{N}^{n+1} \mathrm{E}=0\right)$ the $\mathscr{I}$-filtration $\left(\mathrm{R} i ! \mathrm{E}=\mathrm{N}^{-d} \mathrm{R} i ! \mathrm{E} \supset \cdots \supset \mathrm{N}^{n} \mathrm{R} i ! \mathrm{E} \supset \mathrm{N}^{n+1} \mathrm{R} i ! \mathrm{E}=0\right)$ is the filtration décalée of $(\mathrm{R} i ! \mathrm{E}=$ $\left.\mathrm{R} i{ }^{\prime} \mathrm{N}^{0} \mathrm{E} \supset \cdots \supset \mathrm{R} i^{!} \mathrm{N}^{n} \mathrm{E} \supset \mathrm{R} i^{!} \mathrm{N}^{n+1} \mathrm{E}=0\right)$. To see this we, first, recall an interpretation of the filtration décalée convenient for our purposes.

Let $\operatorname{DF}(\mathscr{C})$ be the filtered derived category of an abelian category $\mathscr{C}$, and let $\mathrm{DF}^{\leq k}(\mathscr{C}) \subset \mathrm{DF}(\mathscr{C})$ (resp. $\mathrm{DF}^{\geq k}(\mathscr{C}) \subset \mathrm{DF}(\mathscr{C})$ ) be the full subcategory whose objects are filtered complexes $\left(\mathrm{E}^{*}, \mathrm{~F}^{*} \mathrm{E}^{*}\right)$ such that, for every integer $n, \mathrm{Gr}^{n} \mathrm{E}^{*}$ has vanishing cohomology in degrees greater than $n+k$ (resp. less then $n+k)$. It is known [1, Appendix], that the subcategories $\operatorname{DF}^{\leq k}(\mathscr{C})$ and $\operatorname{DF}^{\geq k}(\mathscr{C})$ define a $t$-structure on $\operatorname{DF}(\mathscr{C})$ whose heart is the abelian category of complexes $\mathrm{C}(\mathscr{C})$. In particular, the embedding

\footnotetext{
${ }^{11}$ In loc.cit. Katz considers also the $\log$ version of his formula. We shall not do so here.
} 
$\mathrm{DF}^{\leq k}(\mathscr{C}) \rightarrow \mathrm{DF}(\mathscr{C})$ has a right adjoint functor

$$
\tau_{\leq k}: \mathrm{DF}(\mathscr{C}) \rightarrow \mathrm{DF}^{\leq k}(\mathscr{C})
$$

Explicitly,

$$
\mathrm{F}^{m}\left(\tau_{\leq k}\left(\mathrm{E}^{\cdot}, \mathrm{F}^{\cdot} \mathrm{E}^{\cdot}\right)\right)^{i}=\mathrm{F}^{m+i-k} \mathrm{E}^{i}+d\left(\mathrm{~F}^{m+i-k-1} \mathrm{E}^{i-1}\right),
$$

if $i>k$ and

$$
\mathrm{F}^{m}\left(\tau_{\leq k}\left(\mathrm{E}^{\cdot}, \mathrm{F}^{\cdot} \mathrm{E}^{\cdot}\right)\right)^{i}=\mathrm{F}^{m} \mathrm{E}^{i}
$$

otherwise.

The canonical filtration

$$
\cdots \subset \tau_{\leq k}\left(\mathrm{E}^{*}, \mathrm{~F}^{*} \mathrm{E}^{\cdot}\right) \subset \cdots \subset\left(\mathrm{E}^{*}, \mathrm{~F}^{*} \mathrm{E}^{*}\right) .
$$

makes $\mathrm{E}^{*}$ a bifiltered complex. We shall denote this bifiltered complex by

$$
\left(\mathrm{E}^{*}, \mathrm{~F}^{*} \mathrm{E}^{*}\right)^{d e c}:=\left(\mathrm{E}^{*}, \mathrm{~N}^{*} \mathrm{~F}^{*} \mathrm{E}^{*}\right)
$$

so that $\left(\mathrm{E}^{*}, \mathrm{~N}^{-k} \mathrm{~F}^{*} \mathrm{E}^{*}\right)=\tau_{\leq k}\left(\mathrm{E}^{*}, \mathrm{~F}^{*} \mathrm{E}^{*}\right)$. We then have the following generalization of Lemma 3.18.

Lemma 3.21. - Let $\left(\mathrm{E}^{*}, \mathrm{~N}^{*} \mathrm{~F}^{*} \mathrm{E}^{*}\right)$ be a bifiltered complex. Assume that the filtration $\mathrm{N}$ is finite, i.e. there exist integers $a$ and $b$ such that $\mathrm{N}^{a} \mathrm{~F}^{*} \mathrm{E}^{*}=0$ and $\mathrm{N}^{b} \mathrm{~F}^{*} \mathrm{E}^{\cdot}=\mathrm{N}^{b-i} \mathrm{~F}^{*} \mathrm{E}^{*}$ for every $i \geq 0$. Set $\mathrm{F}^{*} \mathrm{E}^{\cdot}:=\mathrm{N}^{b} \mathrm{~F}^{*} \mathrm{E}^{\cdot}$. Assume also that, for every $m$,

$$
\operatorname{Gr}_{\mathrm{N}}^{-m}\left(\mathrm{E}^{\cdot}, \mathrm{N}^{*} \mathrm{~F}^{*} \mathrm{E}^{\cdot}\right) \in \mathrm{DF}^{\leq m}(\mathscr{C}) \cap \mathrm{DF}^{\geq m}(\mathscr{C}),
$$

i.e. $\mathrm{H}^{j}\left(\mathrm{Gr}_{\mathrm{F}}^{k} \operatorname{Gr}_{\mathrm{N}}^{-m}\left(\mathrm{E}^{*}, \mathrm{~N}^{*} \mathrm{~F}^{*} \mathrm{E}^{*}\right)\right)=0$, for every $j \neq k+m$. Then the canonical morphism

$$
\left(\mathrm{E}^{*}, \mathrm{~N}^{*} \mathrm{~F}^{*} \mathrm{E}^{*}\right) \rightarrow\left(\mathrm{E}^{*}, \mathrm{~F}^{*} \mathrm{E}^{*}\right)^{d e c}
$$

defined as in Example 3.17 is a bifiltered quasi-isomorphism.

We omit the proof.

We apply the lemma to the bifiltered complex $\left(\mathrm{R} i ! \mathrm{E}, \mathrm{N}^{*} \mathrm{~F}^{*} \mathrm{R} i ! \mathrm{E}\right)$, where $\mathrm{N}^{k} \mathrm{~F}^{m} \mathrm{R} \imath^{!} \mathrm{E}=\mathrm{N}^{k} \mathrm{R} i^{!} c_{\geq m} w_{\geq m} \mathrm{E}^{12}$. By (3.16.2),

$$
\mathrm{Gr}_{\mathrm{F}}^{k} \mathrm{Gr}_{\mathrm{N}}^{-m} \mathrm{R} i ! \mathrm{E} \simeq \wedge^{k+m} \mathrm{~T}^{*} \otimes_{\mathscr{O}_{\mathrm{Z}}} \mathrm{Gr}^{k} \mathrm{E}[-k-m]
$$

\footnotetext{
${ }^{12}$ Precisely, $\left(\mathrm{R} \imath^{\prime} \mathrm{E}, \mathrm{N}^{*} \mathrm{~F}^{*} \mathrm{R} i^{!} \mathrm{E}\right)$ is defined as $\mathrm{R} i^{!}\left(\mathrm{E}, \mathrm{N}^{*} \mathrm{~F}^{*} \mathrm{E}\right), \mathrm{N}^{k} \mathrm{~F}^{m} \mathrm{E}=\mathrm{N}^{\max (k, m)} \mathrm{E}$ in the bifiltered derived category of $\mathscr{A}$-modules $\left(\mathrm{E}^{*}, \mathrm{~N}^{*} \mathrm{~F}^{*} \mathrm{E}\right)$ such that $\mathscr{I} \mathrm{N}^{k} \mathrm{~F}^{m} \mathrm{E}^{*} \subset \mathrm{N}^{k+1} \mathrm{~F}^{m} \mathrm{E}^{*}$.
} 
Thus we get a canonical bifiltered quasi-isomorphism

$$
\left(\mathrm{R} i ! \mathrm{E}, \mathrm{N}^{*} \mathrm{~F}^{*} \mathrm{R} i ! \mathrm{E}\right) \simeq\left(\mathrm{R} i ! \mathrm{E}, \mathrm{F}^{*} \mathrm{R} i ! \mathrm{E}\right)^{d e c}
$$

\subsection{The derived Cartier transform}

Let $\mathscr{X} / \mathrm{S}$ be a lifting. For any $k$ and $l$ with $l-k<p$, the Cartier transform yields equivalences of categories

$$
\begin{aligned}
& \mathrm{DF}_{[k, l]}\left(\mathrm{F}_{\mathrm{X} / \mathrm{S} *} \mathrm{D}_{\mathrm{X} / \mathrm{S}}, \mathscr{I}_{\mathrm{X}}\right) \stackrel{\mathrm{C}_{\mathscr{X} / \mathrm{S}}}{\longrightarrow} \mathrm{DF}_{[k, l]}\left(\mathrm{S}^{\cdot} \mathrm{T}_{\mathrm{X}^{\prime}}, \mathscr{J}_{\mathrm{X}^{\prime}}\right) \\
& \mathrm{C}_{\mathscr{X} / \mathscr{S}}\left(\mathrm{E}^{\cdot}, \mathrm{N}^{*} \mathrm{E}^{*}\right)=\left(\mathrm{C}_{\mathscr{X} / \mathrm{S}} \mathrm{E}^{*}, \mathrm{C}_{\mathscr{X} / \mathrm{S}} \mathrm{N}^{*} \mathrm{E}^{*}\right) .
\end{aligned}
$$

Theorem 3.22. - a) Let $h: \mathrm{X} \rightarrow \mathrm{Y}$ be a morphism of smooth schemes over $\mathrm{S}$. Then, for any integers $k$ and $l$ with $l-k<p$, a lifting $\tilde{h}^{\prime}: \tilde{\mathrm{X}}^{\prime} \rightarrow \tilde{\mathrm{Y}}^{\prime}$ of $h^{\prime}$ induces an isomorphism:

$$
\begin{aligned}
\mathrm{L}_{\mathrm{DR}^{*}}^{*} \circ \mathrm{C}_{\mathscr{Y} / \mathscr{S}}^{-1} \cong & \mathrm{C}_{\mathscr{X} / \mathscr{S}}^{-1} \circ \mathrm{L} h_{\mathrm{HIG}}^{* *}: \\
& \mathrm{DF}_{[k, l]}\left(\mathrm{S}^{\cdot} \mathrm{T}_{\mathrm{Y}^{\prime}}, \mathscr{J}_{\mathrm{Y}^{\prime}}\right) \rightarrow \mathrm{DF}_{[k, l]}\left(\mathrm{F}_{\mathrm{X} / \mathrm{S} *} \mathrm{D}_{\mathrm{X} / \mathrm{S}}, \mathscr{I}_{\mathrm{X}}\right) .
\end{aligned}
$$

b) If in addition $h$ is smooth of relative dimension $d$ and $l-k-d<p$, then

$$
\begin{aligned}
\mathrm{R} h_{*}^{\mathrm{DR}} \circ \mathrm{C}_{\mathscr{X} / \mathscr{S}}^{-1} & \cong \mathrm{C}_{\mathscr{Y} / \mathscr{S}}^{-1} \circ \mathrm{R}_{*}^{\prime}{ }^{\mathrm{HIG}}: \\
& \mathrm{DF}_{[k, l]}\left(\mathrm{S}^{\circ} \mathrm{T}_{\mathrm{X}^{\prime}}, \mathscr{J}_{\mathrm{X}^{\prime}}\right) \rightarrow \mathrm{DF}_{[k-d, l]}\left(\mathrm{F}_{\mathrm{Y} / \mathrm{S} *} \mathrm{D}_{\mathrm{Y} / \mathrm{S}}, \mathscr{I}_{\mathrm{Y}}\right) .
\end{aligned}
$$

Proof. - a) Define an equivalence of categories

$$
\mathrm{DF}_{[k, l]}\left(i_{h}^{* *}\left(\mathrm{~S}^{\cdot} \mathrm{T}_{\mathrm{X}^{\prime}}\right), \mathscr{J}_{\mathrm{X}^{\prime}}^{\prime}\right) \stackrel{\left(\mathrm{C}_{\mathscr{X} / \mathscr{S}}^{\mathrm{Y}}\right)^{-1}}{\longrightarrow} \mathrm{DF}_{[k, l]}\left(i_{h}^{* *}\left(\mathrm{~F}_{\mathrm{X} / \mathrm{S} *} \mathrm{D}_{\mathrm{X} / \mathrm{S}}\right), \mathscr{I}_{\mathrm{X}}^{\prime}\right)
$$

to be the composition

$$
\left(\mathrm{C}_{\mathscr{X} / \mathscr{S}}^{\mathrm{Y}}\right)^{-1}:=\mathscr{M}_{\mathscr{X} / \mathscr{S}} \circ \iota_{*}
$$

where $\iota_{*}: \mathrm{DF}_{[k, l]}\left(i_{h}^{* *}\left(\mathrm{~S}^{\bullet} \mathrm{T}_{\mathrm{X}^{\prime}}\right), \mathscr{J}_{\mathrm{X}^{\prime}}^{\prime}\right) \rightarrow \mathrm{DF}_{[k, l]}\left(i_{h}^{* *}\left(\mathrm{~S}^{\bullet} \mathrm{T}_{\mathrm{X}^{\prime}}\right), \mathscr{J}_{\mathrm{X}^{\prime}}^{\prime}\right)$ is the involution defined in (2.7.2) and $\mathscr{M}_{\mathscr{X} / \mathrm{S}}$ is the tensor product with the splitting module $\mathrm{F}_{\mathrm{X} / \mathrm{S} *} \mathscr{B}_{\mathscr{X} / \mathscr{S}}$ :

$$
\mathscr{M}_{\mathscr{X} / \mathrm{S}}\left(\mathrm{E}^{*}, \mathrm{~N}^{*} \mathrm{E}^{*}\right)=\left(\mathrm{E}^{*} \otimes_{\hat{\Gamma} \mathrm{T}_{\mathrm{X}^{\prime} / \mathrm{S}}} \mathrm{F}_{\mathrm{X} / \mathrm{S} *} \mathscr{B}_{\mathscr{X} / \mathscr{S}}, \mathrm{N}^{*} \mathrm{E}^{*} \otimes_{\hat{\Gamma} \mathrm{T}_{\mathrm{X}^{\prime} / \mathrm{S}}} \mathrm{F}_{\mathrm{X} / \mathrm{S} *} \mathscr{B}_{\mathscr{X} / \mathscr{S}}\right)
$$

Similarly, the splitting module $h^{\prime *} \mathrm{~F}_{\mathrm{Y} / \mathrm{S} *} \mathscr{B} \mathscr{Y} / \mathscr{S}$ yields an equivalence of categories

$$
\mathrm{DF}_{[k, l]}\left(h^{\prime *}\left(\mathrm{~S}^{*} \mathrm{~T}_{\mathrm{Y}^{\prime}}\right), h^{\prime *} \mathscr{J}_{\mathrm{Y}^{\prime}}\right) \stackrel{\left(\mathrm{C}_{\mathscr{Y} / \mathscr{S}}^{\mathrm{X}}\right)^{-1}}{\longrightarrow} \mathrm{DF}_{[k, l]}\left(h^{\prime *}\left(\mathrm{~F}_{\mathrm{Y} / \mathrm{S} *} \mathrm{D}_{\mathrm{Y} / \mathrm{S}}\right), h^{\prime *} \mathscr{I}_{\mathrm{Y}}\right) .
$$


Lemma 3.23. - A morphism $\left(h, \tilde{h}^{\prime}\right): \mathscr{X} / \mathscr{S} \rightarrow \mathscr{Y} / \mathscr{S}$ induces an isomorphism of functors

$$
\begin{aligned}
& \mathrm{C}_{\mathscr{X} / \mathscr{S}}^{\mathrm{Y}} \simeq \mathrm{C}_{\mathscr{Y} / \mathscr{S}}^{\mathrm{X}} \circ \mathrm{C}_{\mathrm{X}^{\prime} \rightarrow \mathrm{Y}^{\prime}} \\
& \mathrm{DF}_{[k, l]}\left(i_{h}^{* *}\left(\mathrm{~F}_{\mathrm{X} / \mathrm{S} *} \mathrm{D}_{\mathrm{X} / \mathrm{S}}\right), \mathscr{I}_{\mathrm{X}}^{\prime}\right) \stackrel{\mathrm{C}_{\mathrm{X}^{\prime} \rightarrow \mathrm{Y}^{\prime}}}{\longrightarrow} \mathrm{DF}_{[k, l]}\left(h^{\prime *}\left(\mathrm{~F}_{\mathrm{X} / \mathrm{S} *} \mathrm{D}_{\mathrm{Y} / \mathrm{S}}\right), h^{\prime *} \mathscr{I}_{\mathrm{Y}}\right) \\
& \quad \mathrm{C}_{\mathscr{X} / \mathscr{S}}^{\mathrm{Y}} \downarrow \\
& \mathrm{DF}_{[k, l]}\left(i_{h}^{* *}\left(\mathrm{~S}^{\cdot} \mathrm{T}_{\mathrm{X}^{\prime} / \mathscr{S}}\right), \mathscr{J}_{\mathrm{X}^{\prime}}^{\prime}\right) \longrightarrow \mathrm{DF}_{[k, l]}\left(h^{\prime *}\left(\mathrm{~S}^{*} \mathrm{~T}_{\mathrm{Y}^{\prime}}\right), h^{\prime *} \mathscr{J}_{\mathrm{Y}^{\prime}}\right) .
\end{aligned}
$$

Proof. - Recall from Proposition 1.12 that a morphism $\left(h, \tilde{h}^{\prime}\right): \mathscr{X} / \mathscr{S} \rightarrow \mathscr{Y} / \mathscr{S}$ induces an isomorphism

$$
h^{*} \mathscr{A}_{\mathscr{Y} / \mathscr{S}} \stackrel{\cong}{\longrightarrow} \mathscr{H}_{0} m_{\mathrm{F}_{\mathrm{X} / \mathrm{S}}^{*} \Gamma^{*} \mathrm{~T}_{\mathrm{X}^{\prime} / \mathrm{S}}}\left(h^{*} \mathrm{~F}_{\mathrm{Y} / \mathrm{S}}^{*} \Gamma^{\cdot} \mathrm{T}_{\mathrm{Y}^{\prime} / \mathrm{S}}, \mathscr{A}_{\mathscr{X} / \mathscr{S}}\right) .
$$

Dualizing this isomorphism, we find an isomorphism of $\mathrm{D}_{\mathrm{X} / \mathrm{S}}-$ modules

$$
\mathrm{F}_{\mathrm{Y} / \mathrm{S}}^{*} h^{\prime *} \hat{\Gamma} \mathrm{T}_{\mathrm{Y}^{\prime} / \mathrm{S}} \otimes_{\mathrm{F}_{\mathrm{X} / \mathrm{S}}^{*} \hat{\Gamma} \mathrm{T}_{\mathrm{X}^{\prime} / \mathrm{S}}} \mathscr{B} \mathscr{X} / \mathscr{S} \cong h^{*} \mathscr{B} \mathscr{Y} / \mathscr{S} \text {. }
$$

With the notations of Theorem 3.11, we have

$$
\mathrm{F}_{\mathrm{X} / \mathrm{S} *} h^{*} \mathscr{B}_{\mathscr{Y} / \mathscr{S}} \simeq \mathrm{F}_{\mathrm{X} / \mathrm{S} *} \mathrm{D}_{\mathrm{X} \rightarrow \mathrm{Y}} \otimes_{h^{\prime *} \mathrm{~F}_{\mathrm{Y} / \mathrm{S} *} \mathrm{D}_{\mathrm{Y} / \mathrm{S}}} h^{*} \mathrm{~F}_{\mathrm{Y} / \mathrm{S} *} \mathscr{B}_{\mathscr{Y} / \mathscr{S}}
$$

Thus we get an isomorphism of splitting modules for $h^{\prime *} \hat{\Gamma}^{\top} \mathrm{T}_{\mathrm{Y}^{\prime} / \mathrm{S}} \otimes_{\mathrm{S}^{*}} \mathrm{~T}_{\mathrm{X}^{\prime} / \mathrm{S}} \mathrm{F}_{\mathrm{X} / \mathrm{S} *} \mathrm{D}_{\mathrm{X} / \mathrm{S}}$ :

$$
h^{\prime *} \hat{\Gamma} \mathrm{T}_{\mathrm{Y}^{\prime} / \mathrm{S}} \otimes_{\hat{\Gamma}_{\mathrm{X}^{\prime} / \mathrm{S}}} \mathrm{F}_{\mathrm{X} / \mathrm{S} *} \mathscr{B} \mathscr{X} / \mathscr{S} \cong \mathrm{F}_{\mathrm{X} / \mathrm{S} *} \mathrm{D}_{\mathrm{X} \rightarrow \mathrm{Y}} \otimes_{h^{\prime *} \mathrm{~F} / \mathrm{S} * \mathrm{D}_{\mathrm{Y} / \mathrm{S}}} h^{\prime *} \mathrm{~F}_{\mathrm{Y} / \mathrm{S} *} \mathscr{B} \mathscr{\mathscr { Y } / \mathscr { S }}
$$

By definition, the functor $\left(\mathrm{C}_{\mathscr{X} / \mathscr{S}}^{\mathrm{Y}}\right)^{-1}$ is the composition of the involution $\iota_{*}$ and the tensor product over $h^{*} \hat{\Gamma} \mathrm{T}_{\mathrm{Y}^{\prime} / \mathrm{S}}$ with the left-hand side of (3.23.1), and the functor $\left(\mathrm{C}_{\mathscr{Y} / \mathscr{S}}^{\mathrm{X}} \circ \mathrm{C}_{\mathrm{X}^{\prime} \rightarrow \mathrm{Y}^{\prime}}\right)^{-1}$ is the composition of $\iota_{*}$ and the tensor product with the righthand side of (3.23.1). Thus, (3.23.1) induces the desired isomorphism $\left(\mathrm{C}_{\mathscr{X} / \mathscr{S}}^{\mathrm{Y}}\right)^{-1} \simeq$ $\left(\mathrm{C}_{\mathscr{Y} / \mathscr{S}}^{\mathrm{X}} \circ \mathrm{C}_{\mathrm{X}^{\prime} \rightarrow \mathrm{Y}^{\prime}}\right)^{-1}$.

Let us return to the proof of the theorem. Observe the natural isomorphisms of functors:

$$
\begin{aligned}
\mathrm{L}^{\prime *} \circ\left(\mathrm{C}_{\mathscr{Y} / \mathscr{S}}\right)^{-1} & \simeq\left(\mathrm{C}_{\mathscr{Y} / \mathscr{S}}^{\mathrm{X}}\right)^{-1} \circ \mathrm{L} h^{*} \quad \text { and } \\
i_{h *}^{\prime} \circ\left(\mathrm{C}_{\mathscr{X} / \mathscr{S}}^{\mathrm{Y}}\right)^{-1} & \simeq\left(\mathrm{C}_{\mathscr{X} / \mathscr{S}}\right)^{-1} \circ i_{h *}^{\prime} .
\end{aligned}
$$

Hence, by Lemma 3.23

$$
\begin{aligned}
\mathrm{L}_{\mathrm{DR}}^{*} \circ \mathrm{C}_{\mathscr{Y} / \mathscr{S}}^{-1} & \simeq i_{h *}^{\prime} \circ\left(\mathrm{C}_{\mathrm{X}^{\prime} \rightarrow \mathrm{Y}^{\prime}}\right)^{-1} \circ \mathrm{L}^{\prime *} \circ \mathrm{C}_{\mathscr{Y} / \mathscr{S}}^{-1} \\
& \simeq i_{h *}^{\prime} \circ\left(\mathrm{C}_{\mathrm{X}^{\prime} \rightarrow \mathrm{Y}^{\prime}}\right)^{-1} \circ\left(\mathrm{C}_{\mathscr{Y} / \mathscr{S}}^{\mathrm{X}}\right)^{-1} \circ \mathrm{L} h^{\prime *} \\
& \simeq i_{h *}^{\prime} \circ\left(\mathrm{C}_{\mathscr{X} / \mathscr{S}}^{\mathrm{Y}}\right)^{-1} \circ \mathrm{L}^{*} \simeq \mathrm{C}_{\mathscr{X} / \mathscr{S}}^{-1} \circ \mathrm{L}_{\mathrm{HIG}}^{*} .
\end{aligned}
$$

This proves part a) of the theorem. 
b) By Lemma 3.23 it remains to construct an isomorphism of functors:

$$
\begin{aligned}
\mathrm{R} i_{h}^{\prime !} \mathrm{C}_{\mathscr{X} / \mathscr{S}} \cong & \mathrm{C}_{\mathscr{X} / \mathscr{S}}^{\mathrm{Y}} \mathrm{R} i_{h}^{\prime !}: \\
& \mathrm{DF}_{[k, l]}\left(\mathrm{F}_{\mathrm{X} / \mathrm{S} *} \mathrm{D}_{\mathrm{X} / \mathrm{S}}, \mathscr{I}_{\mathrm{X}}\right) \rightarrow \mathrm{DF}_{[k-d, l]}\left(i_{h}^{\prime *}\left(\mathrm{~S}^{\cdot} \mathrm{T}_{\mathrm{X}^{\prime}}\right), \mathscr{J}_{\mathrm{X}^{\prime}}^{\prime}\right) .
\end{aligned}
$$

Let $\mathrm{E}^{\cdot} \in \mathrm{DF}_{[k, l]}\left(\mathrm{F}_{\mathrm{X} / \mathrm{S} *} \mathrm{D}_{\mathrm{X} / \mathrm{S}}, \mathscr{I}_{\mathrm{X}}\right)$ and $\mathrm{E}^{\prime} \in \mathrm{DF}_{[k-d, l]}\left(i_{h}^{* *}\left(\mathrm{~S}^{\cdot} \mathrm{T}_{\mathrm{X}^{\prime}}\right), \mathscr{J}_{\mathrm{X}^{\prime}}^{\prime}\right)$. We then have functorial isomorphisms

$$
\begin{aligned}
\operatorname{Hom}\left(\mathrm{E}^{\prime}, \mathrm{R}_{h}^{\prime !} \mathrm{G}_{\mathscr{X} / \mathscr{S}}\left(\mathrm{E}^{*}\right)\right) & \simeq \operatorname{Hom}\left(\mathrm{C}_{\mathscr{X} / \mathscr{S}}^{-1} i_{h *}^{\prime} \mathrm{E}^{\prime \cdot}, \mathrm{E}^{\cdot}\right) \\
& \simeq \operatorname{Hom}\left(i_{h *}^{\prime}\left(\mathrm{C}_{\mathscr{X} / \mathscr{S}}^{\mathrm{Y}}\right)^{-1} \mathrm{E}^{\prime}, \mathrm{E}^{\cdot}\right) \\
& \simeq \operatorname{Hom}\left(\mathrm{E}^{\prime}, \mathrm{C}_{\mathscr{X} / \mathscr{S}}^{\mathrm{Y}} \mathrm{R}_{h}^{\prime !}\left(\mathrm{E}^{\cdot}\right)\right) .
\end{aligned}
$$

By the Yoneda lemma this yields (3.23.2).

Remark 3.24. - In the absence of the lifting of $h^{\prime}$ the theorem can be modified as follows. Let $\mathscr{L}_{h^{\prime}}$ be the $h^{\prime *} \mathrm{~T}_{\mathrm{Y}^{\prime} / \mathrm{S}}$-torsor of liftings of $h^{\prime}$ and let $\exp \mathscr{L}_{h^{\prime}}$ be the pushforward of $\mathscr{L}_{h^{\prime}}$ via the homomorphism

$$
\exp : h^{\prime *} \mathrm{~T}_{\mathrm{Y}^{\prime}} \rightarrow\left(h^{\prime *} \hat{\Gamma} \mathrm{T}_{\mathrm{Y}^{\prime}}\right)^{*} \text {. }
$$

Thus $\exp \mathscr{L}_{h^{\prime}}$ is an $\left(h^{\prime *} \hat{\Gamma} \mathrm{T}_{\mathrm{Y}^{\prime}}\right)^{*}$-torsor. We denote by $\mathscr{K}_{h^{\prime}}$ the corresponding invertible module over $h^{\prime *} \hat{\Gamma} \mathrm{T}_{\mathrm{Y}^{\prime}}$. Define an autoequivalence

$$
\begin{aligned}
& \tau_{h^{\prime}}: \operatorname{Mod}\left(h^{\prime *} \hat{\Gamma} \mathrm{T}_{\mathrm{Y}^{\prime}}\right) \rightarrow \operatorname{Mod}\left(h^{\prime *} \hat{\Gamma} \mathrm{T}_{\mathrm{Y}^{\prime}}\right) \\
& \tau_{h^{\prime}}(\mathrm{E})=\mathrm{E} \otimes_{h^{\prime *} \hat{\Gamma} \mathrm{T}_{\mathrm{Y}^{\prime}}} \mathscr{K}_{h^{\prime}} .
\end{aligned}
$$

Then, with the notations from the proof of Theorem 3.22, one has

$$
\begin{gathered}
\mathscr{K}_{h^{\prime}} \otimes_{\hat{\Gamma}_{\mathrm{X}} / \mathrm{S}} \mathrm{F}_{\mathrm{X} / \mathrm{S} *} \mathscr{B}_{\mathscr{X} / \mathscr{S}} \cong \mathrm{F}_{\mathrm{X} / \mathrm{S} *} \mathrm{D}_{\mathrm{X} \rightarrow \mathrm{Y}} \otimes_{h^{*} \mathrm{~F}_{\mathrm{Y} / \mathrm{S} *} \mathrm{D}_{\mathrm{Y} / \mathrm{S}}} h^{\prime *} \mathrm{~F}_{\mathrm{Y} / \mathrm{S} *} \mathscr{B}_{\mathscr{Y} / \mathscr{S},} \\
\mathrm{L}_{\mathrm{DR}^{*}}^{*} \circ \mathrm{C}_{\mathscr{Y} / \mathscr{S}}^{-1} \cong \mathrm{C}_{\mathscr{X} / \mathscr{S}}^{-1} \circ i_{h *}^{\prime} \circ \tau_{h^{\prime}}^{-1} \circ \mathrm{L}^{*} \\
\mathrm{DF}_{[k, l]}\left(\mathrm{S}^{\cdot} \mathrm{T}_{\mathrm{Y}^{\prime}}, \mathscr{J}_{\mathrm{Y}^{\prime}}\right) \rightarrow \mathrm{DF}_{[k, l]}\left(\mathrm{F}_{\mathrm{X} / \mathrm{S} *} \mathrm{D}_{\mathrm{X} / \mathrm{S}}, \mathscr{I}_{\mathrm{X}}\right)
\end{gathered}
$$

and if $h$ is smooth of relative dimension $d$ and $l-k+d<p$

$$
\begin{aligned}
\mathrm{R} h_{*}^{\mathrm{DR}} \circ \mathrm{C}_{\mathscr{X} / \mathscr{S}}^{-1} \cong \mathrm{C}_{\mathscr{Y} / \mathscr{S}}^{-1} \circ \mathrm{R} h_{*}^{\prime} \circ \tau_{h^{\prime}} \circ \mathrm{R} i_{h}^{\prime !} \\
\mathrm{DF}_{[k, l]}\left(\mathrm{S}^{\cdot} \mathrm{T}_{\mathrm{X}^{\prime}}, \mathscr{J}_{\mathrm{X}^{\prime}}\right) \rightarrow \mathrm{DF}_{[k-d, l]}\left(\mathrm{F}_{\mathrm{Y} / \mathrm{S} *} \mathrm{D}_{\mathrm{Y} / \mathrm{S}}, \mathscr{I}_{\mathrm{Y}}\right) .
\end{aligned}
$$

Corollary 3.25. - Let $h: \mathrm{X} \rightarrow \mathrm{Y}$ be a smooth morphism of relative dimension $d$ and let $\mathrm{E}^{\cdot}$ be an object of $\mathrm{D}\left(\mathrm{HIG}_{[k, l]}^{\circ}\left(\mathrm{X}^{\prime} / \mathrm{S}\right)\right)$. Assume that $l-k-d<p$ and that there exists $\tilde{h}^{\prime}: \tilde{\mathrm{X}}^{\prime} \rightarrow \tilde{\mathrm{Y}}^{\prime}$. Then the conjugate spectral sequence for $\mathrm{H}^{*}\left(\mathrm{R}_{*}^{\mathrm{DR}}\left(\mathrm{C}_{\mathscr{X} / \mathscr{S}}^{-1} \mathrm{E}^{*}\right)\right)$ degenerates at $\mathrm{E}_{1}$.

Proof. — We have

$$
\mathrm{R} h_{*}^{\mathrm{HIG}}\left(\mathrm{E}^{*}\right) \simeq \mathrm{R} h_{*}^{\mathrm{HIG}}\left(\mathrm{Gr} \mathrm{E}^{*}\right) \simeq \mathrm{Gr} \operatorname{R} h_{*}^{\mathrm{HIG}}\left(\mathrm{E}^{*}\right) .
$$

Here the first isomorphism comes from the grading on $\mathrm{E}^{*}$ and the second one from (3) of Proposition 3.16. It follows that the spectral sequence of the filtered complex 
$\mathrm{R} h_{*}^{\mathrm{HIG}}\left(\mathrm{E}^{*}\right)$ degenerates at $\mathrm{E}_{1}$. Then by Theorem 3.22 the same is true for $\mathrm{R} h_{*}^{*}\left(\mathrm{C}_{\mathscr{X} / \mathscr{S}}^{-1} \mathrm{E}^{*}\right)$.

\section{Applications and examples}

4.1 Local study of the p-curvature

Let $\mathrm{X} / \mathrm{S}$ be a smooth morphism of schemes in characteristic $p>0$ and let

$$
\Psi: \operatorname{MIG}(\mathrm{X} / \mathrm{S}) \rightarrow \mathrm{F}-\mathrm{HIG}(\mathrm{X} / \mathrm{S})
$$

denote the functor taking a module with integrable connection to the corresponding module with F-Higgs field. This functor is not an equivalence or even fully faithful. For example, the category of pairs $(\mathscr{O}, \nabla)$ with vanishing $p$-curvature is equivalent to the category of invertible sheaves $\mathrm{L}$ on $\mathrm{X}^{\prime}$ together with a trivialization $\mathrm{F}_{\mathrm{X} / \mathrm{S}}^{*} \mathrm{~L} \cong \mathscr{O}_{\mathrm{X}}$. However, we show that if $\left(\mathrm{E}_{1}, \nabla_{1}\right)$ and $\left(\mathrm{E}_{2}, \nabla_{2}\right)$ are two noetherian objects of MIC(X/S) with isomorphic images in $\mathrm{F}-\mathrm{HIG}(\mathrm{X} / \mathrm{S})$, then Zariski locally on $\mathrm{X},\left(\mathrm{E}_{1}, \nabla_{1}\right)$ and $\left(\mathrm{E}_{2}, \nabla_{2}\right)$ are isomorphic. Moreover, we can characterize the image of the functor $\Psi$, étale locally on $\mathrm{X}$ : if $\psi$ is an F-Higgs field on a coherent $\mathrm{E}$, then étale locally on $\mathrm{X} / \mathrm{S}, \psi$ comes from a connection if and only if $(\mathrm{E}, \psi)$ descends to a Higgs field on $\mathrm{X}^{\prime} / \mathrm{S}$. Taken together, these results can be interpreted as a nonabelian analog of the well-known exact sequence [23, 4.14]

$$
0 \rightarrow \mathscr{O}_{\mathrm{X}^{\prime}}^{*} \stackrel{\mathrm{F}_{\mathrm{X} / \mathrm{S}}^{*}}{\longrightarrow} \mathrm{F}_{\mathrm{X} / \mathrm{S} *} \mathscr{O}_{\mathrm{X}}^{*} \stackrel{d \log }{\longrightarrow} \mathrm{F}_{\mathrm{X} / \mathrm{S} *} \mathrm{Z}_{\mathrm{X} / \mathrm{S}}^{1} \stackrel{\pi^{*}-\mathrm{C}_{\mathrm{X} / \mathrm{S}}}{\longrightarrow} \Omega_{\mathrm{X}^{\prime} / \mathrm{S}}^{1} \rightarrow 0
$$

where $\mathrm{C}_{\mathrm{X} / \mathrm{S}}$ is the Cartier operator and $\pi: \mathrm{X}^{\prime} \rightarrow \mathrm{X}$ the projection. Indeed, one can recover this sequence by considering the category of connections of the form $\left(\mathscr{O}_{\mathrm{X}}, d+\omega\right)$, where $\omega$ is a closed one-form, and recalling that the $p$-curvature of such a connection is precisely $\pi^{*}(\omega)-\mathrm{C}_{\mathrm{X} / \mathrm{S}}(\omega)$.

Theorem 4.1. - Let $\mathrm{X} / \mathrm{S}$ be a smooth morphism of noetherian schemes in characteristic p.

1. Let $\left(\mathrm{E}_{i}, \nabla_{i}\right), i=1,2$, be objects of $\mathrm{MIC}(\mathrm{X} / \mathrm{S})$, with $\mathrm{E}_{i}$ coherent, and let $\psi_{i}$ denote their p-curvatures. Suppose that there exists an isomorphism $h:\left(\mathrm{E}_{1}, \psi_{1}\right) \rightarrow\left(\mathrm{E}_{2}, \psi_{2}\right)$ in $\mathrm{F}-\mathrm{HIG}(\mathrm{X} / \mathrm{S})$. Then Zariski locally on $\mathrm{X},\left(\mathrm{E}_{1}, \nabla_{1}\right)$ and $\left(\mathrm{E}_{2}, \nabla_{2}\right)$ are isomorphic in $\mathrm{MIC}(\mathrm{X} / \mathrm{S})$.

2. Let $\mathrm{E}$ be a coherent sheaf with an F-Higgs field $\psi: \mathrm{E} \rightarrow \mathrm{E} \otimes \mathrm{F}_{\mathrm{X} / \mathrm{S}}^{*} \Omega_{\mathrm{X}^{\prime} / \mathrm{S}}^{1}$. Then étale locally on $\mathrm{X}$, the following are equivalent:

(a) There exists a connection on $\mathrm{E}$ whose p-curvature is $\psi$.

(b) There exist a coherent sheaf with a Higgs field $\left(\mathrm{E}^{\prime}, \psi^{\prime}\right)$ on $\mathrm{X}^{\prime} / \mathrm{S}$ and an isomorphism $(\mathrm{E}, \psi) \cong \mathrm{F}_{\mathrm{X} / \mathrm{S}}^{*}\left(\mathrm{E}^{\prime}, \psi^{\prime}\right)$. 
Proof. - To prove (1), let $\mathrm{H}:=\operatorname{Hom}\left(\mathrm{E}_{1}, \mathrm{E}_{2}\right)$, with the internal Hom connection and $p$-curvature. Let $\mathrm{H}^{\psi} \subseteq \mathrm{H}$ be the subsheaf annihilated by $\psi$, and let $\mathrm{F}_{\mathrm{X} / \mathrm{S*}}\left(\mathrm{H}^{\nabla}\right)$ be the subsheaf annihilated by $\nabla$. Then by Cartier descent, the natural map $\mathrm{F}_{\mathrm{X} / \mathrm{S}}^{*} \mathrm{~F}_{\mathrm{X} / \mathrm{S}_{*}} \mathrm{H}^{\nabla} \rightarrow \mathrm{H}^{\psi}$ is an isomorphism of $\mathscr{O}_{\mathrm{X}}$-modules.

Let $x$ be a point of $\mathrm{X}$, and let $x^{\prime}$ be its image in $\mathrm{X}^{\prime}$. Then $k(x)$ is a finite and purely inseparable extension of $k\left(x^{\prime}\right)$. The fiber $\mathrm{V}^{\prime}:=\left(\mathrm{F}_{\mathrm{X} / \mathrm{S} *} \mathrm{H}^{\nabla}\right)\left(x^{\prime}\right)$ of $\mathrm{F}_{\mathrm{X} / \mathrm{S} *} \mathrm{H}^{\nabla}$ at $x^{\prime}$ is a finite dimensional $k\left(x^{\prime}\right)$-vector space, the fiber $\mathrm{V}:=\mathrm{H}^{\psi}(x)$ of $\mathrm{H}^{\psi}$ at $x$ is a finite dimensional $k(x)$-vector space, and the natural map $k(x) \otimes_{k\left(x^{\prime}\right)} \mathrm{V}^{\prime} \rightarrow \mathrm{V}$ is an isomorphism. There is also a natural map $\mathrm{V} \rightarrow \operatorname{Hom}_{k(x)}\left(\mathrm{E}_{1}(x), \mathrm{E}_{2}(x)\right)$. Let $\mathbf{V}$ be the affine space over $k(x)$ corresponding to the $k(x)$-vector space $\mathrm{V}$, and let $\mathbf{U}$ denote the Zariski open subset of $\mathbf{V}$ corresponding to those elements which define isomorphisms $\mathrm{E}_{1}(x) \rightarrow \mathrm{E}_{2}(x)$. The isomorphism $h$ lies in $\mathrm{H}^{\psi}$ and hence its image $h(x)$ in $\mathrm{V}$ corresponds to a $k(x)$-rational point of $\mathbf{U}$. Let $\mathbf{V}^{\prime}$ be the affine space over $k\left(x^{\prime}\right)$ corresponding to $\mathbf{V}^{\prime}$. Then $\mathbf{V}$ is the base change of $\mathbf{V}^{\prime}$ to Spec $k(x)$, and since $k\left(x^{\prime}\right) \rightarrow k(x)$ is a purely inseparable extension, the projection mapping $\mathbf{V} \rightarrow \mathbf{V}^{\prime}$ is a homeomorphism and the image $\mathbf{U}^{\prime}$ of $\mathbf{U}$ in $\mathbf{V}^{\prime}$ is a nonempty open subset. If $k\left(x^{\prime}\right)$ is infinite, it follows that the $k\left(x^{\prime}\right)$-rational points of $\mathbf{V}^{\prime}$ are Zariski dense, so $\mathbf{U}^{\prime}$ has a $k\left(x^{\prime}\right)$-rational point. If $k\left(x^{\prime}\right)$ is finite, it is perfect, and it follows that $k(x)=k\left(x^{\prime}\right)$. Thus in either case there is an element $v^{\prime}$ in $\mathrm{V}^{\prime}$ which induces an isomorphism $\mathrm{E}_{1}(x) \rightarrow \mathrm{E}_{2}(x)$. Then there exists an element $g^{\prime}$ in the stalk of the $\mathscr{O}_{\mathrm{X}^{\prime}}$-module $\mathrm{F}_{\mathrm{X} / \mathrm{S} *} \mathrm{H}^{\nabla}$ at $x^{\prime}$ whose image in $\mathrm{V}^{\prime}$ is $v^{\prime}$. Let $h^{\prime}:=\mathrm{F}_{\mathrm{X}}^{*}\left(g^{\prime}\right)$, which defines a horizontal morphism $\mathrm{E}_{1} \rightarrow \mathrm{E}_{2}$ in some neighborhood of $x$. The fiber of $h^{\prime}$ at $x$ is an isomorphism. We know that $\mathrm{E}_{1, x}$ and $\mathrm{E}_{2, x}$ are isomorphic as $\mathscr{O}_{\mathrm{X}, x}$-modules, and in particular their reductions module any power of the maximal ideal have the same finite length. It follows from Nakayama's lemma that $h^{\prime}$ is surjective modulo any power of the maximal ideal, and hence is also an isomorphism modulo any such power. Then it follows that $h^{\prime}$ is an isomorphism in a neighborhood of $x$. This proves (1).

We should remark that (1) could also have been proved from the theory of Azumaya algebras; we preferred to explain the elementary proof above. We do not know of such an elementary proof of (2). Note first that since (2) is a local statement, we may assume that there exists a spitting $\zeta$ of $\mathrm{C}_{\mathrm{X} / \mathrm{S}}^{-1}$ as in (1.9.2).

Suppose that $\left(\mathrm{E}^{\prime}, \psi^{\prime}\right)$ is an object of $\operatorname{HIG}\left(\mathrm{X}^{\prime} / \mathrm{S}\right)$, with $\mathrm{E}^{\prime}$ coherent as an $\mathscr{O}_{\mathrm{X}^{\prime}}$-module. Let $\tilde{\mathrm{E}}^{\prime}$ denote the coherent sheaf on $\mathbf{T}_{\mathrm{X}^{\prime} / \mathrm{S}}^{*}$ corresponding to $\left(\mathrm{E}^{\prime}, \psi^{\prime}\right)$. Let $i^{\prime}: Z^{\prime} \rightarrow \mathbf{T}_{\mathrm{X}^{\prime} / \mathrm{S}}$ be the closed immersion defined by the annihilator of $\tilde{\mathrm{E}}^{\prime}$ in $\mathscr{O}_{\mathbf{T}_{\mathrm{X}^{\prime} / \mathrm{S}}^{*}}$. Since $\mathrm{E}^{\prime}$ is coherent as a sheaf of $\mathscr{O}_{\mathrm{X}^{\prime}}$-modules, $\mathrm{Z}^{\prime}$ is finite over $\mathrm{X}^{\prime}$, and hence the étale covering $\alpha_{\zeta}: \mathbf{T}_{\mathrm{X}^{\prime} / \mathrm{S}} \rightarrow \mathbf{T}_{\mathrm{X}^{\prime} / \mathrm{S}}$ splits over $\mathrm{Z}^{\prime}$, étale locally on $\mathrm{X}^{\prime}$. Thus, after replacing $\mathrm{X}^{\prime}$ by an étale localization, we may assume that there exists a map $j^{\prime}: \mathrm{Z}^{\prime} \rightarrow \mathbf{T}_{\mathrm{X}^{\prime} / \mathrm{S}}^{*}$ such that $\alpha_{\zeta} \circ j^{\prime}=i^{\prime}$. Let $\tilde{\mathrm{E}}^{\prime \prime}:=j_{*}^{\prime} i^{* *} \tilde{\mathrm{E}}^{\prime}$, which corresponds to an object $\left(\mathrm{E}^{\prime \prime}, \psi^{\prime \prime}\right)$ of $\operatorname{HIG}\left(\mathrm{X}^{\prime} / \mathrm{S}\right)$. Then $\tilde{\mathrm{E}}^{\prime} \cong i_{*}^{\prime} i^{* *} \tilde{\mathrm{E}}^{\prime} \cong \alpha_{\zeta *} j_{*}^{\prime} i^{\prime *} \tilde{\mathrm{E}}^{\prime} \cong \alpha_{\zeta *} \tilde{\mathrm{E}}^{\prime \prime}$. Let $(\mathrm{E}, \nabla):=\Psi_{\zeta}^{-1}\left(\mathrm{E}^{\prime \prime}, \psi^{\prime}\right)$ (see Theorem 2.13). By op. cit., the $p$-curvature of $(\mathrm{E}, \nabla)$ is $\mathrm{F}_{\mathrm{X} / \mathrm{S}}^{*} \alpha_{\zeta *}\left(\mathrm{E}^{\prime \prime}, \psi^{\prime \prime}\right) \cong \mathrm{F}_{\mathrm{X} / \mathrm{S}}^{*}\left(\mathrm{E}^{\prime}, \psi^{\prime}\right)$. 
Conversely, suppose that $(\mathrm{E}, \nabla)$ is an object of $\mathrm{MIC}(\mathrm{X} / \mathrm{S})$, with $\mathrm{E}$ coherent as an $\mathscr{O}_{\mathrm{X}}$-module. Its $p$-curvature defines an object $(\mathrm{E}, \psi)$ of $\mathrm{F}-\mathrm{HIG}(\mathrm{X} / \mathrm{S})$, and hence a coherent sheaf $\tilde{\mathrm{E}}$ on $\mathbf{T}_{\mathrm{X}^{\prime} / \mathrm{S}}^{*\left(\mathrm{X}^{\prime}\right)}:=\mathbf{V F}_{\mathrm{X} / \mathrm{S}}^{*} \mathrm{~T}_{\mathrm{X}^{\prime} / \mathrm{S}}$ (see diagram (2.1.1)). The claim is that there exists a coherent sheaf $\tilde{\mathrm{E}}^{\prime}$ on $\mathbf{T}_{\mathrm{X}^{\prime} / \mathrm{S}}$ such that $\pi_{\mathbf{T}}^{*}\left(\tilde{\mathrm{E}}^{\prime}\right) \cong \tilde{\mathrm{E}}$. Since $\mathrm{F}_{\mathrm{X} / \mathrm{S} *} \mathrm{E}$ is coherent as an $\mathscr{O}_{\mathrm{X}^{\prime}}$-module, the scheme-theoretic support $\mathrm{Z}^{\prime}$ of $\pi_{\mathbf{T} *}$ is finite over $\mathrm{X}^{\prime}$, and there exists a section $j^{\prime}$ of $\alpha_{\zeta}$ over $Z^{\prime}$. If we view $\tilde{\mathrm{E}}$ as a module over $\mathrm{S}_{\zeta}^{\cdot} \mathrm{T}_{\mathrm{X}^{\prime} / \mathrm{S}}$ via $j^{\prime \sharp}$, then the action of $\mathrm{S}_{\zeta}^{\cdot} \mathrm{T}_{\mathrm{X}^{\prime} / \mathrm{S}}$ agrees with the action of $\mathrm{S}^{\cdot} \mathrm{T}_{\mathrm{X}^{\prime} / \mathrm{S}}$, and so the action of $\mathrm{D}_{\mathrm{X} / \mathrm{S}}$ on $\mathrm{E}$ extends to an action of $\mathrm{D}_{\zeta}$. Let $\tilde{\mathrm{E}}^{\prime}:=\mathscr{H}_{0 m_{\mathscr{D}_{\zeta}}}\left(\mathscr{B}_{\zeta}, \mathrm{E}\right)$, corresponding to an object $\left(\mathrm{E}^{\prime}, \psi^{\prime}\right) \in \operatorname{HIG}\left(\mathrm{X}^{\prime} / \mathrm{S}\right)$. Then $(\mathrm{E}, \nabla) \cong \Psi_{\zeta}^{-1}\left(\mathrm{E}^{\prime}, \psi^{\prime}\right)$, so $(\mathrm{E}, \psi) \cong \mathrm{F}_{\mathrm{X} / \mathrm{S}}^{*}\left(\alpha_{\zeta^{*}}\left(\mathrm{E}^{\prime}, \psi^{\prime}\right)\right)$ in F-HIG(X/S), by Theorem 2.13.

\subsection{Stacks of liftings and splittings}

In this subsection we discuss relationships between and geometric interpretations of some of the liftings and splittings used in our constructions. In particular, we show that there is a natural equivalence between the gerbe of liftings of $\mathrm{X}^{\prime}$ and the gerbe of tensor splittings of $\mathscr{D} \mathrm{X} / \mathrm{S}$ over the completed divided power envelope $\hat{\mathbf{T}}_{\mathrm{X}^{\prime} / \mathrm{S}}^{* \gamma}$ of the zero section of $\mathbf{T}_{\mathrm{X}^{\prime} / \mathrm{S}}^{*}$.

First we shall study the gerbe of splittings of the Azumaya algebra $\mathscr{D} \mathrm{x} / \mathrm{s}$ on $\mathbf{T}_{\mathrm{X}^{\prime} / \mathrm{S}}^{*}$. Recall from [23] and [9] that the equivalence class of this gerbe can be viewed as the image of $\mathscr{D}_{\mathrm{X} / \mathrm{S}}$ in the cohomological Brauer group $\mathrm{H}^{2}\left(\mathbf{T}_{\mathrm{X}^{\prime} / \mathrm{S}}^{*}, \mathscr{O}_{\mathbf{T}_{\mathrm{X}^{\prime} / \mathrm{S}}^{*}}^{*}\right)$. Our first goal is to provide a simple description of this cohomology class.

Recall from [23, 4.14] that for any smooth Y/S there is an exact sequence of étale sheaves on $\mathrm{Y}^{\prime}$ :

$$
0 \rightarrow \mathscr{O}_{\mathrm{Y}^{\prime}}^{*} \stackrel{\mathrm{F}_{\mathrm{Y} / \mathrm{S}}^{*}}{\longrightarrow} \mathrm{F}_{\mathrm{Y} / \mathrm{S} *} \mathscr{O}_{\mathrm{Y}}^{*} \stackrel{\text { dlog }}{\longrightarrow} \mathrm{F}_{\mathrm{Y} / \mathrm{S} *} \mathrm{Z}_{\mathrm{Y} / \mathrm{S}}^{1} \stackrel{\pi_{\mathrm{Y} / \mathrm{S}}^{*}-\mathrm{CY}_{\mathrm{Y}} \mathrm{S}}{\longrightarrow} \Omega_{\mathrm{Y}^{\prime} / \mathrm{S}}^{1} \rightarrow 0 .
$$

Here $\mathrm{F}_{\mathrm{Y} / \mathrm{S} *} \mathrm{Z}_{\mathrm{Y} / \mathrm{S}}^{1} \subset \mathrm{F}_{\mathrm{Y} / \mathrm{S} *} \Omega_{\mathrm{Y} / \mathrm{S}}^{1}$ is the subsheaf of closed one-forms, $\mathrm{C}_{\mathrm{Y} / \mathrm{S}}$ is the Cartier operator, and $\pi_{\mathrm{Y} / \mathrm{S}}: \mathrm{Y}^{\prime} \rightarrow \mathrm{Y}$ is the morphism induced by the Frobenius on $\mathrm{S}$. As we observed in Section 4.1, the morphism $\pi_{\mathrm{Y} / \mathrm{S}}^{*}-\mathrm{C}_{\mathrm{Y} / \mathrm{S}}: \mathrm{F}_{\mathrm{Y} / \mathrm{S} *} \mathrm{Z}_{\mathrm{Y} / \mathrm{S}}^{1} \rightarrow \Omega_{\mathrm{Y}^{\prime} / \mathrm{S}}^{1}$ can be viewed as the map sending the line bundle $\mathscr{O}_{\mathrm{Y}}$ with integrable connection $\nabla=d+\omega$ to its p-curvature. The exact sequence (4.1.1) induces a morphism:

$$
\phi: \mathrm{H}^{0}\left(\mathrm{Y}^{\prime}, \Omega_{\mathrm{Y}^{\prime} / \mathrm{S}}^{1}\right) \rightarrow \mathrm{H}^{1}\left(\mathrm{Y}^{\prime}, \mathrm{F}_{\mathrm{Y} / \mathrm{S} *}\left(\mathscr{O}_{\mathrm{Y}}^{*}\right) / \mathscr{O}_{\mathrm{Y}^{\prime}}^{*}\right) \rightarrow \mathrm{H}^{2}\left(\mathrm{Y}^{\prime}, \mathscr{O}_{\mathrm{Y}^{\prime}}^{*}\right)=\operatorname{Br}\left(\mathrm{Y}^{\prime}\right) .
$$

As we shall recall below, the cotangent bundle of $\mathrm{X}^{\prime} / \mathrm{S}$ has a canonical global oneform (the "contact form"). We shall see in Proposition 4.4 below that the Brauer class of $\mathscr{D}_{\mathrm{x} / \mathrm{S}}$ can be identified with the image of this one-form under the map $\phi$. We begin with the following convenient geometric description of the map $\phi$.

Proposition 4.2. - Let $\omega^{\prime} \in \mathrm{H}^{0}\left(\mathrm{Y}^{\prime}, \Omega_{\mathrm{Y}^{\prime} / \mathrm{S}}^{1}\right)$ be a one-form. For each étale $\mathrm{U}^{\prime} \rightarrow \mathrm{Y}^{\prime}$, let $\mathrm{U}:=\mathrm{F}_{\mathrm{Y} / \mathrm{S}}^{-1}\left(\mathrm{U}^{\prime}\right) \rightarrow \mathrm{Y}$ and let $\mathscr{P}_{\omega^{\prime}}^{\natural}\left(\mathrm{U}^{\prime}\right)$ be the groupoid of invertible sheaves with integrable 
connection on $\mathrm{U}$ whose p-curvature is equal to $\omega^{\prime}$. Then, $\mathscr{P}_{\omega^{\prime}}^{\natural}$ forms a fibered category which is in fact a gerbe under $\mathscr{O}_{\mathrm{Y}^{\prime}}^{*}$ on $\mathrm{Y}^{\prime}$. The class of $\mathscr{P}_{\omega^{\prime}}^{\natural}$ in $\mathrm{H}^{2}\left(\mathrm{Y}^{\prime}, \mathscr{O}_{\mathrm{Y}^{\prime}}^{*}\right)$ is equal to $\phi\left(\omega^{\prime}\right)$.

Proof. - It is clear that $\mathscr{P}_{\omega^{\prime}}^{\natural}$ forms a stack and that the automorphism group of each object is $\mathscr{O}_{\mathrm{Y}^{\prime}}^{*}$. The local surjectivity of $\pi_{\mathrm{Y} / \mathrm{S}}^{*}-\mathrm{C}_{\mathrm{Y} / \mathrm{S}}$ implies that the class of objects of $\mathscr{P}_{\omega^{\prime}}^{\natural}$ is locally not empty. If $\mathrm{L}_{1}$ and $\mathrm{L}_{2}$ are two objects of $\mathscr{P}_{\omega^{\prime}}^{\natural}$ over some $\mathrm{U}^{\prime}$, then the $p$-curvature of $\mathscr{H} o m\left(\mathrm{~L}_{1}, \mathrm{~L}_{2}\right)$ is zero, and hence locally has a horizontal basis. This implies that any two objects of $\mathscr{P}_{\omega^{\prime}}^{\natural}$ are locally isomorphic, so that $\mathscr{P}_{\omega^{\prime}}^{\natural}$ is indeed a gerbe.

The boundary map associated to the exact sequence

$$
0 \rightarrow \mathrm{F}_{\mathrm{Y} / \mathrm{S} *}\left(\mathscr{O}_{\mathrm{Y}}^{*}\right) / \mathscr{O}_{\mathrm{Y}^{\prime}}^{*} \stackrel{\text { dlog }}{\longrightarrow} \mathrm{F}_{\mathrm{Y} / \mathrm{S} *} \mathrm{Z}_{\mathrm{Y} / \mathrm{S}}^{1} \stackrel{\pi_{\mathrm{Y} / \mathrm{s}}^{*}-\mathrm{CY}_{\mathrm{Y}}}{\longrightarrow} \Omega_{\mathrm{Y}^{\prime} / \mathrm{S}}^{1} \rightarrow 0
$$

takes $\omega^{\prime}$ to the $\left(\mathrm{F}_{\mathrm{Y} / \mathrm{S} *} \mathscr{O}_{\mathrm{Y}}^{*}\right) / \mathscr{O}_{\mathrm{Y}^{\prime}}^{*}$-torsor $\mathscr{T}_{\omega^{\prime}}$ of closed one-forms $\eta$ such that $\pi_{\mathrm{Y} / \mathrm{S}}^{*} \eta-$ $\mathrm{C}_{\mathrm{Y} / \mathrm{S}}(\eta)=\omega^{\prime}$. The boundary map associated to the exact sequence

$$
0 \rightarrow \mathscr{O}_{\mathrm{Y}^{\prime}}^{*} \rightarrow \mathrm{F}_{\mathrm{Y} / \mathrm{S} *}\left(\mathscr{O}_{\mathrm{Y}}^{*}\right) \rightarrow \mathrm{F}_{\mathrm{Y} / \mathrm{S} *}\left(\mathscr{O}_{\mathrm{Y}}^{*}\right) / \mathscr{O}_{\mathrm{Y}^{\prime}}^{*} \rightarrow 0
$$

takes $\mathscr{T}_{\omega^{\prime}}$ to the gerbe $\mathrm{G}_{\omega^{\prime}}$ of $\mathrm{F}_{\mathrm{Y} / \mathrm{S} *}\left(\mathscr{O}_{\mathrm{Y}}^{*}\right)$-torsors $\mathscr{L}$ equipped with an isomorphism $\alpha: \overline{\mathscr{L}} \rightarrow \mathscr{T}_{\omega^{\prime}}$, where $\overline{\mathscr{L}}$ is the $\left(\mathrm{F}_{\mathrm{Y} / \mathrm{S} *} \mathscr{O}_{\mathrm{Y}}^{*}\right) / \mathscr{O}_{\mathrm{Y}^{\prime}}^{*}$-torsor associated to $\mathscr{L}$. Hence $\phi\left(\omega^{\prime}\right)=\mathrm{G}_{\omega^{\prime}}$, and it will suffice to prove that $\mathrm{G}_{\omega^{\prime}}$ is equivalent to $\mathscr{P}_{\omega^{\prime}}^{\natural}$. Let $\mathscr{L}$ be an object of $\mathrm{G}_{\omega^{\prime}}$ over $\mathrm{U}^{\prime}$, let $\mathrm{L}$ be the associated invertible sheaf over $\mathrm{U}$, and let $e$ be a local section of $\mathscr{L}$, i.e., a basis for $\mathrm{L}$ on some open subset $\mathrm{V}$ of $\mathrm{U}$. There is a unique connection $\nabla$ on $\mathrm{L}$ such that $\nabla(e)=e \otimes \alpha(e)$. It follows from the fact that $\alpha$ is a morphism of torsors that $\nabla$ is independent of the choice of $e$, and it is clear that the $p$-curvature of $\nabla$ is $\omega^{\prime}$. This construction defines a functor from the gerbe $\mathrm{G}_{\omega^{\prime}}$ to $\mathscr{P}_{\omega^{\prime}}^{\natural}$, which is easily seen to be an equivalence.

Remark 4.3. - In the context of the above proposition, the form $\omega^{\prime}$ gives a morphism $i: \mathrm{Y}^{\prime} \rightarrow \mathrm{T}_{\mathrm{Y} / \mathrm{S}^{\prime}}^{*}$, and $\mathscr{P}_{\omega^{\prime}}^{\natural}$ is the gerbe of splittings of the Azumaya algebra $i^{*} \mathscr{D}_{\mathrm{Y} / \mathrm{S}}$ on $\mathrm{Y}^{\prime}$.

Let us write $\mathbf{T}^{*}$ for $\mathbf{T}_{\mathrm{X} / \mathrm{S}}^{*}$, and recall that there is an exact sequence

$$
0 \rightarrow p r^{*} \Omega_{\mathrm{X} / \mathrm{S}}^{1} \rightarrow \Omega_{\mathbf{T}_{\mathrm{X} / \mathrm{S}}^{*} / \mathrm{S}}^{1} \rightarrow \Omega_{\mathbf{T}_{\mathrm{X} / \mathrm{S}}^{*} / \mathrm{X}}^{1} \rightarrow 0
$$

Furthermore, $\mathbf{T}^{*}=\mathrm{Spec}_{\mathrm{X}} \mathrm{S}^{*} \mathrm{~T}_{\mathrm{X} / \mathrm{S}}$, so that there is a canonical global section of $p r_{*} p r^{*} \Omega_{\mathrm{X} / \mathrm{S}}^{1} \cong \Omega_{\mathrm{X} / \mathrm{S}}^{1} \otimes \mathrm{S}^{*} \mathrm{~T}_{\mathrm{X} / \mathrm{S}}$, corresponding to the identity element of $\Omega_{\mathrm{X} / \mathrm{S}}^{1} \otimes \mathrm{T}_{\mathrm{X} / \mathrm{S}} \cong$ End $\mathrm{T}_{\mathrm{X} / \mathrm{S}}$. The image of this section in $\Omega_{\mathbf{T}^{*} / \mathrm{S}}^{1}$ is the well-known "contact form" on the cotangent bundle. 
Proposition $4.4([5])$. - Let $\omega^{\prime} \in \Gamma\left(\mathbf{T}_{\mathrm{X}^{\prime} / \mathrm{S}}^{*}, \Omega_{\mathbf{T}_{\mathrm{X}^{\prime} / \mathrm{S}}^{*}}^{1}\right)$ be the contact form and let $\mathscr{P}_{\omega^{\prime}}^{\natural}$ be the corresponding $\mathbf{G}_{m}$-gerbe on $\mathbf{T}_{\mathrm{X}^{\prime} / \mathrm{S}}^{*}$ described in Proposition 4.2. Then the gerbe $\mathscr{P}_{\omega^{\prime}}^{\natural}$ is equivalent to the gerbe $\mathscr{S}$ of splittings of the Azumaya algebra $\mathscr{D} \mathrm{x} / \mathrm{s}$ on $\mathbf{T}_{\mathrm{X}^{\prime} / \mathrm{S}}^{*}$. In particular, the class of $\mathscr{D} \mathrm{X} / \mathrm{S}$ in $\operatorname{Br}\left(\mathbf{T}_{\mathrm{X}^{\prime} / \mathrm{S}}^{*}\right)$ is $\phi\left(\omega^{\prime}\right)$.

Proof. — We have a diagram:

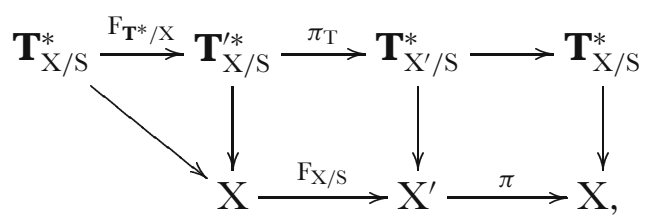

in which both squares are Cartesian and $\mathrm{F}_{\mathbf{T}^{*} / \mathrm{S}}=\pi_{\mathbf{T}} \circ \mathrm{F}_{\mathbf{T}^{*} / \mathrm{X}}$. We identify the pullback of $\mathbf{T}_{\mathrm{X} / \mathrm{S}}^{*}$ by $\mathrm{F}_{\mathrm{S}}$ with $\mathbf{T}_{\mathrm{X}^{\prime} / \mathrm{S}}^{*}$ and use abbreviations:

$$
\mathbf{T}^{*}:=\mathbf{T}_{\mathrm{X} / \mathrm{S}}^{*}, \quad \mathbf{T}^{* \prime}:=\mathbf{T}_{\mathrm{X}^{\prime} / \mathrm{S}}^{*}, \quad \mathbf{T}^{*}:=\mathbf{T}_{\mathrm{X} / \mathrm{S}}^{*} .
$$

Let $\mathrm{U}^{\prime} \rightarrow \mathbf{T}^{* \prime}$ be étale, let $\mathrm{U} \rightarrow \mathbf{T}^{*}$ (resp. $\mathrm{U}^{\prime \prime}$ ) be its pullback via $\mathrm{F}_{\mathbf{T}^{*} / \mathrm{S}}$, (resp. via $\pi_{\mathrm{T}}$ ). Let $(\mathrm{L}, \nabla)$ be an object of $\mathscr{P}_{\omega^{\prime}}^{\natural}\left(\mathrm{U}^{\prime}\right)$, i.e., an invertible sheaf with integrable connection on $\mathrm{U} / \mathrm{S}$ whose $p$-curvature is $\omega^{\prime}$. The connection $\nabla$ defines an action of $\mathrm{D}_{\mathbf{T}^{*} / \mathrm{S}}$ and hence of the subalgebra $D_{\mathbf{T}^{*} / \mathbf{X}}$ on $\mathrm{L}$. Since the projection of $\omega^{\prime}$ to $\Omega_{\mathbf{T}^{* \prime} / \mathbf{X}^{\prime}}^{1}$ is equal to 0 , the $p$-curvature of the corresponding object of $\mathrm{MIC}\left(\mathbf{T}^{*} / \mathrm{X}\right)$ vanishes. Let

$$
\mathrm{L}^{\prime}:=\mathscr{H}_{d \mathrm{R}}^{0}\left(\mathrm{~L} \otimes \Omega_{\mathbf{T}^{*} / \mathrm{X}}^{\cdot}\right):=\operatorname{Ker}\left(\mathrm{L} \stackrel{\nabla}{\rightarrow} \mathrm{L} \otimes \Omega_{\mathbf{T}^{*} / \mathrm{S}}^{1} \rightarrow \mathrm{L} \otimes p r^{*} \Omega_{\mathbf{T}^{*} / \mathrm{X}}^{1}\right) .
$$

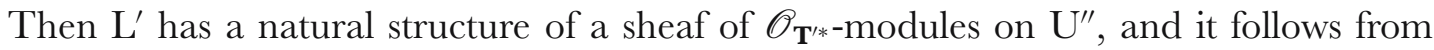
Cartier descent that the natural map $\mathrm{F}_{\mathbf{T}^{*} / \mathrm{X}^{*}} \mathrm{~L}^{\prime} \mathrm{L}$ is an isomorphism. Furthermore, $\nabla$ induces a map $\nabla^{\prime}: \mathrm{L}^{\prime} \rightarrow \mathrm{L}^{\prime} \otimes p r^{*} \Omega_{\mathrm{X} / \mathrm{S}}^{1}$, which defines a $p r^{-1} \mathrm{D}_{\mathrm{X} / \mathrm{S}}$-module structure on $\mathrm{L}^{\prime}$. (This is essentially the Gauss-Manin connection for the morphism $\mathbf{T}^{*} \rightarrow \mathrm{X}$.) The $p$-curvature of this module is still given by the contact form $\omega^{\prime}$, which means that the action of sections of $\mathscr{O}_{\mathbf{T}^{* \prime}}$ via the $p$-curvature is the same as the action via the

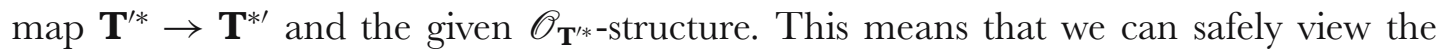
$\mathrm{pr}^{-1} \mathrm{D}_{\mathrm{X} / \mathrm{S}}$-module structure and the $\mathscr{O}_{\mathbf{T}^{* *} \text {-module structure as defining a } \mathscr{D} \mathrm{X} / \mathrm{S} \text {-module }}$ structure on $\mathrm{L}^{\prime}$. Since $\mathrm{L}^{\prime}$ is an invertible sheaf on $\mathbf{T}^{* *}$, it has $\operatorname{rank} p^{d}$ over $\mathbf{T}^{* \prime}$, and thus defines a splitting module for the Azumaya algebra $\mathscr{D}_{\mathrm{X} / \mathrm{s}}$. Thus we have defined a functor $\mathscr{P}_{\omega^{\prime}} \rightarrow \mathscr{S}$. It is clear that this functor is fully faithful, since the automorphisms of objects in either category are just give by units in $\mathscr{O}_{\mathbf{T}^{* \prime}}$. On the other hand, suppose that $\mathrm{M}$ is a splitting module for $\mathscr{D}_{\mathrm{X} / \mathrm{S}}$. Then viewing $\mathscr{O}_{\mathrm{X}} \rightarrow \mathscr{D}_{\mathrm{X} / \mathrm{s}}$ via the action on the left, we can view $\mathrm{M}$ as a module over $\mathbf{T}^{*}$, and by Proposition 2.3 it then

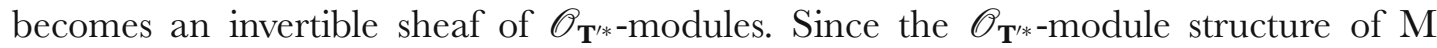
comes from its $p$-curvature, the $p$-curvature of $\mathrm{M}$ is just the contact form $\omega^{\prime}$. A local 
calculation shows that there is a unique extension of the action of $\mathscr{D} \mathrm{x} / \mathrm{S}$ on $\mathrm{M}$ to an action of $\mathrm{D}_{\mathbf{T}^{*} / \mathrm{S}}$ on $\mathrm{F}_{\mathbf{T}^{*} / \mathrm{X}}^{*} \mathrm{M}$ with the property that $\mathrm{M}$ is the annihilator of $\mathrm{D}_{\mathbf{T}^{*} / \mathrm{X}}$. This shows that the functor $\mathscr{P}_{\omega^{\prime}}^{\natural} \rightarrow \mathscr{S}$ is an equivalence. The statement about the Brauer group then follows, as explained in [23].

In this following discussion we will assume that the reader is acquainted with the notion of tensor structure on an Azumaya algebra introduced in Section 5.5. In particular, we explain there that the algebra $\mathscr{D}_{\mathrm{X} / \mathrm{S}}$ has a canonical symmetric tensor structure. Let us consider the following stacks on $\mathrm{X}_{e t}^{\prime}$.

1. The stack $\mathscr{L}$ of liftings of $\mathrm{X}^{\prime}$.

2. The stack $\mathscr{T} \mathscr{S} \mathscr{P}$ of tensor splittings of $\mathscr{D} \mathrm{x} / \mathrm{s}$ over the completed divided power envelope $\hat{\mathbf{T}}_{\mathrm{X}^{\prime} / \mathrm{S}}^{* \gamma}$ of the zero section of $\mathbf{T}_{\mathrm{X}^{\prime} / \mathrm{S}}^{*} \cdot{ }^{13}$

3. The stack $\mathscr{S} \mathscr{P}_{1}$ of pairs $\left(\mathrm{M}_{1}, \alpha\right)$, where $\mathrm{M}_{1}$ is a splitting of $\mathscr{D} \mathrm{x} / \mathrm{S}$ over the first infinitesimal neighborhood $\mathbf{T}_{1}^{*}$ of the zero section of $\mathbf{T}_{\mathrm{X}^{\prime} / \mathrm{S}}^{*}$ and $\alpha: i^{*} \mathrm{M}_{1} \simeq$ $\mathrm{F}_{\mathrm{X} / \mathrm{S} *} \mathscr{O}_{\mathrm{X}}$ is an isomorphism between the restriction of $\mathrm{M}_{1}$ to the zero section and the canonical splitting over $\mathrm{X}^{\prime}$.

4. The stack $\mathscr{E} \mathscr{X}$ of extensions of $\mathrm{F}_{\mathrm{X} / \mathrm{S}}^{*} \Omega_{\mathrm{X}^{\prime} / \mathrm{S}}^{1}$ by $\mathscr{O}_{\mathrm{X}}$ in $\mathrm{MIC}(\mathrm{X} / \mathrm{S})$ such that the graded $p$-curvature mapping $\psi: \mathrm{F}_{\mathrm{X} / \mathrm{S}}^{*} \Omega_{\mathrm{X}^{\prime} / \mathrm{S}}^{1} \rightarrow \mathscr{O}_{\mathrm{X}} \otimes \mathrm{F}_{\mathrm{X} / \mathrm{S}}^{*} \Omega_{\mathrm{X}^{\prime} / \mathrm{S}}^{1}$ is the identity.

In the discussion preparing for Theorem 2.8 we constructed a functor $\mathscr{B}$ associating a tensor splitting $\mathscr{B}_{\mathscr{X} / \mathscr{S}}$ to a lifting $\tilde{\mathrm{X}}^{\prime}$ of $\mathrm{X}^{\prime}$. Furthermore, recall that $\tilde{\mathrm{X}}^{\prime}$ determines an extension (1.4.1) as in (4), so that we also have a functor $\mathscr{E}: \mathscr{L} \rightarrow \mathscr{E} \mathscr{X}$. Recall that for any tensor splitting $\mathrm{M}$ there is a canonical isomorphism $\alpha: i^{*} \mathrm{M} \simeq$ $\mathrm{F}_{\mathrm{X} / \mathrm{S} *} \mathscr{O}_{\mathrm{X}}$, and hence there is a restriction functor $i_{1}^{*}: \mathscr{T} \mathscr{S} \mathscr{P} \rightarrow \mathscr{S} \mathscr{P}_{1}$. The dual of an extension in $\mathscr{E} \mathscr{X}$ is an object of $\mathscr{S} \mathscr{P}_{1}$, so there is also a functor from $\mathscr{E} \mathscr{X} \rightarrow \mathscr{S} \mathscr{P}$. This functor is easily seen to be an equivalence. The following theorem, shows that in fact all the above functors are equivalences.

Theorem 4.5. - The stacks above are in fact gerbes, and the functors

$$
\mathscr{B}: \mathscr{L} \rightarrow \mathscr{T} \mathscr{S} \mathscr{P}, \quad i_{1}^{*}: \mathscr{T} \mathscr{S} \mathscr{P} \rightarrow \mathscr{S} \mathscr{P}_{1}, \quad \text { and } \quad \mathscr{E}: \mathscr{L} \rightarrow \mathscr{E} \mathscr{X}
$$

are equivalences.

Proof. - It is clear that $\mathscr{L}$ and $\mathscr{S} \mathscr{P}_{1}$ are gerbes. The fact that $i_{1}^{*}$ is an equivalence is proven in Proposition 5.30, and it follows that $\mathscr{T} \mathscr{S} \mathscr{P}$ is also a gerbe. Thus, it suffices to prove that the composition $i_{1}^{*} \circ \mathscr{B}: \mathscr{L} \rightarrow \mathscr{S} \mathscr{P}_{1}$ is an equivalence. Let us show that, for any lifting $\tilde{\mathrm{X}}^{\prime}$, the group of automorphisms of $\tilde{\mathrm{X}}^{\prime}$ reducing to the identity on $\mathrm{X}^{\prime}$ maps isomorphically to the group of automorphisms of $\left(i_{1}^{*} \mathscr{B}_{\mathscr{X} / \mathscr{S}}, \alpha\right)$. Indeed, the first group can be identified with the group of vector fields on $\mathrm{X}^{\prime}$, and

\footnotetext{
${ }^{13}$ Note that the étale topologies of $\mathbf{X}, \mathbf{X}^{\prime}$, and $\hat{\mathbf{T}}_{\mathbf{X}^{\prime} / \mathbf{S}}^{* \gamma}$ are the same.
} 
the second one with the group of invertible functions on $\mathrm{X}_{1}^{\prime}$ equal to 1 on $\mathrm{X}^{\prime}$, and the map is the obvious isomorphism between this two groups. The following easy and well known result completes the proof.

Lemma 4.6. - Let $\mathrm{F}: \mathscr{M} \rightarrow \mathscr{N}$ be a morphism of gerbes on $\mathrm{Y}_{\text {ét. }}$. Assume that for every étale morphism $\mathrm{U} \rightarrow \mathrm{Y}$ and every object $\mathrm{C} \in \mathscr{M}(\mathrm{U})$ the induced map

$$
\mathrm{F}_{*}: \operatorname{Aut}(\mathrm{C}) \rightarrow \operatorname{Aut}(\mathrm{F}(\mathrm{C}))
$$

is an isomorphism. Then $\mathrm{F}$ is an equivalence of gerbes.

Let $\theta \in \mathrm{T}_{\mathrm{X}^{\prime} / \mathrm{S}}(\mathrm{U})$ be a vector field on $\mathrm{U} \subset \mathrm{X}^{\prime}$. We may view $\theta$ as a linear function on the cotangent space $\mathbf{T}_{\mathrm{U} / \mathrm{S}}^{*}$. Then the exponential $\exp (\theta)=\sum \frac{\theta^{i}}{i !}$ makes sense as an invertible function on the completed PD envelope $\mathbf{T}_{\mathrm{U} / \mathrm{S}}^{* \gamma} \subset \mathbf{T}_{\mathrm{X}^{\prime} / \mathrm{S}}^{* \gamma}$. Thus we get a homomorphism of sheaves:

$$
\exp : \mathrm{T}_{\mathrm{X}^{\prime} / \mathrm{S}} \rightarrow \mathscr{O}_{\mathrm{T}_{\mathrm{X}^{\prime} / \mathrm{S}}^{* \gamma}}^{*}=\left(\hat{\Gamma} \mathrm{T}_{\mathrm{X}^{\prime} / \mathrm{S}}\right)^{*}
$$

This, in turn, gives a map:

$$
\exp : \mathrm{H}_{\hat{e} t}^{*}\left(\mathrm{X}^{\prime} ; \mathrm{T}_{\mathrm{X}^{\prime} / \mathrm{S}}\right) \rightarrow \mathrm{H}_{e t}^{*}\left(\mathrm{X}^{\prime} ; \mathscr{O}_{\mathbf{T}_{\mathrm{X}^{\prime} / \mathrm{S}}^{* \nu}}^{*}\right) .
$$

In the following corollary we use $\hat{\Gamma} \mathrm{F}_{\mathrm{X} / \mathrm{S}}^{*} \mathrm{~T}_{\mathrm{X}^{\prime} / \mathrm{S}}$-module structure on $\mathscr{B}_{\mathscr{X} / \mathscr{S}}$ as introduced in Subsection 2.

\section{Corollary $\mathbf{4 . 7}$}

1. Let $\theta \in \mathrm{H}^{0}\left(\mathrm{X}^{\prime}, \mathrm{T}_{\mathrm{X}^{\prime} / \mathrm{S}}\right)$ be an automorphism of a lifting $\mathscr{X} / \mathscr{S}$ reducing to the identity on $\mathrm{X}^{\prime}$. Then the induced morphism

$$
\theta_{*}: \mathscr{B}_{\mathscr{X} / \mathscr{S}} \rightarrow \mathscr{B}_{\mathscr{X} / \mathscr{S}}
$$

is the multiplication by $\mathrm{F}_{\mathrm{X} / \mathrm{S}}^{*}(\exp \theta) \in\left(\hat{\Gamma} \mathrm{F}_{\mathrm{X} / \mathrm{S}}^{*} \mathrm{~T}_{\mathrm{X}^{\prime} / \mathrm{S}}\right)^{*}$.

2. Let $(\mathscr{X} / \mathscr{S})_{1},(\mathscr{X} / \mathscr{S})_{2}$ be liftings, and let $\mathscr{L}_{\mathrm{Id}}$ be the $\mathrm{T}_{\mathrm{X}^{\prime} / \mathrm{S}}$-torsor of isomorphisms between $\tilde{\mathrm{X}}_{1}$ and $\tilde{\mathrm{X}}_{2}^{\prime}$ reducing to the identity on $\mathrm{X}^{\prime}$. Denote by exp $\mathscr{L}_{\mathrm{Id}}$ the corresponding $\mathscr{O}_{\mathbf{T}_{\mathrm{X}^{\prime} / \mathrm{S}}^{* \gamma}}^{*}$-torsor and by $\mathscr{K}_{\mathrm{Id}}$ the corresponding invertible sheaf on $\mathbf{T}_{\mathrm{X}^{\prime} / \mathrm{S}}^{* \gamma}$. Then the isomorphism of $\mathrm{F}_{\mathrm{X} / \mathrm{S}}^{*} \mathrm{~T}_{\mathrm{X}^{\prime} / \mathrm{S}}$-torsors $\mathscr{L}_{\mathscr{X} / \mathscr{S}_{1}} \otimes_{\mathrm{F}_{\mathrm{X} / \mathrm{S}}^{*} \mathrm{~T}^{\prime} / \mathrm{S}} \mathrm{F}_{\mathrm{X} / \mathrm{S}}^{*} \mathscr{L}_{\mathrm{Id}} \simeq \mathscr{L}_{\mathscr{X} / \mathscr{S}_{2}}$ induces a tensor isomorphism of splitting modules

$$
\mathscr{B}_{(\mathscr{X} / \mathscr{S})_{1}} \otimes_{\hat{\Gamma}_{\mathrm{X} / \mathrm{S}}^{*} \mathrm{~T}^{\prime} / \mathrm{S}} \mathrm{F}_{\mathrm{X} / \mathrm{S}}^{*} \mathscr{K}_{\mathrm{Id}} \simeq \mathscr{B}_{(\mathscr{X} / \mathscr{S})_{2}} .
$$


3. The class of the Azumaya algebra $\mathscr{D} \mathrm{X} / \mathrm{S}$ restricted to $\mathbf{T}_{\mathrm{X}^{\prime} / \mathrm{S}}^{* \gamma}$ in the cohomological Brauer group $\operatorname{Br}\left(\mathbf{T}_{\mathrm{X}^{\prime} / \mathrm{S}}^{* \gamma}\right)=\mathrm{H}_{\hat{e} t}^{2}\left(\mathrm{X}^{\prime} ; \mathscr{O}_{\mathbf{T}_{\mathrm{X}^{\prime} / \mathrm{S}}^{* \gamma}}^{*}\right)$ is equal to $\exp \delta$, where $\delta \in \mathrm{H}_{\hat{\mathrm{e}} t}^{2}\left(\mathrm{X}^{\prime} ; \mathrm{T}_{\mathrm{X}^{\prime} / \mathrm{S}}\right)$ is the obstruction to lifting of $\mathrm{X}^{\prime}$ over $\tilde{\mathrm{S}}$.

Proof. - Since $\theta_{*}$ and $\exp \theta$ are tensor automorphisms of $\mathscr{B}_{\mathscr{X} / \mathscr{S}}$, by Theorem 4.5 it is enough to check that $\theta_{*}$ and $\exp \theta$ are equal when restricted to $i_{1}^{*} \mathscr{B}_{\mathscr{X} / \mathscr{S}}$. In turn, this follows from the fact that the automorphism of $\mathscr{L}_{\mathscr{X} / \mathscr{S}}$ induced by the automorphism of the lifting $\mathscr{X} / \mathscr{S}$ coincides with the translation by $\mathrm{F}_{\mathrm{X} / \mathrm{S}}^{*} \theta \in \mathrm{H}^{0}\left(\mathrm{X}, \mathrm{F}_{\mathrm{X} / \mathrm{S}}^{*} \mathrm{~T}_{\mathrm{X}^{\prime} / \mathrm{S}}\right)$. This proves (1). The proof of the second claim is similar, and the last claim follows from Proposition 5.32.

Remark 4.8. - The construction of the tensor splittings in the proof of Proposition 5.32 can be viewed in the present setting as follows. Let $\exp \mathscr{L}_{\mathscr{X} / \mathscr{S}}$ be the pushforward of the $\mathrm{F}_{\mathrm{X} / \mathrm{S}}^{*} \mathrm{~T}_{\mathrm{X}^{\prime} / \mathrm{S}}$-torsor $\mathscr{L}_{\mathscr{X} / \mathscr{S}}$ via the homomorphism

$$
\exp : \mathrm{F}_{\mathrm{X} / \mathrm{S}}^{*} \mathrm{~T}_{\mathrm{X}^{\prime} / \mathrm{S}} \rightarrow\left(\hat{\Gamma} \mathrm{F}_{\mathrm{X} / \mathrm{S}}^{*} \mathrm{~T}_{\mathrm{X}^{\prime} / \mathrm{S}}\right)^{*}
$$

The $\left(\hat{\Gamma} \mathrm{F}_{\mathrm{X} / \mathrm{S}}^{*} \mathrm{~T}_{\mathrm{X}^{\prime} / \mathrm{S}}\right)^{*}$-torsor $\exp \mathscr{L}_{\mathscr{X} / \mathscr{S}}$ acquires the induced connection, as does the associated invertible $\hat{\Gamma} \mathrm{F}^{*} \mathrm{~T}_{\mathrm{X}^{\prime} / \mathrm{S}}$-module $\exp \mathscr{L}_{\mathscr{X} / \mathscr{S}} \otimes_{\left(\hat{\Gamma} \mathrm{F}_{\mathrm{X} / \mathrm{S}}^{*} \mathrm{~T}_{\mathrm{X}^{\prime} / \mathrm{S}}\right)^{*}} \hat{\Gamma} \mathrm{F}_{\mathrm{X} / \mathrm{S}}^{*} \mathrm{~T}_{\mathrm{X}^{\prime} / \mathrm{S}}$. We then have a horizontal isomorphism

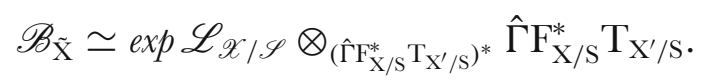

Let us end by explaining the relationships between the various liftings, splittings, and extensions we have been considering. Consider the exact sequence of $\mathscr{O}_{\mathrm{X}^{\prime-}}$ modules:

$$
0 \rightarrow \mathrm{F}_{\mathrm{X} / \mathrm{S} *} \mathrm{~B}_{\mathrm{X} / \mathrm{S}}^{1} \rightarrow \mathrm{F}_{\mathrm{X} / \mathrm{S} *} \mathrm{Z}_{\mathrm{X} / \mathrm{S}}^{1} \rightarrow \mathrm{F}_{\mathrm{X} / \mathrm{S} *} \mathscr{H}_{\mathrm{DR}}^{1}(\mathrm{X} / \mathrm{S}) \rightarrow 0
$$

A splitting of this sequence amounts to lifting $\zeta$ of $\mathrm{C}_{\mathrm{X} / \mathrm{S}}^{-1}$ as in (1.9.2). Let $\mathbf{M}_{\mathrm{X} / \mathrm{S}}$ denote the sheaf on $\mathrm{X}$ which to every open set $\mathrm{U}$ assigns the set of liftings of $\mathrm{C}_{\mathrm{X} / \mathrm{S}}^{-1}$ over $\mathrm{U}$. If $\mathrm{U}$ is an open subset of $\mathrm{X}$, let $\mathbf{L}_{\mathrm{X} / \mathrm{S}}(\mathrm{U})$ denote the category whose objects are morphisms $\tilde{\mathrm{F}}: \tilde{\mathrm{U}} \rightarrow \tilde{\mathrm{U}}^{\prime}$ lifting the relative Frobenius morphism $\mathrm{F}_{\mathrm{U} / \mathrm{S}}: \mathrm{U} \rightarrow \mathrm{U}^{\prime}$ and whose morphisms $\tilde{\mathrm{F}}_{1} \rightarrow \tilde{\mathrm{F}}_{2}$ are commutative diagrams

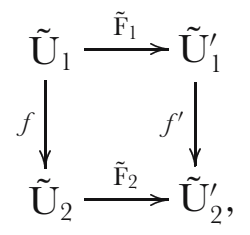

where $f$ and $f^{\prime}$ reduce to the identity modulo $p$. In particular, $f$ and $f^{\prime}$ are necessarily isomorphisms, and $\mathbf{L}_{\mathrm{X} / \mathrm{S}}$ defines a stack over $\mathrm{S}$. As a variant, consider the stack $\mathbf{J}_{\mathrm{X} / \mathrm{S}}$ which over each $\mathrm{U}$ is the category whose objects are pairs $\left(\tilde{\mathbf{U}}^{\prime}, s\right)$, where $\tilde{\mathbf{U}}^{\prime}$ is a lift of 
$\mathrm{U}^{\prime}$ and $s$ is a section of the torsor $\mathscr{L}_{\mathscr{U} / \mathscr{S}}$ defined by $\tilde{\mathrm{U}}^{\prime}$ as in Theorem 1.1 and whose morphisms are those reducing to the identity and compatible with $s$. If we are given a fixed lifting $\tilde{\mathrm{X}}^{\prime} / \tilde{\mathrm{S}}$ of $\mathrm{X}^{\prime} / \mathrm{S}$, then we can also consider the fibered category $\mathbf{L}_{\mathscr{X} / \mathscr{S}}$ which to every open set $\mathrm{U}$ in $\mathrm{X}$ assigns the category of pairs $(\tilde{\mathrm{U}}, \tilde{\mathrm{F}})$, where $\tilde{\mathrm{U}}$ is a lift of $\mathrm{U}$ and $\tilde{\mathrm{F}}: \tilde{\mathrm{U}} \rightarrow \tilde{\mathrm{X}}^{\prime}$ is a lift of $f_{\mathrm{U} / \mathrm{S}}$. Morphisms in this category are diagrams as above, in which $f^{\prime}$ is the identity. If $\tilde{\mathrm{U}}$ is a fixed lifting of $\mathrm{U}$, recall that $\mathscr{L}_{\mathscr{X} / \mathscr{S}}(\tilde{\mathrm{U}})$ is the set of all liftings of $f_{\mathrm{U} / \mathrm{S}}$, so there is a natural map from $\mathscr{L}_{\mathscr{X} / \mathscr{S}}$ to the sheaf of objects of $\mathbf{L}_{\mathscr{X} / \mathscr{S}}$. Finally, if $\tilde{\mathrm{F}}_{\mathrm{S}}: \tilde{\mathrm{S}} \rightarrow \tilde{\mathrm{S}}$ is a lift of the Frobenius endomorphism of $\mathrm{S}$ we can define a more rigid version of $\mathbf{L}_{\mathrm{X} / \mathrm{S}}$. If $\mathrm{U}$ is an open subset of $\mathrm{X}$, let $\mathbf{K}_{\mathrm{X} / \mathrm{S}}(\mathrm{U})$ denote the subcategory of $\mathbf{L}_{\mathrm{X} / \mathrm{S}}(\mathrm{U})$ whose objects are liftings $\tilde{\mathrm{F}}: \tilde{\mathrm{U}} \rightarrow \tilde{\mathrm{U}}^{\prime}$ of $\mathrm{F}_{\mathrm{U} / \mathrm{S}}$ with $\tilde{\mathrm{U}}^{\prime}=\tilde{\mathrm{S}} \times_{\tilde{\mathrm{F}}_{\mathrm{S}}} \tilde{\mathrm{U}}$ and whose morphisms are diagrams as above with $f^{\prime}=f \times_{\tilde{F}_{\mathrm{S}}} \mathrm{id}$.

Proposition 4.9. - Let $\overline{\mathbf{L}}_{\mathrm{X} / \mathrm{S}}$ denote the sheaf associated to the presheaf of isomorphism classes of objects of $\mathbf{L}_{\mathrm{X} / \mathrm{S}}$, and use the analogous notation for $\mathbf{L}_{\mathscr{X} / \mathscr{S}}$.

1. The stack $\mathbf{J}_{\mathrm{X} / \mathrm{S}}$ is rigid, and the natural map $\mathbf{L}_{\mathrm{X} / \mathrm{S}} \rightarrow \mathbf{J}_{\mathrm{X} / \mathrm{S}}$ induces an isomorphism $\overline{\mathbf{L}}_{\mathrm{X} / \mathrm{S}} \rightarrow \mathbf{J}_{\mathrm{X} / \mathrm{S}}$.

2. The map (1.9.3) $\tilde{\mathrm{F}} \mapsto \zeta_{\tilde{\mathrm{F}}}$ induces an isomorphism $\overline{\mathbf{L}}_{\mathrm{X} / \mathrm{S}} \rightarrow \mathbf{M}_{\mathrm{X} / \mathrm{S}}$ and hence also $\mathbf{J}_{\mathrm{X} / \mathrm{S}} \cong \mathbf{M}_{\mathrm{X} / \mathrm{S}}$.

3. The natural map $\mathscr{L}_{\mathscr{X} / \mathscr{S}, \mathrm{X}} \rightarrow \overline{\mathbf{L}}_{\mathscr{X} / \mathscr{S}}$ is an isomorphism.

4. If $\tilde{\mathrm{F}}_{\tilde{\mathrm{S}}}$ lifts $\mathrm{F}_{\mathrm{S}}$, then $\mathbf{K}_{\mathrm{X} / \mathrm{S}}$ is rigid, and if $\mathrm{S}$ is the spectrum of a perfect field, then $\tilde{\mathrm{F}} \mapsto \zeta_{\tilde{\mathrm{F}}}$ induces an isomorphism $\mathbf{K}_{\mathrm{X} / \mathrm{S}} \rightarrow \mathbf{M}_{\mathrm{X} / \mathrm{S}}$.

Proof. - The following lemma follows from standard deformation theory and Remark 1.2; we omit its proof.

Lemma 4.10. - Let $\tilde{\mathrm{X}}$ and $\tilde{\mathrm{X}}^{\prime}$ be liftings of $\mathrm{X}$ and $\mathrm{X}^{\prime}$ respectively. Then

1. The sheaf of liftings $\tilde{\mathrm{F}}: \tilde{\mathrm{X}} \rightarrow \tilde{\mathrm{X}}^{\prime}$ of $\mathrm{F}_{\mathrm{X} / \mathrm{S}}$ is a torsor under $\mathrm{F}_{\mathrm{X} / \mathrm{S}}^{*} \mathrm{~T}_{\mathrm{X}^{\prime} / \mathrm{S}}$, under the standard action.

2. If $\tilde{\mathrm{F}}_{1}$ and $\tilde{\mathrm{F}}_{2}: \tilde{\mathrm{X}} \rightarrow \tilde{\mathrm{X}}^{\prime}$ lift $\mathrm{F}_{\mathrm{X} / \mathrm{S}}$ and differ by a section $h^{\prime}$ of $f_{\mathrm{X} / \mathrm{S}}^{*} \mathrm{~T}_{\mathrm{X}^{\prime} / \mathrm{S}}$, then $\tilde{\mathrm{F}}_{1}$ is isomorphic to $\tilde{\mathrm{F}}_{2}$ in $\mathbf{L}_{\mathrm{X} / \mathrm{S}}$ if and only if $h^{\prime}$ comes from a section of $\mathrm{T}_{\mathrm{X}^{\prime} / \mathrm{S}}$.

3. If $f$ is an automorphism of $\tilde{\mathrm{X}}$ lifting the identity, then $\tilde{\mathrm{F}} \circ f=\tilde{\mathrm{F}}$; if $f^{\prime}$ is an automorphism of $\tilde{\mathrm{X}}^{\prime}$ lifting the identity such that $f^{\prime} \circ \tilde{\mathrm{F}}=\tilde{\mathrm{F}}$, then $f^{\prime}=\mathrm{id}$.

4. The sheaf of automorphisms of an object $\tilde{\mathrm{F}}$ of $\mathbf{L}_{\mathrm{X} / \mathrm{S}}$ is canonically isomorphic to $\mathrm{T}_{\mathrm{X} / \mathrm{s}}$.

Suppose that $\left(\tilde{\mathrm{U}}^{\prime}, s\right)$ is a section of $\mathbf{J}_{\mathrm{X} / \mathrm{S}}$ over $\mathrm{U}$. Then, locally on $\mathrm{U}$, there exist a lift $\tilde{\mathrm{U}}$ of $\mathrm{U}$ and a lift $\tilde{\mathrm{F}}: \tilde{\mathrm{U}} \rightarrow \tilde{\mathrm{U}}^{\prime}$ inducing $s$. Then an automorphism $f$ of $\left(\tilde{\mathrm{U}}^{\prime}, s\right)$ corresponds to an automorphism of $\tilde{\mathbf{U}}^{\prime}$ reducing to the identity and such that $f \circ \tilde{\mathrm{F}}=\tilde{\mathrm{F}}$. By Lemma 4.10, $f$ is the identity, i.e., $\mathbf{J}$ is rigid. It follows that the natural functor $\mathbf{L}_{\mathrm{X} / \mathrm{S}} \rightarrow \mathbf{J}_{\mathrm{X} / \mathrm{S}}$ factors through $\overline{\mathbf{L}}_{\mathrm{X} / \mathrm{S}}$, and the above argument makes it clear that this morphism is surjective. The injectivity follows from the definitions. 
It follows from the lemma that $\overline{\mathbf{L}}_{\mathrm{X} / \mathrm{S}}$ is a torsor under

$$
\mathrm{C}:=\operatorname{Cok}\left(\mathrm{T}_{\mathrm{X}^{\prime} / \mathrm{S}} \rightarrow \mathrm{F}_{\mathrm{X} / \mathrm{S} *} \mathrm{~F}_{\mathrm{X} / \mathrm{S}}^{*} \mathrm{~T}_{\mathrm{X}^{\prime} / \mathrm{S}}\right) \cong \mathscr{H} o m\left(\Omega_{\mathrm{X}^{\prime} / \mathrm{S}}^{1}, \mathrm{~F}_{\mathrm{X} / \mathrm{S} *} \mathrm{~B}_{\mathrm{X} / \mathrm{S}}^{1}\right)
$$

since

$$
\left(\mathrm{F}_{\mathrm{X} / \mathrm{S} *} \mathscr{O}_{\mathrm{X}}\right) / \mathscr{O}_{\mathrm{X}^{\prime}} \cong \mathrm{F}_{\mathrm{X} / \mathrm{S} *} \mathrm{~B}_{\mathrm{X} / \mathrm{S}}^{1} \subseteq \mathrm{F}_{\mathrm{X} / \mathrm{S}}^{*} \Omega_{\mathrm{X} / \mathrm{S}}^{1},
$$

where as before $\mathrm{B}_{\mathrm{X} / \mathrm{S}}$ is the sheaf of locally exact one-forms. The sheaf $\mathbf{M}_{\mathrm{X} / \mathrm{S}}$ is also naturally a torsor under $\mathscr{H} o m\left(\Omega_{\mathrm{X}^{\prime} / \mathrm{S}}^{1}, \mathrm{~F}_{\mathrm{X} / \mathrm{S} *} \mathrm{~B}_{\mathrm{X} / \mathrm{S}}^{1}\right)$, and the map $\tilde{\mathrm{F}} \mapsto \zeta_{\tilde{\mathrm{F}}}$ factors through $\overline{\mathbf{L}}_{\mathrm{X} / \mathrm{S}}$ :

$$
\overline{\mathbf{L}}_{\mathrm{X} / \mathrm{S}} \rightarrow \mathbf{M}_{\mathrm{X} / \mathrm{S}}: \tilde{\mathrm{F}} \mapsto \zeta_{\tilde{\mathrm{F}}}
$$

This map is a morphism of torsors, hence a bijection. Now suppose that $\tilde{\mathrm{F}}_{\tilde{\mathrm{S}}}$ exists and suppose that $\tilde{\mathrm{F}}$ is an object of $\mathbf{K}_{\mathrm{X} / \mathrm{S}}(\mathrm{U})$. Then an automorphism of $\tilde{\mathrm{F}}$ is an automorphism $\tilde{f}$ of $\tilde{\mathrm{U}}$ lifting $\operatorname{id}_{\mathrm{U}}$ such that $\tilde{f^{\prime}} \tilde{\mathrm{F}}=\tilde{\mathrm{F}} \tilde{f}$. where $\tilde{f}^{\prime}:=\tilde{f} \times_{\tilde{\mathrm{F}}_{\tilde{\mathrm{S}}}}$ id. But then it follows from the lemma that $\tilde{f}=\mathrm{id}$, so $\mathbf{K}_{\mathrm{X} / \mathrm{S}}$ is rigid and its presheaf of isomorphism classes is a sheaf. Let $\tilde{\mathrm{F}}_{1}$ and $\tilde{\mathrm{F}}_{2}$ be two objects of $\mathbf{K}_{\mathrm{X} / \mathrm{S}}(\mathrm{U})$. After shrinking $\mathrm{U}$, $\tilde{\mathrm{U}}_{1}$ and $\tilde{\mathrm{U}}_{2}$ become isomorphic; let us assume they are equal. Then $\tilde{\mathrm{F}}_{1}$ is isomorphic to $\tilde{\mathrm{F}}_{2}$ if and only if there exists a lifting $\tilde{f}$ of the identity such that $\tilde{\mathrm{F}}_{2}=\tilde{f}^{\prime} \tilde{\mathrm{F}}_{1} f^{-1}$. But $\tilde{f}^{\prime} \tilde{\mathrm{F}}_{1} f^{-1}=\tilde{f}^{\prime} \tilde{\mathrm{F}}_{1}$, and if $\tilde{f}$ corresponds to an element $\mathrm{D}$ of $\mathrm{T}_{\mathrm{X} / k}, \tilde{f}^{\prime} \tilde{\mathrm{F}}_{1}$ differs from $\tilde{\mathrm{F}}_{1}$ by the action of $\pi^{*} \mathrm{D}$. This shows that $\mathbf{K}_{\mathrm{X} / \mathrm{S}}$ is a torsor under the cokernel of the map

$$
\pi^{1} \mathrm{~T}_{\mathrm{X} / \mathrm{S}} \stackrel{\pi *}{\rightarrow} \mathrm{T}_{\mathrm{X}^{\prime} / \mathrm{S}} \rightarrow \mathrm{F}_{\mathrm{X} / \mathrm{S} *} \mathrm{~F}_{\mathrm{X} / \mathrm{S}}^{*} \mathrm{~T}_{\mathrm{X}^{\prime} / \mathrm{S}}
$$

When $\mathrm{S}$ is the spectrum of a perfect field, $\pi^{*}$ is an isomorphism, and it follows that $\mathbf{K}_{\mathrm{X} / \mathrm{S}}$ is also a torsor under $\mathrm{C}$.

Statement (3) can be checked at the stalks. Let $x$ be a point of $\mathrm{U} \subseteq \mathrm{X}$ and let $\tilde{\mathrm{U}}_{1}$ be a liftings of $\mathrm{U}$. Then the stalk of $\mathscr{L}_{\mathscr{X} / \mathscr{S}, \mathrm{U}}=\mathscr{L}_{\mathscr{X} / \mathscr{S}, \tilde{\mathrm{U}}_{1}}$ at $x$ is the set of germs at $x$ of lifts of $f_{\mathrm{U} / \mathrm{S}}$ to $\tilde{\mathrm{U}}_{1}$, and the stalk of $\overline{\mathbf{L}}_{\mathscr{X} / \mathscr{S}}$ at $x$ is the set of germs of isomorphism classes of of lifts $\left(\tilde{\mathrm{U}}_{2}, \tilde{\mathrm{F}}\right)$ of $f_{\mathrm{U} / \mathrm{s}}$. Let $\tilde{\mathrm{F}}: \tilde{\mathrm{U}}_{2} \rightarrow \tilde{\mathrm{X}}^{\prime}$ be a lift of $f_{\mathrm{U} / \mathrm{S}}$ in some neighborhood of $x$. Then there is an isomorphism $\tilde{\mathrm{U}}_{1} \cong \tilde{\mathrm{U}}_{2}$ near $x$, and this shows that the map is surjective. For the injectivity, observe that if $\tilde{\mathrm{F}}$ and $\tilde{\mathrm{F}}^{\prime}$ are elements of $\mathscr{L}_{\mathscr{X} / \mathscr{S}}\left(\tilde{\mathrm{U}}_{1}\right)$ which become equal in $\overline{\mathbf{L}}_{\mathscr{X} / \mathscr{S}, \mathrm{U}}$, then there is an automorphism of $\tilde{\mathrm{U}}_{1}$ which is the identity $\bmod p$ and which takes $\tilde{\mathrm{F}}$ to $\tilde{\mathrm{F}}^{\prime}$. But then by Remark $1.2, \tilde{\mathrm{F}}=\tilde{\mathrm{F}}^{\prime}$. This shows the injectivity.

\subsection{Line bundles with connection}

We use the following notation. If $\mathrm{X}$ is a scheme over a field $k, \mathrm{E}$ is a coherent sheaf of $\mathscr{O}_{\mathrm{X}}$-modules on $k$, and $\mathrm{S}$ is a $k$-scheme,

$$
\mathbf{H}^{i}(\mathrm{X}, \mathrm{E})(\mathrm{S}):=\mathrm{H}^{0}\left(\mathrm{~S}, \mathscr{O}_{\mathrm{S}}\right) \otimes_{k} \mathrm{H}^{i}(\mathrm{X}, \mathrm{E}) .
$$


If $\mathrm{H}^{i}(\mathrm{X}, \mathrm{E})$ is finite dimensional, the functor $\mathbf{H}^{i}(\mathrm{X}, \mathrm{E})$ is represented by the (vector) $k$-scheme Spec $\mathrm{S}^{\cdot} \mathrm{H}^{i}(\mathrm{X}, \mathrm{E})^{\vee}$.

Let $\mathrm{X} / k$ be a smooth proper geometrically connected scheme over a perfect field of characteristic $p>0$, with a $k$-rational point $x_{0}$. Let $\mathbf{P i c}_{\mathrm{X}}^{\natural}(\mathrm{S})$ denote the set of isomorphism classes of triples $(\mathrm{L}, \nabla, \alpha)$, where $\mathrm{L}$ is an invertible sheaf on $\mathrm{X} \times \mathrm{S}$, $\nabla$ is an integrable connection on $\mathrm{L}$ relative to $\mathrm{S}$, and $\alpha$ is an isomorphism $\mathrm{L} \cong \mathscr{O}_{\mathrm{X}}$ over $x_{0} \times \mathrm{S}$. Forgetting $\nabla$ defines a morphism $b$ from Pic $_{\mathrm{X}}^{\natural}$ to the Picard scheme Pic $\mathrm{X}$ of $\mathrm{X}$. If $\mathrm{L}$ is an invertible sheaf on $\mathrm{X} \times \mathrm{S}$, the set of integrable connections on $\mathrm{L}$ is either empty or a torsor under the group $\mathbf{H}^{0}\left(\mathrm{X}, \mathrm{Z}_{\mathrm{X} \times \mathrm{S} / \mathrm{S}}^{1}\right)$ of closed one-forms on $\mathrm{X} \times \mathrm{S} / \mathrm{S}$. Note that formation of the latter commutes with base change and that $\mathrm{H}^{0}\left(\mathrm{X}, \mathrm{Z}_{\mathrm{X} / k}^{1}\right) \cong \mathrm{H}^{0}\left(\mathrm{X}^{\prime}, \mathrm{F}_{*} \mathrm{Z}_{\mathrm{X} / k}^{1}\right)$. Thus $\mathrm{H}^{0}\left(\mathrm{X}, \mathrm{Z}_{\mathrm{X} \times \mathrm{S} / \mathrm{S}}^{1}\right) \cong \mathbf{H}^{0}\left(\mathrm{X}^{\prime}, \mathrm{F}_{*} \mathrm{Z}_{\mathrm{X} / k}^{1}\right)(\mathrm{S})$. The Chern class map dlog: $\mathscr{O}_{\mathrm{X}}^{*} \rightarrow \mathrm{Z}_{\mathrm{X} / k}^{1}$ defines a morphism $c: \mathbf{P i c}_{\mathrm{X}} \rightarrow \mathbf{H}^{1}\left(\mathrm{X}, \mathrm{Z}_{\mathrm{X} / k}^{1}\right)$, and there is thus an exact sequence:

$$
0 \rightarrow \mathbf{H}^{0}\left(\mathrm{X}, \mathrm{Z}_{\mathrm{X} / k}^{1}\right) \rightarrow \mathbf{P i c}_{\mathrm{X}}^{\natural} \stackrel{b}{\rightarrow} \mathbf{P i c}_{\mathrm{X}} \stackrel{c}{\rightarrow} \mathbf{H}^{1}\left(\mathrm{X}, \mathrm{Z}_{\mathrm{X} / k}^{1}\right) .
$$

The proof of the following is then immediate (and well-known).

Proposition 4.11. - The above sequence is exact as a sequence of sheaves in the flat topology. Furthermore, the functor $\mathbf{P} \mathbf{i c}_{\mathrm{X}}^{\natural}$ is representable, and its tangent space at the origin is canonical isomorphic to $\mathrm{H}_{d \mathrm{R}}^{1}(\mathrm{X} / k)$.

If $(\mathrm{L}, \nabla)$ is an object of $\mathbf{P}$ ic $\mathrm{X}_{\mathrm{X}}^{\natural}(\mathrm{S})$, its $p$-curvature can be viewed as an element of $\mathrm{H}^{0}\left(\mathrm{X}^{\prime} \times \mathrm{S}, \Omega_{\mathrm{X}^{\prime} \times \mathrm{S} / \mathrm{S}}^{1}\right)$. This defines a morphism of group schemes $\psi: \mathbf{P i c}_{\mathrm{X}}^{\natural} \rightarrow$ $\mathbf{H}^{0}\left(\mathrm{X}^{\prime}, \Omega_{\mathrm{X}^{\prime} / k}^{1}\right)$. If $\mathrm{L}^{\prime}$ is an invertible sheaf on $\mathrm{X}^{\prime} \times \mathrm{S}$ trivialized along $x_{0}^{\prime} \times \mathrm{S}$, then $\left(\mathrm{F}_{\mathrm{X} / \mathrm{S}} \times \mathrm{id}_{\mathrm{S}}\right)^{*} \mathrm{~L}$ is an invertible sheaf on $\mathrm{X} \times \mathrm{S}$, and we can equip it with its canonical Frobenius descent connection to obtain an element of $\mathbf{P i c}_{X}^{\natural}(\mathrm{S})$. This defines a morphism of group schemes $\phi: \mathbf{P i c}_{\mathrm{X}^{\prime}} \rightarrow \mathbf{P i c}_{\mathrm{X}}^{\natural}$. An element in the kernel of $b$ is given by an integrable connection on $\mathscr{O}_{\mathrm{X} \times \mathrm{S}}$, relative to $\mathrm{S}$ i.e., a closed one-form $\omega \in \Omega_{\mathrm{X} \times \mathrm{S} / \mathrm{S}}^{1}$, and the $p$-curvature of the corresponding connection is $\pi_{\mathrm{X} \times \mathrm{S} / \mathrm{S}}^{*}(\omega)-\mathrm{C}_{\mathrm{X} \times \mathrm{S} / \mathrm{S}}(\omega)$, where $\mathrm{C}_{\mathrm{X} \times \mathrm{S} / \mathrm{S}}$ is the Cartier operator $[19,7.22]$. Thus there is a commutative diagram:

$(4.11 .1)$

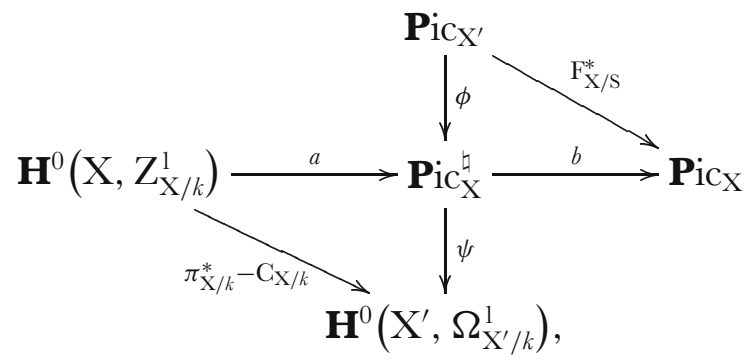

where $\pi_{\mathrm{X} / k}^{*}$ is the composition:

$$
\begin{aligned}
\mathbf{H}^{0}\left(\mathrm{X}, \mathrm{Z}_{\mathrm{X} / k}^{1}\right) & \rightarrow \mathbf{H}^{0}\left(\mathrm{X}, \Omega_{\mathrm{X} / k}^{1}\right) \\
& \rightarrow \mathbf{H}^{0}\left(\mathrm{X}^{\prime}, \Omega_{\mathrm{X}^{\prime} / k}^{1}\right)=\mathbf{H}^{0}\left(\mathrm{X}, \Omega_{\mathrm{X} / k}^{1}\right) \times_{\mathrm{F}_{k}^{*}} k=\mathbf{H}^{0}\left(\mathrm{X}, \Omega_{\mathrm{X} / k}^{1}\right)^{\prime} .
\end{aligned}
$$


Here the map $\mathbf{H}^{0}\left(\mathrm{X}, \Omega_{\mathrm{X} / k}^{1}\right) \rightarrow \mathbf{H}^{0}\left(\mathrm{X}, \Omega_{\mathrm{X} / k}^{1}\right)^{\prime}$ is the relative Frobenius map of the $k$-scheme $\mathbf{H}^{0}\left(\mathrm{X}, \Omega_{\mathrm{X} / k}^{1}\right)$. The map $\mathrm{C}_{\mathrm{X} / k}$ in the diagram is the map of group schemes induced by the linear map of vector spaces

$$
\mathrm{C}_{\mathrm{X} / k}: \mathrm{H}^{0}\left(\mathrm{X}, \mathrm{Z}_{\mathrm{X} / k}^{1}\right) \rightarrow \mathrm{H}^{0}\left(\mathrm{X}^{\prime}, \Omega_{\mathrm{X}^{\prime} / k}^{1}\right)
$$

Recall that there are two spectral sequences converging to de Rham cohomology: the Hodge spectral sequence, with $\mathrm{E}_{1}^{i, j}=\mathrm{H}^{j}\left(\mathrm{X}, \Omega_{\mathrm{X} / k}^{i}\right)$, and the conjugate spectral sequence, with $\mathrm{E}_{2}^{i, j}=\mathrm{H}^{i}\left(\mathrm{X}, \mathscr{H}_{d \mathrm{R}}^{j}\right) \cong \mathrm{H}^{i}\left(\mathrm{X}^{\prime}, \Omega_{\mathrm{X}^{\prime} / k}^{j}\right)$.

Lemma 4.12. - In the diagram of tangent spaces corresponding to Diagram (4.11.1),

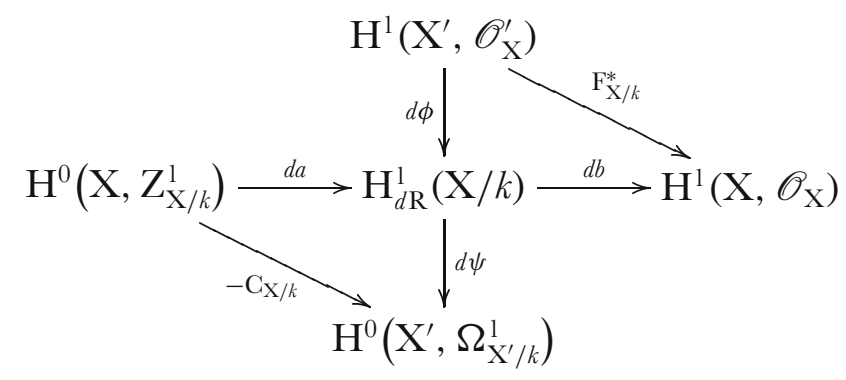

$d b(r e s p .-d \psi)$ is the edge homomorphism coming from the Hodge (resp. conjugate) spectral sequence, and $d \psi \circ d a=-\mathrm{C}_{\mathrm{X} / k}$.

Proof. - Since $\pi_{\mathrm{X} / k}^{*}$ in Diagram (4.11.1) factors through the relative Frobenius map in Formula (4.11.2) above, its differential is zero. Since $\mathrm{C}_{\mathrm{X} / k}$ is $k$-linear, it follows that $d \psi \circ d a=-\mathrm{C}_{\mathrm{X} / k}$. To compute $d \psi$, let $\mathrm{S}:=\operatorname{Spec} k[\epsilon]$, let $\eta \in \mathrm{H}_{\mathrm{DR}}^{1}(\mathrm{X} / k)$ and let $(\mathrm{L}, \nabla)$ be the corresponding line bundle with connection over $\mathrm{X} \times \mathrm{S}$. Then $d \psi(\eta)$ is a section of $\mathrm{H}^{0}\left(\mathrm{X}^{\prime}, \Omega_{\mathrm{X}^{\prime} / k}^{1}\right)$ and is determined by its restriction to any nonempty open subset of $\mathrm{X}^{\prime}$. We can choose an open subset on which $\mathrm{L}$ is trivial, and hence reduce to the previous calculation. This proves the claim.

As we have seen, $\mathrm{F}_{\mathrm{X} / k *} \mathrm{D}_{\mathrm{X} / k}$ defines an Azumaya algebra $\mathscr{D}_{\mathrm{X} / k}$ over $\mathbf{T}_{\mathrm{X}^{\prime} / k}^{*}$; we shall study the splitting of the pullback of this Azumaya algebra along the canonical map $q: \mathrm{X}^{\prime} \times \mathbf{H}^{0}\left(\mathrm{X}^{\prime}, \Omega_{\mathrm{X}^{\prime} / k}^{1}\right) \rightarrow \mathbf{T}_{\mathrm{X}^{\prime} / k}^{*}$. The universal $(\mathrm{L}, \nabla)$ defines a module over the pullback of $\mathscr{D} \mathrm{X} / k$ to $\mathrm{X}^{\prime} \times \mathbf{P i c}_{\mathrm{X}}^{\natural}$, and since it is locally free of rank $p^{\operatorname{dimX}}$, it is a splitting module. More generally, suppose we are given a morphism of $k$-schemes $f: \mathrm{Z} \rightarrow \mathbf{H}^{0}\left(\mathrm{X}^{\prime} / k, \Omega_{\mathrm{X}^{\prime} / k}^{1}\right)$ and a splitting module $\mathrm{L}$ over the pullback of $\mathscr{D} \mathrm{X} / k$ to $\mathrm{X}^{\prime} \times \mathrm{Z}$ via the map $\operatorname{id}_{\mathrm{X}^{\prime}} \times f$. Then $\mathrm{L}$ is a coherent sheaf on $\mathrm{X}^{\prime} \times \mathrm{Z}$ equipped with an action of the differential operators $\mathrm{F}_{\mathrm{X} \times \mathrm{Z} / \mathrm{Z} *}\left(\mathrm{D}_{\mathrm{X} \times \mathrm{Z} / \mathrm{Z}}\right)$, and in particular can be regarded as a coherent sheaf with integrable connection on $\mathrm{X} \times \mathrm{Z} / \mathrm{Z}$ whose $p$-curvature is equal to the section of $\Omega_{\mathrm{X}^{\prime} \times \mathrm{Z} / \mathrm{Z}}^{1}$ defined by $f$. By Proposition 2.3, $\mathrm{L}$ is an invertible sheaf on $\mathrm{X} \times \mathrm{Z}$. By a rigidified splitting of $\mathscr{D} \mathrm{x} / k$ along $f$ we shall mean a pair $(\mathrm{L}, \alpha)$, where $\mathrm{L}$ is 
a splitting module for $\left(\operatorname{id}_{\mathrm{X}^{\prime}} \times f\right)^{*} q^{*} \mathscr{D}_{\mathrm{X} / k}$ and $\alpha$ is a trivialization of the restriction of $\mathrm{L}$ to $x_{0} \times \mathrm{Z}$. Thus the universal $(\mathrm{L}, \nabla, \alpha)$ is a rigidified splitting of $\mathscr{D} \mathrm{x} / k$ along $\psi$.

Proposition 4.13. - Let $f: \mathrm{Z} \rightarrow \mathbf{H}^{0}\left(\mathrm{X}^{\prime}, \Omega_{\mathrm{X} / k}^{1}\right)$ be a morphism and let $(\mathrm{L}, \nabla, \alpha)$ be the universal rigidified line bundle with connection on $\mathrm{X} \times \mathbf{P i c}_{\mathrm{X}}^{\natural}$.

1. The map $f \mapsto f^{*}(\mathrm{~L}, \nabla, \alpha)$ is a bijection between the set of isomorphism classes of rigidified splittings of $\mathscr{D}_{\mathrm{X} / k}$ and the set of maps $\tilde{f}: \mathrm{Z} \rightarrow \mathbf{P i c}_{\mathrm{X}}^{\natural}$ such that $\psi \tilde{f}=f$.

2. If $f$ as above is a morphism of commutative group schemes, then under the the bijection above, the tensor splittings of $(\mathrm{id} \times f)^{*} q^{*} \mathrm{~F}_{*} \mathrm{D}_{\mathrm{X} / k}$ correspond to the group morphisms $\tilde{f}$ with $\psi \tilde{f}=f$.

Proof. - We have seen that a rigidified splitting of $(\mathrm{id} \times f)^{*} q^{*} \mathrm{~F}_{*} \mathrm{D}_{\mathrm{X} / k}$ gives an invertible sheaf $(\mathrm{M}, \nabla, \alpha)$ with connection on $\mathrm{X} \times \mathrm{Z}$ whose $p$-curvature is given by $f$ and a trivialization of $\mathrm{M}$ on $x_{0} \times \mathrm{Z}$. Hence there is a unique map $\tilde{f}: \mathrm{Z} \rightarrow \mathbf{P i c}_{\mathrm{X}}^{\natural}$ such that $\tilde{f}^{*}(\mathrm{~L}, \nabla, \alpha) \cong(\mathrm{M}, \nabla, \alpha)$, and necessarily $\psi \tilde{f}=f$. This completes the proof of (1), and (2) follows immediately.

\subsection{Abelian varieties}

Theorem 4.14. - Let $\mathrm{A}$ be an abelian variety over a perfect field $k$ of characteristic $p$.

1. The Azumaya algebra $\mathrm{F}_{*} \mathrm{D}_{\mathrm{A} / k}$ splits (non-canonically) over the formal completion $\hat{\mathbf{T}}_{\mathrm{A}^{\prime} / k}^{*}{ }^{14}$

2. There exists a tensor splitting of $\mathrm{F}_{*} \mathrm{D}_{\mathrm{A} / k}$ over $\hat{\mathbf{T}}_{\mathrm{A}^{\prime} / k}^{*}$ if and only if $\mathrm{A}$ is ordinary. For an ordinary $\mathrm{A}$, the tensor splitting is unique.

Proof. - It is known [22] that Hodge and conjugate spectral sequences for A degenerate and that $\mathbf{P} i c_{\mathrm{A}}^{\natural}$ is smooth. Thus Lemma 4.12 implies that the differential of $\psi: \mathbf{P i c}_{\mathrm{A}}^{\natural} \rightarrow \mathbf{H}^{0}\left(\mathrm{~A}^{\prime}, \Omega_{\mathrm{A}^{\prime} / k}^{1}\right)$ is surjective, and it follows that $\psi$ is smooth. This implies that $\psi$ has a lifting over the formal completion of $\mathbf{H}^{0}\left(\mathrm{~A}^{\prime}, \Omega_{\mathrm{A} / k}^{1}\right)$ at the origin, and therefore by Proposition 4.13 that $\mathscr{D}_{\mathrm{A} / k}$ splits over $\mathrm{A}^{\prime} \times \hat{\mathbf{H}}^{0}\left(\mathrm{~A}^{\prime}, \Omega_{\mathrm{A}^{\prime} / k}^{1}\right) \cong \hat{\mathbf{T}}_{\mathrm{A}^{\prime} / k}^{*}$.

It follows from Proposition 4.13 that giving a tensor splitting of $\mathrm{F}_{*} \mathrm{D}_{\mathrm{A} / k}$ over $\hat{\mathrm{A}}^{\prime}$ is equivalent to giving a group homomorphism

$$
\tilde{\psi}: \hat{\mathbf{V}} \mathrm{H}^{0}\left(\mathrm{~A}^{\prime}, \Omega_{\mathrm{A}^{\prime}}^{1}\right) \rightarrow \mathbf{P i c}_{\mathrm{A}}^{\natural}
$$

such that $\psi \circ \tilde{\psi}=$ id. The map $b \circ \tilde{\psi}$ necessarily factors through $\mathbf{P} \mathrm{ic}_{\mathrm{A}}^{0}$, and since the latter is $p$-divisible, $b \circ \tilde{\psi}=0$. Hence $\tilde{\psi}$ factors through $a$ in diagram $(4.11 .1)$ and can be viewed as a morphism $\hat{\mathbf{V}} \mathrm{H}^{0}\left(\mathrm{~A}, \Omega_{\mathrm{A}}^{1}\right) \rightarrow \mathbf{H}\left(\mathrm{A}, \mathrm{Z}_{\mathrm{X} / k}^{1}\right)$. Since $\mathrm{H}^{0}\left(\mathrm{~A}, \mathrm{Z}_{\mathrm{X} / k}^{1}\right)=\mathrm{H}^{0}\left(\mathrm{~A}, \Omega_{\mathrm{X} / k}^{1}\right)$, the groups $\hat{\mathbf{V}} \mathrm{H}^{0}\left(\mathrm{~A}, \mathrm{Z}_{\mathrm{A} / k}^{1}\right)$ and $\hat{\mathbf{V}} \mathrm{H}^{0}\left(\mathrm{~A}^{\prime}, \Omega_{\mathrm{A}^{\prime}}^{1}\right)$ are smooth of the same dimension. Thus

14 This result is due to Roman Bezrukavnikov. 
the existence of $\tilde{\psi}$ is equivalent to the differential of $\psi \circ a$ at 0 being an isomorphism. It follows from Lemma 4.12 that this restriction is the negative of the Cartier operator

$$
\mathrm{C}_{\mathrm{A} / k}: \mathrm{H}^{0}\left(\mathrm{~A}, \Omega_{\mathrm{A} / k}^{1}\right) \rightarrow \mathrm{H}^{0}\left(\mathrm{~A}^{\prime}, \Omega_{\mathrm{A}^{\prime} / k}^{1}\right) .
$$

One of the equivalent definitions of an ordinary abelian variety is that $\mathrm{C}_{\mathrm{A} / k}$ is an isomorphism. This proves that lifting $h$ exists if and only if $\mathrm{A}$ is ordinary. Moreover, for an ordinary A the morphism $\psi: \hat{\mathbf{V}} \mathrm{H}^{0}\left(\mathrm{~A}, \Omega_{\mathrm{A}}^{1}\right) \rightarrow \hat{\mathbf{V}} \mathrm{H}^{0}\left(\mathrm{~A}^{\prime}, \Omega_{\mathrm{A}^{\prime}}^{1}\right)$ is an isomorphism. Thus, in this case, the lifting is unique. We could also remark that an ordinary abelian variety over a perfect field of characteristic $p$ has a canonical lifting, together with a lifting of $\mathrm{F}$, and this gives a tensor splitting of $\hat{\mathbf{T}}_{\mathrm{A}^{\prime} / k}^{*}$ by Theorem 2.11.

\subsection{A counterexample: $\mathscr{D}_{\mathrm{X} / k}$ need not split on $\hat{\mathbf{T}}_{\mathrm{X}^{\prime} / \mathrm{S}}$}

In this section, we will construct an example of a smooth proper surface $\mathrm{X} / k$ over a perfect field $k$ which lifts to $\mathrm{W}(k)$ but such that $\mathscr{D}_{\mathrm{x} / k}$ does not split over the formal completion of $\mathrm{T}_{\mathrm{X}^{\prime} / k}^{*}$ along the zero section, or even over the formal completion of $\mathrm{X}^{\prime} \times \mathbf{H}^{0}\left(\mathrm{X}^{\prime}, \Omega_{\mathrm{X}^{\prime} / k}^{1}\right)$ along the zero section.

Lemma 4.15. - Let $\mathrm{X} / k$ be a smooth and proper scheme with a rational point $x_{0}$. Assume that the following properties hold:

1. $\operatorname{dim} \mathrm{H}^{0}\left(\mathrm{X}, \Omega_{\mathrm{X} / k}^{1}\right)=\operatorname{dim} \mathrm{H}^{1}\left(\mathrm{X}, \mathscr{O}_{\mathrm{X}}\right)$,

2. $\mathrm{F}_{\mathrm{X}}$ acts as zero on $\mathrm{H}^{1}\left(\mathrm{X}, \mathscr{O}_{\mathrm{X}}\right)$,

3. The Hodge spectral sequence of $\mathrm{X} / k$ degenerates at $\mathrm{E}_{1}$,

4. $q^{*} \mathrm{~F}_{*} \mathscr{D}_{\mathrm{X} / k}$ splits over the formal completion of $\mathrm{X}^{\prime} \times \mathbf{H}^{0}\left(\mathrm{X}^{\prime}, \Omega_{\mathrm{X}^{\prime} / k}^{1}\right)$ along the zero section.

Then $\mathbf{P i c}_{\mathrm{X}}$ is reduced.

Proof. - It follows from assumption (3) that the Hodge and conjugate spectral sequences of $\mathrm{X} / k$ degenerate at $\mathrm{E}_{1}$ and $\mathrm{E}_{2}$ respectively $[19,2.32]$, and so the row and column of the commutative diagram of Lemma 4.12 are short exact. The map $h:=\mathrm{F}_{\mathrm{X} / k}^{*}$ in the diagram below vanishes by assumption (2). This implies that the map $d \phi$ factors as shown below through $d a$. By (1) and (3) the induced map $h^{\prime}$ is an isomorphism, and it follows that $\mathrm{C}_{\mathrm{X} / k}$ is zero, and hence that $d \psi$ factors through an arrow $h^{\prime \prime}$ as shown.

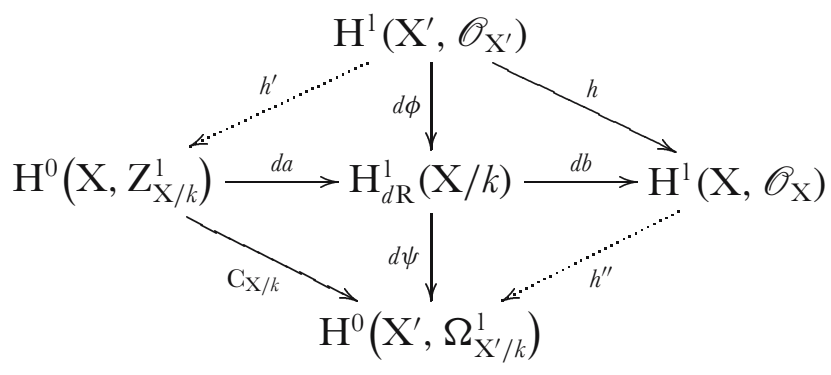


Now suppose that $\mathrm{F}_{\mathrm{X} / k *} \mathrm{D}_{\mathrm{X} / k}$ splits over $\mathrm{X}^{\prime} \times \hat{\mathbf{H}}^{0}\left(\mathrm{X}^{\prime}, \Omega_{\mathrm{X}^{\prime} / k}^{1}\right)$. Choosing a rigidification of the splitting module, we get a lifting $\tilde{\psi}$ of $\psi$ over $\hat{\mathbf{H}}^{0}\left(\mathrm{X}^{\prime}, \Omega_{\mathbf{X}^{\prime} / k}^{1}\right)$, so $d \psi \circ d \tilde{\psi}$ $=$ id. Then $h^{\prime \prime} \circ d b \circ d \tilde{\psi}=$ id, so

$$
d b \circ d \tilde{\psi}: \mathrm{H}^{0}\left(\mathrm{X}^{\prime}, \Omega_{\mathrm{X}^{\prime} / k}^{1}\right) \rightarrow \mathrm{H}^{1}\left(\mathrm{X}, \mathscr{O}_{\mathrm{X}}\right)
$$

is injective. By (1), the source and target have the same dimension, so the differential of the morphism $b \circ \tilde{\psi}$ is an isomorphism. Since $\hat{\mathbf{V}} \mathrm{H}^{0}\left(\mathrm{X}^{\prime}, \Omega_{\mathrm{X}^{\prime} / k}^{1}\right)$ is smooth, this implies that $\mathbf{P}$ ic $_{\mathrm{X}}$ is smooth.

Let $k$ be a perfect field of odd characteristic and let $\mathrm{W}$ be its Witt ring. We construct an example of a smooth projective surface $\tilde{\mathrm{X}} / \mathrm{W}$ whose special fiber $\mathrm{X}$ over $k$ satisfies (1)-(3), but whose Picard scheme is not reduced, using the technique of Serre and its generalization by Raynaud [31, 4.2.3]. Let $\mathrm{E}$ be an elliptic curve over W with supersingular reduction and denote by $\mathrm{G}$ the kernel of multiplication by $p$ in $\mathrm{E}$. By [op. cit.], there exists a projective complete intersection $\tilde{Y}$, flat of relative dimension two over $\mathrm{W}$, with a free action of $\mathrm{G}$ and whose quotient $\tilde{\mathrm{X}}:=\tilde{\mathrm{Y}} / \mathrm{G}$ is smooth over W. By the weak Lefschetz theorem, $\mathbf{P i c}_{\tilde{Y}}^{0}=0$, and it follows that $\mathbf{P} i c_{\tilde{X}}^{0}$ is the Cartier dual of $G$, which can be identified with $G$ itself. Since Pic commutes with base change, the Picard scheme of the special fiber $X$ is the special fiber $G_{0}$ of $G$. In particular $\mathrm{G}_{0}$ is not smooth. Replacing $k$ by a finite extension, we may assume that $\mathrm{X}$ has a rational point. Thus to produce our counterexample, it will suffice to prove that X satisfies (1)-(3) of Lemma 4.15. The degeneration of the Hodge spectral sequence of $\mathrm{X} / k$ follows from its liftability. The endomorphism of $\mathrm{H}^{1}\left(\mathrm{X}, \mathscr{O}_{\mathrm{X}}\right)$ induced by $F_{X}$ corresponds via its identification with the tangent space of $G_{0}$ to the Cartier dual of the endomorphism induced by the Frobenius of $\mathrm{G}_{0}$ which in our case vanishes. Since $\mathbf{P i c}_{\mathrm{X}} \cong \mathrm{G}_{0}, \mathrm{H}^{1}\left(\mathrm{X}, \mathscr{O}_{\mathrm{X}}\right)$ is one-dimensional, and so to prove (1), it will suffice to prove that $\mathrm{H}_{d \mathrm{R}}^{1}(\mathrm{X} / k)$ is two-dimensional. We use Faltings' comparison theorem $[12,5.3]$ which relates the de Rham cohomology $\mathrm{H}_{d \mathrm{R}}^{1}(\mathrm{X} / k)$ to the étale cohomology $\mathrm{H}_{\mathrm{e} t}^{1}\left(\tilde{\mathrm{X}}_{\overline{\mathrm{K}}}, \mathbf{F}_{p}\right)$. In particular, this theorem implies that these have the same dimension. Since $\tilde{\mathrm{X}}_{\overline{\mathrm{K}}}=\tilde{\mathrm{Y}}_{\overline{\mathrm{K}}} / \mathrm{G}_{\overline{\mathrm{K}}}, \mathrm{G}_{\overline{\mathrm{K}}} \cong \mathbf{F}_{p} \oplus \mathbf{F}_{p}$, and $\tilde{\mathrm{Y}}_{\overline{\mathrm{K}}}$ is simply connected, $\mathrm{H}_{\mathrm{e} t}^{1}\left(\tilde{\mathrm{X}}_{\overline{\mathrm{K}}}, \mathbf{F}_{p}\right) \cong \mathbf{F}_{p} \oplus \mathbf{F}_{p}$. Thus $\mathrm{H}_{d \mathrm{R}}^{1}(\mathrm{X} / k)$ is two dimensional, and (2) follows.

\subsection{Fontaine modules}

Throughout this section we assume that $\mathrm{S}$ is a smooth scheme over a field of characteristic $p$.

Definition 4.16. - Let $\mathrm{X} / \mathrm{S}$ be a smooth scheme and let $\mathscr{X} / \mathscr{S}$ be a lifting. Fix integers $k \leq l$ with $l-k<p$. Then a Fontaine module on $\mathscr{X} / \mathscr{S}$ consists of a coherent sheaf with integrable connection $(\mathrm{M}, \nabla)$ of $\mathrm{MIC}(\mathrm{X} / \mathrm{S})$ and $a$ Hodge filtration

$$
0=\mathrm{F}^{l+1} \mathrm{M} \subseteq \mathrm{F}^{l} \mathrm{M} \subseteq \cdots \subseteq \mathrm{F}^{k} \mathrm{M}=\mathrm{M}
$$


satisfying Griffiths transversality, together with an isomorphism

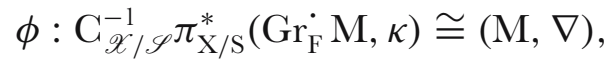

where the Higgs field $\kappa$ is given by the Kodaira-Spencer operator

$$
\mathrm{Gr} \nabla: \mathrm{Gr}_{\mathrm{F}}^{i} \mathrm{M} \rightarrow \mathrm{Gr}_{\mathrm{F}}^{i-1} \mathrm{M} \otimes \Omega_{\mathrm{X} / \mathrm{S}}^{1}
$$

We will denote the category of Fontaine modules by $\mathscr{M} \mathscr{F}_{[k, l]}(\mathscr{X} / \mathscr{S})$. Although we shall not do so here, one can check that if $\mathrm{S}$ is the spectrum of a perfect field $k$, and the lifting $\tilde{\mathrm{X}}^{\prime} \rightarrow \tilde{\mathrm{S}}=$ Spec $\mathrm{W}_{2}(k)$ comes from a smooth formal scheme $\mathrm{X}_{\mathrm{W}(k)}$ over $\mathrm{W}(k)$, the category $\mathscr{M}_{[k, l]}(\mathscr{X} / \mathscr{S})$ is equivalent to the full subcategory of $p$-torsion objects in Faltings' category $\mathscr{M}_{k, l}^{\nabla}\left(\mathrm{X}_{\mathrm{W}(k)}\right)[11]$.

The formula $\mathrm{N}^{m}\left(\mathrm{Gr}_{\mathrm{F}}^{\cdot} \mathrm{M}, \kappa\right)=\bigoplus_{i \leq-m} \operatorname{Gr}_{\mathrm{F}}^{i} \mathrm{M} \subset \mathrm{Gr}_{\mathrm{F}}^{\cdot} \mathrm{M}$ defines a $\mathscr{J}_{\mathrm{X}}$-filtration on $\left(\mathrm{Gr}_{\mathrm{F}} \mathrm{M}, \kappa\right)$. Applying the isomorphism $\phi$ we obtain an $\mathscr{I}_{\mathrm{X}}$-filtration on M:

$$
\mathrm{N}^{m} \mathrm{M}=\mathrm{C}_{\mathscr{X} / \mathscr{S}}^{-1} \pi_{\mathrm{X} / \mathrm{S}}^{*}\left(\bigoplus_{i \leq-m} \mathrm{Gr}_{\mathrm{F}}^{i} \mathrm{M}\right) \subset \mathrm{M}
$$

together with an isomorphism of Higgs modules:

$$
\mathrm{C}_{\mathrm{X} / \mathrm{S}}^{\cdot}\left(\mathrm{Gr}_{\mathrm{N}} \mathrm{M}, \mathrm{Gr} \psi\right) \simeq \pi_{\mathrm{X} / \mathrm{S}}^{*}\left(\operatorname{Gr}_{\mathrm{F}} \mathrm{M}, \mathrm{Gr} \nabla\right)
$$

Theorem 4.17. - Let $\left(\mathrm{M}, \nabla, \mathrm{F}^{*} \mathrm{M}, \phi\right)$ and $\left(\mathrm{M}^{\prime}, \nabla^{\prime}, \mathrm{F}^{*}, \phi^{\prime}\right)$ be Fontaine modules over X. Then

1. For every integer $i$, the $\mathscr{O}_{\mathrm{X}}$-module $\mathrm{Gr}_{\mathrm{F}}^{i} \mathrm{M}$ is locally free. In particular, $\mathrm{M}$ is a locally free $\mathscr{O}_{\mathrm{X}}$-module [11, Theorem 2.1].

2. Every morphism $f: \mathrm{M} \rightarrow \mathbf{M}^{\prime}$ of Fontaine modules is strictly compatible with the Hodge filtration $\mathrm{F}^{\cdot}$. In particular, the category $\mathscr{M}_{\left[{ }_{[k, l]}\right.}(\mathscr{X} / \mathscr{S})$ is abelian [op. cit].

3. Let $h: \mathrm{X} \rightarrow \mathrm{Y}$ be a smooth proper morphism of relative dimension d, let $\tilde{h}^{\prime}: \tilde{\mathrm{X}}^{\prime} \rightarrow \tilde{\mathrm{Y}}^{\prime}$ be a lifting of $h^{\prime}$, and let $\left(\mathrm{M}, \nabla, \mathrm{F}^{*}, \phi\right) \in \mathscr{M}_{[k, l]}(\mathscr{X} / \mathscr{S})$ be a Fontaine module. Assume that $l-k+d<p$. Then, the Hodge spectral sequence for $\mathrm{R} h_{*}^{\mathrm{DR}}\left(\mathrm{M}, \mathrm{F}^{*} \mathrm{M}\right)$ degenerates at $\mathrm{E}_{1}$. Thus, by Theorem 3.22 b), for every integer $i$, we have a canonical isomorphism

$(4.17 .1)$

$$
\phi: \mathrm{C}_{\mathscr{Y} / \mathscr{S}}^{-1} \pi_{\mathrm{Y} / \mathrm{S}}^{*}\left(\mathrm{Gr}_{\mathrm{F}}^{\cdot} \mathrm{R}^{i} h_{*}^{\mathrm{DR}} \mathrm{M}, \kappa\right) \cong\left(\mathrm{R}^{i} h_{*}^{\mathrm{DR}} \mathrm{M}, \nabla\right),
$$

which makes $\left(\mathrm{R}^{i} h_{*}^{\mathrm{DR}} \mathrm{M}, \nabla, \mathrm{F}^{*} \mathrm{R}^{i} h_{*}^{\mathrm{DR}} \mathrm{M}, \phi\right)$ a Fontaine module over $\mathrm{Y}$. In particular, if $d<p$, the $\mathrm{D}_{\mathrm{Y} / \mathrm{S}}$-module $\mathrm{R}^{i} h_{*}^{\mathrm{DR}} \mathscr{O}_{\mathrm{X}}$ is a Fontaine module [11].

4. The Chern classes $c_{i}(\mathrm{M}) \in \mathrm{H}_{\mathrm{e} t}^{2 i}\left(\mathbf{X}, \mathbf{Q}_{\mathfrak{J}}(i)\right), l \neq p, i>0$ are all equal to 0 .

Proof. - The key to parts a) and b) is the following general result, whose proof can be found in [28, 8.2.3]. 
Lemma 4.18. - Let Z be a smooth scheme over a field of characteristic $p$ and let

$$
0=\mathrm{F}^{l+1} \mathrm{M}^{\cdot} \subseteq \mathrm{F}^{l} \mathrm{M}^{\cdot} \subseteq \cdots \subseteq \mathrm{F}^{k} \mathrm{M}^{\cdot}=\mathrm{M}^{\cdot}
$$

be a bounded filtered complex of coherent $\mathscr{O}_{\mathrm{Z}}$-modules. Assume that there exists a not necessarily filtered) quasi-isomorphism

$$
\mathrm{F}_{\mathrm{Z}}^{*} \mathrm{Gr}_{\mathrm{F}} \mathrm{M}^{*} \simeq \mathrm{M}^{*}
$$

Then the differential $\mathrm{M}^{\cdot} \rightarrow \mathrm{M}^{\cdot+1}$ is strictly compatible with the filtration and, for every pair of integers $i$ and $j$, the $\mathscr{O}_{\mathrm{Z}^{-} \text {module }} \mathrm{H}^{j}\left(\mathrm{Gr}_{\mathrm{F}}^{i} \mathrm{M}^{*}\right) \simeq \mathrm{Gr}_{\mathrm{F}}^{i} \mathrm{H}^{j}\left(\mathrm{M}^{*}\right)$ is locally free.

Let us return to the proof of the theorem. Since the claims in parts (1) and (2) are local on $\mathrm{X}$ we may assume that there exists a lifting $\tilde{\mathrm{F}}$ of the Frobenius $\mathrm{F}_{\mathrm{X} / \mathrm{S}}$. By Theorem 2.11, such a lifting induces a natural isomorphism of $\mathscr{O}_{\mathrm{X}}$-modules

$$
\eta_{\tilde{\mathrm{F}}}: \mathrm{C}_{\mathscr{X} / \mathscr{S}}^{-1}(\mathrm{E}) \simeq \mathrm{F}_{\mathrm{X} / \mathrm{S}}^{*} \mathrm{E}
$$

for every $\mathrm{E} \in \mathrm{HIG}_{p-1}\left(\mathrm{X}^{\prime} / \mathrm{S}\right)$. Composing this with (4.16.1) we obtain an isomorphism of $\mathscr{O}_{\mathrm{X}}$-modules

$$
\mathrm{F}_{\mathrm{X}}^{*} \mathrm{Gr}_{\mathrm{F}} \mathrm{M} \simeq \mathrm{M}
$$

Then the statements (1) and (2) follow from the lemma.

By Theorem 3.22 the lifting $\tilde{h}^{\prime}$ induces an quasi-isomorphism

$$
\mathrm{C}_{\mathscr{Y} / \mathscr{S}}^{-1} \pi_{\mathrm{Y} / \mathrm{S}}^{*} \mathrm{R} h_{*}^{\mathrm{HIG}}\left(\mathrm{Gr}_{\mathrm{F}}^{\cdot} \mathrm{M}, \kappa\right) \cong \mathrm{R} h_{*}^{\mathrm{DR}} \mathrm{M} .
$$

Applying (4.18.1) we obtain locally on $\mathrm{Y}$ an isomorphism in the derived category of $\mathscr{O}_{\mathrm{Y}}$-modules

$$
\mathrm{F}_{\mathrm{Y}}^{*} \mathrm{R} h_{*}^{\mathrm{HIG}}\left(\mathrm{Gr}_{\mathrm{F}}^{\cdot} \mathrm{M}, \kappa\right) \cong \mathrm{R} h_{*}^{\mathrm{DR}} \mathrm{M} .
$$

We can compute $\mathrm{R} h_{*}^{\mathrm{HIG}}\left(\mathrm{Gr}_{\mathrm{F}}^{\cdot} \mathrm{M}, \kappa\right)$ as follows. Endow the relative de Rham complex $\Omega_{\mathrm{X} / \mathrm{Y}} \otimes \mathrm{M}$ with the filtration

$$
\mathrm{F}^{i}\left(\Omega_{\mathrm{X} / \mathrm{Y}} \otimes \mathrm{M}\right)=\left(\mathrm{F}^{i} \mathrm{M} \rightarrow \Omega_{\mathrm{X} / \mathrm{Y}}^{1} \otimes \mathrm{F}^{i-1} \mathrm{M} \rightarrow \cdots \rightarrow \Omega_{\mathrm{X} / \mathrm{Y}}^{d} \otimes \mathrm{F}^{i-d} \mathrm{M}\right),
$$

and let $\left(\mathrm{R} h_{*}^{\mathrm{DR}} \mathrm{M}, \mathrm{F}^{*}\right)$ be the filtered derived direct image of $\left(\Omega_{\mathrm{X} / \mathrm{Y}}^{\cdot} \otimes \mathrm{M}, \mathrm{F}^{*}\right)$. We then have an isomorphism in the derived category of $\mathscr{O}_{\mathrm{Y}}$-modules

$$
\mathrm{Gr}_{\mathrm{F}} \mathrm{R} h_{*}^{\mathrm{DR}} \mathrm{M} \simeq \mathrm{R} h_{*}^{\mathrm{HIG}}\left(\mathrm{Gr}_{\mathrm{F}}^{\cdot} \mathrm{M}, \kappa\right)
$$


Thus by Lemma 4.18, applied to the filtered complex of coherent $\mathscr{O}_{\mathrm{Y}}$-modules $\left(\mathrm{R} h_{*}^{\mathrm{DR}} \mathrm{M}, \mathrm{F}^{*}\right)$, the Hodge spectral sequence for $\mathrm{R} h_{*}^{\mathrm{DR}}\left(\mathrm{M}, \mathrm{F}^{*}\right),\left(\mathrm{R} h_{*}^{\mathrm{DR}} \mathrm{M}, \mathrm{F}^{*}\right)$, degenerates at $\mathrm{E}_{1}$. Hence we get a canonical isomorphism of $\mathscr{O}_{\mathrm{Y}}$-modules

$(4.18 .3)$

$$
\mathrm{Gr}_{\mathrm{F}} \mathrm{R}^{i} h_{*}^{\mathrm{DR}} \mathrm{M} \simeq \mathrm{R}^{i} h_{*}^{\mathrm{HIG}}\left(\mathrm{Gr}_{\mathrm{F}}^{\cdot} \mathrm{M}, \kappa\right)
$$

It is well known ${ }^{15}$ that this isomorphism is compatible with the Higgs fields. Thus passing to the cohomology sheaves in (4.18.2) we obtain the desired isomorphism (4.17.1). This completes the proof of statement (3).

For statement (4), we will first prove that for any $\mathscr{O}_{\mathrm{X}}$-coherent $\mathrm{N} \in \mathrm{HIG}_{p-1}(\mathrm{X} / \mathrm{S})$,

$$
\left[\mathrm{C}_{\mathscr{X} / \mathrm{S}}^{-1} \pi_{\mathrm{X} / \mathrm{S}}^{*} \mathrm{~N}\right]=\mathrm{F}_{\mathrm{X}}^{*}[\mathrm{~N}]
$$

where [] denotes the class of a coherent $\mathscr{O}_{\mathrm{X}}$-module in $\mathrm{K}_{0}^{\prime}(\mathrm{X})=\mathrm{K}_{0}(\mathrm{X})$. Indeed, choose any filtration $\mathrm{N}=\mathrm{N}^{0} \supset \mathrm{N}^{1} \supset \cdots \supset \mathrm{N}^{m}=0$ by Higgs submodules such that $\mathrm{N}^{i} / \mathrm{N}^{i+1} \in \mathrm{HIG}_{0}(\mathrm{X} / \mathrm{S})$. Then

$$
\begin{aligned}
\left(\mathrm{C}_{\mathscr{X} / \mathrm{S}}^{-1} \pi_{\mathrm{X} / \mathrm{S}}^{*} \mathrm{~N}^{i}\right) /\left(\mathrm{C}_{\mathscr{X} / \mathrm{S}}^{-1} \pi_{\mathrm{X} / \mathrm{S}}^{*} \mathrm{~N}^{i+1}\right) & \simeq \mathrm{C}_{\mathscr{X} / \mathrm{S}}^{-1} \pi_{\mathrm{X} / \mathrm{S}}^{*}\left(\mathrm{~N}^{i} / \mathrm{N}^{i+1}\right) \\
& \simeq \mathrm{F}_{\mathrm{X}}^{*}\left(\mathrm{~N}^{i} / \mathrm{N}^{i+1}\right) \simeq \mathrm{F}_{\mathrm{X}}^{*} \mathrm{~N}^{i} / \mathrm{F}_{\mathrm{X}}^{*} \mathrm{~N}^{i+1} .
\end{aligned}
$$

This implies the claim.

In particular, for a Fontaine module $\left(\mathrm{M}, \nabla, \mathrm{F}^{*} \mathrm{M}, \phi\right)$ it follows that $[\mathrm{M}]=\mathrm{F}_{\mathrm{X}}^{*}[\mathrm{M}]$. Thus

$$
c_{i}([\mathrm{M}])=c_{i}\left(\mathrm{~F}_{\mathrm{X}}^{*}[\mathrm{M}]\right)=p^{i} c_{i}([\mathrm{M}]),
$$

and we are done.

Proposition 4.19. - Let $\mathrm{X}$ be a smooth projective curve of genus $g$ over a field spec $k=\mathrm{S}$, $\tilde{\mathrm{X}}^{\prime} \rightarrow \tilde{\mathrm{S}}$ a lifting, and let $\left(\mathrm{M}, \nabla, \mathrm{F}^{*}, \phi\right)$ belong to the category $\mathscr{M} \mathscr{F}_{[0, n]}$. Assume that

$$
n(r k \mathrm{M}-1) \max \{2 g-2,1\}<p-1 .
$$

Then $\left(\mathrm{Gr}_{\mathrm{F}}^{\cdot} \mathrm{M}, \kappa\right)$ is a semistable Higgs bundle.

Proof. - We have to show that $\left(\mathrm{Gr}_{\mathrm{F}}^{\circ} \mathrm{M}, \kappa\right)$ has no Higgs subbundles

$$
(\mathrm{L}, \theta) \hookrightarrow\left(\mathrm{Gr}_{\mathrm{F}}^{\cdot} \mathrm{M}, \kappa\right)
$$

of positive degree. Replacing $(\mathrm{L}, \theta)$ by $\wedge^{r k \mathrm{~L}}(\mathrm{~L}, \theta)$ and $\mathrm{M}$ by $\wedge^{r k \mathrm{~L}} \mathrm{M}$ (this is again a Fontaine module) we reduce proposition to the following claim:

15 This fact should be compared with Katz's formula (3.18.3). A conceptual proof of this result can be obtained using an appropriate filtered derived category of D-modules. See, for example [35]. 
For any Fontaine module $\left(\mathrm{M}, \nabla, 0=\mathrm{F}^{n+1} \mathrm{M} \subset \mathrm{F}^{n} \mathrm{M} \subset \cdots \subset \mathrm{F}^{0} \mathrm{M}=\mathrm{M}, \phi\right)$, with $n(2 g-2)<p-1$ the Higgs bundle $\left(\mathrm{Gr}_{\mathrm{F}} \mathrm{M}, \kappa\right)$ does not have one-dimensional Higgs subbundles

$(4.19 .1)$

$$
(\mathrm{L}, 0) \hookrightarrow\left(\mathrm{Gr}_{\mathrm{F}}^{\cdot} \mathrm{M}, \kappa\right)
$$

of positive degree.

Assume that this is not the case and consider such an $\mathrm{L}$ of the largest possible degree $d>0$. Then any morphism $\left(\mathrm{L}^{\prime}, 0\right) \rightarrow\left(\mathrm{Gr}_{\mathrm{F}}^{*} \mathrm{M}, \kappa\right)$, where $\mathrm{L}^{\prime}$ is a line bundle of degree $>d$, is equal to zero. Consider the morphism

$$
\mathrm{F}_{\mathrm{X}}^{*} \mathrm{~L} \simeq \mathrm{C}_{\mathscr{X} / \mathrm{S}}^{-1} \pi_{\mathrm{X} / \mathrm{S}}^{*}(\mathrm{~L}, 0) \hookrightarrow \mathrm{C}_{\mathscr{X} / \mathrm{S}}^{-1} \pi_{\mathrm{X} / \mathrm{S}}^{*}\left(\mathrm{Gr}_{\mathrm{F}}^{\cdot} \mathrm{M}, \kappa\right) \stackrel{\phi}{\simeq} \mathrm{M}
$$

induced by (4.19.1). We will prove by induction on $m$ that the composition

$$
\mathrm{F}_{\mathrm{X}}^{*} \mathrm{~L} \rightarrow \mathrm{M} \rightarrow \mathrm{M} / \mathrm{F}^{m} \mathrm{M}
$$

is 0 . Let us, first, check this for $m=1$. Observe that the Higgs field $\kappa$ restricted to $\mathrm{M} / \mathrm{F}^{\mathrm{l}} \mathrm{M} \hookrightarrow \mathrm{Gr}_{\mathrm{F}}^{\circ} \mathrm{M}$ is 0 . Thus

$$
\left(\mathrm{F}_{\mathrm{X}}^{*} \mathrm{~L}, 0\right) \rightarrow\left(\mathrm{M} / \mathrm{F}^{1} \mathrm{M}, 0\right) \hookrightarrow\left(\mathrm{Gr}_{\mathrm{F}}^{*} \mathrm{M}, \kappa\right)
$$

is a morphism of Higgs bundles. Since $\operatorname{deg} \mathrm{F}_{\mathrm{X}}^{*} \mathrm{~L}=p d>d$, this morphism must be equal to zero.

Assume that the composition $\mathrm{F}_{\mathrm{X}}^{*} \mathrm{~L} \rightarrow \mathrm{M} \rightarrow \mathrm{M} / \mathrm{F}^{m-1} \mathrm{M}$ is 0 . Then (4.19.2) factors through $\mathrm{F}_{\mathrm{X}}^{*} \mathrm{~L} \rightarrow \mathrm{F}^{m-1} \mathrm{M} / \mathrm{F}^{m} \mathrm{M}$. For any $j, 0 \leq j<m$, consider the composition

$$
\rho_{j}: \mathrm{F}_{\mathrm{X}}^{*} \mathrm{~L} \rightarrow \mathrm{F}^{m-1} \mathrm{M} / \mathrm{F}^{m} \mathrm{M} \stackrel{\kappa^{j}}{\longrightarrow} \mathrm{F}^{m-1-j} \mathrm{M} / \mathrm{F}^{m-j} \mathrm{M} \otimes\left(\Omega_{\mathrm{X} / \mathrm{S}}^{1}\right)^{j},
$$

and let $j_{0}$ be the smallest integer less then $m$, such that $\rho_{j_{0}} \neq 0$. Then $\rho_{j_{0}}$ induces a nonzero map of Higgs bundles

$$
\left(\mathrm{F}_{\mathrm{X}}^{*} \mathrm{~L} \otimes\left(\mathrm{T}_{\mathrm{X} / \mathrm{S}}\right)^{j_{0}}, 0\right) \rightarrow\left(\bigoplus_{i \geq j_{0}} \mathrm{~F}^{m-1-i} \mathrm{M} / \mathrm{F}^{m-i} \mathrm{M}, \kappa\right) \hookrightarrow\left(\mathrm{Gr}_{\mathrm{F}}^{\cdot} \mathrm{M}, \kappa\right)
$$

However

$$
\operatorname{deg}\left(\mathrm{F}_{\mathrm{X}}^{*} \mathrm{~L} \otimes\left(\mathrm{T}_{\mathrm{X} / \mathrm{S}}\right)^{j_{0}}\right)=p d-j_{0}(2 g-2) \geq p d-n(2 g-2)>d .
$$

This contradiction completes the proof.

Remark 4.20. - Let $h: \mathrm{Y} \rightarrow \mathrm{X}$ be a smooth proper morphism of relative dimension $d$, and let $\tilde{h}^{\prime}: \tilde{\mathrm{Y}}^{\prime} \rightarrow \tilde{\mathrm{X}}^{\prime}$ be a lifting. Then, for $d<p, \mathrm{M}=\mathrm{R}^{n} h_{*}^{\mathrm{DR}} \mathscr{O}_{\mathrm{Y}}$ is a Fontaine module on $\mathrm{X}$. Thus, by Proposition 4.19, if $n(r k \mathrm{M}-1) \max \{2 g-2,1\}<$ $p-1,\left(\mathrm{Gr}_{\mathrm{F}}^{\cdot} \mathrm{M}, \kappa\right)$ is semistable. By the standard technique this implies the following result over the complex numbers. 
Theorem 4.21. - Let $\mathrm{X}$ be a smooth projective curve over $\mathbf{G}$ and let $h: \mathrm{Y} \rightarrow \mathrm{X}$ be a smooth proper morphism. Then $\left(\mathrm{Gr}_{\mathrm{F}}^{\cdot} \mathrm{R}^{n} h_{*}^{\mathrm{DR}} \mathscr{O}_{\mathrm{Y}}, \kappa\right)$ is a semistable Higgs bundle.

This result was proved by analytic methods (for any polarizable variation of Hodge structure) by Beilinson and Deligne (unpublished) and later, in a greater generality, by Simpson [36] using a similar technique.

\subsection{Proof of a theorem of Barannikov and Kontsevich}

Let us recall the following striking result of Barannikov and Kontsevich, of which the only published proof we know is due to Sabbah [34].

Theorem 4.22. - Let $\mathrm{X} / \mathbf{G}$ be a quasi-projective smooth scheme over $\mathbf{C}$. Suppose that $f \in \Gamma\left(\mathrm{X}, \mathscr{O}_{\mathbf{X}}\right)$ defines a proper morphism to $\mathbf{A}^{1} / \mathbf{G}$. Then the hypercohomologies of the complexes

$$
\begin{aligned}
& \mathscr{O}_{\mathrm{X}} \stackrel{d+\wedge d f}{\longrightarrow} \Omega_{\mathrm{X} / \mathbf{G}}^{1} \stackrel{d+\wedge d f}{\longrightarrow} \Omega_{\mathrm{X} / \mathbf{G}}^{2} \cdots \text { and } \\
& \mathscr{O}_{\mathrm{X}} \stackrel{-\wedge d f}{\longrightarrow} \Omega_{\mathrm{X} / \mathbf{G}}^{1} \stackrel{-\wedge d f}{\longrightarrow} \Omega_{\mathrm{X} / \mathbf{G}}^{2} \cdots
\end{aligned}
$$

have the same finite dimension in every degree.

We shall show how our version of nonabelian Hodge theory can be used to give a proof of this theorem by the technique of reduction modulo $p$. Since any pair $(\mathrm{X} / \mathbf{C}, f)$ as in Theorem 4.22 comes from some "thickened" situation, it is clear that the following result implies Theorem 4.22 by base change $\mathrm{R} \rightarrow \mathbf{G}$.

Theorem 4.23. - Let $\mathscr{S}=\mathrm{Spec} \mathrm{R}$ be an affine, integral, and smooth scheme over $\mathbf{Z}$, let

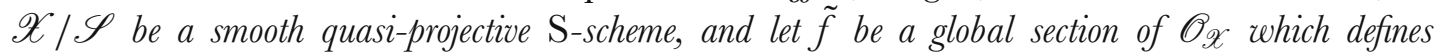
a proper morphism: $\mathscr{X} \rightarrow \mathbf{A}_{\mathscr{S}}^{1}$. Then, after replacing $\mathscr{S}$ by some étale neighborhood of its generic point, the following results are true.

1. The hypercohomology groups

$$
\mathrm{H}^{*}\left(\mathscr{X}, \Omega_{\mathscr{X} / \mathscr{S}}, d+d \tilde{f}\right) \text { and } \mathrm{H}^{*}\left(\mathscr{X}, \Omega_{\mathscr{X} / \mathscr{S}}^{\cdot},-\wedge d \tilde{f}\right)
$$

are finitely generated free $\mathrm{R}$-modules whose formation commutes with base change.

2. Let $p$ be a prime, let $\mathrm{X} / \mathrm{S}$ denote the reduction of $\mathscr{X} / \mathscr{S}$ modulo $p$, and let $\mathrm{X} \stackrel{\mathrm{FX} / \mathrm{s}}{\longrightarrow}$ $\mathrm{X}^{\prime} \stackrel{\pi}{\rightarrow} \mathrm{X}$ be the usual factorization of $\mathrm{F}_{\mathrm{X}}$. Then for every $p$, the complexes of $\mathscr{O}_{\mathrm{X}^{\prime-}}$ modules

$$
\begin{aligned}
& \mathrm{F}_{\mathrm{X} / \mathrm{S} *} \mathscr{O}_{\mathrm{X}} \stackrel{d+\wedge d f}{\longrightarrow} \mathrm{F}_{\mathrm{X} / \mathrm{S} *} \Omega_{\mathrm{X} / \mathrm{S}}^{1} \stackrel{d+\wedge d f}{\longrightarrow} \mathrm{F}_{\mathrm{X} / \mathrm{S} *} \Omega_{\mathrm{X} / \mathrm{S}}^{2} \cdots \\
& \mathscr{O}_{\mathrm{X}^{\prime}} \stackrel{-\wedge d \pi^{*} f}{\longrightarrow} \Omega_{\mathrm{X}^{\prime} / \mathrm{S}}^{1} \stackrel{-\wedge d \pi^{*} f}{\longrightarrow} \Omega_{\mathrm{X}^{\prime} / \mathrm{S}}^{2} \cdots
\end{aligned}
$$

are quasi-isomorphic. 
The rest of this section will be devoted to a proof of Theorem 4.23. Along the way we shall prove some auxiliary results which may be of independent interest, for example the finiteness criterion given in Proposition 4.26 and Corollary 4.27. We begin with a "cleaning" lemma.

Lemma 4.24. - With the notation of Theorem 4.23, let $\mathscr{Z} \subseteq \mathscr{X}$ be the reduced zero locus of $d f$. Then after replacing $\mathscr{S}$ by some étale neighborhood of its generic point, the following conditions are satisfied.

1. The morphism $\mathscr{Z} \rightarrow \mathscr{S}$ is proper, flat, and generically smooth, and for every $p$ the reduction modulo $p$ of $\mathscr{Z}$ is reduced.

2. The restriction of $f$ to each connected component $\mathscr{Z}^{\prime}$ of $\mathscr{Z}$ lies in the image of the map $\Gamma\left(\mathscr{S}, \mathscr{O}_{\mathscr{S}}\right) \rightarrow \Gamma\left(\mathscr{Z}^{\prime}, \mathscr{O}_{\mathscr{Z}}\right)$.

Proof. - Note that formation of $\mathscr{Z}$ commutes with étale base change $\mathscr{S}^{\prime} \rightarrow \mathscr{S}$, so that our statement is not ambiguous. Let $\sigma$ be the generic point of $\mathscr{S}$. The statements are trivial if the generic fiber of $\mathscr{Z}_{\sigma}$ of $\mathscr{Z} / \mathscr{S}$ is empty, so let us assume that this is not the case. By the theorem of generic flatness [13, 6.9.1], we may assume that $\mathscr{Z}$ is flat over $\mathscr{S}$. Then the map from each irreducible component $\mathscr{Z}_{i}$ of $\mathscr{Z}$ to $\mathscr{S}$ is dominant and the generic fiber of $\mathscr{Z}_{i}$ is an irreducible component of $\mathscr{Z}_{\sigma}$. Localizing further if necessary, we may assume that if $\mathscr{Z}_{i}$ and $\mathscr{Z}_{j}$ intersect, then so do their generic fibers. There is a finite extension $k^{\prime}$ of $k(\sigma)$ such that all the connected components of $\mathscr{Z}_{k^{\prime}}$ are geometrically connected and have a $k^{\prime}$-rational point. Replacing $\mathscr{S}$ by an étale neighborhood of $\sigma$, we may assume that $k^{\prime}=k(\sigma)$. Since $\mathscr{Z}_{\sigma}$ is reduced and $k(\sigma)$ is a field of characteristic zero, $\mathscr{Z}_{\sigma} / \sigma$ is generically smooth. Since the differential of $f_{\mathscr{L}_{\sigma}}$ vanishes, its restriction to the smooth locus $\mathscr{Z}_{\sigma}^{s m}$ of $\mathscr{Z}_{\sigma}$ is locally constant. Thus for each irreducible component $\mathscr{Z}_{i}$ of $\mathscr{Z}$, there exists an element $c_{i}$ in $k(\sigma)$ (the value of $f$ at a rational point) such that $f=c_{i}$ on $\mathscr{Z}_{i \sigma}^{s m}$. Since $\mathscr{Z}_{i}$ is reduced, this holds on all of $\mathscr{Z}_{i}$. If $\mathscr{Z}_{i}$ and $\mathscr{Z}_{j}$ intersect, so do $\mathscr{Z}_{i \sigma}$ and $\mathscr{Z}_{j \sigma}$, and it follows that $c_{i}=c_{j}$. Thus $c_{i}$ depends only on the connected component of $\mathscr{Z}_{\sigma}$ containing $\mathscr{Z}_{i}$. Furthermore, localizing on $\tilde{\mathrm{S}}$, we may assume that each $c_{i}$ belongs to R. Thus (2) is proved. Now if $\mathscr{Z}^{\prime}$ is a connected component of $\mathscr{Z}$, the composite $\mathscr{Z}^{\prime} \rightarrow \mathscr{X} \rightarrow \mathbf{A}_{\mathscr{S}}^{1}$ factors through the section of $\mathbf{A}_{\mathscr{S}}^{1} / \mathscr{S}$ defined by the appropriate element of R, Since $\mathrm{X} \rightarrow \mathbf{A}_{\mathscr{S}}^{1}$ is proper, so is each $\mathscr{Z}^{\prime} \rightarrow \mathscr{S}$ and hence the same is true of $\mathscr{Z} \rightarrow \mathscr{S}$.

We have now attained all the desired properties of $\mathscr{Z}$, except for the reducedness of its reductions modulo $p$, which is a consequence of the following (probably standard) lemma.

Lemma 4.25. - Let $\mathscr{Z}$ be a reduced scheme of finite type over $\operatorname{Spec} \mathbf{Z}$. Then for almost all primes $p$, the reduction modulo $p$ of $\mathscr{Z}$ is reduced.

Proof. - In the course of the proof, we may without loss of generality replace $\mathscr{Z}$ by the open subset defined localization by any positive integer. In particular, by 
the theorem of generic flatness, we may assume that $\mathscr{Z}$ is flat over $\mathbf{Z}$. Since $\mathscr{Z} \mathbf{Q} / \mathbf{Q}$ is reduced and of finite type, it is generically smooth over $\mathbf{Z}$. Let $\eta: \mathscr{Y} \rightarrow \mathscr{Z}$ be the normalization mapping. Then $\mathscr{Y}$ is also generically smooth over $\mathbf{Z}$. Thus each irreducible component $\mathscr{Y}^{0}$ of $\mathscr{Y}$ contains a proper closed subscheme $\mathscr{Y}^{1}$ such that $\mathscr{Y}^{0} \backslash \mathscr{Y}^{1} \rightarrow \mathscr{S}$ is smooth. For almost all $p$, the reduction modulo $p$ of $\mathscr{Y}^{1}$ has strictly smaller dimension than that of the reduction modulo $p$ of $\mathscr{Y}^{0}$, and we may assume this is true for all $p$. Then the map $\mathscr{Y} \rightarrow \operatorname{Spec} \mathbf{Z}$ remains generically smooth modulo $p$ for every $p$. By $[13,7.7 .4], \eta$ is finite, and hence the cokernel $Q$ of $\eta^{\sharp}: \mathscr{O}_{\mathscr{Z}} \rightarrow$ $\eta_{*} \mathscr{O}_{\mathscr{Y}}$ is a coherent sheaf of $\mathscr{O}_{\mathscr{Z}}$-modules. Again by the lemma of generic flatness, $\operatorname{Tor}_{1}^{\mathbf{Z}}\left(\mathbf{Q}, \mathbf{F}_{p}\right)=0$ for all but finitely many $p$. Shrinking, we may assume that this is true for all $p$. It then follows that the reduction modulo $p$ of $\eta^{\sharp}$ remains injective for all $p$. Since $\mathscr{Y}$ is normal, it satisfies Serre's condition $\mathrm{S}_{2}$, and since each $p$ defines a nonzero divisor on $\mathscr{Y}$ the fiber $\mathrm{Y}$ of $\mathscr{Y}$ over $p$ satisfies $\mathrm{S}_{1}$. Since $\mathrm{Y}$ is generically smooth over $\mathbf{F}_{p}$, it is generically reduced, and since it satisfies $\mathrm{S}_{1}$, it is reduced. Since $\eta^{\sharp}$ is injective $\bmod p$, the fiber $\mathrm{Z}$ of $\mathscr{Z}$ over $p$ is also reduced.

Let $\mathscr{E}:=\left(\mathscr{O}_{\mathscr{X}}, d+d \tilde{f}\right) \in \operatorname{MIC}(\mathscr{X} / \mathscr{S})$ and let $\mathscr{L}:=\left(\mathscr{O}_{\mathscr{X}}, d \tilde{f}\right) \in \operatorname{HIG}(\mathscr{X} / \mathscr{S})$ we denote by just $\mathrm{E}$ and $\mathrm{L}$ their respective reductions modulo a prime $p$ of $\mathbf{Z}$. Let $\mathrm{J} \subseteq \mathscr{O}_{\mathscr{X}}$ be the ideal of the scheme-theoretic zero locus of $d \tilde{f}$. This is just the ideal generated locally by the set of partial derivatives of $\tilde{f}$ in any set of local coordinates for $\mathscr{X} / \mathscr{S}$. The Higgs complex $\mathscr{L} \otimes \Omega_{\mathscr{X} / \mathscr{S}}$ of $\mathscr{L}$ can be locally identified with the Koszul complex of this sequence of partials, and it follows that the cohomology sheaves of $\mathscr{L} \otimes \Omega_{\mathscr{X} / \mathscr{S}}$ are annihilated by J $[10,17.14]$. Since the closed subscheme of $\mathscr{X}$ defined by the radical of $\mathrm{J}$ is $\mathscr{Z}$, which is proper over $\mathscr{S}$, the hypercohomology groups $\mathrm{H}^{i}\left(\mathscr{L} \otimes \Omega_{\mathscr{X} / \mathscr{S}}\right)$ are finitely generated R-modules. Since $\mathrm{R}$ is reduced, they are free in some neighborhood of the generic point of $\mathscr{S}$, which we may assume is all of $\mathscr{S}$. Since the terms in the complex $\mathscr{L} \otimes \Omega_{\mathscr{X} / \mathscr{S}}$ are flat over $\mathscr{S}$, the formation of its hypercohomology will then commute with all base change. This completes the proof of Theorem 4.23.1 for the Higgs complex.

The proof for the de Rham complex is more difficult; in general, the de Rham cohomology groups of a coherent sheaf with integrable connection on a smooth scheme of finite type over $\mathbf{Z}$ are not finitely generated. (For example, the de Rham cohomology of the trivial connection on $\mathbf{A}_{\mathbf{z}}^{1}$ is not finitely generated.) We will use the technique of logarithmic geometry to study the irregularity of the connection $d+d f$ to obtain the finiteness we need.

Let $\mathrm{Y} / \mathrm{S}$ be a smooth morphism of fine saturated and noetherian log schemes. We just write $\Omega_{\mathrm{Y} / \mathrm{S}}$ for the logarithmic de Rham complex of $\mathrm{Y} / \mathrm{S}$ [17]. If $m$ is a section of $\mathrm{M}_{\mathrm{Y}}$, the set $\mathrm{Y}_{m}$ of all $y \in \mathrm{Y}$ such that $m_{y} \in \mathrm{M}_{\mathrm{Y}, y}^{*}$ is open in $\mathrm{Y}$. In fact, since $\alpha: \mathrm{M}_{\mathrm{Y}} \rightarrow \mathscr{O}_{\mathrm{Y}}$ is a $\log$ structure, $y \in \mathrm{Y}_{m}$ if and only if $\alpha_{\mathrm{Y}}(m) \in \mathscr{O}_{\mathrm{Y}, y}^{*}$. Let us assume that $\alpha_{\mathrm{Y}}(m)$ is a nonzero divisor of $\mathscr{O}_{\mathrm{Y}}$, so that it defines a Cartier divisor D of $\mathrm{Y}$ and 
$\mathrm{Y}_{m}=\mathrm{Y} \backslash \mathrm{D}$. Suppose we are given a torsion free coherent sheaf $\mathrm{E}$ on $\mathrm{Y}$ and an integrable connection $\nabla$ on $j^{*} \mathrm{E}$, where $j: \mathrm{Y}_{m} \rightarrow \mathrm{Y}$ is the inclusion. Then $\nabla$ induces a connection on $j_{*} j^{*} \mathrm{E} \cong \mathrm{E}(*):=\lim _{\longrightarrow} \mathrm{E}(n \mathrm{D})$. If $\nabla$ maps $\mathrm{E}$ to $\mathrm{E} \otimes \Omega_{\mathrm{Y} / \mathrm{S}}^{1}$, then $\mathrm{E}$ has regular singular points along $\mathrm{D}$; we wish to measure the extent to which this fails. Since $\mathrm{E}$ is coherent, $\nabla$ maps $\mathrm{E}$ to $\mathrm{E} \otimes \Omega_{\mathrm{Y} / \mathrm{S}}^{1}(n \mathrm{D})$ for some $n$; replacing $m$ by $m^{n}$ we may assume that $n=1$. Since $d a \in \mathrm{I}_{\mathrm{D}} \Omega_{\mathrm{Y} / \mathrm{S}}^{1}(\mathrm{D})$ for all $a \in \mathscr{O}_{\mathrm{Y}}$, the map

$$
\theta_{\mathrm{D}}: \mathrm{E} \otimes \mathscr{O}_{\mathrm{D}} \rightarrow \mathrm{E} \otimes \Omega_{\mathrm{Y} / \mathrm{S}}^{1}(\mathrm{D})_{\mid \mathrm{D}}
$$

induced by $\nabla$ is $\mathscr{O}_{\mathrm{D}}$-linear. It follows from the integrability of $\nabla$ that $\theta_{\mathrm{D}}$ defines an action of the symmetric algebra $\mathrm{S}^{\cdot}\left(\mathrm{I}_{\mathrm{D}} \mathrm{T}_{\mathrm{Y} / \mathrm{S}}\right)$ on $\mathrm{E}_{\mathrm{l}}$, so that $\mathrm{E}_{\mathrm{l}}$ can be viewed as a module over $\mathbf{V}\left(\mathrm{I}_{\mathrm{D}} \mathrm{T}_{\mathrm{Y} / \mathrm{S}}\right)$.

The following result is inspired by of a result of Deligne [6, II, 6.20] which was pointed out to us by H. Esnault.

Proposition 4.26. - Suppose that in the above situation $\theta_{\mathrm{D}}$ is noncritical, i.e., that the support of the $\mathbf{V}\left(\mathrm{I}_{\mathrm{D}} \mathrm{T}_{\mathrm{Y} / \mathrm{S}}\right)$-module $\mathrm{E}_{\mathrm{D}}$ defined by $\left(\mathrm{E}_{\mathrm{D}}, \theta_{\mathrm{D}}\right)$ is disjoint from the zero section. Suppose further that $\mathrm{Y} / \mathrm{S}$ is proper and that $\mathrm{S}=\operatorname{Spec} \mathrm{R}$. Then for every $i, \mathrm{H}^{i}\left(\mathrm{Y} \backslash \mathrm{D}, \mathrm{E} \otimes \Omega_{\mathrm{Y} / \mathrm{S}}\right)$ is a finitely generated R-module.

Proof. - Let $\Omega_{\mathrm{Y} / \mathrm{S}}^{q}(*):=j_{*} j^{*} \Omega_{\mathrm{Y} / \mathrm{S}}^{q}$ and for each natural number $n$, let

$$
\mathrm{F}_{n}\left(\mathrm{E} \otimes \Omega_{\mathrm{Y} / \mathrm{S}}^{q}\right)(*):=\mathrm{E} \otimes \Omega_{\mathrm{Y} / \mathrm{S}}^{q}((n+q) \mathrm{D}) \subseteq j_{* j} *\left(\mathrm{E} \otimes \Omega_{\mathrm{Y} / \mathrm{S}}^{q}\right) .
$$

Then F. defines an exhaustive filtration of the complex $\mathrm{E} \otimes \Omega_{\mathrm{Y} / \mathrm{S}}(*)$ by coherent sheaves. Since $\mathrm{Y} / \mathrm{S}$ is proper, for each $n$ and $i, \mathrm{H}^{i}\left(\mathrm{~F}_{n} \mathrm{E} \otimes \Omega_{\mathrm{Y} / \mathrm{S}}(*)\right)$ is finitely generated over $\mathrm{R}$. Thus it will suffice to show that for each $n \geq 0$, the natural map

$$
\mathrm{F}_{n} \mathrm{E} \otimes \Omega_{\mathrm{Y} / \mathrm{S}}^{\cdot}(*) \rightarrow \mathrm{E} \otimes \Omega_{\mathrm{Y} / \mathrm{S}}(*)
$$

is a quasi-isomorphism, and for this it will suffice to prove that for each $n \geq 0$, the map

$$
\mathrm{F}_{0} \mathrm{E} \otimes \Omega_{\mathrm{Y} / \mathrm{S}}^{\cdot}(*) \rightarrow \mathrm{F}_{n} \mathrm{E} \otimes \Omega_{\mathrm{Y} / \mathrm{S}}^{\cdot}(*)
$$

is a quasi-isomorphism. This will follow by induction if for every $n>0, \operatorname{Gr}_{n}^{\mathrm{F}} \mathrm{E} \otimes$ $\Omega_{\mathrm{Y} / \mathrm{S}}(*)$ is acyclic.

Multiplication by $g^{n}$ defines an isomorphism $\mathrm{F}_{n} \mathrm{E}(*) \rightarrow \mathrm{F}_{0} \mathrm{E}(*)$ which induces an isomorphism

$$
\mathrm{E}(n \mathrm{D})_{\mid \mathrm{D}}=\mathrm{Gr}_{n}^{\mathrm{F}} \mathrm{E}(*) \rightarrow \mathrm{Gr}_{0}^{\mathrm{F}} \mathrm{E}(*)=\mathrm{E}_{\mid \mathrm{D}} .
$$

If $e \in \mathrm{F}_{n} \mathrm{E}$, then $\nabla(e) \in \mathrm{F}_{n} \mathrm{E} \otimes \Omega_{\mathrm{Y} / \mathrm{S}}^{1}(\mathrm{D})$ and $\nabla\left(g^{n} e\right) \in \mathrm{E} \otimes \Omega_{\mathrm{Y} / \mathrm{S}}^{1}(\mathrm{D})$. Since $g=\alpha(m)$ and $d g=g \operatorname{dlog} m$,

$$
\nabla\left(g^{n} e\right)=n g^{n} e \otimes \operatorname{dlog}(m)+g^{n} \nabla(e) \in \mathrm{E} \otimes \Omega_{\mathrm{Y} / \mathrm{S}}^{1}(\mathrm{D}) .
$$


Since $g^{n} e \otimes \operatorname{dlog}(m) \in \mathrm{E} \otimes \Omega_{\mathrm{Y} / \mathrm{S}}^{1}, \nabla\left(g^{n} e\right)$ reduces to $g^{n} \nabla(e)$ in $\mathrm{E} \otimes \Omega_{\mathrm{Y} / \mathrm{S}}^{1}(\mathrm{D})_{\left.\right|_{\mathrm{D}}}$. Thus multiplication by $g^{n}$ identifies $\operatorname{Gr}_{n}^{\mathrm{F}} \nabla$ with $\theta_{\mathrm{D}}$ for all $n \geq 0$. This identification extends to an isomorphism of complexes

$$
\operatorname{Gr}_{n}^{\mathrm{F}}\left(\mathrm{E} \otimes \Omega_{\mathrm{Y} / \mathrm{S}}^{\cdot}(*)\right) \cong \operatorname{Gr}_{0}^{\mathrm{F}}\left(\mathrm{E} \otimes \Omega_{\mathrm{Y} / \mathrm{S}}^{\cdot}(*)\right) .
$$

But $\operatorname{Gr}_{\mathrm{F}}^{0}\left(\mathrm{E} \otimes \Omega_{\mathrm{Y} / \mathrm{S}}(*)\right)$ is just the Higgs $(\mathrm{Koszul})$ complex of $\theta_{\mathrm{D}}$, whose cohomology sheaves can be identified with $\operatorname{Ext}_{\mathscr{O}_{\mathbf{V}}}^{*}\left(i_{*} \mathscr{O}_{\mathbf{X}}, \mathrm{E}_{\mathrm{D}}\right)$, where $i: \mathrm{X} \rightarrow \mathbf{V}$ is the zero section of $\mathbf{V}:=\mathbf{V}\left(\mathrm{I}_{\mathrm{D}} \mathrm{T}_{\mathrm{Y} / \mathrm{S}}\right)$. These vanish since $\theta_{\mathrm{D}}$ is noncritical.

The following corollary then completes the proof of statement (1) of Theorem 4.23: take $(\mathrm{E}, \nabla)$ to be the constant connection on $\mathscr{X} / \mathscr{S}$.

Corollary 4.27. - Let $\mathrm{X} / \mathrm{S}$ be a smooth quasi-projective scheme over $\mathrm{S}=\mathrm{Spec} \mathrm{R}$, where $\mathrm{R}$ is a flat and finitely generated $\mathbf{Z}$-algebra. Let $(\mathrm{E}, \nabla)$ be a coherent sheaf with integrable connection on $\mathrm{X} / \mathrm{S}$ whose restriction to the generic fiber of $\mathrm{X} / \mathrm{S}$ has regular singularities at infinity. Suppose that $f \in \mathscr{O}_{\mathrm{X}}(\mathrm{X})$ is a global function which defines a proper morphism $\mathrm{X} \rightarrow \mathbf{A}_{\mathrm{S}}^{1}$, and let $\left(\mathrm{E}^{\prime}, \nabla^{\prime}\right)$ be the $d f$-twist of $(\mathrm{E}, \nabla): \mathrm{E}^{\prime}=\mathrm{E}$, and $\nabla^{\prime}:=\nabla+\wedge d f$. Then after replacing $\mathrm{S}$ by some affine neighborhood of the generic point of $\mathrm{S}$, the de Rham cohomology $\mathrm{H}^{*}\left(\mathrm{X}, \mathrm{E}^{\prime} \otimes \Omega_{\mathrm{X} / \mathrm{S}}^{*}\right)$ is finitely generated and free over $\mathrm{R}$.

Proof. - Let $\sigma$ be the generic point of S. We may find a projective compactification $\overline{\mathrm{X}}_{\sigma}$ of $\mathrm{X}_{\sigma}$, and after blowing up $\overline{\mathrm{X}}_{\sigma}$ outside of $\mathrm{X}_{\sigma}$ we may assume that $f$ extends to a morphism $\overline{\mathrm{X}}_{\sigma} \rightarrow \mathbf{P}_{\sigma}^{1}$, which we still denote by $f$. After a further blowing up outside of $\mathrm{X}_{\sigma}$, we may assume that $\overline{\mathrm{X}}_{\sigma}$ is smooth over $\sigma$ and that the complement of $\mathrm{X}_{\sigma}$ in $\overline{\mathrm{X}}_{\sigma}$ is a divisor with strict normal crossings. Then the log scheme $\mathrm{Y}_{\sigma}$ obtained by endowing $\overline{\mathrm{X}}_{\sigma}$ with the $\log$ structure corresponding to the inclusion $\mathrm{X}_{\sigma} \rightarrow \overline{\mathrm{X}}_{\sigma}$ is $(\log )$ smooth. Furthermore, $f$ extends to a morphism of $\log$ schemes $\mathrm{Y}_{\sigma} \rightarrow \mathrm{P}_{\sigma}^{1}$, where $\mathrm{P}_{\sigma}^{1}$ is the log scheme $\mathrm{P}_{\sigma}^{1}$ obtained by endowing $\mathbf{P}_{\sigma}^{1}$ with the $\log$ structure corresponding to the inclusion $\mathbf{A}_{\sigma}^{1} \rightarrow \mathbf{P}_{\sigma}^{1}$.

Let $t$ be the coordinate of $\mathbf{A}_{\sigma}^{1}$ and let $s:=t^{-1}$, which is a local generator of the ideal of $\infty$. There is a unique local section $m$ of the sheaf of monoids $\mathrm{M}_{\mathrm{P}_{\sigma}^{1}}$ over $\mathrm{V}$ with $s:=\alpha_{\mathrm{P}_{\sigma}^{1}}(m)$, and $\operatorname{dog} m$ is basis for the stalk of $\Omega_{\mathrm{P}_{\sigma}^{1}}^{1}$ at $\infty$. Let $y$ be a point of $\mathrm{D}:=f^{-1}(\infty)$. Then in an étale neighborhood of $y$, there exists a system of coordinates $\left(t_{1}, \cdots, t_{n}\right)$ and natural numbers $r, e_{1}, \ldots, e_{r}$ such that such that $f^{*}(s)=t_{1}^{\ell_{1}} \cdots t_{r}^{e_{r}}$. Then $f^{*}(d m)=\sum_{i} e_{i} \mathrm{~d} \log t_{i}$, which is nonvanishing in the fiber of $\Omega_{\mathrm{Y}_{\sigma} / \sigma}^{1}$ at $y$. (This implies that $f$ is $\log$ smooth at $y$.) Since $(\mathrm{E}, \nabla)$ has regular singularities at infinity, there is a coherent (even locally free) extension $\overline{\mathrm{E}}$ of $\mathrm{E}$ to $\mathrm{Y}_{\sigma}$ and a $\log$ connection $\nabla: \overline{\mathrm{E}} \rightarrow \overline{\mathrm{E}} \otimes \Omega_{\mathrm{Y}_{\sigma} / \sigma}^{1}$ extending $\nabla$. Now $d f=f^{*}(d t)=-s^{-2} d s=-s^{-1} f^{*} \operatorname{dlog} m$. Thus $\nabla^{\prime}$ maps $\overline{\mathrm{E}}$ to $\overline{\mathrm{E}} \otimes \Omega_{\mathrm{Y}_{\sigma} / \sigma}^{1}(\mathrm{D})$, and $\theta_{\mathrm{D}}$ is the map $\overline{\mathrm{E}}_{\mathrm{I}_{\mathrm{D}}} \rightarrow \overline{\mathrm{E}}_{\left.\right|_{\mathrm{D}}} \otimes \Omega_{\mathrm{Y}_{\sigma} / \sigma}^{1}$ sending $e$ to $-e \wedge s^{-1} \operatorname{dlog} m$. This is an isomorphism, so $\theta_{\mathrm{D}}$ is noncritical. There exists an affine 
neighborhood of the generic point of $\mathrm{S}$ over which all this remains true, and without loss of generality we may assume they are true for Y/S. Then Proposition 4.26 implies that the de Rham cohomology groups of $(\mathrm{E}, \nabla)$ over $\mathrm{Y} \backslash \mathrm{D}=\mathrm{X}$ are finitely generated over R; shrinking further we may assume they are free.

We now turn to the proof of statement (2) of Theorem 4.23. Assume that $\mathscr{X} / \mathscr{S}$ satisfies the conditions in (1) of Theorem 4.23 and in Lemma 4.24. Fix a prime $p$, let $\mathrm{X} / \mathrm{S}$ be the reduction of $\mathscr{X} / \mathscr{S}$ modulo $p$, and let $\mathscr{S}_{1}$ be the reduction of $\mathscr{S}$ modulo $p^{2}$. Since $\mathscr{S} / \mathbf{Z}$ is smooth and affine, there exists a lifting $\mathrm{F}_{\mathscr{S}}$ of the absolute Frobenius endomorphism of $\mathrm{S}$ to $\mathscr{S}_{1}$ and hence a Cartesian square:

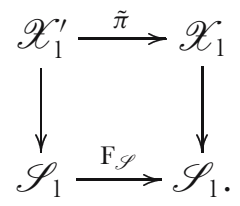

We shall abuse notation and write $\mathrm{C}_{\mathscr{X} / \mathscr{S}}$ for the Cartier transform defined by the lifting $\mathscr{X}_{1}^{\prime} / \mathscr{S}_{1}$ of $\mathrm{X}^{\prime} / \mathrm{S}$.

Let $(\mathrm{E}, \nabla)$ be the restriction of $(\mathscr{E}, \nabla)$ to $\mathrm{X} / \mathrm{S}$. According to $[19,7.22]$, the $p$-curvature $\psi: \mathrm{E} \rightarrow \mathrm{E} \otimes \mathrm{F}_{\mathrm{X} / \mathrm{S}}^{*} \Omega_{\mathrm{X}^{\prime} / \mathrm{S}}^{1}$ is multiplication by

$$
\mathrm{F}_{\mathrm{X}}^{*}(d f)-\mathrm{F}_{\mathrm{X} / \mathrm{S}}^{*} \mathrm{C}_{\mathrm{X} / \mathrm{S}}(d f)=\mathrm{F}_{\mathrm{X} / \mathrm{S}}^{*} \pi^{*}(d f) \text {. }
$$

Since this is not nilpotent, we cannot apply our Cartier transform to it directly. Our approach will be to approximate $\mathrm{E}$ by nilpotent connections, and we shall see that the Cartier transform of these approximations approximate L.

In general, if $(\mathrm{E}, \nabla)$ is a connection on a smooth $\mathrm{X} / \mathrm{S}$ in characteristic $p$, $\mathrm{F}_{\mathrm{X} / \mathrm{S} *}(\mathrm{E})$ becomes an $\mathrm{S}^{*} \mathrm{~T}_{\mathrm{X}^{\prime} / \mathrm{S}}$ module via the $p$-curvature $\psi$, and since $\psi$ acts horizontally, the quotient $\mathrm{E}_{(n)}$ of $\mathrm{E}$ by the $n$th power of the ideal $\mathrm{S}^{+} \mathrm{T}_{\mathrm{X}^{\prime} / \mathrm{S}}$ of $\mathrm{S}^{\cdot} \mathrm{T}_{\mathrm{X}^{\prime} / \mathrm{S}}$ inherits a connection. In fact, this quotient is the maximal quotient of $\mathrm{E}$ on which the connection is nilpotent of level $n-1$. In the situation at hand, we can be quite explicit. Let $\mathrm{J} \subseteq \mathscr{O}_{\mathrm{X}}$ be the ideal of the zeroes of $d f$, i.e., the ideal generated by the partial derivatives of $f$ in any local system of coordinates. Then $\mathrm{E}_{(n)}$ is the quotient of $\mathrm{E}$ by $\mathrm{F}_{\mathrm{X}}^{*}\left(\mathrm{~J}^{n}\right)$. Our next goal is the computation of the Cartier transform of a suitable quotient of $\mathrm{E}_{(n)}$.

Proposition 4.28. - Suppose that $\mathscr{X} / \mathscr{S}$ and $\tilde{f}$ satisfy the conditions of Lemma 4.24, and let $\mathrm{Z}$ be the reduction of $\mathscr{Z}$ modulo $p$. Let $n$ be a natural number and

$$
\begin{aligned}
\mathrm{E} & :=\left(\mathscr{O}_{\mathrm{X}}, d+d f\right) \in \mathrm{MIC}(\mathrm{X} / \mathrm{S}) \\
\mathrm{E}_{n} & :=\left(\mathrm{E} / \mathrm{F}_{\mathrm{X}}^{*}\left(\mathrm{I}_{\mathrm{Z}}^{n}\right) \mathrm{E}, d+d f\right) \in \operatorname{MIC}(\mathrm{X} / \mathrm{S}) \\
\mathrm{L} & :=\left(\mathscr{O}_{\mathrm{X}},-d f\right) \in \mathrm{HIG}(\mathrm{X} / \mathrm{S}) \\
\mathrm{L}_{n} & :=\mathrm{L} / \mathrm{I}_{\mathrm{Z}}^{n} \mathrm{~L} \in \mathrm{HIG}(\mathrm{X} / \mathrm{S}) \\
\mathrm{L}_{n}^{\prime} & :=\pi^{*} \mathrm{~L}_{n} \in \mathrm{HIG}\left(\mathrm{X}^{\prime} / \mathrm{S}\right) .
\end{aligned}
$$


Finally, let $r$ be the maximum codimension of $\mathscr{Z}$ in $\mathscr{X}$. Then if $p>m$, the Cartier transform $\mathrm{C}_{\mathscr{X} / \mathrm{S}}\left(\mathrm{E}_{n}\right)$ of $\mathrm{E}_{n}$ with respect to $\mathscr{X}_{1}^{\prime} / \mathscr{S}_{1}$ is isomorphic to $\mathrm{L}_{n}^{\prime}$.

Proof. - Note that, by definition, $\mathrm{I}_{\mathscr{Z}}$ is the radical of the ideal $\mathrm{J}$, so $\mathrm{E}_{n}$ is indeed a quotient of $\mathrm{E}_{(n)}$ and $\mathrm{C}_{\mathscr{X} / \mathscr{S}}\left(\mathrm{E}_{n}\right)$ is defined. It is enough to prove the proposition after restricting to each connected component of $\mathscr{Z}$. To simplify the notation, we shall assume that $\mathscr{Z}$ is connected. Replacing $\tilde{f}$ by $\tilde{f}-\tilde{c}$, for a suitable $\tilde{c} \in \Gamma\left(\mathscr{O}_{\mathscr{S}}\right)$ as in (2) of Lemma 4.24 , we may assume that the restriction of $\tilde{f}$ to $\mathscr{Z}$ vanishes.

Recall from Proposition 1.14 that the lifting $\tilde{\pi}$ of $\pi: \mathrm{X}^{\prime} \rightarrow \mathrm{X}$ determines a map $\delta_{\tilde{\pi}}: \mathscr{O}_{\mathscr{X}} \rightarrow \mathscr{A}_{\mathscr{X} / \mathscr{S}}$

Claim 4.29. - Let $\alpha:=\delta_{\tilde{\pi}}(\tilde{f}) \in \mathscr{A}_{\mathscr{X} / \mathscr{S}}$ and let

$$
\beta:=1+\alpha+\frac{\alpha^{2}}{2 !}+\cdots+\frac{\alpha^{p-1}}{(p-1) !} \in \mathscr{A}_{\mathscr{X} / \mathscr{S}} .
$$

Then:

1. $\alpha^{r} \in \mathrm{F}_{\mathrm{X}}^{*}\left(\mathrm{I}_{\mathrm{Z}}\right) \mathscr{A}_{\mathscr{X} / \mathscr{S}}$,

2. $\psi_{\mathscr{A}}(\beta)=\left(\beta-\frac{\alpha^{p-1}}{(p-1) !}\right) \otimes \mathrm{F}_{\mathrm{X}}^{*} d f$, and

3. $\nabla_{\mathscr{A}}(\beta)=-\left(\beta-\frac{\alpha^{p-1}}{(p-1) !}\right) f^{p-1} \otimes d f$.

Proof. - By (1) of Lemma 4.24, $\mathrm{Z}$ is reduced and in particular satisfies Serre's condition $\mathrm{S}_{1}$. Since $\mathrm{X}$ is regular, its absolute Frobenius endomorphism is flat, and hence the inverse image $Z^{(p)}$ of $Z$ by $F_{X}$ still satisfies $S_{1}$. (To see this, let $j: U \rightarrow Z$ be the inclusion of any dense open subset of $\mathrm{Z}$ and observe that the map $\mathscr{O}_{\mathrm{Z}} \rightarrow j_{*} j^{*} \mathscr{O}_{\mathrm{Z}}$ is injective, and remains so after pullback by $\mathrm{F}_{\mathrm{X}}$.) Since $\mathrm{Spec}_{\mathrm{X}} \mathscr{A}_{\mathscr{X} / \mathscr{S}}$ is smooth over $\mathrm{X}$, the inverse image of $Z^{(p)}$ in $\operatorname{Spec}_{\mathrm{X}} \mathscr{A}_{\mathscr{X} / \mathscr{S}}$ also satisfies $\mathrm{S}_{1}$. Thus it suffices to check (1) at the generic points of $\mathrm{Z}$, and since $\mathrm{Z} / \mathrm{S}$ is generically smooth, we may assume that it is smooth. We may work in a neighborhood of a point of $\mathrm{Z}$ with the aid of a system of local coordinates $\left(\tilde{t}_{1}, \ldots, \tilde{t}_{n}\right)$ for $\mathscr{X} / \mathscr{S}$ such that $\mathrm{I}_{\mathscr{Z}}=\left(\tilde{t}_{1}, \ldots, \tilde{t}_{s}\right)$. Let $\tilde{\mathrm{F}}: \mathscr{X}_{1} \rightarrow \mathscr{X}_{1}^{\prime}$ be the lift of $\mathrm{F}_{\mathrm{X} / \mathrm{S}}$ sending $\tilde{\pi}^{*} \tilde{t}_{i}$ to $\tilde{t}_{i}^{p}$ for all $i$. This defines a splitting of the fundamental exact sequence (1.4.1), and hence an isomorphism

$$
\mathrm{N}_{1} \mathscr{A}_{\mathscr{X} / \mathscr{S}}=\mathscr{E}_{\mathscr{X} / \mathscr{S}} \cong \mathscr{O}_{\mathrm{X}} \oplus \mathrm{F}_{\mathrm{X} / \mathrm{S}}^{*} \Omega_{\mathrm{X}^{\prime} / \mathrm{S}}^{1}
$$

Proposition 1.14 says that, in terms of this splitting, $\alpha=\left(g, \mathrm{~F}_{\mathrm{X}}^{*} d f\right)$, where $\tilde{\mathrm{F}}^{*} \tilde{\pi}^{*}(\tilde{f})=$ $\tilde{f}^{p}+[p] g$. Since $\tilde{f} \in \mathrm{I}_{\mathscr{Z}} \tilde{f}^{p}$ belongs to $\mathbf{I}_{\mathscr{Z}}^{p}$, and since $\tilde{\mathrm{F}}^{*} \tilde{\pi}^{*}$ maps $\mathbf{I}_{\mathscr{Z}}$ to $\mathbf{I}_{\mathscr{Z}}^{p}$, it follows that $[p] g \in \mathrm{I}_{\mathscr{Z}}^{p}$. It follows from the smoothness of $\mathscr{Z}$ and $\mathscr{X}$ over $\mathscr{S}$ that the closed subscheme of $\mathscr{X}$ defined by $\mathrm{I}_{\mathscr{Z}}^{p}$ is flat over $\mathscr{S}$, and hence that $g \in \mathrm{I}_{\mathrm{Z}}^{p}$. Then $g^{s} \in \mathrm{I}_{\mathrm{Z}}^{p s}$, and since $\mathrm{I}_{\mathrm{Z}}$ has $s$ generators, $\mathrm{I}_{\mathrm{Z}}^{p s} \subseteq \mathrm{F}_{\mathrm{X}}^{*} \mathrm{I}_{\mathrm{Z}} \mathscr{O}_{\mathrm{X}}$, so in fact $g^{s} \in \mathrm{F}_{\mathrm{X}}^{*} \mathrm{I}_{\mathrm{Z}} \mathscr{O}_{\mathrm{X}}$. Since $d f \in \mathrm{I}_{\mathrm{Z}} \Omega_{\mathrm{X} / \mathrm{S}}^{1}$ by hypothesis, $\mathrm{F}_{\mathrm{X}}^{*} d f \in \mathrm{F}_{\mathrm{X}}^{*}(\mathrm{I}) \mathrm{F}_{\mathrm{X}^{\prime} / \mathrm{S}}^{*}\left(\Omega_{\mathrm{X}^{\prime} / \mathrm{S}}^{1}\right)$. Thus $\alpha^{s} \in \mathrm{F}_{\mathrm{X}}^{*}\left(\mathrm{I}_{\mathrm{Z}}\right) \mathscr{A}_{\mathscr{X} / \mathscr{S}}$, and since $s \leq r$, the same is true of $\alpha^{r}$. This proves (1). 
Recall from Proposition 1.14 that $\psi_{\mathscr{A}}(\alpha)=\mathrm{F}_{\mathrm{X}}^{*} d f$. Hence

$$
\begin{aligned}
\psi_{\mathscr{A}}(\beta) & =\left(1+\frac{\alpha}{1 !}+\frac{\alpha^{2}}{2 !}+\cdots+\frac{\alpha^{p-2}}{(p-2) !}\right) \psi(\alpha) \\
& =\left(\beta-\frac{\alpha^{p-1}}{(p-1) !}\right) \otimes \mathrm{F}_{\mathrm{X}}^{*} d f .
\end{aligned}
$$

This proves (2). Proposition 1.14 also says that $\nabla_{\mathscr{A}}(\alpha)=-f^{p-1} d f$, so a similar calculation proves (3).

Recall from Theorem 2.23 that

$$
\mathrm{C}_{\mathscr{X} / \mathscr{S}}^{-1}\left(\mathrm{~L}_{n}^{\prime}\right):=\left(\mathrm{L}_{n}^{\prime} \otimes \mathrm{F}_{\mathrm{X} / \mathrm{S} *} \mathscr{A} \mathscr{X} / \mathscr{S}\right)^{\psi_{t o t}} .
$$

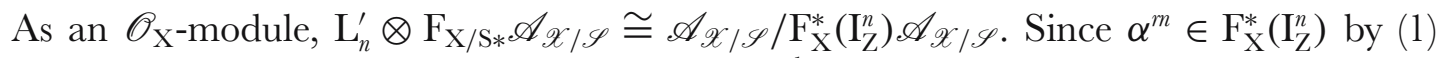
of the claim, this module is annihilated by $\alpha^{p-1}$ if $p>m$. Hence

$$
\psi_{\mathscr{A}}(\beta)=\beta \otimes \mathrm{F}_{\mathrm{X}}^{*} d f \quad \text { in } \quad \mathrm{L}_{n}^{\prime} \otimes \mathscr{A}_{\mathscr{X} / \mathscr{S}} \otimes \mathrm{F}_{\mathrm{X}^{\prime} / \mathrm{S}}^{*} \Omega_{\mathrm{X}^{\prime} / \mathrm{S}}^{1} .
$$

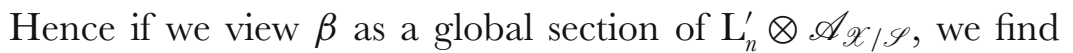

$$
\psi_{t o t}(\beta)=\psi_{\mathrm{L}}(1) \beta+\psi_{\mathscr{A}}(\beta)=-\beta \otimes \pi^{*} d f+\beta \otimes \pi^{*} d f=0 .
$$

Thus $\beta \in \mathrm{C}_{\mathscr{X} / \mathscr{S}}^{-1}\left(\mathrm{~L}_{n}^{\prime}\right)=\left(\mathrm{L}_{n}^{\prime} \otimes \mathscr{A}_{\mathscr{X} / \mathscr{S}}\right)^{\psi_{\text {tot }}}$, and in fact $\beta$ is a basis for $\mathrm{C}_{\mathscr{X} / \mathscr{S}}^{-1}\left(\mathrm{~L}_{n}^{\prime}\right)$ since it is a unit modulo I. Furthermore, it follows from (3) of the claim that

$$
\nabla_{t o t}(\beta)=-\beta \otimes f^{p-1} d f \in \mathrm{E}_{n}^{\prime} \otimes \Omega_{\mathrm{X} / \mathrm{S}}^{1} .
$$

Now consider the Artin-Hasse exponential of $f$, which is given formally by

$$
g:=\exp \left(f+f^{p} / p+f^{p^{2}} / p^{2}+\cdots\right),
$$

and which in fact has $p$-adically integral coefficients. Then

$$
g^{-1} d g=\left(1+f^{p-1}+f^{p^{2}-1}+\cdots\right) d f .
$$

Since $f \in \mathrm{I}_{\mathrm{Z}}$ and $p>n, f^{p^{2}-1}=\mathrm{F}_{\mathrm{X}}^{*}\left(f^{p-1}\right) f^{p-1} \in \mathrm{F}_{\mathrm{X}}^{*}\left(\mathrm{I}_{\mathrm{Z}}^{n}\right)$, so

$$
d g=g\left(1+f^{p-1}\right) d f \quad \bmod \mathrm{F}_{\mathrm{X}}^{*}\left(\mathrm{I}_{\mathrm{Z}}^{n}\right) .
$$

Since $g$ is a unit, $e:=g \beta$ is also a basis for $\mathrm{C}_{\mathscr{X} / \mathscr{S}}^{-1}\left(\mathrm{~L}_{n}^{\prime}\right)$, and

$$
\begin{aligned}
\nabla(e) & =g \nabla(\beta)+\beta \otimes d g \\
& =-g \beta \otimes f^{p-1} d f+g\left(1+f^{p-1} d f\right) \beta \otimes d f \\
& =e \otimes d f .
\end{aligned}
$$

In other words, $\mathrm{C}_{\mathscr{X} / \mathscr{S}}^{-1}\left(\mathrm{~L}_{n}^{\prime}\right)$ is isomorphic to $\mathrm{E}_{n}$, as claimed. 
We shall also need the following general result about morphisms in the derived category.

Proposition 4.30. - Let $\mathrm{X}$ be a noetherian scheme or formal scheme, let $\mathrm{K} \cdot$ be a perfect complex of coherent sheaves of $\mathscr{O}_{\mathrm{X}}$-modules, and let $\mathrm{J}$ be a sheaf of ideals annihilating the cohomology sheaves of $\mathrm{K}$. Then there exists a natural number $n$ such that for all $m>0$, the map in the derived category

$$
\mathrm{J}^{n+m} \stackrel{\mathbf{L}}{\otimes} \mathrm{K}^{\cdot} \rightarrow \mathrm{J}^{m} \stackrel{\mathbf{L}}{\otimes} \mathrm{K}^{\cdot}
$$

induced from the inclusion $\mathrm{J}^{n+m} \rightarrow \mathrm{J}^{n}$ is zero.

Proof. - First we prove the statement for the induced maps on cohomology sheaves. We may cover $\mathrm{X}$ by a finite number of open affines on each of which $\mathrm{K}^{\circ}$ is quasi-isomorphic to a bounded complex $\tilde{\mathrm{K}}^{\cdot}$ of locally free $\mathscr{O}_{\mathrm{X}}$-modules. and it suffices to prove the local statement on each of these open sets. Thus we may assume that $\mathrm{X}=\operatorname{Spec} \mathrm{A}$ and replace $\mathrm{K}^{\cdot}$ by $\Gamma\left(\mathrm{X}, \tilde{\mathrm{K}}^{\cdot}\right)$. Then $\mathrm{J}^{m} \stackrel{\mathbf{L}}{\otimes} \mathrm{K}^{\cdot} \cong \mathrm{J}^{m} \otimes \mathrm{K}^{\cdot}$ for all $m$. Let $\mathrm{B}^{q} \subseteq \mathrm{Z}^{q} \subseteq \mathrm{K}^{q}$ be the boundaries, (resp. cycles, resp. chains) of $\mathrm{K}^{*}$ in degree $q$. By the Artin-Rees lemma, there exists an integer $r$ such that $\mathrm{Z}^{q} \cap \mathrm{J}^{m+r} \mathrm{~K}^{q} \subseteq \mathrm{J}^{m} \mathrm{Z}^{q}$ and $\mathrm{B}^{q} \cap \mathrm{J}^{m+r} \mathrm{~K}^{q} \subseteq \mathrm{J}^{m} \mathrm{~B}^{q}$ for all $m \geq 0$. The hypothesis on $\mathrm{J}$ implies that $\mathrm{JZ}^{q} \subseteq \mathrm{B}^{q}$. Hence if $n>r, \mathrm{Z}^{q} \cap \mathrm{J}^{m+n} \mathrm{~K}^{q} \subseteq \mathrm{JZ}^{q} \subseteq \mathrm{B}^{q}$, so

$$
\mathrm{Z}^{q} \cap \mathrm{J}^{m+n} \mathrm{~K}^{q} \subseteq \mathrm{B}^{q} \cap \mathrm{J}^{m+n} \mathrm{~K}^{q} \subseteq \mathrm{J}^{m} \mathrm{~B}^{q} .
$$

Since $\mathrm{K}^{q}$ is free, $\mathrm{Z}^{q}\left(\mathrm{~J}^{m+n} \mathrm{~K}^{q}\right)=\mathrm{Z}^{q} \cap \mathrm{J}^{m+n} \mathrm{~K}^{q}$ and $\mathrm{J}^{m} \mathrm{~B}^{q}=\mathrm{B}^{q}\left(\mathrm{~J}^{m} \mathrm{~K}\right)$. It follows that the map $\mathrm{H}^{q}\left(\mathrm{~J}^{m+n} \mathrm{~K}\right) \rightarrow \mathrm{H}^{q}\left(\mathrm{~J}^{m} \mathrm{~K}\right)$ is zero.

The following lemma then completes the proof of the proposition.

Lemma 4.31. - Let $\mathrm{K}_{0} \stackrel{f_{0}}{\rightarrow} \mathrm{K}_{1} \stackrel{f_{1}}{\rightarrow} \mathrm{K}_{2} \stackrel{f_{2}}{\rightarrow} \cdots \stackrel{f_{n}}{\rightarrow} \mathrm{K}_{n+1}$ be a sequence of morphisms in the derived category of an abelian category. Suppose that each $\mathrm{K}_{i}$ has cohomological amplitude in $[a, a+n]$ and that the maps $\mathrm{H}^{*}\left(\mathrm{~K}_{i}\right) \rightarrow \mathrm{H}^{*}\left(\mathrm{~K}_{i+1}\right)$ are all zero. Then the composition $\mathrm{K}_{0} \rightarrow$ $\mathrm{K}_{n+1}$ is zero.

Proof. - The proof is by induction on $n$. If $n=0$, there is nothing to prove, since $\mathrm{K}_{i} \cong \mathrm{H}^{a}\left(\mathrm{~K}_{i}\right)$ for all $i$. Let $\tau_{<}$denote the canonical filtration [2], let $f:=f_{1} f_{2} \ldots f_{n+1}$, and consider the following diagram:

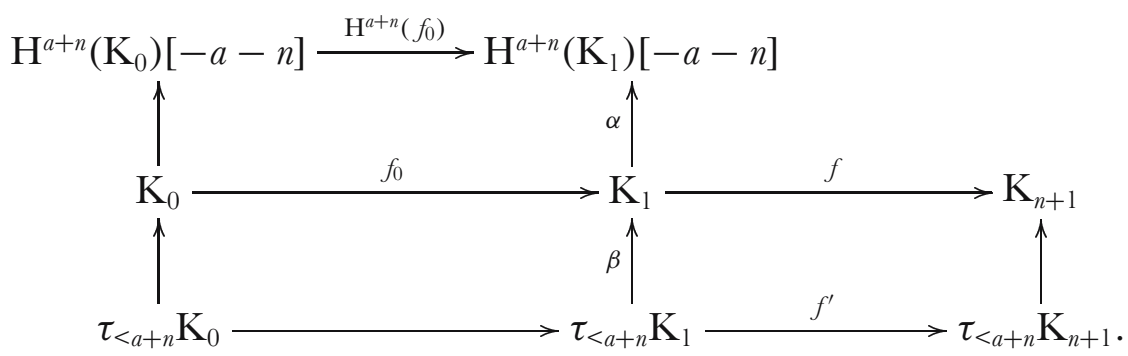


Since $\mathrm{H}^{a+n}\left(f_{0}\right)=0, \alpha f_{0}=0$ and since $\beta$ and $\alpha$ comprise a distinguished triangle, it follows that $f_{0}$ factors through $\beta$. The induction hypothesis implies that $f^{\prime}=0$, and it follows that $f f_{0}=0$. This proves the claim.

Corollary 4.32. - Let $\mathscr{L}:=\left(\mathscr{O}_{\mathscr{X}},-d f\right) \in \operatorname{HIG}(\mathscr{X} / \mathscr{S})$, let $\hat{\mathscr{L}}$ denote its formal completion along $\mathscr{Z}$, and let $b: \mathscr{L} \rightarrow \mathscr{L}_{n}$ denote the projection to the restriction of $\mathscr{L}$ to the nth infinitesimal neighborhood of $\mathscr{Z}$. Then for sufficiently large $n$, there exists a map $s$ in the derived category making the diagram below commute.

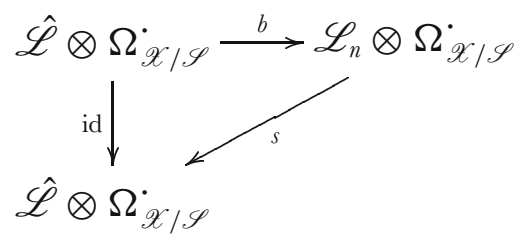

Proof. - Let us write $\hat{\mathscr{L}}^{\cdot}$ for the complex $\hat{\mathscr{L}} \otimes \Omega_{\mathscr{X} / \mathscr{S}}$, and consider for each natural number $n$ the exact sequence of complexes

$$
0 \rightarrow \mathrm{I}_{\mathscr{Z}}^{n} \hat{\mathscr{L}}^{\cdot} \rightarrow \hat{\mathscr{L}}^{\cdot} \rightarrow \mathscr{L}_{n}^{\cdot} \rightarrow 0
$$

There is then a corresponding exact sequence of abelian groups

$$
\operatorname{Ext}^{0}\left(\mathscr{L}_{n}^{\cdot}, \hat{\mathscr{L}}^{\cdot}\right) \rightarrow \operatorname{Ext}^{0}\left(\hat{\mathscr{L}}^{\cdot}, \hat{\mathscr{L}}^{\cdot}\right) \rightarrow \operatorname{Ext}^{0}\left(\mathrm{I}_{\mathscr{L}}^{n} \hat{\mathscr{L}}^{\cdot}, \hat{\mathscr{L}}^{\cdot}\right)
$$

where $\operatorname{Ext}^{0}$ means hyperext, or equivalently, the group of morphisms in the derived category. It will thus suffice to prove that the identity element of $\operatorname{Ext}^{0}\left(\hat{\mathscr{L}}^{\cdot}, \hat{\mathscr{L}}^{\cdot}\right)$ maps to zero in $\operatorname{Ext}^{0}\left(\mathrm{I}_{\mathscr{Z}}^{n} \hat{\mathscr{L}}^{\cdot}, \hat{\mathscr{L}}^{\cdot}\right)$. But the image of the identity element is just the class of the inclusion mapping, which vanishes for $n$ sufficiently large by Proposition 4.30.

Proof of Theorem 4.23. - Choose $n$ as in Corollary 4.32 and localize $\mathscr{S}$ so that all primes less than the maximum of $m$ and $n+\operatorname{dim}(\mathscr{X} / \mathscr{S})$, become invertible. Let $\mathrm{X} / \mathrm{S}$ and $\mathrm{Z} / \mathrm{S}$ denote the reductions of $\mathscr{X} / \mathscr{S}$ and $\mathscr{Z} / \mathscr{S}$ modulo one of the remaining primes $p$. Let $\mathrm{X}_{/ \mathrm{Z}}$ denote the formal completion of $\mathrm{X}$ along $\mathrm{Z}$, let $\hat{\mathrm{E}}:=\mathrm{E}_{/ \mathrm{Z}}$ and $\hat{\mathrm{L}}^{\prime}:=\mathrm{L}_{/ \mathrm{Z}}^{\prime}$, and consider the following diagram:

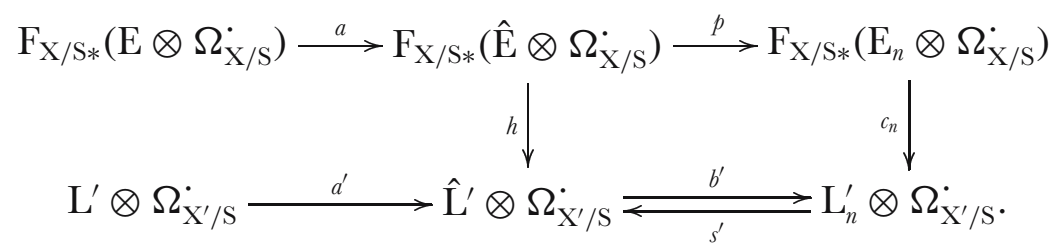


Here $a, p, a^{\prime}$, and $b^{\prime}$ are the obvious maps, $c_{n}$ is the quasi-isomorphism coming from Theorem 2.26, $s^{\prime}$ is the pullback via $\pi$ of the map $s$ of Corollary 4.32, and $h:=$ $s^{\prime} c_{n} p$. Note that we do not know if $b^{\prime} h=c_{n} p$. The arrow $a$ is a quasi-isomorphism by Proposition 2.31 and $a^{\prime}$ is a quasi-isomorphism by a similar (easier) argument. We shall show that $h$ is a quasi-isomorphism, completing the proof of Theorem 4.23.

Since our statement is local, we may restrict to an open affine subset $\mathrm{U}$ of $\mathrm{X}$ and then choose a lifting $\tilde{\mathrm{F}}$ of $\mathrm{F}_{\mathrm{X} / \mathrm{S}} \bmod p^{2}$. Let $\mathrm{C}_{\tilde{\mathrm{F}}}(\hat{\mathrm{E}})$ be the formal Cartier transform of $\hat{\mathrm{E}}$ described in Proposition 2.32 with respect to this lifting.

Claim. - There exists an invertible sheaf $\Lambda$ on $\mathrm{X}_{/ \mathrm{Z}}^{\prime}$ such that $\mathrm{C}_{\tilde{\mathrm{F}}}(\hat{\mathrm{E}}) \cong \mathrm{L}_{\mathscr{O}_{\mathrm{X}^{\prime}}} \Lambda$, where $\Lambda$ is given the trivial Higgs field.

Indeed, the F-Higgs module corresponding to the $p$-curvature of $\mathrm{C}_{\tilde{\mathrm{F}}}^{-1}\left(\hat{\mathrm{L}}^{\prime}\right)$ is $\mathrm{F}_{\mathrm{X} / \mathrm{S}}^{*} \hat{\mathrm{L}}^{\prime}$, and hence the $p$-curvature of $\mathscr{H} 0 m\left(\hat{\mathrm{E}}, \mathrm{C}_{\tilde{\mathrm{F}}}^{-1}\left(\hat{\mathrm{L}}^{\prime}\right)\right)$ is zero. Hence there exists an invertible sheaf $\Lambda$ on $\mathrm{X}^{\prime}$ such that $\mathscr{H} o m\left(\hat{\mathrm{E}}, \mathrm{C}_{\tilde{\mathrm{F}}}^{-1}\left(\hat{\mathrm{L}}^{\prime}\right)\right) \cong \mathrm{F}_{\mathrm{X} / \mathrm{S}}^{*} \Lambda$ with the Frobenius descent connection. Then $\mathrm{C}_{\tilde{\mathrm{F}}}(\hat{\mathrm{E}}) \cong \mathrm{L}^{\prime} \otimes_{\mathscr{O}_{\mathrm{X}^{\prime}}} \Lambda$, where $\Lambda$ has the trivial Higgs field.

By the compatibility of $\mathrm{C}_{\tilde{\mathrm{F}}}$ and $\mathrm{C}_{\mathscr{X} / \mathscr{S}}$, the isomorphism $\alpha_{n}: \mathrm{C}_{\mathscr{X} / \mathscr{S}}\left(\mathrm{E}_{n}\right) \cong \mathrm{L}_{n}^{\prime}$ of Proposition 4.28 defines a trivialization of $\Lambda_{n}$. Restricting to smaller affine if necessary, we may assume that $\Lambda$ is trivial, and choose an extension $\alpha$ of $\alpha_{n}$ to an isomorphism $\mathrm{C}_{\tilde{\mathrm{F}}}(\hat{\mathrm{E}}) \rightarrow \hat{\mathrm{L}}^{\prime}$. Now consider the commutative diagram of maps in the derived category:

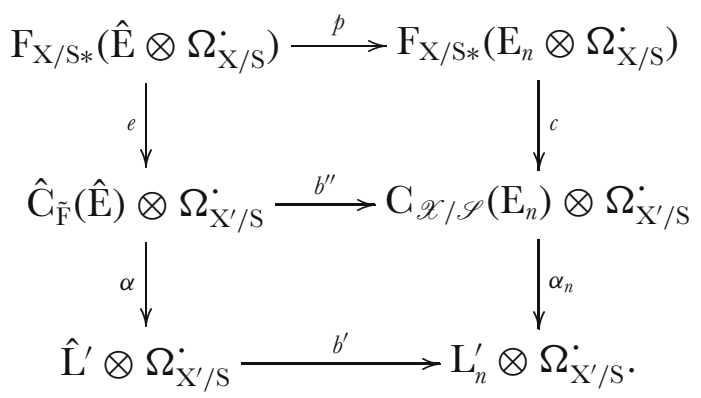

The arrows $e$ and $c$ are quasi-isomorphisms by Proposition 2.32 and Theorem 2.26, respectively, and $\alpha$ and $\alpha_{n}$ are quasi-isomorphisms by construction. Furthermore, $c_{n}=\alpha_{n} c$, so

$$
h=s^{\prime} c_{n} p=s^{\prime} \alpha_{n} c p=s^{\prime} \alpha_{n} b^{\prime \prime} e=s^{\prime} b^{\prime} \alpha e=\alpha e
$$

and hence is a quasi-isomorphism.

\section{Appendix: Higgs fields and Higgs transforms}

\subsection{Higgs fields over group schemes}

Let X/S be a smooth morphism of schemes, let $\Omega_{\mathrm{X} / \mathrm{S}}$ be its sheaf of Kahler differentials and $\mathrm{T}_{\mathrm{X} / \mathrm{S}}$ the dual of $\Omega_{\mathrm{X} / \mathrm{S}}$. Recall that a Higgs field on a sheaf $\mathrm{E}$ of 
$\mathscr{O}_{\mathrm{X}}$-modules is any of the following equivalent sets of data:

1. an $\mathscr{O}_{\mathrm{X}}$-linear map $\theta: \mathrm{E} \rightarrow \mathrm{E} \otimes \Omega_{\mathrm{X} / \mathrm{S}}$ such that the composition of $\theta$ with the map $\mathrm{E} \otimes \Omega_{\mathrm{X} / \mathrm{S}} \rightarrow \mathrm{E} \otimes \Lambda^{2} \Omega_{\mathrm{X} / \mathrm{S}}$ induced by $\theta$ vanishes

2. a linear map $\theta: \mathrm{T}_{\mathrm{X} / \mathrm{S}} \rightarrow \mathscr{E} d_{\mathscr{O}_{\mathrm{X}}}(\mathrm{E})$ with the property that the endomorphisms associated to any two sections of $\mathrm{T}_{\mathrm{X} / \mathrm{S}}$ commute

3. an extension $\theta$ of the $\mathscr{O}_{\mathrm{X}}$-module structure on $\mathrm{E}$ to an $\mathrm{S}^{*} \mathrm{~T}_{\mathrm{X} / \mathrm{S}}$-module structure.

If $\mathrm{E}$ is quasi-coherent, then associated to the $\mathrm{S}^{\cdot} \mathrm{T}_{\mathrm{X} / \mathrm{S}}$-module $\mathrm{E}$ is a quasi-coherent

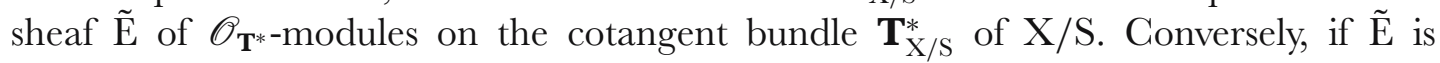
such a sheaf, its direct image on $\mathrm{X}$ is a quasi-coherent sheaf of $\mathscr{O}_{\mathrm{X}}$-modules equipped with a Higgs field.

These definitions make sense with any locally free sheaf $\mathrm{T}$ in place of $\mathrm{T}_{\mathrm{X} / \mathrm{S}}$ and with the vector bundle $\mathbf{V T}:=\operatorname{Spec}_{\mathrm{X}} \mathrm{S}^{\circ} \mathrm{T}$ in place of cotangent bundle. In fact, it will be useful for us to work in an even more general context, in which the vector bundle $\mathbf{T}_{\mathrm{X} / \mathrm{S}}^{*}$ is replaced by any commutative affine group $\mathrm{G}$ scheme over $\mathrm{X}$. Abusing notation, we shall denote by $\mathscr{O}_{\mathrm{G}}$ the sheaf of $\mathscr{O}_{\mathrm{X}}$-bialgebras on $\mathrm{X}$ corresponding to $\mathrm{G}$.

Definition 5.1. - Let $\mathrm{G}$ be a commutative flat affine group scheme over $\mathrm{X}$ and let $\mathrm{E}$ be a sheaf of $\mathscr{O}_{\mathrm{X}}$-modules on $\mathrm{X} . A \mathrm{G}$-field on $\mathrm{E}$ is a structure $\theta$ of an $\mathscr{O}_{\mathrm{G}}$-module on $\mathrm{E}$, compatible with the given $\mathscr{O}_{\mathrm{X}}$-module structure via the map $\mathscr{O}_{\mathrm{X}} \rightarrow \mathscr{O}_{\mathrm{G}}$.

We denote by G-HIG the category whose morphisms are sheaves of $\mathscr{O}_{\mathrm{X}}$-modules $\mathrm{E}$ equipped with a G-field $\theta$ and whose objects are morphisms compatible with the G-fields. We will often omit the $\theta$ from the notation when no confusion seems likely to result. As before, there is an evident equivalence between the category of quasicoherent objects in this category and the category of quasi-coherent sheaves on G. Since we will have to deal with sheaves which are not quasi-coherent, we will not make use of the topological space $\operatorname{Spec}_{\mathrm{X}} \mathscr{O}_{\mathrm{G}}$. Nevertheless we will try to use geometric notation whenever possible. Thus, if $\mathscr{A}$ is a sheaf of $\mathscr{O}_{\mathrm{X}}$-algebras, we denote by $\operatorname{Mod}(\mathscr{A})$ the category of sheaves of $\mathscr{A}$-modules on the topological space X. If $\gamma^{\sharp}: \mathscr{A} \rightarrow \mathscr{B}$ is a homomorphism of sheaves of $\mathscr{O}_{\mathrm{X}}$-algebras, we have functors:

$$
\begin{aligned}
\gamma^{*}: \operatorname{Mod}(\mathscr{A}) \rightarrow \operatorname{Mod}(\mathscr{B}): & \mathrm{M} \mapsto \mathscr{B} \otimes_{\mathscr{A}} \mathrm{M} \\
\gamma_{*}: \operatorname{Mod}(\mathscr{B}) \rightarrow \operatorname{Mod}(\mathscr{A}): & \mathrm{N} \mapsto \mathrm{N}, \quad \text { with an }:=\gamma^{\sharp}(a) n \\
\gamma^{!}: \operatorname{Mod}(\mathscr{A}) \rightarrow \operatorname{Mod}(\mathscr{B}): & \mathrm{M} \mapsto \mathscr{H} o m_{\mathscr{A}}\left(\gamma_{*} \mathscr{B}, \mathrm{M}\right), \\
& \quad \text { with }(b h)\left(b^{\prime}\right):=h\left(b b^{\prime}\right),
\end{aligned}
$$

together with the standard adjunction isomorphisms:

$$
\begin{aligned}
\mathscr{H}_{0} m_{\mathscr{A}}\left(\mathrm{M}, \gamma_{*} \mathrm{~N}\right) & \cong \gamma_{*} \mathscr{H}_{0} m_{\mathscr{B}}\left(\gamma^{*} \mathrm{M}, \mathrm{N}\right) \\
\mathscr{H}_{0} m_{\mathscr{A}}\left(\gamma_{*} \mathrm{~N}, \mathrm{M}\right) & \cong \gamma_{*} \mathscr{H}_{\mathscr{B}}\left(\mathrm{N}, \gamma^{!} \mathrm{M}\right) .
\end{aligned}
$$


Note that even if $\mathscr{A}$ and $\mathscr{B}$ are quasi-coherent, the functor $\gamma^{!}$does not preserve quasicoherence, in general.

In our context we shall consider the following morphisms of $\mathrm{X}$-schemes and the corresponding morphisms of sheaves of $\mathscr{O}_{\mathrm{X}}$-algebras. Here all fiber products are taken in the category of $\mathrm{X}$-schemes and all tensor products in the category of $\mathscr{O}_{\mathrm{X}}$-modules.

Notation 5.2.

$$
\begin{array}{rll}
p_{i}: \mathrm{G} \times \mathrm{G} \rightarrow \mathrm{G}: & \left(g_{1}, g_{2}\right) \mapsto g_{i}, & p_{i}^{\sharp}: \mathscr{O}_{\mathrm{G}} \rightarrow \mathscr{O}_{\mathrm{G}} \otimes \mathscr{O}_{\mathrm{G}} \\
\sigma: \mathrm{G} \times \mathrm{G} \rightarrow \mathrm{G} \times \mathrm{G}: & \left(g_{1}, g_{2}\right) \mapsto\left(g_{2}, g_{1}\right), & \sigma^{\sharp}: \mathscr{O}_{\mathrm{G}} \otimes \mathscr{O}_{\mathrm{G}} \rightarrow \mathscr{O}_{\mathrm{G}} \otimes \mathscr{O}_{\mathrm{G}} \\
\iota: \mathrm{G} \rightarrow \mathrm{G}: & g \mapsto g^{-1}, & \iota^{\sharp}: \mathscr{O}_{\mathrm{G}} \rightarrow \mathscr{O}_{\mathrm{G}} \\
\mu: \mathrm{G} \times \mathrm{G} \rightarrow \mathrm{G}: & \left(g_{1}, g_{2}\right) \mapsto g_{1} g_{2}, & \mu^{\sharp}: \mathscr{O}_{\mathrm{G}} \rightarrow \mathscr{O}_{\mathrm{G}} \otimes \mathscr{O}_{\mathrm{G}} \\
\mu^{\prime}: \mathrm{G} \times \mathrm{G} \rightarrow \mathrm{G}: & \left(g_{1}, g_{2}\right) \mapsto g_{2} g_{1}^{-1}, & \mu^{\prime \sharp}: \mathscr{O}_{\mathrm{G}} \rightarrow \mathscr{O}_{\mathrm{G}} \otimes \mathscr{O}_{\mathrm{G}} \\
i: \mathrm{X} \rightarrow \mathrm{G}: & x \mapsto 0, & i^{\sharp}: \mathscr{O}_{\mathrm{G}} \rightarrow \mathscr{O}_{\mathrm{X}} \\
p: \mathrm{G} \rightarrow \mathrm{X}:\left(g_{1}, \ldots, g_{n}\right) \mapsto p\left(g_{i}\right), & p^{\sharp}: \mathscr{O}_{\mathrm{X}} \rightarrow \mathscr{O}_{\mathrm{G}} \\
j: \mathrm{G} \rightarrow \mathrm{G}: \quad g & g \mapsto 0, & j^{\sharp}: \mathscr{O}_{\mathrm{G}} \rightarrow \mathscr{O}_{\mathrm{G}}
\end{array}
$$

These are the projections $p_{i}$, the inversion mapping $\iota$, the group law $\mu$, the twisted group law $\mu^{\prime}:=\mu \circ \sigma \circ(\iota \times$ id $)$, the augmentation given by the zero section of $\mathrm{G}$, the structure map $\mathrm{G}^{n} \rightarrow \mathrm{X}$, and the map $p \circ i$. Note that since $\iota^{2}=\mathrm{id}_{\mathrm{G}}$, $\iota_{*}=\iota^{*}$. If $\mathrm{E}$ is any object of G-HIG, we let $\mathrm{E}^{\iota}:=\iota_{*} \mathrm{E}=\iota^{*} \mathrm{E}$.

\subsection{Convolution}

Definition 5.3. - Let $\left(\mathrm{E}_{1}, \theta_{1}\right)$ and $\left(\mathrm{E}_{2}, \theta_{2}\right)$ be two objects of G-HIG. Then

1. $\mathrm{E}_{1} \otimes \mathrm{E}_{2}:=p_{1}^{*} \mathrm{E}_{1} \otimes_{\mathscr{O}_{\mathrm{G} \times \mathrm{G}}} p_{2}^{*} \mathrm{E}_{2}$, as an object of $\mathrm{G} \times \mathrm{G}-\mathrm{HIG}$.

2. $\mathrm{E}_{1} \circledast \mathrm{E}_{2}:=\mu_{*}\left(\mathrm{E}_{1} \otimes \mathrm{E}_{2}\right)$, as an object of $\mathrm{G}-\mathrm{HIG}$.

For example, if $\left(\mathrm{E}_{1}, \theta_{1}\right)$ and $\left(\mathrm{E}_{2}, \theta_{2}\right)$ are objects of $\mathrm{HIG}(\mathrm{X} / \mathrm{S})$, then $\mathrm{E}_{1} \circledast \mathrm{E}_{2}$ is the tensor product of $\mathrm{E}_{1}$ and $\mathrm{E}_{2}$ in the category of $\mathscr{O}_{\mathrm{X}}$-modules, with the Higgs field $\theta$ defined by

$$
\theta=\theta_{1} \otimes \mathrm{id}_{\mathrm{E}_{2}}+\mathrm{id}_{\mathrm{E}_{1}} \otimes \theta_{2} .
$$

Geometrically, the object $\left(\mathrm{E}_{1} \otimes \mathrm{E}_{2}, \theta\right)$ corresponds to the convolution of $\mathrm{E}_{1}$ and $\mathrm{E}_{2}$ with respect to the group structure of the cotangent space of $\mathrm{X} / \mathrm{S}$.

The associative law for $\mathrm{G}$ implies that the standard isomorphism

$$
\left(\mathrm{E}_{1} \otimes \mathrm{E}_{2}\right) \otimes \mathrm{E}_{3} \cong \mathrm{E}_{1} \otimes\left(\mathrm{E}_{2} \otimes \mathrm{E}_{3}\right)
$$

induces an isomorphism

$$
\left(\mathrm{E}_{1} \circledast \mathrm{E}_{2}\right) \circledast \mathrm{E}_{3} \cong \mathrm{E}_{1} \circledast\left(\mathrm{E}_{2} \circledast \mathrm{E}_{3}\right) .
$$


Similarly, the commutativity of $\mathrm{G}$ implies that the standard isomorphism $\mathrm{E}_{1} \otimes \mathrm{E}_{2} \cong$ $\mathrm{E}_{2} \otimes \mathrm{E}_{1}$ induces an isomorphism

$$
\mathrm{E}_{1} \circledast \mathrm{E}_{2} \cong \mathrm{E}_{2} \circledast \mathrm{E}_{1} \text {. }
$$

Furthermore, if we let

$$
\mathrm{U}:=i_{*} \mathscr{O}_{\mathrm{X}} \in \mathrm{G}-\mathrm{HIG}
$$

then the fact that $i$ is the identity section implies that the natural isomorphism $\mathscr{O}_{\mathrm{X}} \otimes_{\mathscr{O}_{\mathrm{X}}} \mathrm{E} \cong \mathrm{E}$ induces an isomorphism in G-HIG:

$$
\mathrm{U} \circledast \mathrm{E} \cong \mathrm{E} .
$$

Thus $\circledast$ makes the category G-HIG into an $\mathscr{O}_{\mathrm{X}}$-linear tensor category [9] (ACU tensor category in the terminology of [32]), and $\mathrm{U}$ is its unit object.

Definition 5.4. - Let $\mathrm{E}_{1}$ and $\mathrm{E}_{2}$ be objects of $\mathrm{G}-\mathrm{HIG}$. Then

$$
\begin{aligned}
\mathscr{H} e x\left(\mathrm{E}_{1}, \mathrm{E}_{2}\right) & :=\mathscr{H}_{0} m_{\mathscr{O}_{\mathrm{G} \times \mathrm{G}}}\left(p_{1}^{*} \mathrm{E}_{1}, p_{2}^{!} \mathrm{E}_{2}\right) \\
\mathscr{H}\left(\mathrm{E}_{1}, \mathrm{E}_{2}\right) & :=\mu_{*}^{\prime} \mathscr{H} \operatorname{ex}\left(\mathrm{E}_{1}, \mathrm{E}_{2}\right) .
\end{aligned}
$$

We call $\mathscr{H} e x\left(\mathrm{E}_{1}, \mathrm{E}_{2}\right)$ the external Hom of $\mathrm{E}_{1}$ and $\mathrm{E}_{2}$. Its underlying $\mathscr{O}_{\mathrm{X}}$-module is given by

$$
\begin{aligned}
& \mathscr{H}_{0 m_{\mathrm{X}}}\left(p_{*} \mathrm{E}_{1}, p_{*} \mathrm{E}_{2}\right) \cong p_{*} \mathscr{H}_{0 m_{\mathscr{O}_{\mathrm{G}}}}\left(p^{*} p_{*} \mathrm{E}_{1}, \mathrm{E}_{2}\right) \cong p_{*} \mathscr{H}_{0 m_{\mathscr{O}_{\mathrm{G}}}}\left(p_{2 *} p_{1}^{*} \mathrm{E}_{1}, \mathrm{E}_{2}\right) \\
& \cong p_{*} \mathscr{H}_{0 m_{\mathscr{G}_{\mathrm{G}} \mathrm{G}}}\left(p_{1}^{*} \mathrm{E}_{1}, p_{2}^{\vdots} \mathrm{E}_{2}\right) \cong p_{*} \mathscr{H e x}\left(\mathrm{E}_{1}, \mathrm{E}_{2}\right) \\
& \cong p_{*} \mathscr{H}\left(\mathrm{E}_{1}, \mathrm{E}_{2}\right)
\end{aligned}
$$

and the $\mathscr{O}_{\mathrm{G}} \otimes \mathscr{O}_{\mathrm{G}}$ structure on $\mathscr{H} e x\left(\mathrm{E}_{1}, \mathrm{E}_{2}\right)$ is given by:

$$
(a \otimes b) h: \mathrm{E}_{1} \rightarrow \mathrm{E}_{2} \quad e_{1} \mapsto b h\left(a e_{1}\right) .
$$

Lemma 5.5. - Let $\mathrm{E}_{1}, \mathrm{E}_{2}$, and $\mathrm{E}_{3}$ be objects of $\mathrm{G}-\mathrm{HIG}$. Then the standard adjunction isomorphism in the category of $\mathscr{O}_{\mathrm{X}}$-modules

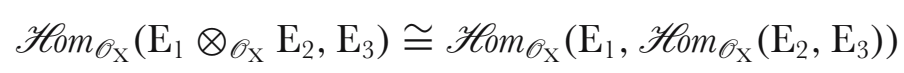

induces isomorphisms

$$
\begin{aligned}
& \operatorname{Hom}_{\mathscr{O}_{\mathrm{G}}}\left(\mathrm{E}_{1} \circledast \mathrm{E}_{2}, \mathrm{E}_{3}\right) \cong \operatorname{Hom}_{\mathscr{O}_{\mathrm{G}}}\left(\mathrm{E}_{1}, \mathscr{H}\left(\mathrm{E}_{2}, \mathrm{E}_{3}\right)\right) \quad \text { (of groups) } \\
&\left.\mathscr{H}\left(\mathrm{E}_{1} \circledast \mathrm{E}_{2}, \mathrm{E}_{3}\right) \cong \mathscr{H}\left(\mathrm{E}_{1}, \mathscr{H}\left(\mathrm{E}_{2}, \mathrm{E}_{3}\right)\right) \quad \text { (in } \mathrm{G}-\mathrm{HIG}\right) .
\end{aligned}
$$


Proof. - By definition,

$$
\begin{aligned}
& \operatorname{Hom}_{\mathscr{O}_{\mathrm{G}}}\left(\mathrm{E}_{1}, \mathscr{H}\left(\mathrm{E}_{2}, \mathrm{E}_{3}\right)\right)=\operatorname{Hom}_{\mathscr{O}_{\mathrm{G}}}\left(\mathrm{E}_{1}, \mu_{*}^{\prime} \mathscr{H}_{0} m_{\mathscr{O}_{\mathrm{G} \times \mathrm{G}}}\left(p_{1}^{*} \mathrm{E}_{2}, p_{2}^{!} \mathrm{E}_{3}\right)\right) \\
& =\operatorname{Hom}_{\mathscr{O}_{\mathrm{G} \times \mathrm{G}}}\left(\mu^{\prime *} \mathrm{E}_{1}, \mathscr{H}_{0 m_{\mathscr{O}_{\mathrm{G} \times \mathrm{G}}}}\left(p_{1}^{*} \mathrm{E}_{2}, p_{2}^{\prime} \mathrm{E}_{3}\right)\right) \\
& \left.=\operatorname{Hom}_{\mathscr{O}_{\mathrm{G} \times \mathrm{G}}}\left(\mu^{\prime *} \mathrm{E}_{1} \otimes_{\mathscr{O}_{\mathrm{G} \times \mathrm{G}}} p_{1}^{*} \mathrm{E}_{2}, p_{2}^{!} \mathrm{E}_{3}\right)\right) \text {. }
\end{aligned}
$$

Let $\alpha: \mathrm{G} \times \mathrm{G} \rightarrow \mathrm{G} \times \mathrm{G}$ denote the map $\left(\mu^{\prime}, p_{1}\right)$, i.e., the map sending $\left(g_{1}, g_{2}\right)$ to $\left(g_{2} g_{1}^{-1}, g_{1}\right)$. Note that $\alpha$ is an isomorphism, whose inverse $\beta=\left(p_{2}, \mu\right)$ sends $(a, b)$ to $(b, a b)$. Thus $\beta_{*}=\alpha^{*}$, and furthermore $\mu^{\prime *}\left(\mathrm{E}_{1}\right) \otimes p_{1}^{*} \mathrm{E}_{2}=\alpha^{*}\left(\mathrm{E}_{1} \otimes \mathrm{E}_{2}\right)$. Hence

$$
\begin{aligned}
\operatorname{Hom}_{\mathscr{O}_{\mathrm{G}}}\left(\mathrm{E}_{1}, \mathscr{H}\left(\mathrm{E}_{2}, \mathrm{E}_{3}\right)\right) & \left.=\operatorname{Hom}_{\mathscr{O}_{\mathrm{G} \times \mathrm{G}}}\left(\alpha^{*}\left(\mathrm{E}_{1} \otimes \mathrm{E}_{2}\right), p_{2}^{!} \mathrm{E}_{3}\right)\right) \\
& \left.=\operatorname{Hom}_{\mathscr{O}_{\mathrm{G} \times \mathrm{G}}}\left(\beta_{*}\left(\mathrm{E}_{1} \otimes \mathrm{E}_{2}\right), p_{2}^{!} \mathrm{E}_{3}\right)\right) \\
& \left.=\operatorname{Hom}_{\mathscr{O}_{\mathrm{G} \times \mathrm{G}}}\left(\mathrm{E}_{1} \otimes \mathrm{E}_{2}, \beta^{!} p_{2}^{!} \mathrm{E}_{3}\right)\right) \\
& \left.=\operatorname{Hom}_{\mathscr{O}_{\mathrm{G} \times \mathrm{G}}}\left(\mathrm{E}_{1} \otimes \mathrm{E}_{2}, \mu ! \mathrm{E}_{3}\right)\right) \\
& \left.=\operatorname{Hom}_{\mathscr{O}_{\mathrm{G}}}\left(\mu_{*}\left(\mathrm{E}_{1} \otimes \mathrm{E}_{2}\right), \mathrm{E}_{3}\right)\right) \\
& =\operatorname{Hom}_{\mathscr{O}_{\mathrm{G}}}\left(\mathrm{E}_{1} \circledast \mathrm{E}_{2}, \mathrm{E}_{3}\right) .
\end{aligned}
$$

This proves the first statement. The second statement just asserts that the standard adjunction morphism is compatible with the G-Higgs fields. It follows formally from the first. Indeed, it will suffice to check that for all E, the adjunction isomorphism induces isomorphisms:

$$
\operatorname{Hom}_{\mathscr{O}_{\mathrm{G}}}\left(\mathrm{E}, \mathscr{H}\left(\mathrm{E}_{1} \circledast \mathrm{E}_{2}, \mathrm{E}_{3}\right)\right) \cong \operatorname{Hom}_{\mathscr{O}_{\mathrm{G}}}\left(\mathrm{E}, \mathscr{H}\left(\mathrm{E}_{1}, \mathscr{H}\left(\mathrm{E}_{2}, \mathrm{E}_{3}\right)\right)\right) .
$$

This follows from the first statement and the associativity of $\circledast$.

Lemma 5.5 shows that $\mathscr{H}$ is the internal Hom functor of the tensor category $(\mathrm{G}-\mathrm{HIG}, \circledast)$ in these sense of [9]. As usual, the dual of an object E of G-HIG is defined by

$$
\mathrm{E}^{\vee}:=\mathscr{H}(\mathrm{E}, \mathrm{U})
$$

The map

$$
\text { ev }: \mathrm{E}^{\vee} \circledast \mathrm{E} \rightarrow \mathrm{U}
$$

is by definition the element of

$$
\operatorname{Hom}_{\mathscr{O}_{\mathrm{G}}}\left(\mathrm{E}^{\vee} \circledast \mathrm{E}, \mathrm{U}\right)=\operatorname{Hom}_{\mathscr{O}_{\mathrm{G}}}\left(\mathrm{E}^{\vee}, \mathscr{H}(\mathrm{E}, \mathrm{U})\right)=\operatorname{Hom}_{\mathscr{O}_{\mathrm{G}}}\left(\mathrm{E}^{\vee}, \mathrm{E}^{\vee}\right)
$$

corresponding to $\mathrm{id}_{\mathrm{E}^{\vee}}$; it corresponds to the usual evaluation map

$$
\mathscr{H}_{0 m_{\mathrm{X}}}\left(\mathrm{E}, \mathscr{O}_{\mathrm{X}}\right) \otimes \mathrm{E} \rightarrow \mathscr{O}_{\mathrm{X}}
$$


For any $\mathrm{E}_{2}$, one gets by functoriality maps

$$
\begin{aligned}
& \mathrm{E}_{2} \cong \mathscr{H}\left(\mathrm{U}, \mathrm{E}_{2}\right) \rightarrow \mathscr{H}\left(\mathrm{E}_{1}^{\vee} \circledast \mathrm{E}_{1}, \mathrm{E}_{2}\right) \\
& \operatorname{Hom}_{\mathscr{O}_{\mathrm{G}}}\left(\mathrm{E}_{2}, \mathscr{H}\left(\mathrm{U}, \mathrm{E}_{2}\right)\right) \rightarrow \operatorname{Hom}_{\mathscr{O}_{\mathrm{G}}}\left(\mathrm{E}_{2}, \mathscr{H}\left(\mathrm{E}_{1}^{\vee} \circledast \mathrm{E}_{1}, \mathrm{E}_{2}\right)\right) \\
& \mathrm{Hom}_{\mathscr{O}_{\mathrm{G}}}\left(\mathrm{E}_{2} \circledast \mathrm{U}, \mathrm{E}_{2}\right) \rightarrow \operatorname{Hom}_{\mathscr{O}_{\mathrm{G}}}\left(\mathrm{E}_{2} \circledast \mathrm{E}_{1}^{\vee} \circledast \mathrm{E}_{1}, \mathrm{E}_{2}\right) \\
& \mathscr{H}_{0} m_{\mathscr{O}_{\mathrm{G}}}\left(\mathrm{E}_{2}, \mathrm{E}_{2}\right) \rightarrow \operatorname{Hom}_{\mathscr{O}_{\mathrm{G}}}\left(\mathrm{E}_{2} \circledast \mathrm{E}_{1}^{\vee}, \mathscr{H}\left(\mathrm{E}_{1}, \mathrm{E}_{2}\right)\right) \\
& \mathscr{H}_{0 m_{\mathscr{O}_{\mathrm{G}}}}\left(\mathrm{E}_{2}, \mathrm{E}_{2}\right) \rightarrow \operatorname{Hom}_{\mathscr{O}_{\mathrm{G}}}\left(\mathrm{E}_{1}^{\vee} \circledast \mathrm{E}_{2}, \mathscr{H}\left(\mathrm{E}_{1}, \mathrm{E}_{2}\right)\right) \text {. }
\end{aligned}
$$

The element of $\operatorname{Hom}\left(\mathrm{E}_{2} \circledast \mathrm{E}_{1}, \mathscr{H}\left(\mathrm{E}_{1}, \mathrm{E}_{2}\right)\right)$ corresponding to $\operatorname{id}_{\mathrm{E}_{2}}$ is the map

$$
\mathrm{E}_{1}^{\vee} \circledast \mathrm{E}_{2} \rightarrow \mathscr{H}\left(\mathrm{E}_{1}, \mathrm{E}_{2}\right)
$$

corresponding to the usual map $\mathrm{E}_{1}^{\vee} \otimes \mathrm{E}_{2} \rightarrow \mathscr{H}_{0} m_{\mathscr{O}_{\mathrm{X}}}\left(\mathrm{E}_{1}, \mathrm{E}_{2}\right)$ in the category of $\mathscr{O}_{\mathrm{X}^{-}}$ modules. In particular it is a homomorphism in G-HIG and commutes with any endomorphism of $\mathrm{E}_{1}$ or $\mathrm{E}_{2}$ in the category G-HIG. For example, any local section of $\mathscr{O}_{\mathrm{G}}$ defines such an endomorphism on each $\mathrm{E}_{i}$. Note that if $\mathrm{E}_{1}$ is locally free and $\mathrm{E}_{1}$ or $\mathrm{E}_{2}$ is of finite presentation as an $\mathscr{O}_{\mathrm{X}}$-module, (5.5.2) an isomorphism. For example, when $\mathrm{G}$ is the cotangent space of $\mathrm{X}$ and $\theta$ is a Higgs field on $\mathrm{X}$, then the Higgs field $\theta^{\vee}$ on $\mathrm{E}^{\vee}$ is given by the usual rule, so that

$$
\left\langle\theta_{\xi}(\phi), e\right\rangle+\left\langle\phi, \theta_{\xi}(e)\right\rangle=0
$$

for sections $\xi$ of $\mathrm{T}, \phi$ of $\mathrm{E}^{\vee}$ and $e$ of $\mathrm{E}$.

Remark 5.6. - If $\mathrm{E}_{1}$ and $\mathrm{E}_{2}$ are objects of $\mathrm{G}-\mathrm{HIG}$, the $\mathscr{O}_{\mathrm{X}}$-module underlying $\mathscr{H}\left(\mathrm{E}_{1}, \mathrm{E}_{2}\right)$ is $\mathscr{H}_{0 m_{\mathscr{O}_{\mathrm{X}}}}\left(\mathrm{E}_{1}, \mathrm{E}_{2}\right)$ and the $\mathscr{O}_{\mathrm{X}}$-module underlying $\mathrm{E}_{1} \circledast \mathrm{E}_{2}$ is $\mathrm{E}_{1} \otimes_{\mathscr{O}_{\mathrm{X}}} \mathrm{E}_{2}$. These $\mathscr{O}_{\mathrm{X}}$-modules also inherit $\mathscr{O}_{\mathrm{G}}$-structures by "transport of structure" from the $\mathscr{O}_{\mathrm{G}}$ module structures of $\mathrm{E}_{1}$ and $\mathrm{E}_{2}$. When necessary we denote by $\theta_{\mathrm{E}_{i}}$ the structure coming from $\mathrm{E}_{i}$ in this way and by $\theta_{t o t}$ the structure defined in (5.3) and (5.4). Thus $\theta_{\mathrm{E}_{i}}$ is the structure on $\mathscr{H}\left(\mathrm{E}_{1}, \mathrm{E}_{2}\right)\left(\right.$ resp. $\left.\mathrm{E}_{1} \circledast \mathrm{E}_{2}\right)$ obtained from the structure on $\mathscr{H}$ ex $\left(\mathrm{E}_{1}, \mathrm{E}_{2}\right)$ (resp. $\mathrm{E}_{1} \otimes \mathrm{E}_{2}$ ) by letting $\mathscr{O}_{\mathrm{G}}$ act via the morphism $p_{i *}$. Note in particular that the $\mathscr{O}_{\mathrm{G}^{-}}$ module structure on $\mathrm{E}^{\vee}$ is not the structure $\theta_{\mathrm{E}}$ corresponding to the action by transport of structure on $\operatorname{Hom}_{\mathscr{O}_{\mathrm{X}}}\left(\mathrm{E}, \mathscr{O}_{\mathrm{X}}\right)$, rather it is given by $\iota_{*} \theta_{\mathrm{E}}$. Indeed, the $\mathscr{O}_{\mathrm{G}} \otimes \mathscr{O}_{\mathrm{G}}{ }^{-}$ module $\mathscr{H e x}\left(\mathrm{E}, \mathscr{O}_{\mathrm{X}}\right)$ is annihilated by the ideal of the graph $\Gamma_{j}$ of the zero morphism $j: \mathrm{G} \rightarrow \mathrm{G}$, and $\mu^{\prime} \circ \Gamma_{j}=\iota$.

Remark 5.7. - A morphism $h: \mathrm{G}^{\prime} \rightarrow \mathrm{G}$ of affine X-schemes induces a pair of adjoint functors

$$
h^{*}: \mathrm{G}-\mathrm{HIG} \rightarrow \mathrm{G}^{\prime}-\mathrm{HIG} \text { and } h_{*}: \mathrm{G}^{\prime}-\mathrm{HIG} \rightarrow \mathrm{G}-\mathrm{HIG} .
$$

If $h$ is a homomorphism of group schemes, these are compatible with $\circledast$ and $\mathscr{H}$. For example, let $f: \mathrm{X} \rightarrow \mathrm{Y}$ be a morphism of schemes, let $\mathrm{G}^{\prime \prime}$ be a commutative 
affine group scheme over $\mathrm{Y}$, and let $f^{-1} \mathrm{G}^{\prime \prime}$ be its pullback to $\mathrm{X}$. If $(\mathrm{E}, \theta)$ is an object of $\mathrm{G}^{\prime \prime}-\mathrm{HIG}$, then $f^{*} \mathrm{E}$ has a natural $f^{-1} \mathrm{G}^{\prime \prime}$-field $f^{*} \theta$. If $\mathrm{G}$ is an affine group scheme over X equipped with a map $h: f^{-1} \mathrm{G}^{\prime} \rightarrow \mathrm{G}$, then one gets by composition with $h^{\#}$ a G-field on $f^{*} \mathrm{E}$. For example, this construction applied to the cotangent bundles, with $h$ the differential of $f$, defines a functor $f^{*}: \mathrm{HIG}(\mathrm{Y} / \mathrm{S}) \rightarrow \mathrm{HIG}(\mathrm{X} / \mathrm{S})$. Finally, note that since $\iota: G \rightarrow G$ is a group homomorphism, we find a canonical isomorphism

$$
\left(\mathrm{E}_{1} \circledast \mathrm{E}_{2}\right)^{\iota} \cong \mathrm{E}_{1}^{\iota} \circledast \mathrm{E}_{2}^{\iota} .
$$

Remark 5.8. - Let $\mathrm{E}_{1}$ and $\mathrm{E}_{2}$ be object of G-HIG. Then there is a natural isomorphism of sheaves of $\mathscr{O}_{\mathrm{X}}$-modules

$$
i \cdot \mathscr{H}\left(\mathrm{E}_{1}, \mathrm{E}_{2}\right) \cong p_{*} \mathscr{H}_{0} m_{\mathscr{O}_{\mathrm{G}}}\left(\mathrm{E}_{1}, \mathrm{E}_{2}\right) .
$$

This follows from the adjointness properties of $\mathscr{H}$ :

$$
\begin{aligned}
& i^{!} \mathscr{H}\left(\mathrm{E}_{1}, \mathrm{E}_{2}\right):=\mathscr{H}_{0} m_{\mathscr{O}_{\mathrm{G}}}\left(i_{*} \mathscr{O}_{\mathrm{X}}, \mathscr{H}\left(\mathrm{E}_{1}, \mathrm{E}_{2}\right)\right) \\
& \cong \mathscr{H}_{0} m_{\mathscr{O}_{\mathrm{G}}}\left(\mathrm{U} \circledast \mathrm{E}_{1}, \mathrm{E}_{2}\right) \cong \mathscr{H}_{0} m_{\mathscr{O}_{\mathrm{G}}}\left(\mathrm{E}_{1}, \mathrm{E}_{2}\right) \text {. }
\end{aligned}
$$

We find a natural map of $\mathscr{O}_{\mathrm{X}}$-modules

$$
\mathscr{H}_{\mathscr{O}_{\mathrm{G}}}\left(\mathrm{E}_{1}, \mathrm{E}_{2}\right) \cong i^{\prime} \mathscr{H}\left(\mathrm{E}_{1}, \mathrm{E}_{2}\right) \subseteq p_{*} \mathscr{H}\left(\mathrm{E}_{1}, \mathrm{E}_{2}\right)
$$

This map is compatible with the actions of $\mathscr{O}_{\mathrm{G}}$ induced by transport of structure via its actions on $\mathrm{E}_{1}$ and $\mathrm{E}_{2}$.

\subsection{Higgs transforms}

We can use an object of G-HIG as a kernel for what we shall call a Higgs transform, of which we consider the following variants.

Definition 5.9. - Let $\mathscr{F}$ be an object of G-HIG. Define functors from G-HIG to itself by:

$$
\begin{aligned}
& \mathscr{H}_{\mathscr{F}}(\mathrm{E}):=\mathscr{H}_{0} m_{\mathscr{O}_{\mathrm{G}}}(\mathscr{F}, \mathrm{E}) \\
& \mathscr{T}_{\mathscr{F}}(\mathrm{E}):=\mathscr{F} \otimes_{\mathscr{O}_{\mathrm{G}}} \mathrm{E} \\
& \mathscr{T} !(\mathrm{E}):=i !(\mathscr{F} \circledast \mathrm{E}) .
\end{aligned}
$$

We view these objects as G-Higgs modules, with the Higgs field induced by transport of structure from the field on $\mathscr{F}$. Note that this is the same as the field induced from $\mathrm{E}$ in the first and second cases, and differs by $\iota$ in the third. Indeed,

$$
i^{\prime}(\mathscr{F} \circledast \mathrm{E})=\operatorname{Hom}_{\mathscr{O}_{\mathrm{G}}}\left(i_{*} \mathscr{O}_{\mathrm{X}}, \mu_{*}(\mathscr{F} \otimes \mathrm{E})\right) \cong \operatorname{Hom}_{\mathscr{O}_{\mathrm{G} \times \mathrm{G}}}\left(\mu^{*} i_{*} \mathscr{O}_{\mathrm{X}}, \mathscr{F} \otimes \mathrm{E}\right) .
$$


This is an $\mathscr{O}_{\mathrm{G} \times \mathrm{G}}$-module, and the action of $\mathscr{O}_{\mathrm{G}}$ by transport of structure via $\mathscr{F}$ corresponds to the action induced by the first projection, while the action via $\mathrm{E}$ is induced by the second projection. We claim these differ by $\iota$. In fact it is enough to check this for the $\mathscr{O}_{\mathrm{G} \times \mathrm{G}}$-module $i_{*} \mu^{*}\left(\mathscr{O}_{\mathrm{X}}\right)$. But this is clear, since the latter is annihilated by the ideal of the graph of $\iota$.

The map (5.5.2) induces a natural map of $\mathscr{O}_{\mathrm{G}}$-modules

$$
\iota^{*} \mathscr{T}_{\mathscr{F} \mathrm{v}}(\mathrm{E}) \rightarrow i !(\mathscr{H}(\mathscr{F}, \mathrm{E})) \cong \mathscr{H}_{\mathscr{F}}(\mathrm{E})
$$

which is an isomorphisms if $\mathscr{F}$ is finitely generated and projective as an $\mathscr{O}_{\mathrm{X}}$-module. The presence of the $\iota$ is due to the fact that $\mathscr{O}_{\mathrm{G}}$ acts on $\mathscr{T}_{\mathscr{F} \vee}(\mathrm{E})$ by transport of structure via $\mathscr{F}^{\vee}$ and on $\mathscr{H}_{\mathscr{F}}(\mathrm{E})$ via $\mathscr{F}$, and these structures differ by $\iota$, as we saw in Remark 5.6.

Let $\mathscr{B}$ be any object of G-HIG. Observe that there are natural transformations:

$$
\eta_{\mathscr{B}}: \mathscr{T}_{\mathscr{B}} \circ \mathscr{H}_{\mathscr{B}} \rightarrow \text { id } \text { and } \zeta_{\mathscr{B}}: \text { id } \rightarrow \mathscr{H}_{\mathscr{B}} \circ \mathscr{T}_{\mathscr{B}}
$$

where for any $\mathrm{E}$,

$$
\eta_{\mathscr{B}, \mathrm{E}}: \mathscr{B} \otimes_{\mathscr{O}_{\mathrm{G}}} \mathscr{H}_{0 m_{\mathscr{O}_{\mathrm{G}}}}(\mathscr{B}, \mathrm{E}) \rightarrow \mathrm{E}
$$

sends $b \otimes h$ to $h(b)$ and

$$
\zeta_{\mathscr{B}, \mathrm{E}}: \mathrm{E} \rightarrow \mathscr{H}_{0} m_{\mathscr{O}_{\mathrm{G}}}\left(\mathscr{B}, \mathscr{B} \otimes_{\mathscr{O}_{\mathrm{G}}} \mathrm{E}\right)
$$

sends $e$ to the homomorphism $b \mapsto b \otimes e$. Then the following result is immediate.

Proposition 5.10. - Let $\mathscr{B}$ be an object of G-HIG which is invertible as an $\mathscr{O}_{\mathrm{G}}$-module. Then $\eta_{\mathscr{B}}$ and $\zeta_{\mathscr{B}}$ are inverse isomorphisms. In particular, $\mathscr{H}_{\mathscr{B}}$ and $\mathscr{T}_{\mathscr{B}}$ are quasi-inverse equivalences of categories.

In our main application, $\mathscr{B}$ will be equipped with the structure of a cocommutative coalgebra with counit in the category G-HIG. That is, it will be provided with morphisms

$$
v: \mathscr{B} \rightarrow \mathscr{B} \circledast \mathscr{B} \quad \text { and } \quad \beta: \mathscr{B} \rightarrow \mathrm{U}
$$

satisfying the usual compatibilities. These data give the corresponding Higgs transform additional structure. We shall be especially interested in the following case.

Definition 5.11. - A character sheaf ${ }^{16}$ in $\mathrm{G}-\mathrm{HIG}$ is a coalgebra $(\mathscr{B}, v, \beta)$ such that the map $\tilde{v}: \mu^{*} \mathscr{B} \rightarrow \mathscr{B} \otimes \mathscr{B}$ corresponding to $v$ is an isomorphism of $\mathscr{O}_{\mathrm{G} \times \mathrm{G}}$-modules and the map $\tilde{\beta}: i^{*} \mathscr{B} \rightarrow \mathscr{O}_{\mathrm{X}}$ induced by $\beta$ is an isomorphism of $\mathscr{O}_{\mathrm{X}}$-modules.

\footnotetext{
16 This terminology is borrowed from G. Lusztig.
} 
Lemma 5.12. - Let $(\mathscr{B}, v, \beta)$ be a character sheaf in $\mathrm{G}$. The map $v$ induces an isomorphism of $\mathscr{O}_{\mathrm{G}}$-modules $\mathscr{B} \otimes_{\mathscr{O}_{\mathrm{G}}} \mathscr{B}^{l} \cong \mathscr{O}_{\mathrm{G}}$. In particular, $\mathscr{B}$ is invertible as an $\mathscr{O}_{\mathrm{G}}$-module, with inverse $\mathscr{B}$.

Proof. — Definition (5.11) implies that $i^{*} \mathscr{B} \cong \mathscr{O}_{\mathrm{X}}$, hence $j^{*} \mathscr{B} \cong \mathscr{O}_{\mathrm{G}}$. Let $\Gamma_{\iota}$ : $\mathrm{G} \rightarrow \mathrm{G} \times \mathrm{G}$ denote the graph of $\iota$. Pulling the isomorphism $\tilde{v}$ back via $\Gamma_{\iota}$, we find an isomorphism:

$$
\mathscr{B} \otimes_{\mathscr{O}_{\mathrm{G}}} \mathscr{B}^{\imath} \cong \Gamma_{\imath}^{*}(\mathscr{B} \bigotimes \mathscr{B}) \cong \Gamma_{\imath}^{*} \mu^{*}(\mathscr{B}) \cong j^{*} \mathscr{B} \cong \mathscr{O}_{\mathrm{G}}
$$

Remark 5.13. - Giving a character sheaf is equivalent to giving a commutative extension of the group $\mathrm{G}$ by the multiplicative group $\mathbf{G}_{m}$. Assume $\mathrm{G}$ is finite and flat over $\mathrm{X}$ and denote by $\mathrm{G}^{\vee}$ its Cartier dual. Then a $\mathrm{G}^{\vee}$-torsor gives rise to a character sheaf. Indeed, $G^{\vee}$ can be identified with the group of automorphisms of the trival extension $\mathbf{E}$ of $G$. Then if $\mathbf{L}$ is a $G^{\vee}$-torsor, we can form the "twist" $\mathbf{L} \otimes_{G^{\vee}} \mathbf{E}$ of $\mathbf{E}$ by $\mathbf{L}$. This defines an extension of $\mathrm{G}$ by $\mathbf{G}_{m}$ and hence a character sheaf $\mathscr{B}$. The functor $\mathbf{L} \mapsto \mathscr{B}$ is an equivalence from the category of $\mathrm{G}^{\vee}$-torsors to the category of character sheaves which are, locally on $\mathrm{X}$, isomorphic to $\mathscr{O}_{\mathrm{G}}$ as character sheaves.

Proposition 5.14. - Let $(\mathscr{B}, v, \beta)$ be a character sheaf on $\mathrm{G}$. If $\mathrm{E}_{1}$ and $\mathrm{E}_{2}$ are objects of $\mathrm{G}-\mathrm{HIG}$, the standard tensor product map $\otimes$ on homomorphisms and the comultiplication $v$ define a commutative diagram:

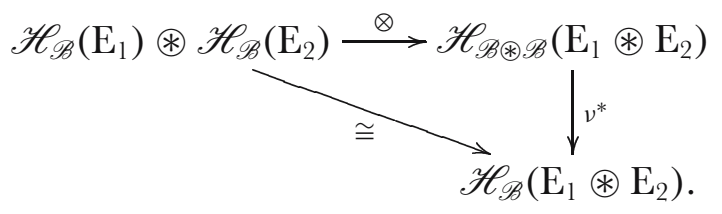

Thus the functor $\mathscr{H}_{\mathscr{B}}$ is an auto-equivalence of the tensor category G-HIG.

Proof. - The diagram above can be expanded as follows.

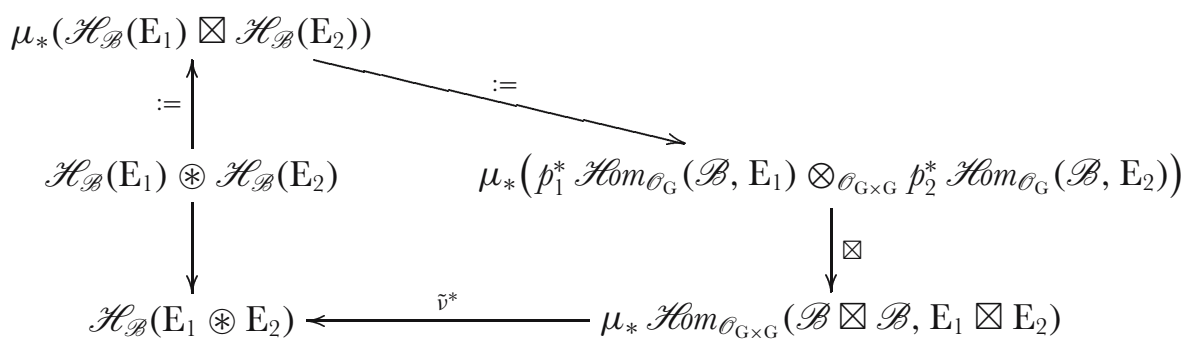

The right vertical map is an isomorphism because $\mathscr{B}$ is invertible, and the map $\tilde{v}^{*}$ is an isomorphism because $\tilde{v}$ is an isomorphism. The cocommutativity of the coalgebra $\mathscr{B}$ implies that $\mathscr{H}_{\mathscr{B}}$ preserves the commutativity constraint of the tensor category G-HIG. 
A change of sign allows us construct an involutive auto-equivalence of G-HIG.

Definition 5.15. - Let $\mathscr{B}$ be an object of G-HIG. Then $\mathscr{H}_{\mathscr{B}}^{\prime}$ is the functor G-HIG $\rightarrow$ $\mathrm{G}-\mathrm{HIG}$ sending an object $\mathrm{E}$ to $\iota_{*} \mathscr{H}_{0} m_{\mathscr{O}_{\mathrm{G}}}(\mathscr{B}, \mathrm{E})$.

For example, $\mathscr{H}_{\mathscr{O}_{\mathrm{G}}}^{\prime}$ can be identified with the involutive functor $\iota_{*}$. More generally, if $(\mathscr{B}, v, \beta)$ is a character sheaf, then by Lemma 5.12:

$$
\begin{aligned}
& \mathscr{H}_{\mathscr{B}}^{\prime}\left(\mathscr{H}_{\mathscr{B}}^{\prime}(\mathrm{E})\right)=\iota_{*} \mathscr{H}_{0} m_{\mathscr{O}_{\mathrm{G}}}\left(\mathscr{B}, \iota_{*} \mathscr{H}_{0 m_{\mathscr{O}_{\mathrm{G}}}}(\mathscr{B}, \mathrm{E})\right) \\
& \cong \iota_{*} \iota_{*} \mathscr{H}_{0 m_{\mathscr{O}_{\mathrm{G}}}}\left(\iota^{*} \mathscr{B}, \mathscr{H} o m(\mathscr{B}, \mathrm{E})\right) \\
& \cong \mathscr{H}_{0} m_{\mathscr{O}_{\mathrm{G}}}\left(\iota^{*} \mathscr{B} \otimes_{\mathscr{O}_{\mathrm{G}}} \mathscr{B}, \mathrm{E}\right) \cong \mathscr{H}_{0} m_{\mathscr{O}_{\mathrm{G}}}\left(\mathscr{O}_{\mathrm{G}}, \mathrm{E}\right) \cong \mathrm{E} .
\end{aligned}
$$

The natural inclusion of $\mathscr{O}_{\mathrm{X}}$-modules $\operatorname{Hom}_{\mathscr{O}_{\mathrm{G}}}(\mathscr{B}, \mathrm{E}) \subseteq \operatorname{Hom}_{\mathscr{O}_{\mathrm{X}}}(\mathscr{B}, \mathrm{E})$ defines morphisms of $\mathscr{O}_{\mathrm{G}}$-modules:

$$
\gamma_{\mathrm{E}}: \mathscr{H}_{\mathscr{B}}^{\prime}(\mathrm{E}) \rightarrow \mathscr{H}\left(\mathscr{B}, j_{*} \mathrm{E}\right) \quad \text { and } \quad j_{*} \mathscr{H}_{\mathscr{B}}^{\prime}(\mathrm{E}) \rightarrow \mathscr{H}(\mathscr{B}, \mathrm{E})
$$

The morphism $\beta: \mathscr{B} \rightarrow \mathrm{U}$ and the isomorphism $\mathscr{H}(\mathrm{U}, \mathrm{E}) \cong \mathrm{E}$ induce maps of $\mathscr{O}_{\mathrm{G}^{-}}$ modules:

$$
\beta_{\mathrm{E}}: \mathrm{E} \rightarrow \mathscr{H}(\mathscr{B}, \mathrm{E}) \quad \text { and } \quad j_{*} \mathrm{E} \rightarrow \mathscr{H}\left(\mathscr{B}, j_{*} \mathrm{E}\right) .
$$

Finally, let us consider the following diagram:

$(5.15 .3)$
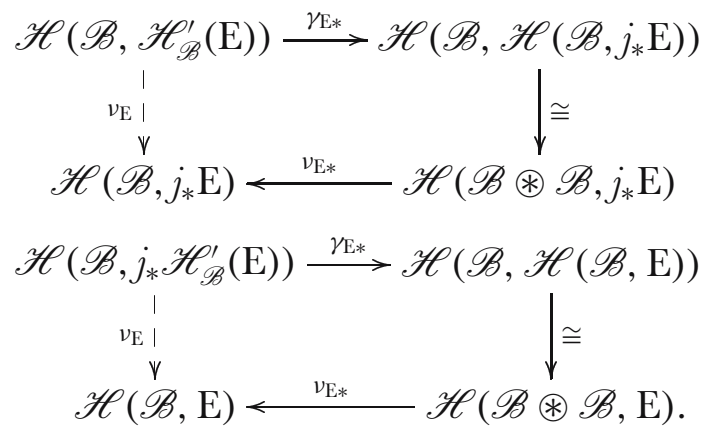

Here the top horizontal arrow is induced by the morphism $\gamma_{\mathrm{E}}(5.15 .1)$, the right arrow is the adjunction map of Lemma 5.5, and the bottom horizontal arrow is induced by $v$; the diagram defines the arrow $v_{\mathrm{E}}$.

Theorem 5.16. - Let $(\mathrm{B}, v, \beta)$ be a character sheaf for $\mathrm{G}$ (5.11). Then for any object $\mathrm{E}$ of $\mathrm{G}-\mathrm{HIG}$, the arrow $\nu_{\mathrm{E}}$ is an isomorphism and induces an isomorphism $\kappa_{\mathrm{E}}:\left(\mathscr{H}_{\mathscr{B}}^{\prime}\left(\mathscr{H}_{\mathscr{B}}^{\prime}(\mathrm{E})\right) \rightarrow \mathrm{E}\right.$ fitting into the following commutative diagram.

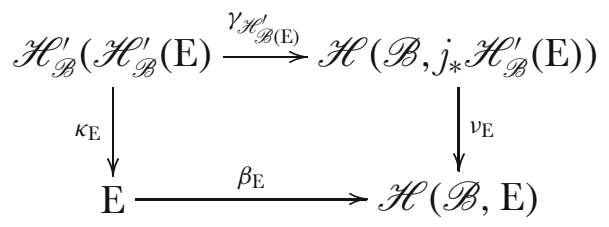


Proof. - Since $(\mathscr{B}, v, \beta)$ is a character sheaf, the map $\tilde{v}$ is an isomorphism, and so induces an isomorphism of $\mathscr{O}_{\mathrm{G}}$-modules:

$$
p_{2 *}(\tilde{v}): p_{2 *} \mu^{*} \mathscr{B} \rightarrow p_{2 *}(\mathscr{B} \otimes \mathscr{B})
$$

Since the diagram

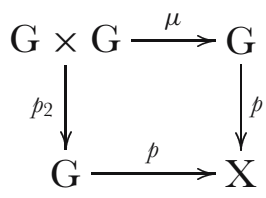

is Cartesian, the natural map $p^{*} p_{*} \mathscr{B} \rightarrow p_{2}^{*} \mu^{*} \mathscr{B}$ is an isomorphism. Composing this map with $p_{2_{*}}(\tilde{v})$, we find that $v$ induces an isomorphism

$$
p^{*} p_{*} \mathscr{B} \rightarrow p_{2 *}(\mathscr{B} \otimes \mathscr{B})
$$

Taking $\mathscr{H}_{0} m_{\mathscr{O}_{\mathrm{G}}}($, E) we find that the top arrow in the diagram below is an isomorphism.

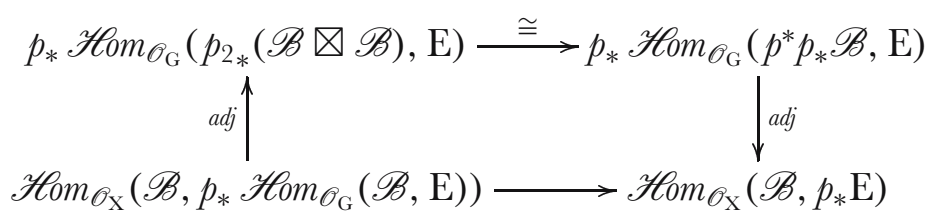

The vertical arrows are the adjunction isomorphisms and the bottom horizontal arrow is $p_{*}\left(v_{\mathrm{E}}\right)$. It follows that $v_{\mathrm{E}}$ is an isomorphism of $\mathscr{O}_{\mathrm{X}}$-modules, and it is compatible with the two pairs of $\mathscr{O}_{\mathrm{G}}$-module structures shown in the diagrams (5.15.3). Applying the functor $i$ to the isomorphism $v_{\mathrm{E}}$ in the left diagram, we find an isomorphism of $\mathscr{O}_{\mathrm{X}}$-modules:

$$
\kappa_{\mathrm{E}}: \mathscr{H}_{\mathscr{B}}^{\prime}\left(\mathscr{H}_{\mathscr{B}}^{\prime}(\mathrm{E})\right) \rightarrow \mathrm{E}
$$

But $\mu_{\mathrm{E}}$ is also compatible with the $\mathscr{O}_{\mathrm{G}}$-module structures in the right diagram. This

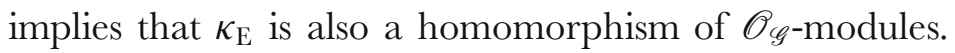

Remark 5.17. - If $\mathscr{B} \rightarrow \mathscr{B}^{\prime}$ is a surjection of invertible $\mathscr{O}_{\mathrm{G}}$-modules and $\mathrm{E}$ is an object of G-HIG which is annihilated by the annihilator of $\mathscr{B}^{\prime}$, then the natural map $\mathscr{H}_{\mathrm{B}^{\prime}}(\mathrm{E}) \rightarrow \mathscr{H}_{\mathscr{B}}(\mathrm{E})$ is an isomorphism.

It is sometimes convenient to use the dual point of view to that taken in Theorem 5.16. With the notation there, let $\left\{\mathrm{I}_{n}: n \in \mathbf{N}\right\}$ denote an inverse system of ideals 
of $\mathscr{O}_{\mathrm{G}}$ defining closed subschemes $\mathrm{G}_{n}$ of $\mathrm{G}$ whose support is the zero section $\mathrm{G}_{0}$. Suppose further that each $\mathscr{O}_{\mathrm{G}_{n}}$ is a locally free $\mathscr{O}_{\mathrm{X}}$-module of finite rank and that for all $m, n$, the comultiplication map fits into commutative diagrams:

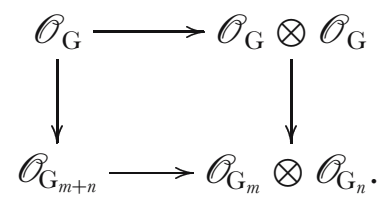

Let $\mathscr{B}_{n}:=\mathscr{B} / \mathrm{I}_{n} \mathscr{B}$ and let $\mathscr{A}_{n}:=\mathscr{H}\left(\mathscr{B}_{n}, \mathscr{O}_{\mathrm{X}}\right)$ Thus the map (5.9.1) induces an isomorphism:

$$
\mathscr{T}_{\mathscr{A}_{n}}^{!} \rightarrow \mathscr{H}_{\mathscr{B}_{n}}^{\prime}:=\iota_{*} \mathscr{H}_{\mathscr{B}_{n}} \text {. }
$$

Let $\mathscr{A}$ be the direct limit of the directed system $\mathscr{A}$., so that we find an injection

$$
\mathscr{T}_{\mathscr{A}}^{!} \cong \lim _{\longrightarrow} \mathscr{T}_{\mathscr{A}_{n}}^{!} \rightarrow \mathscr{H}_{\mathscr{B}}^{\prime} .
$$

The comultiplication maps on $\mathscr{B}$. induce multiplication maps $\mathscr{A}_{n} \otimes \mathscr{A}_{m} \rightarrow \mathscr{A}_{n+m}$, and $\mathscr{A}$ inherits the structure of an algebra in the category G-HIG. The identity element $1_{\mathscr{A}}$ is the dual of the identity section $i^{\sharp}$ of $\mathscr{B}$.

Let us say that an object $\mathrm{E}$ of G-HIG is I.-continuous if each local section $e$ of $\mathrm{E}$ is annihilated by $\mathrm{I}_{n}$ for some $n$, and let us denote the full subcategory of G-HIG consisting of such objects by G.-HIG. If $\mathrm{E}$ is I.-continuous, then any $\mathscr{O}_{\mathrm{G}}$-linear homomorphism $h: \mathscr{B} \rightarrow$ E factors through $\mathscr{B}_{n}$ for some $n$, so that (5.17.1) becomes an isomorphism. Then Theorem 5.16 can be reformulated as follows.

Theorem 5.18. - Let $\mathscr{B}$. and $\mathscr{A}$. be as described above, and let G.-HIG denote the full subcategory of G-HIG consisting of the I.-continuous objects.

1. For any object $\mathrm{E}$ of $\mathrm{G}$.-HIG,

$$
\alpha_{\mathrm{E}}: \mathrm{E} \rightarrow \mathscr{A} \circledast \mathrm{E} \quad e \mapsto 1 \otimes e
$$

defines a locally split injection whose image is the annihilator of I with respect to the Higgs field induced from the Higgs field on $\mathscr{A}$.

2. The functor

$$
\mathscr{T}_{\mathscr{A}}: \mathrm{G} \cdot-\mathrm{HIG} \rightarrow \mathrm{G} \cdot-\mathrm{HIG}
$$

is an involutive equivalence. Furthermore, the isomorphism $\mathscr{T}_{\mathscr{A}}^{!} \circ \mathscr{T}_{\mathscr{A}} \cong \mathrm{id}$ fits into the following commutative diagram

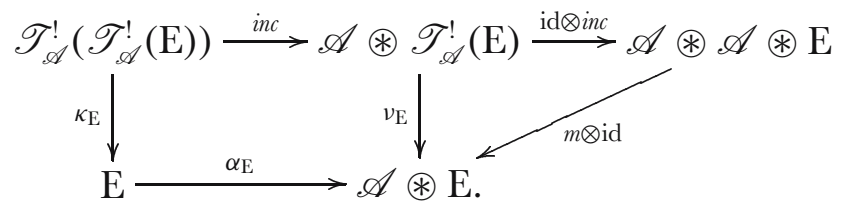


Here $\kappa_{\mathrm{E}}$ is compatible with the $\mathrm{G}$-Higgs fields, and $\nu_{\mathrm{E}}$ is a homomorphism with respect to the following fields:

$$
\mathscr{A} \circledast j_{*} \mathscr{T}_{\mathscr{A}}(\mathrm{E}) \rightarrow \mathscr{A} \circledast \mathrm{E} \quad \text { and } \quad \mathscr{A} \circledast \mathscr{T}_{\mathscr{A}}(\mathrm{E}) \rightarrow \mathscr{A} \circledast j_{*} \mathrm{E} .
$$

3. The functor $\mathscr{T}_{\mathscr{A}}$ is compatible with convolution products in the following sense. For any two objects $\mathrm{E}_{1}$ and $\mathrm{E}_{2}$ of $\mathrm{G} .-\mathrm{HIG}$, one has a canonical commutative diagram

$$
\mathscr{T}_{\mathscr{A}}\left(\mathrm{E}_{1}\right) \circledast \mathscr{T} !\left(\mathrm{E}_{2}\right) \stackrel{\otimes}{\longrightarrow} \mathscr{T} ! \mathfrak{A} \circledast \mathscr{A}\left(\mathrm{E}_{1} \circledast \mathrm{E}_{2}\right)
$$

Proof. - The first statement is clear. The diagram in the second statement is equivalent to the diagram in Theorem 5.16, and hence it follows that the maps indicated are isomorphisms. Let us note, however, that this can also be proved directly by dévissage. When $\mathrm{E}$ is annihilated by $\mathscr{I}$ this is clear. Since the sources and targets of both arrows are exact functors, a dévissage argument implies that the maps are isomorphisms if $\mathrm{E}$ is nilpotent. But any $\mathscr{I}$. continuous $\mathrm{E}$ is, locally on $\mathrm{X}$, a direct limit of nilpotent objects, so the general result follows. Similarly, (3) is a translation of Proposition 5.14, and can also be proved by dévissage.

\subsection{Examples and formulas}

Let us return to the case in which the group scheme is the group underlying a vector bundle $\mathbf{V T}$, where $\mathrm{T}$ is a locally free sheaf of $\mathscr{O}_{\mathrm{X}}$-modules of finite rank, so $\mathscr{O}_{\mathbf{V T}}$ is the symmetric algebra $\mathrm{S}^{\bullet} \mathrm{T}$. The group law in this case is given by the unique algebra homomorphism

$$
\mu^{*}: \mathrm{S}^{\bullet} \mathrm{T} \rightarrow \mathrm{S}^{*} \mathrm{~T} \otimes \mathrm{S}^{\bullet} \mathrm{T}
$$

such that $t \mapsto 1 \otimes t+t \otimes 1$. Let $\mathbf{V}_{\gamma} \mathrm{T}:=\operatorname{Spec} \Gamma$. (T) [3, Al] be the divided power envelope of the ideal of the zero section and $\hat{\mathbf{V}}_{\gamma} \mathrm{T}:=\operatorname{Spec} \hat{\Gamma}$.(T) its completion with respect to the divided power filtration. These are also group schemes, and the group law

$$
\mu^{*}: \Gamma .(\mathrm{T}) \rightarrow \Gamma \cdot(\mathrm{T}) \otimes \Gamma .(\mathrm{T})
$$

is the unique divided power homomorphism sending $t$ to $1 \otimes t+t \otimes 1$.

Let $\Omega$ be the dual of $\mathrm{T}$ and recall from [3, A10] that there is a natural isomorphism

$$
\rho_{n}: \mathrm{S}^{n}(\Omega) \rightarrow \mathscr{H} o m\left(\Gamma_{n}(\mathrm{~T}), \mathscr{O}_{\mathrm{X}}\right)
$$


for all $n$, and hence an isomorphism

$$
\mathrm{S}^{*}(\Omega) \rightarrow \mathscr{H}_{0}{ }^{\prime}\left(\Gamma \cdot(\mathrm{T}), \mathscr{O}_{\mathrm{X}}\right),
$$

where the / signifies the Matlis dual. The following proposition is essentially contained in Theorem V.1 of [33]; we give a slightly simpler proof here for the reader's convenience.

Proposition 5.19. - With respect to the pairing $\rho_{n} \mathrm{~S}^{n} \times \Gamma_{n} \rightarrow \mathscr{O}_{\mathrm{X}}$ defined above,

1. the algebra multiplication $\mathrm{S}^{i}(\Omega) \otimes \mathrm{S}^{j}(\Omega) \rightarrow \mathrm{S}^{i+j}(\Omega)$ is dual to the comultiplication of $\Gamma$.(T) followed by projection:

$$
\Gamma_{i+j}(\mathrm{~T}) \rightarrow \bigoplus_{a+b=i+j} \Gamma_{a}(\mathrm{~T}) \otimes \Gamma_{b}(\mathrm{~T}) \rightarrow \Gamma_{i}(\mathrm{~T}) \otimes \Gamma_{j}(\mathrm{~T})
$$

2. The algebra multiplication $\Gamma_{i}(\Omega) \otimes \Gamma_{j}(\Omega) \rightarrow \Gamma_{i+j}(\Omega)$ is dual to the comultiplication followed by projection:

$$
\mathrm{S}^{i+j}(\mathrm{~T}) \rightarrow \bigoplus_{a+b=i+j} \mathrm{~S}^{a}(\mathrm{~T}) \otimes \mathrm{S}^{b}(\mathrm{~T}) \rightarrow \mathrm{S}^{i}(\mathrm{~T}) \otimes \mathrm{S}^{j}(\mathrm{~T}) .
$$

Proof. - Let $\ell_{i}: \mathrm{T} \rightarrow \Gamma_{i}(\mathrm{~T})$ be the universal polynomial law of degree $i$ [3, A4]. Then if $k=i+j, \ell_{i} \otimes \ell_{j}$ defines a polynomial law $\mathrm{T} \rightarrow \Gamma_{i}(\mathrm{~T}) \otimes \Gamma_{j}(\mathrm{~T})$, and hence a linear map $\mu_{i, j}^{\prime}: \Gamma_{k}(\mathrm{~T}) \rightarrow \Gamma_{i}(\mathrm{~T}) \otimes \Gamma_{j}(\mathrm{~T})$. Adding these up we find a map

$$
\mu^{\prime}: \Gamma .(\mathrm{T}) \rightarrow \Gamma .(\mathrm{T}) \otimes \Gamma .(\mathrm{T}) .
$$

This is the unique $\mathscr{O}_{\mathrm{X}}$-linear map whose restriction to $\Gamma_{k}(\mathrm{~T})$ sends each $\ell_{k}(t)$ to $\sum_{i+j=k} \ell_{i}(t) \otimes \ell_{j}(t)$. On the other hand, the comultiplication $\mu^{*}$ of $\Gamma$. (T) is a divided power homomorphism sending $t$ to $1 \otimes t+t \otimes 1$, so

$$
\mu^{*}\left(\ell_{k}(t)\right)=\mu^{*}\left(t^{[k]}\right)=(t \otimes 1+1 \otimes t)^{[k]}=\sum_{i+j=k} t^{[i]} \otimes t^{[j]} .
$$

Thus, $\mu^{\prime}=\mu^{*}$. Now recall that $\rho_{1}$ is just the standard duality map and that one deduces from $\rho_{1}$ a unique morphism of algebras

$$
\rho: \mathrm{S}^{\cdot}(\Omega) \rightarrow \mathrm{P}\left(\mathrm{T}, \mathscr{O}_{\mathrm{X}}\right) \cong \operatorname{Hom}\left(\Gamma \cdot(\mathrm{T}), \mathscr{O}_{\mathrm{X}}\right),
$$

where $\mathrm{P}\left(\mathrm{T}, \mathscr{O}_{\mathrm{X}}\right)$ means the ring of polynomial laws $\mathrm{T} \rightarrow \mathscr{O}_{\mathrm{X}}$. Thus if $x \in \mathrm{S}^{i}(\Omega)$ and $y \in \mathrm{S}^{j}(\Omega), \rho_{k}(x y)$ is $\rho_{i}(x) \rho_{j}(y)$, where this product is taken in the algebra $\mathrm{P}\left(\mathrm{T}, \mathscr{O}_{\mathrm{X}}\right)$. In other words, the following diagram commutes:

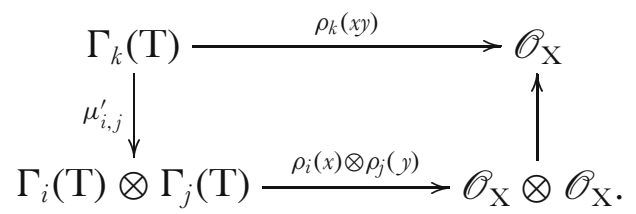

Since $\mu^{\prime}=\mu^{*}$ this proves (1). 
Reversing the roles of $\Omega$ and $\mathrm{T}$, we conclude from (1) that the multiplication map $m_{\mathrm{T}}$ of the algebra $\mathrm{S}^{*}(\mathrm{~T})$ is the dual of the comultiplication map $\mu_{\Omega}^{*}$ of the group law of $\Gamma .(\Omega)$. Since the latter commutes with algebra structure $m_{\Omega}$ of the algebra $\Gamma .(\Omega)$, it follows that the dual $m^{\prime}$ of $m_{\Omega}^{*}$ also commutes with $m_{\mathrm{T}}$. Thus $m^{\prime}$ is an algebra homomorphism $\mathrm{S}^{*}(\mathrm{~T}) \rightarrow \mathrm{S}^{*}(\mathrm{~T}) \otimes \mathrm{S}^{*}(\mathrm{~T})$. The same is true of the group law $\mu_{\mathrm{T}}^{*}$. We claim that these two homomorphisms are equal, and it suffices to check that this is true for elements of degree one. In other words, we have to check that the map $\mu_{\mathrm{T}}^{*}: \mathrm{T} \rightarrow \mathrm{T} \otimes \mathscr{O}_{\mathrm{X}} \oplus \mathscr{O}_{\mathrm{X}} \otimes \mathrm{T}$ is dual to the map $m_{\Omega}: \mathscr{O}_{\mathrm{X}} \otimes \Omega \oplus \Omega \otimes \mathscr{O}_{\mathrm{X}} \rightarrow \Omega$. But this is trivially true.

It will perhaps be helpful to make everything explicit. We shall do this in the case $\mathscr{B}=\mathscr{O}_{\mathrm{G}}$ and $\mathscr{A}=\iota_{*} \mathscr{B}^{\vee}$. Note that $\xi_{i} \mapsto-\xi_{i}$ induces an isomorphism $\mathscr{A} \cong \iota_{*} \mathscr{A}$.

Corollary 5.20. - Let $\mathrm{T}$ and $\Omega$ be as above. Suppose that also that $\mathrm{T}$ is free, and that $\left(\xi_{1}, \ldots, \xi_{m}\right)$ is a basis for $\mathrm{T}$ and $\left(\omega_{1}, \ldots, \omega_{m}\right)$ the dual basis for $\Omega$. Let $\mathrm{I}$. be the I-adic filtration of $\mathrm{S}^{\bullet} \mathrm{T}$ (resp., the PD-filtration of $\left.\Gamma^{\cdot} \mathrm{T}\right)$.

1. If $\mathrm{G}=\mathbf{V T}$ :

$$
\begin{aligned}
\mathscr{O}_{\mathrm{G}} & =\mathrm{S}^{\bullet} \mathrm{T} ; \quad \xi^{\mathrm{I}} \xi^{\mathrm{J}}=\xi^{\mathrm{I}+\mathrm{J}} \\
\iota_{*} \mathscr{O}_{\mathrm{G}}^{\mathrm{V}} & =\hat{\Gamma} \cdot \Omega=\prod \Gamma_{n} \Omega ; \quad \xi^{\mathrm{I}} \omega^{[\mathrm{J}]}=\omega^{[\mathrm{J}-\mathrm{I}]} \\
\theta_{\xi^{n}}\left(e_{1} \circledast e_{2}\right) & =\sum_{i+j=n}\left(\begin{array}{c}
n \\
i
\end{array}\right) \theta_{\xi}^{i}\left(e_{1}\right) \circledast \theta_{\xi}^{j}\left(e_{2}\right) \quad \text { for } \quad e_{1} \circledast e_{2} \in \mathrm{E}_{1} \circledast \mathrm{E}_{2} \\
\theta_{\xi^{n}}(h) & =\sum_{i+j=n}\left(\begin{array}{c}
n \\
i
\end{array}\right)(-1)^{j} \theta_{\xi}^{i} \circ h \circ \theta_{\xi}^{j} \quad \text { for } \quad h \in \mathscr{H}\left(\mathrm{E}_{1}, \mathrm{E}_{2}\right) .
\end{aligned}
$$

2. If $\mathrm{G}=\hat{\mathbf{V}}_{\gamma} \mathrm{T}$ :

$$
\begin{aligned}
& \mathscr{O}_{\mathrm{G}}=\hat{\Gamma} \cdot \mathrm{T} ; \quad \xi^{[\mathrm{I}]} \xi^{[\mathrm{J}]}=\frac{(\mathrm{I}+\mathrm{J}) !}{\mathrm{I} ! \mathrm{J} !} \xi^{[\mathrm{I}+\mathrm{J}]} \\
& \iota_{*} \mathscr{O}_{\mathrm{G}}^{\vee}=\mathrm{S}^{\cdot} \Omega ; \quad \xi^{[\mathrm{I}]} \omega^{\mathrm{J}}=\frac{\mathrm{J} !}{(\mathrm{J}-\mathrm{I}) !(\mathrm{I} !)} \omega^{\mathrm{J}-\mathrm{I}} \\
& \theta_{\xi^{[n]}}\left(e_{1} \circledast e_{2}\right)=\sum_{i+j=n} \theta_{\xi^{[i]}}\left(e_{1}\right) \circledast \theta_{\xi^{[j]}}\left(e_{2}\right) \quad \text { for } \quad e_{1} \circledast e_{2} \in \mathrm{E}_{1} \circledast \mathrm{E}_{2} \\
& \theta_{\xi^{[n]}}(h)=\sum_{i+j}(-1)^{j} \theta_{\xi^{[i]}} \circ h \circ \theta_{\xi^{[j]}} \quad \text { for } \quad h \in \mathscr{H}\left(\mathrm{E}_{1}, \mathrm{E}_{2}\right) .
\end{aligned}
$$

It is also of some interest to give an explicit formula for the inverse to the map $h$ of Theorems 5.16 and 5.18. Let us do this when $\mathrm{G}=\hat{\mathbf{V}}_{\gamma} \mathrm{T}$. Let $\kappa_{i}$ (the "Casimir 
operator") be the element of $\mathrm{S}^{i} \Omega \otimes \Gamma^{i} \mathrm{~T}$ corresponding to the identity homomorphism. In terms of the bases above,

$$
\kappa_{i}:=\sum_{|\mathrm{I}|=i} \omega^{\mathrm{I}} \otimes \xi^{[\mathrm{I}]}:=\sum_{|\mathrm{I}|=i} \omega_{1}^{\mathrm{I}_{1}} \cdots \omega_{d}^{\mathrm{I}_{d}} \otimes \xi_{1}^{\left[\mathrm{I}_{1}\right]} \cdots \xi_{d}^{\left[\mathrm{I}_{d}\right]} .
$$

If $\theta$ is a G-field on $\mathrm{E}, \kappa_{i}$ defines an endomorphism

$$
\kappa_{i, \mathrm{E}}:=\sum_{|\mathrm{I}|=i} \omega^{\mathrm{I}} \theta_{\xi[\mathrm{II}}^{t o t} \quad: \quad \mathrm{S}^{i} \Omega \otimes \mathrm{E} \rightarrow \mathrm{S}^{i} \otimes \mathrm{E} .
$$

If $\theta$ is locally nilpotent, so is $\theta_{t o t}$, and in this case

$$
\kappa_{\mathrm{E}}:=\sum_{i=0}^{\infty}(-1)^{i} \kappa_{i, \mathrm{E}}
$$

is a well-defined endomorphism of $\mathrm{S} \Omega \otimes \mathrm{E}$. The following result is essentially classical; we shall omit the amusing and elementary proof.

Proposition 5.21. — Let $(\mathrm{E}, \theta)$ be a locally nilpotent $\Gamma$.T-module.

1. The Casimir operator $\kappa_{\mathrm{E}}: \mathrm{S} \Omega \otimes \mathrm{E} \rightarrow \mathrm{S} \cdot \Omega \otimes \mathrm{E}$ defined above is a projection operator with image

$$
\mathscr{T}(\mathrm{E}):=\left(\mathrm{S}^{\cdot} \Omega \otimes \mathrm{E}\right)^{\theta_{t o t}}
$$

and factors through the map $\sigma: \mathrm{S} \Omega \otimes \mathrm{E} \rightarrow \mathrm{E}$ defined by the the augmentation $\mathrm{S} \Omega \rightarrow \mathscr{O}_{\mathrm{X}}$.

2. The map

$$
h^{\prime}:=\sum_{\mathrm{J}} \omega^{\mathrm{J}} \otimes\left(\kappa_{\mathrm{E}} \circ \theta_{t^{\mathrm{J} J}}^{t o t}\right) \quad: \quad \mathrm{S} \Omega \otimes \mathrm{E} \rightarrow \mathrm{S} \Omega \otimes \mathscr{T}(\mathrm{E})
$$

is the inverse of the map $h$ of Theorem 5.18.

3. The map

$$
\bar{\kappa}_{\mathrm{E}}: \mathrm{E} \rightarrow \mathscr{T}(\mathrm{E})
$$

induced by $\kappa$ is an isomorphism, inverse to the map

$$
\eta_{\tilde{\mathrm{F}}}:=\sigma_{\tilde{\mathrm{F}}}^{*} \circ j_{\mathrm{E}}: \mathscr{T}_{\mathscr{X} / \mathscr{S}}(\mathrm{E}) \rightarrow \mathrm{E} .
$$

These isomorphisms take the field $\theta$ on $\mathrm{E}$ to $\iota_{*} \theta_{\mathscr{T}}$. That is,

$$
\theta_{t^{[1]}} \circ \bar{\kappa}_{\mathrm{E}}=(-1)^{\mathrm{I}} \bar{\kappa}_{\mathrm{E}} \circ \theta_{t^{[1]}}
$$

for all $\mathrm{I}$. 


\subsection{Azumaya algebras over group schemes}

Recall that if $\mathrm{M}$ is a locally free sheaf of finite rank on a scheme $\mathrm{Z}$, then the (matrix) algebra $\mathscr{E}:=\mathscr{E}_{n} d_{\mathscr{O}_{\mathrm{Z}}}(\mathrm{M})$ is a quasi-coherent sheaf of $\mathscr{O}_{\mathrm{Z}}$-algebras whose center is $\mathscr{O}_{\mathrm{Z}}$. Furthermore, the functor $\mathrm{E} \rightarrow \mathrm{M} \otimes \mathrm{E}$ from the category of sheaves of $\mathscr{O}_{\mathrm{Z}}$-modules to the category of sheaves of left $\mathscr{E}$-modules is an equivalence, with quasiinverse $\mathscr{H}_{0} m_{\mathscr{E}}(\mathrm{M}, \quad)$. A sheaf of algebras over $\mathscr{O}_{\mathrm{Z}}$ which locally for the fppf topology is isomorphic to $\mathscr{E}^{n} d_{\mathscr{O}_{Z}}(\mathrm{M})$ for some locally free $\mathrm{M}$ is called an Azumaya algebra. Note that an Azumaya algebra, viewed as $\mathscr{O}_{\mathrm{Z}}$-module, is locally free of rank $d^{2}$, where $d$ is a locally constant function on $\mathrm{Z}$. The function $d$ is called the index of the Azumaya algebra. An Azumaya algebra $\mathscr{E}$ is said to be split if it is isomorphic to $\mathscr{E}_{\mathscr{O}_{\mathrm{Z}}}(\mathrm{M})$ for some such $\mathrm{M}$; in this case $\mathrm{M}$ is said to be a splitting module. If $\mathscr{A}$ is an Azumaya algebra of rank $d^{2}$ and $\mathrm{M}$ is a sheaf of left $\mathscr{A}$-modules which is locally free of rank $d$ over $\mathscr{O}_{\mathrm{Z}}$, then the natural map $\mathscr{A} \rightarrow \mathscr{E}_{\mathscr{O}_{\mathrm{Z}}}(\mathrm{M})$ is necessarily an isomorphism, so $\mathscr{A}$ is split and $\mathrm{M}$ is a splitting module [23].

Let us note for future reference that if $\mathrm{M}$ is an $\mathscr{A}$-module which is locally free and of finite rank as an $\mathscr{O}_{\mathrm{Z}}$-module, then $\mathrm{M}^{\vee}:=\mathscr{H}_{0} m_{\mathscr{O}_{\mathrm{Z}}}\left(\mathrm{M}, \mathscr{O}_{\mathrm{Z}}\right)$ is a naturally a right $\mathscr{A}$-module, and for any $\mathscr{A}$ module $\mathrm{N}$ there is a natural isomorphism of $\mathscr{O}_{\mathrm{Z}}$-modules:

$$
\mathscr{H}_{0} m_{\mathscr{A}}(\mathrm{M}, \mathrm{N}) \subseteq \mathscr{H}_{0} m_{\mathscr{O}_{\mathrm{Z}}}(\mathrm{M}, \mathrm{N}) \stackrel{\cong}{\longrightarrow} \mathrm{M}^{\vee} \otimes_{\mathscr{O}_{\mathrm{Z}}} \mathrm{N} \rightarrow \mathrm{M}^{\vee} \otimes_{\mathscr{A}} \mathrm{N}
$$

We have found it convenient to use the language of 2-categories to describe the relations among Azumaya algebras.

Definition 5.22. - Let $\mathscr{A}$ and $\mathscr{B}$ be Azumaya algebras over a scheme Z.

1. A 1-morphism $\mathrm{M}: \mathscr{A} \rightarrow \mathscr{B}$ is a module $\mathrm{M}$ over $\mathscr{A}^{\text {op }} \otimes \mathscr{B}$ which is locally free over $\mathscr{O}_{\mathrm{Z}}$ of rank (ind $\left.\mathscr{A}\right)($ ind $\mathscr{B})$, i.e., a splitting of the Azumaya algebra $\mathscr{A}^{\text {op }} \otimes \mathscr{B}$.

2. If $\mathrm{M}: \mathscr{A} \rightarrow \mathscr{B}$ and $\mathrm{N}: \mathscr{B} \rightarrow \mathscr{C}$ are 1-morphisms, then $\mathrm{N} \circ \mathrm{M}:=\mathrm{N} \otimes_{\mathscr{B}} \mathrm{M}$.

3. If $\mathrm{M}$ and $\mathrm{N}$ are 1-morphisms $\mathscr{A} \rightarrow \mathscr{B}$, a 2-morphism: $\mathrm{M} \rightarrow \mathrm{N}$ is an isomorphism of $\mathscr{A}^{\text {op }} \otimes \mathscr{B}$-modules $\mathrm{M} \rightarrow \mathrm{N}$, with the obvious notion of composition.

For any object $\mathscr{A}, \mathrm{id}_{\mathscr{A}}$ is just the bimodule $\mathscr{A}$.

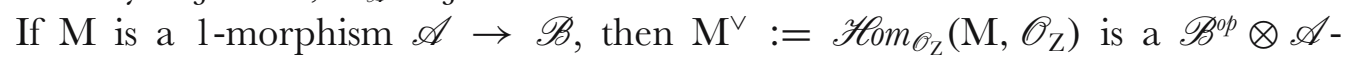

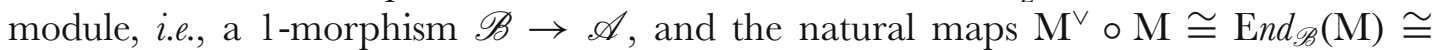
$\mathscr{A}=\mathrm{id}_{\mathscr{A}}$. Thus all 1-morphisms are equivalences (and all 2-morphisms are isomorphisms).

If $\mathscr{A}$ is an Azumaya algebra over Z, let $\operatorname{Mod}(\mathscr{A})$ denote the category of $\mathscr{A}$ modules, which we may view as a stack over Z. A 1 -morphism $\mathrm{M}: \mathscr{A} \rightarrow \mathscr{B}$ gives rise to an equivalence of categories:

$$
\mathrm{C}_{\mathrm{M}}^{-1}: \operatorname{Mod}(\mathscr{A}) \stackrel{\sim}{\longrightarrow} \operatorname{Mod}(\mathscr{B}): \mathrm{E} \mapsto \mathrm{M} \otimes_{\mathscr{A}} \mathrm{E}
$$


together with functorial isomorphisms

$$
\mathrm{C}_{\mathrm{M}}^{-1}\left(\mathrm{E} \otimes_{\mathscr{O}_{\mathrm{Z}}} \mathrm{L}\right) \stackrel{\sim}{\longrightarrow} \mathrm{C}_{\mathrm{M}}^{-1}(\mathrm{E}) \otimes_{\mathscr{O}_{\mathrm{Z}}} \mathrm{L}
$$

for every $\mathscr{O}_{\mathrm{Z}}$-module L. Conversely, every equivalence of stacks, together with such a family of isomorphisms (satisfying suitable compatibilities), comes from a 1-morphism.

Recall that the category of $\mathrm{D}_{\mathrm{X} / \mathrm{S}}$-modules has a tensor structure: the tensor product of $\mathrm{D}_{\mathrm{X} / \mathrm{S}}$-module $\mathrm{M}$ and $\mathrm{N}$ is $\mathrm{M} \otimes_{\mathscr{O}_{\mathrm{X}}} \mathrm{N}$, where the action of the vector fields on $\mathrm{M} \otimes_{\mathscr{O}_{\mathrm{X}}} \mathrm{N}$ is given by the Leibniz rule: $\nabla_{\mathrm{D}}(m \otimes n):=\nabla(m) \otimes n+m \otimes \nabla(n)$. We will see that in characteristic $p$, the tensor structure on $\mathrm{MIC}(\mathrm{X} / \mathrm{S})$ comes from a more rigid structure on the Azumaya algebra $\mathscr{D}_{\mathrm{X} / \mathrm{S}}$ which we will explain below. First we will give an abstract definition on an Azumaya algebra over a group scheme, and then in Example 5.26 we will construct a canonical tensor structure on $\mathscr{D}$ x/s.

Definition 5.23. - Let $\mathrm{G}$ be a flat affine group scheme over a scheme $\mathrm{X}$ and let $\mathscr{A}$ be an Azumaya algebra over $\mathscr{O}_{\mathrm{G}}$. In the notation of (5.2), a tensor structure on $\mathscr{A}$ consists of the following data:

1. A 1-morphism of Azumaya algebras on $\mathrm{G} \times \mathrm{G}$ :

$$
\delta: \mu^{*} \mathscr{A} \rightarrow p_{1}^{*} \mathscr{A} \otimes p_{2}^{*} \mathscr{A} .
$$

2. An associativity 2-morphism $\alpha$ as follows. Note that $\mu \circ\left(\mu, p_{3}\right)=\mu \circ\left(p_{1}, \mu\right)$ is the multiplication morphism $\mu_{3}: \mathrm{G} \times \mathrm{G} \times \mathrm{G} \rightarrow \mathrm{G}$. Then $\alpha$ is a 2-morphism between the 1-morphisms $f$ and $g$ shown below:

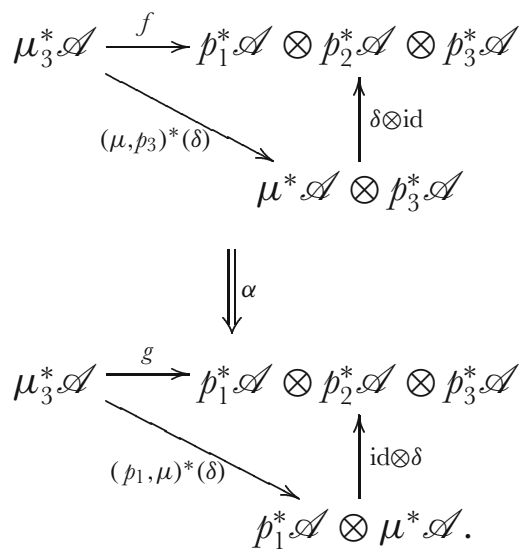

The above data should satisfy the pentagon condition [9, 1.0.1].

If $\mathrm{G}$ is commutative, then a symmetric tensor structure on $\mathscr{A}$ is a tensor structure as above together with an additional datum $\gamma$. Note that $\sigma^{*}\left(p_{1}^{*} \mathscr{A} \otimes p_{2}^{*} \mathscr{A}\right)=$ $\left(p_{2}^{*} \mathscr{A} \otimes p_{1}^{*} \mathscr{A}\right)$, so that the standard commutativity isomorphism for $\otimes$ can be viewed 
as an isomorphism $\gamma_{\otimes}: \sigma^{*}\left(p_{1}^{*} \mathscr{A} \otimes p_{2}^{*} \mathscr{A}\right) \cong p_{1}^{*} \mathscr{A} \otimes p_{2}^{*} \mathscr{A}$. Since $\mathrm{G}$ is commutative, $\mu \circ \sigma=\mu$, and hence the commutative square below exists. Then $\gamma$ is a 2-morphism

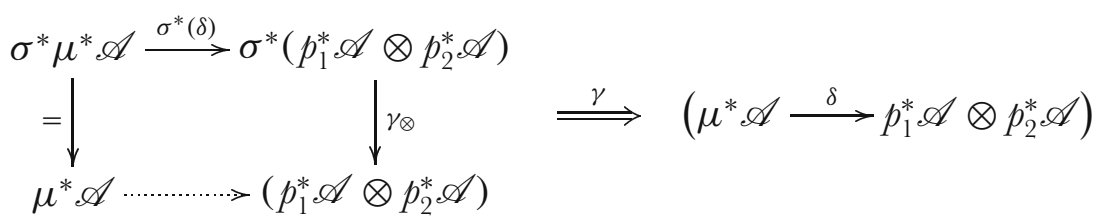

such that $\gamma^{2}=\mathrm{id}$. The associativity morphism $\alpha$ and the commutativity morphism $\gamma$ should also satisfy the hexagon axiom [9, 1.0.2].

Azumaya algebras with (resp. symmetric) tensor structure also form a 2-category: a 1 -morphisms $\mathscr{A} \rightarrow \mathscr{B}$ is by definition a pair $(\mathrm{M}, \lambda)$, where $\mathrm{M}$ is a 1 -morphism of the underlying Azumaya algebras as before and $\lambda$ is a 2-morphism

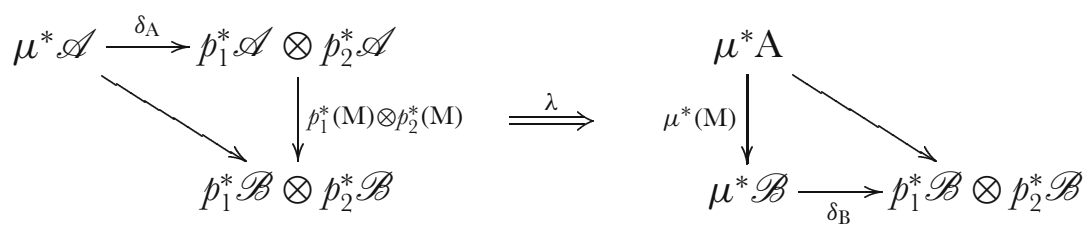

compatible with the associativity (resp. and the commutativity) 2-morphisms.

Let $(\mathscr{A}, \delta, \alpha)$ be an Azumaya algebra with a tensor structure. Then the category $\operatorname{Mod}(\mathscr{A})$ of modules over $\mathscr{A}$ is endowed with tensor structure: given $\mathscr{A}$-modules $\mathrm{M}_{1}$ and $\mathrm{M}_{2}$ we define the tensor product

$$
\mathrm{M}_{1} \circledast \mathrm{M}_{2}=\mu_{*}\left(\delta^{-1}\left(p_{1}^{*} \mathrm{M}_{1} \otimes p_{2}^{*} \mathrm{M}_{2}\right)\right) .
$$

Recall from [9] that a unit object of a category $\mathscr{M}$ with a tensor structure is a pair $(\mathrm{U}, h)$, where $\mathrm{U}$ is an object of $\mathscr{M}$ and $h$ is an isomorphism

$$
h: \mathrm{U} \simeq \mathrm{U} \circledast \mathrm{U},
$$

such that the functor $\circledast \mathrm{U}: \mathscr{M} \rightarrow \mathscr{M}$ sending an object $\mathrm{M}$ to $\mathrm{M} \circledast \mathrm{U}$ is an equivalence of categories. It is shown in (loc. cit.) that the unit object is unique up to a unique isomorphism and that for any object $\mathrm{M}$ there is a functorial isomorphism $\mathrm{M} \circledast \mathrm{U} \simeq \mathrm{M}$.

Lemma 5.24. - Let $\mathscr{A}$ be an Azumaya algebra over $\mathrm{G}$ equipped with a tensor structure $(\delta, \alpha)$ (5.23). Then the restriction $i^{*} \mathscr{A}$ of $\mathscr{A}$ to the zero section has a canonical splitting $\mathrm{N}_{0}$. Moreover, there is an isomorphism $h: i_{*} \mathrm{~N}_{0} \simeq i_{*} \mathrm{~N}_{0} \circledast i_{*} \mathrm{~N}_{0}$, and the pair $\mathrm{U}:=\left(i_{*} \mathrm{~N}_{0}, h\right)$ is the unit object of $\operatorname{Mod}(\mathscr{A})$.

Proof. - Since $\mu \circ i=p_{i} \circ i=i$, the restriction $\delta_{0}: i^{*} \mathscr{A} \rightarrow i^{*} \mathscr{A} \otimes_{\mathscr{O}_{\mathrm{x}}} i^{*} \mathscr{A}$ of $\delta$ to the zero section of $\mathrm{G}$ is an $i^{*}\left(\mathscr{A}^{\text {op }} \otimes \mathscr{A} \otimes \mathscr{A}\right)$-module $\mathrm{P}$. Then the $i^{*} \mathscr{A}$-module $\mathrm{N}_{0}:=\mathscr{H}_{0 m^{*}\left(\mathscr{A}^{\circ)} \otimes \mathscr{A}\right)}\left(i^{*} \mathscr{A}, \mathrm{P}\right)$ gives a splitting of $i^{*} \mathscr{A}$. 
Next we construct the isomorphism $h$. By definition,

$$
\begin{aligned}
\mathrm{U} \circledast \mathrm{U} & =\mu_{*}\left(\delta^{-1}\left(p_{1}^{*} \mathrm{U} \otimes p_{2}^{*} \mathrm{U}\right)\right) \\
& \cong i_{*} \delta_{0}^{-1}\left(\mathrm{~N}_{0} \otimes \mathrm{N}_{0}\right) \cong \mathscr{H} 0 m_{i^{*}(\mathscr{A} \otimes \mathscr{A})}\left(\mathrm{P}, \mathrm{N}_{0} \otimes \mathrm{N}_{0}\right) .
\end{aligned}
$$

Evaluation of homomorphisms defines an isomorphism of $i^{*}\left(\mathrm{~A}^{o p} \otimes \mathrm{A} \otimes \mathrm{A}\right)$-modules $i^{*} \mathscr{A} \otimes \mathrm{N}_{0} \rightarrow$ P. Thus

$$
\begin{aligned}
\mathrm{U} \circledast \mathrm{U} & \cong i_{*} \mathscr{H}_{i^{*}(\mathscr{A} \otimes \mathscr{A})}\left(i^{*} \mathscr{A} \otimes \mathrm{N}_{0}, \mathrm{~N}_{0} \otimes \mathrm{N}_{0}\right) \\
& \cong i_{*} \mathscr{H}_{0 m_{i_{*} \mathscr{A}}}\left(i^{*} \mathscr{A}, \mathrm{N}_{0}\right) \cong \mathrm{U} .
\end{aligned}
$$

Finally, we have to prove that the functor $\circledast \mathrm{U}: \operatorname{Mod}(\mathscr{A}) \rightarrow \operatorname{Mod}(\mathscr{A})$ is an equivalence of categories. Let $\Gamma_{j}: \mathrm{G} \rightarrow \mathrm{G} \times \mathrm{G}$ be the graph of the zero section. Since $\mu \circ \Gamma_{j}=\mathrm{id}, \Gamma_{j}^{*}(\delta)$ is a 1-morphism: $\delta_{1}: \mathscr{A} \rightarrow \mathscr{A} \otimes j^{*} \mathscr{A}$. If $\mathrm{M} \in \operatorname{Mod}(\mathscr{A})$,

$$
\mathrm{M} \circledast \mathrm{U}:=\delta_{1}^{-1}\left(\mathrm{M} \otimes p^{*} \mathrm{~N}_{0}\right) .
$$

Since $p^{*} \mathrm{~N}_{0}$ is a splitting of $j^{*} \mathscr{A}$, the functor $\otimes p^{*} \mathrm{~N}_{0}: \operatorname{Mod}(\mathscr{A}) \rightarrow \operatorname{Mod}\left(\mathscr{A} \otimes j^{*} \mathscr{A}\right)$ is an equivalence. Since $\delta_{1}^{-1}: \operatorname{Mod}\left(\mathscr{A} \otimes j^{*} \mathscr{A}\right) \rightarrow \operatorname{Mod}(\mathscr{A})$ is also an equivalence, the lemma is proved.

Observe that a tensor structure on $\mathscr{A}$ induces a canonical 1 -morphism $\iota^{*} \mathscr{A} \cong$ $\mathscr{A}^{\text {op }}$, obtained by pulling back $\delta$ by the graph of $\iota$, since by Lemma $5.24, i^{*} \mathscr{A}$ is canonically split. It follows that the category $\operatorname{Mod}(\mathscr{A})$ has inner Homs. Let

$$
\delta^{\prime}: \mu^{\prime *} \mathscr{A} \rightarrow p_{1}^{*} \mathscr{A}^{o p} \otimes p_{2}^{*} \mathscr{A}
$$

be the pullback of $\delta$ by $\left(\iota\right.$, id) composed with the 1 -morphism $\iota^{*} \mathscr{A} \otimes \mathscr{A} \rightarrow \mathscr{A}^{o p} \otimes \mathscr{A}$. If $\mathrm{E}_{1}$ and $\mathrm{E}_{2}$ are objects of $\operatorname{Mod}(\mathscr{A}), \mathscr{H} e x\left(\mathrm{E}_{1}, \mathrm{E}_{2}\right)$ is naturally a $p_{1}^{*} \mathscr{A}^{o p} \otimes p_{2}^{*} \mathscr{A}$-module, and

$$
\mathscr{H}\left(\mathrm{E}_{1}, \mathrm{E}_{2}\right)=\mu_{*}^{\prime}\left(\delta^{-1} \mathscr{H e x}\left(\mathrm{E}_{1}, \mathrm{E}_{2}\right)\right) .
$$

If $\mathrm{G}$ is commutative and the tensor structure is endowed with a commutativity morphism, then $\operatorname{Mod}(\mathscr{A})$ becomes a tensor category in the sense of [9]. From now on, we assume this to be the case.

Definition 5.25. - A tensor splitting of an Azumaya algebra $\mathscr{A}$ equipped with a tensor structure over $\mathrm{G}$ is a 1-morphism (in the category of Azumaya algebras with symmetric tensor structure): $\mathscr{O}_{\mathrm{G}} \rightarrow \mathscr{A}$.

Note that, in general, a 1-morphism $\mathrm{M}: \mathscr{A} \rightarrow \mathscr{B}$ gives rise to equivalence of tensor categories:

$$
\mathrm{C}_{\mathrm{M}}^{-1}: \operatorname{Mod}(\mathscr{A}) \sim \operatorname{Mod}(\mathscr{B}), \quad \mathrm{C}_{\mathrm{M}}^{-1}\left(\mathrm{M}_{1} \circledast \mathrm{M}_{2}\right) \cong \mathrm{C}_{\mathrm{M}}^{-1}\left(\mathrm{M}_{1}\right) \circledast \mathrm{C}_{\mathrm{M}}^{-1}\left(\mathrm{M}_{2}\right) .
$$


In particular, a tensor splitting $\mathrm{M}$ gives an equivalence between the tensor categories: $\mathrm{C}_{\mathrm{M}}^{-1}: \operatorname{Mod}\left(\mathscr{O}_{\mathrm{G}}\right) \sim \operatorname{Mod}(\mathscr{A})$. Observe that $\mathscr{O}_{\mathrm{G}}$ is a commutative coalgebra with counit in the category $\operatorname{Mod}\left(\mathscr{O}_{\mathrm{G}}\right)$. Thus, by "transport of structure" the splitting module $\mathrm{M} \cong$ $\mathrm{C}_{\mathrm{M}}^{-1}\left(\mathscr{O}_{\mathrm{G}}\right)$ becomes a commutative coalgebra with counit in $\operatorname{Mod}(\mathscr{A})$. In other words, we have canonical morphisms:

$$
\mathrm{M} \rightarrow \mathrm{M} \circledast \mathrm{M}, e: \mathrm{M} \rightarrow \mathrm{U} .
$$

In the case of the split Azumaya algebra $\mathscr{A}=\mathscr{O}_{\mathrm{G}}$ with the obvious tensor structure, the notion of a tensor splitting boils down to the notion of a character sheaf introduced in Definition 5.11.

Example 5.26. - Let us explain how the above formalism works in the case of the Azumaya algebra of differential operators. First recall the following lemma.

Lemma 5.27. - Let $\mathrm{M}$ and $\mathrm{N}$ be objects of $\mathrm{MIC}(\mathrm{X} / \mathrm{S})$, and let $\psi_{\mathrm{M}}$ and $\psi_{\mathrm{N}}$ be their p-curvatures. Then the p-curvature of the tensor product and internal Hom:

$$
\begin{aligned}
& \psi_{\mathrm{M} \otimes \mathrm{N}}: \mathrm{M} \otimes_{\mathscr{O}_{\mathrm{X}}} \mathrm{N} \rightarrow \mathrm{M} \otimes_{\mathscr{O}_{\mathrm{X}}} \mathrm{N} \otimes \mathrm{F}_{\mathrm{X} / \mathrm{S}}^{*} \Omega_{\mathrm{X}^{\prime} / \mathrm{S}}^{1} \\
& \psi: \mathscr{H}_{0} m_{\mathscr{O}_{\mathrm{X}}}(\mathrm{M}, \mathrm{N}) \rightarrow \mathscr{H}_{0} m_{\mathscr{O}_{\mathrm{X}}}(\mathrm{M}, \mathrm{N}) \otimes \mathrm{F}_{\mathrm{X} / \mathrm{S}}^{*} \Omega_{\mathrm{X}^{\prime} / \mathrm{S}}^{1}
\end{aligned}
$$

are given respectively by the formulas

$$
\begin{aligned}
\psi_{\mathrm{M} \otimes \mathrm{N}}(m \otimes n) & =\psi_{\mathrm{M}}(m) \otimes n+m \otimes \psi_{\mathrm{N}}(n) \\
\psi_{\mathscr{H}}(h) & =\psi_{\mathrm{N}} \circ h-h \circ \psi_{\mathrm{M}} .
\end{aligned}
$$

By definition, giving a tensor structure on $\mathscr{D}_{\mathrm{X} / \mathrm{s}}$ amounts to giving a 1-morphism of the Azumaya algebras on $\mathbf{T}_{\mathrm{X}^{\prime} / \mathrm{S}}^{*} \times_{\mathrm{X}^{\prime}} \mathbf{T}_{\mathrm{X}^{\prime} / \mathrm{S}}^{*}$

$$
\delta: \mu^{*} \mathscr{D} \mathrm{x} / \mathrm{S} \rightarrow p_{1}^{*} \mathscr{D} \mathrm{x} / \mathrm{S} \otimes p_{2}^{*} \mathscr{D} \mathrm{x} / \mathrm{S}
$$

together with the associativity and commutativity 2-morphisms $\alpha$ and $\gamma$. We will construct $\delta$ as follows. View $\mathscr{D}_{\mathrm{X} / \mathrm{s}}$ as a left module over itself, and endow $\Delta:=$ $\mathscr{D}_{\mathrm{x} / \mathrm{s}} \otimes_{\mathscr{O}_{\mathrm{x}}} \mathscr{D} \mathrm{x} / \mathrm{s}$ with the left $\mathscr{D} \mathrm{x} / \mathrm{s}$-module structure defined by the Leibnitz rule above. Note that the right action of $\mathscr{D}_{\mathrm{X} / \mathrm{s}}$ on itself makes $\Delta$ a right module over the alge-

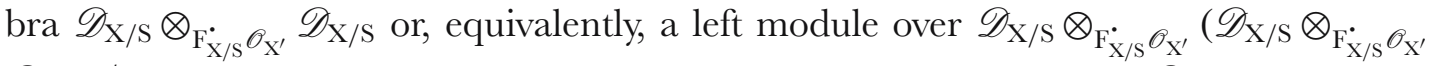

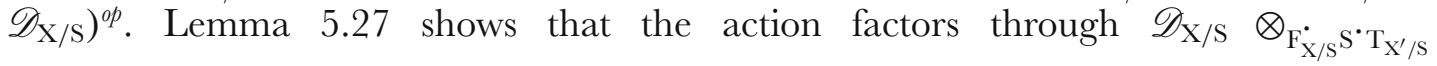
$\left(\mathscr{D}_{\mathrm{X} / \mathrm{S}} \otimes_{\mathrm{F}_{\mathrm{X} / \mathrm{S}} \cdot \mathscr{O}_{\mathrm{X}^{\prime}}} \mathscr{D}_{\mathrm{X} / \mathrm{S}}\right)^{o p}$, where the $\mathrm{F}_{\mathrm{X} / \mathrm{S}}^{\cdot} \mathrm{S}^{\cdot} \mathrm{T}_{\mathrm{X}^{\prime} / \mathrm{S}}$-module structure on $\mathscr{D}_{\mathrm{X} / \mathrm{S}} \otimes_{\mathrm{F}_{\mathrm{X} / \mathrm{s}}} \mathscr{O}_{\mathrm{X}^{\prime}} \mathscr{D}_{\mathrm{X} / \mathrm{S}}$ is given by comultiplication:

$$
\mu^{*}: \mathrm{F}_{\mathrm{X} / \mathrm{S}}^{\cdot} \mathrm{S}^{\cdot} \mathrm{T}_{\mathrm{X}^{\prime} / \mathrm{S}} \rightarrow \mathrm{F}_{\mathrm{X} / \mathrm{S}}^{\cdot}\left(\mathrm{S}^{\cdot} \mathrm{T}_{\mathrm{X}^{\prime} / \mathrm{S}} \otimes_{\mathscr{O}_{\mathrm{X}^{\prime}}} \mathrm{S}^{\cdot} \mathrm{T}_{\mathrm{X}^{\prime} / \mathrm{S}}\right)
$$


Thus, $\Delta$ gives rise to a module over the Azumaya algebra $\mu^{*} \mathscr{D} \mathrm{x} / \mathrm{s} \otimes\left(p_{1}^{*} \mathscr{D} \mathrm{x} / \mathrm{s} \otimes\right.$ $\left.p_{2}^{*} \mathscr{D} \mathrm{x} / \mathrm{S}\right)^{o p}$. It is easy to see using local coordinates that as a module over the center $\mathscr{O}_{\mathbf{T}_{\mathrm{X}^{\prime} / \mathrm{S}}^{*} \times{ }_{\mathrm{X}^{\prime}} \mathbf{T}_{\mathrm{X}^{\prime} / \mathrm{S}}^{*}}$ it is locally free of rank $p^{3 d i m \mathrm{~S}} \mathrm{X}$ which is equal to the index of the Azumaya algebra. Therefore we get a 1-morphism $\delta$.

Next, let us construct the commutativity 2-morphism $\gamma$. Consider the auto-

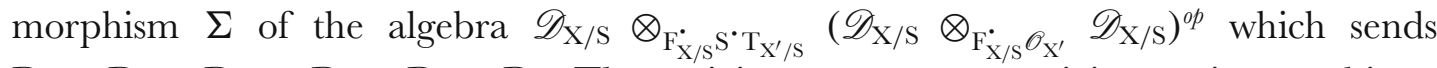
$\mathrm{D}_{1} \otimes \mathrm{D}_{2} \otimes \mathrm{D}_{3}$ to $\mathrm{D}_{1} \otimes \mathrm{D}_{3} \otimes \mathrm{D}_{2}$. Then, giving $\gamma$ amounts to giving an isomorphism

$$
\mathscr{D}_{\mathrm{x} / \mathrm{s}} \otimes_{\mathscr{O}_{\mathrm{x}}} \mathscr{D}_{\mathrm{x} / \mathrm{S}} \rightarrow\left(\mathscr{D}_{\mathrm{x} / \mathrm{s}} \otimes_{\mathscr{O}_{\mathrm{x}}} \mathscr{D}_{\mathrm{x} / \mathrm{S}}\right)^{\Sigma}
$$

of modules over $\mathscr{D}_{\mathrm{X} / \mathrm{S}} \otimes_{\mathrm{F}_{\mathrm{X} / \mathrm{S}}} \mathrm{s}^{\cdot} \mathrm{T}_{\mathrm{X}^{\prime} / \mathrm{S}}\left(\mathscr{D}_{\mathrm{X} / \mathrm{S}} \otimes_{\mathrm{F}_{\mathrm{X} / \mathrm{S}}} \mathscr{O}_{\mathrm{X}^{\prime}} \mathscr{D}_{\mathrm{X} / \mathrm{S}}\right)^{o p}$. The obvious permutation does the trick.

To construct the associativity morphism $\alpha$, we note that the two 1-morphisms:

$$
\mu_{3}^{*} \mathscr{D}_{\mathrm{x} / \mathrm{S}} \rightarrow p_{1}^{*} \mathscr{D}_{\mathrm{x} / \mathrm{s}} \otimes p_{2}^{*} \mathscr{D}_{\mathrm{x} / \mathrm{s}} \otimes p_{3}^{*} \mathscr{D} \mathrm{x} / \mathrm{S}
$$

in Definition (5.23) are given by the same module $\mathscr{D}_{\mathrm{x} / \mathrm{S}} \otimes_{\mathscr{O}_{\mathrm{x}}} \mathscr{D}_{\mathrm{X} / \mathrm{S}} \otimes_{\mathscr{O}_{\mathrm{x}}} \mathscr{D}_{\mathrm{x} / \mathrm{s}}$. With this identification, we let $\alpha$ be the identity morphism.

It remains to show that $\alpha$ and $\gamma$ satisfy the pentagon and hexagon axioms. To save space, we may use the following trick. First, one can easily check the axioms for the restriction of $\mathscr{D} \mathrm{X} / \mathrm{S}$ to the zero section $\mathrm{X}^{\prime} \rightarrow \mathbf{T}_{\mathrm{X}^{\prime} / \mathrm{S}}^{*}$. Furthermore, since the statement is local on $\mathrm{X}$ and stable under a base change $\mathrm{T} \rightarrow \mathrm{S}$, we may assume that $\mathrm{S}$ is reduced. Then any 2-morphism over $\mathbf{T}_{\mathrm{X}^{\prime} / \mathrm{S}}^{*} \times_{\mathrm{X}^{\prime}} \mathbf{T}_{\mathrm{X}^{\prime} / \mathrm{S}}^{*} \times \cdots \times_{\mathrm{X}^{\prime}} \mathbf{T}_{\mathrm{X}^{\prime} / \mathrm{S}}^{*}$ is uniquely determined by its restriction to zero section. This completes the proof.

Remark 5.28. - If the base $\mathrm{S}$ is normal and reduced, one can prove that the tensor structure on $\mathscr{D}_{\mathrm{X} / \mathrm{S}}$ equipped with an isomorphism $\phi: \mathrm{N}_{0} \simeq \mathrm{F}_{\mathrm{X} / \mathrm{S} *} \mathscr{O}_{\mathrm{X}}$ between the canonical splitting module over the zero section $\mathrm{X}^{\prime} \rightarrow \mathbf{T}_{\mathrm{X}^{\prime} / \mathrm{S}}^{*}$ described in Lemma 5.24 and the splitting $\mathrm{F}_{\mathrm{X} / \mathrm{S} *} \mathscr{O}_{\mathrm{X}}$ of Remark 2.2 is unique (up to a unique isomorphism).

Next we shall discuss tensor Azumaya algebras over the formal and PD completion of a group scheme $\mathrm{G}$ along its zero section.

Let $\hat{\mathrm{G}}$ (resp. $\mathscr{G}$ ) denote the formal (resp. PD) completion of $\mathrm{G}$ along its zero section, viewed as a locally ringed space. Let $\hat{\mathrm{G}}^{n}\left(\right.$ resp. $\left.\mathscr{G}^{n}\right)$ be the formal (resp. PD) completion of $\mathrm{G}^{n}$ along its zero section. Then the definition a tensor structure for Azumaya algebras over $\hat{\mathrm{G}}$ (resp. $\mathscr{G}$ ) is the same as before, with these completed fiber products in place of $\mathrm{G}^{n}$.

Denote by $i_{k}: \mathrm{G}_{k} \hookrightarrow \hat{\mathrm{G}}$ (resp. $i_{k}: \mathscr{G}_{k} \hookrightarrow \mathscr{G}$ ) the subscheme defined by the $(k+1)$ st power (resp. divided power) of the ideal of the zero section; if $\mathrm{M}$ is an $\mathscr{O}_{\hat{\mathrm{G}}}$-module (resp. $\mathscr{O}_{\mathscr{G}}-$ module), $\mathrm{M}_{k}:=i_{k *} i_{k}^{*} \mathrm{M}$. The multiplication map $\mu$ gives rise to morphisms 
of schemes: $\mu: \mathrm{G}_{k} \times \mathrm{G}_{l} \rightarrow \mathrm{G}_{k+l}$ (resp. $\left.\mu: \mathscr{G}_{k} \times \mathscr{G}_{l} \rightarrow \mathscr{G}_{k+l}\right)$. The category of $\mathscr{A}$ modules with the convolution product defined in Formula (5.3) is not a tensor category, since the associativity constraint fails in general. However, the subcategory $\operatorname{Mod}(\mathscr{A})$ of I.-continuous modules is stable under the convolution product and is a tensor category. (Recall that a module is said to be I.-continuous if each local section is locally supported on $\mathrm{G}_{k}$ (resp. $\mathscr{G}_{k}$ ), for some $k$.)

Let $\mathrm{M}: \mathscr{O}_{\mathscr{G}} \rightarrow \mathscr{A}$ be a tensor splitting. The splitting module $\mathrm{M}$ inherits a structure of a topological commutative coalgebra with counit, that is, a family of maps $\mathrm{M}_{n} \rightarrow \mathrm{M}_{k} \circledast \mathrm{M}_{l}$, for any $n \geq l+k$, and $e: \mathrm{M} \rightarrow \mathrm{U}$ satisfying the obvious compatibilities.

Proposition 5.29. - Let $\mathscr{A}$ be a tensor Azumaya algebra over $\hat{\mathrm{G}}$ (resp. $\mathscr{G}$ ). Then the following data are equivalent.

\section{A tensor splitting of $\mathscr{A}$.}

2. A splitting module $\mathrm{M}$ for $\mathscr{A}$ with the structure of a topological coalgebra with counit.

3. A splitting module $\mathrm{M}$ for $\mathscr{A}$ whose topological dual $\lim _{\longrightarrow} \mathscr{H}\left(\mathrm{M}_{n}, \mathrm{U}\right)$ is endowed with the structure of an algebra with unit in the tensor category $\operatorname{Mod}^{\cdot}(\mathscr{A})$.

Proof. - We will prove the result for $\mathscr{G}$; the formal case is similar. The equivalence of (2) and (3) is clear, and we have already shown how the data of (1) give the data of (2). It remains to explain how the data of (2) give (1). Let $\mathrm{M}$ be a splitting with a coalgebra structure: $\mathrm{M}_{k+l} \rightarrow \mathrm{M}_{k} \circledast \mathrm{M}_{l}$. By definition, $\mathrm{M}_{k} \circledast \mathrm{M}_{l}=\mu_{*} \delta^{-1}\left(p_{1}^{*} \mathrm{M}_{k} \otimes p_{2}^{*} \mathrm{M}_{l}\right)$. Thus, using the adjointness property of $\mu_{*}$ and $\mu^{*}$, we get a morphism: $\mu^{*} \mathrm{M}_{k+l} \rightarrow$ $\delta^{-1}\left(p_{1}^{*} \mathrm{M}_{k} \otimes p_{2}^{*} \mathrm{M}_{l}\right)$. Let us consider the restriction of the above morphism to $\mathscr{G}_{k} \times \mathscr{G}_{l}$ :

$$
\begin{aligned}
\left(i_{k}, i_{l}\right)^{*} \mu^{*} \mathrm{M}=\left(i_{k}, i_{l}\right)^{*} \mu^{*} \mathrm{M}_{k+l} \rightarrow \delta^{-1} & \left(p_{1}^{*} \mathrm{M}_{k} \otimes p_{2}^{*} \mathrm{M}_{l}\right) \\
& =\left(i_{k}, i_{l}\right)^{*} \delta^{-1}\left(p_{1}^{*} \mathrm{M} \otimes p_{2}^{*} \mathrm{M}\right) .
\end{aligned}
$$

These morphisms are compatible with change of $k$ and $l$, and we claim that they are all isomorphisms. Indeed, both $\left(i_{k}, i_{l}\right)^{*} \mu^{*} \mathrm{M}$ and $\left(i_{k}, i_{l}\right)^{*} \delta^{-1}\left(p_{1}^{*} \mathbf{M} \otimes p_{2}^{*} \mathbf{M}\right)$ are splittings of the Azumaya algebra $\mu^{*} \mathscr{A}$ over $\mathscr{G}_{k} \times \mathscr{G}_{l}$, and, in particular, they are locally free over $\mathscr{G}_{k} \times \mathscr{G}_{l}$. Also, the existence of counit: $\mathrm{M} \rightarrow \mathrm{M}_{0} \simeq \mathrm{U}$ implies that, for $k=l=0$, the morphism (5.29.1) is an isomorphism. Hence (5.29.1) is an isomorphism, for any $k$ and $l$. Thus, we get an isomorphism: $\mu^{*} \mathrm{M} \simeq \delta^{-1}\left(p_{1}^{*} \mathrm{M} \otimes p_{2}^{*} \mathrm{M}\right)$.

Let $\mathscr{A}$ be an Azumaya algebra on $\mathscr{G}$ with a symmetric tensor structure. Let $\mathscr{T} \mathscr{S} \mathscr{P}$ be the stack on $\mathrm{X}$ assigning to a scheme $\mathrm{U}$ étale over $\mathrm{X}$ the groupoid of tensor splittings of the Azumaya algebra $\mathscr{A}_{\mathrm{U}}$ over $\mathscr{G} \times \mathrm{U}$ (i.e. 1-morphisms from $\mathscr{A}_{\mathrm{U}}$ to the trivial Azumaya algebra $\left.\mathscr{O}_{\mathscr{G} \times \mathrm{U}}\right)$. Let $\mathscr{S}_{\mathcal{P}}$ be be the stack sending $\mathrm{U}$ to the groupoid whose objects are pairs $(\mathrm{N}, e)$, where $\mathrm{N}$ is a splitting of the restriction $i_{1}^{*} \mathscr{A}_{\mathrm{U}}$ and $e$ is an isomorphism $e: i^{*} \mathrm{~N} \simeq \mathrm{N}_{0}$. 
Proposition 5.30. - Let $\mathrm{G}$ be a smooth commutative group scheme and $\mathscr{A}$ be an Azumaya algebra on $\mathscr{G}$ with a symmetric tensor structure, which admits a tensor splitting étale locally on $\mathrm{X}$. Then the obvious restriction functor:

$$
i_{1}^{*}: \mathscr{T} \mathscr{S} \mathscr{P} \rightarrow \mathscr{S}_{1}
$$

is an equivalence of stacks.

Remark 5.31. - The stack $\mathscr{S} \mathscr{P}_{1}$ is, in fact, a gerbe. That is, there exists an étale covering of $\mathrm{U}_{i}$ of $\mathrm{X}$ such that, for each $i, \mathscr{S P P}_{1 \mathrm{U}_{i}}$ is non-empty, and, for any étale morphism $\mathrm{U} \rightarrow \mathrm{X}$, any two objects of $\mathscr{S} \mathscr{P}_{1 \mathrm{U}}$ are locally isomorphic. Thus, the proposition implies that the stack $\mathscr{T} \mathscr{S} \mathscr{P}$ is also a gerbe.

Proof. - Recall that, for a smooth commutative group scheme G, the exponential map induces an isomorphism:

$$
\exp : \mathscr{T} \simeq \mathscr{G}
$$

where $\mathscr{T}$ is the completed PD envelope of the zero section $\mathrm{X} \hookrightarrow \mathrm{T}$ of the Lie algebra T. Thus, without loss of generality we can replace $\mathrm{G}$ by the vector group $\mathrm{T}$.

To prove that $i_{1}^{*}$, is an equivalence we will construct the inverse functor exp : $\mathscr{S} \mathscr{P}_{1} \rightarrow \mathscr{T} \mathscr{S} \mathscr{P}$ explicitly. The reason we call it the exponential will be clear in a moment. Given a $\mathscr{A}$-module $\mathrm{M}$ we denote by $\Gamma^{k} \mathrm{M}$ the $\mathscr{A}$-submodule of the tensor power $\mathrm{M}^{\circledast^{k}}$ which consists of $\mathrm{S}_{k}$-invariant sections.

Let $(\mathrm{N}, e)$ be an object of $\mathscr{S} \mathscr{P}_{1}$ over U. Define

$$
\mathrm{M}_{1}=i_{1 *} \mathrm{~N}, \mathrm{M}_{k}=\Gamma^{k} \mathrm{M}_{1} .
$$

We claim that $\mathrm{M}_{k}$ is a splitting of $\mathscr{A}_{\mathrm{U}}$ over $\mathscr{G}_{k}$. Indeed, since the statement is local on $\mathrm{U}$ we may assume that $\mathscr{A}$ has a tensor splitting. A tensor splitting, in turn, gives an equivalence between the tensor category of I-continuous $\mathscr{A}_{\mathrm{U}}$-modules and I.-continuous $\mathscr{O}_{\mathscr{G} \times \mathrm{U}}$-modules. This equivalence takes $\mathrm{M}_{1}$ to a line bundle over $\mathscr{G}_{1} \times \mathrm{X}$ U. Shrinking the base, if necessary, we may assume that the line bundle is trivial. Then the claim follows from the fact that, for smooth $\mathrm{G}$, the $\mathrm{S}_{k}$-invariant multiplication morphism

$$
\mathscr{G}_{1}^{k}=\mathscr{G}_{1} \times \mathscr{G}_{1} \times \cdots \times \mathscr{G}_{1} \rightarrow \mathscr{G}_{k}
$$

induces an isomorphism $\Gamma^{k} \mathscr{O}_{\mathscr{G}_{1}} \simeq \mathscr{O}_{\mathscr{G}_{k}}$.

The morphism $e: \mathrm{M}_{1} \rightarrow \mathrm{U}$ gives the map:

$$
\mathrm{M}_{k} \rightarrow \mathrm{M}_{k-1},
$$

and, moreover, $i_{k-1}^{*} \mathrm{M}_{k} \simeq \mathrm{M}_{k-1}$. 
We define $\exp (\mathrm{N})$ to be

$$
\exp (\mathrm{N})=\lim _{\leftarrow} \mathrm{M}_{k} .
$$

First of all, it is clear that $\mathrm{M}:=\exp (\mathrm{N})$ is a splitting of $\mathscr{A}_{\mathrm{U}}$. Furthermore, it is a coalgebra with counit. The coalgebra structure comes from the canonical morphism:

$$
\mathrm{M}_{k+l}=\Gamma^{k+l} \mathrm{M}_{1} \hookrightarrow \Gamma^{k} \mathrm{M}_{1} \circledast \Gamma^{l} \mathrm{M}_{1}=\mathrm{M}_{k} \circledast \mathrm{M}_{l} .
$$

By Proposition 5.29 it gives a tensor splitting.

It is clear that $i_{1}^{*}(\exp (\mathrm{N}))=\mathrm{N}$, so it remains to construct a canonical isomorphism

$$
\mathrm{M} \simeq \exp \left(i_{1}^{*}(\mathrm{M})\right)
$$

for any tensor splitting $\mathrm{M}$ over $\mathrm{U}$. But this is immediate: the splitting $\mathrm{M}$ gives an equivalence of tensor categories: $\mathrm{C}_{\mathrm{M}}: \operatorname{Mod}\left(\mathscr{A}_{\mathrm{U}}\right) \simeq \operatorname{Mod}\left(\mathscr{O}_{\mathscr{G} \times \mathrm{xU}}\right)$, which takes $\mathrm{M}$ to $\mathscr{O}_{\mathscr{G} \times \mathrm{xU}}$. We define (5.31.1) to be the morphism corresponding under the above equivalence to the canonical isomorphism

$$
\mathscr{O}_{\mathscr{G}} \simeq \lim _{\leftarrow} \Gamma^{k} \mathscr{O}_{\mathscr{G}_{1}}
$$

Denote by $\mathscr{G}^{*}$ the sheaf on $\mathrm{X}$ whose section over a scheme $\mathrm{U}$ étale over $\mathrm{X}$ is the group of homomorphisms: $\operatorname{Hom}_{\mathrm{U}}\left(\mathscr{G} \times \mathrm{U} ; \mathbf{G}_{m}\right)$.

Let $\mathscr{A}$ be an Azumaya algebra on $\mathscr{G}$ with a symmetric tensor structure, which

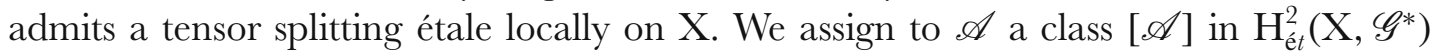
as follows. Choose an étale covering of $\mathrm{U}_{i}$ of $\mathrm{X}$ together with tensor splittings $\mathrm{N}_{i}$ on each $\mathrm{U}_{i}$ and tensor isomorphisms $\phi_{i j}: \mathrm{N}_{i} \simeq \mathrm{N}_{j}$. Then $\phi_{k i} \phi_{j k} \phi_{i j}$ is a Cech cocycle with coefficients in $\mathscr{G}^{*}$. Similarly, given an Azumaya algebra over $\mathscr{G}_{1}$ together with a splitting over the zero section we can construct an element of $\mathrm{H}_{\dot{e} t}^{2}\left(\mathrm{X}, \mathscr{O}_{1, \mathscr{G}_{1}}^{*}\right)=$ $\mathrm{H}_{\mathrm{e} t}^{2}\left(\mathrm{X}, \mathrm{T}_{\mathrm{G} / \mathrm{X}}^{*}\right)$, where $\mathscr{O}_{1, \mathscr{G}_{1}}^{*}$ denotes the group of invertible functions on $\mathscr{G}_{1}$ equal to 1 on $\mathrm{X} \hookrightarrow \mathscr{G}_{1}$.

Recall that for any smooth commutative group scheme $\mathrm{G}$ over $\mathrm{X}$ we have the isomorphism:

$$
\exp : \mathscr{O}_{1, \mathscr{G}_{1}}^{*}=\mathrm{T}_{\mathrm{G} / \mathrm{X}}^{*} \rightarrow \mathscr{G}^{*} .
$$

The inverse map is the restriction $i_{1}^{*}$. Passing to cohomology we get a morphism:

$$
\exp : \mathrm{H}_{\mathrm{e} t}^{2}\left(\mathrm{X}, \mathrm{T}_{\mathrm{G} / \mathrm{X}}^{*}\right) \simeq \mathrm{H}_{\mathrm{e} t}^{2}\left(\mathrm{X}, \mathscr{G}^{*}\right) \rightarrow \mathrm{H}_{\mathrm{e} t}^{2}\left(\mathrm{X}, \mathscr{O}_{\mathscr{G}}^{*}\right) .
$$

Proposition 5.32. - Let $\mathscr{A}$ be an Azumaya algebra on $\mathscr{G}$ with a symmetric tensor structure, which admits a tensor splitting étale locally on $\mathrm{X}$. Then

$$
\exp \left[i_{1}^{*} \mathscr{A}\right]=[\mathscr{A}] .
$$

Since the restriction $i_{1}^{*}: \mathrm{H}_{\mathrm{e} t}^{2}\left(\mathrm{X}, \mathscr{G}^{*}\right) \rightarrow \mathrm{H}_{\mathrm{e} t}^{2}\left(\mathrm{X}, \mathrm{T}_{\mathrm{G} / \mathrm{X}}^{*}\right)$ is an isomorphism, the proposition follows from the obvious fact: $\left[i_{1}^{*} \mathscr{A}\right]=i_{1}^{*}[\mathscr{A}]$. 


\section{REFERENCES}

1. A. Beilinson, On the derived category of perverse sheaves, in K-Theory, Arithmetic and Geometry (Moscow, 1984-1986), Lect. Notes Math., vol. 1289, Springer, Berlin Heidelberg New York, 1987.

2. A. Beilinson, J. Bernstein, and P. Deligne, Faisceaux pervers, Astérisque, 100 (1982), 5-171.

3. P. Berthelot and A. Ogus, Notes on Crystalline Cohomology, Annals of Mathematics Studies, vol. 21, Princeton University Press, Princeton, N.J., 1978.

4. R. Bezrukavnikov, I. Mirković, and D. Rumynin, Localization of modules for a semisimple lie algebra in prime characteristic, Ann. Math., to appear, arXiv:math RT/0205144v5.

5. A. Braverman and R. Bezrukavnikov, Geometric Langlands correspondence for $\mathscr{D}$-modules in prime characteristic: the Gl(n) case, Pure Appl. Math. Q., 3 (2007), 153-179.

6. P. Deligne, Equations Différentielles à Points Singuliers Réguliers, Lect. Notes Math., vol. 163, Springer, Berlin Heidelberg New York, 1970.

7. P. Deligne, Théorie de Hodge II, Publ. Math., Inst. Hautes Étud. Sci., 40 (1972), 5-57.

8. P. Deligne and L. Illusie, Relèvements modulo $p^{2}$ et décomposition du complexe de de Rham, Invent. Math., 89 (1987), 247-270.

9. P. Deligne and J. Milne, Tannakian categories, in Hodge Cycles, Motives, and Shimura Varieties, Lect. Notes Math., vol. 900, Springer, Berlin Heidelberg New York, 1982.

10. D. Eisenbud, Commutative Algebra with a View Toward Algebraic Geometry, Graduate Texts in Mathematics, vol. 150, Springer, New York, 1999.

11. G. Faltings, Crystalline cohomology and p-adic Galois representations, in J.-I. Igusa, ed., Algebraic Analysis, Geometry, and Number Theory, pp. 25-80, The Johns Hopkins University Press, Baltimore London, 1989.

12. G. Faltings, Crystalline cohomology of semistable curve - the $\mathcal{Q}_{p}$-theory, f. Algebr. Geom., 6 (1997), 1-18.

13. A. Grothendieck and J. Dieudonné, Elements de géométrie algébrique: étude locale des schémas et des morphismes des schémas, Publ. Math., Inst. Hautes Étud. Sci., 24 (1964), 5-231.

14. A. Grothendieck and J. Dieudonné, Eléments de Géométrie Algébrique, Grundlehren der mathematischen Wissenschaften, vol. 166, Springer, 1971.

15. L. Illusie, Complexe Cotangent et Déformations I, Lect. Notes Math., vol. 239, Springer, Berlin Heidelberg New York, 1971.

16. K. Joshi and C. S. Rajan, Frobenius splitting and ordinarity, Int. Math. Res. Not., 2 (2003), 109-121.

17. K. Kato, Logarithmic structures of Fontaine-Illusie, in J.-I. Igusa, ed., Algebraic Analysis, Geometry, and Number Theory, Johns Hopkins University Press, Baltimore London, 1989.

18. N. Katz, Nilpotent connections and the monodromy theorem: Applications of a result of Turrittin, Publ. Math., Inst. Hautes Étud. Sci., 39 (1970), 175-232.

19. N. Katz, Algebraic solutions of differential equations ( $p$-curvature and the Hodge filtration), Invent. Math., 18 (1972), 1-118.

20. G. Laumon, Sur la catégorie dérivée des D-modules filtrées, in Algebraic Geometry (Tokyo-Kyoto), pp. 151-237, Springer, Berlin Heidelberg New York, 1983.

21. B. Mazur, Frobenius and the Hodge filtration, Bull. Amer. Math. Soc., 78 (1972), 653-667.

22. B. Mazur and W. Messing, Universal Extensions and One Dimensional Crystalline Cohomology, Lect. Notes Math., vol. 370, Springer, Berlin Heidelberg New York, 1974.

23. J. Milne, Étale Cohomology, Princeton University Press, Princeton, N.J., 1980.

24. A. Neeman, The Grothendieck duality theorem via Bousfield's techniques and Brown representability, f. Amer. Math. Soc., 9 (1996), 205-236.

25. A. Neeman, Triangulated Categories, Annals of Mathematics Studies, vol. 148, Princeton University Press, Princeton, N.J., 2001.

26. A. Ogus, F-crystals and Griffiths transversality. in Proceedings of the International Symposium on Algebraic Geometry, Kyoto 1977, pp. 15-44, Kinokuniya Book-Store, Co., Tokyo, 1977.

27. A. Ogus, Griffiths transversality in crystalline cohomology, Ann. Math., 108 (1978), 395-419.

28. A. Ogus, F-Crystals, Griffiths Transversality, and the Hodge Decomposition, Astérisque, vol. 221, Soc. Math. France, 1994.

29. A. Ogus, Higgs cohomology, p-curvature, and the Cartier isomorphism, Compos. Math., 140 (2004), $145-164$. 
30. B. Osserman, Mochizuki's crys-stable bundles: a lexicon and applications, RIMS Kokyuroku, 43 (2007), 95-119

31. M. Raynaud, "p-torsion” du schéma de Picard, Astérisque, 64 (1978), 87-149.

32. N. S. Rivano, Catégories Tannakiennes, Lect. Notes Math., vol. 265, Springer, 1972.

33. N. Roby, Lois polynômes et lois formelles en théorie des modules, Ann. Éc. Norm. Super., III. Sér., 80 (1963), 213-348.

34. G. SABbah, On a twisted de Rham complex, Tohoku Math. J., 51 (1999), 125-140.

35. M. Saito, Hodge structure via filtered D-modules, Astérisque, 130 (1985), 342-351.

36. C. Simpson, Higgs bundles and local systems, Publ. Math., Inst. Hautes Étud. Sci., 75 (1992), 5-95.

37. V. Srinivas, Decomposition of the de Rham complex, Proc. Indian Acad. Sci., Math. Sci., 100 (1990), 103-106.

38. V. Voevodsky, Homotopy theory of simplicial sheaves in completely decomposable topologies, http://www.math.uiuc.edu/K-theory/443, 2000.

\section{A. O.}

Department of Mathematics, University of California at Berkeley, Berkeley, CA 94720-3840, USA

ogus@math.berkeley.edu

\section{V.}

Department of Mathematics, University of Chicago,

Chicago, IL 60637, USA
Article soumis le 5 juillet 2005

Version révisée le 27 juillet 2007

publié en ligne le 19 janvier 2008. 